\begin{tabular}{|c|l|}
\hline Title & Binary Lennard-Jones atomic clusters: Structural features induced by large sized atoms \\
\hline Author(s) & Takeuchi, Hiroshi \\
\hline Citation & $\begin{array}{l}\text { Computational and Theoretical Chemistry, 1050, 68.73 } \\
\text { https://doi.org/10.1016/.Comptc.2014.10.017 }\end{array}$ \\
\hline Issue Date & 201412-15 \\
\hline Doc URL & http://hdl.handle.net/2115/57327 \\
\hline Type & article(author version) \\
\hline File Information & CTC_1050_68.pdf \\
\hline
\end{tabular}

Instructions for use 


\title{
Binary Lennard-Jones Atomic Clusters: Structural Features Induced by Large-Sized Atoms
}

Hiroshi Takeuchi*

Division of Chemistry, Graduate School of Science, Hokkaido University, Sapporo 060-0810, Japan

Corresponding author phone: +81-11-706-3533; Fax: +81-11-706-3501; e-mail: takehi@sci.hokudai.ac.jp

\begin{abstract}
Global-minimum geometries of binary Lennard-Jones clusters $\left(\mathrm{BLJ}_{N}\right.$ where $N$ is the number of atoms) are previously elucidated when the size ratio of the large B atom to the small A atom is 1.05 to 1.3. In the present study, BLJ clusters for larger size ratios (1.4 to 2.0) are investigated to clarify the structural features. The heuristic method combined with geometrical perturbations and atom-type conversion is developed to search for the global minima of the BLJ clusters. In the lowest-energy geometries of the BLJ clusters $(N \leq 50)$, A and B atoms form cores and outer shells, respectively. The existence of fairly large sized atoms induces complicated structural growth sequence patterns (including icosahedron, triangular orthobicupola, cuboctahedron, etc.). New global minima of $\mathrm{BLJ}_{59}$ for $s=1.15, \mathrm{BLJ}_{68}$ for $s$ $=1.15$ and $\mathrm{BLJ}_{70}$ for $s=1.2$ are also reported.
\end{abstract}

Keyword: Binary clusters; Lennard-Jones potential; Global optimization; Structural growth sequence 


\section{Introduction}

Global optimization plays an important role in a field of computational chemistry including prediction of the lowest-energy structures of biomolecules, clusters, and crystals. However, it is a difficult problem since the optimal geometry of a system must be searched from enormous number of stable geometries. Hence a strategy to efficiently move from a local minimum to the global minimum on the potential energy surface of the system is indispensable.

For atomic and molecular clusters, many investigations on the structures and properties have been performed due to their importance. However, global optimization of the clusters is still challenging. In the field of atomic clusters, Lennard-Jones (LJ) clusters are well elucidated and putative global minima of the LJ clusters up to 1610 atoms are tabulated [1 - 9]. Accordingly the LJ clusters are considered as a test problem for investigating performance of global optimization algorithms. Previously the present author developed an efficient method for geometry optimization of Lennard-Jones clusters [10]. The method optimizes cluster geometries with two types of geometrical perturbations and yielded the global minima of LJ clusters with 10 to 561 atoms reported previously and the new minima for $6 \mathrm{LJ}$ clusters. Then the method is improved to apply it to complicated clusters, molecular homoclusters where molecular orientations are further required as optimized parameters [11, 12]. The first purpose of the present study is to improve the above optimization method for application to another type of complicated clusters, atomic heteroclusters.

For the heteroclusters, the composition of different atoms makes global optimization more difficult [13 - 23]. Since one of the simplest heteroclusters is the binary Lennard-Jones (BLJ) cluster, the putative global minima of the BLJ clusters have been examined [13 - 19]. For the $N$-atom BLJ clusters $\left(\mathrm{BLJ}_{N}\right)$, the potential energy is calculated using the atom-atom interaction potential $V(i, j)$ :

$$
E_{N}=\sum_{i<j}^{N} V(i, j)=4 \sum_{i<j}^{N} \varepsilon_{\alpha \beta}\left[\left(\frac{\sigma_{\alpha \beta}}{r_{i j}}\right)^{12}-\left(\frac{\sigma_{\alpha \beta}}{r_{i j}}\right)^{6}\right]
$$

Here $r_{i j}$ represents the distance between atoms $i$ and $j$, and $\alpha$ and $\beta$ mean the atom types of atoms $i$ and $j$ (represented by A and B). The relation of $\varepsilon_{\mathrm{AA}}=\varepsilon_{\mathrm{BB}}=\varepsilon_{\mathrm{AB}}=\varepsilon$ is used throughout the present 
study as adopted in the previous studies [13 - 19]. The values of $\sigma_{\mathrm{AA}}$ and $\sigma_{\mathrm{BB}}$ are set to be $\sigma$ and $s \sigma$, respectively, and the $\sigma_{\mathrm{AB}}$ value is equal to $\left(\sigma_{\mathrm{AA}}+\sigma_{\mathrm{BB}}\right) / 2$ where the parameter $s$ is a predefined constant of the system representing the size ratio of the B atom to the A atom. The BLJ clusters have been considered as an important model to study performance of optimization methods and structural properties of binary clusters.

The original investigation on the BLJ clusters was performed by Doye and Meyer [13] with basin-hopping $(\mathrm{BH})$ algorithm. The study was aimed at finding the effect of the size of the $\mathrm{B}$ atom $(s=$ 1.05, 1.1, 1.15, 1.2, 1.25, 1.3) on the global minima of the BLJ clusters with 5 to 100 atoms. The lowest energies and the corresponding geometries are tabulated in the CCD (Cambridge Cluster Database $^{7}$ ). Cassioli et al. [14] confirmed a lot of the global minima with the population-based BH method and found 95 new minima. Marques and Pereira [15] proposed an evolutionary algorithm to search for global minima of the BLJ clusters and applied it to the BLJ clusters with 10 to 50 atoms. A new minimum was located for $\mathrm{BLJ}_{38}$ for $s=1.05\left(E_{38} / \varepsilon=-177.260697\right)$. Kolossváry and Bowers [16] developed the hidden-force Monte Carlo (HFMC) algorithm. The results obtained for the geometries of $\mathrm{BLJ}_{90}$ to $\mathrm{BLJ}_{100}$ show that the HFMC method improved 17 global minima. In the study of Sicher et al., [17] the minima hopping method was applied to the clusters with $N=5$ to 100 and yielded 17 new global minima. Most of the new minima were independently obtained with the HFMC algorithm [16]. Tao et al. [18] optimizes the geometries of the BLJ clusters with $N=5$ to 100 . Although 12 new global minima of the BLJ clusters were located, 15 global minima reported previously were missing. After the publication, Wenqi and Tao deposited following new minima in the CCD [7]; BLJ 66 for $s=$ $1.15\left(E_{66} / \varepsilon=-359.49937\right), \mathrm{BLJ}_{89}$ for $s=1.15\left(E_{89} / \varepsilon=-510.052009\right), \mathrm{BLJ}_{72}$ for $s=1.2\left(E_{72} / \varepsilon=\right.$ -404.850951), BLJ 65 for $s=1.25\left(E_{65} / \varepsilon=-363.528091\right)$ and $\mathrm{BLJ}_{99}$ for $s=1.3\left(E_{99} / \varepsilon=-597.679071\right)$. Recently Rondina and Da Silva [19] developed a global optimization method based on the BH algorithm and applied it to several cases including the BLJ clusters with $N=5-100$. Comparison of the results with the data taken from the above-mentioned studies shows that 2 new global minima are 
located with the method: $\mathrm{BLJ}_{38}$ for $s=1.05\left(E_{38} / \varepsilon=-177.260679\right)$ and $\mathrm{BLJ}_{66}$ for $s=1.15\left(E_{66} / \varepsilon=\right.$ -359.749940). However, the global minima of $\mathrm{BLJ}_{99}$ and $\mathrm{BLJ} / 00$ for $s=1.3$ were not found with the method. To enhance the reliability of the global minima, it would be necessary to search for the global minima of the BLJ clusters with different methods.

In the previous study on the LJ clusters by the present author [10], two geometrical perturbations followed by local optimizations efficiently explore geometrical space. For the BLJ clusters, the atom types must be taken into account in the optimization algorithm. A simple way to optimize the type of the atom is to convert $\mathrm{A}$ into $\mathrm{B}$ or vice versa in the algorithm as performed in the literature [18]. In the present study, an optimization method was developed by combination of the two perturbations [10] with the atom-type conversion [18] algorithm. To evaluate the combined method, geometry optimization was performed for the $\mathrm{BLJ}_{5}$ to $\mathrm{BLJ}_{100}$ for $s=1.05,1.1,1.15,1.2,1.25,1.3$. Since these $s$-values were rather limited, the global-minima of $\mathrm{BLJ}_{5}$ to $\mathrm{BLJ}_{50}$ for $s=1.4,1.5,1.6,1.7,1.8,1.9,2.0$ were also calculated. The second purpose of the present study is to examine the structures, relative stability and growth sequence patterns of the BLJ clusters. The results of the present study complement the previous data of the BLJ clusters.

\section{Calculation}

The optimization procedure proposed in the present study starts with a cluster configuration randomly generated. The number of the A atoms $N_{\mathrm{A}}$ is randomly selected from zero to $N$. The atoms are placed within a sphere with the volume of $N_{\mathrm{A}}\left(\sqrt[6]{2} \sigma_{\mathrm{AA}}\right)^{3}+\left(N-N_{\mathrm{A}}\right)\left(\sqrt[6]{2} \sigma_{\mathrm{BB}}\right)^{3}$. The geometry of the cluster is locally optimized with a limited memory quasi-Newton method (L-BFGS [24]). Then the type of an atom is converted from A to B or from B to A. After the conversion, the geometry of the cluster is optimized by the L-BFGS [24] method. If the potential energy of the cluster is improved, the cluster geometry and the type of the atom are updated. This procedure is repeated for every atom and thus the atom-type conversion is $N$ times carried out. 
After performing the above conversion operator, an atom or some atoms with the highest potential energy are moved to positions which are expected to decrease the potential energy of the cluster. The positions are selected from the surface of the cluster or neighborhood of the center of mass of the cluster. The above geometrical perturbations are called the surface and interior operations. By applying local optimization (the L-BFGS [24] method) to geometries created with these operators, new minima are repeatedly searched. The details of the operators are described below.

An atom or $M$ atoms with the highest potential energy are selected as follows: (i) create a list of atoms on the outer shell of the cluster. (ii) the potential energy of an atom $i$ in the list, $E(i)$, is calculated by using the following equation:

$$
E_{\text {select }}(i)=\sum_{j \neq i}^{N} V(i, j)
$$

For all combinations of $M$ atoms (numbering of $M$ atoms is represented by $k_{1}, k_{2}, \ldots, k_{M}$ ), the contribution of $M$ atoms to the potential energy of the cluster $E_{\text {select }}\left(k_{1}, k_{2}, \ldots, k_{M}\right)$ is calculated by the formula:

$$
E_{\text {select }}\left(k_{1}, k_{2}, \cdots, k_{M}\right)=\sum_{i=1}^{M} E_{\text {select }}\left(k_{i}\right)-\sum_{i=1}^{M-1} \sum_{j=i+1}^{M} V\left(k_{i}, k_{j}\right)
$$

(iii) select the atoms with the highest energy contribution from all the combinations.

Many methods ignore the second term in the right-side of eq 3. This is mainly because the computation of the second term is time-consuming for large $M$ values. The neglect of the second terms is a good approximation if the atoms are far from each other. According to our experience on the LJ clusters [10], the second term is often crucial to efficiently search for new minima. The following conditions are used for the BLJ clusters [10]: $M \leq 4$ for the surface operator and $M \leq 5$ for the interior operator, respectively.

The interior operator moves the selected atoms to the surface of the sphere whose center is coincident with the center of mass of the cluster. It takes the radius of $r_{\mathrm{e}} / 2$ where $r_{\mathrm{e}}=\sqrt[6]{2} \sigma_{\mathrm{AA}}$ for the A atoms and $r_{\mathrm{e}}=\sqrt[6]{2} \sigma_{\mathrm{BB}}$ for the $\mathrm{B}$ atoms. The number of atoms surrounding the atoms moved by using the interior operator usually increases compared with that surrounding the atoms at the original surface 
positions. Consequently the potential energy of the atoms obtained after local optimization is expected to be lower than that at the original positions. This leads to a probability that the potential energy of the cluster is improved with the interior operator. This is theoretical background on the development of the operator.

In the geometries created with the interior operator, some atoms are very close to each other. Hence displacement of them caused by local optimization $(\Delta x, \Delta y$, and $\Delta z)$ is large, leading to the possibility of the evaporation. To avoid it, if the absolute values of $\Delta x, \Delta y$, and $\Delta z$ are larger than $\sigma$, these are reduced to $0.1 \sigma$.

If the potential energy of the cluster is not improved during the last ten interior operations followed by local optimizations, the surface operator is carried out. In the operator, stable positions on the surface of the cluster are first examined and the best positions are chosen from them as the positions of the moved atoms as follows: (i) remove the moved atoms from the cluster and prepare the template cluster composed of the $(N-M)$ atoms; (ii) add an atom on the surface of the template at random and optimize the position of the added atom. The surface is constructed by the spheres which have radii of $r_{\mathrm{e}} / 2$ and centers coincident with the positions of the template atoms. A vector generated randomly is used to show the direction from the center of mass of the cluster to the added atom; (iii) the obtained position $P$ of the atom and the potential energy between the atom and the template $E_{\text {template }}(P)$ are stored; (iv) This is repeated $2 N$ times to create a set of stable positions on the surface. The set of stable positions is separately created for the A and B atoms; (v) calculate the potential energy $E_{\text {surface }}$ for all combinations of $M$ independent positions by

$$
E_{\text {surface }}\left(P_{1}, P_{2}, \cdots, P_{M}\right)=\sum_{i=1}^{M} E_{\text {template }}\left(P_{i}\right)+\sum_{i=1}^{M-1} \sum_{j=i+1}^{M} V\left(P_{i}, P_{j}\right)
$$

where numbering of $M$ positions is represented by $P_{1}, P_{2}, \ldots, P_{M}$ and $E_{\text {template }}\left(P_{i}\right)$ is obtained in (iii). If the positions $P_{1}, P_{2}, \ldots, P_{M}$ are similar to the coordinates of the atoms removed in the step (i), the positions are excluded in the calculation of $E_{\text {surface; }}$ and (vi) select the positions with the lowest potential 
energy $E_{\text {surface }}^{\min }$ from all the combinations. This operator does not change the number of the A and B atoms in the cluster.

The cases with $M \geq 2$ must be carefully treated by the following reason. If the distances between the selected positions are close to the equilibrium distance, slight changes of the positions introduce a large energy difference because of the term $V\left(P_{i}, P_{j}\right)$. Therefore, when $M \geq 2$, the following step is carried out instead of (vi): for all the configurations with the potential energy less than $E_{\text {surface }}^{\min }+0.2 \varepsilon$, positions of $M$ atoms are simultaneously optimized and the positions giving the lowest energy are selected.

Stable positions on the surface of the cluster are also used in the previous optimization methods [18, 25]. However, the interactions between $P_{1}, P_{2}, \ldots, P_{M}$ are ignored in the methods and thus the number of the moved atoms ( $N_{\text {move }}=10$ to 40 ) is larger than that in the surface operator. However, the experience on the LJ clusters [10] suggests that the interactions should be taken into account since the efficiency of the optimization method is often enhanced.

The value of $M$ is initially 1 and increases up to 4 at an interval of 1 if the energy of the cluster is not improved. When energy-lowering is observed, the $M$-value is initialized and the cluster geometry is updated. It was found that moving the second or third highest-energy atom by the surface operator improves performance of the method [10]. Hence, if the energy of the cluster is not improved by moving the highest-energy atom, the second highest-energy atom and the third highest-energy atom are separately moved by the surface operator. If the surface operator with 4 moved atoms does not improve the energy of the cluster, optimization algorithm returns to the atom-type conversion operator. In the present algorithm, a series of the atom-type conversion, the interior operator, and the surface operator is repeated. The termination condition of the algorithm is that the lowest energy obtained before the atom-type conversion operator is invariant compared with that obtained after the surface operator. 
A lot of initial geometries were prepared randomly and were optimized with the above algorithm. The computations were performed on dual core $3 \mathrm{GHz}$ Intel Xeon 5160 processors. No parallel computation was carried out. A geometry of the 50-atom BLJ cluster was optimized in $3 \mathrm{~s}$ and the corresponding time needed for the 100-atom BLJ cluster was $40 \mathrm{~s}$.

The global minima of the BLJ clusters for $s=1.0$ to $1.3(N \leq 100)$ and those for $s=1.4$ to $2.0(N \leq 50)$ were searched with the present method (the heuristic method combined with geometrical perturbations and atom-type conversion, HMGPAC). The potential energies of the putative global minima of these clusters are tabulated in supplementary data.

\section{Discussion}

\subsection{Efficiency of the optimization method}

The global minima of the BLJ clusters ( $s=1.05$ to 1.3 and $N \leq 100$ ) reported in the previous studies [7, 13 - 19] were confirmed by HMGPAC except for 7 clusters (3 new minima and 4 missing minima). Three potential energies obtained in the present study $\left(E_{59} / \varepsilon=-315.027359\right.$ for $s=1.15, E_{68} / \varepsilon=$ -372.247969 for $s=1.15$ and $E_{70} / \varepsilon=-391.735161$ for $s=1.2$ ) are lower than the corresponding energies given previously, $-315.023963 \varepsilon$ [19], $-371.831801 \varepsilon$ [7], and $-391.581513 \varepsilon$ [7]. The significant energy differences between the present and previous potential energies indicates that the present method locate new global minima; the geometrical data are deposited in supplementary data. The potential energy of the 72-atom BLJ cluster for $s=1.2,-404.850956 \varepsilon$, was slightly lower than the value of Tao et al. [18], $-404.850951 \varepsilon$. Since the geometries obtained in the present and previous studies are the same, the present potential energy would be obtained with the tighter convergence criterion in the local optimization compared with the criterion in [18].

The global minima of $\mathrm{BLJ}_{93}$ and $\mathrm{BLJ}_{94}$ for $s=1.2$, and $\mathrm{BLJ}_{99}$ and $\mathrm{BLJ}_{100}$ for $s=1.25$ could not be found under the condition that the number of initial geometries was $1 \times 10^{5}$ for each of the clusters. Although HMGPAC is efficient for searching for global minima as shown in the above results of the 
clusters with $N=59,68,70$, any improvement on the optimization algorithm might be necessary to locate the missing global minima.

The number of local optimizations required for searching for the global minimum $N_{\text {opt }}$ is a useful parameter to evaluate the efficiency of the present algorithm. It can be derived from the total number of local optimizations divided by the number of times that the global minimum is located. The results obtained for $s=1.1,1.2,1.3$ are shown in Figure 1 except for the unsuccessful clusters. For the clusters with $N \geq 90$, the $N_{\text {opt }}$ values of the clusters for $s=1.1$ are smaller than the corresponding values of the clusters for $s=1.2$ and 1.3. This trend is observed in the study of Tao et al. [18], suggesting that the difficulty for searching for the global minima for $N \geq 90$ and $s=1.2,1.3$ is due to the complicated potential energy surfaces. For $s=1.1$, the global-minimum geometries with $N \geq 90$ include a lot of Ino decahedra (the notation (10/5) is used for it as described later) whereas the other geometries are based on icosahedra. For $s=1.2,1.3$, the global-minimum geometries take icosahedra as building units. The geometries based on Ino decahedra must reduce the ruggedness of the potential energy surfaces compared with the icosahedron-based geometries.

Figure 2 shows the performance of HMGPAC for the BLJ clusters for $s=1.4,1.6,1.8,2.0$. Compared with the results for the BLJ clusters for $s \leq 1.3$ (Figure 1), computational costs increase for $s$ 21.4. Hence the efficiency of HMGPAC is affected by the difference between the sizes of the A and B atoms.

According to the study of Tao et al. [18], the shapes of the global-minimum geometries affect the efficiency of their optimization method. Among the minima missed by them, the global minima of $\mathrm{BLJ}_{88}$ and $\mathrm{BLJ}_{89}$ for $s=1.3$ take oblate structures whereas those of $\mathrm{BLJ}_{99}$ and $\mathrm{BLJ} \mathrm{J}_{100}$ for $s=1.3$ are prolate. They mention that the method disfavors oblate or prolate structures because a variant of the interior operator used in the study favors spherical shapes. Rondina and Da Silva [19] could not find the minima of $\mathrm{BLJ}_{99}$ and $\mathrm{BLJ}_{100}$ for $s=1.3$ because their geometrical operators did not favor very pronounced prolate structures. In the present study, however, these minima were located by 
HMGPAC. The shapes of the global-minimum structures would not influence the efficiency of HMGPAC.

Most of the clusters show core-shell structures where the core is constructed by small sized A atoms and the shell consists of the B atoms [13]. In the present algorithm, the atom-type conversion operator is first carried out. Consequently core-shell structures would be generated after the conversion operator. Hence the interior operator may move the B atom present in the outer shell to the core region formed by A atoms. This movement cannot lower the potential energy of the cluster since it destroys the A-atom core. The following modification on the interior operator must improve the optimization algorithm: the highest-energy atom in the core is moved to the vicinity of the center of the mass of the cluster.

\subsection{Global minima of $B L J_{5}$ to $B L J_{50}$ for $s=1.4$ to 2.0 .}

The global optimization of the BLJ clusters with $s=1.4$ to 2.0 has been never performed. The optimized geometries are deposited in supplementary data. To clarify relative positions of the A and B atoms, distances between the atoms and the center of mass of the cluster are plotted in Figure $3 . \quad$ The distances for the A atoms are usually shorter than those for the B atoms. Hence the A atoms construct cores of the clusters whereas the B atoms form shells of them. The overlaps of the distributions of the A and B atoms are found for a few clusters. In this case, A atoms may occupy positions in the outer shell. The distribution patterns of the A atoms in the $s=2.0$ clusters are considerably different from the corresponding ones in the $s=1.4$ clusters and the distribution patterns gradually change depending on the parameter s. This is also the case for the distribution patterns of the B atoms. This suggests that the structures of the BLJ clusters are sensitive to the $s$ parameter.

The geometries of the BLJ clusters with $N \leq 12$ are shown in supplementary data. The configurations of the $N=5$, 6 clusters are independent of the $s$ value. However the structures of clusters with $N \geq 7$ are sensitive to the size ratio and thus no common growth sequence is observed for the BLJ clusters. 
For the BLJ clusters with $N \geq 13$, the number of the A atoms in $\operatorname{BLJ}_{N}, N_{\mathrm{A}}(N)$, is shown in Figure 4. In this figure, structural properties of the clusters are also shown by symbols. The number $N_{\mathrm{A}}(N)$ tends to increase with increasing cluster size. The curves for $s=1.4,1.5$ are more rugged than those for larger $s$ values. This indicates that the growth sequence patterns of the clusters for $s \leq 1.5$ are considerably different from those for $s \geq 1.6$.

The number of atoms surrounding a core atom (coordination number for an A atom) was analyzed for the global-minimum structures. In the analysis, the surrounding atom must satisfy the following condition: the distance between it and a core atom is shorter than a tentative cutoff distance which is 1.2 times as long as the equilibrium interatomic distance. The value of $N_{\max }$ is 12 for a lot of the BLJ clusters (closed symbols in Figure 4).

Figure 5 shows the geometries of the coordination atoms and the core atom in the BLJ clusters with $N_{\max }=12$. The polyhedron constructed by the coordination atoms is characterized with the numbers of triangular and square faces ( $r$ and $q$ ) in the surface and the notation $(r / q)$ is used to represent it. The icosahedra are denoted by (20/0). The BLJ clusters for $s=1.4,1.5$ shows both the icosahedral and (10/5) structures. The (8/6) structures are not found for the BLJ clusters with $s \leq 1.5$. These structures are divided into two types I and II, triangular orthobicupola and cuboctahedron, which are included in the hexagonal close-packed and cubic close-packed structures, respectively. The triangular orthobicupolas appear in the clusters for $s \geq 1.6$ whereas the cuboctahedra are found for $s \geq 1.7$. The large sized atoms of $s \geq 1.7$ induce complicated structural transitions in the growth sequence patterns of the BLJ clusters. Hence it is interesting to determine structures of clusters such as a mixture of $\mathrm{CH}_{4}$ and $\mathrm{SiF}_{4}$ molecules $\left(\varepsilon / k=140.42 \mathrm{~K}\right.$ and $\sigma=4.015 \AA$ for $\mathrm{CH}_{4}[26]$ and $\varepsilon / k=140.14 \mathrm{~K}$ and $\sigma=6.692 \AA$ for $\mathrm{SiF}_{4}$ [26] where $k$ is Boltzmann constant). Bimetallic clusters with a significant size difference between constituent metal atoms might display novel structures and properties.

The relative stability of the BLJ clusters is calculated using

$$
S_{N}=E_{N+1}+E_{N-1}-2 E_{N}
$$


The results are shown in Figure 6 where a positive value of $S_{N}$ means that $\mathrm{BLJ}_{N}$ is relatively stable compared with $\mathrm{BLJ}_{N \pm 1}$. The magic numbers of 13, 19, 23, 26, and 29 are observed for the BLJ clusters for $s=1.4$. The BLJ clusters with the magic numbers are stabilized because $1,2,3,4$, and 5 icosahedra are constructed in them. A few BLJ clusters for $s=1.5,1.6$ are relatively stable because of the formation of the icosahedral structures. For $s \geq 1.6$, the magic numbers considerably depend on the size ratio, $s$.

The structural analysis based on the coordination atoms is not useful for understanding stability of non-icosahedral clusters. An example is drawn by using the $s=1.4$ cluster with $N=40$. Each of the $s=1.4$ clusters with $N=36-40$ includes one (20/0) structure and two (10/5) structures. Since the number of these structures is constant, these structures are not responsible for the relative stability of the cluster with $N=40$. We can see another example relating to (8/6) structures, the $s=1.7,1.8$ clusters with $N=44-50$. The clusters with 44 to 50 atoms include 2, 3, 3, 3, 4, 4, and 4 (8/6) structures, respectively. However, the cluster sizes at which the number of the (8/6) structures increases $(N=45$, 48) are different from the magic number of 47.

Stability of the BLJ cluster is related to the structure of the outer shell. In the 40-atom cluster for $s=$ 1.4, 13 A atoms form the (10/5) core structure. It is covered with many triangles formed by $27 \mathrm{~B}$ atoms as shown in Figure 7. The lengths of the sides of them range from $1.54 \sigma$ to $1.65 \sigma$. This indicates that no more atoms can be embedded in the shell. Hence the core is fully surrounded by the $\mathrm{B}$ atoms. This covering due to the $\mathrm{B}$ atoms results in the relative stability of the cluster.

In the 47-atom clusters for $s=1.7,1.8$, the outer shells formed by the B atoms fully cover the cores of the $25 \mathrm{~A}$ atoms. The outer shell consists of triangles and 4-membered rings. This is usually found for many clusters corresponding to the magic numbers. The stability of the BLJ clusters can be explained by the existence of the icosahedra and the stable outer shells formed by the 3-membered and 4-membered rings. 


\section{Conclusions}

The heuristic method combined with the atom-type conversion, interior, and surface operators was applied to the BLJ clusters for $s=1.05$ to 1.3. It reproduced most of the global minima reported previously and located three new global minima. Moreover the method was used to examine the BLJ clusters for $s=1.4$ to 2.0. Hence the structural data of the BLJ clusters with $N \leq 50$ are extended. For $s=1.4,1.5$, icosahedral structures are often observed whereas two close-packed structures appear for $s \geq 1.6$. The clusters for $s \geq 1.6$ show complicated growth sequence patterns including two close-packed structures. The complicated patterns have never been obtained experimentally. It would be interesting to determine structures of clusters with significant differences between atomic sizes.

\section{Appendix A. Supplementary data}

Supplementary data associated with this article can be found, in the online version, at http://dx.doi.org/10.1016/j.comptc.2014.xx.xxx.

\section{References}

[1] D. J. Wales, J.P.K. Doye, Global Optimization by Basin-Hopping and the Lowest Energy Structures of Lennard-Jones Clusters Containing up to 110 Atoms. J. Phys. Chem. A 101 (1997) 5111-5116.

[2] X. Shao, H. Jiang, W. Cai, Parallel Random Tunneling Algorithm for Structural Optimization of Lennard-Jones Clusters up to $N=$ 330. J. Chem. Inf. Comput. Sci. 44 (2004) 193-199.

[3] Y. Xiang, H. Jiang, W. Cai, X. Shao, An Efficient Method Based on Lattice Construction and the Genetic Algorithm for Optimization of Large Lennard-Jones Clusters. J. Phys. Chem. A 108 (2004) 3586-3592. 
[4] Y. Xiang, L. Cheng, W. Cai, X. Shao, Structural Distribution of Lennard-Jones Clusters Containing 562 to 1000 Atoms. J. Phys. Chem. A 108 (2004) 9516-9520.

[5] C. Barrón, S. Gómez, D. Romero, A. Saavedra, A Genetic Algorithm for Lennard-Jones Atomic Clusters. Appl. Math. Lett. 12 (1999) 85-90.

[6] X. Shao, Y. Xiang, W. Cai, Structural Transition from Icosahedra to Decahedra of Large Lennard-Jones Clusters. J. Phys. Chem. A 109 (2005) 5193-5197.

[7] D. J. Wales, J. P. K. Doye, A. Dullweber, M. P. Hodges, F. Y. Naumkin, F. Calvo, J. Hernández-Rojas, T. F. Middleton, The Cambridge Cluster Database, http://www-wales.ch.cam.ac.uk/CCD.html.

[8] D. M. Deaven, N. Tit, J. R. Morris, K. M. Ho, Structural Optimization of Lennard-Jones Clusters by a Genetic Algorithm. Chem. Phys. Lett. 256 (1996) 195-200.

[9] B. Hartke, Global Cluster Geometry Optimization by a Phenotype Algorithm with Niches: Location of Elusive Minima, and Low-Order Scaling with Cluster Size. J. Comput. Chem. 20 (1999) 1752-1759.

[10] H. Takeuchi, Clever and Efficient Method for Searching Optimal Geometries of Lennard-Jones Clusters. J. Chem. Inf. Model. 46 (2006) 2066-2070.

[11] H. Takeuchi, Novel Method for Geometry Optimization of Molecular Clusters: Application to Benzene Clusters. J. Chem. Inf. Model. 47 (2007) 104-109.

[12] H. Takeuchi, Development of an Efficient Geometry Optimization Method for Water Clusters. J. Chem. Inf. Model. 48 (2008) 2226-2233.

[13] J.P.K. Doye, L. Meyer, Mapping the Magic Numbers in Binary Lennard-Jones Clusters. Phys. Rev. Lett. 95 (2005) 063401. 
[14] A. Cassioli, M. Locatelli, F. Schoen, Global Optimization of Binary Lennard-Jones Clusters. Optim.Methods Software 24 (2009) 819-835.

[15] J.M.C. Marques, F.B. Pereira, An Evolutionary Algorithm for Global Minimum Search of Binary Atomic Clusters. Chem. Phys. Lett. 485 (2010) 211-216.

[16] I. Kolossváry, K.J. Bowers, Global Optimization of Additive Potential Energy Functions: Predicting Binary Lennard-Jones Clusters. Phy. Rew. E 82 (2010) 056711.

[17] M. Sicher, S. Mohr, S. Goedecker, Efficient Moves for Global Geometry Optimization Methods and Their Application to Binary Systems. J. Chem. Phys. 134 (2011) 044106.

[18] Y. Tao, X. Ruchu, H. Wenqi, Global Optimization of Binary Lennard-Jones Clusters Using Three Perturbation Operators. J. Chem. Inf. Model. 51 (2011) 572-577.

[19] G.G. Rondina, J.L.F. Da Silva, Revised Basin-Hopping Monte Carlo Algorithm for Structure Optimization of Clusters and Nanoparticles. J. Chem. Inf. Model. 53 (2013) 2282-2298.

[20] X. Wu, Y. Sun, C. Li, W. Yang, Parametric Effects of the Potential Energy Function on the Geometrical Features of Ternary Lennard-Jones Clusters. J. Phys. Chem. A 116 (2012) 8218-8225.

[21] X. Wu, C. Huang, Y. Sun, G. Wu, Structural Distribution in Mixed Ternary Noble Gas and Lennard-Jones Clusters, Chem. Phys. Lett. 415 (2013) 69-75.

[22] X. Wu, Y. Sun, Y. Gao, G. Wu, Structural Transitions in Mixed Ternary Noble Gas Clusters. J. Mol. Model. 19 (2013) 3119-3125.

[23] J.M. Dieterich, B. Hartke, Composition-Induced Structural Transitions in Mixed Lennard-Jones Clusters: Global Reparametrization and Optimization. J. Comput. Chem. 32 (2011) 1377-1385.

[24] D. C. Liu, J. Nocedal, On the Limited Memory BFGS Method for Large Scale Optimization. Math. Prog. 45 (1989) 503-528. 
[25] X. Shao, L. Cheng, W. Cai, A Dynamic Lattice Searching Method for Fast Optimization of Lennard-Jones Clusters. J. Comput. Chem. 25 (2004) 1693-1698.

[26] F. Cuadros, I. Cachadiña, W. Ahumada, Determination of Lennard-Jones Interaction Parameters Using a New Procedure. Mol. Engineering 1996, 6, 319 - 325. 
Figure 1. The number of local optimizations $N_{\text {opt }}$ required for searching for the global minimum of the binary Lennard-Jones cluster: circles, $s=1.1$; squares, $s=1.2$; triangles, $s=1.3$. For clarity the data for $s=1.2,1.3$ are offset vertically by $10^{2}$ and $10^{4}$, respectively.

Figure 2. The number of local optimizations $N_{\text {opt }}$ required for searching for the global minimum of the binary Lennard-Jones cluster: circles, $s=1.4$; squares, $s=1.6$; triangles, $s=1.8$; diamonds, $s=2.0$.

For clarity the data for $s=1.6,1.8,2.0$ are offset vertically by $10^{2}, 10^{4}$ and $10^{6}$, respectively.

Figure 3. Distribution patterns of distances $r(\mathrm{X}-\mathrm{COM})(\mathrm{X}=\mathrm{A}$ or $\mathrm{B})$ between the center of mass (COM) of the binary Lennard-Jones cluster and the A (or B) atoms: (a), $s=1.4$; (b), $s=1.6$; (c), $s=1.8$; (d), $s=2.0$. Bars and dots represent distances obtained for the A and B atoms, respectively.

Figure 4. The number of the A atoms $N_{\mathrm{A}}(N)$ in the global-minimum geometry of the binary Lennard-Jones cluster for $s=1.4$ to 2.0 . The data for $s=1.5$ to 2.0 are offset vertically by $100(s-1.4)$ for clarity. The maximum coordination number $\left(N_{\max }\right)$ obtained for the cluster is also shown in this figure using symbols (see text for the definition of $N_{\max }$ ): $\square, N_{\max }<12 ; \circ, N_{\max }>12$; closed symbols, $N_{\max }=12 . \quad$ For $N_{\max }=12$, five symbols are used to distinguish the geometries formed by 12 coordination atoms: •, (20/0); $\bullet$ mixture of (20/0) and (10/5); $\mathbf{m}$; (10/5), $\boldsymbol{\Delta},(8 / 6)-\mathrm{I} ; \boldsymbol{\nabla},(8 / 6)-\mathrm{II}$ (see the text for the definitions of (20/0), (10/5), and (8/6)).

Figure 5. Stereographic views of typical geometries constructed by the 12 coordination atoms and the core atom in the binary Lennard-Jones clusters with $N \geq 13$. These views are drawn using only the A atoms. In the (20/0) configurations, however, the number of the A atoms ranges from 4 to 12 . The (10/5) configurations include 10 to 12 A atoms. The (8/6) configurations consist of only the A atoms.

Figure 6. Relative stability $S_{N}$ of the global-minimum geometries of the binary Lennard-Jones clusters. The data for $s=1.5$ to 2.0 are offset vertically by $50(s-1.4)$ for clarity. The sizes of the clusters with $S_{N} \geq \varepsilon$ are also described. 
Figure 7. The stereographic views of the $s=1.4$ cluster with $N=40$. The A atoms are shown by spheres and the outer shell is shown by small spheres (the positions of the B atoms) and connectors between them. The connectors with the lengths of $1.54 \sigma$ to $1.65 \sigma$ are drawn to understand the shape of the shell. 


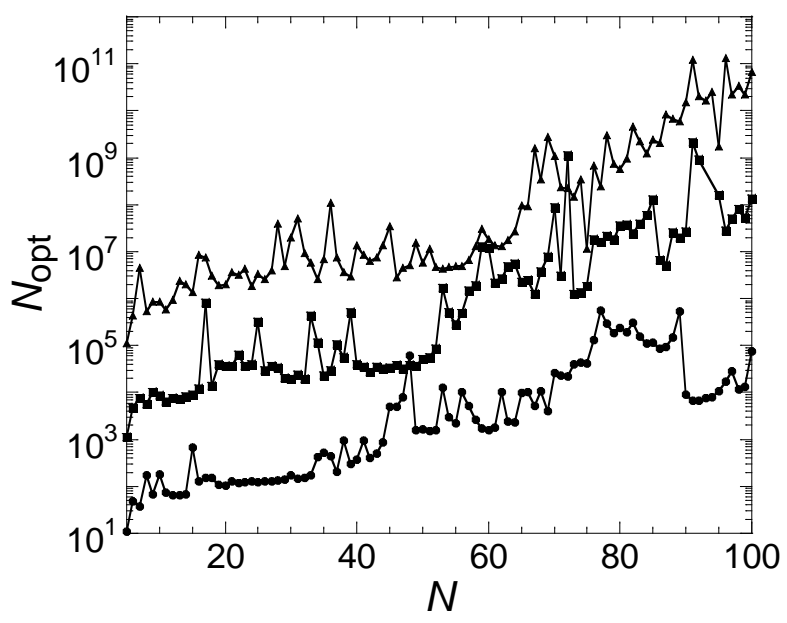

Fig. 1

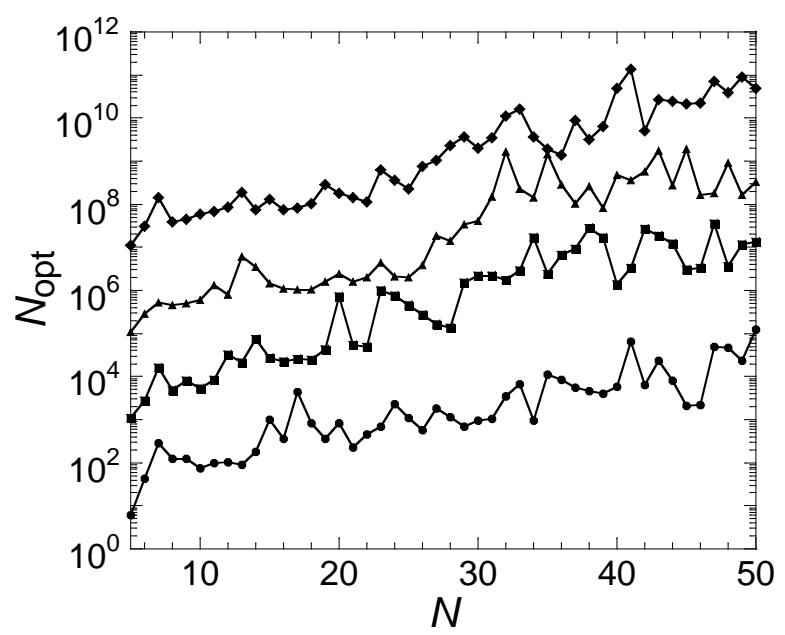

Fig. 2 

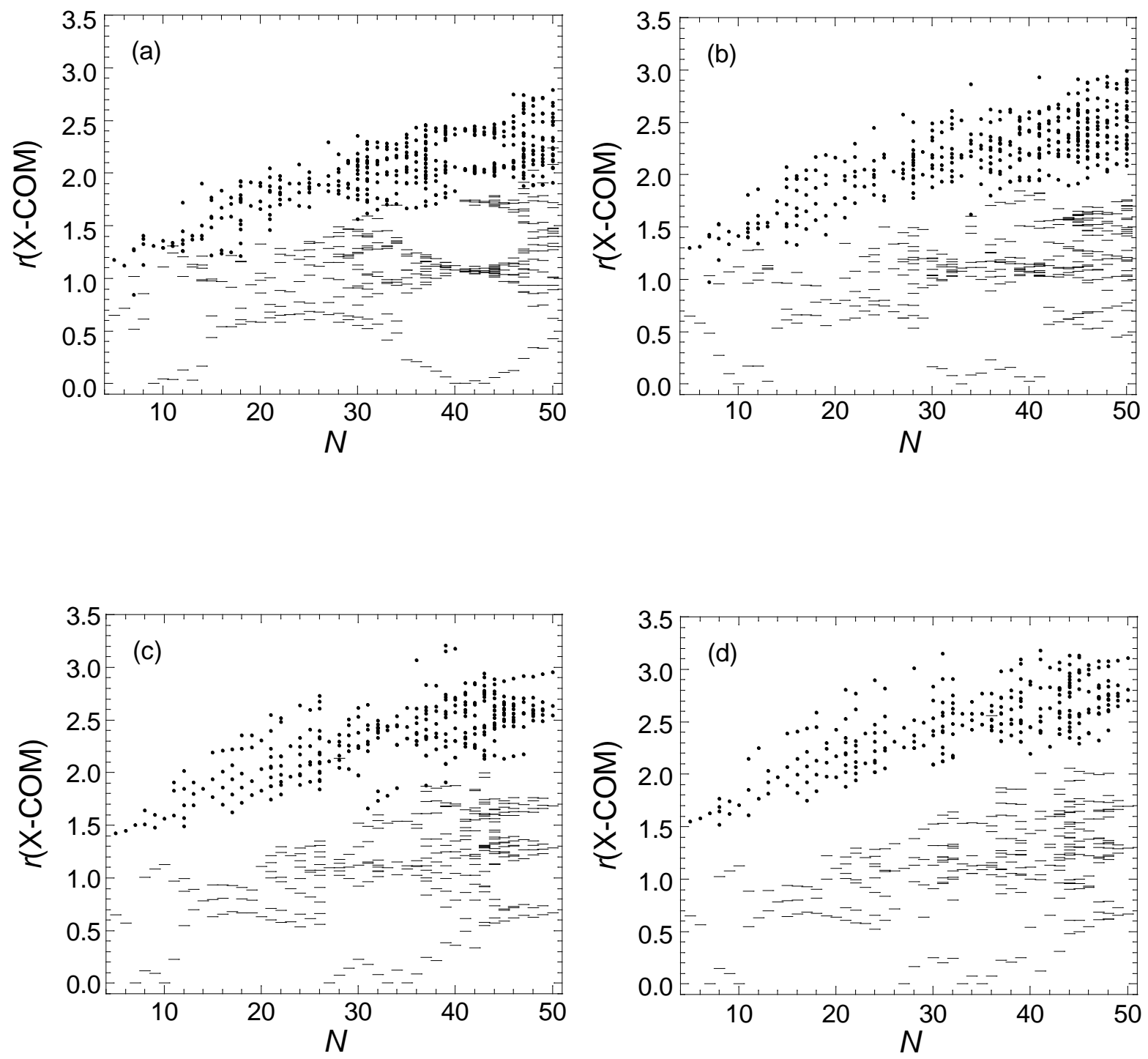

Fig. 3 




Fig. 4 

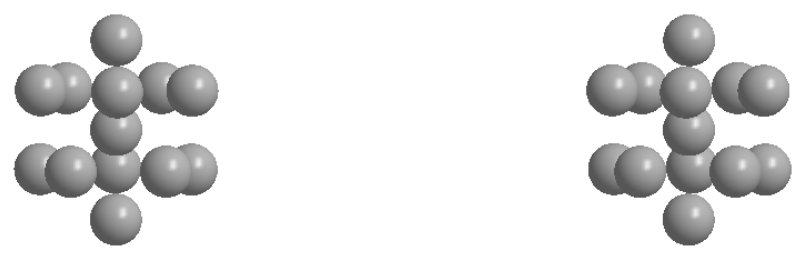

(20/0)
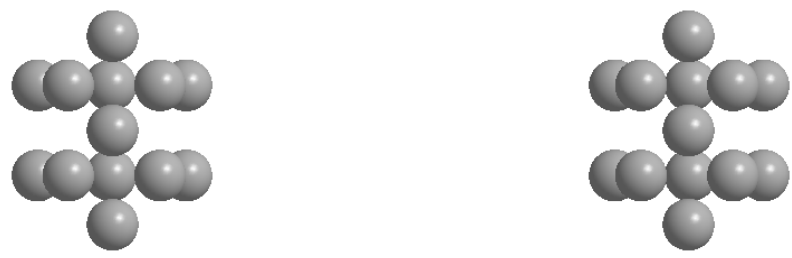

(10/5)
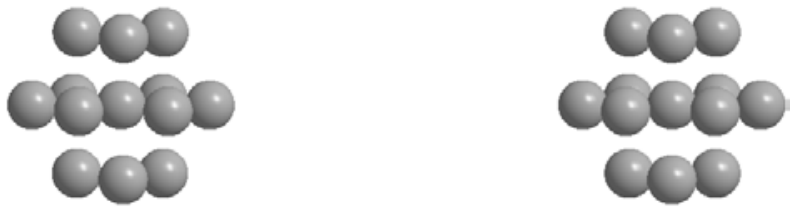

(8/6)-I
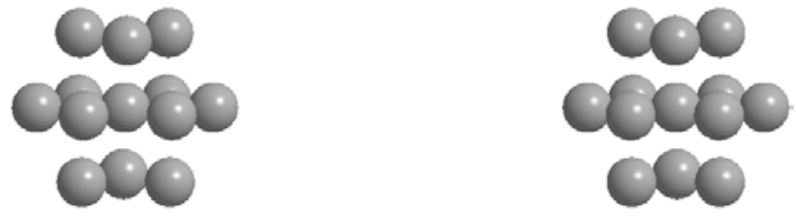

(8/6)-II

Fig. 5 


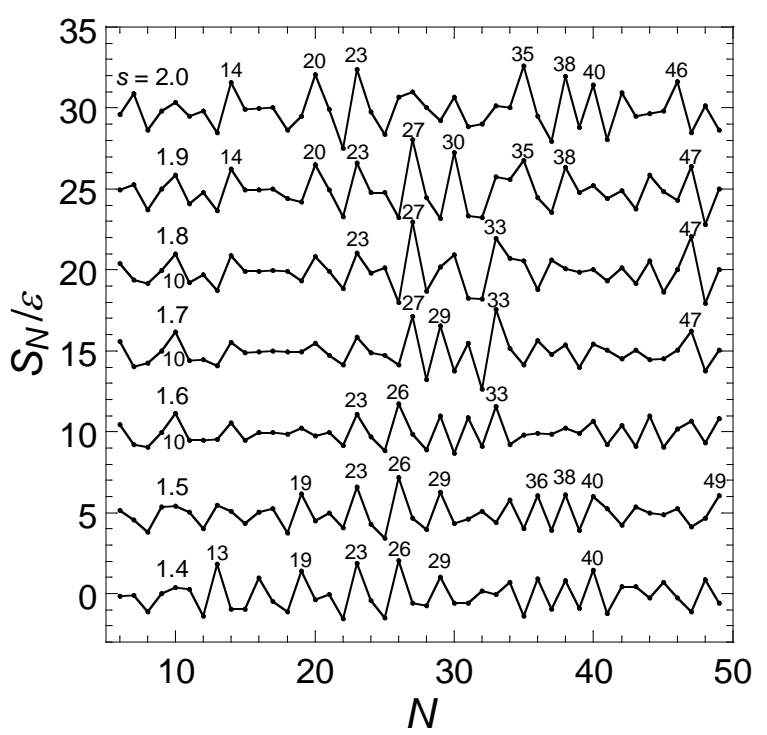

Fig. 6


Fig. 7 


\section{Binary Lennard-Jones Atomic Clusters: Structural Features Induced \\ by Large-Sized Atoms}

Hiroshi Takeuchi

Division of Chemistry, Graduate School of Science, Hokkaido University, Sapporo 060-0810, Japan

Supplementary data

Table S1. The lowest energies of the binary Lennard-Jones clusters for $s=1.05$ to 1.3 obtained in the present study $\left(E_{N} / \varepsilon\right)$.

Table S2. The lowest energies of the binary Lennard-Jones clusters for $s=1.4$ to 2.0 obtained in the present study $\left(E_{N} / \varepsilon\right)$.

Table S3. The Cartesian coordinates of new global minima of $\mathrm{BLJ}_{59}(s=1.15), \mathrm{BLJ}_{68}(s=1.15)$, and $\mathrm{BLJ}_{70}(\mathrm{~s}=1.2)$.

Table S4. The Cartesian coordinates of the lowest energies of the binary Lennard-Jones clusters for $s=$ 1.4 to 2.0 .

Figure S1. Configurations of the binary Lennard-Jones clusters with $N=5-12$. The configurations of the $s=1.8$ clusters are similar to the corresponding ones of the $s=1.9$ clusters. The configurations of the $N=5,6$ clusters are independent of the $s$ value. 
Table S1. The lowest energies of the binary Lennard-Jones clusters for $s=1.05$ to 1.3 obtained in the present study $\left(E_{N} / \varepsilon\right)$.

\begin{tabular}{|c|c|c|c|c|c|c|}
\hline \multirow[b]{2}{*}{$N$} & \multicolumn{6}{|c|}{$E_{N} / \varepsilon$} \\
\hline & $s=1.05$ & $s=1.1$ & $s=1.15$ & $s=1.2$ & $s=1.25$ & $s=1.3$ \\
\hline 5 & -9.111507 & -9.119302 & -9.127219 & -9.135241 & -9.143350 & -9.151532 \\
\hline 6 & 2962 & 734 & 4 & 8 & 7707 & -1 \\
\hline 7 & -16.547241 & -16.557098 & -16.562111 & -16.565375 & -16.572175 & -16.576776 \\
\hline 8 & -19.887476 & -19.948687 & -20.102320 & -20.233378 & -20.333773 & -20.400814 \\
\hline 9 & -24.234145 & -24.300649 & -24.364017 & -24.442494 & -24.533074 & -24.6447 \\
\hline 10 & -28.641948 & -28.771585 & -28.863192 & -28.963939 & -29.502679 & -29.896713 \\
\hline 11 & -33.127634 & -33.371304 & -33.571031 & -33.966602 & -34.345763 & -34.7496 \\
\hline 12 & -38.6295 & -38. & -39 & -39 & $-3 s$ & $-3 s$ \\
\hline 13 & -45.166580 & -45.543272 & -45.577215 & -45.553927 & -45.592206 & -45.601094 \\
\hline 14 & -48.678921 & -49.049816 & -49.252081 & -49.327774 & -49.361851 & -49.421887 \\
\hline 15 & -53.167589 & -53.599281 & -53.874698 & -54.064278 & -54.1 & -54 \\
\hline 16 & -57.758981 & -58.279990 & -58.561496 & -58.855874 & -59.067444 & -59.4706 \\
\hline 17 & -62.419138 & -63.059172 & -63.367529 & -63.715344 & -64.365365 & -64.790023 \\
\hline 18 & -68.022619 & -68.792764 & -69.162203 & -69.601233 & -69.915015 & -70.151377 \\
\hline 19 & -74.524048 & -75. & -75 . & -76.2 & 9260 & -76 \\
\hline 20 & -79.067291 & -80.143826 & -80.606044 & -81.049244 & -81.465579 & -81.777835 \\
\hline 21 & -83.735808 & -84.978975 & -85.558493 & -85.958319 & -86.397935 & -86.806107 \\
\hline 22 & $-\varepsilon$ & - & 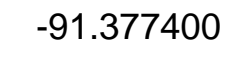 & 5 & 4 & -9 \\
\hline 23 & -95.813706 & -97.429591 & -98.124846 & -98.645805 & -99.186042 & -99.606519 \\
\hline 24 & -100.407430 & -102.241482 & -103.094695 & -103.594194 & -104.215802 & -104.674634 \\
\hline 25 & -105.878 & 200.010 & -108.973 & 50.00 & -110.182559 & -110.621 \\
\hline 26 & -112.438253 & -114.892839 & -115.856394 & -116.459773 & -117.119402 & -117.480017 \\
\hline 27 & -116.926080 & -119.543605 & -120.824602 & -121.413505 & -122.140725 & -122.587527 \\
\hline 28 & -122.322287 & -125.296607 & -126.686572 & -127.341138 & -128.036984 & -128.415202 \\
\hline 29 & -1 & -1 & -1 & 55 & -104.914 & -103.04 \\
\hline 30 & -133.409767 & -136.742249 & -138.632308 & -139.296381 & -139.997093 & -140.582023 \\
\hline 31 & -138.662748 & -142.433255 & -144.488697 & -145.238298 & -145.962936 & -146.516 \\
\hline 32 & -145.044787 & -149.212031 & -151.384854 & -152.149159 & -152.905938 & -153.348 \\
\hline 33 & -150.010109 & -154.633546 & -157.272375 & -158.185112 & -159.258976 & -159.598157 \\
\hline 34 & -156.228179 & -161.360048 & -164.222021 & -165.168631 & -166.318301 & -166.653616 \\
\hline 35 & -161.190333 & -166.185706 & -168.882563 & -170.282025 & -171.344302 & -171.853006 \\
\hline 36 & -166.111652 & -171.524014 & -174.798837 & -176.347917 & -177.367846 & -177.844845 \\
\hline 37 & -172.189967 & -178.167061 & -181.675233 & -183.248506 & -184.345543 & -184.724505 \\
\hline 38 & -177.260697 & -183.371989 & -187.367687 & -189.280007 & -190.778073 & -191.184019 \\
\hline 39 & -183.003251 & -189.903875 & -194.229977 & -196.261792 & -197.847765 & -198.228135 \\
\hline 40 & -188.146744 & -195.024681 & -199.703534 & -203.169138 & -204.897420 & -204.963823 \\
\hline 41 & -193.743072 & -201.346753 & -206.479560 & -210.149549 & -212.023029 & -212.073757 \\
\hline
\end{tabular}


$42-199.257511$

$43-205.003305$

$44 \quad-210.978612$

$45 \quad-216.856331$

$46 \quad-223.210589$

$\begin{array}{ll}47 & -228.725544\end{array}$

$\begin{array}{ll}48 & -235.079224\end{array}$

$49 \quad-242.011604$

$\begin{array}{ll}50 & -247.882695\end{array}$

$\begin{array}{ll}51 & -254.916947\end{array}$

$\begin{array}{ll}52 & -261.953177\end{array}$

$53-268.991573$

$54 \quad-276.058231$

$55 \quad-283.154531$

$\begin{array}{ll}56 & -287.691892\end{array}$

$\begin{array}{ll}57 & -292.490914\end{array}$

$\begin{array}{ll}58 & -298.295002\end{array}$

$\begin{array}{ll}59 & -303.893779\end{array}$

$60-310.001341$

$61-316.112220$

$\begin{array}{ll}62 & -321.789205\end{array}$

$\begin{array}{ll}63 & -327.901806\end{array}$

$\begin{array}{ll}64 & -334.013197\end{array}$

$\begin{array}{ll}65 & -339.910437\end{array}$

$66-346.360633$

$\begin{array}{ll}67 & -352.827543\end{array}$

$\begin{array}{ll}68 & -359.272795\end{array}$

$\begin{array}{ll}69 & -365.744424\end{array}$

$\begin{array}{ll}70 & -372.669868\end{array}$

$\begin{array}{ll}71 & -379.710375\end{array}$

$\begin{array}{ll}72 & -385.168824\end{array}$

$\begin{array}{ll}73 & -391.559518\end{array}$

$74 \quad-397.664635$

75

76

77

78

79

80

81

82

$83 \quad-455.072951$
$-207.024045$

$-213.011525$

$-218.600859$

$-224.652192$

$-229.387288$

$-234.934267$

$-240.845542$

$-247.555272$

$-254.363907$

$-261.263002$

$-266.585540$

$-271.915714$

$-278.194041$

$-284.159496$

$-290.398039$

$-296.039531$

$-302.562491$

$-309.038480$

$-315.474426$

$-321.775834$

$-326.823952$

$-333.289735$

$-339.577727$

$-345.702127$

$-352.051080$

$-358.106731$

$-364.666125$

$-371.201139$

$-377.921655$

$-384.295723$

$-390.588771$

$-396.776526$

$-402.397866$

$-408.569903$

$-414.633801$

$-420.873025$

$-427.333481$

$-436.990405$

$-433.956776$

$-440.436405$

$-446.783469$

$-453.113837$

$-459.576481$

$-212.864370$

$-327.728804$
$-443.855667$

$-450.392713$

$-456.626482$

$-463.282074$

$-219.440909$

$-226.066862$

$-232.763363$

$-237.126607$

$-242.723641$

$-249.375588$

$-254.328385$

$-260.903328$

$-265.962776$

$-272.093082$

$-277.756061$

$-283.532817$

$-289.358203$

$-296.263148$

$-302.288570$

$-309.171713$

$-315.027359$

$-321.988253$

$-334.662475$

$-340.639075$

$-347.239596$

$-352.920771$

$-359.749940$

$-365.780253$

$-372.247969$

$-378.279505$

$-385.326178$

$-391.564061$

$-397.654760$

$-404.095588$

$-410.534023$

$-417.299214$

$-423.812270$

$-430.684586$

$-437.832462$

$-444.864069$

$-451.921407$

$-457.933230$

$-464.450846$

$-471.263190$

$-477.723147$
$-219.151586$

$-219.163369$

$-226.283209-226.239574$

$-233.490683-233.385520$

$-240.774604-240.676356$

$-245.105004-245.364214$

$-251.068364-251.181462$

$-258.051347-258.057115$

$-263.648530-264.146588$

$-270.658086-271.250575$

$-276.007398-277.913411$

$-282.974620-285.100422$

$-289.573568-292.286192$

$-296.605354-299.477866$

$-303.663061-306.733764$

$-310.691065-313.981977$

$-315.238733-318.655257$

$-321.914589-325.589970$

$-327.501569-331.286991$

$-333.705967-338.396340$

$-339.574283-345.440684$

$-346.177037-352.541258$

$-351.339146-357.469508$

$-358.040393-363.997742$

$-363.528091-369.664027$

$-370.103112-376.666635$

$-376.843606-383.146334$

$-383.252132-388.025204$

$-389.872707-393.796485$

$-396.944371-400.540009$

$-403.529486-407.410007$

$-410.107263-414.312666$

$-416.498169-420.923993$

$-423.659482-427.714840$

$-430.250717-434.557994$

$-436.538490-441.662589$

$-443.347693-448.595192$

$-450.147772-455.747811$

$-457.300970-462.413785$

$-464.399834-469.652236$

$-471.122664-476.399256$

$-477.619634-483.437070$

$-484.474951-490.324955$ 


$\begin{array}{rrrrrrr}84 & -461.542943 & -466.338378 & -476.669838 & -484.538812 & -491.591546 & -497.524938 \\ 85 & -468.086930 & -472.943379 & -483.671543 & -491.066290 & -498.453301 & -504.660242 \\ 86 & -474.634175 & -479.520778 & -490.630031 & -497.860282 & -504.842168 & -511.844354 \\ 87 & -481.551013 & -485.876908 & -497.578761 & -504.764331 & -511.400044 & -518.785356 \\ 88 & -488.468369 & -492.216494 & -503.728759 & -511.443323 & -518.256499 & -525.996499 \\ 89 & -494.056891 & -498.342007 & -510.052009 & -517.829589 & -525.381891 & -530.791989 \\ 90 & -500.273073 & -504.977129 & -516.704386 & -524.617913 & -531.763926 & -537.327668 \\ 91 & -506.729133 & -511.845979 & -522.859880 & -531.680233 & -538.411196 & -542.989912 \\ 92 & -513.205038 & -518.431759 & -529.190149 & -538.316013 & -544.507909 & -549.757224 \\ 93 & -519.775813 & -524.964281 & -535.594853 & -544.573272^{\mathrm{a}} & -551.431854 & -556.443498 \\ 94 & -526.614509 & -531.583051 & -542.476905 & -550.802667^{\mathrm{a}} & -558.070885 & -562.801205 \\ 95 & -533.465067 & -538.741277 & -548.732770 & -557.785086 & -564.709518 & -569.480421 \\ 96 & -539.063361 & -544.671597 & -555.145507 & -564.674461 & -571.389275 & -576.517002 \\ 97 & -545.436537 & -551.416081 & -561.876490 & -571.392434 & -578.201634 & -583.871531 \\ 98 & -551.683813 & -558.264685 & -568.603054 & -578.000233 & -584.953816 & -590.787413 \\ 99 & -558.310074 & -564.964519 & -575.609709 & -585.066165 & -592.008931^{\mathrm{a}} & -597.679071 \\ 100 & -564.909753 & -571.812888 & -582.701043 & -591.768143 & -599.043884^{\mathrm{a}} & -604.796307\end{array}$

a The global minima reported previously are not obtained in the present study: the reported lowest energies of the four clusters are $-544.766420 \varepsilon,-551.033813 \varepsilon,-592.138846 \varepsilon$, and $-599.264624 \varepsilon$. 
Table S2. The lowest energies of the binary Lennard-Jones clusters for $s=1.4$ to 2.0 obtained in the present study $\left(E_{N} / \varepsilon\right)$.

\begin{tabular}{|c|c|c|c|c|c|c|c|}
\hline \multirow[b]{2}{*}{$N$} & \multicolumn{7}{|c|}{$E_{N}$} \\
\hline & $s=1.4$ & 1.5 & 1.6 & 1.7 & 1.8 & 1.9 & 2.0 \\
\hline 5 & -9168 & -9.184718 & 01428 & -9.2181 & -9.234711 & -9.2511 & -9267 \\
\hline 6 & -1 & -4 & 5 & 0 & 7 & 5 & 70 \\
\hline 7 & -16.584984 & -16.609636 & -16.634759 & -16.781371 & -17.140302 & -17.672694 & -18.073130 \\
\hline 8 & -20.491743 & -20.714043 & -20.9 & -21.283208 & -21.521513 & -21.634684 & -21.814930 \\
\hline 9 & -2 & -2 & 2 & 2 & -2 & -2 & -2 \\
\hline 10 & -30 & -30.9 & -31 & -31 & -32 & $-3 i$ & -3 \\
\hline 11 & -35.274878 & -35.487787 & -35.603591 & -35.917049 & -36.270022 & -36.549295 & -37.111833 \\
\hline 12 & -39 & -4 & 25 & -40 & 05141 & -41 & -4 \\
\hline 13 & -4 & -45 & -4 & -6 & -46 & -4 & \\
\hline 14 & -49.508949 & -50.489724 & -51.095901 & -51.9879 & -53.222502 & -54.331902 & 33067 \\
\hline 15 & -54 & -55 & -5 & -57.6 & -58 & $-5 c$ & -6 \\
\hline 16 & -6 & -6 & $c_{0}$ &  & -6 & & \\
\hline 17 & -65.332397 & -66.446027 & 9039 & -69.267007 & -70.600218 & -71.481302 & -7 \\
\hline 18 & -7 & 520 & -73 & $-7 !$ & -76 & -77 & -7 \\
\hline 19 & -77. & -10. & 10 & -01 & & & \\
\hline 20 & -82.495693 & 4565 & 3290 & -87 & -89 & -90 & -9 \\
\hline 21 & -88.025471 & -89.330178 & -90.570373 & -92.763166 & -94.850253 & 4037 & -9 \\
\hline 22 & 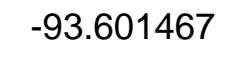 & 3 & -96.512158 & -98.657162 & 5 & -102.128446 & 0 \\
\hline 23 & 0941 & 1346 & 147 & -105.4 & -107.744490 & -109 & -11 \\
\hline 24 & -106.012643 & -107.064064 & -108.932853 & -111.349064 & -113.721598 & -115.433419 & 21863 \\
\hline 25 & 11 & -1 & 07 & -1 & 1 & 1 & 1 \\
\hline 26 & 4648 & 33 & 88 & 6 & 569 & 34324 & -12 \\
\hline 27 & -124.066931 & -125.697620 & -127.430425 & -130.980905 & -133.907416 & -135.837752 & -136.658297 \\
\hline 28 & -129.778684 & -131.301741 & -133.017782 & -136.064944 & -138.966341 & -140.858567 & -142.46091 \\
\hline 29 & 3 & 5 & 3 & 3 & -145.342393 & -146.434540 & \\
\hline 30 & -141.678730 & -143.322385 & -145.480894 & -148.313123 & -151.524277 & -153.819680 & -154.825577 \\
\hline 31 & -147. & 98191 & -152. & -154 & -156 & 4495 & .752462 \\
\hline 32 &  & 2 & -158.700621 & -1 & - & -1 & - \\
\hline 33 & -160. & 866 & -165.7 & -16 & 8008 & -1 & -1 \\
\hline 34 & -167.225215 & -169.250795 & -171.326596 & -175.526184 & -179.450528 & -182.112852 & -183.740999 \\
\hline 35 & -173.011973 & -175.447948 & 17800217 & -181.357766 & 185625002 & 1002110 & 101 \\
\hline 36 & -180. & 32268 & -184 & -188.040054 & 80568 & 28824 & $-1 s$ \\
\hline 37 & -186. & -1 & 208 & -1 & -1 & -20 & -202 \\
\hline 38 & -193 & -195 & -197 & -200 & -204 & -20 & -2 \\
\hline 39 & -200.030446 & -202.040051 & $-204.17 i$ & -206 & -210.627 & -214.480766 & -216.589 \\
\hline 40 & -207.322597 & -209.230681 & -210.847376 & -213.220313 & -216.945765 & -220.807300 & -223.700145 \\
\hline 41 & -213.170471 & -215.407179 & -216.848113 & -219.743606 & -223.258093 & -226.951993 & -229.365627 \\
\hline
\end{tabular}




$\begin{array}{llllllll}42 & -220.223427 & -221.319539 & -223.652390 & -226.250732 & -230.236470 & -233.679219 & -236.995833 \\ 43 & -226.823468 & -227.979704 & -230.062836 & -233.255889 & -237.073585 & -240.538283 & -243.686941 \\ 44 & -232.998720 & -234.297422 & -237.368902 & -240.201249 & -244.756631 & -248.636081 & -250.867906 \\ 45 & -239.434596 & -240.654799 & -243.724171 & -247.677700 & -251.897352 & -255.883412 & -258.409108 \\ 46 & -245.176668 & -247.137015 & -251.048217 & -255.625219 & -260.391381 & -263.282585 & -266.121423 \\ 47 & -251.178900 & -253.365117 & -258.224693 & -263.555682 & -268.881577 & -271.372063 & -272.215887 \\ 48 & -258.325633 & -260.451080 & -264.768267 & -270.247871 & -275.324355 & -278.085201 & -279.832502 \\ 49 & -264.629464 & -267.906522 & -271.973689 & -278.200778 & -283.859545 & -286.985560 & -287.340181 \\ 50 & -271.536410 & -274.312888 & -278.361820 & -286.133713 & -292.387819 & -295.883575 & -296.237038\end{array}$


Table S3. The Cartesian coordinates of new global minima of $\mathrm{BLJ}_{59}(s=1.15), \operatorname{BLJ}_{68}(s=1.15)$, and $\mathrm{BLJ}_{70}(s=1.2)$.

$S=1.15, E 59 / \varepsilon=-315.027359$

atom $\mathrm{x} / \sigma \quad \mathrm{y} / \sigma \quad \mathrm{z} / \sigma$

$\begin{array}{llll}1 \mathrm{~A} & 0.000000 & 0.000000 & 0.000000\end{array}$

2B $\quad 1.140175 \quad 0.000000 \quad 0.000000$

$\begin{array}{lllll}3 \mathrm{~B} & -1.137834 & -0.057027 & 0.000000\end{array}$

$\begin{array}{llll}4 \mathrm{~A} & 0.494187 & -0.979074 & 0.261753\end{array}$

$5 A \quad-0.443904-0.999367-0.269042$

$\begin{array}{lllll}6 \mathrm{~A} & 0.491238 & -0.724123 & -0.753911\end{array}$

$7 A \quad-0.453420-0.757523 \quad 0.749344$

$8 \mathrm{~A} \quad 0.329392-1.754730-0.467342$

9A $\quad-0.240578-1.774761 \quad 0.451201$

$10 A \quad-0.454998-0.288714-1.040820$

$\begin{array}{llll}11 \mathrm{~A} & 0.444840 & 1.002915 & -0.269773\end{array}$

$\begin{array}{llll}12 \mathrm{~A} & 0.469900 & -0.283722 & 1.045810\end{array}$

$\begin{array}{lllll}13 \mathrm{~A} & 0.453347 & 0.725873 & 0.756281\end{array}$

$14 \mathrm{~A} \quad-0.485194 \quad 0.726546-0.750983$

15A $\quad-0.257030 \quad-1.330716-1.274224$



$\begin{array}{lllll}17 A & 0.323572 & -1.333161 & 1.263963\end{array}$

$\begin{array}{llll}18 \mathrm{~A} & -0.472350 & 0.248359 & 1.047400\end{array}$

$\begin{array}{lllll}19 \mathrm{~A} & -0.493856 & 0.983287 & 0.282673\end{array}$

$\begin{array}{lllll}20 \mathrm{~B} & 0.936278 & -2.037827 & 0.510308\end{array}$

21B $\quad-0.833277 \quad-2.075499-0.529333$

$\begin{array}{llll}22 \mathrm{~B} & -0.332862 & -0.571121 & 1.904278\end{array}$

23B $\quad 0.361440 \quad-0.534588-1.906511$

24B $\quad 1.453960-1.255739-0.345379$

$\begin{array}{lllll}25 B & -1.388775 & -1.330603 & 0.334954\end{array}$

$\begin{array}{lllll}26 \mathrm{~B} & -1.401959 & -0.989578 & -0.917156\end{array}$

$\begin{array}{lllll}27 \mathrm{~B} & 1.450746 & -0.930534 & 0.911088\end{array}$

$\begin{array}{llll}28 \mathrm{~B} & -0.847727 & -1.616480 & 1.455926\end{array}$

29B $\quad 0.928114 \quad-1.555597-1.469166$

$\begin{array}{llll}30 \mathrm{~B} & 0.333868 & 0.482580 & 1.924487\end{array}$

31B $\quad-0.361441 \quad 0.478854-1.923385$

32B $\quad 0.295385 \quad 1.402377-1.381990$

$\begin{array}{llll}33 \mathrm{~B} & -0.364885 & 1.385079 & 1.373319\end{array}$

34B $-0.387379 \quad 1.883489-0.443870$

35B $\quad 1.460348-0.328179-1.245316$

$\begin{array}{llll}36 \mathrm{~B} & -1.439191 & -0.418115 & 1.248239\end{array}$

$\begin{array}{lllll}37 \mathrm{~B} & 1.431138 & 1.217419 & 0.339671\end{array}$

$\begin{array}{lllll}38 \mathrm{~B} & 1.430818 & 0.918431 & -0.904357\end{array}$

$\begin{array}{llll}39 \mathrm{~B} & 1.441542 & 0.311080 & 1.259047\end{array}$

$\begin{array}{llll}40 \mathrm{~B} & -1.454125 & 0.255687 & -1.252327\end{array}$

41B $\quad-0.895490-0.695612-2.050873$

$\begin{array}{lllll}42 \mathrm{~B} & 0.929582 & -0.672499 & 2.049860\end{array}$

$\begin{array}{lllll}43 \mathrm{~B} & 0.070335 & -2.812766 & -0.014254\end{array}$

$\begin{array}{llll}44 \mathrm{~A} & 0.326650 & 1.777139 & 0.496090\end{array}$

45B $\quad 0.045420-2.482042-1.341051$

$\begin{array}{llll}46 \mathrm{~B} & 0.078924 & -2.497485 & 1.317160\end{array}$

$\begin{array}{llll}47 \mathrm{~B} & -1.489342 & 1.153296 & -0.326527\end{array}$

$\begin{array}{llll}48 \mathrm{~B} & -1.479030 & 0.823218 & 0.911139\end{array}$

$\begin{array}{llll}49 \mathrm{~B} & 0.922867 & 2.052260 & -0.495873\end{array}$

50B $\quad 0.965160 \quad 0.564896-2.063933$

$\begin{array}{lllll}51 \mathrm{~B} & 0.938886 & 1.505524 & 1.486566\end{array}$

$\begin{array}{lllll}52 \mathrm{~B} & 1.177358 & -2.517976 & -0.656181\end{array}$

$\begin{array}{lllll}53 \mathrm{~B} & -1.049795 & -2.580724 & 0.631567\end{array}$

$\begin{array}{lllll}54 \mathrm{~B} & -0.983047 & 0.503541 & 2.066233\end{array}$

$\begin{array}{llll}55 \mathrm{~B} & -1.014318 & 1.471749 & -1.482597\end{array}$

$\begin{array}{llll}56 \mathrm{~B} & -1.117291 & -1.946052 & -1.759617\end{array}$

$\begin{array}{llll}57 \mathrm{~B} & 1.213697 & -1.908147 & 1.743174\end{array}$

58B $\quad-0.016431-1.662660-2.361797$

$\begin{array}{lllll}59 \mathrm{~B} & 0.099777 & -1.685507 & 2.348008\end{array}$

$s=1.15, E 68 / \varepsilon=-372.247969$

atom $\mathrm{x} / \sigma \quad y / \sigma \quad z / \sigma$

$\begin{array}{llll}1 \mathrm{~A} & 0.000000 & 0.000000 & 0.000000\end{array}$

$\begin{array}{llll}2 B & 1.142436 & 0.000000 & 0.000000\end{array}$

$\begin{array}{llll}3 \mathrm{~B} & -1.140848 & -0.060190 & 0.000000\end{array}$

$\begin{array}{llll}4 \mathrm{~A} & 0.478257 & -0.271482 & 0.984821\end{array}$

$\begin{array}{llll}5 A & -0.463264 & -0.296999 & -0.984632\end{array}$

$\begin{array}{llll}6 A & 0.469029 & 0.268552 & -1.005925\end{array}$

$\begin{array}{llll}7 A & -0.482541 & 0.244179 & 1.005732\end{array}$

$\begin{array}{llll}8 \mathrm{~A} & -0.451447 & -0.761115 & 0.727938\end{array}$

9A $\quad 0.490939-0.736787-0.727420$

$\begin{array}{llll}10 A & -0.478833 & 0.726060 & -0.750603\end{array}$

$\begin{array}{lllll}11 \mathrm{~A} & 0.439906 & 0.750810 & 0.750068\end{array}$

$\begin{array}{lllll}12 \mathrm{~A} & 0.499931 & -1.012039 & 0.264663\end{array}$

13A $-0.445905-1.037160-0.263950$

$14 \mathrm{~A} \quad 0.328880-1.775100-0.467316$

$\begin{array}{llll}15 A & -0.234902 & -1.789632 & 0.468565\end{array}$

16A $-0.253727-1.326826-1.270476$

$\begin{array}{llll}17 A & 0.323263 & -1.310716 & 1.271396\end{array}$

$\begin{array}{llll}18 \mathrm{~A} & -0.497638 & 1.002681 & 0.271389\end{array}$

$\begin{array}{llll}19 A & 0.444129 & 1.027315 & -0.272120\end{array}$

20A $\quad 0.291367 \quad-0.516383-1.747674$

$\begin{array}{llll}21 \mathrm{~A} & -0.263782 & -0.529780 & 1.748032\end{array}$

$\begin{array}{llll}22 \mathrm{~A} & -0.297823 & 0.397142 & -1.775600\end{array}$

$\begin{array}{llll}23 \mathrm{~A} & 0.276459 & 0.413538 & 1.775300\end{array}$

24B $\quad 0.914881-1.518425-1.482884$

$\begin{array}{llll}25 B & -0.833630 & -1.563470 & 1.483962\end{array}$

26B $-0.877050-0.645366-2.018622$

$\begin{array}{llll}27 \mathrm{~B} & 0.909804 & -0.596832 & 2.019049\end{array}$

$\begin{array}{lllll}28 \mathrm{~B} & 0.940297 & -2.052033 & 0.537475\end{array}$

29B $-0.830859-2.099104-0.536019$

$\begin{array}{llll}30 \mathrm{~B} & 0.321871 & 1.352686 & -1.417456\end{array}$

$\begin{array}{llll}31 \mathrm{~B} & -0.392713 & 1.334852 & 1.416492\end{array}$

32B $-1.402969-1.003798-0.915025$

$\begin{array}{lllll}33 \mathrm{~B} & 1.453896 & -0.927837 & 0.915694\end{array}$

34B $1.458018-1.267968-0.345225$

$\begin{array}{lllll}35 \mathrm{~B} & -1.389188 & -1.342781 & 0.346136\end{array}$

$\begin{array}{llll}36 \mathrm{~B} & 1.432659 & -0.354576 & -1.278537\end{array}$

$\begin{array}{lllll}37 \mathrm{~B} & -1.412006 & -0.428661 & 1.278801\end{array}$

$\begin{array}{llll}38 \mathrm{~B} & -1.435426 & 0.250199 & -1.270118\end{array}$

$\begin{array}{lllll}39 B & 1.420233 & 0.326378 & 1.269898\end{array}$

$\begin{array}{lllll}40 \mathrm{~B} & -0.388823 & 1.875813 & -0.533820\end{array}$

$\begin{array}{lllll}41 B & 0.289449 & 1.894071 & 0.532472\end{array}$

$\begin{array}{llll}42 \mathrm{~B} & 0.876321 & 0.454114 & -2.094426\end{array}$

$\begin{array}{lllll}43 B & -0.899063 & 0.408794 & 2.094109\end{array}$

$\begin{array}{llll}44 \mathrm{~B} & 1.435361 & 0.895681 & -0.911482\end{array}$ $\begin{array}{lllll}45 \mathrm{~B} & -1.480570 & 0.819457 & 0.910860\end{array}$ 46B $\quad-1.480160 \quad 1.162607-0.357253$ $\begin{array}{lllll}47 \mathrm{~B} & 1.416849 & 1.239227 & 0.356388\end{array}$ $\begin{array}{llll}48 B & -0.942579 & 1.381160 & -1.603456\end{array}$ $\begin{array}{lllll}49 \mathrm{~B} & 0.868478 & 1.430037 & 1.602447\end{array}$ $\begin{array}{llll}50 \mathrm{~B} & -0.021687 & -0.136440 & -2.805580\end{array}$ $\begin{array}{llll}51 B & 0.028801 & -0.133123 & 2.805663\end{array}$ $\begin{array}{lllll}52 \mathrm{~B} & 0.024429 & -1.464707 & -2.399932\end{array}$ $\begin{array}{llll}53 \mathrm{~B} & 0.052738 & -1.462262 & 2.400955\end{array}$ $\begin{array}{llll}54 \mathrm{~B} & 0.073267 & -2.455767 & 1.384798\end{array}$ $\begin{array}{llll}55 B & 0.056254 & -2.457195 & -1.383073\end{array}$ $\begin{array}{lllll}56 \mathrm{~B} & 0.074415 & -2.822696 & 0.000992\end{array}$ $\begin{array}{llll}57 \mathrm{~B} & 0.936756 & 2.047055 & -0.551376\end{array}$ $\begin{array}{llll}58 \mathrm{~B} & -1.043311 & 1.995248 & 0.549933\end{array}$ $\begin{array}{llll}59 \mathrm{~B} & 1.179281 & -1.855787 & 1.806634\end{array}$ 60B $-1.079826-1.916620-1.805313$ $\begin{array}{llll}61 \mathrm{~B} & 1.126025 & -0.748735 & -2.504677\end{array}$ $\begin{array}{llll}62 B & -1.085053 & -0.805249 & 2.505214\end{array}$ $\begin{array}{lllll}63 \mathrm{~B} & 1.182878 & -2.511194 & -0.668360\end{array}$ $\begin{array}{lllll}64 \mathrm{~B} & -1.048933 & -2.569555 & 0.670144\end{array}$ $\begin{array}{llll}65 \mathrm{~B} & -1.178482 & 0.484521 & -2.526527\end{array}$ $\begin{array}{lllll}66 \mathrm{~B} & 1.151279 & 0.547726 & 2.526147\end{array}$ 67B $\quad-0.085172 \quad 1.165687-2.616060$ $\begin{array}{llll}68 \mathrm{~B} & 0.023597 & 1.170407 & 2.615220\end{array}$

$s=1.2, E 70 / \varepsilon=-391.735161$

$\begin{array}{llll}\text { atom } \mathrm{X} / \sigma & y / \sigma & \mathrm{z} / \sigma\end{array}$

$\begin{array}{llll}1 \mathrm{~A} & 0.000000 & 0.000000 & 0.000000\end{array}$

$\begin{array}{llll}2 \mathrm{~B} & 1.165837 & 0.000000 & 0.000000\end{array}$ $\begin{array}{llll}3 \mathrm{~B} & -1.163824 & -0.046742 & 0.000000\end{array}$ $\begin{array}{llll}4 \mathrm{~A} & 0.474046 & -0.006897 & 1.029543\end{array}$ $\begin{array}{lllll}5 A & 0.474083 & -0.008769 & -1.029535\end{array}$ $\begin{array}{lllll}6 \mathrm{~A} & -0.459050 & -0.546907 & 0.897761\end{array}$ $\begin{array}{lllll}7 A & -0.459015 & -0.548539 & -0.896807\end{array}$ $\begin{array}{lllll}8 \mathrm{~A} & -0.481452 & 0.508523 & 0.909942\end{array}$ $\begin{array}{llll}9 \mathrm{~A} & -0.481417 & 0.506866 & -0.910907\end{array}$ $\begin{array}{lllll}10 A & 0.492439 & -0.919819 & 0.520140\end{array}$ $11 \mathrm{~A} \quad 0.492458-0.920764-0.518473$ $\begin{array}{lllll}12 \mathrm{~A} & -0.450684 & -1.077743 & 0.000959\end{array}$ $\begin{array}{lllll}13 \mathrm{~A} & 0.448779 & 0.932190 & 0.532520\end{array}$ 14A $\quad 0.448799 \quad 0.931220-0.534222$ 15A $\quad 0.308969-0.950669-1.575933$ $\begin{array}{lllll}16 \mathrm{~A} & 0.308909 & -0.947801 & 1.577645\end{array}$ $\begin{array}{llll}17 A & -0.253128 & -1.622866 & -0.910143\end{array}$ $\begin{array}{lllll}18 A & -0.253162 & -1.621208 & 0.913058\end{array}$ $\begin{array}{lllll}19 A & 0.320543 & -1.857887 & 0.001683\end{array}$ $\begin{array}{llll}20 A & -0.276607 & -0.057271 & -1.835432\end{array}$ $\begin{array}{llll}21 \mathrm{~A} & -0.276676 & -0.053933 & 1.835498\end{array}$ $\begin{array}{lllll}22 \mathrm{~A} & -0.495301 & 1.063379 & -0.000989\end{array}$ $\begin{array}{llll}23 \mathrm{~A} & 0.282675 & 0.868498 & -1.624883\end{array}$ $\begin{array}{lllll}24 \mathrm{~A} & 0.282613 & 0.871452 & 1.623286\end{array}$ 25B $\quad-0.874401-1.142043-1.852699$ $\begin{array}{lllll}26 \mathrm{~B} & -0.874472 & -1.138672 & 1.854714\end{array}$ $\begin{array}{lllll}27 \mathrm{~B} & 0.941537 & -1.883275 & 1.068007\end{array}$ 28B $\quad 0.941577-1.885215-1.064571$ $\begin{array}{lllll}29 B & -0.863524 & -2.198720 & 0.001970\end{array}$ $\begin{array}{lllll}30 \mathrm{~B} & 0.919355 & -0.062496 & -2.141127\end{array}$ $\begin{array}{lllll}31 B & 0.919276 & -0.058602 & 2.141246\end{array}$ $\begin{array}{llll}32 \mathrm{~B} & -0.385280 & 1.703274 & 1.027979\end{array}$ 33B $-0.385240 \quad 1.701401-1.031115$ 34B $-1.436581-1.213570-0.664544$ $\begin{array}{lllll}35 \mathrm{~B} & -1.436607 & -1.212359 & 0.666668\end{array}$ $\begin{array}{llll}36 \mathrm{~B} & 1.481177 & -0.680203 & -1.141719\end{array}$ $\begin{array}{lllll}37 \mathrm{~B} & 1.481135 & -0.678124 & 1.142981\end{array}$ $\begin{array}{lllll}38 \mathrm{~B} & 1.489703 & -1.343942 & 0.001237\end{array}$ 39B $-1.454793-0.072068-1.346076$ $\begin{array}{lllll}40 \mathrm{~B} & -1.454845 & -0.069620 & 1.346121\end{array}$ $\begin{array}{llll}41 B & 1.456927 & 0.648915 & -1.153722\end{array}$ $\begin{array}{lllll}42 B & 1.456885 & 0.651012 & 1.152565\end{array}$ $\begin{array}{llll}43 \mathrm{~B} & 0.326467 & 2.021953 & -0.001845\end{array}$ 44B $\quad-0.926728 \quad 0.968446-1.942844$ $\begin{array}{llll}45 B & -0.926803 & 0.971978 & 1.941019\end{array}$ 46B $-1.509043 \quad 1.072714-0.675484$ $\begin{array}{lllll}47 \mathrm{~B} & -1.509069 & 1.073939 & 0.673445\end{array}$ $\begin{array}{lllll}48 B & 1.454506 & 1.327627 & -0.001194\end{array}$ 49B $\quad 0.909086 \quad 1.838947-1.194889$ $\begin{array}{llll}50 \mathrm{~B} & 0.909040 & 1.841117 & 1.191552\end{array}$ $\begin{array}{llll}51 B & 0.015841 & 0.610375 & 2.799768\end{array}$ $\begin{array}{lllll}52 \mathrm{~B} & 0.015947 & 0.605282 & -2.800899\end{array}$ $\begin{array}{lllll}53 \mathrm{~B} & 0.030744 & -0.810787 & 2.747730\end{array}$ $\begin{array}{llll}54 \mathrm{~B} & 0.030847 & -0.815783 & -2.746276\end{array}$ $\begin{array}{lllll}55 \mathrm{~B} & 0.044182 & -2.059222 & 2.006425\end{array}$ $\begin{array}{llll}56 \mathrm{~B} & 0.044257 & -2.062869 & -2.002700\end{array}$ $\begin{array}{lllll}57 \mathrm{~B} & 0.050737 & -2.786654 & 0.736713\end{array}$ $\begin{array}{lllll}58 B & 0.050764 & -2.787989 & -0.731667\end{array}$ $\begin{array}{llll}59 \mathrm{~B} & 1.180371 & -1.367742 & -2.274432\end{array}$ $\begin{array}{lllll}60 \mathrm{~B} & 1.180286 & -1.363602 & 2.276933\end{array}$ $\begin{array}{llll}61 B & -1.055891 & 2.146483 & -0.001985\end{array}$ $\begin{array}{lllll}62 \mathrm{~B} & 1.191670 & -2.665752 & 0.002434\end{array}$ $\begin{array}{llll}63 \mathrm{~B} & -1.098037 & -2.353508 & -1.314892\end{array}$ $\begin{array}{lllll}64 \mathrm{~B} & -1.098086 & -2.351112 & 1.319103\end{array}$ $\begin{array}{llll}65 B & -1.128779 & -0.124609 & -2.672217\end{array}$ $\begin{array}{lllll}66 \mathrm{~B} & -1.128881 & -0.119750 & 2.672369\end{array}$ $\begin{array}{llll}67 \mathrm{~B} & 1.185912 & 1.205338 & -2.351734\end{array}$ $\begin{array}{lllll}68 \mathrm{~B} & 1.185823 & 1.209613 & 2.349555\end{array}$ $\begin{array}{llll}69 \mathrm{~B} & 0.032919 & 1.854078 & -2.271260\end{array}$ $\begin{array}{llll}70 \mathrm{~B} & 0.032832 & 1.858205 & 2.267860\end{array}$ 
Table S4. The Cartesian coordinates of the lowest energies of the binary Lennard-Jones clusters for $s=$ 1.4, 1.5, 1.6, 1.7, 1.8, 1.9, 2.0.

$$
s=1.4
$$

$$
E 5 / \varepsilon=-9.168058
$$

atom $\mathrm{x} / \sigma \quad y / \sigma \quad z / \sigma$

$\begin{array}{llll}1 \mathrm{~A} & 0.000000 & 0.000000 & 0.000000\end{array}$

$\begin{array}{llll}2 \mathrm{~A} & 1.124022 & 0.000000 & 0.000000\end{array}$

$\begin{array}{llll}3 \mathrm{~A} & 0.562011 & -0.973432 & 0.000000\end{array}$

4B $\quad 0.562011-0.324478-1.175919$

5B $\quad 0.562011-0.324478 \quad 1.175919$

\section{$E 6 / \varepsilon=-12.797426$}

\begin{tabular}{cccc} 
atom & $\mathrm{x} / \sigma$ & $y / \sigma$ & \multicolumn{1}{c}{$z / \sigma$} \\
1A & 0.000000 & 0.000000 & 0.000000 \\
2A & 1.428479 & 0.000000 & 0.000000 \\
3B & 0.714240 & -1.123439 & 0.000000 \\
4B & 0.714240 & 0.000000 & -1.123439 \\
5B & 0.714240 & 0.000000 & 1.123439 \\
6B & 0.714240 & 1.123439 & 0.000000
\end{tabular}

\section{$E 7 / \varepsilon=-16.584984$}

\begin{tabular}{cccc} 
atom & $\mathrm{x} / \sigma$ & $y / \sigma$ & \multicolumn{1}{c}{$z / \sigma$} \\
1A & 0.000000 & 0.000000 & 0.000000 \\
2B & 1.357580 & 0.000000 & 0.000000 \\
3B & 0.435909 & -1.268000 & 0.000000 \\
4B & 0.436723 & 0.997830 & 0.782784 \\
5B & 0.436723 & 0.997830 & -0.782784 \\
6A & 0.475301 & -0.330713 & 0.957995 \\
7A & 0.475301 & -0.330713 & -0.957995
\end{tabular}

$E 8 / \varepsilon=-20.491743$

\begin{tabular}{ccrc} 
atom & $\mathrm{x} / \sigma$ & $y / \sigma$ & \multicolumn{1}{c}{$z / \sigma$} \\
1A & 0.000000 & 0.000000 & 0.000000 \\
2A & 1.129529 & 0.000000 & 0.000000 \\
3B & 0.564764 & -1.207598 & 0.000000 \\
4A & 0.564764 & 0.485415 & -0.828600 \\
5B & 1.343739 & -0.545947 & -1.190464 \\
6B & -0.214212 & -0.545946 & -1.190464 \\
7B & 0.564763 & 1.157968 & 0.339096 \\
8B & 0.564763 & -0.174674 & 1.194419
\end{tabular}

$E 9 / \varepsilon=-25.532740$

$\begin{array}{cccc}\text { atom } & x / \sigma & y / \sigma & z / \sigma \\ \text { 1A } & 0.000000 & 0.000000 & 0.000000 \\ \text { 2B } & 1.307282 & 0.000000 & 0.000000 \\ \text { 3B } & 0.341600 & -1.261862 & 0.000000 \\ \text { 4B } & 0.341600 & 0.739019 & 1.022813 \\ \text { 5B } & -0.624083 & -0.522842 & 1.022812 \\ \text { 6B } & 0.341240 & -0.261144 & -1.234644 \\ \text { 7B } & 0.341241 & 1.153693 & -0.511405 \\ \text { 8B } & -1.024440 & -0.630850 & -0.511407 \\ \text { 9B } & -1.024440 & 0.783988 & 0.211831\end{array}$

\section{$E 10 / \varepsilon=-30.595637$}

\begin{tabular}{rrrr} 
atom & $x / \sigma$ & $y / \sigma$ & \multicolumn{1}{c}{$z / \sigma$} \\
1A & 0.000000 & 0.000000 & 0.000000 \\
2B & 1.309426 & 0.000000 & 0.000000 \\
3B & 0.245227 & -1.286259 & 0.000000 \\
4B & -0.818971 & -0.405785 & 0.937669 \\
5B & 0.245230 & 0.880476 & 0.937668 \\
6B & -1.022484 & -0.587101 & -0.609167 \\
7B & 0.385225 & 1.114343 & -0.609168 \\
8B & -1.022483 & 0.845962 & 0.011004 \\
9B & 0.385226 & -0.318722 & -1.229335 \\
10A & 0.500593 & -0.414170 & 0.957050
\end{tabular}

$E 11 / \varepsilon=-35.274878$

atom $\mathrm{x} / \sigma \quad \mathrm{y} / \sigma$ $z / \sigma$

$\begin{array}{|rrrr|}1 \mathrm{~A} & 0.000000 & 0.000000 & 0.000000 \\ 2 \mathrm{~B} & 1.323266 & 0.000000 & 0.000000 \\ 3 \mathrm{~B} & 0.344389 & -1.277665 & 0.000000 \\ 4 \mathrm{~B} & 0.344390 & 0.749961 & 1.034401 \\ 5 \mathrm{~B} & -0.634488 & -0.527703 & 1.034402 \\ 6 \mathrm{~B} & 0.400682 & -0.306981 & -1.218638 \\ 7 \mathrm{~B} & -1.022315 & -0.690543 & -0.466781 \\ 8 \mathrm{~B} & 0.400683 & 1.166804 & -0.466782 \\ 9 \mathrm{~B} & -1.022314 & 0.783242 & 0.285076 \\ 10 \mathrm{~A} & -0.614657 & 0.470916 & -0.923086 \\ 11 \mathrm{~A} & 0.684511 & -0.524435 & 1.027994\end{array}$

\section{$E 12 / \varepsilon=-39.688603$}

atom $\mathrm{x} / \sigma \quad \mathrm{y} / \sigma \quad \mathrm{z} / \sigma$

$\begin{array}{llll}1 \mathrm{~A} & 0.000000 & 0.000000 & 0.000000\end{array}$

$\begin{array}{llll}2 \mathrm{~B} & 1.311588 & 0.000000 & 0.000000\end{array}$

$\begin{array}{llll}3 \mathrm{~B} & 0.364072 & -1.260046 & 0.000000\end{array}$

$\begin{array}{lllll}4 \mathrm{~B} & -0.857166 & 0.644562 & -0.772968\end{array}$

$\begin{array}{lllll}5 A & 0.441371 & 0.363806 & -0.954437\end{array}$

$\begin{array}{llll}6 \mathrm{~A} & -0.226993 & -0.525013 & -0.954437\end{array}$

$\begin{array}{llll}7 \mathrm{~B} & 0.358811 & 1.277244 & 0.008300\end{array}$

$\begin{array}{llll}8 \mathrm{~B} & -1.127452 & -0.699250\end{array}$

$\begin{array}{lll}9 B & -0.845320 & 0.635655\end{array}$

0.008301

$\begin{array}{lll}10 A & -0.205900 & -0.536056\end{array}$

0.793882

$\begin{array}{llll}11 \mathrm{~A} & 0.457837 & 0.346609 & 0.959506\end{array}$

$\begin{array}{llll}12 B & 1.027664 & -0.772772 & -1.322644\end{array}$

\section{$E 13 / \varepsilon=-45.511459$}

atom $\quad \mathrm{x} / \sigma \quad \mathrm{y} / \sigma$

$\begin{array}{lll} & 0.000000 & 0.000000\end{array}$

$\begin{array}{llll}2 \mathrm{~A} & 1.162625 & 0.000000 & 0.000000\end{array}$

$\begin{array}{llll}3 \mathrm{~B} & -1.334847 & -0.000001 & 0.000000\end{array}$

$\begin{array}{llll}4 \mathrm{~A} & 0.599099 & 0.216006 & 0.924718\end{array}$

$\begin{array}{llll}5 A & 0.599099 & 0.946209 & 0.080320\end{array}$

$\begin{array}{lllll}6 \mathrm{~A} & 0.599099 & 0.368784 & -0.875078\end{array}$

$\begin{array}{lllll}7 A & 0.599099 & -0.812710 & 0.491187\end{array}$

$\begin{array}{lllll}8 \mathrm{~A} & 0.599099 & -0.718288 & -0.621148\end{array}$

$\begin{array}{llll}9 \mathrm{~B} & -0.481013 & 1.117323 & -0.675291\end{array}$

$\begin{array}{llll}10 \mathrm{~B} & -0.481013 & 0.987511 & 0.853962\end{array}$

$\begin{array}{llll}11 \mathrm{~B} & -0.481013 & -0.296968 & -1.271314\end{array}$

$\begin{array}{llll}12 \mathrm{~B} & -0.481012 & -0.507009 & 1.203068\end{array}$

$\begin{array}{llll}13 \mathrm{~B} & -0.481012 & -1.300861 & -0.110425\end{array}$

\section{$E 14 / \varepsilon=-49.508949$}

$\begin{array}{llll}\text { atom } & \mathrm{x} / \sigma & \mathrm{y} / \sigma & \mathrm{z} / \sigma\end{array}$

$\begin{array}{llll}1 \mathrm{~A} & 0.000000 & 0.000000 & 0.000000\end{array}$

$\begin{array}{llll}2 \mathrm{~A} & 1.127352 & 0.000000 & 0.000000\end{array}$

$\begin{array}{llll}3 A & 0.592266 & -0.959242 & 0.000000\end{array}$

$\begin{array}{llll}4 \mathrm{~A} & 0.521580 & -0.290949 & -0.914384\end{array}$

$\begin{array}{lllll}5 \mathrm{~B} & 0.673056 & 1.015971 & -0.678662\end{array}$

$\begin{array}{llll}6 \mathrm{~B} & -0.510872 & -1.106442 & -0.678662\end{array}$

$\begin{array}{llll}7 \mathrm{~A} & 0.609418 & -0.339947 & 0.913923\end{array}$

$\begin{array}{llll}8 \mathrm{~B} & -0.645716 & 0.360193 & -1.120994\end{array}$

$\begin{array}{llll}9 \mathrm{~B} & -0.592637 & 1.211545 & 0.188371\end{array}$

$\begin{array}{llll}10 \mathrm{~B} & -1.342227 & -0.132237 & 0.188371\end{array}$

$\begin{array}{llll}11 \mathrm{~A} & -0.324398 & -0.858651 & 0.628930\end{array}$

$\begin{array}{llll}12 \mathrm{~A} & 0.560182 & 0.727125 & 0.628930\end{array}$

$\begin{array}{llll}13 A & -0.352689 & 0.196737 & 1.029165\end{array}$

$\begin{array}{llll}14 \mathrm{~B} & 1.670547 & -0.931868 & -0.780079\end{array}$

\section{$E 15 / \varepsilon=-54.477032$}

$$
\begin{array}{lccc}
\text { atom } & \mathrm{x} / \sigma & y / \sigma & z / \sigma
\end{array}
$$

\section{$z / \sigma$}

$\begin{array}{llll}1 \mathrm{~A} & 0.000000 & 0.000000 & 0.000000\end{array}$

$\begin{array}{llll}2 A & 1.079526 & 0.000000 & 0.000000\end{array}$

$\begin{array}{llll}3 B & 0.556964 & -1.240618 & 0.000000\end{array}$

$\begin{array}{lllll}4 \mathrm{~A} & 0.558971 & -0.302088 & -0.927172\end{array}$

$\begin{array}{lrrr}5 A & 0.558972 & -0.302088 & 0.927172 \\ 6 A & 0.523314 & 0.964169 & 0.000000\end{array}$

$\begin{array}{lll}6 A & 0.523314 & 0.964169\end{array}$ $\begin{array}{rrrr}\text { 7B } & -0.213255 & 0.746781 & 1.084870 \\ \text { 8B } & -0.213255 & 0.746781 & -1.084870 \\ \text { 9B } & -0.692887 & -0.762093 & 0.807401 \\ \text { 10B } & -0.692887 & -0.762094 & -0.807400 \\ \text { 11B } & 1.359862 & 0.766280 & -1.018760 \\ \text { 12B } & 1.359862 & 0.766281 & 1.018760 \\ \text { 13B } & -1.266295 & 0.443607 & 0.000000 \\ \text { 14B } & 1.817039 & -0.738623 & -0.793370 \\ \text { 15B } & 1.817040 & -0.738623 & 0.793369\end{array}$

$E 16 / \varepsilon=-60.382227$

atom $\quad \mathrm{x} / \sigma \quad \mathrm{y} / \sigma$

$\begin{array}{llll}1 \mathrm{~A} & 0.000000 & 0.000000 & 0.000000\end{array}$

$\begin{array}{llll}2 \mathrm{~A} & 1.078297 & 0.000000 & 0.000000\end{array}$

$\begin{array}{llll}3 B & 0.539149 & -1.288155 & 0.000000\end{array}$

$\begin{array}{llll}4 B & 0.539150 & 1.212605 & 0.000000\end{array}$

$\begin{array}{llll}5 A & 0.539149 & -0.361462 & 0.908853\end{array}$

$\begin{array}{lllll}6 \mathrm{~A} & 0.539149 & -0.361462 & -0.908853\end{array}$

$\begin{array}{llll}7 \mathrm{~B} & -0.248183 & 0.646524 & 1.187849\end{array}$

$\begin{array}{llll}8 \mathrm{~B} & -0.248183 & 0.646524 & -1.187849\end{array}$

$\begin{array}{llll}9 B & 1.326481 & 0.646524 & 1.187850\end{array}$

$\begin{array}{llll}10 B & 1.326481 & 0.646524 & -1.187850\end{array}$

$\begin{array}{llll}11 B & 1.800066 & -0.787221 & -0.781957\end{array}$

$\begin{array}{lllll}12 \mathrm{~B} & 1.800066 & -0.787221 & 0.781957\end{array}$

$\begin{array}{llll}13 \mathrm{~B} & -0.721767 & -0.787222 & -0.781958\end{array}$

$\begin{array}{llll}14 \mathrm{~B} & -0.721767 & -0.787222 & 0.781958\end{array}$

$\begin{array}{llll}15 \mathrm{~B} & 2.300474 & 0.491473 & 0.000000\end{array}$ $\begin{array}{llll}16 B & -1.222177 & 0.491471 & 0.000000\end{array}$

$E 17 / \varepsilon=-65.332397$

atom $\mathrm{X} / \sigma \quad \mathrm{yl}$

$\begin{array}{llll}1 \mathrm{~A} & 0.000000 & 0.000000 & 0.000000\end{array}$

$\begin{array}{lllll}2 \mathrm{~A} & 1.077076 & 0.000000 & 0.000000\end{array}$

$\begin{array}{llll}3 \mathrm{~B} & 0.538537 & -1.224158 & 0.000000\end{array}$

$\begin{array}{llll}4 \mathrm{~B} & 0.538537 & 0.712228 & 0.995637\end{array}$

$\begin{array}{lllll}5 \mathrm{~A} & 0.538537 & 0.440928 & -0.857548\end{array}$

$\begin{array}{llll}6 \mathrm{~B} & -0.236599 & -0.608287 & 1.183039\end{array}$

$\begin{array}{llll}7 \mathrm{~B} & 1.313673 & -0.608287 & 1.183039\end{array}$

$\begin{array}{lllll}8 \mathrm{~A} & 1.085593 & 1.106981 & -0.139731\end{array}$

$\begin{array}{lllll}9 A & -0.008519 & 1.106981 & -0.139731\end{array}$

$10 A \quad-0.008519 \quad-0.530406-0.981632$

$\begin{array}{lllll}11 \mathrm{~A} & 1.085593 & -0.530407 & -0.981631\end{array}$

$\begin{array}{lllll}12 B & 1.899563 & 0.495209 & -0.963118\end{array}$

$\begin{array}{lllll}13 B & -0.822488 & 0.495209 & -0.963118\end{array}$

$\begin{array}{lllll}14 \mathrm{~B} & -1.007667 & -0.829216 & -0.144188\end{array}$

$\begin{array}{lllll}15 B & 2.084742 & -0.829216 & -0.144188\end{array}$

$\begin{array}{lllll}16 \mathrm{~B} & 2.084742 & 0.599718 & 0.590531\end{array}$

$\begin{array}{llll}17 \mathrm{~B} & -1.007667 & 0.599717 & 0.590531\end{array}$

$E / 18 \varepsilon=-70.757536$

$\begin{array}{llll}\text { atom } & \mathrm{x} / \sigma & \mathrm{y} / \sigma & \mathrm{z} / \sigma\end{array}$

$\begin{array}{llll}1 \mathrm{~A} & 0.000000 & 0.000000 & 0.000000\end{array}$

$\begin{array}{lllll}2 \mathrm{~A} & 1.068583 & 0.000000 & 0.000000\end{array}$

$\begin{array}{llll}3 \mathrm{~B} & 0.472375 & -1.290041 & 0.000000\end{array}$

$\begin{array}{lllll}4 \mathrm{~A} & 0.539432 & -0.354732 & 0.886332\end{array}$

$\begin{array}{llll}5 \mathrm{~B} & -0.716191 & -0.673692 & 0.892452\end{array}$

$\begin{array}{lllll}6 \mathrm{~B} & 0.509702 & 1.215967 & 0.025333\end{array}$

$\begin{array}{llll}7 \mathrm{~B} & 1.274938 & 0.641774 & 1.219719\end{array}$

$\begin{array}{lllll}8 \mathrm{~B} & 1.755298 & -0.813443 & 0.806258\end{array}$

$\begin{array}{lllll}9 \mathrm{~A} & 0.501198 & -0.371929 & -0.911803\end{array}$

$\begin{array}{llll}10 A & -0.085764 & 0.535832 & 0.977633\end{array}$

$\begin{array}{lllll}11 \mathrm{~B} & 1.307560 & 0.637842 & -1.157045\end{array}$

$\begin{array}{lllll}12 B & -0.272188 & 0.653714 & -1.168568\end{array}$

$\begin{array}{lllll}\text { 13B } & 1.762089 & -0.811401 & -0.759516\end{array}$

$\begin{array}{lllll}14 \mathrm{~B} & -0.818082 & -0.727974 & -0.687868\end{array}$

$\begin{array}{llll}15 \mathrm{~B} & -1.109768 & 0.658640 & 0.137896\end{array}$

$\begin{array}{llll}16 B & 2.284609 & 0.467289 & 0.044596\end{array}$

$\begin{array}{llll}17 \mathrm{~B} & 0.170159 & -0.088561 & 2.114292\end{array}$ 


\begin{tabular}{|c|c|c|c|}
\hline $18 \mathrm{~B}$ & 0.507957 & -1.495441 & 1.532933 \\
\hline \multicolumn{4}{|c|}{$E 19 / \varepsilon=-77.314533$} \\
\hline atom & $\mathrm{x} / \sigma$ & $y / \sigma$ & $z / \sigma$ \\
\hline $1 \mathrm{~A}$ & 0.000000 & 0.000000 & 0.000000 \\
\hline $2 \mathrm{~A}$ & 1.173890 & 0.000000 & 0.000000 \\
\hline $3 \mathrm{~A}$ & 0.586944 & -0.941322 & 0.000000 \\
\hline $4 \mathrm{~A}$ & 0.586945 & 0.761546 & -0.553295 \\
\hline $5 \mathrm{~A}$ & 0.586944 & -0.290884 & -0.895250 \\
\hline $6 \mathrm{~A}$ & 0.586945 & -0.290884 & 0.895251 \\
\hline $7 \mathrm{~A}$ & 0.586946 & 0.761546 & 0.553295 \\
\hline $8 \mathrm{~B}$ & -0.473978 & 0.404933 & -1.246251 \\
\hline 9B & -0.473978 & -1.060124 & -0.770225 \\
\hline $10 B$ & 1.647868 & 0.404931 & 1.246251 \\
\hline $11 \mathrm{~B}$ & 1.647868 & 1.310386 & 0.000000 \\
\hline $2 B$ & 1.647866 & -1.060126 & 0.770226 \\
\hline $13 B$ & 1.647867 & 0.404932 & -1.246252 \\
\hline 14B & 1.647866 & -1.060125 & -0.770227 \\
\hline $15 B$ & -0.473976 & 1.310388 & 0.000001 \\
\hline $16 \mathrm{~B}$ & -0.473977 & -1.060124 & 0.770227 \\
\hline $17 \mathrm{R}$ & -0.473976 & 0.404933 & 1.246253 \\
\hline 1 & -1.324398 & 0.000001 & 0.000002 \\
\hline & 2.498288 & -0.000002 & -0.000001 \\
\hline
\end{tabular}

$E 20 / \varepsilon=-82.495693$

$\begin{array}{llll}1 \mathrm{~A} & 0.000000 & 0.000000 & 0.000000\end{array}$

2A $\quad 1.149505 \quad 0.000000 \quad 0.000000$

$\begin{array}{llll}3 A & 0.574752 & -0.952629 & 0.000000\end{array}$

$\begin{array}{lllll}4 \mathrm{~A} & 0.574751 & -0.340219 & 0.889804\end{array}$

$\begin{array}{lllll}5 \mathrm{~A} & 0.574753 & -0.297721 & -0.893889\end{array}$

$\begin{array}{llll}6 \mathrm{~A} & 0.574752 & 0.728611 & 0.597329\end{array}$

$\begin{array}{lllll}7 A & 0.574753 & 0.772526 & -0.531691\end{array}$

$\begin{array}{llll}8 A & 1.522972 & -0.851866 & 0.586298\end{array}$

$\begin{array}{llll}9 \mathrm{~A} & -0.373470 & -0.851865 & 0.586297\end{array}$

$\begin{array}{llll}10 B & -0.482852 & 0.296434 & 1.276761\end{array}$

$\begin{array}{llll}11 B & 1.632355 & -1.086694 & -0.732863\end{array}$

$\begin{array}{llll}12 B & -0.482852 & -1.086693 & -0.732864\end{array}$

$\begin{array}{llll}13 \mathrm{~B} & 1.632355 & 0.296431 & 1.276764\end{array}$

$\begin{array}{llll}14 \mathrm{~B} & 1.650150 & 0.390721 & -1.205832\end{array}$

$\begin{array}{llll}15 \mathrm{~B} & 1.650150 & 1.265852 & 0.065698\end{array}$

$\begin{array}{llll}16 \mathrm{~B} & -0.500643 & 1.265853 & 0.065696\end{array}$

$\begin{array}{lllll}17 \mathrm{~B} & -0.500643 & 0.390722 & -1.205834\end{array}$

$\begin{array}{llll}18 \mathrm{~B} & -1.329483 & -0.061373 & 0.042240\end{array}$

$\begin{array}{llll}19 \mathrm{~B} & 2.478987 & -0.061376 & 0.042244\end{array}$

$\begin{array}{llll}\text { 20B } & 0.574750 & -1.634602 & 1.125019\end{array}$

$E 21 / \varepsilon=-88.025471$

$\begin{array}{cccc}\text { atom } & \mathrm{x} / \sigma & y / \sigma & z / \sigma \\ 1 \mathrm{~A} & 0.000000 & 0.000000 & 0.000000\end{array}$

$\begin{array}{llll}2 \mathrm{~A} & 1.143552 & 0.000000 & 0.000000\end{array}$

$\begin{array}{llll}3 \mathrm{~A} & 0.631350 & -0.953471 & 0.000000\end{array}$

$\begin{array}{lllll}4 \mathrm{~A} & 0.565963 & -0.304034 & -0.870382\end{array}$

$\begin{array}{lllll}5 B & -0.408481 & -1.163658 & -0.797102\end{array}$

$\begin{array}{llll}6 \mathrm{~B} & 0.744716 & 0.983034 & -0.797103\end{array}$

$\begin{array}{llll}7 \mathrm{~A} & 0.558151 & -0.299836 & 0.889909\end{array}$

$\begin{array}{llll}8 \mathrm{~B} & -0.426612 & -1.162470 & 0.743274\end{array}$

$\begin{array}{llll}9 \mathrm{~B} & 0.733717 & 0.997497 & 0.743273\end{array}$

$\begin{array}{llll}\text { 10B } & 1.948323 & -1.046633 & -0.010071\end{array}$

11B $\quad 1.064478-1.464406-1.196799$

$\begin{array}{llll}12 \mathrm{~B} & 1.808688 & -0.079050 & -1.196799\end{array}$

$\begin{array}{llll}13 \mathrm{~B} & -0.542524 & 0.291441 & -1.267745\end{array}$

$\begin{array}{llll}14 \mathrm{~B} & 1.056713 & -1.470330 & 1.172724\end{array}$

$\begin{array}{llll}15 B & 1.809341 & -0.069303 & 1.172724\end{array}$

$\begin{array}{llll}16 \mathrm{~B} & -0.559582 & 0.300607 & 1.225481\end{array}$

$\begin{array}{llll}17 \mathrm{~B} & -1.330967 & -0.173858 & -0.029460\end{array}$

$\begin{array}{lllll}18 B & -0.589860 & 1.205722 & -0.029461\end{array}$

$\begin{array}{llll}19 B & 2.111990 & 0.881298 & -0.018003\end{array}$

$\begin{array}{lllll}20 \mathrm{~B} & 0.431210 & -2.247497 & -0.018002\end{array}$

21B $\quad 0.580801 \quad-0.312001-2.162446$

$E 22 / \varepsilon=-93.601467$

\begin{tabular}{|c|c|c|c|}
\hline om & $\mathrm{x} / \sigma$ & $y / \sigma$ & $z / \sigma$ \\
\hline $1 \mathrm{~A}$ & 0.000000 & 0.000000 & 0.000000 \\
\hline $2 \mathrm{~A}$ & 1.128779 & 0.000000 & 0.000000 \\
\hline $3 A$ & 0.559560 & -0.984600 & 0.000000 \\
\hline $4 \mathrm{~A}$ & 0.565306 & -0.333589 & 0.878954 \\
\hline $5 A$ & 0.565306 & -0.333589 & -0.878954 \\
\hline $6 \mathrm{~A}$ & 0.564288 & 0.748104 & 0.573595 \\
\hline $7 \mathrm{~A}$ & 0.564288 & 0.748105 & -0.573594 \\
\hline $8 A$ & -0.371480 & -0.858593 & -0.576428 \\
\hline $9 A$ & -0.371481 & -0.858593 & 0.576427 \\
\hline $10 \mathrm{~A}$ & 1.502321 & -0.868461 & -0.563396 \\
\hline $11 \mathrm{~A}$ & 1.502321 & -0.868461 & 0.563396 \\
\hline $12 B$ & -0.506606 & 0.298133 & 1.238203 \\
\hline $13 B$ & -0.506604 & 0.298135 & -1.238203 \\
\hline $14 B$ & 1.636613 & 0.293979 & -1.236513 \\
\hline $15 B$ & 1.636613 & 0.293977 & 1.236514 \\
\hline $16 \mathrm{~B}$ & -1.327696 & -0.141408 & -0.000001 \\
\hline 17B & -0.532614 & 1.226211 & 0.000000 \\
\hline $18 \mathrm{~B}$ & 1.658320 & 1.229384 & 0.000001 \\
\hline $19 B$ & 2.459303 & -0.128964 & 0.000000 \\
\hline $20 B$ & 0.580000 & -1.597472 & 1.190838 \\
\hline . & 0.580001 & -1.597472 & -1.190838 \\
\hline & -0.2966 & -2.0437 & 0.00000 \\
\hline
\end{tabular}

$E 23 / \varepsilon=-100.740941$

atom $\quad \mathrm{x} / \sigma \quad \mathrm{y} / \sigma$

$\begin{array}{llll}1 \mathrm{~A} & 0.000000 & 0.000000 & 0.000000\end{array}$

$\begin{array}{llll}2 \mathrm{~A} & 1.132338 & 0.000000 & 0.000000\end{array}$

$\begin{array}{llll}3 \mathrm{~A} & 0.566169 & -0.980633 & 0.000000\end{array}$

$\begin{array}{llll}4 \mathrm{~A} & 0.566169 & -0.326878 & 0.876160\end{array}$

$\begin{array}{lllll}5 A & 0.566169 & -0.326878 & -0.876160\end{array}$

$\begin{array}{lllll}6 \mathrm{~A} & 1.498873 & -0.865374 & -0.572054\end{array}$

$\begin{array}{lllll}7 A & -0.366534 & -0.865374 & 0.572054\end{array}$

$\begin{array}{llll}8 \mathrm{~A} & 1.498873 & -0.865374 & 0.572054\end{array}$

$\begin{array}{lllll}9 \mathrm{~A} & -0.366534 & -0.865374 & -0.572054\end{array}$

$\begin{array}{lllll}10 A & 0.566169 & 0.750115 & -0.572054\end{array}$

$\begin{array}{llll}11 \mathrm{~A} & 0.566169 & 0.750115 & 0.572054\end{array}$

$\begin{array}{llll}12 \mathrm{~B} & 0.566170 & -1.566992 & 1.236889\end{array}$

$\begin{array}{llll}13 \mathrm{~B} & -0.507801 & 0.293180 & 1.236889\end{array}$

$\begin{array}{lllll}14 \mathrm{~B} & 1.640140 & 0.293180 & -1.236889\end{array}$

$\begin{array}{llll}15 B & -0.507801 & 0.293180 & -1.236889\end{array}$

$\begin{array}{llll}16 \mathrm{~B} & 0.566170 & -1.566992 & -1.236889\end{array}$

$\begin{array}{lllr}17 \mathrm{~B} & 1.640140 & 0.293180 & 1.236889\end{array}$

$\begin{array}{llll}18 \mathrm{~B} & 1.667041 & 1.223017 & 0.000000\end{array}$

$\begin{array}{llll}19 \mathrm{~B} & -0.534702 & 1.223018 & 0.000000\end{array}$

$\begin{array}{llll}20 B & 2.458853 & -0.148441 & 0.000000\end{array}$

$\begin{array}{llll}21 \mathrm{~B} & -0.225643 & -2.055208 & 0.000000\end{array}$

$\begin{array}{llll}22 \mathrm{~B} & 1.357982 & -2.055207 & 0.000000\end{array}$ $\begin{array}{llll}23 \mathrm{~B} & -1.326514 & -0.148441 & 0.000000\end{array}$

\section{$E 24 / \varepsilon=-106.012643$}

$\begin{array}{lll}\text { atom } \quad \mathrm{X} / \sigma & \mathrm{y} / \sigma\end{array}$

$\begin{array}{lll}1 \mathrm{~A} & 0.000000 & 0.000000\end{array}$

$z / \sigma$

2A $\quad 1.135260 \quad 0.000000$

3A $\quad 0.567630 \quad-0.983164$

$\begin{array}{lll}4 \mathrm{~A} & 0.567630 & -0.327721\end{array}$

$\begin{array}{lll}5 \mathrm{~A} & 0.567631 & -0.327721\end{array}$

$\begin{array}{llll}6 \mathrm{~A} & 1.497924 & -0.864827\end{array}$

$\begin{array}{lll}7 A & -0.362664 & -0.864827\end{array}$

$\begin{array}{lll}8 \mathrm{~A} & 0.567630 & 0.746490\end{array}$

$\begin{array}{lll}9 \mathrm{~A} & -0.365453 & -0.866438\end{array}$

$\begin{array}{lll}10 A & 0.567631 & 0.749711\end{array}$

$\begin{array}{lll}11 \mathrm{~A} & 1.500715 & -0.866438\end{array}$

$\begin{array}{lll}12 \mathrm{~B} & -0.508718 & 0.293709\end{array}$

$\begin{array}{lll}13 \mathrm{~B} & 0.567630 & -1.570581\end{array}$

$\begin{array}{lll}14 \mathrm{~B} & 1.643977 & 0.293709\end{array}$

$\begin{array}{lll}15 B & 1.642303 & 0.292740\end{array}$

$\begin{array}{lllll}16 B & 0.567631 & -1.568645 & -1.233754\end{array}$

$\begin{array}{llll}17 \mathrm{~B} & -0.507041 & 0.292740 & -1.233755\end{array}$

$\begin{array}{llll}18 \mathrm{~B} & 2.460307 & -0.151212 & 0.003326\end{array}$

$\begin{array}{llll}19 B & -0.531570 & 1.223130 & 0.003322\end{array}$

$\begin{array}{llll}10 B & -1.325047 & -0.151212 & 0.003323\end{array}$ \begin{tabular}{|llll}
$21 \mathrm{~B}$ & 1.361107 & -2.055082 & 0.003324
\end{tabular} $\begin{array}{llll}22 B & 1.666830 & 1.223130 & 0.003324\end{array}$ $\begin{array}{llll}23 \mathrm{~B} & -0.225847 & -2.055083 & 0.003324\end{array}$ $\begin{array}{llll}24 \mathrm{~B} & 0.567628 & -0.327721 & 2.174920\end{array}$

$E 25 / \varepsilon=-111.721202$

$\begin{array}{llll}\text { atom } \mathrm{x} / \sigma & \mathrm{y} / \sigma & \mathrm{z} / \sigma\end{array}$

$\begin{array}{llll}1 \mathrm{~A} & 0.000000 & 0.000000 & 0.000000\end{array}$

$\begin{array}{llll}2 \mathrm{~A} & 1.112432 & 0.000000 & 0.000000\end{array}$

$\begin{array}{llll}3 \mathrm{~A} & 0.548334 & -0.967902 & 0.000000\end{array}$

$\begin{array}{llll}4 \mathrm{~A} & 0.544591 & -0.317390 & 0.911073\end{array}$

$\begin{array}{lllll}5 \mathrm{~A} & 0.532271 & -0.310210 & -0.885358\end{array}$

$\begin{array}{llll}6 \mathrm{~A} & 0.561764 & 0.736317 & 0.594129\end{array}$

$\begin{array}{llll}7 A & -0.363752 & -0.851719 & 0.594128\end{array}$

$\begin{array}{llll}8 \mathrm{~A} & 1.494042 & -0.870735 & 0.537747\end{array}$

$\begin{array}{lllll}9 \mathrm{~A} & 1.461146 & -0.851563 & -0.606317\end{array}$

$10 \mathrm{~A} \quad-0.401833 \quad-0.851094 \quad-0.551301$

$\begin{array}{llll}11 \mathrm{~A} & 0.542449 & 0.769142 & -0.551300\end{array}$

$\begin{array}{llll}12 \mathrm{~A} & -0.415775 & 0.242316 & 0.961442\end{array}$

$\begin{array}{llll}13 B & 1.683082 & 0.247249 & 1.209860\end{array}$

$\begin{array}{llll}14 \mathrm{~B} & 0.614488 & -1.586283 & 1.209860\end{array}$

$\begin{array}{llll}15 B & -1.332692 & -0.133543 & 0.083200\end{array}$

$\begin{array}{llll}16 \mathrm{~B} & -0.540709 & 1.225372 & 0.083200\end{array}$

$\begin{array}{llll}17 \mathrm{~B} & -0.558078 & 0.325251 & -1.182124\end{array}$

$\begin{array}{llll}18 \mathrm{~B} & 1.595473 & 0.325609 & -1.246980\end{array}$

19B $\quad 0.503128 \quad-1.548683-1.246980$

$\begin{array}{llll}20 \mathrm{~B} & -0.246432 & -2.039275 & 0.017186\end{array}$

$\begin{array}{llll}21 \mathrm{~B} & 1.652857 & 1.219604 & 0.017187\end{array}$

$\begin{array}{llll}22 \mathrm{~B} & 2.446137 & -0.158309 & -0.058210\end{array}$

$\begin{array}{lllll}23 B & 1.343478 & -2.050296 & -0.058209\end{array}$

$\begin{array}{llll}24 \mathrm{~B} & 0.390557 & 0.666372 & 1.899665\end{array}$

$\begin{array}{llll}25 B & -0.387291 & -0.668281 & 1.899663\end{array}$

\section{$E 26 / \varepsilon=-118.914648$}

atom $\mathrm{X} / \sigma \quad \mathrm{y} / \sigma$

$\begin{array}{llll}1 \mathrm{~A} & 0.000000 & 0.000000 & 0.000000\end{array}$

$\begin{array}{llll}2 \mathrm{~A} & 1.106665 & 0.000000 & 0.000000\end{array}$

$\begin{array}{llll}3 \mathrm{~A} & 0.553332 & -0.958401 & 0.000000\end{array}$

$\begin{array}{llll}4 \mathrm{~A} & 0.553332 & -0.319466 & 0.903589\end{array}$

$\begin{array}{lllll}5 \mathrm{~A} & 1.462844 & -0.844574 & 0.597204\end{array}$

$\begin{array}{llll}6 \mathrm{~A} & -0.356180 & -0.844573 & 0.597204\end{array}$

$\begin{array}{llll}7 \mathrm{~A} & 0.553333 & 0.730748 & 0.597204\end{array}$

$\begin{array}{lllll}8 \mathrm{~A} & 0.553332 & -0.319467 & -0.888023\end{array}$

$\begin{array}{lllll}9 \mathrm{~A} & 0.553331 & -1.410964 & 0.997703\end{array}$

$\begin{array}{lllll}10 A & 0.553333 & 0.772031 & -0.545909\end{array}$

$\begin{array}{llll}11 \mathrm{~A} & -0.391933 & -0.865216 & -0.545908\end{array}$

$\begin{array}{lllll}12 \mathrm{~A} & 1.498598 & -0.865217 & -0.545908\end{array}$

$\begin{array}{llll}13 \mathrm{~A} & 1.498598 & 0.226282 & 0.997703\end{array}$

$\begin{array}{llll}14 \mathrm{~A} & -0.391933 & 0.226283 & 0.997703\end{array}$

$\begin{array}{llll}15 \mathrm{~B} & -1.323784 & -0.134588 & 0.095168\end{array}$

$\begin{array}{llll}16 \mathrm{~B} & -0.545335 & 0.314849 & -1.176034\end{array}$

$\begin{array}{lllll}17 \mathrm{~B} & 1.331780 & -2.037537 & 0.095170\end{array}$

$\begin{array}{llll}18 \mathrm{~B} & -0.545335 & 1.213725 & 0.095167\end{array}$

$\begin{array}{llll}19 \mathrm{~B} & 2.430449 & -0.134589 & 0.095169\end{array}$

$\begin{array}{lllll}20 \mathrm{~B} & 0.553332 & -1.588100 & -1.176033\end{array}$

$\begin{array}{llll}21 \mathrm{~B} & 1.652001 & 1.213724 & 0.095168\end{array}$

$\begin{array}{llll}22 \mathrm{~B} & -0.225118 & -2.037536 & 0.095169\end{array}$

$\begin{array}{llll}23 B & 1.652000 & 0.314848 & -1.176033\end{array}$

$\begin{array}{llll}24 \mathrm{~B} & -0.225117 & -0.768903 & 1.889285\end{array}$

$\begin{array}{llll}25 B & 0.553332 & 0.579410 & 1.889285\end{array}$

$\begin{array}{llll}26 B & 1.331781 & -0.768904 & 1.889286\end{array}$

$E 27 / \varepsilon=-124.066931$

atom $\mathrm{x} / \sigma \quad y / \sigma \quad z / \sigma$

$\begin{array}{llll}1 \mathrm{~A} & 0.000000 & 0.000000 & 0.000000\end{array}$

$\begin{array}{llll}2 \mathrm{~A} & 1.111020 & 0.000000 & 0.000000\end{array}$

$\begin{array}{llll}3 \mathrm{~A} & 0.555510 & -0.962171 & 0.000000\end{array}$

$\begin{array}{llll}4 \mathrm{~A} & 0.555510 & -0.320723 & 0.900102\end{array}$

$\begin{array}{lllll}5 A & 0.555510 & -0.320723 & -0.869063\end{array}$

$\begin{array}{llll}6 \mathrm{~A} & -0.355416 & -0.846646 & 0.592316\end{array}$

$\begin{array}{llll}7 A & 1.466436 & -0.846646 & 0.592316\end{array}$

$\begin{array}{llll}8 \mathrm{~A} & 0.555510 & 0.731123 & 0.592315\end{array}$ 
9A $\quad-0.385584 \quad-0.864064 \quad-0.555223$

$\begin{array}{lllll}10 A & 1.496604 & -0.864064 & -0.555223\end{array}$

$\begin{array}{llll}11 \mathrm{~A} & 0.555510 & 0.765958 & -0.555223\end{array}$

$\begin{array}{llll}12 \mathrm{~A} & 0.555510 & -1.414571 & 0.993957\end{array}$

$\begin{array}{llll}13 \mathrm{~A} & -0.391790 & 0.226202 & 0.993957\end{array}$

$\begin{array}{llll}14 \mathrm{~A} & 1.502810 & 0.226201 & 0.993957\end{array}$

15B $\quad-0.532771 \quad 0.307596 \quad-1.192687$

$\begin{array}{lllll}16 \mathrm{~B} & 0.555510 & -1.577361 & -1.192688\end{array}$

$\begin{array}{llll}17 \mathrm{~B} & 1.643791 & 0.307596 & -1.192687\end{array}$

$\begin{array}{llll}18 \mathrm{~B} & -1.319406 & -0.136112 & 0.080691\end{array}$

$\begin{array}{llll}19 B & 1.333090 & -2.036753 & 0.080691\end{array}$

$\begin{array}{llll}20 \mathrm{~B} & -0.541826 & 1.210696 & 0.080691\end{array}$

$\begin{array}{llll}21 B & -0.222070 & -2.036753 & 0.080691\end{array}$

$\begin{array}{llll}22 \mathrm{~B} & 1.652846 & 1.210696 & 0.080691\end{array}$

$\begin{array}{llll}23 B & 2.430426 & -0.136112 & 0.080691\end{array}$

$\begin{array}{llll}24 \mathrm{~B} & -0.223030 & -0.770213 & 1.884415\end{array}$

$\begin{array}{llll}25 \mathrm{~B} & 1.334050 & -0.770213 & 1.884415\end{array}$

$\begin{array}{llll}26 \mathrm{~B} & 0.555510 & 0.578259 & 1.884414\end{array}$

$\begin{array}{llll}27 \mathrm{~B} & 0.555510 & -0.320721 & -2.159459\end{array}$

\section{$E 28 / \varepsilon=-129.778684$}

atom $\mathrm{X} / \sigma$ $y / \sigma$

$\begin{array}{llll}1 \mathrm{~A} & 0.000000 & 0.000000 & 0.000000\end{array}$

$\begin{array}{llll}2 A & 1.092188 & 0.000000 & 0.000000\end{array}$

$\begin{array}{llll}3 \mathrm{~A} & 0.537566 & -0.950736 & 0.000000\end{array}$

$\begin{array}{lllll}4 \mathrm{~A} & 0.515886 & -0.300947 & -0.906316\end{array}$

$\begin{array}{llll}5 \mathrm{~A} & 0.529280 & -0.308763 & 0.910651\end{array}$

$\begin{array}{llll}6 A & -0.391414 & -0.829252 & -0.578692\end{array}$

$\begin{array}{lllll}7 A & 0.529204 & 0.748873 & -0.578689\end{array}$

$\begin{array}{llll}8 \mathrm{~A} & 1.427452 & -0.832720 & -0.625472\end{array}$

$\begin{array}{llll}9 \mathrm{~A} & 0.548145 & 0.755306 & 0.569288\end{array}$

$\begin{array}{llll}10 A & -0.387693 & -0.848910 & 0.569285\end{array}$

$\begin{array}{llll}11 A & 0.502928 & -1.390372 & -1.005262\end{array}$

$\begin{array}{lllll}12 \mathrm{~A} & 1.457839 & 0.246539 & -1.005260\end{array}$

$\begin{array}{lllll}13 \mathrm{~A} & 1.498945 & -0.874428 & 0.511091\end{array}$

$\begin{array}{llll}14 \mathrm{~A} & -0.435265 & 0.253919 & -0.962297\end{array}$

$\begin{array}{llll}15 \mathrm{~A} & -0.442180 & 0.257949 & 0.938308\end{array}$

$\begin{array}{llll}16 \mathrm{~B} & 1.684580 & 0.267993 & 1.156217\end{array}$

$\begin{array}{llll}17 \mathrm{~B} & 0.595850 & -1.598312 & 1.156214\end{array}$

$\begin{array}{llll}18 \mathrm{~B} & -0.549716 & 1.212296 & -0.002783\end{array}$

$\begin{array}{llll}19 B & -1.325853 & -0.118161 & -0.002786\end{array}$

$\begin{array}{llll}20 \mathrm{~B} & -0.249808 & -2.019758 & -0.074406\end{array}$

$\begin{array}{llll}21 B & 1.635219 & 1.211562 & -0.074400\end{array}$

$\begin{array}{llll}22 \mathrm{~B} & 2.415802 & -0.136864 & -0.147896\end{array}$

$\begin{array}{llll}23 \mathrm{~B} & 1.308176 & -2.035560 & -0.147899\end{array}$

24B $\quad 1.266382-0.738755 \quad-1.911490$

$\begin{array}{llll}25 B & -0.291298 & -0.731308 & -1.872568\end{array}$

$\begin{array}{llll}26 \mathrm{~B} & 0.493223 & 0.613520 & -1.872566\end{array}$

$\begin{array}{llll}27 \mathrm{~B} & 0.390605 & 0.665318 & 1.872817\end{array}$

$\begin{array}{llll}28 B & -0.386902 & -0.667486 & 1.872815\end{array}$

$E 29 / \varepsilon=-136.240883$

atom $\mathrm{X} / \sigma$

$y / \sigma$

$\begin{array}{llll}1 \mathrm{~A} & 0.000000 & 0.000000 & 0.000000\end{array}$

$\begin{array}{llll}2 \mathrm{~A} & 1.089984 & 0.000000 & 0.000000\end{array}$

3A $\quad 0.530452 \quad-0.952200 \quad 0.000000$

$\begin{array}{llll}4 \mathrm{~A} & 0.537085 & -0.292453 & 0.911197\end{array}$

$\begin{array}{lllll}5 A & 0.516862 & -0.326867 & -0.911197\end{array}$

$\begin{array}{lllll}6 \mathrm{~A} & 0.548506 & 0.762374 & 0.572722\end{array}$

$\begin{array}{llll}7 \mathrm{~A} & -0.399067 & -0.850187 & -0.572722\end{array}$

$\begin{array}{llll}8 \mathrm{~A} & -0.390741 & -0.817888 & 0.576109\end{array}$

$\begin{array}{lllll}9 \mathrm{~A} & 0.524342 & 0.739382 & -0.576108\end{array}$

$\begin{array}{llll}10 \mathrm{~A} & 1.430292 & -0.867735 & -0.598452\end{array}$

$\begin{array}{llll}11 \mathrm{~A} & 1.454113 & -0.827198 & 0.598452\end{array}$

12B $\quad 0.504200 \quad-1.671104-1.122281$

$\begin{array}{llll}13 B & 1.705237 & 0.372796 & 1.122281\end{array}$

$\begin{array}{llll}14 \mathrm{~A} & 0.501028 & -1.388028 & 0.993919\end{array}$

$\begin{array}{lllll}15 \mathrm{~A} & 1.456399 & 0.237806 & -0.993919\end{array}$

$\begin{array}{llll}16 \mathrm{~A} & -0.436214 & 0.247387 & 0.955473\end{array}$

$\begin{array}{llll}17 A & -0.428403 & 0.260681 & -0.955473\end{array}$

$\begin{array}{llll}18 \mathrm{~B} & -1.326245 & -0.110689 & -0.009649\end{array}$

$\begin{array}{llll}19 \mathrm{~B} & -0.548733 & 1.212465 & 0.009650\end{array}$

$\begin{array}{llll}20 B & 2.404010 & -0.153217 & -0.130100\end{array}$ $\begin{array}{lllll}22 \mathrm{~B} & 1.629747 & 1.232776 & -0.127173\end{array}$

$\begin{array}{llll}23 \mathrm{~B} & -0.283809 & -2.023676 & 0.127174\end{array}$

$\begin{array}{llll}24 \mathrm{~B} & 0.506309 & 0.646108 & -1.860755\end{array}$

$\begin{array}{llll}25 \mathrm{~B} & -0.318033 & -0.756742 & 1.860756\end{array}$

$\begin{array}{llll}26 \mathrm{~B} & 0.417445 & 0.597201 & 1.898308\end{array}$

$\begin{array}{llll}27 \mathrm{~B} & -0.318557 & -0.655310 & -1.898308\end{array}$

$\begin{array}{llll}28 \mathrm{~B} & 1.311249 & -0.671631 & -1.929039\end{array}$

$\begin{array}{llll}29 B & 1.224865 & -0.818639 & 1.929039\end{array}$

$E 30 / \varepsilon=-141.678730$

$\begin{array}{ccc}\text { atom } & \mathrm{x} / \sigma & \mathrm{y} / \sigma\end{array}$

$\begin{array}{llll}1 \mathrm{~A} & 0.000000 & 0.000000 & 0.000000\end{array}$

$\begin{array}{llll}2 \mathrm{~A} & 1.104550 & 0.000000 & 0.000000\end{array}$

$\begin{array}{llll}3 \mathrm{~A} & 0.534062 & -0.955024 & 0.000000\end{array}$

$\begin{array}{lllll}4 \mathrm{~A} & 0.509082 & -0.289117 & 0.906197\end{array}$

$\begin{array}{lllll}5 \mathrm{~A} & 0.531027 & -0.315117 & -0.917908\end{array}$

$\begin{array}{lllll}6 \mathrm{~A} & 0.542789 & 0.732120 & -0.556608\end{array}$

$\begin{array}{lllll}7 \mathrm{~B} & 1.696238 & -0.979322 & -0.673101\end{array}$

$\begin{array}{llll}8 \mathrm{~A} & 0.510401 & 0.752461 & 0.576535\end{array}$

$\begin{array}{llll}9 A & -0.407868 & -0.833984 & -0.555511\end{array}$

$\begin{array}{llll}10 \mathrm{~A} & 1.429483 & -0.831965 & 0.630524\end{array}$

$\begin{array}{llll}11 \mathrm{~A} & -0.409063 & -0.796452 & 0.589526\end{array}$

$\begin{array}{llll}12 \mathrm{~B} & 1.686132 & 0.464380 & -1.122880\end{array}$

$\begin{array}{llll}13 \mathrm{~B} & 0.441874 & -1.676378 & -1.122955\end{array}$

$\begin{array}{llll}14 \mathrm{~A} & -0.442221 & 0.283553 & 0.953179\end{array}$

$\begin{array}{llll}15 \mathrm{~A} & -0.439146 & 0.272113 & -0.932280\end{array}$

$\begin{array}{llll}16 \mathrm{~A} & 1.428967 & 0.272689 & 1.020769\end{array}$

$\begin{array}{llll}17 A & 0.465681 & -1.364048 & 1.026732\end{array}$

$\begin{array}{llll}18 \mathrm{~B} & -0.529193 & 1.249533 & -0.026723\end{array}$

$\begin{array}{llll}19 \mathrm{~B} & 1.582156 & 1.293624 & 0.143286\end{array}$

$\begin{array}{llll}20 \mathrm{~B} & 0.366423 & 0.708850 & -1.860546\end{array}$

$\begin{array}{llll}21 B & -1.330665 & -0.073302 & 0.001640\end{array}$

$\begin{array}{llll}22 B & -0.341958 & -1.997926 & 0.145613\end{array}$

$\begin{array}{llll}23 B & 1.245752 & -0.716062 & 1.917201\end{array}$

$\begin{array}{llll}24 \mathrm{~B} & 0.472324 & 0.643818 & 1.872259\end{array}$

$\begin{array}{llll}25 \mathrm{~B} & -0.315710 & -0.688948 & 1.881295\end{array}$

$\begin{array}{llll}26 \mathrm{~B} & 2.426138 & -0.003800 & 0.223549\end{array}$

$\begin{array}{llll}27 \mathrm{~B} & 1.201361 & -2.096249 & 0.225927\end{array}$

$\begin{array}{lllll}28 \mathrm{~B} & -0.415602 & -0.640769 & -1.863874\end{array}$

$\begin{array}{llll}29 B & 1.123556 & -0.635715 & -2.066949\end{array}$

$\begin{array}{lllll}30 \mathrm{~B} & 0.634713 & 1.956799 & -0.923526\end{array}$

\section{$E 31 / \varepsilon=-147.678325$}

\section{atom $\quad \mathrm{x} / \sigma \quad \mathrm{y} / \sigma$}

$\begin{array}{llll}1 \mathrm{~A} & 0.000000 & 0.000000 & 0.000000\end{array}$

$\begin{array}{llll}2 \mathrm{~A} & 1.110516 & 0.000000 & 0.000000\end{array}$

$\begin{array}{llll}3 \mathrm{~A} & 0.535483 & -0.950830 & 0.000000\end{array}$

$\begin{array}{llll}4 \mathrm{~A} & 0.513076 & -0.281858 & 0.907278\end{array}$

$\begin{array}{lllll}5 A & 0.533021 & -0.300790 & -0.915679\end{array}$

$\begin{array}{llll}6 \mathrm{~A} & 0.520808 & 0.746439 & 0.560672\end{array}$

$\begin{array}{lllll}7 A & 0.545134 & 0.745356 & -0.542368\end{array}$

$\begin{array}{lllll}8 \mathrm{~B} & 1.696495 & -0.975712 & -0.675868\end{array}$

$\begin{array}{llll}9 \mathrm{~A} & 1.428916 & -0.831394 & 0.634827\end{array}$

$\begin{array}{llll}10 \mathrm{~A} & -0.405310 & -0.828351 & -0.560339\end{array}$

$\begin{array}{llll}11 \mathrm{~A} & -0.403796 & -0.792609 & 0.597032\end{array}$

$\begin{array}{llll}12 \mathrm{~B} & -0.556158 & 1.231918 & -0.009506\end{array}$

$\begin{array}{llll}13 B & 1.692507 & 0.472799 & -1.109458\end{array}$

$\begin{array}{llll}14 \mathrm{~B} & 1.617140 & 1.283186 & 0.163844\end{array}$

$\begin{array}{llll}15 A & -0.435286 & 0.294402 & 0.952421\end{array}$

$\begin{array}{llll}16 \mathrm{~B} & 0.442061 & -1.661372 & -1.133347\end{array}$

$\begin{array}{llll}17 \mathrm{~A} & 1.433644 & 0.277769 & 1.021112\end{array}$

$\begin{array}{lrrr}18 A & -0.441306 & 0.281882 & -0.926188\end{array}$

$\begin{array}{llll}19 A & 0.469106 & -1.356243 & 1.032234\end{array}$

$\begin{array}{llll}20 \mathrm{~B} & 0.478781 & 0.690341 & 1.850071\end{array}$

$\begin{array}{llll}21 \mathrm{~B} & 0.370313 & 0.759157 & -1.831357\end{array}$

$\begin{array}{llll}22 \mathrm{~B} & -1.333700 & -0.089411 & 0.007864\end{array}$

$\begin{array}{llll}23 \mathrm{~B} & 1.247260 & -0.690552 & 1.922890\end{array}$

$\begin{array}{llll}24 \mathrm{~B} & -0.339472 & -1.989039 & 0.139639\end{array}$

$\begin{array}{llll}25 B & -0.305511 & -0.657820 & 1.888334\end{array}$

$\begin{array}{llll}26 \mathrm{~B} & 2.434576 & -0.022157 & 0.235311\end{array}$

$\begin{array}{llll}27 \mathrm{~B} & 1.199573 & -2.091356 & 0.223584\end{array}$ $\begin{array}{llll}28 B & -0.407562 & -0.610941 & -1.869212\end{array}$ \begin{tabular}{|llll}
$29 B$ & 1.124057 & -0.601019 & -2.066929
\end{tabular}

$\begin{array}{lllll}\text { 30B } & 0.587449 & 2.011009 & -0.745468\end{array}$

$\begin{array}{llll}31 \mathrm{~B} & 0.446408 & 1.998283 & 0.887277\end{array}$

$E 32 / \varepsilon=-154.290081$

$\begin{array}{llll}\text { atom } & \mathrm{x} / \sigma & \mathrm{y} / \sigma & \mathrm{z} / \sigma\end{array}$

$\begin{array}{llll}1 \mathrm{~A} & 0.000000 & 0.000000 & 0.000000\end{array}$

$\begin{array}{llll}2 \mathrm{~A} & 1.097810 & 0.000000 & 0.000000\end{array}$

$\begin{array}{llll}3 \mathrm{~A} & 0.584491 & -0.953899 & 0.000000\end{array}$

$\begin{array}{lllll}4 \mathrm{~A} & 0.513318 & -0.270704 & -0.914682\end{array}$

$\begin{array}{lllll}5 \mathrm{~A} & 0.511222 & 0.793380 & -0.563917\end{array}$

$\begin{array}{llll}6 \mathrm{~A} & 0.586588 & -0.315582 & 0.920794\end{array}$

$\begin{array}{llll}7 \mathrm{~A} & 0.548905 & 0.748379 & 0.558979\end{array}$

$\begin{array}{llll}8 B & 1.774583 & -0.934088 & 0.653622\end{array}$

$\begin{array}{llll}9 \mathrm{~B} & -0.676773 & 0.361669 & -1.081174\end{array}$

$\begin{array}{llll}10 A & -0.321742 & -0.877000 & 0.582555\end{array}$

$\begin{array}{llll}11 \mathrm{~A} & 1.419551 & 0.309722 & -1.006266\end{array}$

$\begin{array}{llll}12 \mathrm{~A} & -0.372441 & -0.828999 & -0.582138\end{array}$

$\begin{array}{lllll}13 A & 1.470249 & -0.793464 & -0.629714\end{array}$

$\begin{array}{llll}14 \mathrm{~B} & 1.715390 & 0.507797 & 1.095196\end{array}$

$\begin{array}{llll}15 B & -0.617580 & 1.194277 & 0.176117\end{array}$

$\begin{array}{llll}16 \mathrm{~B} & 0.532732 & 0.597019 & -1.938767\end{array}$

$\begin{array}{llll}17 \mathrm{~B} & 0.565077 & -1.689632 & 1.122672\end{array}$

$\begin{array}{lllll}18 \mathrm{~A} & 0.548903 & -1.356949 & -1.013534\end{array}$

$\begin{array}{llll}19 A & -0.396500 & 0.192316 & 0.976362\end{array}$

$\begin{array}{lllll}20 A & 1.494310 & 0.990797 & -0.092670\end{array}$

$\begin{array}{llll}21 \mathrm{~B} & -1.342970 & -0.217070 & 0.149250\end{array}$

$\begin{array}{lllll}22 B & 2.440779 & 0.081511 & -0.250502\end{array}$

$\begin{array}{lllll}23 \mathrm{~B} & 1.334813 & -0.705317 & -1.903969\end{array}$

$\begin{array}{lllll}24 \mathrm{~B} & -0.237007 & -2.025850 & -0.135996\end{array}$

$\begin{array}{llll}25 B & 0.694690 & 1.985452 & 0.137233\end{array}$

$\begin{array}{llll}26 \mathrm{~B} & 0.403121 & 0.695038 & 1.864879\end{array}$

$\begin{array}{llll}27 \mathrm{~B} & 1.310208 & -2.071709 & -0.231199\end{array}$

$\begin{array}{lllll}28 \mathrm{~B} & -0.212402 & -0.809618 & -1.920924\end{array}$

$\begin{array}{lllll}29 B & 1.413610 & 1.623067 & -1.219406\end{array}$

$\begin{array}{llll}30 \mathrm{~B} & -0.315800 & -0.708665 & 1.902391\end{array}$

$\begin{array}{llll}31 \mathrm{~B} & 1.221520 & -0.615601 & 2.054798\end{array}$

$\begin{array}{llll}32 \mathrm{~B} & -0.123710 & 1.795620 & -1.173418\end{array}$

$E 33 / \varepsilon=-160.737338$

atom $\mathrm{x} / \sigma$

$\begin{array}{llc}1 \mathrm{~A} & 0.000000 & 0.000000\end{array}$

$z / \sigma$

$\begin{array}{lllll}3 \mathrm{~A} & -0.462312 & -0.973696 & 0.000000\end{array}$

$\begin{array}{llll}4 \mathrm{~A} & -0.445028 & -0.318459 & 0.936062\end{array}$

$\begin{array}{llll}5 A & 0.478556 & 0.265424 & 0.936062\end{array}$

$\begin{array}{lllll}6 \mathrm{~A} & 0.500436 & -0.791588 & -0.566681\end{array}$

$\begin{array}{llll}7 A & 0.494201 & -0.781725 & 0.541610\end{array}$

$\begin{array}{llll}8 \mathrm{~A} & -0.474808 & 0.751049 & 0.636624\end{array}$

$\begin{array}{llll}9 \mathrm{~A} & -0.488169 & -0.331230 & -0.898236\end{array}$

$\begin{array}{lllll}10 \mathrm{~A} & 0.508596 & 0.298918 & -0.898236\end{array}$

$\begin{array}{llll}11 \mathrm{~A} & 0.476352 & 0.947960 & 0.051508\end{array}$

$\begin{array}{llll}12 \mathrm{~A} & -1.060649 & -0.023721 & 0.051508\end{array}$

$\begin{array}{llll}13 \mathrm{~B} & 0.488872 & -0.773296 & 1.824870\end{array}$

$\begin{array}{llll}14 \mathrm{~B} & 0.384203 & -2.013686 & 0.097223\end{array}$

$\begin{array}{llll}15 \mathrm{~B} & 1.654268 & -1.210760 & 0.097223\end{array}$

$\begin{array}{llll}16 B & -0.328639 & 0.519840 & 1.958989\end{array}$

$\begin{array}{llll}17 \mathrm{~B} & -0.423140 & -1.639120 & -1.139392\end{array}$

$\begin{array}{lllll}18 B & 1.662183 & -0.320794 & -1.139392\end{array}$

$\begin{array}{llll}19 \mathrm{~A} & 1.416318 & -0.287760 & 1.000267\end{array}$

$\begin{array}{llll}20 \mathrm{~A} & -0.347527 & -1.402850 & 1.000268\end{array}$

$\begin{array}{llll}21 \mathrm{~A} & 1.388280 & 0.815907 & 0.676087\end{array}$

$\begin{array}{llll}22 \mathrm{~A} & -1.332495 & -0.904147 & 0.676087\end{array}$

$\begin{array}{llll}23 \mathrm{~B} & -0.614317 & 0.971724 & -0.679313\end{array}$

$\begin{array}{llll}24 \mathrm{~B} & 0.414657 & 1.605432 & 1.162534\end{array}$

$\begin{array}{llll}25 B & -1.628113 & 0.314008 & 1.162534\end{array}$

$\begin{array}{lllll}26 \mathrm{~B} & 1.549775 & 1.118696 & -0.633198\end{array}$

$\begin{array}{lllll}27 \mathrm{~B} & -1.675286 & -0.920164 & -0.633198\end{array}$

$\begin{array}{lllll}28 \mathrm{~B} & 0.342672 & -0.542038 & -1.896621\end{array}$

$\begin{array}{llll}29 B & -1.056197 & -0.873158 & 1.980097\end{array}$

$\begin{array}{llll}30 \mathrm{~B} & 1.241778 & 0.579606 & 1.980097\end{array}$

$\begin{array}{llll}31 \mathrm{~B} & -1.140524 & -2.114591 & 0.192412\end{array}$

$\begin{array}{llll}32 B & 2.399393 & 0.123318 & 0.192412\end{array}$ 
33В $\quad 1.110502-1.756588-1.255554$ $E 34 / \varepsilon=-167.225215$

\begin{tabular}{|c|c|c|c|}
\hline tom & $\mathrm{x} / \sigma$ & $y / \sigma$ & $z / \sigma$ \\
\hline $1 \mathrm{~A}$ & 0.000000 & 0.000000 & 0.000000 \\
\hline $2 A$ & 1.075288 & 0.000000 & 0.000000 \\
\hline $3 A$ & 0.514226 & -0.953485 & 0.000000 \\
\hline $4 \mathrm{~A}$ & 0.514225 & 0.785195 & -0.540928 \\
\hline $5 A$ & 0.494457 & -0.285582 & 0.913768 \\
\hline $6 A$ & 0.494457 & 0.753571 & 0.590474 \\
\hline $7 A$ & 0.518553 & -0.285264 & -0.916913 \\
\hline 8B & 1.686729 & -0.985091 & -0.653501 \\
\hline 9B & 1.686728 & 0.440482 & -1.097016 \\
\hline $10 \mathrm{~A}$ & -0.444732 & 0.298867 & 0.960638 \\
\hline $11 \mathrm{~A}$ & -0.433902 & -0.778047 & 0.587465 \\
\hline $12 \mathrm{~A}$ & -0.433902 & 0.974000 & 0.042380 \\
\hline $13 \mathrm{~A}$ & -0.433048 & -0.809588 & -0.553924 \\
\hline $14 \mathrm{~A}$ & -0.433048 & 0.352445 & -0.915448 \\
\hline $15 \mathrm{~A}$ & 1.413758 & -0.820699 & 0.630394 \\
\hline $16 \mathrm{~A}$ & 1.413758 & 1.033478 & 0.053534 \\
\hline $17 \mathrm{~A}$ & 1.395361 & 0.317553 & 1.020703 \\
\hline 18B & 0.454515 & 0.596444 & 1.917132 \\
\hline 19B & 0.413965 & -1.650877 & -1.124120 \\
\hline $20 B$ & 0.413964 & 0.721766 & -1.862282 \\
\hline $21 \mathrm{~A}$ & 0.441102 & 1.700413 & 0.073207 \\
\hline $22 \mathrm{~A}$ & 0.441102 & -1.358761 & 1.024956 \\
\hline $23 B$ & -1.325594 & 0.000251 & 0.000807 \\
\hline $24 B$ & 2.379995 & 0.071238 & 0.228975 \\
\hline $25 B$ & 1.238871 & 1.679703 & 1.163427 \\
\hline $26 B$ & 1.238870 & -0.723209 & 1.911005 \\
\hline $27 \mathrm{~B}$ & -0.343709 & -0.703068 & 1.877768 \\
\hline $28 \mathrm{~B}$ & -0.343708 & 1.644262 & 1.147483 \\
\hline $29 B$ & -0.372133 & -1.980284 & 0.142209 \\
\hline $30 \mathrm{~B}$ & -0.372133 & 1.711441 & -1.006336 \\
\hline $31 \mathrm{~B}$ & -0.427510 & -0.574514 & -1.846635 \\
\hline $32 B$ & 1.170951 & -2.097349 & 0.231397 \\
\hline $3 \mathrm{~B}$ & 1.170949 & 1.858443 & -0.999304 \\
\hline Th & 1.120712 & -0.639753 & -2.056332 \\
\hline
\end{tabular}

$E 35 / \varepsilon=-173.011973$

atom $x / \sigma$

$\begin{array}{llll}1 \mathrm{~A} & 0.000000 & 0.000000 & 0.000000\end{array}$

$\begin{array}{llll}2 \mathrm{~A} & 1.127618 & 0.000000 & 0.000000\end{array}$

$\begin{array}{llll}3 A & -1.143657 & -0.027534 & 0.000000\end{array}$

$\begin{array}{llll}4 \mathrm{~A} & 0.547053 & -0.935553 & 0.000008\end{array}$

$\begin{array}{llll}5 A & -0.523156 & -0.946944 & 0.000008\end{array}$

$\begin{array}{lllll}6 \mathrm{~A} & 0.540803 & -0.287492 & -0.890212\end{array}$

$\begin{array}{llll}7 A & 0.540802 & -0.287478 & 0.890216\end{array}$

8B $\quad 0.020230-1.483990-1.076166$

$\begin{array}{llll}9 B & 0.020229 & -1.483972 & 1.076189\end{array}$

$\begin{array}{llll}10 A & -0.531549 & -0.299824 & -0.889422\end{array}$

$\begin{array}{llll}11 \mathrm{~A} & -0.531550 & -0.299809 & 0.889427\end{array}$

$\begin{array}{llll}12 \mathrm{~A} & 0.538518 & 0.761864 & 0.551444\end{array}$

$\begin{array}{lllll}13 \mathrm{~A} & 0.538518 & 0.761855 & -0.551456\end{array}$

$\begin{array}{llll}14 \mathrm{~A} & -0.553647 & 0.747971 & 0.551020\end{array}$

$\begin{array}{llll}15 A & -0.553647 & 0.747962 & -0.551033\end{array}$

$\begin{array}{lllll}16 \mathrm{~B} & 1.574497 & -1.076670 & 0.783048\end{array}$

$\begin{array}{llll}17 \mathrm{~B} & 1.574497 & -1.076683 & -0.783030\end{array}$

$\begin{array}{llll}18 \mathrm{~B} & -0.003598 & 0.591461 & 1.726298\end{array}$

$\begin{array}{llll}19 B & -0.003598 & 0.591433 & -1.726307\end{array}$

$\begin{array}{llll}20 B & -1.546365 & -1.112229 & -0.784120\end{array}$

$\begin{array}{llll}21 B & -1.546365 & -1.112217 & 0.784138\end{array}$

$\begin{array}{llll}22 \mathrm{~B} & 1.580266 & 0.402001 & 1.256926\end{array}$

$\begin{array}{lllll}23 \mathrm{~B} & 1.580267 & 0.401981 & -1.256932\end{array}$

$\begin{array}{llll}24 \mathrm{~B} & -1.582704 & 0.367244 & -1.263120\end{array}$

$\begin{array}{llll}25 B & -1.582705 & 0.367264 & 1.263114\end{array}$

$\begin{array}{llll}26 \mathrm{~B} & 1.586463 & 1.309896 & -0.000010\end{array}$

$\begin{array}{llll}27 \mathrm{~B} & -0.019462 & 1.817562 & -0.000016\end{array}$

$\begin{array}{llll}28 \mathrm{~B} & 0.829951 & -2.222095 & 0.000018\end{array}$

$\begin{array}{llll}29 \mathrm{~B} & -0.770711 & -2.240837 & 0.000018\end{array}$

$\begin{array}{lllll}30 \mathrm{~B} & 0.810614 & -0.670250 & -2.113983\end{array}$

$\begin{array}{llll}31 \mathrm{~B} & 0.810613 & -0.670217 & 2.113994\end{array}$ $\begin{array}{llll}32 \mathrm{~B} & -1.613299 & 1.273190 & -0.000011\end{array}$ \begin{tabular}{|llll}
$33 B$ & -0.783872 & -0.689485 & -2.115799
\end{tabular} $\begin{array}{llll}34 \mathrm{~B} & -0.783872 & -0.689450 & 2.115810\end{array}$ $\begin{array}{llll}35 B & 2.429289 & -0.020407 & 0.000001\end{array}$

\section{$E 36 / \varepsilon=-180.176688$}

atom $\mathrm{x} / \sigma \quad \mathrm{y} / \sigma$

$\begin{array}{llll}1 \mathrm{~A} & 0.000000 & 0.000000 & 0.000000\end{array}$

$\begin{array}{llll}2 \mathrm{~A} & 1.126202 & 0.000000 & 0.000000\end{array}$

$\begin{array}{llll}3 \mathrm{~A} & -1.125875 & -0.027117 & 0.000000\end{array}$

$\begin{array}{llll}4 \mathrm{~A} & 0.546086 & -0.935893 & 0.000152\end{array}$

$\begin{array}{llll}5 A & -0.523393 & -0.948770 & 0.000153\end{array}$

$\begin{array}{lllll}6 \mathrm{~A} & -0.532399 & -0.300917 & -0.890255\end{array}$

$\begin{array}{lllll}7 \mathrm{~A} & 0.539490 & -0.288010 & -0.890255\end{array}$

$\begin{array}{lllll}8 \mathrm{~A} & 0.539488 & -0.287720 & 0.890349\end{array}$

$\begin{array}{llll}9 \mathrm{~A} & -0.532402 & -0.300627 & 0.890349\end{array}$

$\begin{array}{llll}\text { 10B } & 0.017877 & -1.484728 & -1.076101\end{array}$

$\begin{array}{llll}11 \mathrm{~B} & 0.017875 & -1.484377 & 1.076583\end{array}$

$\begin{array}{llll}12 \mathrm{~A} & -0.555099 & 0.748701 & -0.551985\end{array}$

$\begin{array}{lllll}13 A & 0.536910 & 0.761849 & -0.551986\end{array}$

$\begin{array}{llll}14 \mathrm{~A} & -0.555101 & 0.748880 & 0.551737\end{array}$

$\begin{array}{llll}15 A & 0.536909 & 0.762029 & 0.551736\end{array}$

$\begin{array}{llll}16 \mathrm{~B} & -1.546296 & -1.115096 & -0.783302\end{array}$

$\begin{array}{llll}17 \mathrm{~B} & 1.572695 & -1.077285 & 0.783657\end{array}$

$\begin{array}{llll}18 \mathrm{~B} & 1.572696 & -1.077540 & -0.783306\end{array}$

$\begin{array}{llll}19 B & -1.546297 & -1.114841 & 0.783660\end{array}$

$\begin{array}{llll}20 B & -0.007109 & 0.590295 & -1.726549\end{array}$

$\begin{array}{llll}21 B & -0.007113 & 0.590857 & 1.726354\end{array}$

$\begin{array}{lllll}22 \mathrm{~B} & -1.587666 & 0.363027 & -1.257431\end{array}$

$\begin{array}{lllll}23 B & 1.578462 & 0.401150 & -1.257435\end{array}$

$\begin{array}{llll}24 \mathrm{~B} & 1.578460 & 0.401560 & 1.257303\end{array}$

$\begin{array}{llll}25 B & -1.587669 & 0.363436 & 1.257307\end{array}$

$\begin{array}{llll}26 \mathrm{~B} & -1.616067 & 1.270584 & -0.000210\end{array}$

$\begin{array}{llll}27 \mathrm{~B} & 1.585005 & 1.309128 & -0.000214\end{array}$

$\begin{array}{llll}28 \mathrm{~B} & -0.021883 & 1.817376 & -0.000297\end{array}$

$\begin{array}{lllll}29 \mathrm{~B} & 0.827246 & -2.222451 & 0.000362\end{array}$

$\begin{array}{llll}30 \mathrm{~B} & -0.773492 & -2.241726 & 0.000363\end{array}$

$\begin{array}{llll}31 \mathrm{~B} & 0.806417 & -0.670913 & -2.114245\end{array}$

$\begin{array}{llll}32 \mathrm{~B} & -0.790034 & -0.690136 & -2.114243\end{array}$

$\begin{array}{llll}33 \mathrm{~B} & -0.790038 & -0.689448 & 2.114464\end{array}$

$\begin{array}{llll}34 \mathrm{~B} & 0.806415 & -0.670225 & 2.114462\end{array}$

$\begin{array}{llll}35 \mathrm{~B} & 2.427570 & -0.021351 & 0.000004\end{array}$ $\begin{array}{llll}36 \mathrm{~B} & -2.426352 & -0.079797 & 0.000010\end{array}$

\section{$E 37 / \varepsilon=-186.439022$}

$$
\begin{array}{lll}
\text { atom } \quad \mathrm{x} / \sigma & \mathrm{y} / \sigma
\end{array}
$$

$\begin{array}{llll}1 \mathrm{~A} & 0.000000 & 0.000000 & 0.000000\end{array}$

$\begin{array}{llll}2 \mathrm{~A} & 1.127757 & 0.000000 & 0.000000\end{array}$

$\begin{array}{llll}3 \mathrm{~A} & -1.123774 & -0.022392 & 0.000000\end{array}$

$\begin{array}{lllll}4 \mathrm{~A} & 0.546169 & -0.928218 & -0.110752\end{array}$

$\begin{array}{lllll}5 \mathrm{~A} & -0.523987 & -0.940288 & -0.111940\end{array}$

$\begin{array}{llll}6 \mathrm{~A} & 0.540748 & -0.390886 & 0.850502\end{array}$

$\begin{array}{llll}7 A & -0.531132 & -0.402989 & 0.848909\end{array}$

$\begin{array}{llll}8 \mathrm{~A} & 0.539702 & -0.178723 & -0.917748\end{array}$

$\begin{array}{lllll}9 \mathrm{~A} & -0.532687 & -0.190434 & -0.918803\end{array}$

$\begin{array}{llll}\text { 10B } & 0.018938 & -1.600419 & 0.892643\end{array}$

$\begin{array}{llll}11 \mathrm{~B} & 0.016724 & -1.343928 & -1.244492\end{array}$

$\begin{array}{llll}12 \mathrm{~A} & 0.537033 & 0.685639 & 0.633930\end{array}$

$\begin{array}{llll}13 \mathrm{~A} & -0.548972 & 0.682823 & 0.640432\end{array}$

$\begin{array}{llll}14 \mathrm{~B} & -0.010892 & 0.372962 & 1.787240\end{array}$

$\begin{array}{llll}15 \mathrm{~A} & 0.538857 & 0.822475 & -0.456969\end{array}$

$\begin{array}{llll}16 \mathrm{~A} & -0.552638 & 0.810581 & -0.456845\end{array}$

$\begin{array}{llll}17 \mathrm{~B} & 1.573924 & -1.160101 & 0.650373\end{array}$

$\begin{array}{llll}18 \mathrm{~B} & 1.573709 & -0.974312 & -0.904579\end{array}$

$\begin{array}{llll}19 B & -1.547268 & -1.195338 & 0.646773\end{array}$

$\begin{array}{llll}20 \mathrm{~B} & -1.548396 & -1.008561 & -0.907841\end{array}$

$\begin{array}{llll}21 \mathrm{~B} & 1.577707 & 0.252356 & 1.297728\end{array}$

$\begin{array}{llll}22 \mathrm{~B} & -0.006307 & 0.794274 & -1.642388\end{array}$

$\begin{array}{llll}23 B & -1.581544 & 0.216750 & 1.295667\end{array}$

$\begin{array}{llll}24 \mathrm{~B} & 1.579647 & 0.549033 & -1.199942\end{array}$

$\begin{array}{llll}25 B & -1.586587 & 0.515951 & -1.201401\end{array}$

$\begin{array}{llll}26 B & 1.585596 & 1.301205 & 0.154730\end{array}$

$\begin{array}{llll}27 \mathrm{~B} & -0.019236 & 1.807482 & 0.208212\end{array}$ $\begin{array}{llll}28 \mathrm{~B} & -1.608748 & 1.268393 & 0.156198\end{array}$ $\begin{array}{llll}29 B & 0.827311 & -2.204841 & -0.264251\end{array}$

$\begin{array}{llll}30 \mathrm{~B} & -0.775210 & -2.222851 & -0.265237\end{array}$

$\begin{array}{llll}31 \mathrm{~B} & 0.809047 & -0.915544 & 2.020164\end{array}$

$\begin{array}{llll}32 \mathrm{~B} & -0.791322 & -0.940343 & 2.017188\end{array}$

$\begin{array}{lllll}33 \mathrm{~B} & 0.805938 & -0.412610 & -2.178254\end{array}$

$\begin{array}{lllll}34 \mathrm{~B} & -0.791402 & -0.429683 & -2.179667\end{array}$

$\begin{array}{lllll}35 \mathrm{~B} & 2.428264 & -0.016811 & 0.001058\end{array}$

$\begin{array}{lllll}36 \mathrm{~B} & -2.424044 & -0.070121 & -0.004108\end{array}$

$\begin{array}{llll}37 \mathrm{~B} & 0.844799 & 1.628427 & 1.494076\end{array}$

$E 38 / \varepsilon=-193.649484$

$\begin{array}{cccc}\text { atom } & \mathrm{x} / \sigma & y / \sigma & z / \sigma \\ 1 \mathrm{~A} & 0.000000 & 0.000000 & 0.000000\end{array}$

$\begin{array}{llll}2 \mathrm{~A} & 1.124935 & 0.000000 & 0.000000\end{array}$

$\begin{array}{llll}3 A & -1.124842 & -0.014460 & 0.000000\end{array}$

$\begin{array}{lllll}4 \mathrm{~A} & -0.531146 & -0.763093 & -0.550332\end{array}$

$\begin{array}{lllll}5 \mathrm{~A} & 0.540910 & -0.756203 & -0.550332\end{array}$

$\begin{array}{llll}6 \mathrm{~A} & -0.531146 & -0.763081 & 0.550348\end{array}$

$\begin{array}{llll}7 \mathrm{~A} & 0.540910 & -0.756191 & 0.550348\end{array}$

$\begin{array}{llll}8 \mathrm{~A} & -0.538764 & 0.285201 & 0.889357\end{array}$

$\begin{array}{lllll}9 \mathrm{~A} & -0.538763 & 0.285182 & -0.889364\end{array}$

$\begin{array}{llll}10 A & 0.535053 & 0.292102 & 0.889357\end{array}$

$\begin{array}{lllll}11 \mathrm{~A} & 0.535053 & 0.292083 & -0.889364\end{array}$

$\begin{array}{llll}12 B & 0.011763 & -1.830324 & 0.000019\end{array}$

$\begin{array}{llll}13 \mathrm{~B} & 0.003625 & -0.564005 & -1.738717\end{array}$

$\begin{array}{llll}14 \mathrm{~B} & 0.003625 & -0.563967 & 1.738729\end{array}$

$\begin{array}{llll}15 \mathrm{~A} & -0.551529 & 0.932054 & -0.000010\end{array}$

$\begin{array}{llll}16 \mathrm{~A} & 0.539503 & 0.939067 & -0.000010\end{array}$

$\begin{array}{llll}17 \mathrm{~B} & -1.555807 & -1.346106 & 0.000014\end{array}$

$\begin{array}{llll}18 \mathrm{~B} & 1.572980 & -1.325997 & 0.000014\end{array}$

$\begin{array}{lllll}19 B & 1.569079 & -0.408228 & -1.264774\end{array}$

$\begin{array}{llll}19 B & 1.569080 & -0.408201 & 1.264781\end{array}$

$\begin{array}{llll}21 \mathrm{~B} & -1.563703 & -0.428335 & 1.264782\end{array}$

$\begin{array}{llll}22 \mathrm{~B} & -1.563703 & -0.428362 & -1.264774\end{array}$

$\begin{array}{llll}23 B & -0.009623 & 1.497206 & 1.042924\end{array}$

$\begin{array}{llll}24 \mathrm{~B} & -0.009622 & 1.497183 & -1.042957\end{array}$

$\begin{array}{lllll}25 \mathrm{~B} & 1.577881 & 1.064124 & -0.778356\end{array}$

$\begin{array}{llll}26 \mathrm{~B} & -1.591429 & 1.043755 & -0.778356\end{array}$

$\begin{array}{llll}27 \mathrm{~B} & 1.577881 & 1.064141 & 0.778332\end{array}$

$\begin{array}{llll}28 B & -1.591429 & 1.043772 & 0.778333\end{array}$

$\begin{array}{llll}29 B & -0.793159 & -1.804763 & -1.306745\end{array}$

$\begin{array}{llll}30 \mathrm{~B} & -0.793159 & -1.804735 & 1.306782\end{array}$

$\begin{array}{llll}31 \mathrm{~B} & 0.816290 & -1.794392 & 1.306782\end{array}$

$\begin{array}{lllll}32 \mathrm{~B} & 0.816290 & -1.794420 & -1.306744\end{array}$

$\begin{array}{llll}33 \mathrm{~B} & -0.805357 & 0.692647 & -2.102470\end{array}$

$\begin{array}{llll}34 \mathrm{~B} & -0.805358 & 0.692692 & 2.102455\end{array}$

$\begin{array}{lllll}35 B & 0.796388 & 0.702941 & -2.102470\end{array}$

$\begin{array}{llll}36 \mathrm{~B} & 0.796388 & 0.702987 & 2.102454\end{array}$

$\begin{array}{llll}37 \mathrm{~B} & -2.423376 & -0.042342 & -0.000001\end{array}$

$\begin{array}{llll}38 \mathrm{~B} & 2.423720 & -0.011190 & -0.000001\end{array}$

\section{$E 39 / \varepsilon=-200.030446$}

atom $\mathrm{X} / \sigma$

$y / \sigma$

$\begin{array}{llll}1 \mathrm{~A} & 0.000000 & 0.000000 & 0.000000\end{array}$

2A $\quad 1.126000 \quad 0.000000 \quad 0.000000$

$\begin{array}{llll}3 A & -1.123254 & -0.011001 & 0.000000\end{array}$

$\begin{array}{llll}4 \mathrm{~A} & 0.540971 & -0.755697 & 0.550106\end{array}$

$\begin{array}{lllll}5 \mathrm{~A} & 0.540971 & -0.755557 & -0.550298\end{array}$

$\begin{array}{llll}6 \mathrm{~A} & -0.531426 & -0.762236 & 0.550267\end{array}$

$\begin{array}{llll}7 \mathrm{~A} & -0.531427 & -0.762097 & -0.550459\end{array}$

$\begin{array}{lllll}8 \mathrm{~A} & 0.536080 & 0.292675 & 0.889191\end{array}$

$\begin{array}{lllll}9 \mathrm{~A} & 0.536079 & 0.292900 & -0.889117\end{array}$

$\begin{array}{llll}10 \mathrm{~A} & -0.537818 & 0.285257 & 0.889508\end{array}$

$\begin{array}{llll}11 \mathrm{~A} & -0.537819 & 0.285482 & -0.889434\end{array}$

$\begin{array}{lllll}12 \mathrm{~B} & 0.010779 & -1.829406 & -0.000231\end{array}$

$\begin{array}{llll}13 \mathrm{~B} & 0.005191 & -0.563591 & 1.738343\end{array}$

$\begin{array}{llll}14 \mathrm{~B} & 0.005189 & -0.563150 & -1.738485\end{array}$

$\begin{array}{llll}15 A & 0.539505 & 0.934695 & 0.000118\end{array}$

$\begin{array}{llll}16 \mathrm{~A} & -0.546864 & 0.935883 & 0.000119\end{array}$

$\begin{array}{llll}17 \mathrm{~B} & -0.014554 & 1.491942 & 1.051102\end{array}$

$\begin{array}{llll}18 B & -0.014555 & 1.492208 & -1.050723\end{array}$

$\begin{array}{lllll}19 B & 1.573153 & -1.325387 & -0.000168\end{array}$ $\begin{array}{llll}20 B & -1.557099 & -1.343120 & -0.000169\end{array}$ 


$\begin{array}{rrrr}\text { 21B } & 1.569444 & -0.408775 & 1.264531 \\ 22 B & 1.569443 & -0.408454 & -1.264636 \\ 23 B & -1.564273 & -0.425973 & 1.263751 \\ 24 B & -1.564274 & -0.425653 & -1.263857 \\ \text { 25B } & 1.580249 & 1.061363 & 0.778920 \\ 26 B & 1.580248 & 1.061561 & -0.778652 \\ 27 B & -1.586491 & 1.048091 & 0.778563 \\ \text { 28B } & -1.586492 & 1.048288 & -0.778295 \\ \text { 29B } & 0.816007 & -1.794633 & 1.305860 \\ \text { 30B } & 0.816005 & -1.794302 & -1.306315 \\ \text { 31B } & -0.794116 & -1.803018 & 1.306761 \\ \text { 32B } & -0.794117 & -1.802687 & -1.307217 \\ \text { 33B } & 0.798887 & 0.703545 & 2.101891 \\ \text { 34B } & 0.798885 & 0.704077 & -2.101713 \\ \text { 35B } & 2.424544 & -0.010449 & -0.000002 \\ \text { 36B } & -0.807006 & 0.687863 & 2.105824 \\ \text { 37B } & -0.807008 & 0.688397 & -2.105648 \\ \text { 38B } & -2.421674 & -0.035829 & -0.000003 \\ \text { 39B } & 0.865048 & 2.211801 & 0.000280\end{array}$

$E 40 / \varepsilon=-207.322597$

$\begin{array}{llll}1 \mathrm{~A} & 0.000000 & 0.000000 & 0.000000\end{array}$

2A $\quad 1.123359 \quad 0.000000 \quad 0.000000$

$\begin{array}{llll}3 \mathrm{~A} & -1.123359 & 0.000000 & 0.000000\end{array}$

$\begin{array}{llll}4 \mathrm{~A} & 0.537062 & -0.935832 & -0.000288\end{array}$

$\begin{array}{lllll}5 \mathrm{~A} & -0.537061 & -0.935832 & -0.000288\end{array}$

$\begin{array}{llll}6 A & 0.537062 & -0.289461 & 0.889940\end{array}$

$\begin{array}{lllll}7 A & 0.537062 & -0.288914 & -0.890118\end{array}$

$\begin{array}{llll}8 \mathrm{~A} & -0.537061 & -0.289462 & 0.889940\end{array}$

$\begin{array}{llll}9 \mathrm{~A} & -0.537061 & -0.288914 & -0.890118\end{array}$

$\begin{array}{llll}10 \mathrm{~A} & 0.537061 & 0.756935 & 0.550301\end{array}$

$\begin{array}{lllll}11 \mathrm{~A} & 0.537061 & 0.757273 & -0.549835\end{array}$

$\begin{array}{llll}12 \mathrm{~A} & -0.537062 & 0.756934 & 0.550301\end{array}$

$\begin{array}{llll}13 \mathrm{~A} & -0.537062 & 0.757273 & -0.549835\end{array}$

$\begin{array}{llll}14 \mathrm{~B} & 0.000000 & 1.826899 & 0.000562\end{array}$

$\begin{array}{llll}15 \mathrm{~B} & 0.000000 & 0.564009 & 1.737658\end{array}$

$\begin{array}{llll}16 \mathrm{~B} & 0.000000 & 0.565077 & -1.737311\end{array}$

$\begin{array}{llll}17 \mathrm{~B} & 0.000000 & -1.478323 & 1.073370\end{array}$

$\begin{array}{llll}18 \mathrm{~B} & 0.000000 & -1.477662 & -1.074279\end{array}$

$\begin{array}{llll}19 B & 1.570538 & 1.325050 & 0.000408\end{array}$

$\begin{array}{llll}20 B & 1.570538 & 0.409076 & 1.260324\end{array}$

$\begin{array}{llll}21 B & 1.570538 & 0.409851 & -1.260071\end{array}$

$\begin{array}{llll}22 \mathrm{~B} & -1.570537 & -1.071749 & -0.779174\end{array}$

$\begin{array}{llll}23 \mathrm{~B} & 1.570538 & -1.072228 & 0.778516\end{array}$

$\begin{array}{llll}24 \mathrm{~B} & 1.570538 & -1.071749 & -0.779174\end{array}$

$\begin{array}{lllll}25 \mathrm{~B} & -1.570538 & 0.409850 & -1.260071\end{array}$

$\begin{array}{llll}26 \mathrm{~B} & -1.570537 & -1.072228 & 0.778516\end{array}$

$\begin{array}{llll}27 \mathrm{~B} & -1.570538 & 1.325050 & 0.000408\end{array}$

$\begin{array}{llll}28 B & -1.570538 & 0.409075 & 1.260324\end{array}$

$\begin{array}{llll}29 B & -0.808693 & -0.686528 & 2.110708\end{array}$

$\begin{array}{llll}30 \mathrm{~B} & -0.808693 & -0.685230 & -2.111129\end{array}$

$\begin{array}{lllll}31 \mathrm{~B} & 0.808693 & -0.685230 & -2.111129\end{array}$

$\begin{array}{llll}32 \mathrm{~B} & 0.808693 & -0.686528 & 2.110708\end{array}$

$\begin{array}{llll}33 \mathrm{~B} & 0.808693 & 1.796056 & -1.304067\end{array}$

$\begin{array}{llll}34 \mathrm{~B} & 0.808693 & 1.795254 & 1.305171\end{array}$

$\begin{array}{llll}35 \mathrm{~B} & -0.808694 & 1.795253 & 1.305172\end{array}$

$\begin{array}{llll}36 \mathrm{~B} & -0.808693 & 1.796056 & -1.304067\end{array}$

$\begin{array}{lllll}37 \mathrm{~B} & 0.808693 & -2.219551 & -0.000682\end{array}$

$\begin{array}{llll}38 \mathrm{~B} & -0.808693 & -2.219551 & -0.000682\end{array}$

$\begin{array}{llll}39 \mathrm{~B} & 2.420332 & 0.000000 & 0.000000\end{array}$

$\begin{array}{llll}40 \mathrm{~B} & -2.420332 & -0.000001 & 0.000000\end{array}$

$E 41 / \varepsilon=-213.170471$

atom $\mathrm{x} / \sigma \quad \mathrm{yl}$

$\begin{array}{llll}1 \mathrm{~A} & 0.000000 & 0.000000 & 0.000000\end{array}$

$\begin{array}{llll}2 \mathrm{~A} & 1.068072 & 0.000000 & 0.000000\end{array}$

$\begin{array}{llll}3 \mathrm{~A} & -0.444792 & -0.971050 & 0.000000\end{array}$

$\begin{array}{llll}4 \mathrm{~A} & 0.444793 & 0.971049 & 0.000000\end{array}$

$\begin{array}{llll}5 \mathrm{~A} & -1.068071 & -0.000001 & 0.000000\end{array}$

$\begin{array}{llll}6 \mathrm{~A} & 0.501927 & -0.781985 & 0.548298\end{array}$

$\begin{array}{llll}7 A & -0.501926 & 0.781984 & -0.548299\end{array}$

$\begin{array}{llll}8 \text { A } & 0.496979 & -0.774277 & -0.587465\end{array}$ $\begin{array}{|llll|}9 A & -0.496978 & 0.774276 & 0.587465\end{array}$

$\begin{array}{llll}10 \mathrm{~A} & 0.468693 & 0.301249 & -0.902770\end{array}$

$\begin{array}{llll}11 \mathrm{~A} & 0.469069 & 0.300664 & 0.902770\end{array}$

$\begin{array}{llll}12 \mathrm{~A} & -0.469069 & -0.300665 & -0.902770\end{array}$

$\begin{array}{llll}13 \mathrm{~A} & -0.468693 & -0.301250 & 0.902770\end{array}$

$\begin{array}{llll}14 \mathrm{~B} & -1.660579 & 1.167383 & -0.008813\end{array}$

$\begin{array}{llll}15 B & 0.369803 & -1.995884 & 0.008813\end{array}$

$\begin{array}{llll}16 \mathrm{~B} & -0.369802 & 1.995883 & -0.008813\end{array}$

$\begin{array}{llll}17 \mathrm{~B} & 1.660579 & -1.167384 & 0.008812\end{array}$

$\begin{array}{lllll}18 \mathrm{~B} & -1.588215 & 0.238449 & -1.235068\end{array}$

$\begin{array}{llll}19 \mathrm{~B} & 0.444613 & 1.543244 & -1.235068\end{array}$

$\begin{array}{llll}20 B & 1.588216 & -0.238450 & 1.235067\end{array}$

$\begin{array}{llll}21 B & -0.444612 & -1.543245 & 1.235068\end{array}$

$\begin{array}{llll}22 \mathrm{~B} & -1.612681 & 0.245824 & 1.209008\end{array}$

$\begin{array}{llll}23 \mathrm{~B} & 1.612681 & -0.245825 & -1.209008\end{array}$

$\begin{array}{llll}24 \mathrm{~B} & -0.448097 & -1.568561 & -1.209007\end{array}$

$\begin{array}{lllr}25 \mathrm{~B} & 0.448097 & 1.568559 & 1.209008\end{array}$

$\begin{array}{llll}26 \mathrm{~A} & 1.378621 & 0.882021 & -0.568073\end{array}$

$\begin{array}{llll}27 \mathrm{~A} & 1.376019 & 0.886077 & 0.568072\end{array}$

$\begin{array}{llll}28 \mathrm{~A} & -1.378620 & -0.882022 & 0.568073\end{array}$

$\begin{array}{llll}29 A & -1.376018 & -0.886077 & -0.568072\end{array}$

$\begin{array}{llll}30 \mathrm{~B} & -0.315579 & 0.491662 & 1.951783\end{array}$

$\begin{array}{lllll}31 B & 0.315579 & -0.491663 & -1.951783\end{array}$

$\begin{array}{lllll}32 \mathrm{~A} & -0.364405 & 0.567731 & -1.608659\end{array}$

$\begin{array}{llll}33 \mathrm{~A} & 0.364406 & -0.567732 & 1.608659\end{array}$

$\begin{array}{lllll}34 \mathrm{~B} & -2.389618 & -0.241451 & -0.015918\end{array}$

$\begin{array}{llll}35 \mathrm{~B} & -1.214658 & -2.071999 & 0.015919\end{array}$

$\begin{array}{llll}36 \mathrm{~B} & 2.389619 & 0.241450 & 0.015917\end{array}$

$\begin{array}{llll}37 \mathrm{~B} & 1.214659 & 2.071998 & -0.015919\end{array}$

$\begin{array}{llll}38 \mathrm{~B} & -1.079161 & 1.681297 & -1.354217\end{array}$

$\begin{array}{llll}39 B & 1.079162 & -1.681297 & 1.354216\end{array}$

$\begin{array}{llll}40 \mathrm{~B} & 1.090929 & -1.699633 & -1.334517\end{array}$

$\begin{array}{llll}41 B & -1.090929 & 1.699631 & 1.334518\end{array}$

\section{$E 42 / \varepsilon=-220.223427$}

atom $\mathrm{X} / \sigma$ $y / \sigma$

1A $\quad 0.000000 \quad 0.000000$

2A $1.068792 \quad 0.000000$

3A $\quad 0.466512 \quad-0.961605$

$\begin{array}{llll}4 \mathrm{~A} & 0.453264 & -0.283893\end{array}$

$\begin{array}{lll}5 \mathrm{~A} & -0.487641 & 0.951227\end{array}$

$\begin{array}{lll}6 A & -1.068678 & 0.023540\end{array}$

$\begin{array}{lll}7 \mathrm{~A} & 0.505004 & 0.761620\end{array}$

$\begin{array}{llll}8 \mathrm{~A} & -0.464812 & -0.786794\end{array}$

$\begin{array}{llll}9 \mathrm{~A} & 0.495760 & -0.310509\end{array}$

$\begin{array}{lll}10 A & -0.497820 & 0.311799\end{array}$

$\begin{array}{lll}11 \mathrm{~A} & 0.458726 & 0.765653\end{array}$

$\begin{array}{lll}12 \mathrm{~A} & -0.488640 & -0.746917\end{array}$

$\begin{array}{lll}13 \mathrm{~A} & -0.448574 & 0.280955\end{array}$

14B $1.622663-1.016321$

$\begin{array}{lll}15 \mathrm{~B} & -1.633958 & 1.023394\end{array}$

$\begin{array}{lll}16 \mathrm{~B} & 1.647682 & 0.430279\end{array}$

$\begin{array}{lll}17 \mathrm{~B} & 0.332060 & -1.670250\end{array}$

$\begin{array}{llll}18 \mathrm{~A} & 1.357577 & -0.850290\end{array}$

19B $\quad-1.532256 \quad 0.959696$

$\begin{array}{lll}20 B & -0.459857 & -1.955447\end{array}$

$\begin{array}{lll}21 \mathrm{~B} & 1.558618 & 1.267261\end{array}$

$\begin{array}{lll}22 \mathrm{~B} & -1.634024 & -0.430952\end{array}$

$\begin{array}{lllll}23 B & -0.325495 & 1.658253 & -1.129219\end{array}$

$\begin{array}{llll}24 \mathrm{~B} & -0.455305 & -0.606378 & 1.880012\end{array}$

$\begin{array}{llll}25 \mathrm{~B} & 0.346831 & 0.674318 & 1.880012\end{array}$

$\begin{array}{llll}26 \mathrm{~B} & 0.463754 & 0.612783 & -1.887419\end{array}$

$\begin{array}{llll}27 \mathrm{~B} & -0.348907 & -0.684717 & -1.887419\end{array}$

$\begin{array}{llll}28 \mathrm{~A} & -1.400260 & -1.016692 & 0.027800\end{array}$

$\begin{array}{llll}29 A & 0.303537 & 1.703601 & 0.027799\end{array}$

$\begin{array}{llll}30 A & -1.378724 & -0.366186 & 0.975403\end{array}$

$\begin{array}{llll}31 \mathrm{~A} & -0.272331 & 1.400289 & 0.975402\end{array}$

$\begin{array}{lllll}32 \mathrm{~A} & 1.385807 & 0.251871 & -1.020682\end{array}$

$\begin{array}{lllll}33 \mathrm{~A} & 0.378272 & -1.356765 & -1.020681\end{array}$

$\begin{array}{lllll}34 \mathrm{~B} & 1.201016 & -0.752231 & -1.909299\end{array}$

$\begin{array}{lllll}35 \mathrm{~B} & 1.084142 & -2.119558 & -0.226879\end{array}$

$\begin{array}{llll}36 \mathrm{~B} & 2.380202 & -0.050261 & -0.226879\end{array}$

$\begin{array}{llll}37 \mathrm{~B} & 0.978342 & 1.860701 & 1.144306\end{array}$ \begin{tabular}{|llll}
$38 \mathrm{~B}$ & -1.247063 & -1.692394 & 1.144306
\end{tabular}

$\begin{array}{llll}39 \mathrm{~B} & -0.939413 & 2.195153 & 0.165932\end{array}$

$\begin{array}{llll}40 \mathrm{~B} & -2.385044 & -0.112951 & 0.165933\end{array}$

$\begin{array}{llll}41 \mathrm{~B} & 1.080020 & -0.676448 & 2.061764\end{array}$

$\begin{array}{llll}42 \mathrm{~B} & -1.081616 & 0.677445 & -2.062147\end{array}$

$E 43 / \varepsilon=-226.823468$

atom $\mathrm{X} / \sigma$

$y / \sigma$

$\begin{array}{lll}1 \mathrm{~A} & 0.000000 & 0.000000\end{array}$

$z / \sigma$

$\begin{array}{llll}3 \mathrm{~A} & 0.451932 & -0.954598 & 0.000000\end{array}$

$\begin{array}{lllll}4 \mathrm{~A} & -0.451932 & 0.954598 & -0.000001\end{array}$

$\begin{array}{llll}5 \mathrm{~A} & -1.056172 & 0.000000 & 0.000001\end{array}$

$\begin{array}{lllll}6 \mathrm{~A} & 0.481482 & -0.267142 & -0.917061\end{array}$

$\begin{array}{lllll}7 \mathrm{~A} & 0.447474 & -0.320868 & 0.917062\end{array}$

$\begin{array}{llll}8 \mathrm{~A} & -0.481481 & 0.267142 & 0.917062\end{array}$

$\begin{array}{lllll}9 \mathrm{~A} & -0.447474 & 0.320867 & -0.917062\end{array}$

10A $\quad 0.507020 \quad 0.801005-0.552306$

$\begin{array}{llll}11 \mathrm{~A} & -0.507019 & -0.801005 & 0.552306\end{array}$

$\begin{array}{llll}12 \mathrm{~A} & 0.480128 & 0.758522 & 0.567410\end{array}$

$\begin{array}{llll}13 \mathrm{~A} & -0.480129 & -0.758522 & -0.567410\end{array}$

$\begin{array}{llll}14 \mathrm{~B} & 0.395892 & -1.648834 & 1.119233\end{array}$

$\begin{array}{lllll}15 B & 1.659664 & 0.347710 & -1.119232\end{array}$

$\begin{array}{lllll}16 \mathrm{~B} & -0.395892 & 1.648834 & -1.119234\end{array}$

$\begin{array}{llll}17 \mathrm{~B} & -1.659663 & -0.347710 & 1.119234\end{array}$

$\begin{array}{llll}18 B & 0.383423 & 0.605742 & -1.905221\end{array}$

$\begin{array}{llll}19 B & -0.383422 & -0.605742 & 1.905221\end{array}$

$\begin{array}{llll}20 B & 0.434072 & 0.685762 & 1.837822\end{array}$

$\begin{array}{llll}21 \mathrm{~B} & -0.434072 & -0.685761 & -1.837822\end{array}$

$\begin{array}{lllll}22 \mathrm{~A} & -1.346711 & 0.878300 & -0.608759\end{array}$

$\begin{array}{llll}23 A & -1.370085 & 0.841374 & 0.608758\end{array}$

$\begin{array}{llll}24 \mathrm{~A} & 1.346711 & -0.878301 & 0.608759\end{array}$

$\begin{array}{lllll}25 \mathrm{~A} & 1.370086 & -0.841374 & -0.608757\end{array}$

$\begin{array}{lllll}26 \mathrm{~B} & -0.364042 & -1.996890 & -0.150584\end{array}$

$\begin{array}{lllll}27 \mathrm{~B} & -1.649072 & -1.183493 & -0.150583\end{array}$

$\begin{array}{llll}28 \mathrm{~B} & 1.649072 & 1.183493 & 0.150585\end{array}$

$\begin{array}{llll}29 B & 0.364042 & 1.996890 & 0.150584\end{array}$

$\begin{array}{llll}30 \mathrm{~A} & -1.394787 & -0.220713 & -1.004932\end{array}$

$\begin{array}{llll}31 \mathrm{~A} & -0.397337 & 1.355090 & 1.004932\end{array}$

$\begin{array}{llll}32 A & 1.394786 & 0.220713 & 1.004934\end{array}$

$\begin{array}{lllll}33 \mathrm{~A} & 0.397337 & -1.355090 & -1.004933\end{array}$

$\begin{array}{llll}34 \mathrm{~B} & -1.201015 & 2.031190 & 0.132083\end{array}$

$\begin{array}{llll}35 \mathrm{~B} & -2.349756 & 0.216372 & -0.132083\end{array}$

$\begin{array}{lllll}36 \mathrm{~B} & 1.201015 & -2.031190 & -0.132083\end{array}$

$\begin{array}{llll}37 \mathrm{~B} & 2.349756 & -0.216372 & 0.132086\end{array}$

$\begin{array}{llll}38 \mathrm{~B} & 1.118413 & -0.807677 & -1.948028\end{array}$

$\begin{array}{llll}39 B & 1.208563 & -0.665250 & 1.948030\end{array}$

$\begin{array}{llll}40 \mathrm{~B} & -1.118412 & 0.807677 & 1.948029\end{array}$

$\begin{array}{llll}41 \mathrm{~B} & -1.208563 & 0.665249 & -1.948030\end{array}$

$\begin{array}{llll}42 \mathrm{~B} & -1.132580 & -1.789285 & 1.189849\end{array}$

$\begin{array}{llll}\text { 43B } & 1.132580 & 1.789285 & -1.189848\end{array}$

\section{$E 44 / \varepsilon=-232.998720$}

$$
\begin{array}{llll}
\text { atom } \mathrm{x} / \sigma & \mathrm{y} / \sigma & \mathrm{z} / \sigma
\end{array}
$$

$\begin{array}{llll}1 \mathrm{~A} & 0.000000 & 0.000000 & 0.000000\end{array}$

$\begin{array}{llll}2 \mathrm{~A} & 1.060415 & 0.000000 & 0.000000\end{array}$

$\begin{array}{llll}3 \mathrm{~A} & 0.454311 & -0.967622 & 0.000000\end{array}$

$\begin{array}{llll}4 \mathrm{~A} & -0.447160 & 0.324442 & -0.906437\end{array}$

$\begin{array}{lllll}5 \mathrm{~A} & 0.487269 & -0.282752 & -0.904941\end{array}$

$\begin{array}{llll}6 \mathrm{~A} & 0.462757 & -0.314572 & 0.901838\end{array}$

$\begin{array}{llll}7 \mathrm{~A} & 0.494332 & 0.771195 & 0.572929\end{array}$

$\begin{array}{llll}8 \mathrm{~A} & -1.062930 & 0.009857 & -0.005240\end{array}$

$\begin{array}{llll}9 A & -0.433809 & 0.975443 & 0.020071\end{array}$

$\begin{array}{llll}10 \mathrm{~A} & 0.514740 & 0.798058 & -0.528295\end{array}$

$\begin{array}{llll}11 \mathrm{~A} & -0.491163 & -0.771156 & -0.557424\end{array}$

$\begin{array}{llll}12 \mathrm{~A} & -0.501432 & -0.781566 & 0.575769\end{array}$

$\begin{array}{llll}13 A & -0.464146 & 0.282748 & 0.912568\end{array}$

$\begin{array}{llll}14 \mathrm{~B} & -0.398751 & 1.592375 & -1.173572\end{array}$

15B $\quad 0.466752 \quad-1.534203-1.218356$

$\begin{array}{llll}16 \mathrm{~B} & 1.633277 & 0.264986 & -1.176328\end{array}$

$\begin{array}{llll}17 \mathrm{~B} & -1.574061 & -0.220721 & -1.231255\end{array}$

$\begin{array}{llll}18 \mathrm{~B} & 1.672433 & 1.172448 & 0.039524\end{array}$ $\begin{array}{llll}19 B & 0.399194 & 1.999924 & 0.071958\end{array}$ 


\begin{tabular}{|c|c|c|c|}
\hline$B$ & -1.658687 & -1.159976 & -0.036087 \\
\hline $21 B$ & 0.411699 & -1.596198 & 93571 \\
\hline 2B & -0.374157 & -1.993586 & -0.035741 \\
\hline $3 B$ & -0.397511 & 1.503135 & 1.284641 \\
\hline 4 & 1.359493 & -0.856792 & 0.626860 \\
\hline $5 \mathrm{~A}$ & 1.390828 & 0.289152 & 0.990698 \\
\hline $26 \mathrm{~B}$ & -1.620782 & -0.254626 & 1.193752 \\
\hline 27B & -0.337683 & -0.518766 & 1.948648 \\
\hline $28 \mathrm{~A}$ & -0.329579 & -0.526043 & -1.601792 \\
\hline $29 A$ & 0.394638 & 0.588833 & -1.578335 \\
\hline $30 A$ & -1.350478 & 0.907389 & -0.569368 \\
\hline $31 \mathrm{~A}$ & 1.402545 & -0.867414 & -0.548500 \\
\hline $32 \mathrm{~A}$ & -1.360977 & 0.883524 & 0.585895 \\
\hline $33 \mathrm{~A}$ & 0.426018 & 0.492804 & 1.625065 \\
\hline $34 \mathrm{~B}$ & 2.358309 & -0.196079 & 0.136628 \\
\hline $35 B$ & 1.227319 & -0.549790 & 1.959246 \\
\hline $36 \mathrm{~B}$ & 1.241754 & -2.046628 & 0.007288 \\
\hline $7 \mathrm{~B}$ & -1.175149 & 2.076673 & 0.062516 \\
\hline $3 \mathrm{~B}$ & 1.152203 & 1.560613 & 1.413301 \\
\hline B & -2.376341 & 0.272518 & -0.024793 \\
\hline $\mathrm{OB}$ & -1.048855 & -1.644421 & -1.395643 \\
\hline $1 \mathrm{~B}$ & 1.112040 & 1.699261 & -1.305645 \\
\hline $2 \mathrm{E}$ & -0.717594 & 0.539279 & -2.248310 \\
\hline & 0.797299 & -0.448830 & -2.250780 \\
\hline & -1.118289 & -1.71256 & 1.30517 \\
\hline
\end{tabular}

$E 45 / \varepsilon=-239.434596$

atom $\mathrm{x} / \sigma \quad \mathrm{yl}$

$\begin{array}{llll}1 \mathrm{~A} & 0.000000 & 0.000000 & 0.000000\end{array}$

$\begin{array}{llll}2 \mathrm{~A} & 1.089679 & 0.000000 & 0.000000\end{array}$

$\begin{array}{llll}3 A & -0.409841 & -1.009668 & 0.000000\end{array}$

$\begin{array}{llll}4 \mathrm{~A} & 0.512288 & 0.264418 & 0.914231\end{array}$

$\begin{array}{llll}5 \mathrm{~A} & -0.437680 & -0.375221 & 0.914231\end{array}$

$\begin{array}{lllll}6 \mathrm{~A} & 0.512288 & 0.264418 & -0.914231\end{array}$

$\begin{array}{llll}7 A & -0.437680 & -0.375221 & -0.914231\end{array}$

$\begin{array}{llll}8 \mathrm{~A} & 0.511547 & -0.759729 & 0.533373\end{array}$

$\begin{array}{lllll}9 \mathrm{~A} & 0.511547 & -0.759729 & -0.533373\end{array}$

$\begin{array}{llll}10 \mathrm{~A} & 0.513777 & 0.944192 & 0.000000\end{array}$

$\begin{array}{llll}11 \mathrm{~A} & -1.068101 & -0.120929 & 0.000000\end{array}$

$\begin{array}{llll}12 \mathrm{~A} & -0.485356 & 0.720835 & 0.606184\end{array}$

$\begin{array}{llll}13 \mathrm{~A} & -0.485356 & 0.720835 & -0.606184\end{array}$

$\begin{array}{lllll}14 \mathrm{~B} & -0.382023 & 1.919667 & 0.000000\end{array}$

$\begin{array}{llll}15 \mathrm{~B} & -1.635028 & 1.075985 & 0.000000\end{array}$

$\begin{array}{llll}16 \mathrm{~B} & -0.299901 & 0.445404 & -1.949129\end{array}$

$\begin{array}{llll}17 \mathrm{~B} & -0.299901 & 0.445404 & 1.949129\end{array}$

$\begin{array}{llll}18 \mathrm{~B} & -1.457822 & -1.243898 & -0.751179\end{array}$

$\begin{array}{llll}19 \mathrm{~B} & -1.457822 & -1.243898 & 0.751179\end{array}$

$\begin{array}{llll}20 \mathrm{~B} & 1.700871 & 0.882934 & -0.751179\end{array}$

$\begin{array}{llll}21 B & 1.700871 & 0.882934 & 0.751179\end{array}$

$\begin{array}{llll}22 \mathrm{~B} & 0.432027 & 1.536912 & 1.226325\end{array}$

$\begin{array}{llll}23 \mathrm{~B} & -1.586551 & 0.177749 & -1.226326\end{array}$

$\begin{array}{llll}24 \mathrm{~B} & -1.586551 & 0.177749 & 1.226326\end{array}$

$\begin{array}{llll}25 \mathrm{~B} & 0.432027 & 1.536912 & -1.226325\end{array}$

$\begin{array}{llll}26 \mathrm{~A} & 0.474018 & -1.704546 & 0.000000\end{array}$

$\begin{array}{llll}27 A & 1.401104 & -1.080314 & 0.000000\end{array}$

$\begin{array}{llll}28 \mathrm{~A} & 1.431241 & -0.406181 & 0.964921\end{array}$

$\begin{array}{llll}29 A & -0.161951 & -1.478919 & -0.964922\end{array}$

$\begin{array}{lllll}30 \mathrm{~A} & 1.431241 & -0.406181 & -0.964921\end{array}$

$\begin{array}{llll}31 \mathrm{~A} & -0.161951 & -1.478919 & 0.964922\end{array}$

$\begin{array}{llll}32 \mathrm{~A} & 0.425878 & -0.632497 & -1.586262\end{array}$

$\begin{array}{llll}33 \mathrm{~A} & 0.425878 & -0.632497 & 1.586262\end{array}$

34B $\quad 1.139963-1.693030-1.167785$

$\begin{array}{llll}35 B & 1.139963 & -1.693030 & 1.167785\end{array}$

$\begin{array}{llll}36 \mathrm{~B} & 2.396037 & -0.265178 & 0.000000\end{array}$

$\begin{array}{llll}37 \mathrm{~B} & -0.655473 & -2.319841 & 0.000000\end{array}$

$\begin{array}{llll}38 \mathrm{~B} & -0.747529 & -1.000613 & 2.063637\end{array}$

$\begin{array}{llll}39 \mathrm{~B} & 1.208298 & 0.316298 & 2.063637\end{array}$

$\begin{array}{llll}40 \mathrm{~B} & -0.747529 & -1.000613 & -2.063637\end{array}$

$\begin{array}{llll}41 B & 1.208298 & 0.316298 & -2.063637\end{array}$

$\begin{array}{llll}42 \mathrm{~B} & -2.403770 & -0.274229 & 0.000000\end{array}$

$\begin{array}{llll}43 \mathrm{~B} & 1.158184 & 2.124130 & 0.000000\end{array}$

$\begin{array}{llll}44 \mathrm{~B} & -1.104798 & 1.640807 & 1.347343\end{array}$

$\begin{array}{lllll}45 B & -1.104798 & 1.640807 & -1.347343\end{array}$
$E 46 / \varepsilon=-245.176668$

atom $\quad \mathrm{x} / \sigma \quad \mathrm{g} / \sigma$

$\begin{array}{lllc}1 \mathrm{~A} & 0.000000 & 0.000000 & 0.000000\end{array}$

$\begin{array}{lllll}2 \mathrm{~A} & 1.094799 & 0.000000 & 0.000000\end{array}$

$\begin{array}{llll}3 \mathrm{~A} & -0.417732 & -1.011972 & 0.000000\end{array}$

$\begin{array}{llll}4 \mathrm{~A} & 0.491261 & 0.298070 & 0.911173\end{array}$

$\begin{array}{llll}5 A & -0.462964 & -0.340363 & 0.911173\end{array}$

$\begin{array}{lllll}6 A & 0.526295 & -0.786621 & -0.527297\end{array}$

$\begin{array}{lllll}7 \mathrm{~A} & 0.518756 & 0.274389 & -0.890711\end{array}$

$\begin{array}{llll}8 A & -0.451568 & -0.374814 & -0.890710\end{array}$

$\begin{array}{llll}9 \mathrm{~A} & 0.493319 & -0.737334 & 0.555445\end{array}$

$\begin{array}{llll}10 A & -0.491346 & 0.734383 & -0.583238\end{array}$

$\begin{array}{llll}11 \mathrm{~A} & -1.075805 & -0.096974 & -0.003154\end{array}$

$\begin{array}{llll}12 \mathrm{~A} & 0.500120 & 0.957412 & -0.003155\end{array}$

$\begin{array}{llll}13 A & -0.499039 & 0.745882 & 0.581453\end{array}$

$\begin{array}{llll}14 \mathrm{~B} & 1.614909 & -0.546744 & -1.155050\end{array}$

$\begin{array}{llll}15 B & -0.110807 & -1.701348 & -1.155049\end{array}$

$\begin{array}{llll}16 \mathrm{~B} & 0.396844 & 1.522522 & -1.244381\end{array}$

$\begin{array}{llll}17 \mathrm{~B} & -1.558757 & 0.214112 & -1.244380\end{array}$

$\begin{array}{llll}18 \mathrm{~B} & -0.398227 & 1.949089 & -0.029810\end{array}$

$\begin{array}{llll}19 \mathrm{~B} & -1.649683 & 1.111792 & -0.029809\end{array}$

$\begin{array}{llll}\text { 20B } & 1.666981 & 0.923740 & 0.740246\end{array}$

$\begin{array}{llll}21 B & -1.489906 & -1.188402 & 0.740248\end{array}$

$\begin{array}{llll}22 \mathrm{~B} & -0.336323 & 0.502682 & 1.933287\end{array}$

$\begin{array}{lllll}23 B & 1.686420 & 0.899819 & -0.771230\end{array}$

$\begin{array}{llll}24 \mathrm{~B} & -1.475215 & -1.215498 & -0.771228\end{array}$

$\begin{array}{llll}25 \mathrm{~B} & 0.404405 & 1.581274 & 1.204039\end{array}$

$\begin{array}{llll}26 \mathrm{~B} & -1.615946 & 0.229541 & 1.204040\end{array}$

$\begin{array}{llll}27 \mathrm{~A} & 0.450177 & -1.703545 & 0.086490\end{array}$

$\begin{array}{llll}28 A & 1.402891 & -1.066123 & 0.086489\end{array}$

$\begin{array}{llll}29 A & -0.284540 & 0.425281 & -1.631620\end{array}$

$\begin{array}{llll}30 A & 1.397460 & -0.364539 & 1.002670\end{array}$

$\begin{array}{llll}31 \mathrm{~A} & -0.196256 & -1.430828 & 1.002670\end{array}$

$\begin{array}{llll}32 \mathrm{~A} & 0.358974 & -0.536540 & -1.579180\end{array}$

$\begin{array}{llll}33 \mathrm{~A} & 0.385925 & -0.576818 & 1.605114\end{array}$

$\begin{array}{llll}34 \mathrm{~B} & 1.102687 & -1.648118 & 1.249138\end{array}$

$\begin{array}{llll}35 \mathrm{~B} & -0.780216 & -0.733834 & -2.149378\end{array}$

$\begin{array}{llll}36 \mathrm{~B} & 0.976010 & 0.441184 & -2.149379\end{array}$

$\begin{array}{llll}37 \mathrm{~B} & 2.411302 & -0.238006 & 0.117567\end{array}$

$\begin{array}{llll}38 \mathrm{~B} & -0.700058 & -2.319687 & 0.117569\end{array}$

$\begin{array}{lllll}39 B & 1.340958 & -2.004249 & -0.826419\end{array}$

$\begin{array}{llll}40 \mathrm{~B} & -0.804722 & -0.932651 & 2.068999\end{array}$

$\begin{array}{llll}41 \mathrm{~B} & 1.169140 & 0.387979 & 2.068998\end{array}$

$\begin{array}{llll}42 \mathrm{~B} & -2.415951 & -0.229563 & -0.038615\end{array}$

$\begin{array}{llll}43 \mathrm{~B} & 1.134025 & 2.145578 & -0.038618\end{array}$

$\begin{array}{llll}44 \mathrm{~B} & -1.128709 & 1.687011 & 1.310153\end{array}$

$\begin{array}{llll}45 B & -1.090342 & 1.629665 & -1.396976\end{array}$ $\begin{array}{llll}46 \mathrm{~B} & 0.965353 & -1.442858 & -2.261084\end{array}$

\section{$E 47 / \varepsilon=-251.178900$}

$$
\text { atom } \mathrm{x} / \sigma \quad y / \sigma \quad z / \sigma
$$

$\begin{array}{llll}1 \mathrm{~A} & 0.000000 & 0.000000 & 0.000000\end{array}$

$\begin{array}{llll}2 \mathrm{~A} & 1.073779 & 0.000000 & 0.000000\end{array}$

$\begin{array}{llll}3 \mathrm{~A} & 0.484381 & -0.931986 & 0.000000\end{array}$

$\begin{array}{lllll}4 \mathrm{~A} & 0.542583 & -0.270697 & 0.898067\end{array}$

$\begin{array}{lllll}5 A & 0.484491 & 0.761944 & -0.561859\end{array}$

$\begin{array}{lllll}6 \mathrm{~A} & 0.505054 & -0.299264 & -0.904195\end{array}$

$\begin{array}{llll}7 A & -0.414492 & -0.781214 & 0.609545\end{array}$

$\begin{array}{lllll}8 A & 0.520864 & 0.757510 & 0.555779\end{array}$

$\begin{array}{lllll}9 A & -0.449957 & 0.963899 & 0.020238\end{array}$

$\begin{array}{llll}10 A & -0.474741 & 0.299426 & -0.909377\end{array}$

$\begin{array}{llll}11 \mathrm{~A} & -0.463007 & -0.808485 & -0.535662\end{array}$

$\begin{array}{llll}12 \mathrm{~A} & -1.062970 & -0.004180 & 0.076064\end{array}$

$\begin{array}{llll}13 \mathrm{~A} & 1.419883 & -0.862988 & 0.587806\end{array}$

$\begin{array}{llll}14 \mathrm{~A} & -0.410893 & 0.297330 & 0.936140\end{array}$

$\begin{array}{llll}15 \mathrm{~B} & 1.665004 & 0.391539 & 1.117849\end{array}$

$\begin{array}{llll}16 \mathrm{~A} & 0.470672 & -1.350307 & 1.002248\end{array}$

$\begin{array}{llll}17 \mathrm{~A} & 1.416521 & -0.862348 & -0.548383\end{array}$

$\begin{array}{llll}18 \mathrm{~B} & -0.214345 & -2.036562 & 0.118398\end{array}$

$\begin{array}{lllll}19 \mathrm{~B} & 0.531653 & -1.637465 & -1.117539\end{array}$

$\begin{array}{llll}20 \mathrm{~B} & -1.602521 & -0.404649 & -1.081037\end{array}$ $\begin{array}{llll}21 \mathrm{~B} & 0.494323 & 0.622252 & -1.871843\end{array}$ \begin{tabular}{|llll}
$22 \mathrm{~B}$ & -0.300504 & 1.657867 & -1.126220
\end{tabular}

$\begin{array}{llll}23 \mathrm{~B} & -1.530302 & -1.283975 & 0.165175\end{array}$

$\begin{array}{llll}24 \mathrm{~B} & -1.585379 & 1.044000 & -0.618876\end{array}$

$\begin{array}{llll}25 B & -0.312077 & -0.647255 & -1.872219\end{array}$

$\begin{array}{llll}26 \mathrm{~B} & 0.382051 & 0.638314 & 1.894269\end{array}$

$\begin{array}{llll}27 \mathrm{~A} & 0.395122 & 1.689620 & 0.000082\end{array}$

$\begin{array}{lllll}28 \mathrm{~A} & 1.424251 & 0.369252 & -0.953543\end{array}$

$\begin{array}{llll}29 \mathrm{~A} & 1.417213 & 1.032451 & -0.004978\end{array}$

$\begin{array}{lllll}30 \mathrm{~B} & 2.367032 & -0.057727 & -0.136751\end{array}$

$\begin{array}{llll}31 \mathrm{~B} & -1.433001 & -0.377258 & 1.381041\end{array}$

$\begin{array}{llll}32 \mathrm{~B} & -1.464212 & 1.058024 & 0.897308\end{array}$

$\begin{array}{llll}33 \mathrm{~B} & 1.132116 & -0.752945 & 1.964675\end{array}$

$\begin{array}{llll}34 \mathrm{~A} & -0.153841 & -0.557109 & 1.661630\end{array}$

$\begin{array}{llll}35 A & -0.195637 & 1.429033 & 0.991381\end{array}$

$\begin{array}{llll}36 \mathrm{~A} & 1.090118 & -1.803740 & 0.072178\end{array}$

$\begin{array}{llll}37 \mathrm{~B} & 1.092161 & 1.811313 & 1.090451\end{array}$

$\begin{array}{llll}38 \mathrm{~B} & 1.236389 & 1.722949 & -1.079414\end{array}$

$\begin{array}{lllll}39 \mathrm{~B} & 1.397700 & -0.611030 & -1.840484\end{array}$

$\begin{array}{llll}40 B & -0.711083 & -1.731311 & 1.510436\end{array}$

$\begin{array}{llll}41 \mathrm{~B} & 1.577268 & -2.037771 & 1.252234\end{array}$

$\begin{array}{llll}42 \mathrm{~B} & 2.373380 & -1.608101 & -0.052558\end{array}$

$\begin{array}{llll}43 \mathrm{~B} & -0.818556 & 2.243844 & 0.168667\end{array}$

$\begin{array}{lllll}44 \mathrm{~B} & -1.012745 & -1.837902 & -1.187207\end{array}$

$\begin{array}{lllll}45 B & -1.062973 & 0.687270 & -2.043130\end{array}$

$\begin{array}{llll}46 \mathrm{~B} & -2.383765 & 0.006417 & 0.207387\end{array}$

$\begin{array}{llll}47 \mathrm{~B} & 2.558041 & -0.829127 & 1.264520\end{array}$

\section{$E 48 / \varepsilon=-258.325633$}

atom $\mathrm{X} / \sigma$

1A $\quad 0.000000 \quad 0.000000$

$z / \sigma$

$\begin{array}{llll}1.073996 & 0.000000 & 0.000000\end{array}$

$\begin{array}{llll}3 A & 0.466208 & -0.930545 & 0.000000\end{array}$

$\begin{array}{lllll}4 \mathrm{~A} & 0.493288 & -0.303804 & -0.897767\end{array}$

$\begin{array}{lllll}5 \mathrm{~A} & 0.493288 & -0.303804 & 0.897767\end{array}$

$\begin{array}{llll}6 \mathrm{~A} & -0.464423 & -0.819003 & -0.551002\end{array}$

$\begin{array}{llll}7 \mathrm{~A} & -0.464424 & -0.819003 & 0.551002\end{array}$

$\begin{array}{llll}8 A & -1.063230 & -0.085460 & -0.000001\end{array}$

$\begin{array}{llll}9 \mathrm{~A} & -0.500794 & 0.927349 & 0.000000\end{array}$

$\begin{array}{llll}10 A & 0.464763 & 0.774308 & -0.583771\end{array}$

$\begin{array}{lllll}11 \mathrm{~A} & 0.464763 & 0.774308 & 0.583770\end{array}$

$\begin{array}{llll}12 \mathrm{~A} & 1.370762 & -0.915332 & 0.588577\end{array}$

$\begin{array}{lllll}13 \mathrm{~A} & 1.370763 & -0.915333 & -0.588577\end{array}$

$\begin{array}{llll}14 \mathrm{~A} & -0.473880 & 0.245745 & -0.913020\end{array}$

$\begin{array}{llll}15 A & -0.473880 & 0.245745 & 0.913019\end{array}$

$\begin{array}{llll}16 \mathrm{~B} & 1.598766 & 0.300740 & 1.197839\end{array}$

$\begin{array}{llll}17 \mathrm{~B} & 1.598766 & 0.300740 & -1.197840\end{array}$

$\begin{array}{llll}18 B & -0.229507 & -2.052683 & 0.000000\end{array}$

$\begin{array}{llll}19 A & 0.379043 & -1.397646 & 0.963969\end{array}$

$\begin{array}{lllll}20 \mathrm{~A} & 0.379043 & -1.397646 & -0.963969\end{array}$

$\begin{array}{llll}21 \mathrm{~B} & 1.596577 & 1.216596 & 0.000000\end{array}$

$\begin{array}{llll}22 \mathrm{~B} & -1.563779 & -1.317346 & -0.000001\end{array}$

$\begin{array}{llll}23 \mathrm{~B} & 2.370782 & -0.197167 & 0.000000\end{array}$

$\begin{array}{llll}24 \mathrm{~B} & 0.269844 & 1.981624 & -0.000001\end{array}$

$\begin{array}{lllll}25 \mathrm{~B} & -1.533722 & -0.399278 & -1.239976\end{array}$

$\begin{array}{llll}26 \mathrm{~B} & -1.533723 & -0.399278 & 1.239975\end{array}$

$\begin{array}{llll}27 B & 0.294468 & 0.487014 & 1.952703\end{array}$

$\begin{array}{lllll}28 \mathrm{~B} & 0.294469 & 0.487014 & -1.952703\end{array}$

$\begin{array}{llll}29 B & -0.530432 & 1.512783 & 1.210415\end{array}$

$\begin{array}{llll}30 \mathrm{~B} & -0.530431 & 1.512783 & -1.210416\end{array}$

$\begin{array}{llll}31 \mathrm{~B} & 1.015741 & -0.881669 & 1.968762\end{array}$

$\begin{array}{lllll}32 \mathrm{~B} & 1.015742 & -0.881670 & -1.968763\end{array}$

$\begin{array}{llll}33 \mathrm{~A} & 1.037176 & -1.817013 & 0.000000\end{array}$

$\begin{array}{llll}34 \mathrm{~B} & 2.326127 & -1.710446 & 0.000000\end{array}$

$\begin{array}{llll}35 \mathrm{~A} & -1.421561 & 0.762558 & -0.578401\end{array}$

$\begin{array}{llll}36 \mathrm{~A} & -1.421562 & 0.762558 & 0.578399\end{array}$

$\begin{array}{llll}37 \mathrm{~A} & -0.257851 & -0.648496 & -1.605032\end{array}$

$\begin{array}{llll}38 \mathrm{~A} & -0.257852 & -0.648495 & 1.605031\end{array}$

$\begin{array}{lllll}39 B & 1.429799 & -2.146742 & -1.195701\end{array}$

$\begin{array}{llll}40 B & 1.429799 & -2.146742 & 1.195701\end{array}$

41B $-0.847041-1.793192-1.363510$

$\begin{array}{llll}42 \mathrm{~B} & -0.847042 & -1.793192 & 1.363509\end{array}$

$\begin{array}{llll}43 \mathrm{~B} & -2.373603 & 0.034038 & -0.000001\end{array}$

$\begin{array}{llll}44 \mathrm{~B} & -1.348057 & 1.959487 & -0.000001\end{array}$ 
$\begin{array}{llll}45 B & 1.004869 & 1.724971 & -1.341707\end{array}$ $\begin{array}{llll}46 \mathrm{~B} & 1.004869 & 1.724972 & 1.341706\end{array}$ $\begin{array}{lllll}47 \mathrm{~B} & 2.449068 & -0.974680 & -1.357947\end{array}$ $\begin{array}{llll}48 B & 2.449068 & -0.974680 & 1.357947\end{array}$

\section{$E 49 / \varepsilon=-264.629464$}

$\begin{array}{cccc}\text { atom } & \mathrm{x} / \sigma & y / \sigma & z / \sigma \\ & 0.0000 & 0.00000 & 0.000000\end{array}$ $\begin{array}{llll}2 \mathrm{~A} & 1.072956 & 0.000000 & 0.000000\end{array}$ $\begin{array}{llll}3 A & -0.429039 & -0.983443 & 0.000000\end{array}$

$\begin{array}{llll}4 \mathrm{~A} & 0.503948 & -0.769671 & 0.565302\end{array}$

$\begin{array}{lllll}5 \mathrm{~A} & 0.496567 & -0.758398 & -0.533498\end{array}$

$\begin{array}{lllll}6 \mathrm{~A} & 0.492280 & 0.235566 & -0.936457\end{array}$

$\begin{array}{llll}7 A & -0.412760 & -0.357015 & -0.936457\end{array}$

$\begin{array}{lllll}8 \mathrm{~A} & 0.478911 & 0.950225 & -0.073288\end{array}$

$\begin{array}{lllll}9 A & -1.062452 & -0.058994 & -0.073288\end{array}$

$\begin{array}{llll}10 \mathrm{~A} & 0.485422 & 0.335622 & 0.889656\end{array}$

$\begin{array}{llll}11 \mathrm{~A} & -0.501726 & -0.310721 & 0.889656\end{array}$

$\begin{array}{llll}12 \mathrm{~A} & -0.524571 & 0.801168 & 0.463012\end{array}$

$\begin{array}{llll}13 \mathrm{~A} & 1.406571 & -1.066793 & -0.055966\end{array}$

$\begin{array}{llll}14 \mathrm{~A} & 0.415353 & -1.715800 & -0.055966\end{array}$

$\begin{array}{llll}15 \mathrm{~A} & -0.463495 & 0.707888 & -0.652100\end{array}$

$\begin{array}{llll}16 \mathrm{~B} & -0.325273 & -1.675809 & 1.097704\end{array}$

$\begin{array}{llll}17 \mathrm{~B} & 1.666068 & -0.371963 & 1.097703\end{array}$

$\begin{array}{lllll}18 \mathrm{~B} & 0.486024 & -0.742296 & -1.868283\end{array}$

$\begin{array}{llll}19 \mathrm{~A} & -0.216421 & -1.426284 & -0.992869\end{array}$

$\begin{array}{lllll}20 \mathrm{~A} & 1.393834 & -0.371958 & -0.992869\end{array}$

$\begin{array}{llll}21 B & -0.441858 & 0.674843 & 1.789257\end{array}$

$\begin{array}{llll}22 \mathrm{~B} & 0.394798 & -0.602967 & 1.867000\end{array}$

$\begin{array}{llll}23 \mathrm{~B} & -1.608533 & -1.097496 & 0.592537\end{array}$

$\begin{array}{llll}24 \mathrm{~B} & 1.649134 & 1.035487 & 0.592536\end{array}$

$\begin{array}{llll}25 \mathrm{~B} & 1.171713 & -1.789537 & 1.074708\end{array}$

$\begin{array}{llll}26 \mathrm{~B} & 0.354070 & 1.695951 & 0.992408\end{array}$

$\begin{array}{llll}27 \mathrm{~B} & -1.696044 & 0.353623 & 0.992408\end{array}$

$\begin{array}{lllll}28 \mathrm{~B} & -0.352426 & 0.538254 & -1.931198\end{array}$

$\begin{array}{llll}29 \mathrm{~A} & -1.280490 & -0.965659 & -0.680572\end{array}$

$\begin{array}{lllll}30 A & 1.397123 & 0.787529 & -0.680572\end{array}$

$\begin{array}{llll}31 B & 2.375852 & -0.039978 & -0.221835\end{array}$

$\begin{array}{llll}32 \mathrm{~B} & -0.913381 & -2.193629 & -0.221835\end{array}$

$\begin{array}{llll}33 \mathrm{~B} & -0.378689 & 1.959825 & -0.312243\end{array}$

$\begin{array}{llll}34 \mathrm{~B} & -1.644898 & 1.130765 & -0.312242\end{array}$

$\begin{array}{llll}35 \mathrm{~A} & 1.012563 & -1.546469 & -1.003871\end{array}$

$\begin{array}{llll}36 \mathrm{~B} & 1.583574 & -2.418564 & -0.236230\end{array}$

$\begin{array}{llll}37 \mathrm{~A} & 0.414960 & 1.285405 & -1.131827\end{array}$

$\begin{array}{lllll}38 \mathrm{~A} & -1.344096 & 0.133650 & -1.131826\end{array}$

$\begin{array}{llll}39 \mathrm{~B} & 1.237400 & 0.591443 & -1.954299\end{array}$

$\begin{array}{lllll}40 \mathrm{~B} & -1.036897 & -0.897668 & -1.954298\end{array}$

41B $\quad 0.293045 \quad-2.630137-1.076008$

$\begin{array}{llll}42 B & 2.293536 & -1.320302 & -1.076007\end{array}$

$\begin{array}{lllll}43 \mathrm{~B} & -2.360675 & -0.222569 & -0.380942\end{array}$

$\begin{array}{lllll}44 \mathrm{~B} & 1.147956 & 2.074734 & -0.380942\end{array}$

$\begin{array}{llll}45 \mathrm{~B} & 1.091672 & 0.765479 & 2.000590\end{array}$

$\begin{array}{llll}46 \mathrm{~B} & -1.138141 & -0.694508 & 2.000590\end{array}$

$\begin{array}{llll}47 \mathrm{~B} & -1.186692 & 1.812415 & 1.013141\end{array}$

$\begin{array}{llll}48 \mathrm{~B} & 2.570345 & -1.439245 & 0.454118\end{array}$

$\begin{array}{llll}\text { 49B } & 0.291380 & -2.931416 & 0.454118\end{array}$

$E 50 / \varepsilon=-271.536410$

$\begin{array}{cccc}\text { atom } & x / \sigma & y / \sigma & z / \sigma \\ 1 \mathrm{~A} & 0.000000 & 0.000000 & 0.000000 \\ \text { 2A } & 1.075219 & 0.000000 & 0.000000 \\ \text { 3A } & 0.492675 & -0.953435 & 0.000000 \\ \text { 4A } & 0.484257 & -0.298973 & 0.865150 \\ \text { 5A } & 0.490337 & 0.745395 & 0.598184 \\ \text { 6A } & -0.446297 & 0.250442 & 0.956655 \\ \text { 7A } & -0.446098 & -0.788267 & 0.593817 \\ \text { 8A } & -1.062651 & -0.037173 & 0.105511 \\ \text { 9A } & -0.520553 & 0.297484 & -0.860718 \\ \text { 10A } & 0.455030 & 0.807565 & -0.529316 \\ 11 \mathrm{~A} & 0.454967 & -0.305076 & -0.917284 \\ \text { 12A } & 1.373971 & -0.861674 & 0.683472 \\ \text { 13A } & 1.374401 & 0.250991 & 1.073017 \\ \text { 14A } & -0.481810 & 0.938475 & 0.087056\end{array}$
$\begin{array}{llll}16 \mathrm{~A} & 0.402604 & -1.341351 & 1.032978\end{array}$ $\begin{array}{llll}17 \mathrm{~A} & 0.395415 & 0.392006 & 1.669454\end{array}$ $\begin{array}{llll}18 \mathrm{~A} & -0.210581 & -0.574671 & 1.643211\end{array}$ $\begin{array}{llll}19 \mathrm{~A} & 0.954636 & -0.611079 & 1.753367\end{array}$ $\begin{array}{llll}20 B & 1.590892 & 1.227031 & 0.116661\end{array}$ $\begin{array}{llll}21 \mathrm{~B} & 1.593308 & -1.038088 & -0.671383\end{array}$ $\begin{array}{llll}22 \mathrm{~B} & -1.512721 & -0.450215 & 1.294490\end{array}$ $\begin{array}{llll}23 B & 1.583009 & 0.397602 & -1.148220\end{array}$ $\begin{array}{llll}24 \mathrm{~B} & 2.367874 & -0.069213 & 0.190285\end{array}$ $\begin{array}{llll}25 \mathrm{~B} & 0.248112 & 0.643886 & -1.857303\end{array}$ $\begin{array}{llll}26 \mathrm{~B} & -1.524113 & 1.019870 & 0.834405\end{array}$ $\begin{array}{llll}27 \mathrm{~B} & -1.516989 & -1.325601 & 0.031314\end{array}$ $\begin{array}{llll}28 \mathrm{~B} & 1.013698 & 1.538980 & 1.501137\end{array}$ $\begin{array}{llll}29 B & 0.279380 & -1.698721 & -1.071175\end{array}$ $\begin{array}{llll}30 \mathrm{~B} & 0.280832 & 1.995314 & 0.209427\end{array}$ $\begin{array}{llll}31 B & 1.020553 & -2.145454 & 0.215931\end{array}$ $\begin{array}{llll}32 \mathrm{~B} & -0.549737 & 1.631867 & -1.009453\end{array}$ $\begin{array}{llll}33 \mathrm{~B} & -0.548530 & -0.658128 & -1.804350\end{array}$ $\begin{array}{llll}34 \mathrm{~B} & 2.284312 & -0.569616 & 1.622588\end{array}$ $\begin{array}{llll}35 \mathrm{~A} & -1.436549 & -0.316738 & -0.874122\end{array}$ $\begin{array}{llll}36 \mathrm{~A} & -1.438125 & 0.785381 & -0.493892\end{array}$ $\begin{array}{llll}37 \mathrm{~A} & -0.247872 & -1.747240 & 0.142219\end{array}$ $\begin{array}{llll}38 \mathrm{~B} & -0.769044 & -1.792435 & 1.374373\end{array}$ $\begin{array}{llll}39 \mathrm{~A} & -0.254106 & 1.286771 & 1.186703\end{array}$ $\begin{array}{llll}40 \mathrm{~B} & -0.799313 & 0.585050 & 2.171477\end{array}$ $\begin{array}{llll}\text { 41B } & 1.447257 & -1.838367 & 1.653293\end{array}$ $\begin{array}{llll}42 \mathrm{~B} & 1.478054 & 0.426243 & 2.425530\end{array}$ $\begin{array}{llll}43 B & 0.232958 & -0.330348 & 2.843076\end{array}$ $\begin{array}{llll}44 \mathrm{~B} & -2.368460 & -0.017248 & 0.032798\end{array}$ $\begin{array}{llll}45 \mathrm{~B} & -1.378110 & 0.627843 & -1.816577\end{array}$ $\begin{array}{lllll}46 \mathrm{~B} & 0.983835 & -0.714569 & -2.058726\end{array}$ $\begin{array}{lllll}47 \mathrm{~B} & 0.983916 & 1.834417 & -1.174499\end{array}$ $\begin{array}{llll}\text { 48B } & 2.454957 & -1.602810 & 0.480411\end{array}$ $\begin{array}{llll}49 B & 2.463615 & 0.957621 & 1.356683\end{array}$ $\begin{array}{llll}50 A & 0.281477 & -1.436219 & 2.123550\end{array}$

\section{$s=1.5$}

$E 5 / \varepsilon=-9.184718$

$\begin{array}{cccc}\text { atom } & x / \sigma & y / \sigma & z / \sigma \\ \text { 1A } & 0.000000 & 0.000000 & 0.000000 \\ \text { 2A } & 1.123998 & 0.000000 & 0.000000 \\ \text { 3A } & 0.562000 & -0.973411 & 0.000000 \\ \text { 4B } & 0.562000 & -0.324470 & 1.239161 \\ \text { 5B } & 0.562000 & -0.324470 & -1.239161\end{array}$ $E 6 / \varepsilon=-12.968869$

$\begin{array}{cccc}\text { atom } & x / \sigma & y / \sigma & z / \sigma \\ \text { 1A } & 0.000000 & 0.000000 & 0.000000 \\ \text { 2A } & 1.184180 & 0.000000 & 0.000000 \\ \text { 3B } & 0.592090 & -1.242964 & 0.000000 \\ \text { 4B } & 0.592090 & 0.000000 & 1.242964 \\ \text { 5B } & 0.592090 & 0.000000 & -1.242964 \\ \text { 6B } & 0.592090 & 1.242964 & 0.000000\end{array}$

$E 7 / \varepsilon=-16.609636$

$\begin{array}{cccc}\text { atom } & \mathrm{X} / \sigma & y / \sigma & \mathrm{z} / \sigma \\ 1 \mathrm{~A} & 0.000000 & 0.000000 & 0.000000\end{array}$ $\begin{array}{llll}2 \mathrm{~B} & 1.409860 & 0.000000 & 0.000000\end{array}$ $\begin{array}{llll}\text { 3B } & 0.394643 & -1.341452 & 0.000000\end{array}$ $\begin{array}{llll}4 B & 0.396357 & 1.048084 & 0.837272\end{array}$ $\begin{array}{llll}5 \mathrm{~B} & 0.396357 & 1.048084 & -0.837272\end{array}$ $\begin{array}{lllll}6 \mathrm{~A} & 0.454748 & -0.338539 & 0.966493\end{array}$ $\begin{array}{lllll}7 \mathrm{~A} & 0.454748 & -0.338539 & -0.966493\end{array}$

$E 8 / \varepsilon=-20.714043$

$\begin{array}{llll}\text { atom } & \mathrm{x} / \sigma & \mathrm{y} / \sigma & \mathrm{z} / \sigma\end{array}$ $\begin{array}{cccc}1 \mathrm{~A} & 0.000000 & 0.000000 & 0.000000\end{array}$ $\begin{array}{llll}2 \mathrm{~B} & 1.372614 & 0.000000 & 0.000000\end{array}$ $\begin{array}{llll}3 \mathrm{~B} & 0.234630 & -1.361777 & 0.000000\end{array}$ $\begin{array}{lllll}4 \mathrm{~B} & 0.234631 & 0.916203 & -1.007477\end{array}$ $\begin{array}{lllll}5 A & 0.494380 & -0.428603 & -0.969103\end{array}$

$\begin{array}{llll}6 \mathrm{~B} & 0.359385 & 1.191352 & 0.630439\end{array}$

$\begin{array}{llll}7 \mathrm{~B} & 0.359385 & -0.335126 & 1.305551\end{array}$

$\begin{array}{lllll}8 B & -0.886536 & -0.420728 & -0.951297\end{array}$

$E 9 / \varepsilon=-25.991730$

$\begin{array}{llll}\text { atom } \quad \mathrm{x} / \sigma & \mathrm{y} / \sigma & \mathrm{z} / \sigma\end{array}$

$\begin{array}{llll}1 \mathrm{~A} & 0.000000 & 0.000000 & 0.000000\end{array}$

$\begin{array}{llll}\text { 2B } & 1.381971 & 0.000000 & 0.000000\end{array}$

3B $\quad 0.365941-1.332641 \quad 0.000000$

$\begin{array}{llll}4 \mathrm{~B} & -0.650092 & -0.558001 & -1.084371\end{array}$

$\begin{array}{lllll}5 B & 0.365939 & 0.774640 & -1.084373\end{array}$

$\begin{array}{llll}6 \mathrm{~B} & 0.352503 & -0.268752 & 1.308953\end{array}$

$\begin{array}{lllll}7 B & -1.084383 & 0.826753 & -0.224581\end{array}$

$\begin{array}{llll}8 \mathrm{~B} & 0.352503 & 1.221321 & 0.542183\end{array}$

$\begin{array}{llll}9 B & -1.084381 & -0.663321 & 0.542187\end{array}$

$E 10 / \varepsilon=-30.931901$

atom $\mathrm{x} / \sigma \quad \mathrm{g} / \sigma \quad \mathrm{z} / \sigma$

$\begin{array}{llll}1 \mathrm{~A} & 0.000000 & 0.000000 & 0.000000\end{array}$

$\begin{array}{llll}2 \mathrm{~B} & 1.377337 & 0.000000 & 0.000000\end{array}$

$\begin{array}{llll}\text { 3B } & 0.300770 & -1.344097 & 0.000000\end{array}$

$\begin{array}{llll}4 \mathrm{~B} & 0.321982 & 1.018262 & 0.855047\end{array}$

$\begin{array}{llll}5 B & -0.923376 & -0.536571 & 0.855046\end{array}$

$\begin{array}{llll}6 \mathrm{~B} & 0.321988 & 1.018267 & -0.855040\end{array}$

$\begin{array}{llll}7 \mathrm{~B} & -0.923379 & -0.536577 & -0.855040\end{array}$

$\begin{array}{llll}8 \mathrm{~B} & -1.099417 & 0.880588 & -0.000002\end{array}$

$\begin{array}{lllll}9 \mathrm{~A} & 0.446546 & -0.357665 & -0.967696\end{array}$

$\begin{array}{llll}10 \mathrm{~A} & 0.446550 & -0.357669 & 0.967696\end{array}$

$E 11 / \varepsilon=-35.487787$

atom $\mathrm{X} / \sigma$

$y / \sigma$

$\begin{array}{llll}1 \mathrm{~A} & 0.000000 & 0.000000 & 0.000000\end{array}$

2B $\quad 1.401505 \quad 0.000000 \quad 0.000000$

$\begin{array}{llll}\text { 3B } & 0.386899 & -1.347044 & 0.000000\end{array}$

$\begin{array}{llll}4 \mathrm{~B} & 0.386899 & 0.764212 & -1.109282\end{array}$

$\begin{array}{lllll}5 \mathrm{~B} & -0.627707 & -0.582832 & -1.109283\end{array}$

$\begin{array}{llll}6 \mathrm{~B} & 0.404461 & -0.304646 & 1.294483\end{array}$

$\begin{array}{llll}7 \mathrm{~B} & 0.404462 & 1.238831 & 0.483520\end{array}$

$\begin{array}{llll}8 \mathrm{~B} & -1.079036 & -0.730738 & 0.483518\end{array}$

$\begin{array}{lllll}9 \mathrm{~B} & -1.079036 & 0.812740 & -0.327446\end{array}$

$\begin{array}{llll}10 A & -0.644098 & 0.485139 & 0.923348\end{array}$

$\begin{array}{lllll}11 \mathrm{~A} & 0.750149 & -0.565019 & -1.075380\end{array}$

$E 12 / \varepsilon=-40.000211$

atom $\mathrm{x} / \sigma \quad y / \sigma \quad z / \sigma$

$\begin{array}{llll}1 \mathrm{~A} & 0.000000 & 0.000000 & 0.000000\end{array}$

$\begin{array}{llll}2 B & 1.353576 & 0.000000 & 0.000000\end{array}$

$\begin{array}{llll}3 \mathrm{~B} & -1.011156 & -0.943526 & 0.000000\end{array}$

$\begin{array}{lllll}4 \mathrm{~B} & -1.011156 & 0.599713 & -0.728411\end{array}$

$\begin{array}{lllll}5 A & 0.398240 & 0.409974 & -0.949630\end{array}$

$\begin{array}{lllll}6 \mathrm{~A} & 0.398241 & -0.993708 & -0.287090\end{array}$

$\begin{array}{llll}7 A & -0.233240 & -0.485510 & -1.028619\end{array}$

$\begin{array}{llll}8 \mathrm{~B} & 0.249759 & 1.383608 & 0.007340\end{array}$

$\begin{array}{llll}9 \mathrm{~B} & 0.249760 & -0.873768 & 1.072826\end{array}$

$\begin{array}{llll}10 \mathrm{~B} & -0.887621 & 0.443736 & 0.940118\end{array}$

$\begin{array}{llll}11 \mathrm{~A} & 0.490164 & 0.457250 & 0.968745\end{array}$

$\begin{array}{llll}12 \mathrm{~B} & 1.054580 & -0.698142 & -1.479108\end{array}$

$E 13 / \varepsilon=-45.475297$

atom $\mathrm{X} / \sigma$

$0.000000-0.000000$

$\begin{array}{llll}2 \mathrm{~A} & 1.148432 & 0.000000 & 0.000000\end{array}$

$\begin{array}{llll}3 \mathrm{~A} & 0.608462 & -0.973997 & 0.000000\end{array}$

$\begin{array}{llll}4 \mathrm{~B} & -1.399472 & -0.189028 & -0.000001\end{array}$

$\begin{array}{llll}5 \mathrm{~B} & -0.581152 & 1.287056 & 0.000000\end{array}$

$\begin{array}{llll}6 B & -0.532776 & 0.295362 & 1.314231\end{array}$

$\begin{array}{lllll}7 \mathrm{~B} & -0.532774 & 0.295363 & -1.314233\end{array}$

$\begin{array}{lllll}8 \mathrm{~A} & 0.596870 & -0.330896 & -0.894069\end{array}$

$\begin{array}{llll}9 \mathrm{~A} & 0.596869 & -0.330896 & 0.894069\end{array}$

$\begin{array}{llll}10 \mathrm{~A} & 0.568801 & 0.751724 & 0.570125\end{array}$ $\begin{array}{llll}11 \mathrm{~A} & 0.568802 & 0.751725 & -0.570124\end{array}$ 
$\begin{array}{llll}12 A & -0.336183 & -0.880685 & -0.570125\end{array}$ $\begin{array}{llll}13 \mathrm{~A} & -0.336183 & -0.880685 & 0.570124\end{array}$

$E 14 / \varepsilon=-50.489724$

atom $\quad x / \sigma$

$\begin{array}{llll}1 \mathrm{~A} & 0.000000 & 0.000000 & 0.000000\end{array}$

$\begin{array}{llll}2 A & 1.094025 & 0.000000 & 0.000000\end{array}$

$\begin{array}{llll}3 B & 0.547011 & -1.254801 & 0.000000\end{array}$

$\begin{array}{lllll}4 \mathrm{~B} & 0.547013 & 0.004236 & -1.254794\end{array}$

$\begin{array}{llll}5 A & 0.547012 & -0.187034 & 0.941602\end{array}$

$\begin{array}{llll}6 \mathrm{~A} & 0.547013 & 0.942228 & -0.183855\end{array}$

$\begin{array}{llll}7 \mathrm{~B} & -0.283451 & 0.941508 & 0.944690\end{array}$

$\begin{array}{llll}8 \mathrm{~B} & 1.377474 & 0.941507 & 0.944691\end{array}$

$\begin{array}{llll}9 B & 1.849169 & -0.676771 & 0.915917\end{array}$

$\begin{array}{llll}10 \mathrm{~B} & -0.755145 & 0.918197 & -0.673676\end{array}$

$\begin{array}{llll}11 B & 1.849171 & 0.918195 & -0.673674\end{array}$

$\begin{array}{llll}12 \mathrm{~B} & -0.755146 & -0.676770 & 0.915916\end{array}$

$\begin{array}{llll}13 \mathrm{~B} & -0.862167 & -0.757487 & -0.760051\end{array}$

$\begin{array}{llll}14 \mathrm{~B} & 1.956191 & -0.757490 & -0.760050\end{array}$

$E 15 / \varepsilon=-55.390724$

$\begin{array}{cccc}\text { atom } & \mathrm{x} / \sigma & y / \sigma & z / \sigma \\ 1 \mathrm{~A} & 0.000000 & 0.000000 & 0.000000\end{array}$

$\begin{array}{llll}2 \mathrm{~A} & 1.122549 & 0.000000 & 0.000000\end{array}$

$\begin{array}{llll}3 B & 0.598787 & -1.252248 & 0.000000\end{array}$

$\begin{array}{llll}4 \mathrm{~B} & 0.598788 & -0.069271 & 1.250331\end{array}$

$\begin{array}{llll}5 B & -0.257103 & 0.966310 & -0.914256\end{array}$

$\begin{array}{lllll}6 \mathrm{~A} & 0.579437 & -0.148324 & -0.935716\end{array}$

$\begin{array}{lllll}7 A & 0.579437 & 0.926079 & 0.199859\end{array}$

$\begin{array}{lllll}8 \mathrm{~B} & 1.412328 & 0.970903 & -0.918600\end{array}$

$\begin{array}{lllll}9 \mathrm{~B} & -0.632827 & -0.774610 & -0.990758\end{array}$

$\begin{array}{llll}10 \mathrm{~B} & -0.632828 & 0.946391 & 0.828229\end{array}$

$\begin{array}{llll}11 B & -0.830798 & -0.784893 & 0.742611\end{array}$

$\begin{array}{llll}12 \mathrm{~B} & 1.868914 & -0.652634 & -0.947016\end{array}$

$\begin{array}{llll}13 \mathrm{~B} & 1.868914 & 0.909463 & 0.704021\end{array}$

$\begin{array}{llll}14 \mathrm{~B} & 2.028662 & -0.758148 & 0.717306\end{array}$

$\begin{array}{llll}15 A & -1.133718 & 0.194065 & -0.183613\end{array}$

$E 16 / \varepsilon=-60.928253$

$\begin{array}{llcc}\text { atom } \quad \mathrm{x} / \sigma & \mathrm{y} / \sigma & \mathrm{z} / \sigma\end{array}$

$\begin{array}{llll}1 \mathrm{~A} & 0.000000 & 0.000000 & 0.000000\end{array}$

$\begin{array}{llll}2 \mathrm{~A} & 1.064317 & 0.000000 & 0.000000\end{array}$

$\begin{array}{llll}3 \mathrm{~B} & 0.532160 & -1.285279 & 0.000000\end{array}$

$\begin{array}{llll}4 \mathrm{~B} & 0.532158 & 1.349602 & -0.000001\end{array}$

$\begin{array}{lllll}5 \mathrm{~A} & 0.492746 & 0.365301 & -0.917049\end{array}$

$\begin{array}{llll}6 \mathrm{~A} & 0.571572 & 0.365303 & 0.917049\end{array}$

$\begin{array}{lllll}7 \mathrm{~B} & 1.827087 & 0.694077 & -0.908862\end{array}$

$\begin{array}{llll}8 \mathrm{~B} & -0.762771 & 0.694076 & 0.908860\end{array}$

$\begin{array}{llll}9 \mathrm{~B} & 1.306307 & -0.702544 & 1.305576\end{array}$

$\begin{array}{llll}10 B & -0.241987 & -0.702546 & -1.305577\end{array}$

$\begin{array}{llll}11 \mathrm{~A} & -0.063660 & -0.526681 & 0.984620\end{array}$

$\begin{array}{llll}12 \mathrm{~A} & 1.127979 & -0.526681 & -0.984621\end{array}$

$\begin{array}{llll}13 \mathrm{~B} & -0.853744 & 0.743508 & -0.757964\end{array}$

$\begin{array}{llll}14 \mathrm{~B} & 1.918061 & 0.743511 & 0.757962\end{array}$

$\begin{array}{llll}15 B & -1.123959 & -0.749962 & 0.104479\end{array}$

$\begin{array}{llll}16 B & 2.188279 & -0.749959 & -0.104480\end{array}$

$E 17 / \varepsilon=-66.446027$

\begin{tabular}{crrr} 
atom & $x / \sigma$ & $y / \sigma$ & \multicolumn{1}{c}{$z / \sigma$} \\
$1 \mathrm{~A}$ & 0.000000 & 0.000000 & 0.000000 \\
2B & 1.358945 & 0.000000 & 0.000000 \\
3A & 0.440918 & -1.001977 & 0.000000 \\
$4 \mathrm{~A}$ & 0.361735 & -0.438820 & -0.917340 \\
5B & -0.875867 & -0.983423 & -0.453749 \\
$6 \mathrm{~B}$ & 0.332215 & -1.835488 & -1.122328 \\
$7 \mathrm{~B}$ & -0.687997 & 0.482927 & -1.122327 \\
8A & -0.306922 & -0.733061 & 0.777358 \\
9B & 0.296164 & 0.421626 & 1.282122 \\
$10 \mathrm{~B}$ & 0.951863 & -1.068438 & 1.282121 \\
$11 \mathrm{~B}$ & 1.571511 & -0.805777 & -1.465421 \\
$12 \mathrm{~B}$ & 0.908428 & 0.701067 & -1.465420 \\
$13 \mathrm{~B}$ & 0.258759 & 1.338920 & -0.075413
\end{tabular}

$\begin{array}{llll}\text { 13B } & 0.258759 & 1.338920 & -0.075413\end{array}$ \begin{tabular}{|llll}
$14 B$ & 1.602932 & -1.715689 & -0.075415
\end{tabular} $\begin{array}{llll}15 \mathrm{~B} & -1.185784 & 0.380180 & 0.499586\end{array}$ $\begin{array}{llll}16 \mathrm{~B} & -0.079820 & -2.133106 & 0.499585\end{array}$ $\begin{array}{llll}17 B & 0.029909 & -0.584839 & -2.212341\end{array}$

\section{$E 18 / \varepsilon=-71.703520$}

atom $\mathrm{x} / \sigma \quad \mathrm{yl} \sigma$

$\begin{array}{llll}1 \mathrm{~A} & 0.000000 & 0.000000 & 0.000000\end{array}$

$\begin{array}{llll}2 \mathrm{~A} & 1.066074 & 0.000000 & 0.000000\end{array}$

$\begin{array}{llll}3 \mathrm{~B} & 0.532339 & -1.344414 & 0.000000\end{array}$

$\begin{array}{lllll}4 \mathrm{~A} & 0.568306 & -0.339225 & -0.909851\end{array}$

$\begin{array}{llll}5 B & 0.540400 & 1.284827 & 0.003268\end{array}$

$\begin{array}{lllll}6 \mathrm{~B} & -0.740246 & -0.708806 & -0.927625\end{array}$

$\begin{array}{lllll}7 B & 1.308140 & 0.715200 & -1.310797\end{array}$

$\begin{array}{llll}8 \mathrm{~B} & 1.890910 & -0.748084 & -0.793595\end{array}$

$\begin{array}{lllll}9 \mathrm{~A} & 0.497238 & -0.366538 & 0.917182\end{array}$

$\begin{array}{llll}10 \mathrm{~A} & -0.071312 & 0.544733 & -0.970674\end{array}$

$\begin{array}{llll}11 \mathrm{~B} & 1.833420 & -0.706415 & 0.884917\end{array}$

$\begin{array}{llll}12 \mathrm{~B} & -0.852566 & -0.754735 & 0.743173\end{array}$

$\begin{array}{llll}13 B & -0.247130 & 0.695568 & 1.293786\end{array}$

$\begin{array}{lllll}14 \mathrm{~B} & -1.139207 & 0.725392 & -0.112702\end{array}$

$\begin{array}{llll}15 \mathrm{~A} & 1.136875 & 0.521481 & 0.984590\end{array}$

$\begin{array}{llll}16 \mathrm{~B} & 2.194667 & 0.739070 & 0.092547\end{array}$

$\begin{array}{lllll}17 \mathrm{~B} & 0.119538 & -0.033830 & -2.202529\end{array}$

$\begin{array}{llll}18 \mathrm{~B} & 0.624179 & -1.503827 & -1.636823\end{array}$

\section{$E 19 / \varepsilon=-78.191386$}

atom $\mathrm{x} / \sigma \quad \mathrm{y} / \sigma$

$\begin{array}{llll}\text { 1A } & 0.000000 & 0.000000 & 0.000000\end{array}$

$\begin{array}{llll}2 \mathrm{~A} & 1.106133 & 0.000000 & 0.000000\end{array}$

$\begin{array}{llll}3 A & 0.553067 & -0.922179 & 0.000000\end{array}$

$\begin{array}{llll}4 \mathrm{~B} & 0.553068 & 0.562672 & -1.157945\end{array}$

$\begin{array}{llll}5 B & 0.553066 & 0.562672 & 1.157945\end{array}$

$\begin{array}{llll}6 \mathrm{~B} & -0.781595 & -1.271141 & -0.000001\end{array}$

$\begin{array}{llll}7 \mathrm{~B} & 1.887729 & -1.271141 & 0.000002\end{array}$

$\begin{array}{llll}8 \mathrm{~A} & 0.006883 & -0.666108 & 0.923839\end{array}$

$\begin{array}{llll}9 \mathrm{~A} & 1.099249 & -0.666108 & 0.923840\end{array}$

$\begin{array}{llll}10 \mathrm{~A} & 1.099251 & -0.666109 & -0.923839\end{array}$

$\begin{array}{llll}11 \mathrm{~A} & 0.006885 & -0.666108 & -0.923840\end{array}$

$\begin{array}{llll}12 \mathrm{~B} & 1.392642 & 1.374024 & 0.000000\end{array}$

$\begin{array}{llll}13 B & -0.286508 & 1.374024 & -0.000001\end{array}$

$\begin{array}{llll}14 \mathrm{~B} & 0.553066 & -1.957967 & 0.854950\end{array}$

$\begin{array}{llll}15 B & 0.553068 & -1.957968 & -0.854950\end{array}$

$\begin{array}{lllll}16 \mathrm{~B} & -1.079203 & 0.169300 & -0.840167\end{array}$

$\begin{array}{llll}17 \mathrm{~B} & 2.185336 & 0.169301 & 0.840166\end{array}$

$\begin{array}{llll}18 B & 2.185338 & 0.169300 & -0.840164\end{array}$ $\begin{array}{llll}19 B & -1.079204 & 0.169300 & 0.840164\end{array}$

$E 20 / \varepsilon=-83.514565$

atom $\mathrm{x} / \sigma \quad \mathrm{yl}$

$\begin{array}{lll}1 \mathrm{~A} & 0.000000 & 0.000000\end{array}$

$\begin{array}{lll}2 \mathrm{~A} & 1.197703 & 0.000000\end{array}$

$z / \sigma$

उA $\quad 0.598852-0.912246$

$\begin{array}{lll}4 \mathrm{~A} & 0.598852 & -0.295459\end{array}$

$\begin{array}{lll}5 A & 0.598852 & -0.295459\end{array}$

$\begin{array}{lll}6 \mathrm{~A} & 0.598852 & 0.751774\end{array}$

$\begin{array}{lll}7 A & 0.598852 & 0.751774\end{array}$

8B $-0.433863-1.188890$

$9 B-0.433863-1.188890$

10B $\quad 1.631567-1.188889$

$\begin{array}{llll}11 \mathrm{~B} & 1.631567 & -1.188889\end{array}$

$\begin{array}{lll}12 B & 1.679741 & 0.394639\end{array}$

$\begin{array}{lll}13 \mathrm{~B} & -0.482038 & 0.394639\end{array}$

$\begin{array}{lll}14 \mathrm{~B} & 1.679741 & 0.394639\end{array}$

$\begin{array}{lll}15 B & -0.482038 & 0.394639\end{array}$

$\begin{array}{lll}16 \mathrm{~B} & 1.692956 & 1.362047\end{array}$

$\begin{array}{lll}17 \mathrm{~B} & -0.495252 & 1.362047\end{array}$

$\begin{array}{lll}18 \mathrm{~B} & -1.370553 & -0.077970\end{array}$

$\begin{array}{lll}19 \mathrm{~B} & 2.568256 & -0.077969\end{array}$

20B $\quad 0.598852-2.261996$

$E 21 / \varepsilon=-89.330178$

\begin{tabular}{|crrr} 
atom & $x / \sigma$ & $y / \sigma$ & \multicolumn{1}{c}{$z / \sigma$} \\
1A & 0.000000 & 0.000000 & 0.000000 \\
2A & 1.198969 & 0.000000 & 0.000000 \\
3A & 0.599485 & -0.917149 & 0.000000 \\
4A & 0.599485 & -0.289503 & -0.870259 \\
5B & 1.604291 & -1.202290 & -0.867111 \\
6B & -0.405320 & -1.202291 & -0.867114 \\
7A & 0.599485 & 0.752681 & -0.563269 \\
8A & 0.599484 & -0.296883 & 0.891998 \\
9A & 0.599484 & 0.756326 & 0.545475 \\
10B & 1.646633 & -1.188716 & 0.799331 \\
11B & -0.447665 & -1.188717 & 0.799328 \\
12B & 1.646634 & 0.383238 & -1.380256 \\
13B & -0.447663 & 0.383237 & -1.380258 \\
14B & 1.701885 & 0.390351 & 1.293750 \\
15B & -0.502918 & 0.390350 & 1.293748 \\
16B & -0.502917 & 1.350821 & -0.037989 \\
17B & 1.701886 & 1.350821 & -0.037987 \\
18B & -1.364218 & -0.091215 & -0.065789 \\
19B & 2.563187 & -0.091213 & -0.065785 \\
20B & 0.599486 & -2.266918 & -0.014293 \\
21B & 0.599487 & -0.729130 & -2.146507 \\
\hline & & &
\end{tabular}

\section{$E 22 / \varepsilon=-95.143253$}

\begin{tabular}{rrrr} 
atom & \multicolumn{1}{c}{$\mathrm{X} / \sigma$} & \multicolumn{1}{c}{$y / \sigma$} & \multicolumn{1}{c}{$z / \sigma$} \\
1A & 0.000000 & 0.000000 & 0.000000 \\
2A & 1.078302 & 0.000000 & 0.000000 \\
3A & 0.513393 & -0.948242 & 0.000000 \\
4B & 1.290068 & -0.768547 & 1.161497 \\
5A & -0.035671 & -0.608753 & 0.905029 \\
6A & 0.518346 & 0.321207 & 0.905027 \\
7B & 0.377391 & 1.323254 & -0.001144 \\
8B & -0.983968 & -0.961889 & -0.001138 \\
9B & -0.851960 & 0.507551 & 0.919351 \\
10B & 1.274692 & -0.759391 & -1.155039 \\
11B & 0.096449 & -1.952827 & 0.862660 \\
12B & 1.763208 & 0.844953 & 0.862655 \\
13A & -0.061938 & -0.598133 & -0.923409 \\
14A & 0.496498 & 0.339243 & -0.923411 \\
15B & 0.158467 & -0.094399 & 2.168585 \\
16B & -0.902937 & 0.537918 & -0.821507 \\
17B & 1.537972 & -1.898995 & 0.003978 \\
18B & 2.402194 & -0.448334 & 0.003976 \\
19B & 1.769287 & 0.852091 & -0.824232 \\
20B & 0.093061 & -1.961577 & -0.824228 \\
21B & -1.109911 & -1.026460 & 1.644587 \\
22B & 0.374212 & 1.464757 & 1.644580
\end{tabular}

$E 23 / \varepsilon=-101.901346$

atom $\quad \mathrm{x} / \sigma \quad \mathrm{y} / \sigma$

$\begin{array}{llll}1 \mathrm{~A} & 0.000000 & 0.000000 & 0.000000\end{array}$

$\begin{array}{llll}2 \mathrm{~A} & 1.161114 & 0.000000 & 0.000000\end{array}$

$\begin{array}{llll}3 \mathrm{~A} & 0.580557 & -1.005554 & 0.000000\end{array}$

$\begin{array}{llll}4 \mathrm{~A} & 0.580557 & -0.335185 & -0.857079\end{array}$

$\begin{array}{lllll}5 \mathrm{~A} & 0.580557 & -0.335184 & 0.857079\end{array}$

$\begin{array}{lllll}6 \mathrm{~A} & 0.580557 & 0.740722 & -0.571010\end{array}$

$\begin{array}{lllll}7 \mathrm{~A} & 0.580557 & 0.740723 & 0.571009\end{array}$

$\begin{array}{llll}8 \mathrm{~A} & -0.351206 & -0.873138 & 0.571010\end{array}$

$\begin{array}{lllll}9 A & -0.351206 & -0.873138 & -0.571009\end{array}$

$\begin{array}{llll}10 \mathrm{~A} & 1.512319 & -0.873138 & 0.571010\end{array}$

$\begin{array}{lllll}11 \mathrm{~A} & 1.512319 & -0.873138 & -0.571010\end{array}$

$\begin{array}{llll}12 \mathrm{~B} & -0.504936 & 0.291524 & -1.324520\end{array}$

$\begin{array}{llll}13 \mathrm{~B} & -0.504935 & 0.291525 & 1.324519\end{array}$

$\begin{array}{llll}14 \mathrm{~B} & 1.666049 & 0.291525 & 1.324519\end{array}$

$\begin{array}{llll}15 B & 1.666050 & 0.291524 & -1.324519\end{array}$

$\begin{array}{llll}16 \mathrm{~B} & 0.580557 & -1.588603 & 1.324520\end{array}$

$\begin{array}{llll}17 \mathrm{~B} & 0.580556 & -1.588604 & -1.324519\end{array}$

$\begin{array}{lllll}18 \mathrm{~B} & 1.424022 & -2.122218 & 0.000000\end{array}$

$\begin{array}{llll}19 \mathrm{~B} & -1.388792 & -0.172131 & 0.000000\end{array}$

$\begin{array}{llll}20 \mathrm{~B} & -0.262908 & -2.122218 & 0.000000\end{array}$

$\begin{array}{llll}21 B & 2.549905 & -0.172131 & 0.000000\end{array}$

$\begin{array}{llll}22 B & -0.545327 & 1.288794 & -0.000001\end{array}$ 
23B $\quad 1.706441 \quad 1.288793 \quad-0.000001$

$E 24 / \varepsilon=-107.064064$

atom $\mathrm{x} / \sigma \quad \mathrm{y} / \sigma \quad \mathrm{z} / \sigma$

1A $\quad 0.000000 \quad 0.000000 \quad 0.000000$

$\begin{array}{llll}2 \mathrm{~A} & 1.157329 & 0.000000 & 0.000000\end{array}$

$\begin{array}{llll}3 A & 0.590476 & -1.009004 & 0.000000\end{array}$

$\begin{array}{llll}4 \mathrm{~A} & 0.588234 & -0.333039 & 0.858882\end{array}$

$\begin{array}{llll}5 \mathrm{~A} & 0.576931 & -0.339653 & -0.858341\end{array}$

$\begin{array}{llll}6 \mathrm{~A} & -0.318850 & -0.863871 & 0.554668\end{array}$

$\begin{array}{llll}7 \mathrm{~A} & -0.350938 & -0.882649 & -0.563555\end{array}$

$\begin{array}{lllll}8 \mathrm{~A} & 1.510674 & -0.872480 & 0.584872\end{array}$

$\begin{array}{llll}9 \mathrm{~A} & 0.569734 & 0.735395 & 0.584872\end{array}$

10A $1.517175 \quad-0.879530 \quad-0.563330$

$\begin{array}{llll}11 \mathrm{~A} & 0.566771 & 0.744517 & -0.563329\end{array}$

$\begin{array}{llll}12 \mathrm{~B} & 0.544797 & -1.569333 & 1.358834\end{array}$

$\begin{array}{llll}13 \mathrm{~B} & -0.510889 & 0.234621 & 1.358834\end{array}$

$\begin{array}{llll}14 \mathrm{~B} & -1.401601 & -0.232672 & 0.046091\end{array}$

$\begin{array}{llll}15 B & -0.298871 & -2.117012 & 0.046090\end{array}$

$\begin{array}{llll}16 \mathrm{~B} & -0.534599 & 0.281005 & -1.291047\end{array}$

$\begin{array}{llll}17 \mathrm{~B} & 0.573623 & -1.612721 & -1.291048\end{array}$

$\begin{array}{llll}18 \mathrm{~B} & 1.663621 & 0.296285 & 1.336716\end{array}$

$\begin{array}{llll}19 B & 1.662708 & 0.295752 & -1.311590\end{array}$

$\begin{array}{llll}\text { 20B } & -0.579679 & 1.254272 & 0.044233\end{array}$

$\begin{array}{llll}21 \mathrm{~B} & 1.400075 & -2.128725 & 0.044232\end{array}$

$\begin{array}{llll}22 \mathrm{~B} & 1.691927 & 1.291587 & 0.012718\end{array}$

$\begin{array}{llll}23 B & 2.545226 & -0.166528 & 0.012717\end{array}$

$\begin{array}{llll}24 \mathrm{~B} & -1.198357 & -1.378563 & 1.403470\end{array}$

$E 25 / \varepsilon=-112.963841$

$\begin{array}{cccc}\text { atom } & x / \sigma & y / \sigma & z / \sigma \\ 1 \mathrm{~A} & 0.000000 & 0.000000 & 0.000000\end{array}$

2A $\quad 1.139519 \quad 0.000000 \quad 0.000000$

$\begin{array}{llll}3 A & 0.565278 & -0.999312 & 0.000000\end{array}$

$\begin{array}{llll}4 \mathrm{~A} & 0.573240 & -0.332085 & 0.865833\end{array}$

$\begin{array}{lllll}5 \mathrm{~A} & 0.550263 & -0.330668 & -0.865672\end{array}$

$\begin{array}{llll}6 \mathrm{~A} & -0.334091 & -0.856908 & 0.578485\end{array}$

$\begin{array}{llll}7 A & 0.567087 & 0.721468 & 0.624596\end{array}$

8B $\quad 0.543270 \quad-1.557292 \quad 1.378233$

$\begin{array}{lllll}9 \mathrm{~A} & 1.491287 & -0.874797 & 0.575815\end{array}$

$\begin{array}{llll}10 A & -0.387209 & -0.867912 & -0.545783\end{array}$

$\begin{array}{llll}11 \mathrm{~A} & -0.383862 & 0.194944 & 1.002629\end{array}$

$\begin{array}{llll}12 \mathrm{~A} & 1.484919 & -0.876878 & -0.576035\end{array}$

$\begin{array}{llll}13 \mathrm{~A} & 0.552260 & 0.758868 & -0.543819\end{array}$

$\begin{array}{llll}14 \mathrm{~B} & 1.695163 & 0.270053 & 1.319818\end{array}$

$\begin{array}{llll}15 \mathrm{~B} & -1.399267 & -0.176630 & 0.122253\end{array}$

$\begin{array}{llll}16 \mathrm{~B} & -0.570540 & 0.317257 & -1.245579\end{array}$

$\begin{array}{llll}\text { 17B } & 0.529441 & -1.605480 & -1.288921\end{array}$

$\begin{array}{llll}18 \mathrm{~B} & -0.306149 & -2.107689 & 0.060343\end{array}$

19B $\quad 1.633220 \quad 0.312139-1.312476$

$\begin{array}{llll}20 B & -0.567090 & 1.265832 & 0.119551\end{array}$

$\begin{array}{llll}21 B & 1.376889 & -2.123590 & 0.038019\end{array}$

$\begin{array}{llll}22 \mathrm{~B} & 1.680824 & 1.287200 & 0.028012\end{array}$

$\begin{array}{lllll}23 \mathrm{~B} & 2.531109 & -0.185378 & -0.018754\end{array}$

$\begin{array}{llll}24 \mathrm{~B} & 0.316156 & -0.044672 & 2.163237\end{array}$

$\begin{array}{llll}25 B & -1.058539 & -0.838260 & 1.695343\end{array}$

\section{$E 26 / \varepsilon=-120.425633$}

\begin{tabular}{crrr} 
atom & $x / \sigma$ & $y / \sigma$ & \multicolumn{1}{c}{$z / \sigma$} \\
$1 \mathrm{~A}$ & 0.000000 & 0.000000 & 0.000000 \\
2A & 1.118544 & 0.000000 & 0.000000 \\
3A & 0.559271 & -0.968687 & 0.000000 \\
4A & 0.559271 & -0.322896 & 0.913287 \\
5A & 0.559272 & 0.720572 & 0.597243 \\
$6 \mathrm{~A}$ & 0.559272 & -0.322895 & -0.878443 \\
7A & -0.344399 & -0.844629 & 0.597243 \\
8A & 1.462941 & -0.844630 & 0.597243 \\
9A & 0.559270 & -1.415077 & 1.000610 \\
$10 A$ & 0.559273 & 0.769286 & -0.543967 \\
$11 \mathrm{~A}$ & -0.386585 & 0.223196 & 1.000610 \\
$12 \mathrm{~A}$ & 1.505128 & 0.223194 & 1.000611 \\
$13 \mathrm{~A}$ & 1.505128 & -0.868986 & -0.543967
\end{tabular}

$\begin{array}{llll}13 \mathrm{~A} & 1.505128 & -0.868986 & -0.543967\end{array}$ $\begin{array}{llll}15 \mathrm{~B} & 1.390851 & -2.097898 & 0.110000\end{array}$

$\begin{array}{llll}16 \mathrm{~B} & -0.272310 & -2.097897 & 0.109999\end{array}$

$\begin{array}{llll}17 \mathrm{~B} & 0.559272 & 0.637331 & 1.941250\end{array}$

$\begin{array}{llll}18 \mathrm{~B} & 2.512258 & -0.155566 & 0.110001\end{array}$

$\begin{array}{llll}19 B & 1.680679 & 0.324548 & -1.247964\end{array}$

$\begin{array}{llll}20 B & -1.393715 & -0.155562 & 0.110000\end{array}$

$\begin{array}{llll}21 B & -0.562134 & 0.324550 & -1.247965\end{array}$

$\begin{array}{llll}22 \mathrm{~B} & 1.680679 & 1.284774 & 0.110002\end{array}$

$\begin{array}{llll}23 \mathrm{~B} & 1.390851 & -0.803009 & 1.941250\end{array}$

$\begin{array}{llll}24 \mathrm{~B} & -0.272310 & -0.803008 & 1.941249\end{array}$

$\begin{array}{llll}25 \mathrm{~B} & -0.562133 & 1.284777 & 0.110001\end{array}$

$\begin{array}{llll}26 \mathrm{~B} & 0.559271 & -1.617783 & -1.247965\end{array}$

$E 27 / \varepsilon=-125.697620$

atom $\mathrm{x} / \sigma \quad \mathrm{yl}$

$\begin{array}{llll}1 \mathrm{~A} & 0.000000 & 0.000000 & 0.000000\end{array}$

$\begin{array}{lllll}2 \mathrm{~A} & 1.119144 & 0.000000 & 0.000000\end{array}$

$\begin{array}{llll}3 A & 0.559572 & -0.969208 & 0.000000\end{array}$

$\begin{array}{lllll}4 \mathrm{~A} & 0.559572 & -0.323070 & 0.913182\end{array}$

$\begin{array}{lllll}5 A & 0.559572 & -0.323069 & -0.876086\end{array}$

$\begin{array}{llll}6 \mathrm{~A} & 1.462517 & -0.844385 & 0.596550\end{array}$

$\begin{array}{llll}7 \mathrm{~A} & -0.343372 & -0.844385 & 0.596550\end{array}$

$\begin{array}{lllll}8 A & 0.559572 & 0.719561 & 0.596550\end{array}$

$\begin{array}{llll}9 \mathrm{~A} & -0.383820 & -0.867737 & -0.548724\end{array}$

$\begin{array}{lllll}10 A & 1.502964 & -0.867737 & -0.548725\end{array}$

$\begin{array}{llll}11 \mathrm{~A} & 0.559572 & 0.766266 & -0.548724\end{array}$

$\begin{array}{llll}12 \mathrm{~A} & 1.505079 & 0.222819 & 1.001119\end{array}$

$\begin{array}{llll}13 A & -0.385935 & 0.222819 & 1.001119\end{array}$

$\begin{array}{llll}14 \mathrm{~A} & 0.559572 & -1.414847 & 1.001118\end{array}$

$\begin{array}{llll}15 \mathrm{~B} & 1.692751 & 0.331172 & -1.235277\end{array}$

$\begin{array}{llll}16 \mathrm{~B} & -0.573609 & 0.331173 & -1.235275\end{array}$

$\begin{array}{llll}17 \mathrm{~B} & 0.559571 & -1.631553 & -1.235276\end{array}$

$\begin{array}{llll}18 B & -0.274469 & -2.097746 & 0.114972\end{array}$

$\begin{array}{llll}19 \mathrm{~B} & -0.560323 & 1.286569 & 0.114973\end{array}$

$\begin{array}{llll}20 B & 2.513507 & -0.158031 & 0.114972\end{array}$

$\begin{array}{llll}21 B & 1.393613 & -2.097746 & 0.114971\end{array}$

$\begin{array}{llll}22 \mathrm{~B} & 1.679466 & 1.286569 & 0.114971\end{array}$

$\begin{array}{llll}23 \mathrm{~B} & -1.394364 & -0.158032 & 0.114973\end{array}$

$\begin{array}{llll}24 \mathrm{~B} & -0.272233 & -0.803314 & 1.940815\end{array}$

$\begin{array}{llll}25 \mathrm{~B} & 1.391378 & -0.803313 & 1.940815\end{array}$

$\begin{array}{llll}26 \mathrm{~B} & 0.559572 & 0.637416 & 1.940816\end{array}$

$\begin{array}{llll}27 \mathrm{~B} & 0.559570 & -0.323069 & -2.250492\end{array}$

\section{$E 28 / \varepsilon=-131.301741$}

atom $\quad \mathrm{X} / \sigma$ $y / \sigma$

1A $\quad 0.000000 \quad 0.000000$

$\begin{array}{lll}2 \mathrm{~A} & 1.124603 & 0.000000\end{array}$

3A $\quad 0.562301 \quad-0.963801$

$\begin{array}{lll}4 \mathrm{~A} & 0.562300 & -0.313790\end{array}$

$\begin{array}{llll}5 \mathrm{~A} & 0.562301 & -0.313844\end{array}$

$\begin{array}{lll}6 \mathrm{~A} & 0.562301 & 0.751682\end{array}$

$\begin{array}{lll}7 A & 0.562301 & 0.726461\end{array}$

$\begin{array}{lll}8 \mathrm{~A} & 1.464206 & -0.840672\end{array}$

9A $-0.339605-0.840671$

$\begin{array}{lll}10 \mathrm{~B} & 1.721039 & 0.306148\end{array}$

$\begin{array}{lll}11 \mathrm{~B} & -0.596438 & 0.306147\end{array}$

$\begin{array}{lll}12 \mathrm{~A} & -0.383956 & -0.869556\end{array}$

$\begin{array}{llll}13 \mathrm{~A} & 1.508557 & -0.869556\end{array}$

$\begin{array}{llll}14 \mathrm{~A} & 0.562300 & -1.406078\end{array}$

$\begin{array}{lll}15 A & 1.505476 & 0.239229\end{array}$

$\begin{array}{lll}16 \mathrm{~A} & -0.380875 & 0.239229\end{array}$

$\begin{array}{lll}17 \mathrm{~B} & 0.562300 & -1.612533\end{array}$

$\begin{array}{lll}18 \mathrm{~B} & -0.546031 & 1.294628\end{array}$

$\begin{array}{lll}19 B & 1.670632 & 1.294628\end{array}$

$\begin{array}{lll}20 \mathrm{~B} & 2.518564 & -0.151917\end{array}$

21B $-1.393963-0.151917$

$\begin{array}{llll}1.392518 & -2.093931 & -0.113360\end{array}$

$\begin{array}{llll}23 B & -0.267918 & -2.093931 & -0.113360\end{array}$

$\begin{array}{llll}24 \mathrm{~B} & -0.269014 & -0.783259 & -1.942985\end{array}$

$\begin{array}{llll}25 B & 1.393614 & -0.783259 & -1.942985\end{array}$

$\begin{array}{llll}26 \mathrm{~B} & 0.562300 & 0.657394 & -1.934244\end{array}$

$\begin{array}{llll}27 \mathrm{~B} & 0.562300 & -0.270900 & 2.249663\end{array}$
|28B $\quad 0.562300 \quad 1.368918 \quad 1.752056$

$E 29 / \varepsilon=-137.929835$

$\begin{array}{llll}\text { atom } \mathrm{x} / \sigma & \mathrm{y} / \sigma & \mathrm{z} / \sigma\end{array}$

$\begin{array}{llll}1 \mathrm{~A} & 0.000000 & 0.000000 & 0.000000\end{array}$

$\begin{array}{llll}2 \mathrm{~A} & 1.091230 & 0.000000 & 0.000000\end{array}$

$\begin{array}{lllll}3 \mathrm{~A} & 0.545615 & -0.945033 & 0.000000\end{array}$

$\begin{array}{llll}4 \mathrm{~A} & 0.545616 & -0.315010 & -0.910215\end{array}$

$\begin{array}{llll}5 \mathrm{~A} & 0.545613 & -0.315011 & 0.910215\end{array}$

$\begin{array}{llll}6 \mathrm{~A} & -0.367975 & -0.842472 & -0.578446\end{array}$

$\begin{array}{llll}7 \mathrm{~A} & 0.545615 & 0.739912 & 0.578445\end{array}$

$\begin{array}{llll}8 \mathrm{~A} & 1.459204 & -0.842472 & 0.578446\end{array}$

$\begin{array}{llll}9 \mathrm{~A} & -0.367976 & -0.842473 & 0.578443\end{array}$

$\begin{array}{lllll}10 A & 0.545616 & 0.739912 & -0.578444\end{array}$

$\begin{array}{lllll}11 \mathrm{~A} & 1.459205 & -0.842473 & -0.578444\end{array}$

$\begin{array}{lllll}12 \mathrm{~A} & 1.501942 & 0.237126 & 0.974081\end{array}$

$\begin{array}{llll}13 \mathrm{~A} & 0.545613 & -1.419285 & 0.974080\end{array}$

$\begin{array}{lllll}14 \mathrm{~A} & 0.545616 & -1.419284 & -0.974081\end{array}$

$\begin{array}{llll}15 A & -0.410716 & 0.237126 & 0.974079\end{array}$

$\begin{array}{llll}16 A & -0.410713 & 0.237127 & -0.974081\end{array}$

$\begin{array}{lllll}17 A & 1.501946 & 0.237126 & -0.974079\end{array}$

$\begin{array}{llll}18 \mathrm{~B} & -1.381226 & -0.145774 & -0.000003\end{array}$

$\begin{array}{lllll}19 B & -0.271243 & -2.068322 & -0.000002\end{array}$

$\begin{array}{llll}20 B & 1.655599 & 1.269063 & 0.000002\end{array}$

$\begin{array}{llll}21 \mathrm{~B} & -0.564368 & 1.269064 & -0.000001\end{array}$

$\begin{array}{llll}22 B & 1.362472 & -2.068322 & 0.000001\end{array}$

$\begin{array}{llll}23 \mathrm{~B} & 2.472455 & -0.145776 & 0.000003\end{array}$

$\begin{array}{llll}24 \mathrm{~B} & -0.285861 & -0.795061 & 1.921471\end{array}$

$\begin{array}{llll}25 \mathrm{~B} & 0.545611 & 0.645091 & 1.921473\end{array}$

$\begin{array}{lllll}26 \mathrm{~B} & -0.285855 & -0.795060 & -1.921474\end{array}$

$\begin{array}{llll}27 \mathrm{~B} & 1.377082 & -0.795061 & 1.921474\end{array}$

$\begin{array}{llll}28 \mathrm{~B} & 0.545617 & 0.645092 & -1.921472\end{array}$

$\begin{array}{lllll}29 B & 1.377088 & -0.795061 & -1.921472\end{array}$

$E 30 / \varepsilon=-143.322385$

atom $\mathrm{x} / \sigma \quad \mathrm{y} / \sigma \quad \mathrm{z} / \sigma$

$\begin{array}{llll}1 \mathrm{~A} & 0.000000 & 0.000000 & 0.000000\end{array}$

$\begin{array}{llll}2 \mathrm{~A} & 1.107729 & 0.000000 & 0.000000\end{array}$

$\begin{array}{llll}3 A & 0.537017 & -0.940517 & 0.000000\end{array}$

$\begin{array}{lllll}4 \mathrm{~A} & 0.521180 & -0.319450 & 0.913820\end{array}$

$\begin{array}{lllll}5 \mathrm{~A} & 0.536804 & -0.324356 & -0.921839\end{array}$

$\begin{array}{lllll}6 \mathrm{~A} & 0.554122 & 0.716324 & -0.584682\end{array}$

$\begin{array}{llll}7 A & 0.531109 & 0.729409 & 0.581423\end{array}$

$\begin{array}{llll}8 \mathrm{~A} & -0.389668 & -0.841646 & -0.553673\end{array}$

$\begin{array}{llll}9 A & -0.387993 & -0.835434 & 0.575650\end{array}$

10B $\quad 1.752089 \quad 0.408443 \quad-1.167824$

$\begin{array}{llll}11 \mathrm{~A} & 1.460184 & -0.843584 & -0.588759\end{array}$

$\begin{array}{lllll}12 \mathrm{~A} & 1.426049 & -0.860310 & 0.599258\end{array}$

$\begin{array}{lllll}13 \mathrm{~A} & -0.438493 & 0.217793 & -0.957394\end{array}$

$\begin{array}{llll}14 \mathrm{~B} & -0.535123 & 1.292176 & -0.042032\end{array}$

$\begin{array}{llll}15 \mathrm{~A} & 1.470438 & 0.207932 & 1.011196\end{array}$

$\begin{array}{llll}16 \mathrm{~A} & 0.509449 & -1.422412 & 0.975404\end{array}$

$\begin{array}{llll}17 A & -0.425209 & 0.252688 & 0.965506\end{array}$

$\begin{array}{llll}18 A & 0.493599 & -1.435436 & -0.955075\end{array}$

$\begin{array}{llll}19 B & 1.668236 & 1.296018 & 0.181787\end{array}$

$\begin{array}{llll}20 \mathrm{~B} & 0.371133 & 0.605308 & -1.975226\end{array}$

$\begin{array}{llll}21 \mathrm{~B} & -1.392853 & -0.111875 & 0.013244\end{array}$

$\begin{array}{lllll}22 B & 1.334509 & -2.070670 & -0.006538\end{array}$

$\begin{array}{llll}23 B & -0.302031 & -2.070478 & 0.018956\end{array}$

$\begin{array}{llll}24 \mathrm{~B} & 2.491908 & -0.186853 & 0.151626\end{array}$

$\begin{array}{llll}25 B & 0.519135 & 0.646165 & 1.926642\end{array}$

$\begin{array}{llll}26 \mathrm{~B} & 1.327958 & -0.816585 & 1.943489\end{array}$

$\begin{array}{llll}27 \mathrm{~B} & -0.330331 & -0.783002 & 1.916258\end{array}$

$\begin{array}{lllll}28 B & -0.392429 & -0.848069 & -1.885644\end{array}$

$\begin{array}{lllll}29 B & 1.251590 & -0.910946 & -1.966467\end{array}$

$\begin{array}{llll}30 \mathrm{~B} & 0.747904 & 1.956061 & -1.048390\end{array}$

$E 31 / \varepsilon=-149.398191$
atom $\mathrm{x} / \sigma$
$y / \sigma$
$\begin{array}{llll}1 \mathrm{~A} & 0.000000 & 0.000000 & 0.000000\end{array}$
$\begin{array}{llll}2 \mathrm{~A} & 1.099627 & 0.000000 & 0.000000\end{array}$
$\begin{array}{llll}3 A & 0.549814 & -0.939102 & 0.000000\end{array}$ 
$\begin{array}{llll}4 \mathrm{~A} & 0.549813 & -0.299276 & -0.910130\end{array}$

$\begin{array}{lllll}5 \mathrm{~A} & 0.549813 & -0.299275 & 0.910130\end{array}$

$\begin{array}{lllll}6 \mathrm{~A} & 0.549813 & 0.744576 & -0.557218\end{array}$

$\begin{array}{llll}7 \mathrm{~A} & 0.549813 & 0.744577 & 0.557216\end{array}$

$\begin{array}{lllll}8 \mathrm{~B} & -0.597325 & 1.251339 & -0.000002\end{array}$

$\begin{array}{llll}9 \mathrm{~B} & 1.696951 & 1.251340 & -0.000002\end{array}$

$\begin{array}{llll}10 \mathrm{~A} & -0.360181 & -0.836621 & -0.586318\end{array}$

$\begin{array}{llll}11 \mathrm{~A} & -0.360180 & -0.836620 & 0.586318\end{array}$

$\begin{array}{lllll}12 \mathrm{~A} & 1.459807 & -0.836621 & -0.586319\end{array}$

$\begin{array}{llll}13 \mathrm{~A} & 1.459807 & -0.836620 & 0.586319\end{array}$

$\begin{array}{lllll}14 \mathrm{~A} & 1.505509 & 0.254089 & -0.970058\end{array}$

$\begin{array}{lllll}15 A & -0.405884 & 0.254089 & -0.970057\end{array}$

$\begin{array}{llll}16 \mathrm{~A} & 1.505509 & 0.254091 & 0.970057\end{array}$

$\begin{array}{llll}17 \mathrm{~A} & -0.405884 & 0.254091 & 0.970056\end{array}$

$\begin{array}{llll}18 \mathrm{~A} & 0.549813 & -1.403306 & -0.982203\end{array}$

$\begin{array}{llll}19 \mathrm{~A} & 0.549813 & -1.403305 & 0.982204\end{array}$

$\begin{array}{llll}\text { 20B } & 0.549812 & 0.696064 & -1.896743\end{array}$

$\begin{array}{llll}21 B & 0.549812 & 0.696068 & 1.896741\end{array}$

$\begin{array}{llll}22 \mathrm{~B} & -1.382464 & -0.167468 & 0.000000\end{array}$

$\begin{array}{llll}23 \mathrm{~B} & 2.482090 & -0.167467 & 0.000000\end{array}$

$\begin{array}{llll}24 \mathrm{~B} & -0.266345 & -2.058232 & 0.000001\end{array}$

$\begin{array}{llll}25 \mathrm{~B} & 1.365972 & -2.058232 & 0.000001\end{array}$

$\begin{array}{llll}26 \mathrm{~B} & -0.278359 & -0.755761 & -1.929185\end{array}$

$\begin{array}{llll}27 \mathrm{~B} & -0.278359 & -0.755757 & 1.929186\end{array}$

$\begin{array}{llll}28 B & 1.377983 & -0.755761 & -1.929186\end{array}$

$\begin{array}{llll}29 B & 1.377983 & -0.755757 & 1.929187\end{array}$

$\begin{array}{llll}30 \mathrm{~B} & 0.549813 & 2.065096 & -0.868167\end{array}$

$\begin{array}{llll}31 B & 0.549813 & 2.065099 & 0.868161\end{array}$

$E 32 / \varepsilon=-155.872192$

atom $\quad x / \sigma \quad y / \sigma$

$\begin{array}{llcc}\text { 1A } & 0.000000 & 0.000000 & 0.000000\end{array}$

$\begin{array}{llll}2 \mathrm{~A} & 1.143923 & 0.000000 & 0.000000\end{array}$

$\begin{array}{llll}3 \mathrm{~A} & -1.143923 & -0.000871 & 0.000000\end{array}$

$\begin{array}{llll}4 \mathrm{~A} & -0.541869 & 0.947053 & 0.000199\end{array}$

$\begin{array}{llll}5 \mathrm{~A} & 0.541148 & 0.947465 & 0.000199\end{array}$

$\begin{array}{lllll}6 \mathrm{~A} & 0.548495 & 0.281982 & 0.888285\end{array}$

$\begin{array}{lllll}7 A & -0.548709 & 0.281937 & -0.888167\end{array}$

$\begin{array}{llll}8 \mathrm{~A} & -0.548709 & 0.281565 & 0.888285\end{array}$

$\begin{array}{lllll}9 \mathrm{~A} & 0.548495 & 0.282355 & -0.888167\end{array}$

$\begin{array}{llll}10 A & -0.545384 & -0.764705 & 0.550041\end{array}$

$\begin{array}{llll}11 \mathrm{~A} & 0.545966 & -0.764290 & 0.550041\end{array}$

$\begin{array}{llll}12 \mathrm{~A} & -0.545384 & -0.764475 & -0.550360\end{array}$

$\begin{array}{llll}13 \mathrm{~A} & 0.545966 & -0.764060 & -0.550360\end{array}$

$\begin{array}{llll}14 \mathrm{~B} & -0.000566 & 1.487471 & 1.173636\end{array}$

15B $-0.000566 \quad 1.487963-1.173010$

$\begin{array}{llll}16 \mathrm{~B} & 1.622236 & 1.116512 & -0.826587\end{array}$

$\begin{array}{llll}17 \mathrm{~B} & -1.623085 & 1.115277 & -0.826588\end{array}$

$\begin{array}{llll}18 \mathrm{~B} & -1.623084 & 1.114931 & 0.827057\end{array}$

$\begin{array}{llll}19 B & 1.622235 & 1.116165 & 0.827058\end{array}$

$\begin{array}{llll}\text { 20B } & 0.000222 & -0.583033 & 1.799973\end{array}$

21B $\quad 0.000222 \quad-0.582279-1.800216$

$\begin{array}{lllll}22 \mathrm{~B} & 0.000722 & -1.899234 & -0.000398\end{array}$

$\begin{array}{llll}23 \mathrm{~B} & 1.617039 & -0.444798 & -1.333963\end{array}$

$\begin{array}{llll}24 \mathrm{~B} & -1.616699 & -0.446028 & -1.333963\end{array}$

$\begin{array}{llll}25 B & -1.616698 & -0.446586 & 1.333779\end{array}$

$\begin{array}{llll}26 \mathrm{~B} & 1.617038 & -0.445356 & 1.333779\end{array}$

$\begin{array}{llll}27 \mathrm{~B} & -1.615457 & -1.410686 & -0.000294\end{array}$

$\begin{array}{llll}28 B & 1.616530 & -1.409456 & -0.000294\end{array}$

$\begin{array}{llll}29 B & 0.826771 & 2.312008 & 0.000486\end{array}$

$\begin{array}{llll}30 \mathrm{~B} & -0.828529 & 2.311378 & 0.000485\end{array}$

$\begin{array}{lllll}31 \mathrm{~B} & -2.523068 & -0.014280 & -0.000001\end{array}$

$\begin{array}{llll}32 \mathrm{~B} & 2.523078 & -0.012361 & 0.000000\end{array}$

$E 33 / \varepsilon=-162.262866$

$\begin{array}{cccc}\text { atom } & x / \sigma & y / \sigma & z / \sigma \\ \text { 1A } & 0.000000 & 0.000000 & 0.000000 \\ \text { 2B } & 1.321573 & 0.000000 & 0.000000 \\ \text { 3A } & 0.049854 & -1.130394 & 0.000000 \\ \text { 4A } & 0.049853 & 1.130396 & 0.000000 \\ \text { 5B } & -1.840696 & 0.000000 & 0.000001 \\ \text { 6A } & -0.720427 & 0.548135 & 0.573103 \\ \text { 7A } & -0.720428 & 0.548135 & -0.573102\end{array}$

$\begin{array}{llll}7 A & -0.720428 & 0.548135 & -0.573102\end{array}$ $\begin{array}{llll}8 A & -0.720427 & -0.548134 & 0.573103\end{array}$

$\begin{array}{lllll}9 A & -0.720428 & -0.548134 & -0.573102\end{array}$

$\begin{array}{llll}10 \mathrm{~B} & -0.425575 & 0.000001 & 1.832113\end{array}$

$\begin{array}{llll}11 B & -0.425577 & 0.000001 & -1.832113\end{array}$

$\begin{array}{llll}12 \mathrm{~B} & -1.287936 & 1.645046 & 0.000001\end{array}$

$\begin{array}{llll}13 \mathrm{~B} & -1.287935 & -1.645045 & 0.000001\end{array}$

$\begin{array}{llll}14 \mathrm{~A} & 0.348844 & 0.538346 & 0.859409\end{array}$

$\begin{array}{llll}15 A & 0.348844 & -0.538345 & 0.859409\end{array}$

$\begin{array}{lllll}16 \mathrm{~A} & 0.348843 & 0.538346 & -0.859409\end{array}$

$\begin{array}{lllll}17 A & 0.348843 & -0.538345 & -0.859410\end{array}$

$\begin{array}{llll}18 B & -0.301670 & -1.620676 & -1.332293\end{array}$

$\begin{array}{llll}19 B & -0.301669 & 1.620677 & 1.332293\end{array}$

$\begin{array}{llll}20 B & -0.301670 & 1.620676 & -1.332293\end{array}$

$\begin{array}{llll}21 B & -0.301669 & -1.620675 & 1.332294\end{array}$

$\begin{array}{llll}22 \mathrm{~B} & 1.279881 & 1.491046 & -0.814053\end{array}$

$\begin{array}{lllll}23 B & 1.279881 & -1.491045 & -0.814053\end{array}$

$\begin{array}{llll}24 \mathrm{~B} & 1.279882 & -1.491045 & 0.814053\end{array}$

$\begin{array}{llll}25 B & 1.279882 & 1.491046 & 0.814053\end{array}$

$\begin{array}{llll}26 \mathrm{~B} & 1.286963 & 0.000001 & 1.685024\end{array}$

$\begin{array}{llll}27 \mathrm{~B} & 1.286961 & 0.000001 & -1.685024\end{array}$

$\begin{array}{llll}28 \mathrm{~B} & -1.779308 & 0.847093 & 1.403087\end{array}$

$\begin{array}{llll}29 B & -1.779307 & -0.847091 & 1.403087\end{array}$

$\begin{array}{llll}30 \mathrm{~B} & -1.779309 & 0.847092 & -1.403085\end{array}$

$\begin{array}{llll}31 \mathrm{~B} & -1.779309 & -0.847091 & -1.403085\end{array}$

$\begin{array}{llll}32 \mathrm{~B} & 0.187752 & 2.486328 & 0.000000\end{array}$

$\begin{array}{llll}33 \mathrm{~B} & 0.187753 & -2.486327 & 0.000001\end{array}$

$E 34 / \varepsilon=-169.250795$

$\begin{array}{ccc}\text { atom } & x / \sigma & y / \sigma \\ 1 \mathrm{~A} & 0.000000 & 0.00000\end{array}$

$\begin{array}{llll}2 A & 1.000000000 & 0.000000 & 0.000000\end{array}$

$\begin{array}{ll}0.000000 & 0.000000\end{array}$

$\begin{array}{llll}3 A & -1.141385 & -0.002418 & 0.000000\end{array}$

$\begin{array}{llll}4 \mathrm{~B} & -0.001998 & 1.885038 & -0.000573\end{array}$

$\begin{array}{lllll}5 A & -0.543776 & 0.753781 & -0.564192\end{array}$

$\begin{array}{llll}6 \mathrm{~A} & -0.543777 & 0.754124 & 0.563733\end{array}$

$\begin{array}{lllll}7 \mathrm{~A} & 0.542177 & 0.754932 & -0.564192\end{array}$

$\begin{array}{lllll}8 \mathrm{~A} & 0.542177 & 0.755274 & 0.563733\end{array}$

$\begin{array}{llll}9 A & -0.547965 & -0.305340 & -0.883521\end{array}$

$\begin{array}{llll}10 A & -0.547965 & -0.304803 & 0.883706\end{array}$

$\begin{array}{llll}11 \mathrm{~A} & 0.548610 & -0.304178 & -0.883521\end{array}$

$\begin{array}{llll}12 \mathrm{~A} & 0.548610 & -0.303641 & 0.883706\end{array}$

$\begin{array}{llll}13 A & -0.543322 & -0.949334 & 0.000289\end{array}$

$\begin{array}{llll}14 \mathrm{~A} & 0.545332 & -0.948181 & 0.000289\end{array}$

$\begin{array}{llll}15 B & 1.619341 & 1.375352 & -0.000417\end{array}$

$\begin{array}{llll}16 \mathrm{~B} & -1.622253 & 1.371917 & -0.000417\end{array}$

$\begin{array}{llll}17 \mathrm{~B} & -0.000518 & 0.488208 & -1.831811\end{array}$

$\begin{array}{llll}18 \mathrm{~B} & -0.000519 & 0.489322 & 1.831514\end{array}$

$\begin{array}{llll}19 \mathrm{~B} & 1.623368 & 0.398100 & 1.332301\end{array}$

$\begin{array}{lllll}20 \mathrm{~B} & -1.624207 & 0.393849 & -1.332541\end{array}$

$\begin{array}{llll}21 B & 1.623369 & 0.397290 & -1.332541\end{array}$

$\begin{array}{llll}22 \mathrm{~B} & -1.624208 & 0.394659 & 1.332301\end{array}$

$\begin{array}{llll}23 \mathrm{~B} & 0.001631 & -1.539116 & -1.118184\end{array}$

$\begin{array}{llll}24 \mathrm{~B} & 0.001630 & -1.538436 & 1.119120\end{array}$

$\begin{array}{llll}25 B & -1.612360 & -1.162071 & 0.821949\end{array}$

$\begin{array}{llll}26 \mathrm{~B} & -1.612360 & -1.162570 & -0.821241\end{array}$

$\begin{array}{llll}27 \mathrm{~B} & 1.614820 & -1.159151 & -0.821242\end{array}$

$\begin{array}{llll}28 \mathrm{~B} & 1.614819 & -1.158651 & 0.821949\end{array}$

$\begin{array}{llll}29 B & -0.839356 & 1.843433 & 1.399148\end{array}$

$\begin{array}{lllll}30 \mathrm{~B} & 0.835448 & 1.844357 & -1.400268\end{array}$

$\begin{array}{llll}31 \mathrm{~B} & 0.835447 & 1.845208 & 1.399147\end{array}$

$\begin{array}{llll}32 \mathrm{~B} & -0.839355 & 1.842582 & -1.400268\end{array}$

$\begin{array}{llll}33 \mathrm{~B} & -2.521052 & -0.029854 & 0.000009\end{array}$ $\begin{array}{llll}34 \mathrm{~B} & 2.521110 & -0.024512 & 0.000009\end{array}$

$E 35 / \varepsilon=-175.447948$

atom $\mathrm{x} / \sigma \quad \mathrm{y} / \sigma$

$\begin{array}{llll}1 \mathrm{~A} & 0.000000 & 0.000000 & 0.000000\end{array}$

$\begin{array}{llll}2 A & 1.141995 & 0.000000 & 0.000000\end{array}$

$\begin{array}{llll}3 A & -1.142920 & -0.004446 & 0.000000\end{array}$

$\begin{array}{llll}3 \mathrm{~B} & -0.003624 & 1.888661 & 0.002970\end{array}$

$\begin{array}{llll}5 \mathrm{~A} & 0.541020 & 0.756915 & 0.563935\end{array}$

$\begin{array}{lllll}6 \mathrm{~A} & 0.541018 & 0.758685 & -0.561552\end{array}$

$\begin{array}{llll}7 \mathrm{~A} & -0.545000 & 0.756105 & 0.563365\end{array}$ $\begin{array}{lllll}8 A & -0.545003 & 0.757873 & -0.560979\end{array}$

$\begin{array}{lllll}9 \mathrm{~A} & 0.544367 & -0.953878 & -0.001499\end{array}$

$\begin{array}{lllll}10 A & -0.533885 & -0.960675 & -0.001507\end{array}$

$\begin{array}{llll}11 \mathrm{~A} & 0.550142 & -0.300520 & 0.878980\end{array}$

$\begin{array}{llll}12 \mathrm{~A} & 0.550138 & -0.297755 & -0.879921\end{array}$

$\begin{array}{llll}13 \mathrm{~A} & -0.550897 & -0.299545 & 0.877515\end{array}$

$\begin{array}{llll}14 \mathrm{~A} & -0.550904 & -0.296784 & -0.878446\end{array}$

$\begin{array}{llll}15 \mathrm{~B} & 1.618613 & 1.377352 & 0.002163\end{array}$

$\begin{array}{llll}16 \mathrm{~B} & -1.625168 & 1.372146 & 0.002163\end{array}$

$\begin{array}{llll}17 \mathrm{~B} & -0.001528 & 0.488977 & -1.829705\end{array}$

$\begin{array}{llll}18 \mathrm{~B} & -0.001519 & 0.483223 & 1.831237\end{array}$

$\begin{array}{llll}19 \mathrm{~B} & 1.622889 & 0.399088 & 1.333178\end{array}$

$\begin{array}{llll}20 \mathrm{~B} & 1.622883 & 0.403276 & -1.331920\end{array}$

$\begin{array}{llll}21 \mathrm{~B} & -1.626249 & 0.392461 & 1.332804\end{array}$

$\begin{array}{llll}22 \mathrm{~B} & -1.626257 & 0.396650 & -1.331550\end{array}$

$\begin{array}{llll}23 \mathrm{~B} & -0.007386 & -1.495799 & -1.182695\end{array}$

$\begin{array}{llll}24 \mathrm{~B} & -0.007380 & -1.499510 & 1.177991\end{array}$

$\begin{array}{llll}25 B & 1.614321 & -1.154842 & 0.823016\end{array}$

$\begin{array}{llll}26 \mathrm{~B} & 1.614317 & -1.152249 & -0.826646\end{array}$

$\begin{array}{llll}27 \mathrm{~B} & -1.608850 & -1.163708 & 0.817991\end{array}$

$\begin{array}{llll}28 \mathrm{~B} & -1.608856 & -1.161130 & -0.821633\end{array}$

$\begin{array}{llll}29 \mathrm{~B} & -0.840873 & 1.839989 & 1.403150\end{array}$

$\begin{array}{llll}30 \mathrm{~B} & -0.840879 & 1.844391 & -1.397351\end{array}$

$\begin{array}{llll}31 \mathrm{~B} & 0.832623 & 1.843210 & 1.403239\end{array}$

$\begin{array}{llll}32 \mathrm{~B} & 0.832617 & 1.847612 & -1.397438\end{array}$

$\begin{array}{lllll}33 \mathrm{~B} & 2.520262 & -0.024028 & -0.000041\end{array}$

$\begin{array}{lllll}34 \mathrm{~B} & -2.522069 & -0.031799 & -0.000041\end{array}$

$\begin{array}{lllll}35 B & 0.764167 & -2.329880 & -0.003662\end{array}$

$E 36 / \varepsilon=-182.632268$

atom $\quad \mathrm{x} / \sigma \quad \mathrm{y} / \sigma$

$\begin{array}{llll}1 \mathrm{~A} & 0.000000 & 0.000000 & 0.000000\end{array}$

$\begin{array}{llll}2 A & 1.142878 & 0.000000 & 0.000000\end{array}$

$\begin{array}{llll}3 \mathrm{~A} & -1.142878 & -0.000431 & 0.000000\end{array}$

$\begin{array}{lllll}4 \mathrm{~A} & 0.542529 & 0.747295 & -0.578072\end{array}$

$\begin{array}{lllll}5 A & -0.542810 & 0.747091 & -0.578072\end{array}$

$\begin{array}{llll}6 \mathrm{~A} & -0.542815 & 0.771521 & 0.545030\end{array}$

$\begin{array}{llll}7 \mathrm{~A} & 0.542524 & 0.771725 & 0.545030\end{array}$

$\begin{array}{llll}8 \mathrm{~B} & -0.000356 & 1.891210 & -0.041140\end{array}$

$\begin{array}{llll}9 \mathrm{~A} & 0.540603 & -0.957529 & 0.020829\end{array}$

$\begin{array}{llll}10 A & -0.540242 & -0.957732 & 0.020829\end{array}$

$\begin{array}{lllll}11 \mathrm{~A} & -0.550628 & -0.315884 & -0.871872\end{array}$

$\begin{array}{lllll}12 \mathrm{~A} & 0.550747 & -0.315677 & -0.871872\end{array}$

$\begin{array}{llll}13 A & 0.550742 & -0.277465 & 0.884775\end{array}$

$\begin{array}{llll}14 \mathrm{~A} & -0.550637 & -0.277673 & 0.884775\end{array}$

$\begin{array}{llll}15 \mathrm{~B} & -1.619148 & 1.382025 & -0.030072\end{array}$

$\begin{array}{llll}16 \mathrm{~B} & 1.618628 & 1.382633 & -0.030071\end{array}$

$\begin{array}{llll}17 \mathrm{~B} & -0.000100 & 0.529535 & 1.818024\end{array}$

$\begin{array}{llll}18 B & -0.000084 & 0.449979 & -1.839335\end{array}$

$\begin{array}{llll}19 B & 0.000277 & -1.471139 & 1.208239\end{array}$

$\begin{array}{llll}20 \mathrm{~B} & 0.000287 & -1.522288 & -1.143129\end{array}$

$\begin{array}{lllll}21 B & 1.623078 & 0.379382 & -1.342095\end{array}$

$\begin{array}{llll}22 \mathrm{~B} & -1.623220 & 0.378772 & -1.342096\end{array}$

$\begin{array}{llll}23 \mathrm{~B} & -1.623233 & 0.436773 & 1.324336\end{array}$

$\begin{array}{llll}24 \mathrm{~B} & 1.623068 & 0.437384 & 1.324337\end{array}$

$\begin{array}{lllll}25 B & 1.620304 & -1.157727 & -0.800183\end{array}$

$\begin{array}{lllll}26 \mathrm{~B} & -1.619868 & -1.158336 & -0.800183\end{array}$

$\begin{array}{llll}27 \mathrm{~B} & 1.620298 & -1.121836 & 0.849777\end{array}$

$\begin{array}{llll}28 \mathrm{~B} & -1.619875 & -1.122446 & 0.849777\end{array}$

$\begin{array}{llll}29 B & -0.835404 & 1.819170 & -1.440342\end{array}$

$\begin{array}{llll}30 \mathrm{~B} & 0.834721 & 1.819485 & -1.440340\end{array}$

$\begin{array}{llll}31 \mathrm{~B} & 0.834709 & 1.880395 & 1.359863\end{array}$

$\begin{array}{llll}32 \mathrm{~B} & -0.835417 & 1.880081 & 1.359863\end{array}$

$\begin{array}{llll}33 \mathrm{~B} & -2.523359 & -0.012562 & 0.000260\end{array}$

$\begin{array}{llll}34 \mathrm{~B} & 2.523364 & -0.011613 & 0.000261\end{array}$

$\begin{array}{llll}35 \mathrm{~B} & 0.823299 & -2.326991 & 0.050620\end{array}$

$\begin{array}{llll}36 \mathrm{~B} & -0.822423 & -2.327301 & 0.050619\end{array}$

$E 37 / \varepsilon=-188.761434$

atom $\quad \mathrm{x} / \sigma \quad \mathrm{y} / \sigma$

$\begin{array}{llll}1 \mathrm{~A} & 0.000000 & 0.000000 & 0.000000\end{array}$

$\begin{array}{llll}2 \mathrm{~A} & 1.138623 & 0.000000 & 0.000000\end{array}$

$\begin{array}{llll}3 \mathrm{~A} & -1.143771 & -0.004669 & 0.000000\end{array}$

$\begin{array}{llll}4 \mathrm{~A} & 0.540354 & 0.506129 & 0.794287\end{array}$ 


\begin{tabular}{|c|c|c|c|}
\hline B & -0.002822 & -0 . & 3866 \\
\hline $6 \mathrm{~A}$ & -0.547466 & 0.500080 & 6730 \\
\hline $7 \mathrm{~A}$ & -0.544218 & -0.616249 & 0.722958 \\
\hline $8 \mathrm{~A}$ & 0.538507 & 0.052897 & -0952905 \\
\hline $9 \mathrm{~A}$ & 0.539966 & -0.614537 & 0723284 \\
\hline LOA & -0.544064 & 0.046383 & -0.954421 \\
\hline 11 & 0.550619 & 0.907394 & -0.244763 \\
\hline $12 B$ & -0.019128 & 1.772026 & 0.642849 \\
\hline $13 B$ & -0.017303 & 1.227810 & -1.446318 \\
\hline $14 \mathrm{~A}$ & -0.548795 & 0.894247 & -0.242524 \\
\hline $15 \mathrm{~A}$ & 0.548297 & -0.862544 & -0.346447 \\
\hline $16 \mathrm{~A}$ & -0.551715 & -0.864075 & -0.345954 \\
\hline 17B & 1.614103 & -0.104543 & 1.386496 \\
\hline 18B & -1.623872 & -0.103257 & 1.380875 \\
\hline $19 B$ & -0.002248 & -1.865420 & 0.373582 \\
\hline $20 B$ & -0.002328 & -1.097269 & -1.559020 \\
\hline $21 B$ & 1.631165 & 1.278557 & 0.504556 \\
\hline $22 \mathrm{~B}$ & 1.628077 & 0.863083 & -1.086159 \\
\hline $23 B$ & -1.626664 & 1.290043 & 0.494295 \\
\hline $24 \mathrm{~B}$ & -1.622942 & 0.877559 & -1.086007 \\
\hline $25 B$ & 1.616286 & -1.371608 & 0.327752 \\
\hline $26 \mathrm{~B}$ & -1.623187 & -1.370278 & 0.325998 \\
\hline $27 \mathrm{~B}$ & 1.614577 & -0.774799 & -1.186655 \\
\hline $28 \mathrm{~B}$ & -1.622757 & -0.770028 & -1.184736 \\
\hline $9 \mathrm{~B}$ & 0.833976 & 1.263248 & 1.947410 \\
\hline $30 \mathrm{~B}$ & 2.521375 & -0.024011 & -0.006388 \\
\hline $31 B$ & 0.821030 & 0.118094 & -2.324448 \\
\hline $32 B$ & -0.837659 & -1.526579 & 1.759520 \\
\hline $33 B$ & 0.829207 & -1.528190 & 1.760643 \\
\hline $34 \mathrm{~B}$ & -2.525576 & -0.014422 & -0.010573 \\
\hline $35 \mathrm{~B}$ & -0.851693 & 1.255164 & 1.955432 \\
\hline $36 \mathrm{~B}$ & -0.833829 & 0.108689 & -2.329675 \\
\hline $37 \mathrm{~B}$ & 0.911337 & 2.266727 & -0.600699 \\
\hline
\end{tabular}

$E 38 / \varepsilon=-195.963276$

atom $\mathrm{X} / \sigma$

$\begin{array}{llll}1 \mathrm{~A} & 0.000000 & 0.000000 & 0.000000\end{array}$

$\begin{array}{lllll}2 \mathrm{~A} & 1.138584 & 0.000000 & 0.000000\end{array}$

$\begin{array}{lrrr}3 A & -1.138580 & -0.003196 & 0.000000\end{array}$

$\begin{array}{llll}4 \mathrm{~B} & -0.002641 & 1.880919 & 0.001930\end{array}$

$\begin{array}{llll}5 \mathrm{~A} & 0.543922 & 0.753050 & 0.561971\end{array}$

$\begin{array}{lllll}6 A & -0.546034 & 0.751520 & 0.561971\end{array}$

$\begin{array}{lllll}7 A & 0.543920 & 0.754201 & -0.560425\end{array}$

$\begin{array}{lllll}8 A & -0.546036 & 0.752671 & -0.560425\end{array}$

$\begin{array}{llll}9 \mathrm{~B} & -0.000791 & 0.562674 & -1.809950\end{array}$

$\begin{array}{llll}10 B & -0.000785 & 0.558962 & 1.811102\end{array}$

$\begin{array}{llll}11 \mathrm{~A} & -0.541524 & -0.313280 & 0.900434\end{array}$

$\begin{array}{llll}12 \mathrm{~A} & 0.542400 & -0.311759 & 0.900434\end{array}$

$\begin{array}{llll}13 \mathrm{~A} & -0.541527 & -0.311434 & -0.901071\end{array}$

$\begin{array}{llll}14 \mathrm{~A} & 0.542398 & -0.309912 & -0.901071\end{array}$

$\begin{array}{llll}15 A & -0.548807 & -0.932754 & -0.000955\end{array}$

$\begin{array}{llll}16 \mathrm{~A} & 0.551422 & -0.931210 & -0.000955\end{array}$

$\begin{array}{llll}17 \mathrm{~B} & -1.626341 & 1.371892 & 0.001410\end{array}$

$\begin{array}{llll}18 \mathrm{~B} & 1.622482 & 1.376452 & 0.001410\end{array}$

$\begin{array}{llll}19 B & 1.620271 & 0.403182 & 1.329609\end{array}$

$\begin{array}{llll}20 B & -1.621398 & 0.398632 & 1.329609\end{array}$

$\begin{array}{lllll}21 B & -1.621402 & 0.401357 & -1.328782\end{array}$

$\begin{array}{llll}22 B & 1.620267 & 0.405908 & -1.328782\end{array}$

$\begin{array}{llll}23 B & 0.002249 & -1.602858 & -1.035703\end{array}$

$\begin{array}{llll}24 \mathrm{~B} & 0.002252 & -1.604978 & 1.032416\end{array}$

$\begin{array}{llll}25 \mathrm{~B} & 1.621646 & -1.159322 & 0.810381\end{array}$

$\begin{array}{llll}26 \mathrm{~B} & -1.618386 & -1.163870 & 0.810381\end{array}$

$\begin{array}{llll}27 \mathrm{~B} & 1.621644 & -1.157658 & -0.812759\end{array}$

28B $\quad-1.618389-1.162206-0.812759$

$\begin{array}{llll}29 B & -2.520567 & -0.026370 & -0.000022\end{array}$

$\begin{array}{llll}30 \mathrm{~B} & 2.520630 & -0.019294 & -0.000022\end{array}$

$\begin{array}{llll}31 \mathrm{~B} & -0.856299 & 1.872635 & 1.384452\end{array}$

$\begin{array}{llll}32 \mathrm{~B} & 0.851038 & 1.875032 & 1.384452\end{array}$

$\begin{array}{llll}33 \mathrm{~B} & -0.856303 & 1.875469 & -1.380605\end{array}$

$\begin{array}{llll}34 \mathrm{~B} & 0.851033 & 1.877866 & -1.380605\end{array}$

$\begin{array}{llll}35 \mathrm{~B} & -0.832320 & -0.786580 & 2.200393\end{array}$

$\begin{array}{llll}36 \mathrm{~B} & -0.832326 & -0.782068 & -2.201997\end{array}$

$\begin{array}{llll}37 \mathrm{~B} & 0.834522 & -0.784241 & 2.200393\end{array}$
|38B $\quad 0.834516 \quad-0.779728 \quad-2.201997$ $E 39 / \varepsilon=-202.040051$

$\begin{array}{llll}\text { atom } & \mathrm{x} / \sigma & \mathrm{y} / \sigma & \mathrm{z} / \sigma\end{array}$

$\begin{array}{llll}1 \mathrm{~A} & 0.000000 & 0.000000 & 0.000000\end{array}$

$\begin{array}{llll}2 \mathrm{~A} & 1.134713 & 0.000000 & 0.000000\end{array}$

$\begin{array}{llll}3 A & -1.140312 & -0.001014 & 0.000000\end{array}$

$\begin{array}{lllll}4 \mathrm{~A} & 0.540368 & 0.303371 & -0.898998\end{array}$

$\begin{array}{lllll}5 A & 0.540368 & 0.304736 & 0.898536\end{array}$

$\begin{array}{llll}6 \mathrm{~B} & -0.006694 & -1.889625 & 0.001435\end{array}$

$\begin{array}{llll}7 \mathrm{~A} & -0.548157 & -0.759862 & -0.557521\end{array}$

$\begin{array}{llll}8 A & -0.548157 & -0.759015 & 0.558674\end{array}$

$\begin{array}{lllll}9 \mathrm{~A} & 0.540972 & -0.760447 & -0.557879\end{array}$

$\begin{array}{llll}10 A & 0.540972 & -0.759599 & 0.559034\end{array}$

$\begin{array}{llll}11 B & -0.003295 & -0.573247 & 1.806997\end{array}$

$\begin{array}{llll}12 \mathrm{~B} & -0.003295 & -0.575991 & -1.806124\end{array}$

$\begin{array}{llll}13 A & -0.546191 & 0.298658 & -0.901132\end{array}$

$\begin{array}{llll}14 \mathrm{~A} & -0.546191 & 0.300026 & 0.900677\end{array}$

$\begin{array}{llll}15 A & 0.551929 & 0.945682 & -0.000718\end{array}$

$\begin{array}{llll}16 \mathrm{~B} & -0.016202 & 1.571354 & 1.068460\end{array}$

$\begin{array}{llll}17 \mathrm{~B} & -0.016202 & 1.569730 & -1.070844\end{array}$

$\begin{array}{llll}18 \mathrm{~A} & -0.546560 & 0.932015 & -0.000708\end{array}$

$\begin{array}{llll}19 \mathrm{~B} & 1.614998 & -1.392375 & 0.001058\end{array}$

$\begin{array}{llll}20 \mathrm{~B} & -1.628260 & -1.383881 & 0.001051\end{array}$

$\begin{array}{llll}21 \mathrm{~B} & 1.614903 & -0.424208 & 1.331377\end{array}$

$\begin{array}{llll}22 \mathrm{~B} & 1.614903 & -0.426230 & -1.330730\end{array}$

$\begin{array}{llll}23 \mathrm{~B} & -1.626082 & -0.416755 & -1.326915\end{array}$

$\begin{array}{llll}24 \mathrm{~B} & -1.626083 & -0.414739 & 1.327546\end{array}$

$\begin{array}{llll}25 \mathrm{~B} & 1.630713 & 1.126613 & -0.819384\end{array}$

$\begin{array}{llll}26 \mathrm{~B} & 1.630713 & 1.127856 & 0.817672\end{array}$

$\begin{array}{llll}27 \mathrm{~B} & -1.621434 & 1.147125 & -0.814125\end{array}$

$\begin{array}{llll}28 \mathrm{~B} & -1.621434 & 1.148360 & 0.812382\end{array}$

$\begin{array}{llll}29 B & 2.519615 & -0.007425 & 0.000006\end{array}$

$\begin{array}{llll}30 \mathrm{~B} & -2.523938 & 0.013439 & -0.000010\end{array}$

$\begin{array}{llll}31 \mathrm{~B} & 0.834722 & 0.757188 & -2.210841\end{array}$

$\begin{array}{llll}32 \mathrm{~B} & 0.834721 & 0.760546 & 2.209689\end{array}$

$\begin{array}{llll}33 \mathrm{~B} & -0.860363 & -1.885846 & 1.383031\end{array}$

$\begin{array}{llll}34 \mathrm{~B} & -0.860363 & -1.887945 & -1.380166\end{array}$

$\begin{array}{llll}35 \mathrm{~B} & 0.844817 & -1.893878 & -1.380380\end{array}$

$\begin{array}{llll}36 \mathrm{~B} & 0.844817 & -1.891779 & 1.383255\end{array}$

$\begin{array}{llll}37 \mathrm{~B} & -0.848216 & 0.750555 & -2.218123\end{array}$

$\begin{array}{llll}38 \mathrm{~B} & -0.848217 & 0.753923 & 2.216981\end{array}$

$\begin{array}{llll}39 \mathrm{~B} & 0.919001 & 2.366230 & -0.001796\end{array}$

\section{$E 40 / \varepsilon=-209.230681$}

atom $\mathrm{X} / \sigma$ $y / \sigma$

$\begin{array}{llll}1 \mathrm{~A} & 0.000000 & 0.000000 & 0.000000\end{array}$

$\begin{array}{llll}2 \mathrm{~A} & 1.132965 & 0.000000 & 0.000000\end{array}$

$\begin{array}{llll}3 A & -1.132936 & -0.008220 & 0.000000\end{array}$

$\begin{array}{llll}4 \mathrm{~A} & -0.537776 & -0.770854 & 0.533362\end{array}$

$\begin{array}{llll}5 \mathrm{~A} & 0.543353 & -0.766932 & 0.533362\end{array}$

$\begin{array}{llll}6 \mathrm{~A} & -0.537777 & -0.770652 & -0.533651\end{array}$

$\begin{array}{lllll}7 \mathrm{~A} & 0.543352 & -0.766731 & -0.533651\end{array}$

$\begin{array}{llll}8 B & -0.005367 & 1.479223 & -1.139144\end{array}$

$\begin{array}{llll}9 \mathrm{~B} & -0.005365 & 1.478794 & 1.139702\end{array}$

$\begin{array}{llll}10 \mathrm{~A} & -0.542217 & 0.241335 & 0.914188\end{array}$

$\begin{array}{llll}11 \mathrm{~A} & -0.542218 & 0.241680 & -0.914096\end{array}$

$\begin{array}{llll}12 \mathrm{~A} & 0.540450 & 0.245263 & 0.914189\end{array}$

$\begin{array}{lllll}13 \mathrm{~A} & 0.540449 & 0.245607 & -0.914096\end{array}$

$\begin{array}{llll}14 \mathrm{~A} & -0.548772 & 0.923289 & 0.000175\end{array}$

$\begin{array}{llll}15 A & 0.542058 & 0.927247 & 0.000175\end{array}$

$\begin{array}{llll}16 \mathrm{~B} & 0.002839 & -0.782824 & -1.760434\end{array}$

$\begin{array}{llll}17 \mathrm{~B} & 0.002842 & -0.783487 & 1.760139\end{array}$

$\begin{array}{llll}18 \mathrm{~B} & -1.635764 & 1.039359 & 0.827534\end{array}$

$\begin{array}{llll}19 B & 1.628179 & 1.051199 & 0.827533\end{array}$

$\begin{array}{llll}\text { 20B } & -1.635766 & 1.039671 & -0.827137\end{array}$

$\begin{array}{llll}21 \mathrm{~B} & 1.628178 & 1.051511 & -0.827138\end{array}$

$\begin{array}{llll}22 \mathrm{~B} & -1.613603 & -0.560191 & 1.295686\end{array}$

$\begin{array}{llll}23 \mathrm{~B} & 1.617624 & -0.548470 & 1.295686\end{array}$

$\begin{array}{llll}24 \mathrm{~B} & -1.613606 & -0.559702 & -1.295893\end{array}$

$\begin{array}{lllll}25 B & 1.617622 & -0.547981 & -1.295894\end{array}$

$\begin{array}{lllll}26 \mathrm{~A} & 0.005603 & -1.544721 & -0.000291\end{array}$ $\begin{array}{llll}27 \mathrm{~A} & 1.456871 & -1.072494 & -0.000203\end{array}$ $\begin{array}{llll}28 A & -1.449053 & -1.083035 & -0.000202\end{array}$ $\begin{array}{llll}29 \mathrm{~B} & -0.875383 & -2.022412 & 0.898063\end{array}$ $\begin{array}{llll}30 \mathrm{~B} & -0.875384 & -2.022073 & -0.898823\end{array}$ $\begin{array}{lllll}31 \mathrm{~B} & 0.890031 & -2.016009 & 0.898062\end{array}$ $\begin{array}{lllll}32 \mathrm{~B} & 0.890030 & -2.015670 & -0.898822\end{array}$ $\begin{array}{lllll}33 \mathrm{~B} & 2.505750 & -0.110772 & -0.000022\end{array}$ $\begin{array}{lllll}34 \mathrm{~B} & -2.504882 & -0.128948 & -0.000021\end{array}$ $\begin{array}{llll}35 \mathrm{~B} & 0.833392 & 0.573638 & 2.224754\end{array}$ $\begin{array}{llll}36 \mathrm{~B} & -0.837533 & 0.567576 & 2.224754\end{array}$ $\begin{array}{lllll}37 \mathrm{~B} & 0.833388 & 0.574476 & -2.224538\end{array}$ $\begin{array}{llll}38 \mathrm{~B} & -0.837537 & 0.568415 & -2.224537\end{array}$ $\begin{array}{llll}39 \mathrm{~B} & 0.848678 & 2.281771 & 0.000430\end{array}$ $\begin{array}{llll}\text { 40B } & -0.865212 & 2.275554 & 0.000430\end{array}$

\section{$E 41 / \varepsilon=-215.407179$}

$$
\text { atom } \mathrm{x} / \sigma \quad \mathrm{y} / \sigma \quad \mathrm{z} / \sigma
$$

$\begin{array}{llll}1 \mathrm{~A} & 0.000000 & 0.000000 & 0.000000\end{array}$

$\begin{array}{llll}2 \mathrm{~A} & 1.133834 & 0.000000 & 0.000000\end{array}$

$\begin{array}{llll}3 A & -1.130450 & -0.003499 & 0.000000\end{array}$

$\begin{array}{llll}4 \mathrm{~A} & 0.529580 & -0.776333 & 0.531613\end{array}$

$\begin{array}{lllll}5 A & 0.529579 & -0.775005 & -0.533547\end{array}$

$\begin{array}{lllll}6 A & -0.548384 & -0.766292 & -0.547942\end{array}$

$\begin{array}{llll}7 \mathrm{~A} & -0.548381 & -0.767656 & 0.546033\end{array}$

$\begin{array}{llll}8 \mathrm{~A} & 0.553613 & 0.259022 & 0.905428\end{array}$

$\begin{array}{lllll}9 A & 0.553611 & 0.261279 & -0.904779\end{array}$

$\begin{array}{llll}10 A & -0.531936 & 0.263348 & 0.911534\end{array}$

$\begin{array}{lllll}11 \mathrm{~A} & -0.531939 & 0.265620 & -0.910871\end{array}$

$\begin{array}{llll}12 B & 0.021791 & 1.503219 & -1.125043\end{array}$

$\begin{array}{llll}13 B & 0.021794 & 1.500409 & 1.128791\end{array}$

$\begin{array}{llll}14 \mathrm{~A} & 0.554666 & 0.932083 & 0.001163\end{array}$

$\begin{array}{llll}15 A & -0.535567 & 0.933983 & 0.001166\end{array}$

$\begin{array}{llll}16 \mathrm{~B} & 0.039657 & -0.678832 & 1.783979\end{array}$

$\begin{array}{lllll}17 \mathrm{~B} & 0.039652 & -0.674381 & -1.785664\end{array}$

$\begin{array}{lllll}18 \mathrm{~A} & -0.057901 & -1.568938 & -0.001955\end{array}$

$\begin{array}{lllll}19 B & 1.618568 & -0.529666 & -1.290151\end{array}$

$\begin{array}{llll}20 \mathrm{~B} & 1.618571 & -0.532882 & 1.288824\end{array}$

$\begin{array}{llll}21 \mathrm{~B} & 1.649720 & 1.062394 & -0.816996\end{array}$

$\begin{array}{llll}22 \mathrm{~B} & 1.649722 & 1.060353 & 0.819641\end{array}$

$\begin{array}{llll}23 \mathrm{~B} & -1.609890 & 1.084989 & 0.830739\end{array}$

$\begin{array}{lllll}24 \mathrm{~B} & -1.609892 & 1.087057 & -0.828025\end{array}$

$\begin{array}{llll}25 \mathrm{~B} & -1.612316 & -1.425124 & -0.001774\end{array}$

$\begin{array}{llll}26 \mathrm{~B} & -1.599481 & -0.495756 & 1.340203\end{array}$

$\begin{array}{llll}27 \mathrm{~B} & -1.599485 & -0.492413 & -1.341430\end{array}$

$\begin{array}{llll}28 \mathrm{~B} & 0.939422 & -2.008372 & 0.856037\end{array}$

$\begin{array}{lllll}29 B & 0.939420 & -2.006231 & -0.861043\end{array}$

$\begin{array}{lllll}30 \mathrm{~A} & 1.465177 & -1.048088 & -0.001308\end{array}$

$\begin{array}{lllll}31 \mathrm{~B} & -0.689735 & -1.977444 & -1.202281\end{array}$

$\begin{array}{llll}32 \mathrm{~B} & -0.689732 & -1.980436 & 1.197350\end{array}$

$\begin{array}{lllll}33 \mathrm{~B} & 2.517953 & -0.105474 & -0.000134\end{array}$

$\begin{array}{llll}34 \mathrm{~B} & -2.503965 & -0.039366 & -0.000046\end{array}$

$\begin{array}{llll}35 \mathrm{~B} & -0.810267 & 0.646922 & 2.235158\end{array}$

$\begin{array}{llll}36 \mathrm{~B} & -0.810272 & 0.652495 & -2.233534\end{array}$

$\begin{array}{llll}37 \mathrm{~B} & -0.832455 & 2.307450 & 0.002879\end{array}$

$\begin{array}{llll}38 B & 0.885195 & 2.298186 & 0.002866\end{array}$

$\begin{array}{llll}39 \mathrm{~B} & 0.882916 & 0.643066 & 2.222437\end{array}$

$\begin{array}{lllll}40 \mathrm{~B} & 0.882912 & 0.648606 & -2.220827\end{array}$

$\begin{array}{llll}41 B & -0.141191 & -2.955065 & -0.003683\end{array}$

\section{$E 42 / \varepsilon=-221.319539$}

atom $\mathrm{X} / \sigma$

$y / \sigma$

$\begin{array}{llll}1 \mathrm{~A} & 0.000000 & 0.000000 & 0.000000\end{array}$

$\begin{array}{llll}2 \mathrm{~A} & 1.066727 & 0.000000 & 0.000000\end{array}$

$\begin{array}{llll}3 \mathrm{~A} & 0.453774 & -0.952288 & 0.000000\end{array}$

$\begin{array}{lllll}4 \mathrm{~A} & 0.485722 & -0.281128 & -0.903408\end{array}$

$\begin{array}{llll}5 A & -0.462493 & 0.955018 & 0.020844\end{array}$

$\begin{array}{lllll}6 \mathrm{~A} & -1.064421 & -0.021418 & -0.017058\end{array}$

$\begin{array}{llll}7 \mathrm{~A} & 0.453557 & -0.319748 & 0.917584\end{array}$

$\begin{array}{llll}8 \mathrm{~A} & -0.465717 & 0.246337 & 0.925913\end{array}$

$\begin{array}{llll}9 \mathrm{~A} & -0.495764 & -0.784454 & -0.558251\end{array}$

$\begin{array}{llll}10 A & 0.498239 & 0.804137 & -0.553182\end{array}$

$\begin{array}{llll}11 \mathrm{~A} & -0.492906 & -0.799154 & 0.544595\end{array}$

$\begin{array}{llll}12 \mathrm{~A} & 0.481875 & 0.754416 & 0.580407\end{array}$ $\begin{array}{llll}13 \mathrm{~A} & -0.459944 & 0.317123 & -0.901014\end{array}$ 


$\begin{array}{rrrr}\text { 14B } & 1.700673 & 0.325712 & -1.186277 \\ \text { 15B } & 0.432056 & 0.649036 & 1.915590 \\ \text { 16B } & -1.601134 & -0.249679 & -1.287902 \\ \text { 17B } & -0.448066 & -0.773468 & 1.880743 \\ \text { 18A } & -1.352233 & 0.793569 & 0.640756 \\ \text { 19A } & 1.336583 & -0.878498 & 0.609905 \\ \text { 20B } & -0.337829 & -2.080394 & 0.068343 \\ \text { 21B } & -0.523123 & 1.667512 & -1.155973 \\ \text { 22B } & 1.707868 & 1.212401 & 0.177983 \\ \text { 23B } & 0.333968 & 2.062137 & 0.193205 \\ \text { 24A } & 1.359846 & -0.869658 & -0.607323 \\ \text { 25B } & 0.316842 & 0.569843 & -1.993979 \\ \text { 26B } & -1.698869 & -1.241313 & 0.019424 \\ \text { 27A } & 0.327855 & -1.397699 & -0.962983 \\ \text { 28A } & -1.418383 & -0.287095 & 0.984722 \\ \text { 29A } & -0.429808 & 1.323423 & 1.052437 \\ \text { 30A } & 1.410058 & 0.182955 & 1.022837 \\ \text { 31A } & -1.417764 & 0.880695 & -0.503420 \\ \text { 32A } & -0.277223 & -0.594257 & -1.606180 \\ \text { 33A } & 0.418475 & -1.411853 & 0.987326 \\ \text { 34B } & 1.268399 & -2.077274 & -0.021962 \\ \text { 35B } & 2.430955 & -0.286024 & 0.141298 \\ \text { 36B } & 1.036670 & -0.933958 & -1.998896 \\ \text { 37B } & -1.242821 & 0.682267 & 1.984775 \\ \text { 38B } & -1.314464 & 2.067317 & 0.240671 \\ \text { 39B } & 1.218016 & -0.821494 & 1.962579 \\ \text { 40B } & -2.444026 & 0.206717 & 0.120258 \\ \text { 41B } & -0.939461 & -1.772028 & -1.420527 \\ \text { 42B } & 1.110727 & 1.849765 & -1.250149\end{array}$

$E 43 / \varepsilon=-227.979704$

\begin{tabular}{|c|c|c|c|}
\hline & $\mathrm{x} / \sigma$ & $y$ & $z / \sigma$ \\
\hline $1 \mathrm{~A}$ & 0.000000 & 0.000000 & 0.000000 \\
\hline $2 \mathrm{~A}$ & 1.071204 & 0.000000 & 0.000000 \\
\hline $3 A$ & 0.496923 & -0.948970 & 0.000000 \\
\hline $4 \mathrm{~A}$ & -0.466250 & 0.282157 & 0.953503 \\
\hline $5 \mathrm{~A}$ & 0.466685 & 0.776693 & 0.579253 \\
\hline $6 \mathrm{~A}$ & -0.471575 & -0.773734 & 0.579252 \\
\hline $7 \mathrm{~A}$ & 0.466832 & -0.282509 & 0.886211 \\
\hline $8 \mathrm{~A}$ & -0.466154 & 0.952654 & 0.059461 \\
\hline $9 A$ & -1.060193 & -0.028965 & 0.059460 \\
\hline $10 \mathrm{~A}$ & -0.485919 & 0.294061 & -0.888978 \\
\hline $11 \mathrm{~A}$ & 0.485390 & -0.293739 & -0.929036 \\
\hline $12 \mathrm{~A}$ & 0.471889 & 0.774989 & -0.548972 \\
\hline $13 \mathrm{~A}$ & -0.467651 & -0.777553 & -0.548972 \\
\hline 14B & -1.605771 & -0.370481 & 1.285026 \\
\hline 15B & -0.416697 & 1.594403 & 1.285027 \\
\hline $16 B$ & 1.701015 & -1.029389 & -0.661433 \\
\hline 17B & 0.440742 & 2.016687 & -0.057951 \\
\hline 18B & -1.582110 & -1.325973 & -0.057952 \\
\hline $19 A$ & -1.370968 & 0.829659 & -0.520561 \\
\hline $20 A$ & 1.374754 & -0.831949 & 0.676109 \\
\hline $21 \mathrm{~A}$ & 1.347746 & 0.295572 & 1.039790 \\
\hline $22 \mathrm{~A}$ & 0.363363 & -1.331071 & 1.039789 \\
\hline $23 \mathrm{~A}$ & -0.252070 & -0.583099 & 1.635353 \\
\hline $24 \mathrm{~A}$ & 0.399629 & 0.493801 & 1.635353 \\
\hline $25 B$ & 0.310394 & -1.743375 & -1.115832 \\
\hline $26 B$ & 1.688431 & 0.533763 & -1.115831 \\
\hline 27B & -0.555912 & -0.626050 & -1.907564 \\
\hline $28 B$ & 0.296731 & 0.782900 & -1.907563 \\
\hline $29 A$ & -1.396788 & 0.845284 & 0.626982 \\
\hline $30 A$ & -0.450305 & 1.386521 & -0.933755 \\
\hline $31 \mathrm{~A}$ & -1.437200 & -0.244272 & -0.933756 \\
\hline $32 \mathrm{~A}$ & -0.252143 & -1.733164 & 0.132270 \\
\hline $33 A$ & 1.418430 & 1.027372 & 0.132271 \\
\hline 34B & 1.077507 & -0.652067 & 2.027398 \\
\hline $35 B$ & 1.227571 & 1.601988 & 1.381865 \\
\hline $36 B$ & -0.849729 & -1.830644 & 1.381863 \\
\hline $37 \mathrm{~B}$ & -2.429972 & 0.141192 & -0.043628 \\
\hline $38 \mathrm{~B}$ & -1.252323 & 2.087196 & -0.043626 \\
\hline $39 B$ & -1.356455 & 0.820877 & -1.857665 \\
\hline $40 B$ & 1.071695 & -2.187849 & 0.300998 \\
\hline 418 & 2.435349 & 0.065520 & 0.300999 \\
\hline
\end{tabular}
$\begin{array}{llll}41 B & 2.435349 & 0.065520 & 0.300999\end{array}$ \begin{tabular}{|llll}
$42 \mathrm{~B}$ & 1.081654 & -0.654574 & -2.132678
\end{tabular} $\begin{array}{llll}43 B & -0.726405 & 0.439593 & 2.394677\end{array}$

$E 44 / \varepsilon=-234.297422$

$\begin{array}{cccc}\text { atom } & x / \sigma & y / \sigma & z / \sigma \\ 1 \mathrm{~A} & 0.000000 & 0.000000 & 0.000000\end{array}$

$\begin{array}{llll}2 \mathrm{~A} & 1.073556 & 0.000000 & 0.000000\end{array}$

$\begin{array}{llll}3 \mathrm{~A} & 0.472550 & -0.971526 & 0.000000\end{array}$

$\begin{array}{llll}4 \mathrm{~A} & -0.469799 & -0.804174 & -0.591771\end{array}$

$\begin{array}{lllll}5 A & 0.492505 & 0.773822 & -0.576145\end{array}$

$\begin{array}{llll}6 \mathrm{~A} & -1.057677 & -0.042125 & -0.063024\end{array}$

$\begin{array}{lllll}7 A & -0.462572 & 0.962506 & -0.028301\end{array}$

$\begin{array}{lllll}8 \mathrm{~A} & 0.474496 & -0.304567 & -0.873322\end{array}$

$\begin{array}{llll}9 \mathrm{~A} & -0.478651 & -0.818354 & 0.514919\end{array}$

$\begin{array}{llll}10 \mathrm{~A} & -0.480633 & 0.285857 & 0.889120\end{array}$

$\begin{array}{llll}11 \mathrm{~A} & -0.451463 & 0.271808 & -0.930438\end{array}$

$\begin{array}{llll}12 \mathrm{~A} & 0.495848 & 0.785571 & 0.572327\end{array}$

$\begin{array}{llll}13 \mathrm{~A} & 0.476073 & -0.294306 & 0.896402\end{array}$

$\begin{array}{llll}14 \mathrm{~B} & -1.583872 & -0.280675 & -1.372072\end{array}$

$\begin{array}{llll}15 B & -0.475984 & 1.524982 & -1.364255\end{array}$

$\begin{array}{llll}16 \mathrm{~B} & -0.316096 & -2.093386 & -0.028375\end{array}$

$\begin{array}{llll}17 \mathrm{~B} & 1.726321 & 1.196043 & -0.064895\end{array}$

$\begin{array}{llll}18 \mathrm{~B} & -0.473499 & 1.617332 & 1.219948\end{array}$

$\begin{array}{llll}19 B & -1.686424 & -1.273364 & -0.109107\end{array}$

$\begin{array}{llll}20 \mathrm{~B} & 0.365017 & 2.062016 & -0.092263\end{array}$

$\begin{array}{llll}21 B & 0.503895 & -1.564411 & 1.274655\end{array}$

$\begin{array}{llll}22 \mathrm{~A} & 1.383977 & 0.255582 & -1.031170\end{array}$

$\begin{array}{lllll}23 \mathrm{~A} & 0.387895 & -1.376957 & -1.022195\end{array}$

$\begin{array}{lllll}24 \mathrm{~A} & 0.388018 & 0.439940 & -1.631435\end{array}$

$\begin{array}{llll}25 A & -0.227322 & -0.556533 & -1.637906\end{array}$

$\begin{array}{lllll}26 \mathrm{~A} & 1.352140 & -0.857043 & -0.664818\end{array}$

$\begin{array}{llll}27 A & -1.375239 & 0.803405 & 0.570764\end{array}$

$\begin{array}{llll}28 A & -1.395180 & -0.364205 & 0.905915\end{array}$

$\begin{array}{llll}29 B & 1.709723 & 0.197202 & 1.218928\end{array}$

$\begin{array}{llll}30 B & 0.330015 & 0.465010 & 2.021412\end{array}$

$\begin{array}{llll}31 \mathrm{~A} & -1.388750 & 0.827414 & -0.605314\end{array}$

$\begin{array}{llll}32 \mathrm{~B} & 1.090126 & -0.686808 & -2.016977\end{array}$

$\begin{array}{llll}33 \mathrm{~A} & -0.431207 & -0.555747 & 1.568115\end{array}$

$\begin{array}{llll}34 \mathrm{~A} & 1.435544 & -0.907298 & 0.486074\end{array}$

$\begin{array}{llll}35 \mathrm{~B} & -2.427244 & 0.166274 & 0.040391\end{array}$

$\begin{array}{llll}36 \mathrm{~B} & -1.343733 & 0.449314 & 1.964106\end{array}$

$\begin{array}{llll}37 \mathrm{~B} & 2.450674 & -0.250922 & -0.213945\end{array}$

$\begin{array}{llll}38 \mathrm{~B} & -0.856215 & -1.755222 & -1.530834\end{array}$

$\begin{array}{llll}39 B & 1.293391 & -2.103778 & -0.169162\end{array}$

$\begin{array}{llll}40 B & 1.158696 & 1.547326 & -1.540246\end{array}$

$\begin{array}{llll}41 \mathrm{~B} & -1.165552 & -1.673785 & 1.378137\end{array}$

$\begin{array}{llll}42 B & -1.349026 & 2.062303 & -0.062847\end{array}$

$\begin{array}{llll}\text { 43B } & 1.148874 & 1.752484 & 1.360174\end{array}$ $\begin{array}{llll}44 \mathrm{~B} & -0.620267 & 0.378577 & -2.536969\end{array}$

\section{$E 45 / \varepsilon=-240.654799$}

$$
\text { atom } \mathrm{x} / \sigma \quad \mathrm{l} / \sigma \quad \mathrm{z} / \sigma
$$

$\begin{array}{llll}1 \mathrm{~A} & 0.000000 & 0.000000 & 0.000000\end{array}$

$\begin{array}{llll}2 \mathrm{~A} & 1.079134 & 0.000000 & 0.000000\end{array}$

$\begin{array}{llll}3 \mathrm{~A} & 0.473022 & -0.969938 & 0.000000\end{array}$

$\begin{array}{llll}4 \mathrm{~A} & 0.509199 & 0.772326 & 0.589571\end{array}$

$\begin{array}{llll}5 A & -0.470976 & -0.796212 & 0.589570\end{array}$

$\begin{array}{lllll}6 \mathrm{~A} & 0.484561 & -0.302801 & 0.871092\end{array}$

$\begin{array}{llll}7 \mathrm{~A} & -0.441340 & 0.971637 & 0.046898\end{array}$

$\begin{array}{llll}8 \mathrm{~A} & -1.066773 & -0.029221 & 0.046897\end{array}$

$\begin{array}{lllll}9 A & -0.436180 & 0.272567 & 0.936463\end{array}$

$\begin{array}{lllll}10 A & -0.474978 & 0.296812 & -0.890266\end{array}$

$\begin{array}{llll}11 \mathrm{~A} & 0.508397 & 0.798090 & -0.524090\end{array}$

$\begin{array}{llll}12 \mathrm{~A} & -0.494484 & -0.806784 & -0.524090\end{array}$

$\begin{array}{llll}13 \mathrm{~A} & 0.488154 & -0.305046 & -0.891372\end{array}$

$\begin{array}{llll}14 \mathrm{~B} & -0.422619 & 1.528195 & 1.386723\end{array}$

$\begin{array}{llll}15 \mathrm{~B} & -1.558808 & -0.290007 & 1.386722\end{array}$

$\begin{array}{llll}16 \mathrm{~B} & 0.564918 & -1.600882 & -1.236769\end{array}$

$\begin{array}{llll}17 \mathrm{~B} & 1.686516 & 0.193968 & -1.236768\end{array}$

$\begin{array}{llll}18 B & -0.485911 & 1.600140 & -1.217207\end{array}$

$\begin{array}{llll}19 \mathrm{~B} & -1.651216 & -0.264655 & -1.217207\end{array}$

$\begin{array}{llll}20 \mathrm{~B} & -0.311718 & -2.086988 & 0.045543\end{array}$ $\begin{array}{llll}21 B & 1.739172 & 1.194976 & 0.045543\end{array}$
22B $-1.687834-1.253566$

$\begin{array}{lll}23 \mathrm{~B} & 0.386883 & 2.066527\end{array}$

0.087039

$\begin{array}{llll}4 \text { A } & 0.391138 & -1.370431 & 1.024977\end{array}$

$\begin{array}{llll}25 A & 1.403209 & 0.249148 & 1.024978\end{array}$

$\begin{array}{llll}26 \mathrm{~A} & 0.413567 & 0.434003 & 1.638579\end{array}$

$\begin{array}{llll}27 A & -0.208807 & -0.561958 & 1.638579\end{array}$

$\begin{array}{llll}28 \mathrm{~A} & 1.363139 & -0.851822 & 0.662570\end{array}$

$\begin{array}{llll}29 A & -0.332778 & -0.566633 & -1.566000\end{array}$

$\begin{array}{lllll}30 \mathrm{~A} & 0.363428 & 0.547481 & -1.566000\end{array}$

$\begin{array}{llll}31 \mathrm{~A} & -1.380913 & 0.862929 & -0.497280\end{array}$

$\begin{array}{lllll}32 \mathrm{~A} & -1.347372 & 0.841969 & 0.646692\end{array}$

$\begin{array}{llll}33 \mathrm{~B} & 1.110067 & -0.693678 & 2.012676\end{array}$

$\begin{array}{lllll}34 \mathrm{~A} & 1.449875 & -0.906023 & -0.473300\end{array}$

$\begin{array}{lllll}35 \mathrm{~B} & 2.462240 & -0.246998 & 0.198987\end{array}$

$\begin{array}{llll}36 \mathrm{~B} & 1.301291 & -2.104822 & 0.198987\end{array}$

$\begin{array}{llll}37 \mathrm{~B} & 1.206597 & 1.533281 & 1.537580\end{array}$

$\begin{array}{llll}38 \mathrm{~B} & -0.849239 & -1.756595 & 1.537579\end{array}$

$\begin{array}{llll}39 \mathrm{~B} & -2.454909 & 0.250052 & 0.113580\end{array}$

$\begin{array}{llll}40 B & -1.300823 & 2.096894 & 0.113581\end{array}$

$\begin{array}{llll}41 \mathrm{~B} & -0.729687 & 0.455980 & -2.365903\end{array}$

$\begin{array}{lllll}42 \mathrm{~B} & 1.117881 & 1.713444 & -1.395302\end{array}$

$\begin{array}{llll}43 \mathrm{~B} & -1.050057 & -1.755827 & -1.395302\end{array}$

$\begin{array}{lllll}44 \mathrm{~B} & 0.727260 & -0.454463 & -2.404789\end{array}$

$\begin{array}{llll}45 B & -0.593430 & 0.370832 & 2.544795\end{array}$

\section{$E 46 / \varepsilon=-247.137015$}

\section{$\begin{array}{lll}\text { atom } \quad x / \sigma & y / \sigma & z / \sigma\end{array}$}

$\begin{array}{llll}1 \mathrm{~A} & 0.000000 & 0.000000 & 0.000000\end{array}$

$\begin{array}{llll}2 \mathrm{~A} & 1.174039 & 0.000000 & 0.000000\end{array}$

$\begin{array}{llll}3 \mathrm{~A} & 0.500979 & -1.006225 & 0.000000\end{array}$

$\begin{array}{llll}4 \mathrm{~A} & -0.509323 & 1.010035 & 0.000000\end{array}$

$\begin{array}{llll}5 A & 0.551231 & -0.318951 & -0.849854\end{array}$

$\begin{array}{llll}6 \mathrm{~A} & 0.551231 & -0.318952 & 0.849853\end{array}$

$\begin{array}{llll}7 \mathrm{~A} & 0.574923 & 0.888081 & 0.000000\end{array}$

$\begin{array}{lllll}8 \mathrm{~A} & 0.016317 & 0.625478 & -0.896836\end{array}$

$\begin{array}{llll}9 \mathrm{~A} & 0.016318 & 0.625478 & 0.896837\end{array}$

$\begin{array}{llll}10 \mathrm{~B} & -0.421254 & -0.267387 & -1.817091\end{array}$

$\begin{array}{llll}11 B & -0.421253 & -0.267388 & 1.817091\end{array}$

$\begin{array}{llll}12 \mathrm{~A} & -0.415004 & -0.823761 & -0.566507\end{array}$

$\begin{array}{llll}13 A & -0.415003 & -0.823762 & 0.566507\end{array}$

$\begin{array}{llll}14 A & -0.912190 & 0.143950 & -0.567007\end{array}$

$\begin{array}{llll}15 A & -0.912190 & 0.143949 & 0.567007\end{array}$

$\begin{array}{llll}16 \mathrm{~B} & -1.661588 & -0.858099 & 0.000000\end{array}$

$\begin{array}{llll}17 \mathrm{~B} & 0.458384 & -1.586365 & 1.309322\end{array}$

$\begin{array}{lllll}18 \mathrm{~B} & 0.458383 & -1.586364 & -1.309323\end{array}$

$\begin{array}{llll}19 \mathrm{~B} & 1.811499 & 1.385841 & -0.000001\end{array}$

$\begin{array}{llll}20 \mathrm{~A} & 1.091630 & 0.628768 & 0.929291\end{array}$

$\begin{array}{llll}21 \mathrm{~A} & 1.091629 & 0.628769 & -0.929292\end{array}$

$\begin{array}{llll}22 \mathrm{~A} & 1.430443 & -0.903990 & 0.560942\end{array}$

$\begin{array}{lllll}23 \mathrm{~A} & 1.430442 & -0.903990 & -0.560942\end{array}$

$\begin{array}{llll}24 \mathrm{~B} & 0.441383 & 1.935463 & -0.817401\end{array}$

$\begin{array}{llll}25 \mathrm{~B} & 0.441384 & 1.935462 & 0.817401\end{array}$

$\begin{array}{llll}26 \mathrm{~B} & 2.339793 & 0.061951 & -0.819180\end{array}$

$\begin{array}{llll}27 \mathrm{~B} & 2.339794 & 0.061951 & 0.819177\end{array}$

$\begin{array}{llll}28 \mathrm{~B} & -0.415209 & -2.070790 & -0.000001\end{array}$

$\begin{array}{llll}29 B & -1.926691 & 0.855626 & 0.000001\end{array}$

$\begin{array}{llll}30 \mathrm{~B} & -1.052405 & 1.294833 & -1.329618\end{array}$

$\begin{array}{llll}31 \mathrm{~B} & -1.052404 & 1.294832 & 1.329619\end{array}$

$\begin{array}{lllll}32 \mathrm{~B} & 1.273362 & -2.158372 & -0.000001\end{array}$

$\begin{array}{llll}33 \mathrm{~B} & 1.265512 & -0.275161 & 1.980094\end{array}$

$\begin{array}{lllll}34 \mathrm{~B} & 1.265509 & -0.275159 & -1.980097\end{array}$

$\begin{array}{llll}35 \mathrm{~B} & 0.379123 & 1.080644 & 2.174140\end{array}$

$\begin{array}{llll}36 \mathrm{~B} & 0.379120 & 1.080646 & -2.174141\end{array}$

$\begin{array}{lllll}37 \mathrm{~B} & 2.669580 & -1.308984 & -0.000002\end{array}$

$\begin{array}{llll}38 \mathrm{~B} & -0.982012 & 2.282486 & 0.000001\end{array}$

$\begin{array}{llll}39 \mathrm{~B} & -1.993678 & -0.083285 & 1.398661\end{array}$

$\begin{array}{llll}40 \mathrm{~B} & -1.993679 & -0.083283 & -1.398660\end{array}$

$\begin{array}{llll}41 \mathrm{~B} & -1.222245 & -1.616410 & 1.381112\end{array}$

$\begin{array}{llll}42 \mathrm{~B} & -1.222246 & -1.616408 & -1.381112\end{array}$

$\begin{array}{llll}43 \mathrm{~B} & 1.897876 & 1.429259 & -1.664827\end{array}$

$\begin{array}{llll}44 \mathrm{~B} & 1.897877 & 1.429261 & 1.664823\end{array}$

$\begin{array}{llll}45 \mathrm{~B} & 2.101907 & -1.621868 & -1.499297\end{array}$

$\begin{array}{llll}46 B & 2.101909 & -1.621871 & 1.499293\end{array}$ 
$E 47 / \varepsilon=-253.365117$

atom $x / \sigma$

$\begin{array}{llll}1 \mathrm{~A} & 0.000000 & 0.000000 & 0.000000\end{array}$

2A $\quad 1.108782 \quad 0.000000 \quad 0.000000$

$\begin{array}{llll}3 \mathrm{~A} & 0.513684 & -0.962192 & 0.000000\end{array}$

$\begin{array}{llll}4 \mathrm{~A} & -0.438103 & 0.268841 & -0.973515\end{array}$

$\begin{array}{llll}5 A & -0.439590 & -0.791365 & -0.605676\end{array}$

$\begin{array}{llll}6 \mathrm{~A} & 0.487419 & -0.307372 & -0.848446\end{array}$

$\begin{array}{lllll}7 \mathrm{~A} & 0.505358 & 0.737613 & -0.586085\end{array}$

$\begin{array}{llll}8 \mathrm{~A} & 0.509170 & 0.770619 & 0.534051\end{array}$

$\begin{array}{lllll}9 A & -0.481721 & 0.946139 & -0.097353\end{array}$

$\begin{array}{llll}10 A & 0.522000 & -0.295014 & 0.894900\end{array}$

$\begin{array}{llll}11 \mathrm{~A} & -1.080762 & -0.028238 & -0.070765\end{array}$

$\begin{array}{llll}12 \mathrm{~A} & -0.468328 & -0.791165 & 0.513442\end{array}$

$\begin{array}{llll}13 \mathrm{~A} & -0.498845 & 0.299086 & 0.866484\end{array}$

$\begin{array}{llll}14 \mathrm{~A} & 0.459143 & -1.338323 & -1.088933\end{array}$

$\begin{array}{llll}15 A & -0.152702 & -0.574022 & -1.663620\end{array}$

$\begin{array}{lllll}16 \mathrm{~A} & 1.394948 & -0.849366 & -0.676638\end{array}$

$\begin{array}{lllll}17 A & 0.442585 & 0.398280 & -1.636662\end{array}$

$\begin{array}{lllll}18 \mathrm{~A} & 1.379062 & 0.231746 & -1.043471\end{array}$

$\begin{array}{lllll}19 B & 1.642896 & 1.321126 & -0.221637\end{array}$

$\begin{array}{lllll}20 A & 0.997654 & -0.575702 & -1.724139\end{array}$

21B $\quad-0.112152 \quad 1.635159-1.345996$

$\begin{array}{llll}22 \mathrm{~B} & 1.683550 & -1.080338 & 0.678408\end{array}$

$\begin{array}{llll}23 \mathrm{~B} & -1.522384 & -0.506556 & -1.373221\end{array}$

$\begin{array}{llll}24 \mathrm{~B} & 1.688435 & 0.463844 & 1.162852\end{array}$

$\begin{array}{llll}25 B & -1.569119 & 1.045103 & -0.924805\end{array}$

$\begin{array}{llll}26 \mathrm{~B} & 0.244523 & -1.744664 & 1.124962\end{array}$

$\begin{array}{llll}27 \mathrm{~B} & 0.227049 & 0.705414 & 1.912372\end{array}$

$\begin{array}{llll}28 \mathrm{~B} & -1.526482 & -1.445156 & -0.039533\end{array}$

$\begin{array}{llll}29 \mathrm{~B} & -1.611282 & 1.062806 & 0.717909\end{array}$

$\begin{array}{lllll}30 \mathrm{~A} & 0.332073 & 1.683375 & -0.047289\end{array}$

$\begin{array}{llll}31 \mathrm{~A} & -0.298427 & 1.426361 & 0.865204\end{array}$

$\begin{array}{lllll}32 \mathrm{~B} & 2.482634 & -0.080717 & -0.316798\end{array}$

$\begin{array}{llll}33 \mathrm{~B} & -0.704033 & 0.488204 & -2.352456\end{array}$

34B $\quad-0.714151 \quad-1.912062-1.419116$

$\begin{array}{lllll}35 \mathrm{~B} & 1.058391 & -2.230789 & -0.271465\end{array}$

$\begin{array}{llll}36 \mathrm{~B} & -1.579006 & -0.466550 & 1.255176\end{array}$

$\begin{array}{llll}37 \mathrm{~A} & -0.207751 & -1.750444 & -0.163019\end{array}$

$\begin{array}{llll}38 \mathrm{~B} & 1.424460 & 1.308779 & -1.862524\end{array}$

$\begin{array}{llll}39 \mathrm{~A} & -0.269643 & -0.543243 & 1.590517\end{array}$

$\begin{array}{llll}40 \mathrm{~B} & 0.844593 & 0.103547 & -2.891950\end{array}$

$\begin{array}{lllll}41 \mathrm{~B} & 2.263833 & -0.157721 & -1.985540\end{array}$

42B $\quad 1.745121 \quad-1.712057-1.664302$

43B $\quad 0.374353 \quad-1.458332 \quad-2.550153$

$\begin{array}{llll}44 \mathrm{~B} & 0.944979 & 1.967296 & 1.136053\end{array}$

$\begin{array}{llll}45 \mathrm{~B} & -0.874772 & 2.306525 & -0.043716\end{array}$

$\begin{array}{llll}46 \mathrm{~B} & 1.011040 & -0.708532 & 2.140211\end{array}$

$\begin{array}{llll}47 \mathrm{~B} & -2.473718 & -0.083353 & -0.096142\end{array}$

$E 48 / \varepsilon=-260.451080$

atom $\mathrm{x} / \sigma \quad \mathrm{y} / \sigma$

$\begin{array}{llll}1 \mathrm{~A} & 0.000000 & 0.000000 & 0.000000\end{array}$

2A $\quad 1.095930 \quad 0.000000 \quad 0.000000$

$\begin{array}{llll}3 \mathrm{~A} & 0.541814 & -0.950976 & 0.000000\end{array}$

$\begin{array}{lllll}4 \mathrm{~A} & 0.550579 & 0.988193 & -0.002180\end{array}$

$\begin{array}{llll}5 A & -0.587370 & -0.959479 & -0.000991\end{array}$

$\begin{array}{llll}6 \mathrm{~A} & 1.667833 & -0.970621 & 0.001045\end{array}$

$\begin{array}{llll}7 \mathrm{~A} & 0.547240 & 0.311124 & -0.866425\end{array}$

$\begin{array}{lllll}8 \mathrm{~A} & 0.001703 & -0.627354 & -0.867906\end{array}$

$\begin{array}{llll}9 \mathrm{~A} & 0.546983 & 0.312332 & 0.866065\end{array}$

$\begin{array}{llll}10 \mathrm{~A} & -0.000544 & -0.626686 & 0.866244\end{array}$

$\begin{array}{llll}11 \mathrm{~A} & 1.085569 & -0.630593 & -0.867080\end{array}$

$\begin{array}{llll}12 \mathrm{~A} & 1.083844 & -0.629928 & 0.867878\end{array}$

$\begin{array}{lllll}13 \mathrm{~A} & -0.368008 & 0.840832 & -0.579012\end{array}$

$\begin{array}{llll}14 \mathrm{~A} & -0.909562 & -0.091862 & -0.583907\end{array}$

$\begin{array}{llll}15 \mathrm{~B} & -0.338887 & 0.201628 & -1.861733\end{array}$

$\begin{array}{llll}16 \mathrm{~A} & -0.365096 & 0.842623 & 0.573434\end{array}$

$\begin{array}{llll}17 \mathrm{~B} & -1.613398 & 0.929838 & 0.005119\end{array}$

$\begin{array}{llll}18 \mathrm{~A} & -0.918016 & -0.089072 & 0.585443\end{array}$

$\begin{array}{llll}19 \mathrm{~B} & -0.350464 & 0.203814 & 1.854563\end{array}$

20B $\quad 0.552060 \quad 1.565106-1.330520$ $\begin{array}{llll}21 \mathrm{~A} & 1.468351 & 0.841238 & -0.584991\end{array}$ 22B $\quad-1.084451 \quad-1.251160 \quad-1.335191$ $\begin{array}{llll}23 \mathrm{~A} & 1.467753 & 0.842481 & 0.582551\end{array}$ $\begin{array}{llll}24 \mathrm{~A} & -0.007374 & -1.688430 & -0.583898\end{array}$ $\begin{array}{llll}25 A & -0.010114 & -1.688140 & 0.583696\end{array}$ $\begin{array}{llll}26 \mathrm{~B} & 0.552958 & 1.568149 & 1.325923\end{array}$ $\begin{array}{llll}27 \mathrm{~A} & 2.009058 & -0.105268 & -0.584149\end{array}$ $\begin{array}{llll}28 A & 2.008096 & -0.104489 & 0.585418\end{array}$ $\begin{array}{llll}29 A & 1.082854 & -1.693458 & -0.584577\end{array}$ $\begin{array}{llll}30 \mathrm{~A} & 1.081040 & -1.693144 & 0.585569\end{array}$ $\begin{array}{llll}31 \mathrm{~B} & -0.312754 & 2.079809 & -0.003284\end{array}$ $\begin{array}{llll}32 \mathrm{~B} & 1.453939 & 0.206112 & -1.849106\end{array}$ $\begin{array}{llll}33 \mathrm{~B} & 0.540916 & -1.364641 & -1.849653\end{array}$ $\begin{array}{llll}34 \mathrm{~B} & 1.453164 & 0.208695 & 1.848611\end{array}$ $\begin{array}{llll}35 \mathrm{~B} & 0.535741 & -1.365020 & 1.848475\end{array}$ $\begin{array}{llll}36 \mathrm{~B} & -1.096573 & -1.242515 & 1.325765\end{array}$ $\begin{array}{lllll}37 \mathrm{~B} & -1.959964 & -0.752377 & -0.011367\end{array}$ $\begin{array}{llll}38 \mathrm{~B} & 2.170490 & -1.263104 & -1.329793\end{array}$ $\begin{array}{llll}39 \mathrm{~B} & 2.167740 & -1.262276 & 1.332494\end{array}$ $\begin{array}{llll}40 \mathrm{~B} & 1.400237 & 2.081105 & -0.003081\end{array}$ $\begin{array}{llll}41 \mathrm{~B} & -1.118959 & -2.240088 & -0.003078\end{array}$ $\begin{array}{llll}42 \mathrm{~B} & 2.183265 & -2.259836 & 0.001887\end{array}$ $\begin{array}{llll}43 B & 3.043995 & -0.784554 & 0.002038\end{array}$ $\begin{array}{llll}44 \mathrm{~B} & 2.704053 & 0.921654 & -0.000090\end{array}$ $\begin{array}{llll}45 \mathrm{~B} & 0.530777 & -2.803567 & 0.000521\end{array}$ $\begin{array}{llll}46 \mathrm{~B} & -1.110910 & 1.616131 & -1.419025\end{array}$ $\begin{array}{llll}47 \mathrm{~B} & -1.949442 & 0.168697 & -1.430035\end{array}$ $\begin{array}{llll}48 \mathrm{~B} & -1.110817 & 1.616246 & 1.417370\end{array}$

\section{$E 49 / \varepsilon=-267.906522$}

$\begin{array}{cccc}\text { atom } & \mathrm{X} / \sigma & y / \sigma & z / \sigma \\ 1 \mathrm{~A} & 0.000000 & 0.000000 & 0.000000\end{array}$

$\begin{array}{llll}2 \mathrm{~A} & 1.094017 & 0.000000 & 0.000000\end{array}$

$\begin{array}{llll}3 \mathrm{~A} & 0.539861 & -0.951537 & 0.000000\end{array}$

$\begin{array}{llll}4 \mathrm{~A} & -0.589381 & -0.962357 & 0.000000\end{array}$

$\begin{array}{llll}5 \mathrm{~A} & 0.546184 & 0.987513 & 0.000000\end{array}$

$\begin{array}{llll}6 \mathrm{~A} & 1.665823 & -0.970141 & 0.000001\end{array}$

$\begin{array}{llll}7 \mathrm{~A} & -0.001952 & -0.627536 & -0.866554\end{array}$

$\begin{array}{llll}8 A & 0.544845 & 0.311366 & -0.866555\end{array}$

$\begin{array}{llll}9 A & -0.001953 & -0.627536 & 0.866554\end{array}$

$\begin{array}{llll}10 \mathrm{~A} & 0.544845 & 0.311366 & 0.866554\end{array}$

$\begin{array}{llll}11 \mathrm{~A} & 1.082297 & -0.630308 & 0.867467\end{array}$

$\begin{array}{llll}12 \mathrm{~A} & 1.082298 & -0.630308 & -0.867466\end{array}$

$\begin{array}{llll}13 \mathrm{~B} & -1.611266 & 0.938368 & -0.000001\end{array}$

$\begin{array}{llll}14 \mathrm{~A} & -0.913184 & -0.092337 & 0.578600\end{array}$

$\begin{array}{llll}15 A & -0.370314 & 0.839820 & -0.578601\end{array}$

$\begin{array}{llll}16 \mathrm{~A} & -0.370315 & 0.839820 & 0.578600\end{array}$

$\begin{array}{llll}17 A & -0.913183 & -0.092337 & -0.578601\end{array}$

$\begin{array}{llll}18 \mathrm{~B} & -0.344737 & 0.200767 & 1.859063\end{array}$

$\begin{array}{llll}19 B & -0.344736 & 0.200767 & -1.859064\end{array}$

$\begin{array}{llll}\text { 20B } & 0.548957 & 1.565920 & 1.328623\end{array}$

21B $\quad-1.091089-1.250194 \quad-1.328623$

$\begin{array}{llll}22 \mathrm{~B} & -1.091090 & -1.250193 & 1.328623\end{array}$

$\begin{array}{llll}23 \mathrm{~B} & 0.548958 & 1.565920 & -1.328623\end{array}$

$\begin{array}{llll}24 \mathrm{~A} & -0.009898 & -1.689535 & 0.583590\end{array}$

$\begin{array}{llll}25 \mathrm{~A} & -0.009898 & -1.689536 & -0.583590\end{array}$

$\begin{array}{llll}26 \mathrm{~A} & 1.464613 & 0.842339 & 0.583590\end{array}$

$\begin{array}{llll}27 \mathrm{~A} & 1.464614 & 0.842339 & -0.583590\end{array}$

$\begin{array}{llll}28 \mathrm{~B} & -1.963316 & -0.748895 & -0.000001\end{array}$

$\begin{array}{llll}29 B & -0.317469 & 2.077177 & -0.000001\end{array}$

$\begin{array}{llll}30 \mathrm{~A} & 1.080522 & -1.693375 & 0.585173\end{array}$

$\begin{array}{llll}31 \mathrm{~A} & 1.080523 & -1.693375 & -0.585171\end{array}$

$\begin{array}{llll}32 \mathrm{~A} & 2.006039 & -0.104176 & 0.585173\end{array}$

$\begin{array}{llll}33 \mathrm{~A} & 2.006040 & -0.104176 & -0.585171\end{array}$

$\begin{array}{llll}34 \mathrm{~B} & 0.535519 & -1.364208 & -1.849153\end{array}$

$\begin{array}{llll}35 B & 1.450800 & 0.207415 & -1.849153\end{array}$

$\begin{array}{llll}36 \mathrm{~B} & 0.535517 & -1.364207 & 1.849154\end{array}$

$\begin{array}{llll}37 \mathrm{~B} & 1.450798 & 0.207416 & 1.849155\end{array}$

$\begin{array}{llll}38 B & 2.166993 & -1.262013 & 1.331345\end{array}$

$\begin{array}{llll}39 \mathrm{~B} & 2.166995 & -1.262014 & -1.331341\end{array}$

$\begin{array}{llll}40 B & 1.394985 & 2.081389 & 0.000000\end{array}$

$\begin{array}{llll}41 \mathrm{~B} & -1.121940 & -2.240405 & 0.000000\end{array}$

$\begin{array}{llll}42 B & 3.041643 & -0.783203 & 0.000002\end{array}$ \begin{tabular}{|llll}
$43 B$ & 2.182153 & -2.259029 & 0.000002
\end{tabular}

$\begin{array}{llll}44 \mathrm{~B} & 2.700517 & 0.922842 & 0.000001\end{array}$

$\begin{array}{llll}45 \mathrm{~B} & 0.529961 & -2.804206 & 0.000001\end{array}$

$\begin{array}{lllll}46 \mathrm{~B} & -1.111540 & 1.616970 & -1.421920\end{array}$

$\begin{array}{lllll}47 \mathrm{~B} & -1.954891 & 0.168858 & -1.421921\end{array}$

$\begin{array}{llll}48 \mathrm{~B} & -1.954892 & 0.168858 & 1.421919\end{array}$

$\begin{array}{llll}49 \mathrm{~B} & -1.111542 & 1.616970 & 1.421919\end{array}$

$E 50 / \varepsilon=-274.312888$

$\begin{array}{llll}1 \mathrm{~A} & 0.000000 & 0.000000 & 0.000000\end{array}$

$\begin{array}{llll}2 \mathrm{~A} & 1.093271 & 0.000000 & 0.000000\end{array}$

$\begin{array}{llll}3 \mathrm{~A} & 0.542471 & -0.950805 & 0.000000\end{array}$

$\begin{array}{lllll}4 \mathrm{~A} & 0.542216 & 0.989942 & -0.001169\end{array}$

$\begin{array}{llll}5 A & -0.586131 & -0.964094 & 0.001154\end{array}$

$\begin{array}{lllll}6 \mathrm{~A} & 1.669753 & -0.963572 & -0.003390\end{array}$

$\begin{array}{lllll}7 A & 0.543167 & 0.312010 & -0.866595\end{array}$

$\begin{array}{llll}8 \mathrm{~A} & -0.000602 & -0.628054 & -0.866292\end{array}$

$\begin{array}{llll}9 \mathrm{~A} & 0.545060 & 0.313583 & 0.865457\end{array}$

$\begin{array}{llll}10 A & 0.000900 & -0.626908 & 0.867077\end{array}$

$\begin{array}{lllll}11 \mathrm{~A} & 1.083360 & -0.627805 & -0.867591\end{array}$

$\begin{array}{llll}12 \mathrm{~A} & 1.084664 & -0.628280 & 0.867495\end{array}$

$\begin{array}{lllll}13 \mathrm{~B} & -1.612862 & 0.935001 & -0.000162\end{array}$

$\begin{array}{lllll}14 \mathrm{~A} & -0.373044 & 0.838208 & -0.579735\end{array}$

$\begin{array}{llll}15 A & -0.371944 & 0.839959 & 0.577665\end{array}$

$\begin{array}{llll}16 A & -0.913055 & -0.095372 & -0.577981\end{array}$

$\begin{array}{llll}17 \mathrm{~A} & -0.911949 & -0.094028 & 0.579126\end{array}$

$\begin{array}{llll}18 \mathrm{~B} & -0.346164 & 0.198405 & -1.859334\end{array}$

$\begin{array}{llll}19 B & -0.342602 & 0.202986 & 1.858549\end{array}$

$\begin{array}{llll}20 B & 0.542581 & 1.563374 & -1.332876\end{array}$

$\begin{array}{lllll}21 \mathrm{~A} & 1.456998 & 0.839439 & -0.576586\end{array}$

$\begin{array}{llll}22 \mathrm{~B} & 1.430809 & 0.196828 & -1.862073\end{array}$

$\begin{array}{lllll}23 \mathrm{~A} & 2.006374 & -0.093132 & -0.593285\end{array}$

$\begin{array}{llll}24 \mathrm{~A} & 1.462001 & 0.846577 & 0.579492\end{array}$

$\begin{array}{llll}25 \mathrm{~B} & 0.549229 & 1.571034 & 1.321512\end{array}$

$\begin{array}{llll}26 \mathrm{~B} & -1.088388 & -1.253979 & -1.326984\end{array}$

$\begin{array}{llll}27 \mathrm{~B} & -1.086092 & -1.251571 & 1.330310\end{array}$

$\begin{array}{llll}28 B & -0.319372 & 2.077339 & -0.004950\end{array}$

$\begin{array}{llll}29 A & -0.005082 & -1.689982 & -0.583219\end{array}$

$\begin{array}{llll}30 \mathrm{~A} & -0.003554 & -1.689520 & 0.584787\end{array}$

$\begin{array}{llll}31 \mathrm{~A} & 2.004868 & -0.099631 & 0.583872\end{array}$

$\begin{array}{lllll}32 \mathrm{~A} & 1.085778 & -1.690021 & -0.586719\end{array}$

$\begin{array}{llll}33 \mathrm{~B} & -1.960794 & -0.753748 & 0.002203\end{array}$

$\begin{array}{lllll}34 \mathrm{~A} & 1.087818 & -1.690092 & 0.583095\end{array}$

$\begin{array}{lllll}35 \mathrm{~B} & 0.537764 & -1.363123 & -1.849453\end{array}$

$\begin{array}{llll}36 \mathrm{~B} & 1.453374 & 0.213957 & 1.845863\end{array}$

$\begin{array}{llll}37 \mathrm{~B} & 0.541581 & -1.363427 & 1.848893\end{array}$

$\begin{array}{lllll}38 \mathrm{~B} & 2.169820 & -1.252072 & -1.335829\end{array}$

$\begin{array}{llll}39 B & 1.396582 & 2.082806 & -0.012564\end{array}$

$\begin{array}{llll}\text { 40B } & 2.173720 & -1.255361 & 1.327804\end{array}$

$\begin{array}{llll}41 \mathrm{~B} & 2.702271 & 0.924593 & 0.016286\end{array}$

$\begin{array}{llll}42 \mathrm{~B} & -1.114807 & -2.243317 & 0.002524\end{array}$

$\begin{array}{lllll}43 \mathrm{~B} & 3.043337 & -0.769571 & -0.006537\end{array}$

$\begin{array}{lllll}44 \mathrm{~B} & 2.191272 & -2.250067 & -0.004881\end{array}$

$\begin{array}{lllll}45 \mathrm{~B} & 0.539481 & -2.802337 & -0.000235\end{array}$

$\begin{array}{lllll}46 \mathrm{~B} & -1.116239 & 1.613113 & -1.423598\end{array}$

$\begin{array}{lllll}47 \mathrm{~B} & -1.955696 & 0.162870 & -1.420803\end{array}$

$\begin{array}{llll}48 \mathrm{~B} & -1.111895 & 1.617929 & 1.419681\end{array}$

$\begin{array}{llll}49 B & -1.952599 & 0.166878 & 1.423172\end{array}$

$\begin{array}{llll}50 B & 2.212557 & 1.595409 & -1.419669\end{array}$

$s=1.6$

$E 5 / \varepsilon=-9.201428$

$\begin{array}{llll}\text { atom } & \mathrm{X} / \sigma & \mathrm{y} / \sigma & \mathrm{z} / \sigma\end{array}$

$\begin{array}{llll}1 \mathrm{~A} & 0.000000 & 0.000000 & 0.000000\end{array}$

$\begin{array}{llll}2 \mathrm{~A} & 1.123972 & 0.000000 & 0.000000\end{array}$

$\begin{array}{llll}3 \mathrm{~A} & 0.561986 & -0.973388 & 0.000000\end{array}$

$\begin{array}{llll}4 \mathrm{~B} & 0.561986 & -0.324462 & 1.301696\end{array}$

$\begin{array}{llll}5 B & 0.561986 & -0.324462 & -1.301696\end{array}$

$E 6 / \varepsilon=-13.138655$ 


$\begin{array}{cccc}\text { atom } & x / \sigma & y / \sigma & z / \sigma \\ \text { 1A } & 0.000000 & 0.000000 & 0.000000 \\ \text { 2A } & 1.161602 & 0.000000 & 0.000000 \\ \text { 3B } & 0.580800 & -1.312441 & 0.000000 \\ \text { 4B } & 0.580800 & 0.000000 & 1.312441 \\ \text { 5B } & 0.580800 & 0.000000 & -1.312441 \\ \text { 6B } & 0.580800 & 1.312441 & 0.000000\end{array}$

$E 7 / \varepsilon=-16.634759$

$\begin{array}{cccc}\text { atom } & x / \sigma & y / \sigma & z / \sigma \\ \text { 1A } & 0.000000 & 0.000000 & 0.000000 \\ \text { 2B } & 1.462464 & 0.000000 & 0.000000 \\ \text { 3B } & 0.351120 & -1.413250 & 0.000000 \\ \text { 4B } & 0.354329 & 1.096111 & -0.891534 \\ \text { 5B } & 0.354329 & 1.096111 & 0.891534 \\ \text { 6A } & 0.435898 & -0.346080 & 0.973719 \\ \text { 7A } & 0.435898 & -0.346080 & -0.973719\end{array}$

$E 8 / \varepsilon=-20.902561$

$\begin{array}{ccrc}\text { atom } & x / \sigma & y / \sigma & z / \sigma \\ \text { 1A } & 0.000000 & 0.000000 & 0.000000 \\ \text { 2B } & 1.422607 & 0.000000 & 0.000000 \\ \text { 3B } & 0.210330 & -1.439661 & 0.000000 \\ \text { 4B } & 0.210329 & 0.964521 & 1.068795 \\ \text { 5A } & 0.454391 & -0.431815 & 0.971339 \\ \text { 6B } & -0.989290 & -0.415740 & 0.935180 \\ \text { 7B } & 0.313550 & -0.351685 & -1.382000 \\ \text { 8B } & 0.313550 & 1.261604 & -0.664802\end{array}$

$E 9 / \varepsilon=-26.148194$

\begin{tabular}{crrr} 
atom & $x / \sigma$ & $y / \sigma$ & \multicolumn{1}{c}{$z / \sigma$} \\
1A & 0.000000 & 0.000000 & 0.000000 \\
2B & 1.411875 & 0.000000 & 0.000000 \\
3B & 0.113788 & -1.407282 & 0.000000 \\
4B & -1.043285 & -0.295742 & 0.906118 \\
5B & 0.210696 & 1.063726 & -0.906118 \\
6B & 0.210696 & 1.063726 & 0.906118 \\
7B & -1.043285 & -0.295741 & -0.906118 \\
8A & 0.405251 & -0.373806 & 0.974336 \\
9A & 0.405251 & -0.373806 & -0.974336
\end{tabular}

$E 10 / \varepsilon=-31.451692$

$\begin{array}{crrc}\text { atom } & x / \sigma & y / \sigma & z / \sigma \\ \text { 1A } & 0.000000 & 0.000000 & 0.000000 \\ \text { 2B } & 1.418215 & 0.000000 & 0.000000 \\ \text { 3B } & 0.152584 & -1.409983 & 0.000000 \\ \text { 4B } & 0.240116 & 1.057487 & 0.914001 \\ \text { 5B } & -1.025516 & -0.352496 & 0.914001 \\ \text { 6B } & 0.240117 & 1.057487 & -0.914002 \\ \text { 7B } & -1.025515 & -0.352497 & -0.914001 \\ \text { 8A } & -0.835835 & 0.750264 & -0.000001 \\ \text { 9A } & 0.417918 & -0.375132 & -0.972696 \\ 10 A & 0.417917 & -0.375132 & 0.972696\end{array}$

$E 11 / \varepsilon=-35.603591$

$\begin{array}{cccc}\text { atom } & \mathrm{x} / \sigma & y / \sigma & z / \sigma \\ & 0.0000 & 0.00000 & 0.000000\end{array}$ $\begin{array}{llll}2 \mathrm{~B} & 1.457006 & 0.000000 & 0.000000\end{array}$ $\begin{array}{llll}3 B & -0.989940 & -1.064991 & 0.000000\end{array}$ $\begin{array}{llll}4 \mathrm{~B} & -0.989941 & 0.380077 & -0.994858\end{array}$ $\begin{array}{lllll}5 A & 0.423851 & 0.005391 & -1.007602\end{array}$ $\begin{array}{lllll}6 \mathrm{~A} & 0.423851 & -0.943174 & -0.354561\end{array}$ $\begin{array}{llll}7 \mathrm{~B} & 0.328819 & -0.817361 & 1.125619\end{array}$ $\begin{array}{lllll}8 \mathrm{~B} & 0.328820 & 1.343198 & -0.361822\end{array}$ $\begin{array}{llll}9 B & -0.999143 & 0.557312 & 0.809518\end{array}$ $\begin{array}{llll}10 A & 0.444109 & 0.587216 & 0.852952\end{array}$ 11B $\quad-0.243561 \quad-1.113469-1.617352$ $E 12 / \varepsilon=-40.269325$

atom $\quad \mathrm{x} / \sigma \quad \mathrm{y} / \sigma \quad z / \sigma$ $\begin{array}{llll}1 \mathrm{~A} & 0.000000 & 0.000000 & 0.000000\end{array}$ $\begin{array}{llll}2 B & 1.428326 & 0.000000 & 0.000000\end{array}$ $\begin{array}{llll}3 B & -1.053626 & -1.006925 & 0.000000\end{array}$ \begin{tabular}{|llll}
$4 \mathrm{~B}$ & -1.053626 & 0.619471 & -0.793825
\end{tabular} $\begin{array}{lllll}5 A & 0.407656 & -0.992294 & -0.290954\end{array}$ $\begin{array}{lllll}6 \mathrm{~A} & 0.407656 & 0.381091 & -0.961287\end{array}$ $\begin{array}{llll}7 \mathrm{~B} & 0.288479 & -0.913985 & 1.130890\end{array}$ $\begin{array}{lllll}8 \mathrm{~B} & 0.288480 & 1.453846 & -0.024820\end{array}$ $\begin{array}{llll}9 \mathrm{~A} & -0.230310 & -0.506631 & -1.037992\end{array}$ $\begin{array}{llll}10 \mathrm{~B} & -0.933819 & 0.477277 & 0.977846\end{array}$ $\begin{array}{llll}11 \mathrm{~A} & 0.491027 & 0.470707 & 0.964387\end{array}$ $\begin{array}{llll}12 B & 1.077587 & -0.760542 & -1.558207\end{array}$

$E 13 / \varepsilon=-45.438215$

$\begin{array}{cccc}\text { atom } & \mathrm{x} / \sigma & y / \sigma & z / \sigma \\ 1 \mathrm{~A} & 0.000000 & 0.000000 & 0.000000\end{array}$

$\begin{array}{llll}2 B & 1.482393 & 0.000000 & 0.000000\end{array}$

$\begin{array}{llll}3 \mathrm{~B} & 0.396676 & -1.428334 & 0.000000\end{array}$

$\begin{array}{llll}4 \mathrm{~A} & -0.470450 & 1.057273 & 0.000000\end{array}$

$\begin{array}{llll}5 A & -1.144606 & 0.170375 & 0.000000\end{array}$

$\begin{array}{llll}6 \mathrm{~B} & 0.494120 & -0.375595 & 1.398251\end{array}$

$\begin{array}{llll}7 \mathrm{~B} & 0.494120 & -0.375595 & -1.398251\end{array}$

$\begin{array}{llll}8 \mathrm{~A} & -0.551530 & 0.419233 & 0.891251\end{array}$

$\begin{array}{lllll}9 A & -0.551530 & 0.419233 & -0.891251\end{array}$

$\begin{array}{llll}10 A & -0.679210 & -0.655097 & -0.570594\end{array}$

$\begin{array}{llll}11 \mathrm{~A} & -0.679210 & -0.655097 & 0.570593\end{array}$

$\begin{array}{lllll}12 \mathrm{~A} & 0.449455 & 0.829740 & -0.570593\end{array}$

$\begin{array}{llll}13 A & 0.449455 & 0.829740 & 0.570593\end{array}$

$E 14 / \varepsilon=-51.095901$

$\begin{array}{llll}1 \mathrm{~A} & 0.000000 & 0.000000 & 0.000000\end{array}$

$\begin{array}{llll}2 \mathrm{~A} & 1.069407 & 0.000000 & 0.000000\end{array}$

$\begin{array}{lllll}3 A & 0.534703 & -0.972693 & 0.000000\end{array}$

$\begin{array}{lllll}4 \mathrm{~A} & 0.534703 & -0.333352 & -0.913788\end{array}$

$\begin{array}{lllll}5 A & 0.534703 & 0.333353 & 0.913787\end{array}$

$\begin{array}{lllll}6 \mathrm{~A} & 0.534703 & 0.972693 & -0.000002\end{array}$

$\begin{array}{llll}7 \mathrm{~B} & -0.368819 & -0.778883 & 1.113231\end{array}$

$\begin{array}{llll}8 \mathrm{~B} & -0.368820 & 0.778882 & -1.113232\end{array}$

$\begin{array}{llll}9 \mathrm{~B} & 1.438224 & -0.778883 & 1.113232\end{array}$

$\begin{array}{llll}\text { 10B } & 1.438225 & 0.778883 & -1.113232\end{array}$

$\begin{array}{llll}11 \mathrm{~B} & -0.754620 & -0.966557 & -0.676261\end{array}$

$\begin{array}{llll}12 B & -0.754621 & 0.966557 & 0.676260\end{array}$

$\begin{array}{llll}13 B & 1.824025 & 0.966558 & 0.676261\end{array}$

$\begin{array}{lllll}14 \mathrm{~B} & 1.824026 & -0.966557 & -0.676262\end{array}$

$E 15 / \varepsilon=-56.181783$

atom $\mathrm{x} / \sigma \quad \mathrm{y} / \sigma$

$\begin{array}{lll}\text { 1A } & 0.000000 & 0.000000\end{array}$

$\begin{array}{lll}2 \mathrm{~A} & 1.150794 & 0.000000\end{array}$

3B $\quad 0.575396-1.315325$

$\begin{array}{lll}4 \mathrm{~B} & 0.575396 & -0.042743\end{array}$

$\begin{array}{lll}5 \mathrm{~A} & 0.575397 & -0.155419\end{array}$

$\begin{array}{lll}6 \mathrm{~B} & -0.300233 & 0.989926\end{array}$

$\begin{array}{lll}7 \mathrm{~B} & 1.451029 & 0.989925\end{array}$

$\begin{array}{lll}8 \mathrm{~A} & 0.575397 & 0.925001\end{array}$

$\begin{array}{lll}8 \mathrm{~B} & -0.711676 & -0.718679\end{array}$

10B $\quad 1.862470-0.718680$

$\begin{array}{lll}11 B & -0.750447 & 0.997973\end{array}$

$\begin{array}{lll}12 B & 1.901240 & 0.997972\end{array}$

$\begin{array}{lll}13 \mathrm{~B} & 2.115973 & -0.774525\end{array}$

$\begin{array}{lll}14 \mathrm{~B} & -0.965181 & -0.774524\end{array}$

15B $\quad 0.575399 \quad-0.000195$

$z / \sigma$

0.000000

0.000000

0.000000

1.302121

$-0.897578$

$-1.010308$

$-1.010307$

0.148999

$-1.070075$

$-1.070074$

0.710125

0.710127

0.710306

0.710305

$E 16 / \varepsilon=-61.799365$

atom $\mathrm{X} / \sigma$

$y / \sigma$

$\begin{array}{ccc}1 \mathrm{~A} & 0.000000 & 0.000000\end{array}$

$-2.278691$

$\begin{array}{lll}2 \mathrm{~A} & 1.092841 & 0.000000\end{array}$

$\begin{array}{lll}3 \mathrm{~A} & 0.540055 & -0.950073\end{array}$

$\begin{array}{lll}4 \mathrm{~B} & 0.476565 & -0.277281\end{array}$

$\begin{array}{lll}5 B & 0.480136 & 1.303232\end{array}$

$\begin{array}{lll}6 \mathrm{~B} & -0.895711 & -1.061437\end{array}$

$\begin{array}{lll}7 \mathrm{~B} & 1.380720 & -0.803345\end{array}$

$\begin{array}{lll}8 \mathrm{~A} & 0.538168 & 0.333024\end{array}$

$\begin{array}{lll}9 \mathrm{~A} & -0.023564 & -0.632432\end{array}$
10B $\quad 1.875394 \quad-1.091172$

$\begin{array}{llll}11 B & -0.898209 & 0.522610 & -0.937409\end{array}$

$\begin{array}{lllll}12 \mathrm{~B} & 0.189434 & -2.066890 & -0.820913\end{array}$

$\begin{array}{lllll}13 \mathrm{~B} & 1.890488 & 0.856728 & -0.820910\end{array}$

$\begin{array}{llll}14 \mathrm{~B} & 0.410162 & -2.017891 & 0.948801\end{array}$

$\begin{array}{llll}15 B & 1.956978 & 0.640605 & 0.948800\end{array}$

$\begin{array}{llll}16 \mathrm{~B} & -1.009681 & 0.587464 & 0.845461\end{array}$

$E 17 / \varepsilon=-67.449039$

$\begin{array}{lcc}\text { atom } & \mathrm{x} / \sigma & y / \sigma\end{array}$

$0.000000 \quad 0.000000$

$\begin{array}{llll}2 \mathrm{~A} & 1.085938 & 0.000000 & 0.000000\end{array}$

$\begin{array}{llll}3 A & 0.542969 & -0.940450 & 0.000000\end{array}$

$\begin{array}{llll}4 \mathrm{~B} & 0.542969 & -0.313483 & 1.276333\end{array}$

$\begin{array}{llll}5 A & -0.014554 & -0.635370 & -0.897392\end{array}$

$\begin{array}{lllll}6 \mathrm{~A} & 1.100493 & -0.635370 & -0.897392\end{array}$

$\begin{array}{lllll}7 A & 0.542969 & 0.330290 & -0.897392\end{array}$

$\begin{array}{llll}8 \mathrm{~B} & 0.542969 & 1.295469 & 0.166473\end{array}$

$\begin{array}{llll}9 B & 1.936363 & -1.117959 & 0.166473\end{array}$

$\begin{array}{llll}10 B & -0.850424 & -1.117959 & 0.166473\end{array}$

$\begin{array}{llll}11 B & 1.960157 & 0.504730 & -0.977814\end{array}$

12B $\quad-0.874218 \quad 0.504730 \quad-0.977815$

$\begin{array}{lllll}\text { 13B } & 0.542970 & -1.949910 & -0.977814\end{array}$

$\begin{array}{lllll}14 \mathrm{~B} & 0.542970 & -0.313481 & -2.164474\end{array}$

$\begin{array}{llll}15 B & -0.975732 & 0.563339 & 0.840733\end{array}$

$\begin{array}{llll}16 \mathrm{~B} & 0.542970 & -2.067128 & 0.840733\end{array}$

$\begin{array}{llll}17 \mathrm{~B} & 2.061671 & 0.563339 & 0.840733\end{array}$

$E 18 / \varepsilon=-73.165711$

$\begin{array}{llll}\text { atom } & \mathrm{x} / \sigma & \mathrm{y} / \sigma & \mathrm{z} / \sigma\end{array}$

$\begin{array}{llll}1 \mathrm{~A} & 0.000000 & 0.000000 & 0.000000\end{array}$

$\begin{array}{llll}2 \mathrm{~A} & 1.093196 & 0.000000 & 0.000000\end{array}$

$\begin{array}{llll}3 \mathrm{~A} & 0.546599 & -0.936196 & 0.000000\end{array}$

$\begin{array}{llll}4 \mathrm{~A} & 0.546599 & 0.301581 & 0.886291\end{array}$

$\begin{array}{lllll}5 \mathrm{~B} & 0.546598 & -0.294839 & -1.274867\end{array}$

$\begin{array}{lllll}6 B & 0.546598 & 1.301887 & -0.131557\end{array}$

$\begin{array}{llll}7 \mathrm{~A} & 1.108507 & -0.644818 & 0.900541\end{array}$

$\begin{array}{llll}8 A & -0.015308 & -0.644819 & 0.900541\end{array}$

9B $-0.848071-1.113101-0.173369$

$\begin{array}{llll}10 \mathrm{~B} & -0.848072 & 0.522693 & 0.997919\end{array}$

$\begin{array}{llll}11 \mathrm{~B} & 1.941270 & -1.113099 & -0.173369\end{array}$

$\begin{array}{llll}12 \mathrm{~B} & 1.941270 & 0.522696 & 0.997916\end{array}$

$\begin{array}{llll}13 \mathrm{~B} & 0.546601 & -1.958603 & 0.963004\end{array}$

$\begin{array}{llll}14 \mathrm{~B} & 0.546600 & -0.280735 & 2.164415\end{array}$

$\begin{array}{llll}15 B & -0.972928 & 0.587369 & -0.820308\end{array}$

$\begin{array}{lllll}16 \mathrm{~B} & 2.066123 & 0.587370 & -0.820310\end{array}$

$\begin{array}{lllll}17 \mathrm{~B} & 0.546600 & -2.051610 & -0.856304\end{array}$

$\begin{array}{llll}18 \mathrm{~B} & 0.546598 & 1.471553 & 1.666399\end{array}$

$E 19 / \varepsilon=-79.045725$

atom $\quad \mathrm{x} / \sigma \quad \mathrm{y} / \sigma \quad z / \sigma$

$\begin{array}{llll}1 \mathrm{~A} & 0.000000 & 0.000000 & 0.000000\end{array}$

$\begin{array}{llll}2 A & 1.094865 & 0.000000 & 0.000000\end{array}$

$\begin{array}{llll}3 A & -0.007244 & -1.094842 & 0.000000\end{array}$

$\begin{array}{llll}4 \mathrm{~A} & 0.543810 & -0.547421 & 0.776744\end{array}$

$\begin{array}{lllll}5 A & 0.543810 & -0.547421 & -0.776744\end{array}$

$\begin{array}{llll}6 \mathrm{~B} & 1.401250 & -1.410551 & 0.000000\end{array}$

$\begin{array}{llll}7 \mathrm{~B} & 0.475301 & 0.892473 & 0.966182\end{array}$

$\begin{array}{lllll}8 \mathrm{~B} & 0.475301 & 0.892473 & -0.966182\end{array}$

$\begin{array}{llll}9 \mathrm{~B} & -0.895599 & -0.469388 & 0.966181\end{array}$

$\begin{array}{llll}10 \mathrm{~B} & -0.895599 & -0.469388 & -0.966181\end{array}$

$\begin{array}{lllll}11 \mathrm{~B} & 1.874829 & -0.200230 & -1.188968\end{array}$

$\begin{array}{llll}12 \mathrm{~B} & 0.187822 & -1.876114 & 1.188968\end{array}$

$\begin{array}{llll}13 B & 1.874829 & -0.200230 & 1.188968\end{array}$

$\begin{array}{lllll}14 \mathrm{~B} & 0.187822 & -1.876114 & -1.188968\end{array}$

$\begin{array}{llll}15 \mathrm{~B} & -1.013067 & 1.019790 & 0.000000\end{array}$

$\begin{array}{llll}16 \mathrm{~B} & -1.149659 & -1.948798 & 0.000000\end{array}$

$\begin{array}{llll}17 \mathrm{~B} & 1.956359 & 1.136741 & 0.000000\end{array}$

$\begin{array}{llll}18 \mathrm{~B} & 0.403349 & -0.406027 & 2.189058\end{array}$

$\begin{array}{llll}\text { 19B } & 0.403349 & -0.406027 & -2.189058\end{array}$

$E 20 / \varepsilon=-84.683290$ 


\begin{tabular}{rrrr} 
atom & $x / \sigma$ & $y / \sigma$ & \multicolumn{1}{c}{$z / \sigma$} \\
1A & 0.000000 & 0.000000 & 0.000000 \\
2A & 1.089526 & 0.000000 & 0.000000 \\
3A & 0.000000 & -1.089526 & 0.000000 \\
4A & 1.089526 & -1.089526 & 0.000000 \\
5A & 0.544763 & -0.544763 & -0.770411 \\
6A & 0.544763 & -0.544763 & 0.770411 \\
7B & 0.544763 & 0.856273 & -0.990681 \\
8B & 0.544763 & 0.856273 & 0.990681 \\
9B & -0.856273 & -0.544762 & 0.990682 \\
10B & -0.856273 & -0.544762 & -0.990682 \\
11B & 1.945799 & -0.544763 & -0.990682 \\
12B & 0.544763 & -1.945799 & 0.990681 \\
13B & 1.945799 & -0.544763 & 0.990682 \\
14B & 0.544763 & -1.945799 & -0.990681 \\
15B & -0.986360 & 0.986360 & 0.000000 \\
16B & 0.544762 & -0.544762 & 2.165335 \\
17B & 0.544762 & -0.544762 & -2.165335 \\
18B & 2.075886 & 0.986360 & 0.000000 \\
19B & 2.075886 & -2.075886 & 0.000000 \\
20B & -0.986362 & -2.075884 & 0.000000
\end{tabular}

$E 21 / \varepsilon=-90.570373$

$\begin{array}{cccc}\text { atom } & \mathrm{x} / \sigma & y / \sigma & z / \sigma \\ 1 \mathrm{~A} & 0.000000 & 0.000000 & 0.000000\end{array}$

$\begin{array}{llll}2 \mathrm{~A} & 1.115920 & 0.000000 & 0.000000\end{array}$

$\begin{array}{llll}3 \mathrm{~A} & 0.557960 & -0.910668 & 0.000000\end{array}$

$\begin{array}{lllll}4 \mathrm{~A} & 0.557960 & 0.368390 & -0.832829\end{array}$

$\begin{array}{llll}5 B & 0.557960 & 0.730814 & 1.122384\end{array}$

$\begin{array}{llll}6 \mathrm{~A} & 1.093899 & -0.607693 & -0.933294\end{array}$

$\begin{array}{lllll}7 A & 0.022022 & -0.607693 & -0.933294\end{array}$

8B $\quad \begin{array}{llll}1.974579 & -1.191211 & -0.016313\end{array}$

$\begin{array}{llll}9 B & 1.974580 & 0.466958 & -1.095990\end{array}$

$\begin{array}{llll}10 \mathrm{~B} & -0.858659 & 0.466958 & -1.095991\end{array}$

$\begin{array}{llll}11 B & -0.858659 & -1.191211 & -0.016313\end{array}$

$\begin{array}{llll}12 \mathrm{~A} & 1.110667 & -0.578570 & 0.932155\end{array}$

$\begin{array}{llll}13 \mathrm{~A} & 0.005253 & 1.086527 & -0.152034\end{array}$

$\begin{array}{llll}14 \mathrm{~A} & 0.005253 & -0.578570 & 0.932154\end{array}$

$\begin{array}{lllll}15 \mathrm{~A} & 1.110667 & 1.086527 & -0.152034\end{array}$

$\begin{array}{llll}16 \mathrm{~B} & 0.557960 & -1.946179 & -0.938923\end{array}$

$\begin{array}{llll}17 \mathrm{~B} & 0.557960 & -0.071389 & -2.159650\end{array}$

$\begin{array}{llll}18 B & 0.557961 & 1.630802 & -1.399651\end{array}$

$\begin{array}{llll}19 \mathrm{~B} & 0.557960 & -1.939722 & 0.925213\end{array}$

$\begin{array}{llll}20 \mathrm{~B} & -1.123347 & 0.445231 & 0.683788\end{array}$

$\begin{array}{llll}21 B & 2.239267 & 0.445231 & 0.683789\end{array}$

$E 22 / \varepsilon=-96.512158$

atom $\quad x / \sigma$

$\begin{array}{cccc}1 \mathrm{~A} & 0.000000 & 0.000000 & 0.000000\end{array}$

$\begin{array}{llll}2 \mathrm{~A} & 1.210237 & 0.000000 & 0.000000\end{array}$

$\begin{array}{llll}3 \mathrm{~A} & 0.605118 & -0.926448 & 0.000000\end{array}$

$\begin{array}{llll}4 \mathrm{~A} & 0.605118 & -0.289333 & 0.876166\end{array}$

$\begin{array}{lllll}5 \mathrm{~A} & 0.605118 & -0.289333 & -0.876166\end{array}$

$\begin{array}{llll}6 \mathrm{~B} & -0.464180 & -1.219483 & 0.879925\end{array}$

$\begin{array}{llll}7 \mathrm{~B} & -0.464181 & -1.219483 & -0.879924\end{array}$

$\begin{array}{lllll}8 \mathrm{~B} & 1.674413 & -1.219487 & 0.879925\end{array}$

$\begin{array}{lllll}9 B & 1.674414 & -1.219487 & -0.879925\end{array}$

$\begin{array}{llll}10 \mathrm{~A} & 0.605120 & 0.751026 & -0.548082\end{array}$

$\begin{array}{llll}11 \mathrm{~A} & 0.605120 & 0.751026 & 0.548082\end{array}$

$\begin{array}{llll}12 \mathrm{~B} & 1.671507 & 0.456721 & 1.428819\end{array}$

$\begin{array}{llll}\text { 13B } & 1.671507 & 0.456721 & -1.428818\end{array}$

$\begin{array}{llll}14 \mathrm{~B} & -0.461268 & 0.456725 & -1.428818\end{array}$

$\begin{array}{llll}15 \mathrm{~B} & -0.461268 & 0.456725 & 1.428819\end{array}$

$\begin{array}{llll}16 \mathrm{~B} & 1.675653 & 1.493587 & 0.000000\end{array}$

$\begin{array}{llll}17 \mathrm{~B} & -0.465410 & 1.493592 & 0.000000\end{array}$

$\begin{array}{llll}18 \mathrm{~B} & 2.634041 & -0.008756 & 0.000000\end{array}$

$\begin{array}{llll}19 \mathrm{~B} & -1.423803 & -0.008747 & 0.000001\end{array}$

$\begin{array}{llll}20 \mathrm{~B} & 0.605114 & -2.349172 & 0.000000\end{array}$

$\begin{array}{llll}21 B & 0.605117 & -0.746398 & -2.225541\end{array}$

$\begin{array}{llll}22 \mathrm{~B} & 0.605117 & -0.746398 & 2.225541\end{array}$

$E 23 / \varepsilon=-103.276147$

\begin{tabular}{|cccc||rrrr} 
atom & $x / \sigma$ & $y / \sigma$ & $z / \sigma$ & $19 \mathrm{~A}$ & 0.008424 & 1.075047 & -0.238337 \\
1A & 0.000000 & 0.000000 & 0.000000 & 20B & 0.552324 & -2.286537 & 0.513649 \\
2A & 1.210250 & 0.000000 & 0.000000 & $21 \mathrm{~B}$ & -1.151715 & 0.664941 & 0.513656 \\
3A & 0.605124 & -1.048108 & 0.000000 & $22 \mathrm{~B}$ & 2.256360 & 0.664942 & 0.513651 \\
4A & 0.605124 & -0.349369 & 0.826000 & $23 \mathrm{~B}$ & 2.184060 & -1.260966 & -1.510704 \\
5A & 0.605125 & -0.349369 & -0.825999 & $24 \mathrm{~B}$ & 0.552319 & 1.565291 & -1.510696 \\
6A & -0.326605 & -0.887303 & 0.567184 & $25 \mathrm{~B}$ & -1.079420 & -1.260968 & -1.510699 \\
7A & -0.326605 & -0.887303 & -0.567186 & & & &
\end{tabular}

$\begin{array}{llll}6 A & -0.326605 & -0.887303 & 0.567184\end{array}$

$\begin{array}{llll}8 \mathrm{~A} & 1.536853 & -0.887304 & 0.567185\end{array}$

$\begin{array}{lllll}9 A & 0.605124 & 0.726499 & 0.567185\end{array}$

$\begin{array}{llll}10 A & 1.536853 & -0.887304 & -0.567185\end{array}$

$\begin{array}{llll}11 A & 0.605125 & 0.726499 & -0.567186\end{array}$

$\begin{array}{lllll}12 B & 0.605124 & -1.603096 & -1.413658\end{array}$

$\begin{array}{lllll}13 \mathrm{~B} & 1.690884 & 0.277494 & -1.413657\end{array}$

$\begin{array}{lllll}14 \mathrm{~B} & -0.480634 & 0.277494 & -1.413658\end{array}$

$\begin{array}{llll}15 \mathrm{~B} & -0.480636 & 0.277495 & 1.413655\end{array}$

$\begin{array}{llll}16 \mathrm{~B} & 0.605123 & -1.603097 & 1.413656\end{array}$

$\begin{array}{llll}17 \mathrm{~B} & 1.690884 & 0.277495 & 1.413656\end{array}$

$\begin{array}{llll}18 \mathrm{~B} & 1.501393 & -2.195604 & -0.000001\end{array}$

$\begin{array}{llll}19 \mathrm{~B} & 2.652145 & -0.202446 & 0.000000\end{array}$

$\begin{array}{llll}20 \mathrm{~B} & -0.545625 & 1.349942 & -0.000001\end{array}$

$\begin{array}{llll}21 B & -0.291148 & -2.195603 & -0.000001\end{array}$

$\begin{array}{llll}22 \mathrm{~B} & 1.755875 & 1.349941 & -0.000001\end{array}$

$\begin{array}{llll}23 \mathrm{~B} & -1.441897 & -0.202445 & -0.000002\end{array}$

$E 24 / \varepsilon=-108.932853$

atom $\mathrm{x} / \sigma$ $y / \sigma$

$\begin{array}{lll}1 \mathrm{~A} & 0.000000 & 0.000000\end{array}$

2A $1.217398 \quad 0.000000$

3A 0.608698 - 0.044293

$\begin{array}{llll}4 A & 0.608699 & -0.352781\end{array}$

$\begin{array}{lll}5 A & 0.608699 & -0.344442\end{array}$

$\begin{array}{lll}6 \mathrm{~A} & 0.608699 & 0.703938\end{array}$

$\begin{array}{lll}7 A & 0.608699 & 0.732035\end{array}$

$\begin{array}{lll}8 \mathrm{~B} & -0.447072 & 0.294688\end{array}$

$\begin{array}{lll}9 B & 1.664469 & 0.294687\end{array}$

$10 A-0.322879-0.881022$

$\begin{array}{llll}11 \mathrm{~A} & 1.540276 & -0.881022\end{array}$

$12 \mathrm{~A} \quad 1.546846-0.883012$

$\begin{array}{lll}13 A & -0.329450 & -0.883012\end{array}$

$\begin{array}{lll}14 \mathrm{~B} & -0.517358 & 1.375848\end{array}$

$\begin{array}{lll}15 B & 1.734756 & 1.375848\end{array}$

$\begin{array}{lll}16 \mathrm{~B} & -0.487701 & 0.297950\end{array}$

$\begin{array}{lllll}17 B & 1.705098 & 0.297950 & -1.387466\end{array}$

$\begin{array}{llll}18 \mathrm{~B} & 0.608698 & -1.597188 & 1.426569\end{array}$

$\begin{array}{llll}19 B & 0.608698 & -1.604417 & -1.400653\end{array}$

$\begin{array}{llll}20 \mathrm{~B} & 2.649390 & -0.174483 & 0.033153\end{array}$

$\begin{array}{llll}21 \mathrm{~B} & -1.431993 & -0.174482 & 0.033153\end{array}$

$\begin{array}{llll}22 \mathrm{~B} & 1.505344 & -2.190092 & 0.013679\end{array}$

$\begin{array}{llll}23 \mathrm{~B} & -0.287948 & -2.190092 & 0.013678\end{array}$

$\begin{array}{llll}24 \mathrm{~B} & 0.608698 & 1.787590 & 1.431848\end{array}$

\section{$E 25 / \varepsilon=-114.879107$}

atom $x / \sigma$

$\begin{array}{llll}1 \mathrm{~A} & 0.000000 & 0.000000 & 0.000000\end{array}$

$\begin{array}{llll}2 \mathrm{~A} & 1.104644 & 0.000000 & 0.000000\end{array}$

$\begin{array}{llll}3 A & 0.552322 & -0.956649 & 0.000000\end{array}$

$\begin{array}{llll}4 \mathrm{~A} & 1.105582 & -0.638308 & -0.869473\end{array}$

$\begin{array}{llll}5 A & -0.000940 & -0.638308 & -0.869472\end{array}$

$\begin{array}{lllll}6 \mathrm{~A} & 0.552321 & 0.319969 & -0.869471\end{array}$

$\begin{array}{lllll}7 \mathrm{~B} & -0.816274 & 0.471276 & -1.235863\end{array}$

$\begin{array}{lllll}8 \mathrm{~B} & 1.920914 & 0.471277 & -1.235867\end{array}$

$\begin{array}{llll}9 \mathrm{~B} & 0.552321 & -1.899198 & -1.235869\end{array}$

$\begin{array}{llll}10 B & 0.552323 & 0.728600 & 1.076815\end{array}$

$\begin{array}{llll}11 B & -0.354825 & -0.842628 & 1.076813\end{array}$

$\begin{array}{llll}12 \mathrm{~B} & 1.459474 & -0.842628 & 1.076811\end{array}$

$\begin{array}{llll}13 B & 0.552319 & -0.318881 & -2.137622\end{array}$

$\begin{array}{llll}14 \mathrm{~A} & 1.487552 & -1.486876 & -0.238343\end{array}$

$\begin{array}{llll}15 A & -0.382907 & -1.486877 & -0.238340\end{array}$

$\begin{array}{llll}16 \mathrm{~A} & 1.096219 & 1.075046 & -0.238338\end{array}$

$\begin{array}{llll}17 \mathrm{~A} & -0.926805 & -0.544820 & -0.238338\end{array}$

$\begin{array}{lllll}18 A & 2.031449 & -0.544819 & -0.238343\end{array}$
$E 26 / \varepsilon=-122.006208$

$\begin{array}{llll}1 \mathrm{~A} & 0.000000 & 0.000000 & 0.000000\end{array}$

$\begin{array}{llll}2 A & 1.129285 & 0.000000 & 0.000000\end{array}$

$\begin{array}{llll}3 \mathrm{~A} & 0.564642 & -0.977990 & 0.000000\end{array}$

$\begin{array}{llll}4 \mathrm{~A} & 0.564642 & -0.325997 & 0.922058\end{array}$

$\begin{array}{lllll}5 A & -0.333259 & -0.844399 & 0.597081\end{array}$

$\begin{array}{llll}7 \mathrm{~A} & 1.462542 & -0.844400 & 0.597081\end{array}$

$\begin{array}{lllll}8 \mathrm{~A} & 0.564642 & -0.325997 & -0.869185\end{array}$

$\begin{array}{llll}9 A & -0.381527 & 0.220275 & 1.003058\end{array}$

$\begin{array}{llll}10 A & 0.564641 & -1.418538 & 1.003058\end{array}$

$\begin{array}{lllll}12 A & 0.564642 & 0.766545 & -0.542029\end{array}$

$\begin{array}{llll}13 \mathrm{~A} & 1.510811 & 0.220274 & 1.003059\end{array}$

$\begin{array}{llll}14 \mathrm{~A} & -0.381528 & -0.872267 & -0.542029\end{array}$

$\begin{array}{llll}15 \mathrm{~B} & -0.321550 & -2.155437 & 0.126388\end{array}$

$\begin{array}{llll}16 \mathrm{~B} & 1.450830 & -2.155438 & 0.126388\end{array}$

$\begin{array}{llll}17 \mathrm{~B} & 1.705890 & 1.356186 & 0.126387\end{array}$

$\begin{array}{lllll}18 B & 0.564640 & -1.643796 & -1.320753\end{array}$

$\begin{array}{llll}19 B & 2.592079 & -0.178739 & 0.126388\end{array}$

$\begin{array}{llll}20 B & 0.564641 & 0.697286 & 1.990037\end{array}$

$\begin{array}{llll}21 \mathrm{~B} & 1.450830 & -0.837639 & 1.990037\end{array}$

$\begin{array}{llll}22 \mathrm{~B} & -0.576605 & 0.332903 & -1.320754\end{array}$

$\begin{array}{llll}23 \mathrm{~B} & -1.462795 & -0.178738 & 0.126387\end{array}$

$\begin{array}{llll}24 \mathrm{~B} & -0.321550 & -0.837639 & 1.990037\end{array}$

$\begin{array}{llll}25 B & 1.705890 & 0.332903 & -1.320754\end{array}$

$\begin{array}{llll}26 \mathrm{~B} & -0.576605 & 1.356187 & 0.126387\end{array}$

$E 27 / \varepsilon=-127.430425$

atom $\quad \mathrm{x} / \sigma \quad \mathrm{y} / \sigma \quad \mathrm{z} / \sigma$

$\begin{array}{llll}1 \mathrm{~A} & 0.000000 & 0.000000 & 0.000000\end{array}$

$\begin{array}{llll}2 \mathrm{~A} & 1.126458 & 0.000000 & 0.000000\end{array}$

$\begin{array}{llll}3 \mathrm{~A} & 0.560490 & -0.977118 & 0.000000\end{array}$

$\begin{array}{llll}4 \mathrm{~A} & 0.570968 & -0.330718 & 0.922477\end{array}$

$\begin{array}{llll}5 \mathrm{~A} & 0.568125 & 0.709409 & 0.599911\end{array}$

$\begin{array}{llll}6 \mathrm{~A} & -0.332678 & -0.845786 & 0.599911\end{array}$

$\begin{array}{llll}7 A & 0.552726 & -0.320151 & -0.870640\end{array}$

$\begin{array}{lllll}8 \mathrm{~A} & 1.462524 & -0.847126 & 0.599264\end{array}$

$\begin{array}{llll}9 \mathrm{~A} & -0.355743 & 0.206053 & 0.991281\end{array}$

$\begin{array}{llll}10 A & -0.398351 & -0.867712 & -0.529368\end{array}$

$\begin{array}{lllll}11 \mathrm{~A} & 0.554468 & 0.777286 & -0.529367\end{array}$

$\begin{array}{llll}12 \mathrm{~A} & 1.506105 & 0.226076 & 1.008134\end{array}$

$\begin{array}{llll}13 \mathrm{~A} & 0.553286 & -1.418922 & 1.008133\end{array}$

$\begin{array}{lllll}14 \mathrm{~A} & 1.503250 & -0.870715 & -0.545375\end{array}$

$\begin{array}{llll}15 \mathrm{~B} & -1.473715 & -0.167386 & 0.187106\end{array}$

$\begin{array}{llll}16 \mathrm{~B} & -0.588081 & 1.361622 & 0.187108\end{array}$

$\begin{array}{llll}17 \mathrm{~B} & -0.358133 & -0.813556 & 1.989484\end{array}$

$\begin{array}{llll}18 \mathrm{~B} & 0.527501 & 0.715451 & 1.989484\end{array}$

$\begin{array}{llll}19 B & -0.607710 & 0.351999 & -1.276118\end{array}$

$\begin{array}{llll}20 B & 1.419667 & -0.822304 & 1.999391\end{array}$

$\begin{array}{llll}21 \mathrm{~B} & -0.334361 & -2.155504 & 0.131405\end{array}$

$\begin{array}{llll}22 B & 1.703370 & 1.362544 & 0.131406\end{array}$

$\begin{array}{llll}23 B & 1.436801 & -2.155988 & 0.124136\end{array}$

$\begin{array}{llll}24 \mathrm{~B} & 0.544862 & -1.639357 & -1.316913\end{array}$

$\begin{array}{llll}25 B & 2.585064 & -0.173565 & 0.124137\end{array}$

$\begin{array}{llll}26 \mathrm{~B} & 1.693125 & 0.343066 & -1.316913\end{array}$

$\begin{array}{llll}27 \mathrm{~B} & -1.328676 & 0.769596 & 1.795513\end{array}$

$E 28 / \varepsilon=-133.017782$

atom $\quad \mathrm{x} / \sigma \quad \mathrm{y} / \sigma$

$\begin{array}{llll}1 \mathrm{~A} & 0.000000 & 0.000000 & 0.000000\end{array}$

$\begin{array}{llll}2 \mathrm{~A} & 1.097111 & 0.000000 & 0.000000\end{array}$

$\begin{array}{llll}3 A & 0.494536 & -0.970528 & 0.000000\end{array}$ atom $\mathrm{x} / \sigma \quad \mathrm{y} / \sigma$

$\begin{array}{llll}6 \mathrm{~A} & 0.564642 & 0.710810 & 0.597081\end{array}$

$\begin{array}{lllll}11 \mathrm{~A} & 1.510811 & -0.872268 & -0.542029\end{array}$ 
$\begin{array}{llll}4 \mathrm{~A} & 0.475929 & -0.277290 & 0.937182\end{array}$ $\begin{array}{lllll}5 \mathrm{~A} & 0.524061 & -0.314605 & -0.880621\end{array}$ $\begin{array}{llll}6 \mathrm{~B} & -0.811580 & 0.532315 & -0.976871\end{array}$ $\begin{array}{llll}7 B & -0.871442 & 0.562919 & 0.905943\end{array}$ $\begin{array}{lllll}8 \mathrm{~A} & 0.553419 & 0.737526 & -0.569985\end{array}$ $\begin{array}{llll}9 \mathrm{~A} & 0.527017 & 0.749718 & 0.566301\end{array}$ $\begin{array}{llll}10 A & 1.368764 & -0.833311 & 0.625946\end{array}$ $\begin{array}{llll}11 \mathrm{~A} & -0.422775 & -0.792525 & 0.580397\end{array}$ $\begin{array}{llll}12 \mathrm{~B} & 1.711312 & 0.271737 & -1.324940\end{array}$ $\begin{array}{llll}13 \mathrm{~A} & -0.413233 & -0.820124 & -0.598581\end{array}$ $\begin{array}{llll}14 \mathrm{~B} & 1.674478 & 1.357359 & 0.059031\end{array}$ $\begin{array}{llll}15 A & 1.454610 & -0.890567 & -0.516771\end{array}$ $\begin{array}{llll}16 \mathrm{~A} & 0.449339 & -1.375770 & 1.013734\end{array}$ $\begin{array}{llll}17 \mathrm{~A} & 1.445191 & 0.245610 & 1.001557\end{array}$ $\begin{array}{lllll}18 \mathrm{~B} & 0.531184 & -1.645709 & -1.316940\end{array}$ $\begin{array}{llll}19 \mathrm{~B} & -0.438915 & -2.094726 & 0.096391\end{array}$ $\begin{array}{llll}20 B & 2.532359 & -0.205335 & 0.169042\end{array}$ $\begin{array}{llll}21 B & 1.337244 & -2.155643 & 0.178828\end{array}$ $\begin{array}{llll}22 B & 1.346153 & -0.800694 & 2.012286\end{array}$ $\begin{array}{llll}23 \mathrm{~B} & 0.522046 & 0.764451 & 1.971656\end{array}$ $\begin{array}{llll}24 B & -0.421645 & -0.765486 & 1.984952\end{array}$ $\begin{array}{llll}25 B & -0.091578 & 1.873446 & -0.021010\end{array}$ $\begin{array}{llll}26 \mathrm{~B} & -1.696608 & -0.714491 & -0.012858\end{array}$ $\begin{array}{llll}27 \mathrm{~B} & 0.220720 & -0.131133 & -2.234236\end{array}$ $\begin{array}{llll}28 B & 0.522582 & 1.562278 & -1.693405\end{array}$

\section{$E 29 / \varepsilon=-139.739463$}

atom $\mathrm{x} / \sigma \quad \mathrm{g} / \sigma$

$\begin{array}{llll}1 \mathrm{~A} & 0.000000 & 0.000000 & 0.000000\end{array}$

$\begin{array}{llll}2 \mathrm{~A} & 1.094292 & 0.000000 & 0.000000\end{array}$

$\begin{array}{llll}3 \mathrm{~A} & 0.547146 & -0.947685 & 0.000000\end{array}$

$\begin{array}{llll}4 \mathrm{~A} & 0.547147 & -0.315895 & 0.919466\end{array}$

$\begin{array}{lllll}5 A & 0.547146 & -0.315895 & -0.919466\end{array}$

$\begin{array}{llll}6 \mathrm{~A} & -0.361611 & -0.840567 & 0.579040\end{array}$

$\begin{array}{llll}7 A & -0.361612 & -0.840567 & -0.579039\end{array}$

$\begin{array}{lllll}8 \mathrm{~A} & 0.547146 & 0.733448 & -0.579040\end{array}$

$\begin{array}{llll}9 A & 0.547146 & 0.733448 & 0.579040\end{array}$

$\begin{array}{lllll}10 A & 1.455904 & -0.840566 & -0.579040\end{array}$

$\begin{array}{llll}11 \mathrm{~A} & 1.455904 & -0.840567 & 0.579039\end{array}$

$\begin{array}{lllll}12 \mathrm{~A} & 1.504658 & 0.236925 & -0.974509\end{array}$

13A $\quad 0.547146 \quad-1.421535 \quad 0.974509$

$\begin{array}{llll}14 \mathrm{~A} & 0.547146 & -1.421535 & -0.974509\end{array}$

$\begin{array}{llll}15 A & -0.410366 & 0.236925 & 0.974509\end{array}$

$\begin{array}{llll}16 \mathrm{~A} & -0.410367 & 0.236924 & -0.974508\end{array}$

$\begin{array}{llll}17 \mathrm{~A} & 1.504660 & 0.236925 & 0.974509\end{array}$

$\begin{array}{llll}18 \mathrm{~B} & -1.450358 & -0.166051 & 0.000000\end{array}$

$\begin{array}{llll}19 B & -0.581376 & 1.339071 & 0.000000\end{array}$

$\begin{array}{llll}20 B & 1.416129 & -2.120707 & -0.000001\end{array}$

$\begin{array}{llll}21 \mathrm{~B} & 1.675669 & 1.339071 & 0.000000\end{array}$

$\begin{array}{llll}22 \mathrm{~B} & -0.321836 & -2.120707 & 0.000000\end{array}$

$\begin{array}{llll}23 \mathrm{~B} & 2.544651 & -0.166051 & -0.000001\end{array}$

$\begin{array}{llll}24 \mathrm{~B} & -0.337466 & -0.826626 & 1.970744\end{array}$

$\begin{array}{llll}25 \mathrm{~B} & -0.337468 & -0.826627 & -1.970744\end{array}$

$\begin{array}{llll}26 \mathrm{~B} & 0.547147 & 0.705567 & 1.970744\end{array}$

$\begin{array}{llll}27 \mathrm{~B} & 0.547143 & 0.705568 & -1.970744\end{array}$

$\begin{array}{llll}28 B & 1.431759 & -0.826627 & 1.970744\end{array}$

$\begin{array}{llll}29 B & 1.431757 & -0.826625 & -1.970745\end{array}$

$E 30 / \varepsilon=-145.480894$

atom $\mathrm{X} / \sigma$

$\begin{array}{llll}1 \mathrm{~A} & 0.000000 & 0.000000 & 0.000000\end{array}$

$\begin{array}{llll}2 \mathrm{~A} & 1.110012 & 0.000000 & 0.000000\end{array}$

$\begin{array}{llll}3 \mathrm{~A} & 0.575570 & -0.951018 & 0.000000\end{array}$

$\begin{array}{lllll}4 \mathrm{~A} & 0.553540 & 0.967676 & -0.000001\end{array}$

$\begin{array}{llll}5 A & -0.557873 & 0.959112 & 0.000000\end{array}$

$\begin{array}{llll}6 \mathrm{~B} & 1.343723 & -0.762382 & 1.250581\end{array}$

$\begin{array}{llll}7 B & 1.343722 & -0.762384 & -1.250581\end{array}$

$\begin{array}{llll}8 \mathrm{~A} & -0.538559 & -0.972859 & 0.000001\end{array}$

$\begin{array}{llll}9 \mathrm{~A} & -1.111378 & -0.011720 & 0.000001\end{array}$

$\begin{array}{llll}10 \mathrm{~A} & 0.532116 & 0.322046 & -0.884782\end{array}$

$\begin{array}{lllll}11 \mathrm{~A} & 0.532116 & 0.322047 & 0.884781\end{array}$

$\begin{array}{llll}12 \mathrm{~A} & 0.000429 & -0.625584 & 0.884606\end{array}$

$\begin{array}{llll}13 \mathrm{~A} & 0.000427 & -0.625586 & -0.884605\end{array}$

$\begin{array}{|llll|}14 A & -0.552085 & 0.303626 & 0.883196\end{array}$

$\begin{array}{llll}15 A & -0.552086 & 0.303624 & -0.883196\end{array}$

$\begin{array}{llll}16 \mathrm{~B} & -0.031372 & 1.578491 & -1.186247\end{array}$

$\begin{array}{llll}17 \mathrm{~B} & -0.031371 & 1.578493 & 1.186245\end{array}$

$\begin{array}{llll}18 \mathrm{~B} & 1.741811 & 0.987932 & 0.884137\end{array}$

$\begin{array}{llll}19 \mathrm{~B} & 1.741811 & 0.987931 & -0.884139\end{array}$

$\begin{array}{llll}20 \mathrm{~B} & -1.377059 & -0.819581 & -1.151288\end{array}$

$\begin{array}{llll}21 B & -1.377057 & -0.819580 & 1.151291\end{array}$

$\begin{array}{llll}22 \mathrm{~B} & 0.032029 & -2.006462 & 0.878355\end{array}$

$\begin{array}{llll}23 \mathrm{~B} & 0.032027 & -2.006464 & -0.878351\end{array}$

$\begin{array}{llll}24 \mathrm{~B} & -0.031393 & 0.005594 & 2.159577\end{array}$

$\begin{array}{llll}25 B & -0.031395 & 0.005591 & -2.159576\end{array}$

$\begin{array}{llll}26 \mathrm{~B} & -1.750912 & 0.986234 & 0.876720\end{array}$

$\begin{array}{llll}27 \mathrm{~B} & -1.750912 & 0.986233 & -0.876720\end{array}$

$\begin{array}{llll}28 \mathrm{~B} & 2.498238 & -0.371923 & -0.000001\end{array}$

$\begin{array}{llll}29 B & 1.596463 & -1.957796 & 0.000001\end{array}$

$\begin{array}{llll}30 \mathrm{~B} & 1.014092 & 2.341037 & -0.000002\end{array}$

\section{$E 31 / \varepsilon=-152.530780$}

atom $\quad \mathrm{x} / \sigma \quad \mathrm{yl}$

$\begin{array}{llll}1 \mathrm{~A} & 0.000000 & 0.000000 & 0.000000\end{array}$

$\begin{array}{llll}2 \mathrm{~A} & 1.111655 & 0.000000 & 0.000000\end{array}$

$\begin{array}{llll}3 \mathrm{~A} & 0.549155 & -0.966542 & 0.000000\end{array}$

$\begin{array}{llll}4 \mathrm{~A} & -0.543364 & -0.968461 & 0.000000\end{array}$

$\begin{array}{llll}5 A & 0.573624 & 0.950853 & 0.000000\end{array}$

$\begin{array}{llll}6 \mathrm{~B} & 1.345605 & 0.766203 & 1.249307\end{array}$

$\begin{array}{llll}7 \mathrm{~B} & -0.001465 & -1.548455 & -1.249307\end{array}$

$\begin{array}{lllll}8 \mathrm{~B} & 1.345605 & 0.766203 & -1.249307\end{array}$

$\begin{array}{llll}9 \mathrm{~B} & -0.001465 & -1.548455 & 1.249307\end{array}$

$\begin{array}{llll}10 \mathrm{~A} & -1.110490 & -0.004729 & 0.000000\end{array}$

$\begin{array}{llll}11 \mathrm{~A} & -0.544464 & 0.967869 & 0.000000\end{array}$

$\begin{array}{llll}12 \mathrm{~A} & 0.537141 & -0.312600 & -0.883861\end{array}$

$\begin{array}{llll}13 \mathrm{~A} & 0.537141 & -0.312600 & 0.883861\end{array}$

$\begin{array}{lllll}14 \mathrm{~A} & -0.002246 & 0.627668 & -0.882948\end{array}$

$\begin{array}{llll}15 A & -0.002246 & 0.627668 & 0.882948\end{array}$

$\begin{array}{llll}16 \mathrm{~A} & -0.546844 & -0.308111 & 0.882948\end{array}$

$\begin{array}{llll}17 A & -0.546844 & -0.308111 & -0.882948\end{array}$

$\begin{array}{llll}18 \mathrm{~B} & 1.731064 & -1.007432 & -0.878621\end{array}$

$\begin{array}{llll}19 B & 1.731064 & -1.007432 & 0.878621\end{array}$

$\begin{array}{llll}20 \mathrm{~B} & -1.384138 & 0.805532 & -1.146673\end{array}$

$\begin{array}{llll}21 B & -1.384138 & 0.805532 & 1.146673\end{array}$

$\begin{array}{llll}22 \mathrm{~B} & 0.026032 & 2.006569 & -0.877599\end{array}$

$\begin{array}{llll}23 \mathrm{~B} & 0.026032 & 2.006569 & 0.877599\end{array}$

$\begin{array}{llll}24 \mathrm{~B} & -1.731781 & -1.013867 & 0.877599\end{array}$

$\begin{array}{llll}25 \mathrm{~B} & -1.731781 & -1.013867 & -0.877599\end{array}$

$\begin{array}{llll}26 \mathrm{~B} & -0.034039 & 0.019812 & -2.161164\end{array}$

$\begin{array}{llll}27 \mathrm{~B} & -0.034039 & 0.019812 & 2.161164\end{array}$

$\begin{array}{llll}28 \mathrm{~B} & 2.496930 & 0.358889 & 0.000000\end{array}$

$\begin{array}{llll}29 B & 0.921427 & -2.348281 & 0.000000\end{array}$

$\begin{array}{llll}30 \mathrm{~B} & 1.593947 & 1.958895 & 0.000000\end{array}$

$\begin{array}{llll}31 \mathrm{~B} & -0.915789 & -2.353563 & 0.000000\end{array}$

\section{$E 32 / \varepsilon=-158.700621$}

atom $\mathrm{x} / \sigma \quad \mathrm{y} / \sigma$

$\begin{array}{lll}1 \mathrm{~A} & 0.000000 & 0.000000\end{array}$

$\begin{array}{lll}2 \mathrm{~A} & 1.109419 & 0.000000\end{array}$

3A $\quad-0.546424 \quad-0.969308$

$\begin{array}{llll}4 \mathrm{~A} & 0.573324 & -0.953146\end{array}$

$\begin{array}{lll}5 A & -1.111540 & -0.033924\end{array}$

$\begin{array}{lll}6 \mathrm{~A} & 0.546824 & 0.970669\end{array}$

$\begin{array}{lll}7 \mathrm{~A} & -0.571168 & 0.951843\end{array}$

$\begin{array}{llll}8 \mathrm{~B} & 1.348077 & -0.760762\end{array}$

$\begin{array}{lll}9 \mathrm{~B} & 1.348077 & -0.760762\end{array}$

10B $-1.328880-0.801599$

$\begin{array}{lll}11 B & -1.328880 & -0.801599\end{array}$

$\begin{array}{llll}12 \mathrm{~A} & 0.008753 & -0.623296\end{array}$

13A $0.008753 \quad-0.623296$

$\begin{array}{lll}14 \mathrm{~A} & 0.534477 & 0.326489\end{array}$

$\begin{array}{lll}15 \mathrm{~A} & 0.534477 & 0.326489\end{array}$

$\begin{array}{lll}16 \mathrm{~A} & -0.549212 & 0.303555\end{array}$

$\begin{array}{lll}17 \mathrm{~A} & -0.549212 & 0.303555\end{array}$

$\begin{array}{lll}18 \mathrm{~B} & -0.042727 & 1.582394\end{array}$

$\begin{array}{lll}19 B & -0.042727 & 1.582394\end{array}$
$-0.878582$

$\begin{array}{llll}22 \mathrm{~B} & 1.741787 & 0.991782 & -0.883207\end{array}$

$\begin{array}{llll}23 \mathrm{~B} & 1.741787 & 0.991782 & 0.883207\end{array}$

$\begin{array}{lllll}24 \mathrm{~B} & -1.762319 & 0.959537 & -0.876624\end{array}$

$\begin{array}{llll}25 B & -1.762319 & 0.959537 & 0.876624\end{array}$

$\begin{array}{llll}26 \mathrm{~B} & -0.001363 & 0.028465 & 2.160625\end{array}$

$\begin{array}{llll}27 \mathrm{~B} & -0.001363 & 0.028465 & -2.160625\end{array}$

$\begin{array}{llll}28 B & 1.591467 & -1.959546 & 0.000000\end{array}$

$\begin{array}{lllll}29 \mathrm{~B} & -1.537647 & -2.003470 & 0.000000\end{array}$

$\begin{array}{llll}30 \mathrm{~B} & 2.500099 & -0.368701 & 0.000000\end{array}$

$\begin{array}{llll}31 \mathrm{~B} & -2.489404 & -0.431081 & 0.000000\end{array}$

$\begin{array}{llll}32 \mathrm{~B} & 1.012489 & 2.343453 & 0.000000\end{array}$

\section{$E 33 / \varepsilon=-165.783355$}

atom $\mathrm{x} / \sigma \quad \mathrm{yl} \sigma$

$\begin{array}{llll}1 \mathrm{~A} & 0.000000 & 0.000000 & 0.000000\end{array}$

$\begin{array}{llll}2 \mathrm{~A} & 1.110416 & 0.000000 & 0.000000\end{array}$

$\begin{array}{llll}3 A & -0.555210 & -0.961648 & 0.000000\end{array}$

$\begin{array}{lllll}4 \mathrm{~A} & -0.555208 & 0.961650 & -0.000002\end{array}$

$\begin{array}{lllll}5 \mathrm{~A} & 0.540981 & -0.969723 & 0.000000\end{array}$

$\begin{array}{llll}6 A & -1.110296 & 0.016358 & -0.000002\end{array}$

$\begin{array}{llll}7 \mathrm{~A} & 0.569313 & 0.953366 & -0.000001\end{array}$

$\begin{array}{llll}8 \mathrm{~B} & -0.011430 & -1.551391 & 1.245128\end{array}$

$\begin{array}{llll}9 B & -0.011429 & -1.551392 & -1.245128\end{array}$

$\begin{array}{llll}10 B & -1.337832 & 0.785596 & 1.245124\end{array}$

$\begin{array}{llll}11 B & 1.349259 & 0.765798 & -1.245128\end{array}$

$\begin{array}{lllll}12 \mathrm{~B} & -1.337830 & 0.785591 & -1.245132\end{array}$

$\begin{array}{llll}13 B & 1.349257 & 0.765800 & 1.245127\end{array}$

$\begin{array}{llll}14 \mathrm{~A} & 0.004606 & 0.625384 & 0.881184\end{array}$

$\begin{array}{llll}15 A & -0.543901 & -0.308702 & -0.881186\end{array}$

$\begin{array}{llll}16 A & 0.004607 & 0.625382 & -0.881187\end{array}$

$\begin{array}{llll}17 A & -0.543902 & -0.308700 & 0.881185\end{array}$

$\begin{array}{lllll}18 A & 0.539294 & -0.316682 & -0.881186\end{array}$

$\begin{array}{lllll}19 A & 0.539293 & -0.316680 & 0.881186\end{array}$

$\begin{array}{lllll}20 \mathrm{~B} & 0.014763 & 2.004044 & -0.877172\end{array}$

$\begin{array}{llll}21 \mathrm{~B} & -1.742937 & -0.989236 & 0.877166\end{array}$

$\begin{array}{llll}22 \mathrm{~B} & -1.742936 & -0.989238 & -0.877169\end{array}$

$\begin{array}{llll}23 B & 0.014760 & 2.004047 & 0.877163\end{array}$

$\begin{array}{llll}24 \mathrm{~B} & 1.728172 & -1.014807 & 0.877168\end{array}$

$\begin{array}{lllll}25 \mathrm{~B} & 1.728172 & -1.014808 & -0.877167\end{array}$

$\begin{array}{lllll}26 \mathrm{~B} & 0.000001 & -0.000002 & -2.163218\end{array}$

$\begin{array}{llll}27 \mathrm{~B} & -0.000003 & 0.000002 & 2.163216\end{array}$

$\begin{array}{llll}28 \mathrm{~B} & 2.498055 & 0.353020 & 0.000000\end{array}$

$\begin{array}{lllll}29 B & 1.583853 & 1.963750 & -0.000002\end{array}$

$\begin{array}{llll}30 \mathrm{~B} & -2.492584 & 0.389783 & -0.000004\end{array}$

$\begin{array}{llll}31 \mathrm{~B} & 0.908728 & -2.353532 & 0.000001\end{array}$

$\begin{array}{llll}32 \mathrm{~B} & -0.943306 & -2.339889 & 0.000000\end{array}$ $\begin{array}{llll}33 \mathrm{~B} & -1.554753 & 1.986870 & -0.000007\end{array}$

$E 34 / \varepsilon=-171.326596$

atom $\mathrm{x} / \sigma \quad \mathrm{y} / \sigma$

$\begin{array}{llll}1 \mathrm{~A} & 0.000000 & 0.000000 & 0.000000\end{array}$

$\begin{array}{llll}2 \mathrm{~B} & 1.618302 & 0.000000 & 0.000000\end{array}$

$\begin{array}{llll}3 \mathrm{~B} & -1.618285 & -0.008059 & 0.000000\end{array}$

$\begin{array}{llll}4 \mathrm{~A} & -0.544558 & -0.761444 & -0.554486\end{array}$

$\begin{array}{llll}5 \mathrm{~A} & -0.544557 & -0.761736 & 0.554087\end{array}$

$\begin{array}{llll}6 \mathrm{~A} & 0.548341 & -0.759015 & 0.554087\end{array}$

$\begin{array}{lllll}7 \mathrm{~A} & 0.548341 & -0.758724 & -0.554486\end{array}$

$\begin{array}{llll}8 \mathrm{~A} & -0.547642 & 0.292006 & 0.894201\end{array}$

$\begin{array}{lllll}9 \mathrm{~A} & -0.547644 & 0.292477 & -0.894046\end{array}$

$\begin{array}{lllll}10 \mathrm{~A} & 0.546178 & 0.295201 & -0.894046\end{array}$

$\begin{array}{llll}11 \mathrm{~A} & 0.546179 & 0.294730 & 0.894202\end{array}$

$\begin{array}{llll}12 \mathrm{~A} & -0.548320 & 0.940366 & 0.000249\end{array}$

$\begin{array}{llll}13 \mathrm{~A} & 0.543627 & 0.943087 & 0.000249\end{array}$

$\begin{array}{llll}14 \mathrm{~A} & 0.003797 & -1.525526 & -0.000401\end{array}$

$\begin{array}{llll}15 B & 0.004593 & -1.845978 & 1.365047\end{array}$

$\begin{array}{lllll}16 \mathrm{~B} & 0.004591 & -1.845259 & -1.366018\end{array}$

$\begin{array}{lllll}17 \mathrm{~B} & -1.380424 & -1.771869 & -0.000463\end{array}$

$\begin{array}{llll}18 \mathrm{~B} & 1.389227 & -1.764974 & -0.000463\end{array}$

$\begin{array}{llll}19 \mathrm{~A} & -0.003098 & 1.243455 & -0.900533\end{array}$

$\begin{array}{llll}20 \mathrm{~A} & -0.003097 & 1.242981 & 0.901190\end{array}$

$\begin{array}{llll}21 \mathrm{~A} & 0.001157 & -0.465120 & 1.458638\end{array}$ 


\begin{abstract}
$\begin{array}{llll}22 \mathrm{~A} & 0.001155 & -0.464352 & -1.458881\end{array}$
$\begin{array}{llll}23 \mathrm{~B} & -0.005738 & 2.302391 & 0.000607\end{array}$

$\begin{array}{llll}24 \mathrm{~B} & -0.001789 & 0.717492 & 2.188162\end{array}$

$\begin{array}{llll}25 \mathrm{~B} & -0.001791 & 0.718644 & -2.187783\end{array}$

$\begin{array}{llll}26 \mathrm{~B} & -1.393761 & -0.547120 & 1.671351\end{array}$

$\begin{array}{llll}27 B & -1.393764 & -0.546239 & -1.671636\end{array}$

28B $\quad 1.396465 \quad-0.539290-1.671636$

$\begin{array}{llll}29 \mathrm{~B} & 1.396466 & -0.540172 & 1.671352\end{array}$

$\begin{array}{llll}30 \mathrm{~B} & -1.401657 & 1.418966 & 1.028970\end{array}$

31B $\quad-1.401659 \quad 1.419507 \quad-1.028219$

$\begin{array}{llll}\text { 32B } & 1.394570 & 1.425929 & 1.028971\end{array}$

$\begin{array}{lllll}33 \mathrm{~B} & 1.394569 & 1.426470 & -1.028220\end{array}$

$\begin{array}{llll}34 \mathrm{~B} & 0.007355 & -2.954436 & -0.000778\end{array}$
\end{abstract}

\section{$E 35 / \varepsilon=-177.660242$}

\begin{tabular}{|c|c|c|c|}
\hline tom & $\mathrm{x} / \sigma$ & $y / \sigma$ & $z / \sigma$ \\
\hline $1 \mathrm{~A}$ & 0.000000 & 0.000000 & 0.000000 \\
\hline $2 A$ & 1.109480 & 0.000000 & 0.000000 \\
\hline $3 A$ & -0.554485 & -0.960986 & 0.000000 \\
\hline $4 \mathrm{~A}$ & 0.565808 & -0.954264 & 0.013740 \\
\hline $5 A$ & 0.543769 & 0.966690 & -0.027756 \\
\hline $6 \mathrm{~A}$ & -0.554485 & 0.960100 & -0.041226 \\
\hline $7 A$ & -1.109067 & -0.013312 & -0.027210 \\
\hline $8 B$ & -1.379087 & -0.773058 & 1.183843 \\
\hline $9 B$ & -0.023736 & 1.605884 & 1.149589 \\
\hline 10B & 1.358816 & -0.756628 & 1.217422 \\
\hline $11 \mathrm{~A}$ & -0.554745 & 0.328621 & 0.872832 \\
\hline $12 \mathrm{~A}$ & 0.529189 & 0.335126 & 0.886126 \\
\hline $13 \mathrm{~A}$ & -0.007394 & -0.606695 & 0.899687 \\
\hline 14B & 1.357617 & -0.791298 & -1.237771 \\
\hline $15 B$ & -1.317261 & -0.807347 & -1.270578 \\
\hline 16B & 0.006892 & 1.516830 & -1.304044 \\
\hline $17 \mathrm{~A}$ & -0.529736 & 0.288811 & -0.902534 \\
\hline $18 \mathrm{~A}$ & 0.014590 & -0.641338 & -0.875827 \\
\hline $19 A$ & 0.548208 & 0.295278 & -0.889314 \\
\hline $20 \mathrm{~B}$ & -1.763793 & 1.016012 & 0.772887 \\
\hline $21 B$ & 0.001462 & -2.000467 & 0.859496 \\
\hline $22 \mathrm{~B}$ & 1.731990 & 1.036988 & 0.815761 \\
\hline 23B & 1.734919 & 0.988625 & -0.928359 \\
\hline $24 B$ & -1.723367 & 0.967874 & -0.970773 \\
\hline 25B & 0.022953 & -2.016249 & -0.885093 \\
\hline $26 B$ & 0.026958 & -0.046680 & -2.175177 \\
\hline $27 B$ & 0.849314 & 0.562066 & 2.265423 \\
\hline $28 \mathrm{~B}$ & -0.022247 & -0.967717 & 2.287450 \\
\hline $29 B$ & -0.911300 & 0.551501 & 2.243830 \\
\hline 30B & -0.951384 & 2.343862 & -0.115327 \\
\hline $31 B$ & 0.925734 & 2.355125 & -0.092304 \\
\hline $32 \mathrm{~B}$ & -2.502566 & -0.378803 & -0.076122 \\
\hline 33 & -1.554683 & -1.998552 & -0.029617 \\
\hline $34 \mathrm{~B}$ & 1.578809 & -1.979750 & 0.008815 \\
\hline & & & \\
\hline
\end{tabular}

$\begin{array}{lll}2.508045 & -0.348738 & -0.014670\end{array}$

\section{$E 36 / \varepsilon=-184.177344$}

\begin{tabular}{|c|c|c|c|}
\hline atom & $\mathrm{x} / \sigma$ & $y / \sigma$ & $z / \sigma$ \\
\hline $1 \mathrm{~A}$ & 0.000000 & 0.000000 & 0.000000 \\
\hline $2 A$ & 1.154512 & 0.000000 & 0.000000 \\
\hline $3 A$ & -1.154496 & -0.006374 & 0.000000 \\
\hline $4 \mathrm{~A}$ & -0.552026 & 0.596382 & 0.709352 \\
\hline $5 A$ & 0.548723 & 0.599421 & 0.709352 \\
\hline $6 \mathrm{~A}$ & -0.546362 & -0.478976 & 0.806677 \\
\hline $7 \mathrm{~A}$ & 0.548998 & -0.475952 & 0.806677 \\
\hline $8 B$ & -0.005195 & 1.881359 & 0.632465 \\
\hline $9 A$ & -0.550136 & 0.886881 & -0.346966 \\
\hline $10 \mathrm{~A}$ & 0.545230 & 0.889905 & -0.346966 \\
\hline $11 \mathrm{~A}$ & 0.551522 & -0.931716 & -0.176642 \\
\hline $12 \mathrm{~A}$ & -0.546370 & -0.934747 & -0.176642 \\
\hline $13 \mathrm{~A}$ & 0.558494 & -0.093719 & -0.914764 \\
\hline $14 \mathrm{~A}$ & -0.557969 & -0.096802 & -0.914764 \\
\hline $15 B$ & -0.002587 & 0.936753 & -1.695580 \\
\hline $16 B$ & -1.679966 & 1.379866 & 0.443093 \\
\hline 17B & 1.672320 & 1.389122 & 0.443092 \\
\hline $18 \mathrm{~B}$ & 0.004901 & -1.775037 & 0.886541 \\
\hline $19 \mathrm{~A}$ & -0.000355 & 0.128598 & 1.518807 \\
\hline
\end{tabular}
$\begin{array}{llll}19 \mathrm{~A} & -0.000355 & 0.128598 & 1.518807\end{array}$ $\begin{array}{lllll}21 B & 1.693406 & 0.730569 & -1.188683\end{array}$

$\begin{array}{llll}22 \mathrm{~B} & 1.666056 & -1.321570 & 0.677619\end{array}$

$\begin{array}{llll}23 B & -1.658733 & -1.330750 & 0.677619\end{array}$

$\begin{array}{llll}24 \mathrm{~A} & -1.463557 & 0.075049 & 1.065316\end{array}$

$\begin{array}{llll}25 \mathrm{~A} & 1.463120 & 0.083130 & 1.065316\end{array}$

$\begin{array}{llll}26 \mathrm{~B} & 0.908563 & 1.077137 & 1.998487\end{array}$

$\begin{array}{llll}27 \mathrm{~B} & -0.914499 & 1.072102 & 1.998488\end{array}$

$\begin{array}{llll}28 \mathrm{~B} & -0.899643 & -0.742490 & 2.151832\end{array}$

$\begin{array}{llll}29 B & 0.903730 & -0.737509 & 2.151832\end{array}$

30B $\quad 0.003561 \quad-1.290045 \quad-1.453559$

$\begin{array}{lllll}31 \mathrm{~B} & 1.686007 & -0.988827 & -1.029253\end{array}$

$\begin{array}{llll}32 \mathrm{~B} & -1.680523 & -0.998122 & -1.029253\end{array}$

$\begin{array}{lllll}33 \mathrm{~B} & 0.864834 & 2.232744 & -0.859561\end{array}$

$\begin{array}{llll}34 \mathrm{~B} & -0.877151 & 2.227934 & -0.859560\end{array}$

$\begin{array}{llll}35 B & -2.619140 & -0.020379 & 0.129931\end{array}$

$\begin{array}{llll}36 B & 2.619211 & -0.005916 & 0.129930\end{array}$

\section{$E 37 / \varepsilon=-190.811208$}

$\begin{array}{llll}\text { atom } & \mathrm{x} / \sigma & \mathrm{g} / \sigma & \mathrm{z} / \sigma\end{array}$

$\begin{array}{llll}1 \mathrm{~A} & 0.000000 & 0.000000 & 0.000000\end{array}$

$\begin{array}{lllll}2 \mathrm{~A} & 1.150537 & 0.000000 & 0.000000\end{array}$

$\begin{array}{llll}3 \mathrm{~A} & -1.149780 & -0.041732 & 0.000000\end{array}$

$\begin{array}{llll}4 \mathrm{~A} & -0.552248 & 0.226903 & 0.905705\end{array}$

$\begin{array}{llll}5 A & 0.543656 & 0.246785 & 0.905704\end{array}$

$\begin{array}{lllll}6 \mathrm{~A} & 0.538184 & 0.923707 & 0.066199\end{array}$

$\begin{array}{llll}7 \mathrm{~A} & -0.571334 & 0.903578 & 0.066200\end{array}$

$\begin{array}{lllll}8 \mathrm{~B} & -0.031558 & 1.739423 & -0.913514\end{array}$

$\begin{array}{llll}9 \mathrm{~A} & -0.554498 & 0.342633 & -0.881853\end{array}$

$\begin{array}{lllll}10 A & 0.541704 & 0.362520 & -0.881853\end{array}$

$\begin{array}{llll}11 B & 0.010416 & -0.574227 & -1.865591\end{array}$

$\begin{array}{llll}12 \mathrm{~A} & 0.552614 & -0.767811 & 0.541150\end{array}$

$\begin{array}{llll}13 A & -0.524400 & -0.787350 & 0.541150\end{array}$

$\begin{array}{llll}14 \mathrm{~A} & 0.559113 & -0.719087 & -0.565667\end{array}$

$\begin{array}{llll}15 A & -0.532664 & -0.738894 & -0.565666\end{array}$

$\begin{array}{llll}16 \mathrm{~B} & 0.010268 & -0.565858 & 1.924832\end{array}$

$\begin{array}{llll}17 \mathrm{~B} & -1.713489 & 1.199826 & -0.682384\end{array}$

$\begin{array}{llll}18 \mathrm{~B} & 1.668839 & 1.261189 & -0.682388\end{array}$

$\begin{array}{llll}19 \mathrm{~A} & -0.021219 & 1.169626 & 0.966478\end{array}$

$\begin{array}{llll}20 B & -1.669776 & -0.441465 & -1.348102\end{array}$

$\begin{array}{llll}21 \mathrm{~B} & 1.684687 & -0.380608 & -1.348105\end{array}$

$\begin{array}{llll}22 \mathrm{~B} & 1.650716 & -0.466021 & 1.410198\end{array}$

$\begin{array}{llll}23 \mathrm{~B} & -1.632723 & -0.525591 & 1.410201\end{array}$

$\begin{array}{llll}24 \mathrm{~B} & -1.542472 & -1.567734 & -0.009070\end{array}$

$\begin{array}{llll}25 \mathrm{~B} & 1.598322 & -1.510753 & -0.009072\end{array}$

$\begin{array}{llll}26 \mathrm{~A} & -1.489316 & 0.760168 & 0.685376\end{array}$

$\begin{array}{llll}27 \mathrm{~A} & 1.460765 & 0.813689 & 0.685373\end{array}$

$\begin{array}{llll}28 \mathrm{~B} & -0.932614 & 0.995798 & 2.023689\end{array}$

$\begin{array}{llll}29 B & 0.895884 & 1.028972 & 2.023687\end{array}$

$\begin{array}{llll}\text { 30B } & 0.882847 & 2.178457 & 0.612067\end{array}$

$\begin{array}{llll}31 B & -0.961282 & 2.145000 & 0.612069\end{array}$

$\begin{array}{llll}32 \mathrm{~B} & 0.036384 & -2.005551 & -0.809700\end{array}$

$\begin{array}{llll}33 \mathrm{~B} & 0.036708 & -2.023345 & 0.930529\end{array}$

$\begin{array}{lllll}34 \mathrm{~B} & 0.861027 & 0.926029 & -2.196418\end{array}$

$\begin{array}{llll}35 B & -0.894055 & 0.894189 & -2.196416\end{array}$

$\begin{array}{llll}36 \mathrm{~B} & -2.600681 & -0.067816 & 0.083844\end{array}$

$\begin{array}{llll}37 \mathrm{~B} & 2.601429 & 0.026562 & 0.083839\end{array}$

\section{$E 38 / \varepsilon=-197.608976$}

atom $\mathrm{x} / \sigma \quad \mathrm{y} / \sigma$

$\begin{array}{llll}1 \mathrm{~A} & 0.000000 & 0.000000 & 0.000000\end{array}$

$\begin{array}{llll}2 \mathrm{~A} & 1.155549 & 0.000000 & 0.000000\end{array}$

$\begin{array}{llll}3 A & -1.155547 & -0.003219 & 0.000000\end{array}$

$\begin{array}{llll}4 \mathrm{~A} & -0.548423 & -0.759408 & -0.539851\end{array}$

$\begin{array}{llll}5 A & -0.548423 & -0.759340 & 0.539946\end{array}$

$\begin{array}{lllll}6 \mathrm{~A} & 0.550534 & -0.757878 & -0.539851\end{array}$

$\begin{array}{llll}7 \mathrm{~A} & 0.550534 & -0.757811 & 0.539946\end{array}$

$\begin{array}{llll}8 \mathrm{~B} & 0.001114 & -0.801061 & 1.826460\end{array}$

$\begin{array}{llll}9 \mathrm{~B} & 0.001114 & -0.801289 & -1.826360\end{array}$

$\begin{array}{llll}10 A & -0.547396 & 0.262185 & 0.923629\end{array}$

$\begin{array}{lllll}11 \mathrm{~A} & -0.547396 & 0.262070 & -0.923662\end{array}$

$\begin{array}{lllll}12 \mathrm{~A} & 0.546662 & 0.263594 & -0.923662\end{array}$ $\begin{array}{llll}13 A & 0.546662 & 0.263709 & 0.923630\end{array}$ \begin{tabular}{|llll}
$14 \mathrm{~A}$ & -0.562478 & 0.912410 & -0.000057
\end{tabular}

$\begin{array}{lllll}15 A & 0.559933 & 0.913974 & -0.000057\end{array}$

$\begin{array}{llll}16 \mathrm{~B} & -0.002230 & 1.600563 & 1.094585\end{array}$

$\begin{array}{llll}17 \mathrm{~B} & -0.002231 & 1.600427 & -1.094784\end{array}$

$\begin{array}{llll}18 \mathrm{~B} & -1.671833 & -0.572444 & 1.354807\end{array}$

$\begin{array}{llll}19 B & -1.671833 & -0.572613 & -1.354736\end{array}$

$\begin{array}{lllll}20 \mathrm{~B} & 1.673419 & -0.567954 & -1.354736\end{array}$

$\begin{array}{llll}21 \mathrm{~B} & 1.673418 & -0.567786 & 1.354808\end{array}$

$\begin{array}{llll}22 \mathrm{~A} & 0.002117 & -1.521390 & 0.000095\end{array}$

$\begin{array}{llll}23 \mathrm{~B} & 1.693393 & 1.116275 & -0.856765\end{array}$

$\begin{array}{llll}24 \mathrm{~B} & 1.693394 & 1.116381 & 0.856627\end{array}$

$\begin{array}{llll}25 \mathrm{~B} & -1.696499 & 1.111660 & 0.856626\end{array}$

$\begin{array}{llll}26 \mathrm{~B} & -1.696499 & 1.111552 & -0.856765\end{array}$

$\begin{array}{llll}27 \mathrm{~A} & 1.463687 & -1.068858 & 0.000067\end{array}$

$\begin{array}{llll}28 A & -1.460707 & -1.072931 & 0.000067\end{array}$

$\begin{array}{llll}29 \mathrm{~B} & -0.903758 & -2.088446 & -0.905070\end{array}$

$\begin{array}{llll}30 \mathrm{~B} & -0.903758 & -2.088333 & 0.905331\end{array}$

$\begin{array}{lllll}31 \mathrm{~B} & 0.909568 & -2.085921 & -0.905070\end{array}$

$\begin{array}{llll}32 \mathrm{~B} & 0.909568 & -2.085808 & 0.905330\end{array}$

$\begin{array}{llll}33 \mathrm{~B} & 2.623797 & -0.128552 & 0.000008\end{array}$

$\begin{array}{llll}34 \mathrm{~B} & -2.623433 & -0.135860 & 0.000008\end{array}$

$\begin{array}{llll}35 \mathrm{~B} & 0.867431 & 0.651884 & 2.315249\end{array}$

$\begin{array}{lllll}36 \mathrm{~B} & 0.867431 & 0.651596 & -2.315329\end{array}$

$\begin{array}{lllll}37 \mathrm{~B} & -0.869247 & 0.649176 & -2.315330\end{array}$

$\begin{array}{llll}38 \mathrm{~B} & -0.869247 & 0.649466 & 2.315248\end{array}$

$E 39 / \varepsilon=-204.172478$

atom $\quad \mathrm{x} / \sigma \quad \mathrm{y} / \sigma$

$\begin{array}{llll}1 \mathrm{~A} & 0.000000 & 0.000000 & 0.000000\end{array}$

$\begin{array}{llll}2 \mathrm{~A} & 1.136410 & 0.000000 & 0.000000\end{array}$

$\begin{array}{llll}3 \mathrm{~A} & -1.143537 & -0.002641 & 0.000000\end{array}$

$\begin{array}{llll}4 \mathrm{~A} & 0.538178 & 0.773548 & -0.537200\end{array}$

$\begin{array}{llll}5 \mathrm{~A} & 0.538178 & 0.773564 & 0.537177\end{array}$

$\begin{array}{lllll}6 \mathrm{~A} & 0.548433 & -0.257702 & -0.883426\end{array}$

$\begin{array}{llll}7 A & 0.548433 & -0.257677 & 0.883433\end{array}$

$\begin{array}{lllll}8 A & 0.540885 & -0.946045 & 0.000013\end{array}$

$\begin{array}{llll}9 \mathrm{~A} & -0.547538 & 0.764642 & -0.536506\end{array}$

$\begin{array}{llll}10 A & -0.547538 & 0.764657 & 0.536483\end{array}$

$\begin{array}{llll}11 \mathrm{~A} & -0.543943 & -0.266603 & -0.881634\end{array}$

$\begin{array}{llll}12 \mathrm{~A} & -0.543943 & -0.266578 & 0.881642\end{array}$

$\begin{array}{llll}13 \mathrm{~A} & -0.551512 & -0.946508 & 0.000013\end{array}$

$\begin{array}{llll}14 \mathrm{~B} & -0.009551 & -1.487040 & 1.271062\end{array}$

$\begin{array}{llll}15 \mathrm{~B} & -0.009551 & -1.487076 & -1.271021\end{array}$

$\begin{array}{llll}16 \mathrm{~B} & -0.054985 & 1.972658 & -0.000029\end{array}$

$\begin{array}{llll}17 \mathrm{~B} & 1.679712 & -1.075571 & 0.888129\end{array}$

$\begin{array}{llll}18 \mathrm{~B} & 1.679712 & -1.075596 & -0.888098\end{array}$

$\begin{array}{llll}19 B & 1.623520 & 1.494286 & -0.000022\end{array}$

$\begin{array}{llll}20 B & -1.741000 & 1.317435 & -0.000020\end{array}$

$\begin{array}{llll}21 \mathrm{~A} & -0.007169 & 0.503796 & -1.437652\end{array}$

$\begin{array}{llll}22 \mathrm{~A} & -0.007170 & 0.503838 & 1.437637\end{array}$

$\begin{array}{llll}23 \mathrm{~B} & -1.720512 & -1.028916 & 0.875359\end{array}$

$\begin{array}{llll}24 \mathrm{~B} & -1.720512 & -1.028940 & -0.875330\end{array}$

$\begin{array}{llll}25 \mathrm{~A} & 1.468090 & 0.376603 & 0.978114\end{array}$

$\begin{array}{lllll}26 \mathrm{~A} & 1.468090 & 0.376574 & -0.978125\end{array}$

$\begin{array}{llll}27 \mathrm{~B} & 0.931836 & -0.178252 & 2.230395\end{array}$

$\begin{array}{lllll}28 \mathrm{~B} & 0.931836 & -0.178317 & -2.230391\end{array}$

$\begin{array}{llll}29 B & 0.901036 & 1.568928 & 1.626183\end{array}$

$\begin{array}{llll}\text { 30B } & 0.901037 & 1.568880 & -1.626230\end{array}$

$\begin{array}{llll}31 \mathrm{~B} & -0.891673 & -0.216592 & 2.250495\end{array}$

$\begin{array}{llll}32 \mathrm{~B} & -0.891673 & -0.216658 & -2.250489\end{array}$

$\begin{array}{llll}33 B & -0.904933 & 1.586391 & 1.606767\end{array}$

$\begin{array}{llll}34 \mathrm{~B} & -0.904931 & 1.586345 & -1.606814\end{array}$

$\begin{array}{llll}35 \mathrm{~A} & -1.433638 & 0.363338 & 1.034265\end{array}$

$\begin{array}{lllll}36 \mathrm{~A} & -1.433637 & 0.363309 & -1.034276\end{array}$

$\begin{array}{lllll}37 \mathrm{~B} & 0.853293 & -2.355343 & 0.000033\end{array}$

$\begin{array}{llll}38 \mathrm{~B} & 2.584732 & 0.100668 & -0.000001\end{array}$

$\begin{array}{llll}39 \mathrm{~B} & -0.907274 & -2.335082 & 0.000032\end{array}$

$E 40 / \varepsilon=-210.847376$

$\begin{array}{llll}\text { atom } & \mathrm{x} / \sigma & \mathrm{l} / \sigma & \mathrm{\sigma} / \sigma\end{array}$

$\begin{array}{cccc}1 \mathrm{~A} & 0.000000 & 0.000000 & 0.000000\end{array}$

$\begin{array}{llll}2 \mathrm{~A} & 1.144330 & 0.000000 & 0.000000\end{array}$

$\begin{array}{llll}3 \mathrm{~A} & -1.144293 & -0.009329 & 0.000000\end{array}$

$\begin{array}{llll}4 \mathrm{~A} & 0.542524 & 0.770811 & -0.536078\end{array}$ 
$\begin{array}{llll}6 \mathrm{~A} & -0.548790 & 0.766305 & 0.536162\end{array}$

$\begin{array}{lllll}7 A & -0.548791 & 0.766363 & -0.536078\end{array}$

$\begin{array}{lllll}8 A & 0.550508 & -0.258313 & -0.880661\end{array}$

$\begin{array}{llll}9 A & -0.548384 & -0.262887 & 0.880632\end{array}$

$\begin{array}{llll}10 A & -0.548385 & -0.262791 & -0.880660\end{array}$

$\begin{array}{lllll}11 A & 0.550509 & -0.258409 & 0.880632\end{array}$

$\begin{array}{llll}12 \mathrm{~A} & -0.541928 & -0.950186 & -0.000051\end{array}$

$\begin{array}{lllll}13 \mathrm{~A} & 0.549654 & -0.945737 & -0.000052\end{array}$

14B $\quad 0.006046 \quad-1.483861 \quad-1.273857$

$\begin{array}{llll}15 \mathrm{~B} & 0.006048 & -1.484000 & 1.273695\end{array}$

$\begin{array}{llll}16 \mathrm{~B} & -0.008054 & 1.975970 & 0.000107\end{array}$

$\begin{array}{llll}17 \mathrm{~B} & 1.687869 & -1.070780 & 0.892077\end{array}$

$\begin{array}{llll}18 \mathrm{~B} & -1.679086 & -1.084404 & -0.892194\end{array}$

$\begin{array}{llll}19 \mathrm{~B} & -1.679084 & -1.084502 & 0.892079\end{array}$

$\begin{array}{llll}\text { 20B } & 1.687867 & -1.070683 & -0.892197\end{array}$

$\begin{array}{llll}21 B & 1.644248 & 1.476813 & 0.000079\end{array}$

$\begin{array}{llll}22 \mathrm{~B} & -1.656231 & 1.463362 & 0.000080\end{array}$

$\begin{array}{llll}23 \mathrm{~A} & -0.002048 & 0.502298 & -1.433953\end{array}$

$\begin{array}{llll}24 \mathrm{~A} & -0.002046 & 0.502142 & 1.434008\end{array}$

$\begin{array}{lllll}25 \mathrm{~A} & 1.470605 & 0.374303 & -0.981848\end{array}$

$\begin{array}{llll}26 \mathrm{~A} & -1.473607 & 0.362197 & 0.981888\end{array}$

$\begin{array}{lllll}27 A & -1.473609 & 0.362304 & -0.981847\end{array}$

$\begin{array}{llll}28 \mathrm{~A} & 1.470606 & 0.374196 & 0.981888\end{array}$

$\begin{array}{llll}29 B & -0.921378 & -0.189469 & 2.232891\end{array}$

$\begin{array}{llll}30 \mathrm{~B} & 0.922890 & -0.181709 & -2.232911\end{array}$

$\begin{array}{llll}31 \mathrm{~B} & -0.921381 & -0.189227 & -2.232910\end{array}$

$\begin{array}{llll}32 \mathrm{~B} & 0.922893 & -0.181953 & 2.232890\end{array}$

$\begin{array}{llll}33 \mathrm{~B} & 0.900681 & 1.570707 & -1.622090\end{array}$

$\begin{array}{llll}34 \mathrm{~B} & -0.913455 & 1.563136 & 1.622260\end{array}$

$\begin{array}{llll}35 \mathrm{~B} & -0.913458 & 1.563311 & -1.622090\end{array}$

$\begin{array}{llll}36 \mathrm{~B} & 0.900684 & 1.570530 & 1.622260\end{array}$

$\begin{array}{lllll}37 \mathrm{~B} & 0.883144 & -2.351986 & -0.000129\end{array}$

$\begin{array}{llll}38 \mathrm{~B} & -0.863945 & -2.359107 & -0.000128\end{array}$

$\begin{array}{llll}39 \mathrm{~B} & 2.601826 & 0.088001 & 0.000003\end{array}$

$\begin{array}{llll}40 B & -2.602458 & 0.066791 & 0.000007\end{array}$

$E 41 / \varepsilon=-216.848113$

atom $\mathrm{x} / \sigma \quad y / \sigma \quad z / \sigma$

$\begin{array}{llll}1 \mathrm{~A} & 0.000000 & 0.000000 & 0.000000\end{array}$

$\begin{array}{llll}2 \mathrm{~A} & 1.140841 & 0.000000 & 0.000000\end{array}$

$\begin{array}{llll}3 A & -1.140761 & -0.005391 & 0.000000\end{array}$

$\begin{array}{llll}4 \mathrm{~A} & 0.526343 & 0.557824 & 0.780325\end{array}$

$\begin{array}{llll}5 \mathrm{~A} & 0.538910 & -0.531443 & 0.752808\end{array}$

$\begin{array}{lllll}6 \mathrm{~A} & 0.553749 & 0.895774 & -0.253561\end{array}$

$\begin{array}{llll}7 A & -0.541168 & 0.900826 & -0.274735\end{array}$

$\begin{array}{llll}8 A & -0.534478 & 0.043311 & -0.929723\end{array}$

$\begin{array}{llll}9 \mathrm{~A} & -0.551755 & -0.560085 & 0.740905\end{array}$

$\begin{array}{llll}10 \mathrm{~A} & 0.563634 & 0.050496 & -0.923694\end{array}$

$\begin{array}{llll}11 \mathrm{~A} & -0.553513 & 0.570727 & 0.749324\end{array}$

$\begin{array}{llll}12 \mathrm{~A} & 0.563377 & -0.882515 & -0.341952\end{array}$

$\begin{array}{llll}13 \mathrm{~B} & 0.038142 & -0.952281 & -1.733237\end{array}$

$\begin{array}{llll}14 \mathrm{~A} & -0.531198 & -0.890830 & -0.346136\end{array}$

$\begin{array}{llll}15 \mathrm{~B} & 0.050698 & -1.849455 & 0.577274\end{array}$

$\begin{array}{llll}16 \mathrm{~B} & 0.016708 & 1.946126 & 0.489965\end{array}$

$\begin{array}{llll}17 \mathrm{~A} & -0.070667 & -0.000376 & 1.546151\end{array}$

$\begin{array}{llll}18 \mathrm{~B} & -1.657813 & 0.034370 & 1.437117\end{array}$

$\begin{array}{llll}19 \mathrm{~B} & 1.693281 & -1.298702 & 0.455340\end{array}$

$\begin{array}{llll}20 B & -1.663878 & -1.358424 & 0.394740\end{array}$

21B $1.716956-0.690529-1.213193$

$\begin{array}{llll}22 \mathrm{~B} & -1.652380 & -0.726934 & -1.257305\end{array}$

$\begin{array}{llll}23 \mathrm{~B} & 1.641799 & 1.390221 & 0.494780\end{array}$

$\begin{array}{llll}24 \mathrm{~B} & -1.646911 & 1.440383 & 0.406154\end{array}$

$\begin{array}{llll}25 \mathrm{~A} & 0.020733 & 0.945432 & -1.198266\end{array}$

$\begin{array}{llll}26 \mathrm{~B} & 0.954904 & -0.907062 & 2.025769\end{array}$

$\begin{array}{llll}27 \mathrm{~B} & 0.995472 & 0.872680 & 2.064413\end{array}$

$\begin{array}{llll}28 \mathrm{~A} & -1.453838 & 0.659805 & -0.836583\end{array}$

$\begin{array}{lllll}29 \mathrm{~A} & 1.486317 & 0.675444 & -0.798160\end{array}$

$\begin{array}{llll}30 \mathrm{~A} & 1.480763 & 0.019525 & 1.028027\end{array}$

$\begin{array}{lllll}31 B & 0.942776 & 1.999237 & -1.047848\end{array}$

$\begin{array}{llll}32 \mathrm{~B} & -0.882687 & 0.558850 & -2.197260\end{array}$

$\begin{array}{llll}33 \mathrm{~B} & -0.892786 & 2.001535 & -1.091503\end{array}$

$\begin{array}{llll}34 \mathrm{~B} & 0.951203 & 0.574782 & -2.177146\end{array}$ \begin{tabular}{|llll}
$35 B$ & -0.673550 & 1.351445 & 1.937894
\end{tabular} $\begin{array}{llll}36 \mathrm{~B} & -0.754799 & -1.312153 & 1.970887\end{array}$ $\begin{array}{lllll}37 \mathrm{~B} & -2.591971 & 0.084691 & -0.069387\end{array}$ $\begin{array}{llll}38 \mathrm{~B} & -0.824696 & -2.225283 & -0.887339\end{array}$ $\begin{array}{llll}39 \mathrm{~B} & 2.613113 & 0.093509 & 0.031197\end{array}$ $\begin{array}{llll}\text { 40B } & 0.938148 & -2.205765 & -0.875082\end{array}$ $\begin{array}{llll}41 B & -0.271373 & 0.013807 & 2.975001\end{array}$

\section{$E 42 / \varepsilon=-223.652390$}

atom $\mathrm{x} / \sigma$

$\begin{array}{cccc}1 \mathrm{~A} & 0.000000 & 0.000000 & 0.000000\end{array}$

$\begin{array}{llll}2 \mathrm{~A} & 1.079447 & 0.000000 & 0.000000\end{array}$

$\begin{array}{llll}3 A & 0.539723 & -0.995012 & 0.000000\end{array}$

$\begin{array}{llll}4 \mathrm{~A} & 0.539723 & -0.321118 & 0.896033\end{array}$

$\begin{array}{lllll}5 \mathrm{~A} & 0.539724 & -0.321118 & -0.896032\end{array}$

$\begin{array}{llll}6 \mathrm{~A} & 0.539724 & 0.923037 & 0.000000\end{array}$

$\begin{array}{lll}7 \mathrm{~A} & 1.614951 & 1.017331\end{array}$

0.000000

$\begin{array}{llll}8 \mathrm{~A} & -0.535504 & 1.017332 & -0.000001\end{array}$

$\begin{array}{llll}9 \mathrm{~A} & 0.001601 & 0.621520 & 0.898884\end{array}$

$\begin{array}{llll}10 \mathrm{~A} & 1.077846 & 0.621519 & 0.898884\end{array}$

$\begin{array}{llll}11 \mathrm{~A} & 0.001602 & 0.621520 & -0.898884\end{array}$

$\begin{array}{lllll}12 \mathrm{~A} & 1.077847 & 0.621519 & -0.898884\end{array}$

$\begin{array}{lllll}13 \mathrm{~B} & 0.539724 & 1.989826 & 0.867888\end{array}$

$\begin{array}{llll}14 \mathrm{~B} & 0.539725 & 1.989826 & -0.867887\end{array}$

$\begin{array}{llll}15 \mathrm{~A} & -0.921060 & 0.141479 & -0.559048\end{array}$

$\begin{array}{llll}16 \mathrm{~A} & 2.000508 & 0.141478 & -0.559047\end{array}$

$\begin{array}{llll}17 \mathrm{~A} & -0.921061 & 0.141479 & 0.559047\end{array}$

$\begin{array}{llll}18 \mathrm{~A} & 2.000508 & 0.141478 & 0.559048\end{array}$

$\begin{array}{llll}19 A & -0.387013 & -0.841443 & -0.557660\end{array}$

$\begin{array}{lllll}20 \mathrm{~A} & 1.466460 & -0.841444 & -0.557659\end{array}$

$\begin{array}{llll}21 \mathrm{~A} & 1.466459 & -0.841443 & 0.557659\end{array}$

$\begin{array}{llll}22 \mathrm{~A} & -0.387013 & -0.841443 & 0.557659\end{array}$

$\begin{array}{lllll}23 \mathrm{~B} & 1.641452 & -0.333741 & -1.847459\end{array}$

$\begin{array}{lllll}24 \mathrm{~B} & -0.562003 & -0.333740 & -1.847460\end{array}$

$\begin{array}{llll}25 \mathrm{~B} & 1.641452 & -0.333741 & 1.847460\end{array}$

$\begin{array}{llll}26 \mathrm{~B} & -0.562006 & -0.333741 & 1.847459\end{array}$

$\begin{array}{llll}27 \mathrm{~B} & 0.539723 & -1.628457 & -1.377154\end{array}$

$\begin{array}{llll}28 \mathrm{~B} & 0.539722 & -1.628456 & 1.377154\end{array}$

$\begin{array}{llll}29 B & 2.140820 & 1.334209 & -1.413776\end{array}$

$\begin{array}{llll}\text { 30B } & -1.061372 & 1.334210 & 1.413776\end{array}$

$\begin{array}{llll}31 \mathrm{~B} & -1.061370 & 1.334210 & -1.413777\end{array}$

$\begin{array}{llll}32 \mathrm{~B} & 2.140818 & 1.334209 & 1.413777\end{array}$

$\begin{array}{llll}33 \mathrm{~B} & 2.776614 & -0.911491 & 0.000001\end{array}$

$\begin{array}{llll}34 \mathrm{~B} & -1.697167 & -0.911489 & 0.000000\end{array}$

$\begin{array}{llll}35 \mathrm{~B} & 0.539723 & 0.942280 & 2.211803\end{array}$

$\begin{array}{llll}36 \mathrm{~B} & 0.539725 & 0.942282 & -2.211803\end{array}$

$\begin{array}{llll}37 \mathrm{~B} & 1.459799 & -2.152966 & 0.000001\end{array}$

$\begin{array}{llll}38 \mathrm{~B} & -0.380354 & -2.152965 & 0.000000\end{array}$

$\begin{array}{llll}39 \mathrm{~B} & 3.078280 & 0.883781 & 0.000001\end{array}$

$\begin{array}{llll}40 \mathrm{~B} & -1.998832 & 0.883783 & -0.000001\end{array}$

$\begin{array}{llll}41 \mathrm{~B} & -0.957071 & 2.375869 & -0.000001\end{array}$

$\begin{array}{llll}42 \mathrm{~B} & 2.036521 & 2.375868 & 0.000000\end{array}$

\section{$E 43 / \varepsilon=-230.062836$}

\section{atom $\mathrm{x} / \sigma$}

$\begin{array}{llll}1 \mathrm{~A} & 0.000000 & 0.000000 & 0.000000\end{array}$

$\begin{array}{llll}2 \mathrm{~A} & 1.091241 & 0.000000 & 0.000000\end{array}$

$\begin{array}{llll}3 A & 0.545620 & -0.995625 & 0.000000\end{array}$

$\begin{array}{lllll}4 \mathrm{~A} & 0.545620 & 0.925731 & -0.037968\end{array}$

$\begin{array}{llll}5 A & -0.558158 & 1.002593 & -0.000912\end{array}$

$\begin{array}{lllll}6 A & 1.649398 & 1.002593 & -0.000913\end{array}$

$\begin{array}{llll}7 \mathrm{~B} & 0.545621 & 2.049912 & 0.695760\end{array}$

$\begin{array}{llll}8 \mathrm{~A} & 0.545621 & -0.305620 & 0.884513\end{array}$

$\begin{array}{lllll}9 A & 0.545620 & -0.323758 & -0.875085\end{array}$

$\begin{array}{lllll}10 \mathrm{~A} & 1.097743 & 0.612831 & -0.893883\end{array}$

$\begin{array}{llll}11 \mathrm{~A} & -0.006503 & 0.612831 & -0.893883\end{array}$

$\begin{array}{llll}12 \mathrm{~A} & 0.011088 & 0.648636 & 0.873861\end{array}$

$\begin{array}{llll}13 \mathrm{~A} & 1.080153 & 0.648636 & 0.873861\end{array}$

$\begin{array}{llll}14 \mathrm{~A} & -0.381824 & -0.841263 & -0.576753\end{array}$

$\begin{array}{llll}15 A & 1.473065 & -0.841263 & -0.576754\end{array}$

$\begin{array}{llll}16 A & -0.924324 & 0.118295 & -0.566526\end{array}$

$\begin{array}{llll}17 A & 2.015564 & 0.118295 & -0.566527\end{array}$ $\begin{array}{llll}18 \mathrm{~A} & -0.377612 & -0.841271 & 0.563390\end{array}$ \begin{tabular}{|llll}
$19 A$ & 1.468853 & -0.841271 & 0.563389
\end{tabular}

$\begin{array}{llll}20 A & -0.913306 & 0.131081 & 0.569621\end{array}$

$\begin{array}{llll}21 \mathrm{~A} & 2.004547 & 0.131082 & 0.569620\end{array}$

$\begin{array}{llll}22 \mathrm{~B} & -0.417439 & -0.260031 & -1.904414\end{array}$

$\begin{array}{lllll}23 B & 1.508678 & -0.260031 & -1.904415\end{array}$

$\begin{array}{llll}24 \mathrm{~B} & -0.489767 & -0.282226 & 1.862788\end{array}$

$\begin{array}{llll}25 \mathrm{~B} & 1.581009 & -0.282226 & 1.862788\end{array}$

$\begin{array}{llll}26 \mathrm{~B} & 0.545619 & 1.317641 & -1.972492\end{array}$

$\begin{array}{llll}27 \mathrm{~B} & 0.545621 & -1.618934 & 1.378379\end{array}$

$\begin{array}{lllll}28 \mathrm{~B} & 0.545620 & -1.633319 & -1.377647\end{array}$

$\begin{array}{lllll}29 B & 2.233286 & 1.274808 & -1.403638\end{array}$

$\begin{array}{llll}30 \mathrm{~B} & -1.142048 & 1.274809 & -1.403636\end{array}$

$\begin{array}{lllll}31 \mathrm{~A} & 1.082446 & 1.683706 & -0.650473\end{array}$

$\begin{array}{lllll}32 \mathrm{~A} & 0.008793 & 1.683706 & -0.650473\end{array}$

$\begin{array}{llll}33 \mathrm{~B} & -1.055428 & 1.352969 & 1.393076\end{array}$

$\begin{array}{llll}34 \mathrm{~B} & 2.146669 & 1.352970 & 1.393074\end{array}$

$\begin{array}{llll}35 \mathrm{~B} & 0.545621 & 1.091799 & 2.139981\end{array}$

$\begin{array}{llll}36 \mathrm{~B} & -1.684868 & -0.934837 & 0.014654\end{array}$

$\begin{array}{llll}37 \mathrm{~B} & 2.776109 & -0.934837 & 0.014652\end{array}$

$\begin{array}{llll}38 \mathrm{~B} & 1.460259 & -2.152250 & 0.001298\end{array}$

$\begin{array}{llll}39 \mathrm{~B} & -0.369018 & -2.152250 & 0.001299\end{array}$

$\begin{array}{llll}40 \mathrm{~B} & 3.113684 & 0.829824 & 0.030024\end{array}$

$\begin{array}{llll}41 B & -2.022444 & 0.829823 & 0.030026\end{array}$

$\begin{array}{llll}42 \mathrm{~B} & 2.153210 & 2.365138 & -0.022472\end{array}$

$\begin{array}{llll}43 \mathrm{~B} & -1.061971 & 2.365138 & -0.022470\end{array}$

$E 44 / \varepsilon=-237.368902$

atom $\mathrm{x} / \sigma \quad y / \sigma$

$\begin{array}{llll}1 \mathrm{~A} & 0.000000 & 0.000000 & 0.000000\end{array}$

$\begin{array}{llll}2 \mathrm{~A} & 1.071802 & 0.000000 & 0.000000\end{array}$

$\begin{array}{llll}3 \mathrm{~A} & 0.512712 & -0.941215 & 0.000000\end{array}$

$\begin{array}{llll}4 \mathrm{~B} & -0.948092 & 0.563175 & 0.767588\end{array}$

$\begin{array}{lllll}5 \mathrm{~A} & 1.646268 & -0.977897 & -0.036770\end{array}$

$\begin{array}{llll}6 \mathrm{~A} & -0.634431 & -0.909000 & 0.039983\end{array}$

$\begin{array}{llll}7 A & 0.494759 & 0.991966 & 0.039982\end{array}$

$\begin{array}{lllll}8 \mathrm{~A} & 1.082206 & -0.642839 & 0.871997\end{array}$

$\begin{array}{lllll}9 \mathrm{~A} & 1.043342 & -0.619754 & -0.885706\end{array}$

$\begin{array}{lllll}10 A & 0.519736 & 0.330863 & -0.872022\end{array}$

$\begin{array}{llll}11 \mathrm{~A} & -0.041928 & -0.614685 & -0.872022\end{array}$

$\begin{array}{llll}12 A & -0.009906 & -0.616021 & 0.898621\end{array}$

$\begin{array}{llll}13 \mathrm{~A} & 0.536227 & 0.303383 & 0.898621\end{array}$

$\begin{array}{llll}14 \mathrm{~B} & -0.441293 & 0.262131 & -1.900688\end{array}$

$\begin{array}{lllll}15 A & 1.968139 & -0.088771 & -0.610358\end{array}$

$\begin{array}{lllll}16 A & 1.019443 & -1.685879 & -0.610358\end{array}$

$\begin{array}{lllll}17 \mathrm{~A} & 1.418402 & 0.867278 & -0.565026\end{array}$

$\begin{array}{llll}18 A & -0.083096 & -1.660462 & -0.565026\end{array}$

$\begin{array}{llll}19 A & 1.058690 & -1.704810 & 0.532165\end{array}$

$\begin{array}{llll}20 \mathrm{~A} & 2.003538 & -0.114179 & 0.532164\end{array}$

$\begin{array}{llll}21 \mathrm{~A} & 1.441396 & 0.843838 & 0.573804\end{array}$

$\begin{array}{llll}22 \mathrm{~A} & -0.051512 & -1.669441 & 0.573805\end{array}$

$\begin{array}{llll}23 \mathrm{~A} & -0.384903 & 0.853248 & -0.578725\end{array}$

$\begin{array}{lllll}24 \mathrm{~A} & -0.933414 & -0.070158 & -0.578724\end{array}$

$\begin{array}{llll}25 \mathrm{~B} & 1.434260 & 0.259867 & -1.918322\end{array}$

$\begin{array}{lllll}26 \mathrm{~B} & 0.457893 & -1.383823 & -1.918322\end{array}$

$\begin{array}{llll}27 \mathrm{~B} & 1.617153 & 0.246004 & 1.850568\end{array}$

$\begin{array}{llll}28 B & 0.557559 & -1.537799 & 1.850569\end{array}$

$\begin{array}{lllll}29 B & -1.216964 & -1.230075 & -1.357306\end{array}$

$\begin{array}{llll}30 \mathrm{~B} & 0.498052 & 1.657114 & -1.357308\end{array}$

$\begin{array}{llll}31 \mathrm{~B} & 2.223746 & -1.320923 & 1.318452\end{array}$

$\begin{array}{llll}32 \mathrm{~B} & 2.149156 & -1.276618 & -1.439686\end{array}$

$\begin{array}{llll}33 B & 0.468235 & 1.574212 & 1.428062\end{array}$

$\begin{array}{llll}34 \mathrm{~B} & -1.158424 & -1.164232 & 1.428064\end{array}$

$\begin{array}{llll}35 \mathrm{~B} & -0.083531 & 0.049620 & 2.177798\end{array}$

$\begin{array}{lllll}36 \mathrm{~B} & 0.448893 & -2.863222 & -0.023576\end{array}$

$\begin{array}{llll}37 \mathrm{~B} & 2.729105 & 0.975465 & -0.023578\end{array}$

$\begin{array}{llll}38 \mathrm{~B} & 2.170468 & -2.353694 & -0.079401\end{array}$

$\begin{array}{llll}39 B & 3.105198 & -0.780097 & -0.079402\end{array}$

$\begin{array}{llll}40 B & -1.243656 & -2.241041 & 0.055178\end{array}$

$\begin{array}{llll}41 \mathrm{~B} & 1.373074 & 2.164166 & 0.055176\end{array}$

$\begin{array}{llll}42 \mathrm{~B} & -0.460381 & 2.107991 & 0.046154\end{array}$

$\begin{array}{llll}43 \mathrm{~B} & -2.071385 & -0.604100 & 0.046156\end{array}$ $\begin{array}{llrr}44 \mathrm{~B} & -1.763339 & 1.047438 & -0.775909\end{array}$ 


\begin{tabular}{|c|c|c|c|}
\hline \multicolumn{4}{|c|}{$E 45 / \varepsilon=-243.724171$} \\
\hline atom & $\mathrm{x} / \sigma$ & $y / \sigma$ & $z / \sigma$ \\
\hline $1 \mathrm{~A}$ & 0.000000 & 0.000000 & 0.000000 \\
\hline $2 \mathrm{~A}$ & 1.095586 & 0.000000 & 0.000000 \\
\hline $3 B$ & 0.549776 & -2.164716 & 0.000000 \\
\hline $4 \mathrm{~A}$ & 0.548740 & -0.861015 & 0.327050 \\
\hline $5 A$ & 0.545758 & 0.942487 & -0.315102 \\
\hline $6 \mathrm{~A}$ & -0.558142 & -0.950999 & 0.320286 \\
\hline $7 \mathrm{~A}$ & 1.655247 & -0.950275 & 0.318352 \\
\hline $8 \mathrm{~A}$ & 0.551327 & 0.003201 & -0.935250 \\
\hline $9 A$ & -0.005424 & -0.292629 & 1.041882 \\
\hline $10 \mathrm{~A}$ & 0.546775 & 0.587057 & 0.722029 \\
\hline $11 \mathrm{~A}$ & 1.096559 & -0.291323 & 1.044222 \\
\hline $12 \mathrm{~A}$ & 0.015898 & -0.896253 & -0.620985 \\
\hline $3 \mathrm{~A}$ & 1.085136 & -0.895558 & -0.621106 \\
\hline $14 \mathrm{~A}$ & -0.927175 & 0.067783 & 0.574351 \\
\hline $5 B$ & -0.353606 & 0.837929 & 1.765762 \\
\hline $16 \mathrm{~A}$ & -0.394579 & 0.977052 & 0.286340 \\
\hline 17B & 0.553247 & -0.674599 & 2.285477 \\
\hline 18B & -1.121487 & -0.779173 & 1.749182 \\
\hline 19A & 1.475761 & 0.980458 & 0.280539 \\
\hline $20 A$ & 2.017782 & 0.066036 & 0.578943 \\
\hline $21 \mathrm{~A}$ & -0.909162 & -0.316094 & -0.497842 \\
\hline $22 \mathrm{~A}$ & 1.471689 & 0.616175 & -0.801804 \\
\hline $23 \mathrm{~A}$ & 2.010037 & -0.307642 & -0.496616 \\
\hline $24 \mathrm{~A}$ & -0.365832 & 0.609817 & -0.807424 \\
\hline $25 \mathrm{~A}$ & 0.014299 & -1.374568 & 1.137682 \\
\hline $26 \mathrm{~A}$ & 1.087915 & -1.373377 & 1.138383 \\
\hline $27 B$ & 1.510460 & 0.849800 & 1.725283 \\
\hline $28 B$ & -0.488921 & -0.337021 & -1.853141 \\
\hline $29 B$ & 1.588421 & -0.335615 & -1.851776 \\
\hline $30 B$ & 2.229435 & -0.769734 & 1.741810 \\
\hline 31B & 0.550667 & 1.087637 & -1.825093 \\
\hline $32 B$ & 0.541568 & 1.985022 & 0.785720 \\
\hline $33 B$ & -2.013660 & -0.805216 & 0.251748 \\
\hline 4B & -1.045708 & -1.741719 & -0.864921 \\
\hline B & -1.680959 & 0.858031 & -0.397510 \\
\hline $36 B$ & 2.143494 & -1.742917 & -0.866932 \\
\hline 37B & 0.549411 & -1.724692 & -1.674801 \\
\hline $38 \mathrm{~B}$ & -0.368729 & 2.030758 & -0.687869 \\
\hline $39 B$ & 2.779151 & 0.877620 & -0.306876 \\
\hline $40 B$ & 1.459799 & 2.036697 & -0.686212 \\
\hline $41 B$ & 3.111497 & -0.807869 & 0.246509 \\
\hline $42 B$ & -1.028918 & -2.253941 & 0.804847 \\
\hline $43 B$ & 2.128299 & -2.253876 & 0.804512 \\
\hline $44 \mathrm{~B}$ & 0.550261 & -2.504616 & 1.778491 \\
\hline $45 B$ & -2.028350 & 0.662199 & 1.294033 \\
\hline
\end{tabular}

$E 46 / \varepsilon=-251.048217$

atom $x / \sigma$

$\begin{array}{cccc}1 \mathrm{~A} & 0.000000 & 0.000000 & 0.000000\end{array}$

$\begin{array}{llll}2 \mathrm{~A} & 1.096126 & 0.000000 & 0.000000\end{array}$

$\begin{array}{llll}3 B & 0.560782 & -2.165423 & 0.000000\end{array}$

$\begin{array}{llll}4 \mathrm{~A} & 0.551329 & -0.861354 & -0.326656\end{array}$

$\begin{array}{llll}5 \mathrm{~A} & 0.547697 & 0.940826 & 0.318518\end{array}$

$\begin{array}{llll}6 \mathrm{~A} & -0.556120 & -0.950132 & -0.321746\end{array}$

$\begin{array}{lllll}7 A & 1.657999 & -0.949627 & -0.319642\end{array}$

$\begin{array}{llll}8 A & 0.552700 & 0.000703 & 0.935918\end{array}$

$\begin{array}{lllll}9 \mathrm{~A} & 0.544605 & 0.588134 & -0.720938\end{array}$

$\begin{array}{llll}10 A & -0.004297 & -0.294873 & -1.041021\end{array}$

11A $\quad 1.096971 \quad-0.289889-1.043916$

$\begin{array}{llll}12 \mathrm{~B} & -0.361831 & 0.829750 & -1.763356\end{array}$

$\begin{array}{llll}13 \mathrm{~A} & 0.018624 & -0.897812 & 0.619892\end{array}$

$\begin{array}{llll}14 \mathrm{~A} & -0.391341 & 0.980634 & -0.278730\end{array}$

$\begin{array}{llll}15 \mathrm{~A} & 1.088606 & -0.896107 & 0.620601\end{array}$

$\begin{array}{lllll}16 \mathrm{~A} & -0.927026 & 0.073607 & -0.578416\end{array}$

$\begin{array}{llll}17 \mathrm{~B} & 0.555092 & -0.674522 & -2.285184\end{array}$

$\begin{array}{llll}18 \mathrm{~B} & -1.114567 & -0.794906 & -1.748636\end{array}$

$\begin{array}{lllll}19 \mathrm{~A} & 1.476734 & 0.979615 & -0.278166\end{array}$

$\begin{array}{llll}19 \mathrm{~B} & 0.553797 & 1.984809 & -0.776075\end{array}$

$\begin{array}{llll}21 \mathrm{~A} & 2.018528 & 0.067443 & -0.579877\end{array}$

$\begin{array}{llll}22 \mathrm{~A} & -0.907086 & -0.317925 & 0.497086\end{array}$ $\begin{array}{llll}23 \mathrm{~A} & -0.365688 & 0.608301 & 0.809222\end{array}$ $\begin{array}{|llll|}24 \mathrm{~A} & 1.473841 & 0.612708 & 0.803248\end{array}$ $\begin{array}{llll}25 \mathrm{~A} & 2.012831 & -0.307861 & 0.494050\end{array}$ $\begin{array}{llll}26 \mathrm{~B} & -1.682406 & 0.851108 & 0.389838\end{array}$ $\begin{array}{llll}27 \mathrm{~B} & 1.505208 & 0.851599 & -1.725967\end{array}$ $\begin{array}{lllll}28 \mathrm{~A} & 0.020103 & -1.377978 & -1.134731\end{array}$ $\begin{array}{lllll}29 A & 1.092693 & -1.371959 & -1.139749\end{array}$ $\begin{array}{llll}30 \mathrm{~B} & -0.489247 & -0.341192 & 1.852334\end{array}$ $\begin{array}{llll}31 B & 1.597796 & -0.340573 & 1.849885\end{array}$ $\begin{array}{llll}32 \mathrm{~B} & 2.231170 & -0.764228 & -1.744098\end{array}$ $\begin{array}{llll}33 \mathrm{~B} & 0.558942 & 1.074835 & 1.835850\end{array}$ $\begin{array}{llll}34 \mathrm{~B} & -2.005944 & -0.819374 & -0.247794\end{array}$ $\begin{array}{llll}35 \mathrm{~B} & -0.352104 & 2.026726 & 0.714904\end{array}$ $\begin{array}{llll}36 \mathrm{~B} & -1.037184 & -1.748276 & 0.867881\end{array}$ $\begin{array}{llll}37 \mathrm{~B} & 2.148651 & -1.743620 & 0.862299\end{array}$ $\begin{array}{llll}38 \mathrm{~B} & 0.555463 & -1.724991 & 1.674744\end{array}$ $\begin{array}{llll}39 \mathrm{~B} & 1.464648 & 2.031667 & 0.697578\end{array}$ $\begin{array}{llll}40 \mathrm{~B} & 2.781054 & 0.877484 & 0.306996\end{array}$ $\begin{array}{lllll}41 \mathrm{~B} & 3.114682 & -0.805169 & -0.249983\end{array}$ $\begin{array}{lllll}42 \mathrm{~B} & -1.017638 & -2.263237 & -0.799553\end{array}$ $\begin{array}{llll}43 \mathrm{~B} & 2.135921 & -2.250868 & -0.810430\end{array}$ $\begin{array}{lllll}44 \mathrm{~B} & 0.557256 & -2.504386 & -1.779977\end{array}$ $\begin{array}{lllll}45 B & -2.062064 & 0.603269 & -1.308708\end{array}$ $\begin{array}{llll}46 \mathrm{~B} & -1.205891 & 2.054251 & -0.819538\end{array}$

$E 47 / \varepsilon=-258.224693$

$\begin{array}{lccc}\text { atom } & \mathrm{x} / \sigma & y / \sigma & \mathrm{z} / \sigma\end{array}$ $\begin{array}{llll}1 \mathrm{~A} & 0.000000 & 0.000000 & 0.000000\end{array}$ $\begin{array}{llll}2 A & 1.099025 & 0.000000 & 0.000000\end{array}$ $\begin{array}{llll}3 \mathrm{~A} & 0.554605 & -0.948825 & 0.000000\end{array}$ $\begin{array}{llll}4 \mathrm{~A} & 0.547016 & 0.997605 & 0.000349\end{array}$ $\begin{array}{llll}5 \mathrm{~A} & -0.585223 & -0.975683 & 0.000350\end{array}$ $\begin{array}{llll}6 \mathrm{~A} & 1.693966 & -0.971969 & 0.001835\end{array}$ $\begin{array}{llll}7 A & 0.003269 & -0.633246 & 0.867557\end{array}$ $\begin{array}{llll}8 \mathrm{~A} & 0.548353 & 0.316736 & 0.867557\end{array}$

$\begin{array}{lllll}9 A & 1.097637 & -0.629805 & 0.871332\end{array}$ $\begin{array}{llll}10 A & 0.010500 & -0.635303 & -0.871347\end{array}$ $\begin{array}{lllll}11 \mathrm{~A} & 0.553777 & 0.311529 & -0.871347\end{array}$ $\begin{array}{llll}12 \mathrm{~A} & 1.103220 & -0.633010 & -0.869787\end{array}$ $\begin{array}{llll}13 \mathrm{~B} & -0.348661 & 0.200057 & 1.928054\end{array}$ $\begin{array}{llll}14 A & -0.923633 & -0.098103 & 0.579510\end{array}$ $\begin{array}{llll}15 A & -0.381401 & 0.846909 & 0.579509\end{array}$ $\begin{array}{llll}16 A & -0.910415 & -0.111076 & -0.585472\end{array}$ $\begin{array}{llll}17 A & -0.363532 & 0.842043 & -0.585473\end{array}$ $\begin{array}{llll}18 \mathrm{~B} & 0.564933 & 1.623510 & 1.378616\end{array}$ $\begin{array}{llll}19 B & -1.116545 & -1.307002 & 1.378619\end{array}$ $\begin{array}{llll}20 \mathrm{~A} & 0.010272 & -1.698387 & 0.572628\end{array}$ $\begin{array}{llll}21 \mathrm{~A} & 1.471458 & 0.848196 & 0.572626\end{array}$ $\begin{array}{llll}22 \mathrm{~A} & 0.010514 & -1.696405 & -0.578354\end{array}$ $\begin{array}{lllll}23 \mathrm{~A} & 1.469868 & 0.846985 & -0.578356\end{array}$ $\begin{array}{lllll}24 \mathrm{~A} & 1.105413 & -1.694880 & -0.576179\end{array}$ $\begin{array}{lllll}25 \mathrm{~A} & 2.021075 & -0.099047 & -0.576180\end{array}$ $\begin{array}{llll}26 \mathrm{~A} & 1.104355 & -1.694202 & 0.577243\end{array}$ $\begin{array}{llll}27 \mathrm{~A} & 2.019956 & -0.098474 & 0.577242\end{array}$ $\begin{array}{lllll}28 \mathrm{~B} & -1.673060 & 0.959973 & -0.075698\end{array}$ $\begin{array}{llll}29 B & 1.497766 & 0.230951 & 1.901988\end{array}$ $\begin{array}{llll}30 \mathrm{~B} & 0.556437 & -1.409615 & 1.901990\end{array}$ $\begin{array}{llll}31 B & -0.385398 & 0.221133 & -1.909762\end{array}$ $\begin{array}{llll}32 B & 0.562554 & -1.405890 & -1.906301\end{array}$ $\begin{array}{lllll}33 \mathrm{~B} & 1.497634 & 0.223786 & -1.906303\end{array}$ $\begin{array}{llll}34 \mathrm{~B} & -1.116005 & -1.295639 & -1.397085\end{array}$ $\begin{array}{llll}35 \mathrm{~B} & 0.555393 & 1.617305 & -1.397088\end{array}$ $\begin{array}{llll}36 \mathrm{~B} & 2.234221 & -1.281958 & 1.386830\end{array}$ $\begin{array}{llll}37 \mathrm{~B} & 2.239379 & -1.284919 & -1.382984\end{array}$ $\begin{array}{llll}38 \mathrm{~B} & -0.345620 & 2.154836 & -0.018616\end{array}$ $\begin{array}{lllll}39 B & -2.034754 & -0.789019 & -0.018613\end{array}$ $\begin{array}{llll}40 \mathrm{~B} & 1.453667 & 2.159660 & -0.010578\end{array}$ $\begin{array}{llll}41 \mathrm{~B} & -1.130936 & -2.344838 & -0.010573\end{array}$ $\begin{array}{lllll}42 \mathrm{~B} & 3.150172 & -0.765841 & 0.002887\end{array}$ $\begin{array}{llll}43 B & 2.250860 & -2.333180 & 0.002888\end{array}$ $\begin{array}{lllll}44 \mathrm{~B} & 0.561480 & -2.882844 & -0.001539\end{array}$ $\begin{array}{llll}45 \mathrm{~B} & 2.772197 & 0.970036 & -0.001541\end{array}$ $\begin{array}{llll}46 \mathrm{~B} & -1.182581 & 1.686944 & 1.458000\end{array}$ $\begin{array}{llll}47 \mathrm{~B} & -2.053165 & 0.169673 & 1.458001\end{array}$
$E 48 / \varepsilon=-264.768267$

$\begin{array}{ccc}\text { atom } & \mathrm{x} / \sigma & y / \sigma \\ 1 \mathrm{~A} & 0.000000 & 0.0000\end{array}$

$0.000000 \quad 0.000000$

$\begin{array}{llll}2 \mathrm{~A} & 1.097566 & 0.000000 & 0.000000\end{array}$

$\begin{array}{llll}3 \mathrm{~B} & 0.548780 & -2.166060 & 0.000000\end{array}$

$\begin{array}{lllll}4 \mathrm{~A} & 0.548784 & 0.939782 & 0.318824\end{array}$

$\begin{array}{llll}5 \mathrm{~A} & 0.548782 & -0.861152 & -0.326706\end{array}$

$\begin{array}{lllll}6 \mathrm{~A} & -0.559829 & -0.948190 & -0.323345\end{array}$

$\begin{array}{lllll}7 A & 1.657391 & -0.948192 & -0.323347\end{array}$

$\begin{array}{lllll}8 \mathrm{~A} & 0.548783 & -0.005237 & 0.936911\end{array}$

$\begin{array}{lllll}9 \mathrm{~A} & 0.548782 & 0.588938 & -0.717850\end{array}$

10A $\quad 1.100695 \quad-0.292088-1.040859$

$\begin{array}{lllll}11 \mathrm{~A} & -0.003133 & -0.292087 & -1.040858\end{array}$

$\begin{array}{lllll}12 B & -0.367091 & 0.834444 & -1.756109\end{array}$

$\begin{array}{lllll}13 B & 1.464652 & 0.834443 & -1.756112\end{array}$

$\begin{array}{lllll}14 \mathrm{~A} & -0.391347 & 0.980746 & -0.274905\end{array}$

$\begin{array}{lllll}15 \mathrm{~A} & 1.488913 & 0.980745 & -0.274908\end{array}$

$\begin{array}{llll}16 \mathrm{~A} & 0.013554 & -0.900281 & 0.618338\end{array}$

$\begin{array}{llll}17 \mathrm{~A} & 1.084010 & -0.900281 & 0.618337\end{array}$

$\begin{array}{llll}18 \mathrm{~A} & -0.927957 & 0.076023 & -0.577559\end{array}$

$\begin{array}{lllll}19 A & 2.025521 & 0.076021 & -0.577563\end{array}$

$\begin{array}{llll}20 B & 0.548783 & 1.982605 & -0.765255\end{array}$

$\begin{array}{lllll}21 \mathrm{~B} & 0.548779 & -0.677538 & -2.283625\end{array}$

$\begin{array}{llll}22 \mathrm{~B} & -1.118903 & -0.787580 & -1.747437\end{array}$

$\begin{array}{lllll}23 B & 2.216463 & -0.787583 & -1.747442\end{array}$

$\begin{array}{llll}24 \mathrm{~A} & -0.365282 & 0.605896 & 0.810354\end{array}$

$\begin{array}{llll}25 A & 1.462850 & 0.605895 & 0.810353\end{array}$

$\begin{array}{llll}26 \mathrm{~A} & -0.909077 & -0.317202 & 0.495072\end{array}$

$\begin{array}{llll}27 \mathrm{~A} & 2.006643 & -0.317204 & 0.495070\end{array}$

$\begin{array}{llll}28 \mathrm{~B} & 2.780492 & 0.851668 & 0.395069\end{array}$

$\begin{array}{llll}29 B & -1.682925 & 0.851671 & 0.395074\end{array}$

$\begin{array}{llll}30 \mathrm{~A} & 1.084370 & -1.376463 & -1.135203\end{array}$

$\begin{array}{lllll}31 \mathrm{~A} & 0.013190 & -1.376462 & -1.135201\end{array}$

$\begin{array}{llll}32 \mathrm{~B} & -0.501054 & -0.346520 & 1.850327\end{array}$

$\begin{array}{llll}33 \mathrm{~B} & 1.598622 & -0.346523 & 1.850325\end{array}$

$\begin{array}{llll}34 \mathrm{~B} & 0.548786 & 1.060267 & 1.848360\end{array}$

$\begin{array}{llll}35 \mathrm{~B} & 1.451041 & 2.022765 & 0.725519\end{array}$

$\begin{array}{llll}36 \mathrm{~B} & -0.353471 & 2.022766 & 0.725522\end{array}$

$\begin{array}{llll}37 \mathrm{~B} & -2.010135 & -0.815192 & -0.247477\end{array}$

$\begin{array}{lllll}38 \mathrm{~B} & 3.107697 & -0.815197 & -0.247482\end{array}$

$\begin{array}{llll}39 B & 2.141311 & -1.749664 & 0.864400\end{array}$

$\begin{array}{llll}40 \mathrm{~B} & -1.043748 & -1.749660 & 0.864404\end{array}$

$\begin{array}{llll}41 \mathrm{~B} & 0.548782 & -1.725165 & 1.674687\end{array}$

$\begin{array}{lllll}42 \mathrm{~B} & -1.027248 & -2.259718 & -0.804295\end{array}$

$\begin{array}{lllll}43 \mathrm{~B} & 2.124807 & -2.259721 & -0.804299\end{array}$

$\begin{array}{lllll}44 \mathrm{~B} & 0.548778 & -2.504171 & -1.781013\end{array}$

$\begin{array}{lllll}45 \mathrm{~B} & 2.305132 & 2.058349 & -0.809090\end{array}$

$\begin{array}{llll}46 \mathrm{~B} & -1.207566 & 2.058351 & -0.809085\end{array}$

$\begin{array}{lllll}47 \mathrm{~B} & 3.163505 & 0.609913 & -1.303016\end{array}$

$\begin{array}{llll}48 \mathrm{~B} & -2.065943 & 0.609918 & -1.303010\end{array}$

\section{$E 49 / \varepsilon=-271.973689$}

atom $\mathrm{x} / \sigma \quad \mathrm{y} / \sigma$

$\begin{array}{llll}1 \mathrm{~A} & 0.000000 & 0.000000 & 0.000000\end{array}$

$\begin{array}{llll}2 \mathrm{~A} & 1.104542 & 0.000000 & 0.000000\end{array}$

$\begin{array}{llll}3 \mathrm{~A} & 0.567341 & -0.947701 & 0.000000\end{array}$

$\begin{array}{lllll}4 \mathrm{~A} & 0.544068 & 0.994643 & -0.000001\end{array}$

$\begin{array}{llll}5 A & -0.573951 & -0.977703 & 0.000000\end{array}$

$\begin{array}{llll}6 \mathrm{~A} & 1.707621 & -0.967960 & 0.000001\end{array}$

$\begin{array}{llll}7 \mathrm{~A} & 0.018343 & -0.638755 & 0.868337\end{array}$

$\begin{array}{llll}8 \mathrm{~A} & 0.557476 & 0.312354 & 0.868337\end{array}$

$\begin{array}{lllll}9 A & 0.018343 & -0.638756 & -0.868337\end{array}$

$\begin{array}{lllll}10 \mathrm{~A} & 0.557476 & 0.312353 & -0.868337\end{array}$

$\begin{array}{lllll}11 \mathrm{~A} & 1.112199 & -0.630447 & 0.871128\end{array}$

$\begin{array}{llll}12 \mathrm{~A} & 1.112200 & -0.630447 & -0.871128\end{array}$

$\begin{array}{llll}13 \mathrm{~B} & -1.666965 & 0.944914 & -0.000001\end{array}$

$\begin{array}{llll}14 \mathrm{~B} & -0.324287 & 0.183822 & 1.939863\end{array}$

$\begin{array}{llll}15 B & -0.324286 & 0.183820 & -1.939864\end{array}$

$\begin{array}{llll}16 \mathrm{~A} & -0.910521 & -0.111898 & 0.595469\end{array}$

$\begin{array}{llll}17 A & -0.371674 & 0.838706 & -0.595470\end{array}$

$\begin{array}{llll}18 A & -0.371674 & 0.838706 & 0.595469\end{array}$ $\begin{array}{llll}19 A & -0.910521 & -0.111898 & -0.595469\end{array}$ 


$\begin{array}{rrrr}20 \mathrm{~B} & -1.090991 & -1.325027 & -1.389456 \\ 21 \mathrm{~B} & 0.576498 & 1.616664 & -1.389457 \\ 22 \mathrm{~B} & -1.090991 & -1.325026 & 1.389456 \\ 23 \mathrm{~B} & 0.576497 & 1.616666 & 1.389455 \\ 24 \mathrm{~A} & 0.028367 & -1.701005 & -0.571645 \\ 25 \mathrm{~A} & 0.028367 & -1.701005 & 0.571646 \\ 26 \mathrm{~A} & 1.474040 & 0.849371 & 0.571646 \\ 27 \mathrm{~A} & 1.474040 & 0.849370 & -0.571646 \\ 28 \mathrm{~B} & -0.343050 & 2.143455 & -0.000001 \\ 29 \mathrm{~B} & -2.015299 & -0.806633 & 0.000000 \\ 30 \mathrm{~A} & 1.122240 & -1.693126 & 0.574702 \\ 31 \mathrm{~A} & 2.029140 & -0.093224 & 0.574702 \\ 32 \mathrm{~A} & 2.029140 & -0.093224 & -0.574701 \\ 33 \mathrm{~A} & 1.122240 & -1.693127 & -0.574700 \\ 34 \mathrm{~B} & 0.580605 & -1.416885 & 1.900730 \\ 35 \mathrm{~B} & 1.513917 & 0.229612 & 1.900730 \\ 36 \mathrm{~B} & 0.580606 & -1.416887 & -1.900729 \\ 37 \mathrm{~B} & 1.513918 & 0.229610 & -1.900729 \\ 38 \mathrm{~B} & 2.253906 & -1.277620 & -1.382513 \\ 39 \mathrm{~B} & 2.253905 & -1.277619 & 1.382516 \\ 40 \mathrm{~B} & 1.453524 & 2.163181 & -0.000001 \\ 41 \mathrm{~B} & -1.109426 & -2.358232 & 0.000001 \\ 42 \mathrm{~B} & 3.164016 & -0.753476 & 0.000002 \\ 43 \mathrm{~B} & 2.271659 & -2.327721 & 0.000002 \\ 44 \mathrm{~B} & 2.776407 & 0.979780 & 0.000000 \\ 45 \mathrm{~B} & 0.585426 & -2.885426 & 0.000002 \\ 46 \mathrm{~B} & -2.032980 & 0.138483 & -1.501668 \\ 47 \mathrm{~B} & -1.163046 & 1.673172 & -1.501669 \\ 48 \mathrm{~B} & -1.163046 & 1.673174 & 1.501666 \\ 49 \mathrm{~B} & -2.032980 & 0.138485 & 1.501667\end{array}$

$E 50 / \varepsilon=-278.361820$

atom $\quad x / \sigma \quad y / \sigma$

$\begin{array}{cccc}1 \mathrm{~A} & 0.000000 & 0.000000 & 0.000000\end{array}$

$\begin{array}{llll}2 \mathrm{~A} & 1.105597 & 0.000000 & 0.000000\end{array}$

$\begin{array}{llll}3 \mathrm{~A} & 0.565175 & -0.947598 & 0.000000\end{array}$

$\begin{array}{lllll}4 \mathrm{~A} & 0.546246 & 0.994536 & 0.000854\end{array}$

$\begin{array}{llll}5 \mathrm{~A} & -0.575824 & -0.976992 & -0.000036\end{array}$

$\begin{array}{llll}6 \mathrm{~A} & 1.707219 & -0.966528 & 0.003223\end{array}$

$\begin{array}{llll}7 \mathrm{~A} & 0.559434 & 0.313576 & 0.867074\end{array}$

$\begin{array}{llll}8 \mathrm{~A} & 0.017872 & -0.637445 & 0.868648\end{array}$

$\begin{array}{lllll}9 \mathrm{~A} & 0.555437 & 0.310115 & -0.868839\end{array}$

10A $\quad 0.015269 \quad-0.639686-0.868629$

$\begin{array}{llll}11 \mathrm{~A} & 1.107639 & -0.633850 & -0.871642\end{array}$

$\begin{array}{llll}12 \mathrm{~A} & 1.112395 & -0.631202 & 0.868274\end{array}$

$\begin{array}{llll}13 \mathrm{~B} & -0.335137 & 0.186077 & 1.934601\end{array}$

$\begin{array}{lllll}14 \mathrm{~B} & -1.666683 & 0.947482 & -0.003064\end{array}$

$\begin{array}{llll}15 B & -0.325110 & 0.182788 & -1.940963\end{array}$

$\begin{array}{llll}16 \mathrm{~A} & -0.371835 & 0.839233 & 0.593147\end{array}$

$\begin{array}{llll}17 A & -0.370431 & 0.839494 & -0.595340\end{array}$

$\begin{array}{llll}18 A & -0.912318 & -0.110546 & 0.593055\end{array}$

19A $\quad-0.911058 \quad-0.110465 \quad-0.595992$

$\begin{array}{llll}20 \mathrm{~B} & 0.572175 & 1.613067 & 1.388464\end{array}$

$\begin{array}{llll}21 \mathrm{~A} & 1.481851 & 0.851576 & 0.569334\end{array}$

$\begin{array}{llll}22 B & 1.469182 & 0.192822 & 1.929550\end{array}$

$\begin{array}{llll}23 \mathrm{~A} & 2.033826 & -0.081595 & 0.577979\end{array}$

$\begin{array}{lllll}24 \mathrm{~A} & 1.468308 & 0.845652 & -0.576474\end{array}$

$\begin{array}{llll}25 \mathrm{~B} & -0.338612 & 2.144378 & -0.001936\end{array}$

$\begin{array}{lllll}26 \mathrm{~B} & 0.577208 & 1.614762 & -1.392521\end{array}$

$\begin{array}{llll}27 \mathrm{~B} & -1.094805 & -1.322559 & 1.386839\end{array}$

$\begin{array}{lllll}28 A & 0.026593 & -1.701272 & -0.571483\end{array}$

$\begin{array}{llll}29 \mathrm{~A} & 0.025820 & -1.700547 & 0.570672\end{array}$

$\begin{array}{llll}30 \mathrm{~B} & -1.093576 & -1.324858 & -1.390117\end{array}$

$\begin{array}{lllll}31 B & -2.017482 & -0.804630 & -0.002888\end{array}$

$\begin{array}{llll}32 \mathrm{~A} & 1.121146 & -1.693809 & -0.572044\end{array}$

$\begin{array}{llll}33 \mathrm{~A} & 1.120430 & -1.693581 & 0.574475\end{array}$

$\begin{array}{llll}34 \mathrm{~A} & 2.021312 & -0.102625 & -0.582030\end{array}$

$\begin{array}{llll}35 \mathrm{~B} & 0.576358 & -1.423852 & 1.896507\end{array}$

$\begin{array}{llll}36 \mathrm{~B} & 1.511410 & 0.225163 & -1.902800\end{array}$

$\begin{array}{llll}37 \mathrm{~B} & 0.577218 & -1.420700 & -1.900233\end{array}$

$\begin{array}{llll}38 \mathrm{~B} & 2.253530 & -1.273462 & 1.382268\end{array}$

$\begin{array}{llll}39 \mathrm{~B} & 2.250261 & -1.283589 & -1.383540\end{array}$

$\begin{array}{lllll}40 \mathrm{~B} & 1.457471 & 2.159574 & -0.005281\end{array}$ $\begin{array}{|llll|}41 B & 2.787235 & 0.968748 & -0.093910\end{array}$ $\begin{array}{llll}42 \mathrm{~B} & -1.112012 & -2.357635 & -0.000746\end{array}$ $\begin{array}{llll}43 \mathrm{~B} & 3.160452 & -0.754751 & -0.001467\end{array}$ $\begin{array}{llll}44 \mathrm{~B} & 2.270036 & -2.328395 & 0.003111\end{array}$ $\begin{array}{llll}45 \mathrm{~B} & 0.582852 & -2.885863 & 0.001149\end{array}$ $\begin{array}{llll}46 \mathrm{~B} & -1.166273 & 1.677134 & 1.497017\end{array}$ $\begin{array}{llll}47 \mathrm{~B} & -1.161084 & 1.674153 & -1.503403\end{array}$ $\begin{array}{llll}48 \mathrm{~B} & -2.033215 & 0.140330 & -1.503880\end{array}$ $\begin{array}{llll}49 \mathrm{~B} & -2.039897 & 0.141540 & 1.496064\end{array}$ $\begin{array}{llll}50 B & 2.338086 & 1.622684 & 1.452093\end{array}$

$s=1.7$

$E 5 / \varepsilon=-9.218112$

atom $\quad \mathrm{x} / \sigma \quad \mathrm{g} / \sigma \quad z / \sigma$

$\begin{array}{llll}1 \mathrm{~A} & 0.000000 & 0.000000 & 0.000000\end{array}$

$\begin{array}{llll}2 \mathrm{~A} & 1.123943 & 0.000000 & 0.000000\end{array}$

$\begin{array}{llll}3 A & 0.561972 & -0.973363 & 0.000000\end{array}$

$\begin{array}{llll}4 \mathrm{~B} & 0.561972 & -0.324454 & 1.363623\end{array}$

$\begin{array}{llll}5 \mathrm{~B} & 0.561972 & -0.324454 & -1.363623\end{array}$

$E 6 / \varepsilon=-13.273980$

\begin{tabular}{cccc} 
atom & $x / \sigma$ & $y / \sigma$ & \multicolumn{1}{c}{$z / \sigma$} \\
1A & 0.000000 & 0.000000 & 0.000000 \\
2A & 1.147934 & 0.000000 & 0.000000 \\
3B & 0.573968 & -1.380046 & 0.000000 \\
4B & 0.573968 & 0.000000 & -1.380046 \\
5B & 0.573968 & 0.000000 & 1.380046 \\
6B & 0.573968 & 1.380046 & 0.000000
\end{tabular}

$E 7 / \varepsilon=-16.781371$

$\begin{array}{cccc}\text { atom } & \mathrm{x} / \sigma & y / \sigma & z / \sigma \\ 1 \mathrm{~A} & 0.000000 & 0.000000 & 0.000000\end{array}$

$\begin{array}{llll}2 \mathrm{~B} & 1.494896 & 0.000000 & 0.000000\end{array}$

$\begin{array}{llll}3 \mathrm{~B} & 0.226754 & -1.477598 & 0.000000\end{array}$

$\begin{array}{lllll}4 \mathrm{~A} & 0.431686 & -0.370492 & -0.985394\end{array}$

$\begin{array}{lllll}5 \mathrm{~B} & 0.212747 & 1.111237 & -0.957496\end{array}$

$\begin{array}{llll}6 \mathrm{~B} & -1.066107 & -0.378840 & -0.957498\end{array}$

$\begin{array}{llll}7 \mathrm{~B} & 0.268773 & -0.230673 & 1.444600\end{array}$

$E 8 / \varepsilon=-21.283208$

$\begin{array}{llll}\text { atom } & \mathrm{x} / \sigma & \mathrm{y} / \sigma & \mathrm{z} / \sigma\end{array}$

$\begin{array}{cccc}1 \mathrm{~A} & 0.000000 & 0.000000 & 0.000000\end{array}$

$\begin{array}{llll}2 B & 1.476483 & 0.000000 & 0.000000\end{array}$

$\begin{array}{llll}\text { 3B } & 0.189751 & -1.464238 & 0.000000\end{array}$

$\begin{array}{lllll}4 B & 0.181498 & 1.137998 & -0.923048\end{array}$

$\begin{array}{llll}5 B & -1.105234 & -0.326240 & -0.923053\end{array}$

$\begin{array}{lllll}6 \mathrm{~A} & 0.398347 & -0.350055 & -0.990428\end{array}$

$\begin{array}{llll}7 \mathrm{~B} & 0.233847 & 1.071423 & 0.992854\end{array}$

$\begin{array}{llll}8 B & -1.032489 & -0.369599 & 0.992850\end{array}$

$E 9 / \varepsilon=-26.529040$

$\begin{array}{llll}\text { atom } \mathrm{x} / \sigma & \mathrm{y} / \sigma & \mathrm{z} / \sigma\end{array}$

$\begin{array}{llll}1 \mathrm{~A} & 0.000000 & 0.000000 & 0.000000\end{array}$

$\begin{array}{llll}2 \mathrm{~B} & 1.478641 & 0.000000 & 0.000000\end{array}$

$\begin{array}{llll}3 B & 0.131687 & -1.472765 & 0.000000\end{array}$

$\begin{array}{llll}\text { 4B } & 0.207750 & 1.118229 & -0.950898\end{array}$

$\begin{array}{llll}5 B & 0.207750 & 1.118229 & 0.950898\end{array}$

$\begin{array}{llll}6 \mathrm{~B} & -1.095284 & -0.306511 & -0.950898\end{array}$

$\begin{array}{llll}7 \mathrm{~B} & -1.095284 & -0.306511 & 0.950898\end{array}$

$\begin{array}{lllll}8 \mathrm{~A} & 0.405574 & -0.370928 & 0.980663\end{array}$

$\begin{array}{lllll}9 A & 0.405574 & -0.370928 & -0.980663\end{array}$

$E 10 / \varepsilon=-31.799676$

$\begin{array}{llll}\text { atom } & \mathrm{X} / \sigma & \mathrm{y} / \sigma & \mathrm{z} / \sigma\end{array}$

$\begin{array}{llll}1 \mathrm{~A} & 0.000000 & 0.000000 & 0.000000\end{array}$

$\begin{array}{llll}2 \mathrm{~B} & 1.488577 & 0.000000 & 0.000000\end{array}$

$\begin{array}{llll}\text { 3B } & 0.173092 & -1.478479 & 0.000000\end{array}$

$\begin{array}{llll}4 \mathrm{~B} & 0.242325 & 1.108858 & -0.963106\end{array}$

$\begin{array}{llll}5 B & -1.073159 & -0.369619 & -0.963106\end{array}$

$\begin{array}{llll}6 \mathrm{~B} & 0.242325 & 1.108861 & 0.963103\end{array}$

$\begin{array}{llll}7 \mathrm{~B} & -1.073160 & -0.369619 & 0.963104\end{array}$ $\begin{array}{llll}8 A & -0.842779 & 0.749868 & -0.000001\end{array}$

$\begin{array}{lllll}9 A & 0.421389 & -0.374933 & 0.976951\end{array}$

$\begin{array}{lllll}10 \mathrm{~A} & 0.421390 & -0.374935 & -0.976951\end{array}$

$E 11 / \varepsilon=-35.917049$

$\begin{array}{llll}\text { atom } & \mathrm{x} / \sigma & \mathrm{y} / \sigma & \mathrm{z} / \sigma\end{array}$

$\begin{array}{llll}1 \mathrm{~A} & 0.000000 & 0.000000 & 0.000000\end{array}$

$\begin{array}{llll}2 \mathrm{~A} & 1.109981 & 0.000000 & 0.000000\end{array}$

$\begin{array}{llll}3 \mathrm{~A} & 0.550457 & -0.963875 & 0.000000\end{array}$

$\begin{array}{lllll}4 \mathrm{~A} & 0.550457 & 0.324801 & -0.907502\end{array}$

$\begin{array}{llll}5 A & -0.009067 & -0.639074 & -0.907502\end{array}$

$\begin{array}{llll}6 \mathrm{~B} & -0.934490 & -1.117627 & 0.176158\end{array}$

$\begin{array}{llll}7 \mathrm{~B} & -0.934490 & 0.542467 & -0.992899\end{array}$

$\begin{array}{llll}8 B & 0.507086 & -0.294360 & 1.345217\end{array}$

$\begin{array}{llll}9 \mathrm{~B} & 0.507085 & 1.365732 & 0.176160\end{array}$

$\begin{array}{llll}10 \mathrm{~B} & -1.073661 & 0.623252 & 0.885036\end{array}$

$\begin{array}{llll}11 \mathrm{~B} & 1.446211 & -0.839517 & -1.192136\end{array}$

$E 12 / \varepsilon=-40.619462$

atom $\quad \mathrm{x} / \sigma \quad \mathrm{y} / \sigma \quad \mathrm{z} / \sigma$

$\begin{array}{llll}1 \mathrm{~A} & 0.000000 & 0.000000 & 0.000000\end{array}$

$\begin{array}{llll}2 \mathrm{~A} & 1.111221 & 0.000000 & 0.000000\end{array}$

$\begin{array}{llll}3 \mathrm{~A} & 0.558816 & -0.960488 & 0.000000\end{array}$

$\begin{array}{llll}4 \mathrm{~B} & 0.477618 & -0.274691 & 1.353294\end{array}$

$\begin{array}{lllll}5 A & 0.548765 & 0.330028 & -0.902350\end{array}$

$\begin{array}{llll}6 \mathrm{~A} & -0.009296 & -0.640293 & -0.902349\end{array}$

$\begin{array}{llll}7 \mathrm{~B} & -0.917482 & -1.127058 & 0.193252\end{array}$

$\begin{array}{llll}8 \mathrm{~B} & 0.512788 & 1.359809 & 0.193251\end{array}$

$\begin{array}{llll}9 B & -0.932567 & 0.536348 & -1.002646\end{array}$

$\begin{array}{llll}10 \mathrm{~B} & 1.431292 & -0.823180 & -1.224818\end{array}$

$\begin{array}{llll}11 \mathrm{~B} & -1.092795 & 0.628498 & 0.865257\end{array}$

$\begin{array}{llll}12 B & 1.949159 & -1.121019 & 0.558597\end{array}$

\section{$E 13 / \varepsilon=-45.844938$}

$\begin{array}{llll}\text { atom } & \mathrm{x} / \sigma & \mathrm{y} / \sigma & \mathrm{z} / \sigma\end{array}$

$\begin{array}{llll}1 \mathrm{~A} & 0.000000 & 0.000000 & 0.000000\end{array}$

$\begin{array}{llll}2 \mathrm{~A} & 1.098475 & 0.000000 & 0.000000\end{array}$

$\begin{array}{llll}3 \mathrm{~A} & 0.565285 & -0.962062 & 0.000000\end{array}$

$\begin{array}{llll}4 \mathrm{~A} & 0.565284 & 0.325900 & 0.905181\end{array}$

$\begin{array}{llll}5 \mathrm{~B} & 0.659059 & 1.367074 & -0.200141\end{array}$

$\begin{array}{llll}6 \mathrm{~B} & 0.659059 & -0.274791 & -1.354045\end{array}$

$\begin{array}{llll}7 \mathrm{~B} & -0.278410 & -0.865782 & 1.231902\end{array}$

$\begin{array}{lllll}8 \mathrm{~A} & 1.147460 & -0.632389 & 0.899814\end{array}$

$\begin{array}{llll}9 \mathrm{~B} & -0.826410 & 0.889660 & 0.878687\end{array}$

$\begin{array}{llll}10 \mathrm{~B} & -0.826408 & -1.128111 & -0.539403\end{array}$

$\begin{array}{llll}11 B & 2.054716 & -1.076162 & -0.221597\end{array}$

$\begin{array}{llll}12 \mathrm{~B} & 2.054715 & 0.573049 & 0.937470\end{array}$

$\begin{array}{llll}13 B & -0.915931 & 0.695361 & -0.989416\end{array}$

$E 14 / \varepsilon=-51.987964$

atom $\mathrm{x} / \sigma \quad \mathrm{y} / \sigma \quad \mathrm{z} / \sigma$

$\begin{array}{llll}1 \mathrm{~A} & 0.000000 & 0.000000 & 0.000000\end{array}$

$\begin{array}{lllll}2 \mathrm{~A} & 1.084094 & 0.000000 & 0.000000\end{array}$

$\begin{array}{llll}3 \mathrm{~A} & 0.542048 & -0.965664 & 0.000000\end{array}$

$\begin{array}{lllll}4 \mathrm{~A} & 0.542048 & 0.315693 & -0.912604\end{array}$

$\begin{array}{llll}5 \mathrm{~A} & 0.542047 & 0.965664 & 0.000000\end{array}$

$\begin{array}{llll}6 \mathrm{~A} & 0.542046 & -0.315693 & 0.912604\end{array}$

$\begin{array}{llll}7 \mathrm{~B} & -0.408088 & -0.821624 & -1.153616\end{array}$

$\begin{array}{llll}8 \mathrm{~B} & 1.492184 & 0.821623 & 1.153615\end{array}$

$\begin{array}{llll}9 \mathrm{~B} & 1.492187 & -0.821623 & -1.153614\end{array}$

$\begin{array}{llll}10 B & -0.408091 & 0.821623 & 1.153614\end{array}$

$\begin{array}{llll}11 \mathrm{~B} & -0.776883 & 1.013946 & -0.722151\end{array}$

$\begin{array}{llll}12 \mathrm{~B} & -0.776884 & -1.013947 & 0.722148\end{array}$

$\begin{array}{llll}13 \mathrm{~B} & 1.860978 & 1.013947 & -0.722148\end{array}$

$\begin{array}{llll}\text { 14B } & 1.860978 & -1.013946 & 0.722151\end{array}$

$E 15 / \varepsilon=-57.624738$

atom $\quad \mathrm{x} / \sigma \quad \mathrm{y} / \sigma$

$\begin{array}{llll}1 \mathrm{~A} & 0.000000 & 0.000000 & 0.000000\end{array}$

$\begin{array}{llll}2 A & 1.100216 & 0.000000 & 0.000000\end{array}$

$\begin{array}{llll}3 \mathrm{~A} & 0.541721 & -0.957608 & 0.000000\end{array}$

$\begin{array}{llll}4 \mathrm{~A} & 0.541722 & 0.325724 & 0.900509\end{array}$ 


$\begin{array}{rrrr}\text { 5A } & -0.016772 & -0.631884 & 0.900509 \\ \text { 6A } & 1.088811 & -0.635014 & 0.904970 \\ \text { 7B } & -0.914081 & 0.533110 & 1.033898 \\ \text { 8B } & 0.553983 & -0.323093 & -1.333194 \\ \text { 9B } & -0.914082 & -1.153582 & -0.149647 \\ \text { 10B } & 0.553985 & 1.363598 & -0.149648 \\ \text { 11B } & 1.997365 & -1.164901 & -0.133156 \\ \text { 12B } & 0.529600 & -1.995220 & 1.050150 \\ \text { 13B } & 0.529602 & -0.308872 & 2.233454 \\ \text { 14B } & 1.997367 & 0.521447 & 1.050149 \\ \text { 15B } & -1.040411 & 0.606787 & -0.864740\end{array}$

$E 16 / \varepsilon=-63.402124$

$\begin{array}{llll}1 \mathrm{~A} & 0.000000 & 0.000000 & 0.000000\end{array}$

$\begin{array}{llll}2 \mathrm{~A} & 1.092802 & 0.000000 & 0.000000\end{array}$

$\begin{array}{llll}3 A & 0.546400 & -0.957918 & 0.000000\end{array}$

$\begin{array}{llll}4 \mathrm{~A} & 0.546401 & 0.329363 & 0.899515\end{array}$

$\begin{array}{llll}5 A & 1.099424 & -0.630159 & 0.901810\end{array}$

$\begin{array}{llll}6 \mathrm{~A} & -0.006623 & -0.630159 & 0.901810\end{array}$

$\begin{array}{llll}7 \mathrm{~B} & 0.546401 & 1.367243 & -0.147652\end{array}$

$\begin{array}{llll}8 \mathrm{~B} & 0.546401 & -0.331453 & -1.334651\end{array}$

$\begin{array}{llll}9 \mathrm{~B} & -0.909303 & 0.531810 & 1.035867\end{array}$

$\begin{array}{llll}10 \mathrm{~B} & 2.002104 & 0.531810 & 1.035868\end{array}$

11B $\quad-0.909303 \quad-1.155565 \quad-0.143221$

12B $\quad 2.002103 \quad-1.155566 \quad-0.143222$

$\begin{array}{llll}13 \mathrm{~B} & 0.546400 & -0.303710 & 2.231805\end{array}$

$\begin{array}{llll}\text { 14B } & 0.546400 & -1.991309 & 1.052559\end{array}$

$\begin{array}{llll}15 \mathrm{~B} & -1.039787 & 0.604163 & -0.864607\end{array}$

$\begin{array}{llll}16 \mathrm{~B} & 2.132589 & 0.604161 & -0.864607\end{array}$

\section{$E 17 / \varepsilon=-69.267007$}

$\begin{array}{rrrr}\text { atom } & x / \sigma & y / \sigma & z / \sigma \\ \text { 1A } & 0.000000 & 0.000000 & 0.000000 \\ \text { 2A } & 1.095706 & 0.000000 & 0.000000 \\ \text { 3A } & 0.547853 & -0.948909 & 0.000000 \\ \text { 4B } & 0.547853 & -0.316303 & 1.336609 \\ \text { 5A } & 1.101603 & -0.636012 & -0.900052 \\ \text { 6A } & -0.005899 & -0.636012 & -0.900052 \\ \text { 7A } & 0.547852 & 0.323113 & -0.900052 \\ \text { 8B } & 2.003727 & -1.156853 & 0.145759 \\ \text { 9B } & 0.547853 & 1.364797 & 0.145759 \\ \text { 10B } & -0.908022 & -1.156852 & 0.145761 \\ \text { 11B } & -0.908447 & 0.524492 & -1.038369 \\ \text { 12B } & 0.547852 & -1.997893 & -1.038369 \\ \text { 13B } & 2.004151 & 0.524492 & -1.038370 \\ \text { 14B } & 0.547851 & -0.316303 & -2.230094 \\ \text { 15B } & -1.041823 & 0.601498 & 0.862083 \\ \text { 16B } & 2.137530 & 0.601497 & 0.862082 \\ \text { 17B } & 0.547852 & -2.151903 & 0.862083\end{array}$

$E 18 / \varepsilon=-75.146699$

\begin{tabular}{crrr} 
atom & \multicolumn{1}{c}{$\mathrm{X} / \sigma$} & \multicolumn{1}{c}{$y / \sigma$} & \multicolumn{1}{c}{$z / \sigma$} \\
1A & 0.000000 & 0.000000 & 0.000000 \\
2A & 1.098807 & 0.000000 & 0.000000 \\
3A & 0.549403 & -0.947064 & 0.000000 \\
4A & 0.549404 & 0.313261 & 0.893755 \\
5B & 0.549403 & -0.308909 & -1.335734 \\
$6 \mathrm{~B}$ & 0.549403 & 1.362725 & -0.150302 \\
7A & -0.005203 & -0.638880 & 0.900916 \\
8A & 1.104010 & -0.638881 & 0.900916 \\
9B & 2.005997 & -1.155522 & -0.145957 \\
10B & 2.005999 & 0.519951 & 1.042200 \\
11B & -0.907190 & 0.519954 & 1.042200 \\
12B & -0.907192 & -1.155521 & -0.145954 \\
13B & 0.549405 & -0.315503 & 2.229550 \\
14B & 0.549402 & -1.999692 & 1.035214 \\
15B & 2.141990 & 0.606241 & -0.854890 \\
16B & -1.043184 & 0.606241 & -0.854889 \\
17B & 0.549401 & -2.146192 & -0.866354 \\
18B & 0.549406 & 1.527487 & 1.738820
\end{tabular}

\begin{tabular}{|crrr}
\multicolumn{5}{|c}{$E 19 / \varepsilon=-81.113225$} \\
atom & $x / \sigma$ & $y / \sigma$ & \multicolumn{1}{c}{$z / \sigma$} \\
1A & 0.000000 & 0.000000 & 0.000000 \\
2A & 1.097440 & 0.000000 & 0.000000 \\
3A & 0.551390 & -0.948865 & 0.000000 \\
4A & 0.551391 & 0.314240 & -0.895320 \\
5A & 0.005339 & -0.634624 & -0.895320 \\
6B & -0.904958 & -1.156397 & 0.146057 \\
7B & -0.904958 & 0.520786 & -1.042770 \\
8B & 0.545158 & 1.363454 & 0.146056 \\
9B & 0.545158 & -0.313727 & 1.334884 \\
10A & 1.109139 & -0.638285 & -0.900484 \\
11B & 0.555409 & -1.997717 & -1.039902 \\
12B & 0.555409 & -0.319625 & -2.229374 \\
13B & 2.006317 & 0.523499 & -1.039901 \\
14B & 2.006316 & -1.154589 & 0.149570 \\
15B & -1.047331 & 0.602715 & 0.850303 \\
16B & 2.135405 & 0.608309 & 0.858193 \\
17B & 0.546947 & 1.522433 & -1.746294 \\
18B & 0.546946 & -2.151940 & 0.858192 \\
19B & -1.041519 & -1.237814 & -1.746293
\end{tabular}

$E 20 / \varepsilon=-87.167631$

atom $\quad \mathrm{x} / \sigma \quad y / \sigma \quad z / \sigma$

$\begin{array}{llll}1 \mathrm{~A} & 0.000000 & 0.000000 & 0.000000\end{array}$

$\begin{array}{llll}2 \mathrm{~A} & 1.551213 & 0.000000 & 0.000000\end{array}$

$\begin{array}{llll}3 \mathrm{~A} & 0.775607 & -0.775607 & 0.000000\end{array}$

$\begin{array}{lllll}4 \mathrm{~A} & 0.775607 & 0.775607 & 0.000000\end{array}$

$\begin{array}{lllll}5 A & 0.775607 & 0.000000 & -0.775607\end{array}$

$\begin{array}{llll}6 \mathrm{~A} & 0.775606 & 0.000000 & 0.775607\end{array}$

$\begin{array}{llll}7 B & 1.804352 & -1.028745 & 1.028748\end{array}$

$\begin{array}{llll}8 \mathrm{~B} & -0.253139 & 1.028747 & -1.028747\end{array}$

$\begin{array}{llll}9 B & 1.804353 & 1.028746 & 1.028747\end{array}$

$\begin{array}{llll}10 \mathrm{~B} & -0.253139 & -1.028747 & -1.028747\end{array}$

$\begin{array}{llll}11 B & -0.253141 & 1.028748 & 1.028745\end{array}$

$\begin{array}{llll}12 \mathrm{~B} & -0.253140 & -1.028747 & 1.028745\end{array}$

$\begin{array}{llll}13 \mathrm{~B} & 1.804354 & -1.028746 & -1.028746\end{array}$

$\begin{array}{lllll}14 \mathrm{~B} & 1.804354 & 1.028746 & -1.028747\end{array}$

$\begin{array}{llll}15 \mathrm{~B} & -1.476414 & 0.000000 & -0.000001\end{array}$

$\begin{array}{llll}16 \mathrm{~B} & 3.027627 & 0.000000 & 0.000001\end{array}$

$\begin{array}{llll}17 \mathrm{~B} & 0.775609 & 2.252021 & -0.000001\end{array}$

$\begin{array}{llll}18 \mathrm{~B} & 0.775608 & -2.252021 & 0.000000\end{array}$

$\begin{array}{llll}19 B & 0.775603 & 0.000001 & 2.252021\end{array}$

$\begin{array}{lllll}20 \mathrm{~B} & 0.775607 & 0.000000 & -2.252021\end{array}$

\section{$E 21 / \varepsilon=-92.763166$}

$3 A$

\begin{tabular}{lll}
$3 \mathrm{~A}$ & 0.537051 & 0.037195 \\
\hline $\mathrm{A}$ & 0.539366 & 0.313202
\end{tabular}

$\begin{array}{lll}4 \mathrm{~A} & 0.539366 & 0.313202\end{array}$

$\begin{array}{llll}5 A & -0.003578 & -0.623697\end{array}$

$\begin{array}{lll}6 \mathrm{~B} & 0.537718 & 1.433746\end{array}$

7B $\quad-0.976626 \quad-1.179392$

$\begin{array}{lll}8 \mathrm{~B} & -0.925255 & 0.536197\end{array}$

$\begin{array}{lll}9 \mathrm{~A} & 1.090877 & -0.632176\end{array}$

10B $2.060393-1.194022$

$11 \mathrm{~A} \quad 1.091881-0.632758$

$12 \mathrm{~A}$

$13 \mathrm{~A}$

$14 \mathrm{~B}$

$15 \mathrm{~B}$

$16 \mathrm{~B}$

$17 \mathrm{~B}$

$18 \mathrm{~B}$

$19 \mathrm{~B}$

20B

$21 \mathrm{~B}$

$22 B$

0.5442790 .314472

$-0.002238-0.628591$

$\begin{array}{ll}0.994172 & 0.546795\end{array}$

$0.517064-2.002086$

$0.624682-0.362010$

$\begin{array}{ll}-0.927411 & 0.537446\end{array}$

$0.538760-2.014147$

2.0154240 .533967

$0.556237-0.322346$

$0.519161 \quad 1.463225$

$-1.011431-1.177947$
$E 23 / \varepsilon=-105.416880$

$\begin{array}{lcr}\text { atom } & \mathrm{x} / \sigma & y / \sigma\end{array}$

$\begin{array}{lll}1 \mathrm{~A} & 0.000000 & 0.000000\end{array}$

$y / \sigma$

0.000000

$\begin{array}{llll}2 \mathrm{~A} & 1.195733 & 0.000000 & 0.000000\end{array}$

$\begin{array}{llll}3 \mathrm{~A} & 0.597867 & -0.883559 & 0.000000\end{array}$

$\begin{array}{lllll}4 \mathrm{~A} & 0.597866 & 0.441778 & -0.765185\end{array}$

$\begin{array}{lllll}5 \mathrm{~A} & 0.597866 & 0.441781 & 0.765184\end{array}$

$\begin{array}{lllll}6 \mathrm{~B} & -0.894416 & 0.580791 & -1.005966\end{array}$

$\begin{array}{llll}7 \mathrm{~B} & -0.894415 & -1.161588 & 0.000000\end{array}$

$\begin{array}{llll}8 \mathrm{~B} & -0.894417 & 0.580794 & 1.005962\end{array}$

$\begin{array}{llll}9 B & 2.090147 & 0.580796 & 1.005964\end{array}$

10B $\quad 2.090149 \quad 0.580793 \quad-1.005964$

$\begin{array}{llll}11 B & 2.090149 & -1.161586 & 0.000003\end{array}$

$\begin{array}{llll}12 \mathrm{~A} & 0.059043 & -0.544232 & -0.942636\end{array}$

$\begin{array}{lllll}13 \mathrm{~A} & 1.136690 & -0.544230 & 0.942637\end{array}$

$\begin{array}{llll}14 \mathrm{~A} & 1.136690 & 1.088462 & -0.000002\end{array}$

$\begin{array}{lllll}15 \mathrm{~A} & 1.136692 & -0.544232 & -0.942635\end{array}$

$\begin{array}{lllll}16 A & 0.059041 & 1.088462 & -0.000002\end{array}$

$\begin{array}{llll}17 \mathrm{~A} & 0.059041 & -0.544230 & 0.942636\end{array}$

$\begin{array}{lllll}18 B & 0.597867 & -1.939277 & 0.994039\end{array}$

$\begin{array}{llll}19 \mathrm{~B} & 0.597868 & 0.108774 & -2.176484\end{array}$

$\begin{array}{lllll}20 \mathrm{~B} & 0.597868 & -1.939280 & -0.994035\end{array}$

$\begin{array}{llll}21 \mathrm{~B} & 0.597865 & 0.108781 & 2.176483\end{array}$

$\begin{array}{llll}22 \mathrm{~B} & 0.597866 & 1.830498 & -1.182450\end{array}$

$\begin{array}{llll}23 \mathrm{~B} & 0.597864 & 1.830502 & 1.182443\end{array}$

$E 24 / \varepsilon=-111.349064$

atom $\mathrm{x} / \sigma \quad \mathrm{y} / \sigma$

$\begin{array}{lll}1 \mathrm{~A} & 0.000000 & 0.000000\end{array}$

$z / \sigma$

$\begin{array}{llll}2 \mathrm{~A} & 1.194980 & 0.000000 & 0.000000\end{array}$

$\begin{array}{llll}3 \mathrm{~A} & 0.599952 & -0.884471 & 0.000000\end{array}$

$\begin{array}{llll}4 \mathrm{~A} & 0.599952 & 0.440134 & 0.767184\end{array}$

$\begin{array}{lllll}5 \mathrm{~A} & 0.594547 & 0.442608 & -0.764199\end{array}$

$\begin{array}{llll}6 \mathrm{~B} & -0.892109 & 0.569130 & 1.014782\end{array}$

$\begin{array}{llll}7 \mathrm{~B} & -0.892108 & -1.163428 & 0.011321\end{array}$

$\begin{array}{llll}8 \mathrm{~A} & 0.078809 & -0.540325 & 0.932915\end{array}$

$\begin{array}{llll}9 B & -0.902176 & 0.577205 & -0.996592\end{array}$

$\begin{array}{llll}10 B & 2.091484 & 0.586002 & 1.002862\end{array}$

$\begin{array}{llll}11 \mathrm{~B} & 2.091484 & -1.161484 & -0.009246\end{array}$

$\begin{array}{lllll}12 \mathrm{~B} & 2.083263 & 0.584250 & -1.008756\end{array}$

$\begin{array}{llll}13 \mathrm{~A} & 1.151207 & -0.546477 & 0.943537\end{array}$

$\begin{array}{llll}14 \mathrm{~A} & 0.054110 & -0.546348 & -0.940055\end{array}$

$\begin{array}{llll}15 \mathrm{~A} & 0.054110 & 1.087273 & 0.006104\end{array}$

$\begin{array}{llll}16 \mathrm{~A} & 1.131655 & 1.089105 & 0.001578\end{array}$

$\begin{array}{lllll}17 A & 1.131656 & -0.543334 & -0.943897\end{array}$

$\begin{array}{lllll}18 B & 0.604632 & -1.937691 & 0.998087\end{array}$

$\begin{array}{llll}19 \mathrm{~B} & 0.604631 & 0.098508 & 2.177412\end{array}$

$\begin{array}{llll}\text { 20B } & 0.592311 & -1.941520 & -0.989661\end{array}$

$\begin{array}{llll}21 B & 0.592310 & 1.824572 & 1.191581\end{array}$

$\begin{array}{llll}22 \mathrm{~B} & 0.586196 & 0.110160 & -2.174964\end{array}$ $\begin{array}{llll}23 B & 0.586196 & 1.831729 & -1.177867\end{array}$ 
24B $\quad-0.850991 \quad-1.111039 \quad 1.918299$ $E 25 / \varepsilon=-117.418348$

\begin{tabular}{|c|c|c|c|}
\hline & $x / \sigma$ & $y / \sigma$ & $z / \sigma$ \\
\hline $1 \mathrm{~A}$ & 0.000000 & 0.000000 & 0.000000 \\
\hline $2 A$ & 1.196787 & 0.000000 & 0.000000 \\
\hline $3 A$ & 0.598393 & -0.884357 & 0.000000 \\
\hline $4 \mathrm{~A}$ & 0.598393 & 0.436607 & -0.769065 \\
\hline $5 A$ & 0.598393 & 0.443549 & 0.761851 \\
\hline $6 B$ & -0.893475 & -1.163850 & -0.005175 \\
\hline $7 \mathrm{~B}$ & -0.893476 & 0.570092 & -1.014675 \\
\hline $8 B$ & 2.090260 & 0.570092 & -1.014678 \\
\hline $9 B$ & 2.090260 & -1.163850 & -0.005177 \\
\hline $10 \mathrm{~A}$ & 1.131087 & -0.543107 & -0.932854 \\
\hline $11 \mathrm{~A}$ & 0.065697 & -0.543108 & -0.932854 \\
\hline $12 B$ & 0.598392 & -1.939568 & -0.996389 \\
\hline 13B & 0.598391 & 0.091073 & -2.178629 \\
\hline 14B & -0.894783 & 0.581493 & 0.998788 \\
\hline 15B & 2.091569 & 0.581494 & 0.998788 \\
\hline $16 \mathrm{~A}$ & 0.059310 & -0.545589 & 0.940706 \\
\hline $17 \mathrm{~A}$ & 1.137477 & 1.087425 & -0.010035 \\
\hline $18 \mathrm{~A}$ & 1.137477 & -0.545589 & 0.940706 \\
\hline $19 \mathrm{~A}$ & 0.059309 & 1.087425 & -0.010034 \\
\hline $20 \mathrm{~B}$ & 0.598391 & 1.816378 & -1.203319 \\
\hline 21B & 0.598393 & -1.943192 & 0.985501 \\
\hline $22 B$ & 0.598392 & 1.833598 & 1.170165 \\
\hline $23 B$ & 0.598393 & 0.112363 & 2.172267 \\
\hline & 2.048133 & -1.118184 & -1.92062 \\
\hline & & & \\
\hline
\end{tabular}

$E 26 / \varepsilon=-123.754916$

atom $\quad x / \sigma \quad 0.0000$

$\begin{array}{cccc}1 \mathrm{~A} & 0.000000 & 0.000000 & 0.000000\end{array}$

$\begin{array}{llll}2 \mathrm{~A} & 1.139234 & 0.000000 & 0.000000\end{array}$

$\begin{array}{llll}3 \mathrm{~A} & 0.569616 & -0.986607 & 0.000000\end{array}$

$\begin{array}{lllll}4 \mathrm{~A} & 0.569617 & -0.328869 & -0.930182\end{array}$

$\begin{array}{llll}5 \mathrm{~A} & 0.569617 & -0.328869 & 0.860239\end{array}$

$\begin{array}{llll}6 \mathrm{~A} & -0.322638 & -0.844012 & -0.596807\end{array}$

$\begin{array}{llll}7 A & 1.461871 & -0.844013 & -0.596806\end{array}$

$\begin{array}{lllll}8 \mathrm{~A} & 0.569617 & 0.701419 & -0.596807\end{array}$

$\begin{array}{llll}9 \mathrm{~A} & 1.515944 & -0.875232 & 0.540130\end{array}$

$\begin{array}{llll}10 A & -0.376711 & 0.217495 & -1.005219\end{array}$

$11 \mathrm{~A} \quad 0.569616 \quad-1.421595-1.005220$

$\begin{array}{llll}12 \mathrm{~A} & 1.515946 & 0.217494 & -1.005219\end{array}$

$\begin{array}{llll}13 \mathrm{~A} & -0.376712 & -0.875232 & 0.540128\end{array}$

$\begin{array}{llll}14 \mathrm{~A} & 0.569617 & 0.763857 & 0.540129\end{array}$

$\begin{array}{llll}15 B & 2.669819 & -0.204100 & -0.144317\end{array}$

$\begin{array}{llll}16 \mathrm{~B} & -0.372431 & -2.210082 & -0.144320\end{array}$

$\begin{array}{llll}17 \mathrm{~B} & 1.511663 & -0.872761 & -2.035579\end{array}$

$\begin{array}{llll}18 B & -0.588540 & 0.339792 & 1.394036\end{array}$

$\begin{array}{llll}19 B & -0.588539 & 1.427573 & -0.144318\end{array}$

$\begin{array}{lllll}20 \mathrm{~B} & 1.727774 & 1.427573 & -0.144318\end{array}$

21B $\quad 1.511661 \quad-2.210083-0.144318$

$\begin{array}{llll}22 \mathrm{~B} & -0.372430 & -0.872759 & -2.035580\end{array}$

$\begin{array}{llll}23 \mathrm{~B} & 1.727772 & 0.339792 & 1.394037\end{array}$

$\begin{array}{llll}24 \mathrm{~B} & -1.530586 & -0.204098 & -0.144320\end{array}$

$\begin{array}{llll}25 \mathrm{~B} & 0.569618 & 0.758912 & -2.035580\end{array}$

$\begin{array}{llll}26 \mathrm{~B} & 0.569614 & -1.666192 & 1.394037\end{array}$

\section{$E 27 / \varepsilon=-130.980905$}

\begin{tabular}{rrrr} 
atom & $x / \sigma$ & $y / \sigma$ & \multicolumn{1}{c}{$z / \sigma$} \\
1A & 0.000000 & 0.000000 & 0.000000 \\
2A & 1.096518 & 0.000000 & 0.000000 \\
3A & 0.548259 & -0.949613 & 0.000000 \\
4A & 0.548259 & 0.316538 & -0.895303 \\
5A & -0.548259 & -0.316537 & 0.895304 \\
6A & -0.548260 & 0.316539 & -0.895303 \\
7A & -0.000001 & -0.633074 & -0.895303 \\
8A & -0.548258 & 0.949613 & 0.000001 \\
9A & -0.548260 & -0.949612 & 0.000000 \\
10A & 0.000001 & 0.633075 & 0.895304 \\
11A & -1.096518 & 0.000001 & 0.000001
\end{tabular}

$\begin{array}{llll}11 \mathrm{~A} & -1.096518 & 0.000001 & 0.000001\end{array}$ $\begin{array}{llll}13 \mathrm{~A} & 0.548260 & 0.949613 & 0.000000 \\ 14 \mathrm{~B} & -1.439447 & 0.831067 & 1.175307\end{array}$

$\begin{array}{llll}14 \mathrm{~B} & -1.439447 & 0.831067 & 1.175307\end{array}$

$\begin{array}{llll}15 \mathrm{~B} & 1.439449 & 0.831066 & 1.175305\end{array}$

$\begin{array}{llll}16 \mathrm{~B} & 0.000000 & 1.662133 & -1.175304\end{array}$

$\begin{array}{llll}17 \mathrm{~B} & -1.439450 & -0.831064 & -1.175304\end{array}$

$\begin{array}{lllll}18 \mathrm{~B} & 1.439447 & -0.831065 & -1.175305\end{array}$

$\begin{array}{llll}19 B & 0.000000 & -1.662132 & 1.175305\end{array}$

$\begin{array}{llll}\text { 20B } & 0.000001 & 2.099190 & 0.742176\end{array}$

$\begin{array}{llll}21 B & 0.000002 & 0.000000 & 2.226527\end{array}$

$\begin{array}{llll}22 \mathrm{~B} & 1.817952 & 1.049595 & -0.742176\end{array}$

$\begin{array}{llll}23 \mathrm{~B} & -1.817952 & 1.049597 & -0.742173\end{array}$

$\begin{array}{llll}24 \mathrm{~B} & -1.817952 & -1.049593 & 0.742177\end{array}$

$\begin{array}{llll}25 \mathrm{~B} & 1.817951 & -1.049595 & 0.742176\end{array}$

$\begin{array}{llll}26 \mathrm{~B} & -0.000001 & 0.000001 & -2.226527\end{array}$

$\begin{array}{llll}27 \mathrm{~B} & -0.000002 & -2.099189 & -0.742176\end{array}$

$E 28 / \varepsilon=-136.064944$

$\begin{array}{lccc}\text { atom } & \mathrm{x} / \sigma & y / \sigma & z / \sigma\end{array}$

$\begin{array}{llll}1 \mathrm{~A} & 0.000000 & 0.000000 & 0.000000 \\ 2 \mathrm{~A} & 1.094810 & 0.000000 & 0.000000\end{array}$

$\begin{array}{rrrr}2 \mathrm{~A} & 1.094810 & 0.000000 & 0.000000 \\ 3 \mathrm{~A} & 0.546122 & -0.950607 & 0.000000\end{array}$

$\begin{array}{llll}4 A & 0.546123 & 0.950607 & -0.000001\end{array}$

$\begin{array}{llll}5 \mathrm{~A} & 0.546123 & 0.323893 & 0.893727\end{array}$

$\begin{array}{lllll}6 A & 0.546122 & -0.323892 & -0.893727\end{array}$

$\begin{array}{lllll}7 A & 0.012423 & -0.630473 & 0.899088\end{array}$

$\begin{array}{lllll}8 \mathrm{~A} & 0.012424 & 0.630474 & -0.899088\end{array}$

$\begin{array}{llll}9 \mathrm{~A} & -0.549226 & -0.315030 & -0.893240\end{array}$

$\begin{array}{llll}10 A & -0.549225 & 0.315032 & 0.893240\end{array}$

$\begin{array}{llll}11 \mathrm{~A} & -0.549225 & 0.947131 & -0.008165\end{array}$

$\begin{array}{llll}12 \mathrm{~A} & -0.549226 & -0.947129 & 0.008166\end{array}$

$\begin{array}{llll}13 B & 1.476086 & 0.803249 & -1.145474\end{array}$

$\begin{array}{llll}14 \mathrm{~B} & 1.476086 & -0.803249 & 1.145474\end{array}$

$\begin{array}{llll}15 \mathrm{~A} & -1.098110 & 0.000001 & 0.000000\end{array}$

$\begin{array}{llll}16 \mathrm{~B} & -0.037500 & -1.668964 & -1.170340\end{array}$

$\begin{array}{llll}17 \mathrm{~B} & -0.037497 & 1.668965 & 1.170340\end{array}$

$\begin{array}{llll}18 \mathrm{~B} & -1.430408 & 0.830838 & -1.184815\end{array}$

$\begin{array}{llll}19 B & -1.430409 & -0.830835 & 1.184815\end{array}$

$\begin{array}{llll}\text { 20B } & 1.754018 & 1.144839 & 0.802804\end{array}$

$\begin{array}{llll}21 \mathrm{~B} & 1.754016 & -1.144840 & -0.802804\end{array}$

$\begin{array}{llll}22 \mathrm{~B} & 0.015404 & 2.095208 & -0.749787\end{array}$

$\begin{array}{llll}23 \mathrm{~B} & 0.015401 & -0.008958 & -2.225308\end{array}$

$\begin{array}{llll}24 \mathrm{~B} & 0.015401 & -2.095206 & 0.749788\end{array}$

$\begin{array}{llll}25 B & 0.015403 & 0.008960 & 2.225308\end{array}$

$\begin{array}{llll}26 \mathrm{~B} & -1.825893 & 1.043567 & 0.731787\end{array}$

$\begin{array}{llll}27 \mathrm{~B} & -1.825895 & -1.043562 & -0.731786\end{array}$

$\begin{array}{llll}28 \mathrm{~A} & 2.199118 & 0.000000 & 0.000000\end{array}$

$E 29 / \varepsilon=-142.945508$

$$
\text { at }
$$

$\begin{array}{cccc}\text { atom } & \mathrm{x} / \sigma & y / \sigma & z / \sigma \\ 1 \mathrm{~A} & 0.000000 & 0.000000 & 0.000000\end{array}$

$\begin{array}{llll}2 \mathrm{~A} & 1.098350 & 0.000000 & 0.000000\end{array}$

$\begin{array}{llll}3 \mathrm{~A} & 0.556309 & -0.947044 & 0.000000\end{array}$

$\begin{array}{llll}4 \mathrm{~A} & 0.556309 & -0.318403 & -0.891915\end{array}$

$\begin{array}{llll}5 B & -0.042519 & -1.665055 & -1.173567\end{array}$

$\begin{array}{llll}6 \mathrm{~B} & 1.414147 & 0.880005 & -1.173566\end{array}$

$\begin{array}{llll}7 \mathrm{~B} & 1.414146 & -0.809387 & 1.223339\end{array}$

$\begin{array}{llll}8 \mathrm{~A} & -0.547297 & -0.315840 & -0.893217\end{array}$

$\begin{array}{llll}9 \mathrm{~A} & 0.539693 & 0.951761 & 0.002852\end{array}$

$\begin{array}{llll}10 \mathrm{~A} & 0.539693 & 0.322675 & 0.895399\end{array}$

$\begin{array}{llll}11 \mathrm{~A} & -0.004873 & -0.628780 & 0.895398\end{array}$

$\begin{array}{llll}12 \mathrm{~A} & -0.004872 & 0.631875 & -0.893216\end{array}$

$\begin{array}{llll}13 A & -0.547297 & -0.947408 & 0.002852\end{array}$

$\begin{array}{llll}14 \mathrm{~A} & -1.098561 & -0.001197 & -0.000843\end{array}$

$\begin{array}{llll}15 A & -0.555383 & 0.947833 & -0.000843\end{array}$

$\begin{array}{llll}16 A & -0.555383 & 0.317874 & 0.892941\end{array}$

$\begin{array}{llll}17 \mathrm{~B} & -0.018584 & 1.666677 & 1.174710\end{array}$

$\begin{array}{llll}18 \mathrm{~B} & -1.446494 & -0.828138 & 1.174710\end{array}$

$\begin{array}{lllll}19 B & -1.446493 & 0.827902 & -1.174876\end{array}$

$\begin{array}{llll}\text { 20B } & 1.791667 & 1.079373 & 0.760767\end{array}$

$\begin{array}{llll}21 \mathrm{~B} & -0.023212 & -2.091549 & 0.760766\end{array}$

$\begin{array}{llll}22 \mathrm{~B} & -0.023212 & 0.013287 & -2.225571\end{array}$ $\begin{array}{llll}23 \mathrm{~B} & -0.022486 & 0.012870 & 2.227549\end{array}$ \begin{tabular}{|llll}
$24 B$ & -0.022486 & 2.102206 & -0.736797
\end{tabular} $\begin{array}{lllll}25 \mathrm{~B} & -1.824001 & -1.045367 & -0.736798\end{array}$ $\begin{array}{llll}26 \mathrm{~B} & -1.827786 & 1.046135 & 0.737339\end{array}$ $\begin{array}{lllll}27 \mathrm{~B} & 1.571883 & -0.899667 & -1.781663\end{array}$ $\begin{array}{lllll}28 \mathrm{~B} & 1.571883 & -1.980423 & -0.248289\end{array}$ $\begin{array}{lllll}29 B & 2.503756 & -0.352271 & -0.248288\end{array}$

\section{$E 30 / \varepsilon=-148.313123$}

atom $\mathrm{x} / \sigma \quad \mathrm{y} / \sigma \quad \mathrm{z} / \sigma$

$\begin{array}{llll}1 \mathrm{~A} & 0.000000 & 0.000000 & 0.000000\end{array}$

$\begin{array}{llll}2 \mathrm{~A} & 1.098428 & 0.000000 & 0.000000\end{array}$

$\begin{array}{llll}3 \mathrm{~A} & 0.530294 & -0.961942 & 0.000000\end{array}$

$\begin{array}{lllll}4 \mathrm{~A} & 0.530285 & -0.313190 & -0.909532\end{array}$

$\begin{array}{llll}5 B & -0.018592 & -1.648552 & -1.175879\end{array}$

$\begin{array}{lllll}6 \mathrm{~B} & 1.434733 & 0.812168 & -1.175876\end{array}$

$\begin{array}{llll}7 \mathrm{~B} & 1.434738 & -0.847374 & 1.150765\end{array}$

$\begin{array}{llll}8 \mathrm{~A} & 0.549737 & 0.948046 & 0.003070\end{array}$

$\begin{array}{llll}9 \mathrm{~A} & 0.549739 & 0.311571 & 0.895391\end{array}$

$\begin{array}{llll}10 A & -0.564846 & -0.939123 & 0.003067\end{array}$

$11 \mathrm{~A} \quad-0.007458 \quad-0.631852 \quad 0.895390$

$\begin{array}{llll}12 \mathrm{~A} & -0.564853 & -0.302864 & -0.888948\end{array}$

$\begin{array}{lllll}13 \mathrm{~A} & -0.007466 & 0.640884 & -0.888947\end{array}$

$\begin{array}{lllll}14 \mathrm{~A} & -1.100588 & 0.014573 & 0.010401\end{array}$

$\begin{array}{llll}15 \mathrm{~A} & -0.544100 & 0.956798 & 0.010402\end{array}$

$\begin{array}{llll}16 \mathrm{~A} & -0.544097 & 0.321348 & 0.901283\end{array}$

$\begin{array}{llll}17 \mathrm{~B} & 0.002221 & 1.662637 & 1.185931\end{array}$

$\begin{array}{llll}18 \mathrm{~B} & -1.454975 & -0.804629 & 1.185926\end{array}$

$\begin{array}{llll}19 B & -1.454986 & 0.859332 & -1.146892\end{array}$

$\begin{array}{llll}20 \mathrm{~B} & 1.788286 & 1.085460 & 0.774235\end{array}$

$\begin{array}{llll}21 \mathrm{~B} & -0.087246 & -2.090116 & 0.774231\end{array}$

$\begin{array}{lllll}22 \mathrm{~B} & -0.087263 & 0.051541 & -2.228308\end{array}$

$\begin{array}{llll}23 \mathrm{~A} & 1.418598 & -0.837842 & -0.597632\end{array}$

$\begin{array}{llll}24 \mathrm{~B} & 0.013469 & -0.007957 & 2.227772\end{array}$

$\begin{array}{lllll}25 \mathrm{~B} & 0.013460 & 2.103797 & -0.732845\end{array}$

$\begin{array}{lllll}26 \mathrm{~B} & -1.835887 & -1.027449 & -0.732850\end{array}$

$\begin{array}{llll}27 \mathrm{~B} & -1.809064 & 1.068452 & 0.762118\end{array}$

$\begin{array}{lllll}28 \mathrm{~B} & 2.657351 & -0.255069 & -0.181933\end{array}$

$\begin{array}{lllll}29 B & 1.506282 & -2.204020 & -0.181932\end{array}$

$\begin{array}{lllll}30 \mathrm{~B} & 1.506264 & -0.889610 & -2.024717\end{array}$

\section{$E 31 /=-154.937882$}

atom $\mathrm{x} / \sigma \quad \mathrm{y} / \sigma$

$\begin{array}{llll}1 \mathrm{~A} & 0.000000 & 0.000000 & 0.000000\end{array}$

$\begin{array}{llll}2 \mathrm{~B} & 2.031831 & 0.000000 & 0.000000\end{array}$

$\begin{array}{llll}3 \mathrm{~A} & 0.769048 & -0.780275 & 0.000000\end{array}$

$\begin{array}{llll}4 \mathrm{~A} & 0.769048 & 0.013329 & 0.780161\end{array}$

$\begin{array}{lllll}5 \mathrm{~A} & 0.769047 & -0.013329 & -0.780161\end{array}$

$\begin{array}{llll}6 \mathrm{~A} & 0.769047 & 0.780275 & 0.000000\end{array}$

$\begin{array}{lllll}7 \mathrm{~A} & 0.001294 & 0.787410 & 0.774072\end{array}$

$\begin{array}{lllll}8 \mathrm{~A} & 0.001294 & -0.787410 & -0.774072\end{array}$

$\begin{array}{llll}9 \mathrm{~B} & -0.052073 & -2.041023 & 0.048516\end{array}$

$\begin{array}{llll}10 \mathrm{~B} & -0.052073 & -0.013643 & 2.041554\end{array}$

$\begin{array}{llll}11 B & -0.052073 & 2.041023 & -0.048515\end{array}$

$\begin{array}{llll}12 B & -0.052073 & 0.013642 & -2.041554\end{array}$

$\begin{array}{llll}13 A & -0.006098 & -0.765755 & 0.778950\end{array}$

$\begin{array}{llll}14 \mathrm{~A} & -0.006098 & 0.765755 & -0.778950\end{array}$

$\begin{array}{llll}15 A & -0.778887 & 0.008792 & 0.770854\end{array}$

$\begin{array}{llll}16 \mathrm{~A} & -0.778887 & -0.770892 & 0.004378\end{array}$

$\begin{array}{lllll}17 A & -0.778887 & -0.008792 & -0.770854\end{array}$

$\begin{array}{llll}18 A & -0.778887 & 0.770892 & -0.004377\end{array}$

$\begin{array}{llll}19 B & -2.044411 & 0.000000 & 0.000000\end{array}$

$\begin{array}{llll}20 \mathrm{~B} & 1.252810 & -1.287643 & 1.309831\end{array}$

$\begin{array}{llll}21 \mathrm{~B} & 1.252810 & 1.287643 & -1.309831\end{array}$

$\begin{array}{llll}22 \mathrm{~B} & -1.325792 & 1.280999 & 1.259301\end{array}$

$\begin{array}{llll}23 \mathrm{~B} & -1.325792 & -1.281000 & -1.259300\end{array}$

$\begin{array}{llll}24 \mathrm{~B} & -1.309131 & -1.265506 & 1.287312\end{array}$

$\begin{array}{llll}25 \mathrm{~B} & -1.309131 & 1.265507 & -1.287311\end{array}$

$\begin{array}{llll}26 B & 1.742902 & 1.794356 & 0.445880\end{array}$

$\begin{array}{lllll}27 \mathrm{~B} & 1.742902 & -0.476468 & -1.786476\end{array}$

$\begin{array}{llll}28 \mathrm{~B} & 1.742902 & 0.476467 & 1.786477\end{array}$

$\begin{array}{llll}29 B & 1.742902 & -1.794356 & -0.445879\end{array}$

$\begin{array}{llll}20 \mathrm{~B} & 0.413007 & -1.795939 & -1.765516\end{array}$

$\begin{array}{llll}\text { 31B } & 0.413007 & 1.795938 & 1.765517\end{array}$ 


\begin{tabular}{|c|c|c|c|}
\hline \multicolumn{4}{|c|}{$E 32 / \varepsilon=-161.073978$} \\
\hline atom & $\mathrm{x} / \sigma$ & $y_{10}$ & \\
\hline $1 \mathrm{~A}$ & 0.000000 & 0.000000 & 0.000000 \\
\hline $2 B$ & 1.703492 & 0.000000 & 0.000000 \\
\hline $3 B$ & -1.703486 & -0.004558 & 0.000000 \\
\hline $4 \mathrm{~A}$ & -0.551229 & -0.269446 & 0.901102 \\
\hline $5 A$ & -0.551229 & -0.269443 & -0.901103 \\
\hline $6 \mathrm{~A}$ & 0.551947 & -0.267968 & -0.901103 \\
\hline $7 \mathrm{~A}$ & 0.551947 & -0.267970 & 0.901103 \\
\hline $8 \mathrm{~A}$ & -0.553814 & -0.931997 & -0.000002 \\
\hline $9 \mathrm{~A}$ & 0.556306 & -0.930512 & -0.000001 \\
\hline $10 \mathrm{~A}$ & 0.548583 & 0.773400 & 0.539414 \\
\hline $11 \mathrm{~A}$ & 0.548583 & 0.773401 & -0.539412 \\
\hline $12 \mathrm{~A}$ & -0.550650 & 0.771931 & -0.539412 \\
\hline $13 \mathrm{~A}$ & -0.550651 & 0.771929 & 0.539414 \\
\hline 14B & 0.003142 & -2.347590 & -0.000004 \\
\hline $15 B$ & 0.000920 & -0.688488 & 2.250927 \\
\hline $16 B$ & 0.000920 & -0.688482 & -2.250930 \\
\hline $17 \mathrm{~A}$ & 0.001621 & -1.211894 & -0.898907 \\
\hline $18 \mathrm{~A}$ & 0.001621 & -1.211896 & 0.898903 \\
\hline $19 A$ & -0.000694 & 0.518579 & 1.446864 \\
\hline $20 \mathrm{~A}$ & -0.000694 & 0.518583 & -1.446863 \\
\hline $21 B$ & -0.002638 & 1.971567 & -1.197668 \\
\hline $22 \mathrm{~B}$ & -0.002638 & 1.971564 & 1.197671 \\
\hline $23 B$ & -1.417572 & -1.469155 & 1.091663 \\
\hline $24 B$ & -1.417572 & -1.469150 & -1.091669 \\
\hline $25 B$ & 1.421498 & -1.465351 & -1.091670 \\
\hline $26 B$ & 1.421498 & -1.465353 & 1.091667 \\
\hline $27 \mathrm{~B}$ & -1.428423 & 0.609198 & -1.727051 \\
\hline $28 \mathrm{~B}$ & -1.428423 & 0.609191 & 1.727053 \\
\hline $29 B$ & 1.426787 & 0.613011 & 1.727053 \\
\hline $30 \mathrm{~B}$ & 1.426787 & 0.613013 & -1.727053 \\
\hline 31B & -1.449533 & 1.820421 & 0.000001 \\
\hline $32 B$ & 1.444657 & 1.824293 & 0.000001 \\
\hline
\end{tabular}

$E 33 / \varepsilon=-169.571951$

\begin{tabular}{rrrr} 
atom & $x / \sigma$ & \multicolumn{1}{c}{$y / \sigma$} & \multicolumn{1}{c}{$z / \sigma$} \\
1A & 0.000000 & 0.000000 & 0.000000 \\
2B & 1.698927 & 0.000000 & 0.000000 \\
3B & -1.698927 & 0.000000 & 0.000000 \\
4A & -0.551860 & -0.904135 & -0.263812 \\
5A & -0.551859 & 0.886526 & -0.318009 \\
6A & 0.551859 & -0.028494 & -0.941406 \\
7A & 0.551859 & 0.576397 & 0.744865 \\
8A & -0.551859 & -0.530293 & 0.778362 \\
9A & 0.551859 & -0.530293 & 0.778362 \\
10A & 0.551859 & 0.886525 & -0.318010 \\
11A & -0.551859 & 0.576396 & 0.744866 \\
12A & 0.551859 & -0.904135 & -0.263812 \\
13A & -0.551859 & -0.028493 & -0.941406 \\
14B & -0.000002 & -2.272880 & -0.663189 \\
15B & 0.000001 & 1.448987 & 1.872496 \\
16B & 0.000001 & -0.071629 & -2.366575 \\
17B & -0.000001 & 2.228612 & -0.799435 \\
18B & 0.000001 & -1.333089 & 1.956701 \\
19A & 0.000001 & 0.046162 & 1.525175 \\
20A & 0.000000 & -0.933821 & -1.206759 \\
21A & 0.000000 & 0.859129 & -1.261026 \\
22A & 0.000000 & 1.464792 & 0.427402 \\
23A & 0.000000 & -1.436262 & 0.515208 \\
24B & -1.425402 & 0.055439 & 1.831645 \\
25B & 1.425402 & -1.724867 & 0.618732 \\
26B & 1.425402 & 1.759130 & 0.513283 \\
27B & -1.425403 & -1.121462 & -1.449248 \\
28B & -1.425402 & 1.759129 & 0.513287 \\
29B & 1.425402 & 1.031763 & -1.514418 \\
30B & 1.425402 & -1.121465 & -1.449246 \\
31B & -1.425402 & 1.031762 & -1.514419 \\
32B & -1.425403 & -1.724866 & 0.618736 \\
33B & 1.425403 & 0.055439 & 1.831645 \\
& & &
\end{tabular}
$\begin{array}{llll}33 \mathrm{~B} & 1.425403 & 0.055439 & 1.831645\end{array}$
$E 34 / \varepsilon=-175.526184$

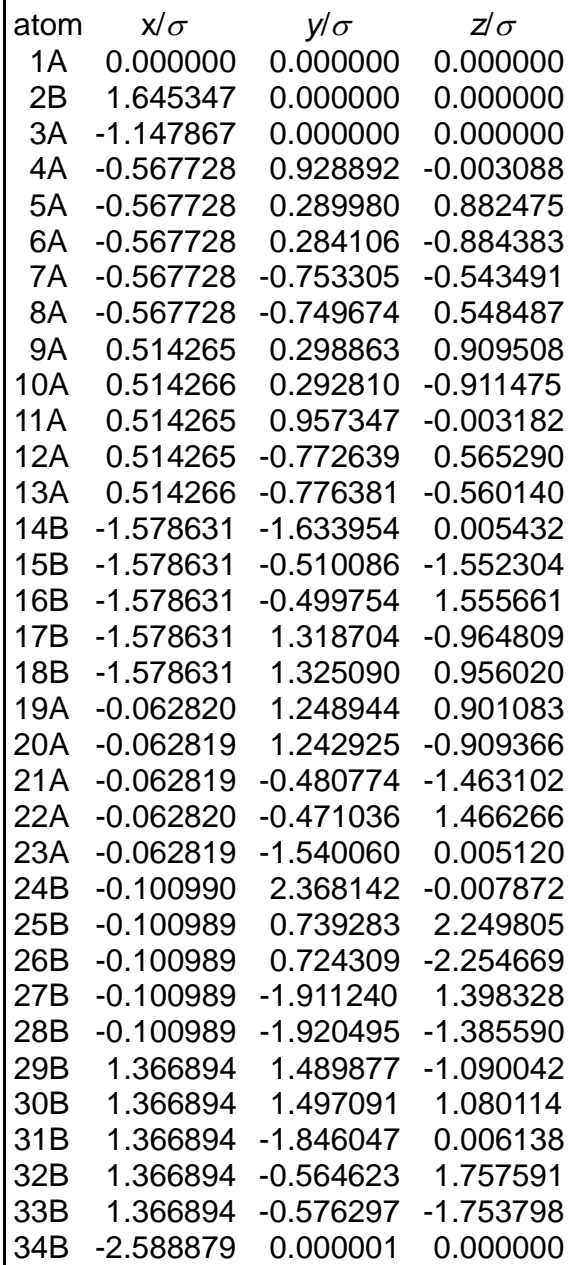

$E 35 / \varepsilon=-181.357766$

atom $\quad \mathrm{x} / \sigma \quad \mathrm{y} / \sigma$

$\begin{array}{llll}1 \mathrm{~A} & 0.000000 & 0.000000 & 0.000000\end{array}$

$\begin{array}{llll}2 \mathrm{~B} & 1.644227 & 0.000000 & 0.000000\end{array}$

$\begin{array}{llll}3 A & -0.562714 & -0.931722 & 0.000000\end{array}$

$\begin{array}{llll}4 \mathrm{~A} & -0.562715 & -0.290606 & 0.885242\end{array}$

$\begin{array}{llll}5 A & -1.146309 & -0.004781 & 0.003461\end{array}$

$\begin{array}{llll}6 A & -0.572486 & 0.752609 & -0.545062\end{array}$

$\begin{array}{llll}7 \mathrm{~A} & -0.570550 & 0.750841 & 0.543964\end{array}$

$\begin{array}{llll}8 \mathrm{~A} & -0.570549 & -0.282642 & -0.883049\end{array}$

$\begin{array}{lllll}9 \mathrm{~A} & 0.517313 & -0.957232 & -0.001610\end{array}$

$\begin{array}{llll}10 \mathrm{~A} & 0.517312 & -0.297032 & 0.909982\end{array}$

$\begin{array}{lllll}11 \mathrm{~A} & 0.509416 & 0.778419 & -0.563753\end{array}$

$\begin{array}{llll}12 \mathrm{~A} & 0.513518 & -0.286749 & -0.912606\end{array}$

$\begin{array}{llll}13 A & 0.513517 & 0.777644 & 0.557087\end{array}$

$\begin{array}{llll}14 \mathrm{~B} & -1.557542 & -1.343233 & 0.972803\end{array}$

$\begin{array}{llll}15 \mathrm{~A} & -0.060541 & -1.251697 & 0.906513\end{array}$

$\begin{array}{llll}16 \mathrm{~B} & -1.573052 & -1.328469 & -0.955235\end{array}$

$\begin{array}{llll}17 \mathrm{~B} & -1.573056 & 0.493231 & 1.560132\end{array}$

$\begin{array}{llll}18 \mathrm{~B} & -1.589328 & 0.501055 & -1.546693\end{array}$

$\begin{array}{llll}19 B & -1.589330 & 1.625813 & 0.006351\end{array}$

$\begin{array}{llll}20 \mathrm{~B} & -0.080548 & -2.363618 & -0.049007\end{array}$

$\begin{array}{llll}21 B & -0.080551 & -0.690653 & 2.260993\end{array}$

$\begin{array}{llll}22 B & 1.356989 & -1.506154 & 1.090801\end{array}$

$\begin{array}{llll}23 \mathrm{~A} & -0.068699 & 1.542577 & -0.000781\end{array}$

$\begin{array}{lllll}24 \mathrm{~A} & -0.068698 & 0.481872 & -1.465383\end{array}$

$\begin{array}{llll}25 A & -0.058959 & 0.487095 & 1.454188\end{array}$

$\begin{array}{llll}26 \mathrm{~A} & -0.058957 & -1.229720 & -0.916359\end{array}$

$\begin{array}{llll}27 \mathrm{~B} & -0.112952 & 1.920800 & -1.391098\end{array}$

$\begin{array}{llll}28 B & -0.106880 & -0.718502 & -2.261161\end{array}$

$\begin{array}{llll}29 B & -0.106882 & 1.924260 & 1.387916\end{array}$

$\begin{array}{llll}30 \mathrm{~B} & 1.368853 & -1.480704 & -1.098223\end{array}$

$\begin{array}{llll}31 \mathrm{~B} & 1.368851 & 0.581602 & 1.749376\end{array}$

$\begin{array}{llll}32 \mathrm{~B} & 1.362797 & 0.582208 & -1.755056\end{array}$

$\begin{array}{lllll}33 \mathrm{~B} & 1.362796 & 1.849095 & -0.005761\end{array}$ $\begin{array}{llll}34 \mathrm{~B} & -2.585727 & -0.022514 & 0.016299\end{array}$
|35B $-0.201658-2.465318 \quad 1.785450$

$E 36 / \varepsilon=-188.040054$

$\begin{array}{llll}\text { atom } \mathrm{x} / \sigma & \mathrm{y} / \sigma & \mathrm{z} \sigma\end{array}$

$\begin{array}{llll}1 \mathrm{~A} & 0.000000 & 0.000000 & 0.000000\end{array}$

$\begin{array}{llll}\text { 2B } & 1.718279 & 0.000000 & 0.000000\end{array}$

$\begin{array}{llll}3 B & -1.717912 & -0.035490 & 0.000000\end{array}$

$\begin{array}{lllll}4 \mathrm{~A} & -0.602771 & 0.892156 & -0.000068\end{array}$

$\begin{array}{lllll}5 \mathrm{~A} & 0.584216 & 0.904416 & -0.000068\end{array}$

$\begin{array}{llll}6 \mathrm{~A} & -0.547492 & -0.745423 & -0.565785\end{array}$

$\begin{array}{llll}7 A & -0.547493 & -0.745338 & 0.565895\end{array}$

$\begin{array}{lllll}8 \mathrm{~A} & 0.562772 & -0.733956 & -0.565785\end{array}$

$\begin{array}{llll}9 \mathrm{~A} & 0.562771 & -0.733871 & 0.565895\end{array}$

$\begin{array}{llll}10 A & -0.548581 & 0.311177 & -0.899989\end{array}$

$\begin{array}{lllll}11 \mathrm{~A} & 0.542037 & 0.322441 & -0.899989\end{array}$

$\begin{array}{llll}12 \mathrm{~A} & -0.548582 & 0.311312 & 0.899941\end{array}$

$\begin{array}{llll}13 A & 0.542036 & 0.322576 & 0.899941\end{array}$

$\begin{array}{llll}14 \mathrm{~A} & -0.013513 & 1.308363 & 0.785023\end{array}$

$\begin{array}{llll}15 A & -0.013512 & 1.308245 & -0.785220\end{array}$

$\begin{array}{llll}16 \mathrm{~B} & -0.008907 & 0.862418 & -2.177872\end{array}$

$\begin{array}{llll}17 \mathrm{~B} & -0.008911 & 0.862744 & 2.177742\end{array}$

$\begin{array}{llll}18 \mathrm{~B} & -1.466326 & 1.451759 & 1.077402\end{array}$

$\begin{array}{llll}19 \mathrm{~B} & 1.436028 & 1.481736 & 1.077402\end{array}$

$\begin{array}{llll}20 \mathrm{~B} & -1.466324 & 1.451598 & -1.077624\end{array}$

$\begin{array}{llll}21 \mathrm{~B} & 1.436029 & 1.481575 & -1.077623\end{array}$

$\begin{array}{lllll}\text { 22B } & 0.019410 & -1.879188 & -1.397942\end{array}$

$\begin{array}{lllll}23 \mathrm{~B} & 0.019405 & -1.878978 & 1.398223\end{array}$

$\begin{array}{llll}24 \mathrm{~A} & 0.004462 & -0.431985 & 1.471086\end{array}$

$\begin{array}{lllll}25 \mathrm{~A} & 0.004464 & -0.432205 & -1.471022\end{array}$

$\begin{array}{llll}26 \mathrm{~A} & 0.015389 & -1.489967 & 0.000112\end{array}$

$\begin{array}{lllll}27 \mathrm{~A} & 0.520560 & 1.977726 & -0.000148\end{array}$

$\begin{array}{llll}28 A & -0.561298 & 1.966552 & -0.000148\end{array}$

$\begin{array}{llll}29 B & -1.419026 & -0.521408 & 1.761399\end{array}$

$\begin{array}{llll}30 \mathrm{~B} & 1.429493 & -0.491989 & 1.761399\end{array}$

$\begin{array}{lllll}31 \mathrm{~B} & -1.419023 & -0.521671 & -1.761326\end{array}$

$\begin{array}{lllll}32 \mathrm{~B} & 1.429496 & -0.492250 & -1.761325\end{array}$

$\begin{array}{lllll}33 \mathrm{~B} & -1.390492 & -1.847627 & 0.000138\end{array}$

$\begin{array}{llll}34 \mathrm{~B} & 1.428358 & -1.818513 & 0.000140\end{array}$

$\begin{array}{llll}35 \mathrm{~B} & -0.027907 & 2.701876 & 1.193773\end{array}$

$\begin{array}{llll}36 \mathrm{~B} & -0.027903 & 2.701697 & -1.194178\end{array}$

$E 37 / \varepsilon=-194.081783$

\begin{tabular}{lll} 
atom $\quad \mathrm{x} / \sigma \quad \mathrm{y} / \sigma$ \\
\hline $\mathrm{A}$
\end{tabular}

$\begin{array}{llll}1 \mathrm{~A} & 0.000000 & 0.000000 & 0.000000\end{array}$

$\begin{array}{llll}2 \mathrm{~B} & 1.719197 & 0.000000 & 0.000000\end{array}$

$\begin{array}{llll}3 \mathrm{~B} & -1.718786 & -0.037621 & 0.000000\end{array}$

$\begin{array}{llll}4 \mathrm{~A} & 0.538989 & 0.510524 & -0.811484\end{array}$

$\begin{array}{lllll}5 A & -0.550031 & 0.498607 & -0.811485\end{array}$

$\begin{array}{lllll}6 \mathrm{~A} & 0.560722 & -0.591742 & -0.714735\end{array}$

$\begin{array}{llll}7 \mathrm{~A} & -0.547640 & -0.603870 & -0.714734\end{array}$

$\begin{array}{llll}8 \mathrm{~A} & -0.605322 & 0.868297 & 0.195148\end{array}$

$\begin{array}{lllll}9 \mathrm{~A} & 0.586175 & 0.881335 & 0.195149\end{array}$

$\begin{array}{llll}10 A & -0.546619 & -0.849912 & 0.393061\end{array}$

$\begin{array}{llll}11 \mathrm{~A} & 0.565085 & -0.837747 & 0.393061\end{array}$

$\begin{array}{llll}12 \mathrm{~A} & 0.543927 & 0.119738 & 0.948533\end{array}$

$\begin{array}{llll}13 \mathrm{~A} & -0.546417 & 0.107807 & 0.948533\end{array}$

$\begin{array}{llll}14 \mathrm{~A} & -0.012102 & 1.105864 & 1.048944\end{array}$

$\begin{array}{llll}15 A & -0.015835 & 1.447043 & -0.476616\end{array}$

$\begin{array}{llll}16 \mathrm{~B} & -0.014581 & 1.332635 & -1.927011\end{array}$

$\begin{array}{llll}17 \mathrm{~B} & -0.004053 & 0.370401 & 2.313632\end{array}$

$\begin{array}{llll}18 B & -1.470939 & 1.647320 & -0.731837\end{array}$

$\begin{array}{llll}19 \mathrm{~B} & 1.434538 & 1.679115 & -0.731834\end{array}$

$\begin{array}{llll}\text { 20B } & 0.016861 & -1.541039 & -1.761880\end{array}$

$\begin{array}{llll}21 \mathrm{~B} & -1.464205 & 1.181720 & 1.371654\end{array}$

$\begin{array}{llll}22 B & 1.437994 & 1.213480 & 1.371653\end{array}$

$\begin{array}{lllll}23 \mathrm{~A} & 0.001111 & -0.101518 & -1.534850\end{array}$

$\begin{array}{lllll}24 \mathrm{~B} & -1.432622 & -0.128630 & -1.819031\end{array}$

$\begin{array}{lllll}25 \mathrm{~B} & 1.435094 & -0.097252 & -1.819030\end{array}$

$\begin{array}{llll}26 \mathrm{~B} & 0.023390 & -2.137636 & 0.958047\end{array}$

$\begin{array}{llll}27 \mathrm{~A} & 0.008110 & -0.741176 & 1.343254\end{array}$

$\begin{array}{lllll}28 A & 0.015885 & -1.451768 & -0.319252\end{array}$

$\begin{array}{llll}29 A & 0.520127 & 1.929474 & 0.428887\end{array}$ $\begin{array}{llll}30 A & -0.562228 & 1.917630 & 0.428887\end{array}$ 
$\begin{array}{llll}31 \mathrm{~B} & 1.432924 & -0.861323 & 1.612811\end{array}$ $\begin{array}{llll}32 \mathrm{~B} & -1.413733 & -0.892472 & 1.612812\end{array}$ $\begin{array}{llll}33 \mathrm{~B} & 1.428155 & -1.771979 & -0.402479\end{array}$ $\begin{array}{lllll}34 \mathrm{~B} & -1.389039 & -1.802806 & -0.402479\end{array}$ $\begin{array}{llll}35 \mathrm{~B} & -0.031676 & 2.894691 & -0.579148\end{array}$ $\begin{array}{llll}36 \mathrm{~B} & -0.026008 & 2.376670 & 1.752561\end{array}$ $\begin{array}{llll}37 \mathrm{~B} & 0.001867 & -0.170570 & -3.041564\end{array}$

$E 38 / \varepsilon=-200.368418$

$\begin{array}{llll}1 \mathrm{~A} & 0.000000 & 0.000000 & 0.000000\end{array}$

$\begin{array}{llll}2 \mathrm{~A} & 1.137213 & 0.000000 & 0.000000\end{array}$

$\begin{array}{llll}3 \mathrm{~A} & -1.137199 & -0.005664 & 0.000000\end{array}$

$\begin{array}{llll}4 \mathrm{~B} & 0.005080 & -2.039816 & 0.000072\end{array}$

$\begin{array}{llll}5 A & -0.541954 & -0.778384 & 0.538207\end{array}$

$\begin{array}{llll}6 \mathrm{~A} & -0.541954 & -0.778422 & -0.538152\end{array}$

$\begin{array}{llll}7 \mathrm{~A} & 0.545825 & -0.775675 & 0.538207\end{array}$

$\begin{array}{lllll}8 \mathrm{~A} & 0.545824 & -0.775713 & -0.538152\end{array}$

$\begin{array}{llll}9 A & -0.547915 & 0.261424 & 0.889862\end{array}$

$\begin{array}{llll}10 A & -0.547915 & 0.261361 & -0.889881\end{array}$

$\begin{array}{lllll}11 \mathrm{~A} & 0.546606 & 0.264087 & -0.889881\end{array}$

$\begin{array}{lllll}12 \mathrm{~A} & 0.546605 & 0.264150 & 0.889862\end{array}$

$\begin{array}{llll}13 \mathrm{~B} & -0.003806 & 1.527952 & -1.332985\end{array}$

$\begin{array}{llll}14 \mathrm{~B} & -0.003806 & 1.528047 & 1.332875\end{array}$

$\begin{array}{llll}15 \mathrm{~A} & -0.555119 & 0.950102 & -0.000034\end{array}$

$\begin{array}{lllll}16 \mathrm{~A} & 0.550379 & 0.952855 & -0.000034\end{array}$

$\begin{array}{llll}17 \mathrm{~B} & 1.805752 & -1.356259 & 0.000048\end{array}$

$\begin{array}{llll}18 \mathrm{~B} & -1.798974 & -1.365237 & 0.000048\end{array}$

$\begin{array}{llll}19 A & 0.001273 & -0.511348 & 1.439376\end{array}$

$\begin{array}{llll}20 \mathrm{~A} & 0.001273 & -0.511450 & -1.439340\end{array}$

$\begin{array}{lllll}21 B & 1.787982 & 1.047550 & -0.913217\end{array}$

$\begin{array}{llll}22 \mathrm{~B} & 1.787982 & 1.047615 & 0.913141\end{array}$

$\begin{array}{llll}23 \mathrm{~B} & -1.793178 & 1.038631 & -0.913216\end{array}$

$\begin{array}{llll}24 \mathrm{~B} & -1.793179 & 1.038695 & 0.913142\end{array}$

$\begin{array}{llll}25 B & 0.939400 & -1.635747 & -1.659277\end{array}$

$\begin{array}{llll}26 B & 0.939400 & -1.635629 & 1.659393\end{array}$

$\begin{array}{llll}27 \mathrm{~B} & -0.931242 & -1.640405 & -1.659277\end{array}$

$\begin{array}{llll}28 \mathrm{~B} & -0.931242 & -1.640287 & 1.659393\end{array}$

$\begin{array}{llll}29 \mathrm{~B} & 0.937356 & 0.174803 & -2.330139\end{array}$

$\begin{array}{llll}30 \mathrm{~B} & 0.937356 & 0.174969 & 2.330126\end{array}$

$\begin{array}{llll}31 \mathrm{~B} & -0.938216 & 0.170132 & -2.330139\end{array}$

$\begin{array}{llll}32 \mathrm{~B} & -0.938216 & 0.170297 & 2.330126\end{array}$

$\begin{array}{llll}33 \mathrm{~A} & -1.429576 & -0.379020 & 1.032124\end{array}$

$\begin{array}{llll}34 \mathrm{~A} & -1.429576 & -0.379092 & -1.032098\end{array}$

$\begin{array}{llll}35 \mathrm{~A} & 1.431446 & -0.371894 & 1.032124\end{array}$

$\begin{array}{llll}36 \mathrm{~A} & 1.431446 & -0.371967 & -1.032098\end{array}$

$\begin{array}{lllll}37 \mathrm{~B} & 0.928734 & 2.429357 & -0.000087\end{array}$

$\begin{array}{lllll}38 \mathrm{~B} & -0.940824 & 2.424701 & -0.000087\end{array}$

$E 39 / \varepsilon=-206.281050$

\begin{tabular}{crrr|} 
atom & $x / \sigma$ & $y / \sigma$ & $z / \sigma$ \\
$1 \mathrm{~A}$ & 0.000000 & 0.000000 & 0.000000 \\
$2 \mathrm{~A}$ & 1.076031 & 0.000000 & 0.000000 \\
$3 \mathrm{~A}$ & 0.523664 & -0.934425 & 0.000000 \\
$4 \mathrm{~A}$ & 0.407631 & 0.835267 & -0.533327 \\
$5 \mathrm{~A}$ & -0.471304 & -0.749066 & -0.610432 \\
$6 \mathrm{~A}$ & -0.487715 & -0.747547 & 0.587823 \\
$7 \mathrm{~A}$ & 0.488882 & -0.270255 & -0.902861 \\
$8 \mathrm{~A}$ & 0.494818 & -0.272325 & 0.918095 \\
$9 \mathrm{~A}$ & 0.454457 & 0.772509 & 0.564055 \\
$10 \mathrm{~A}$ & -0.477011 & 0.292482 & -0.927862 \\
$11 \mathrm{~A}$ & -0.526261 & 0.950263 & 0.036712 \\
$12 \mathrm{~B}$ & 1.713951 & -1.041307 & -0.944029 \\
$13 \mathrm{~A}$ & -1.057920 & -0.012639 & -0.025597 \\
$14 \mathrm{~A}$ & -0.462927 & 0.282888 & 0.936936 \\
$15 \mathrm{~B}$ & 1.726132 & -1.025683 & 0.923753 \\
$16 \mathrm{~B}$ & 0.314301 & 2.168338 & 0.255551 \\
$17 \mathrm{~B}$ & -0.177951 & -0.653923 & -2.118981 \\
$18 \mathrm{~A}$ & 1.338932 & 0.414894 & -0.951840 \\
$19 \mathrm{~A}$ & 1.319231 & 1.075917 & -0.021225 \\
$20 \mathrm{~B}$ & -1.837470 & -0.273412 & 1.145893 \\
$21 \mathrm{~A}$ & -0.305351 & -1.626994 & -0.007558 \\
$22 \mathrm{~B}$ & -0.597785 & 1.679593 & -1.298319
\end{tabular}
$\begin{array}{llll}22 B & -0.597785 & 1.679593 & -1.298319\end{array}$ $\begin{array}{llll}24 \mathrm{~B} & -0.286694 & -0.692707 & 2.086084\end{array}$ $\begin{array}{llll}25 \mathrm{~B} & 0.560461 & 0.925814 & 1.984753\end{array}$ $\begin{array}{lllll}26 \mathrm{~B} & 2.565076 & 0.356123 & -0.084733\end{array}$ $\begin{array}{llll}27 \mathrm{~B} & 1.283907 & 1.807316 & -1.316723\end{array}$ $\begin{array}{llll}28 \mathrm{~A} & -1.325696 & -1.102730 & -0.026534\end{array}$ $\begin{array}{llll}29 A & 0.392310 & 0.600032 & -1.580810\end{array}$ $\begin{array}{llll}30 A & 0.301068 & -1.429120 & -0.948730\end{array}$ $\begin{array}{lllll}31 \mathrm{~A} & 1.433699 & 0.424381 & 0.919692\end{array}$ $\begin{array}{llll}32 \mathrm{~A} & 0.285718 & -1.429984 & 0.940621\end{array}$ $\begin{array}{lllll}33 \mathrm{~B} & 0.883492 & -2.436439 & -0.000104\end{array}$ $\begin{array}{llll}34 \mathrm{~B} & -1.032720 & -2.067799 & -1.164410\end{array}$ $\begin{array}{llll}35 \mathrm{~B} & -1.107094 & -2.046142 & 1.108110\end{array}$ $\begin{array}{llll}36 \mathrm{~B} & -1.068844 & 1.525574 & 1.346069\end{array}$ $\begin{array}{llll}37 \mathrm{~B} & -1.988779 & 1.118509 & -0.185978\end{array}$ $\begin{array}{lllll}38 \mathrm{~B} & 1.538690 & 0.153479 & -2.376259\end{array}$ $\begin{array}{llll}39 \mathrm{~B} & 1.973991 & 1.796002 & 1.065461\end{array}$

\section{$E 40 / \varepsilon=-213.220313$}

atom $\quad \mathrm{x} / \sigma \quad \mathrm{y} / \sigma$

$\begin{array}{llll}\text { 1A } & 0.000000 & 0.000000 & 0.000000\end{array}$ $\begin{array}{llll}2 \mathrm{~A} & 1.085044 & 0.000000 & 0.000000\end{array}$

$\begin{array}{llll}3 \mathrm{~A} & -0.451604 & -0.986597 & 0.000000\end{array}$

$\begin{array}{lllll}4 \mathrm{~A} & 0.449191 & 0.346518 & -0.929894\end{array}$

$\begin{array}{lllll}5 A & -0.502035 & -0.264212 & -0.929893\end{array}$

$\begin{array}{lllll}6 \mathrm{~A} & 0.456239 & -0.710603 & -0.592579\end{array}$

$\begin{array}{llll}7 \mathrm{~A} & 0.510057 & 0.306482 & 0.902828\end{array}$

$\begin{array}{llll}8 \mathrm{~A} & -0.490964 & -0.336218 & 0.902828\end{array}$

$\begin{array}{llll}9 \mathrm{~A} & 0.498420 & -0.776300 & 0.522980\end{array}$

$\begin{array}{llll}10 \mathrm{~A} & 0.491982 & 0.938376 & 0.013885\end{array}$

$\begin{array}{llll}11 \mathrm{~A} & -1.058004 & -0.056784 & 0.013886\end{array}$

$\begin{array}{llll}12 B & 1.729689 & -0.647282 & 1.249130\end{array}$

$\begin{array}{llll}13 B & -0.131357 & -1.842157 & 1.249130\end{array}$

$\begin{array}{llll}14 \mathrm{~A} & -0.492205 & 0.766621 & -0.570437\end{array}$

$\begin{array}{llll}15 \mathrm{~A} & -0.488698 & 0.761160 & 0.523591\end{array}$

$\begin{array}{llll}16 \mathrm{~B} & 1.776149 & 1.132608 & 0.704312\end{array}$

$\begin{array}{llll}17 \mathrm{~B} & -1.769092 & -1.143596 & 0.704314\end{array}$

$\begin{array}{llll}18 \mathrm{~B} & 1.114134 & -1.735291 & -1.427192\end{array}$

$\begin{array}{llll}19 B & -0.489558 & 0.762496 & -2.039311\end{array}$

$\begin{array}{lllll}20 A & 1.367501 & -1.043463 & -0.140662\end{array}$

$\begin{array}{llll}21 \mathrm{~A} & 0.379623 & -1.677725 & -0.140662\end{array}$

$\begin{array}{lllll}22 A & 0.310701 & -0.483926 & -1.648845\end{array}$

$\begin{array}{llll}23 \mathrm{~A} & 1.321735 & -0.314929 & -1.043210\end{array}$

$\begin{array}{llll}24 \mathrm{~A} & -0.263763 & -1.332889 & -1.043211\end{array}$

$\begin{array}{llll}25 \mathrm{~B} & -1.949669 & 0.351547 & -1.001760\end{array}$

$\begin{array}{llll}26 \mathrm{~B} & 0.491818 & 1.919090 & -1.001762\end{array}$

$\begin{array}{llll}27 \mathrm{~B} & -1.779733 & 0.644372 & 1.124848\end{array}$

$\begin{array}{llll}28 B & 0.154833 & 1.886450 & 1.124846\end{array}$

$\begin{array}{llll}29 A & -0.331837 & 0.516846 & 1.574635\end{array}$

$\begin{array}{lllll}30 \mathrm{~A} & 1.419740 & 0.774509 & -0.684099\end{array}$

$\begin{array}{llll}31 \mathrm{~A} & -1.295146 & -0.968568 & -0.684097\end{array}$

$\begin{array}{llll}32 \mathrm{~A} & 0.311514 & -0.485189 & 1.615095\end{array}$

$\begin{array}{llll}33 \mathrm{~B} & -0.906338 & -2.413674 & -0.355630\end{array}$

$\begin{array}{lllll}34 \mathrm{~B} & 2.571905 & -0.180487 & -0.355630\end{array}$

$\begin{array}{llll}35 \mathrm{~B} & -1.229676 & 1.915250 & -0.144279\end{array}$

$\begin{array}{llll}36 B & 1.329900 & 0.589032 & -2.156637\end{array}$

$\begin{array}{llll}37 \mathrm{~B} & -1.089109 & -0.964076 & -2.156635\end{array}$

$\begin{array}{llll}38 \mathrm{~B} & -0.975573 & -0.563182 & 2.330319\end{array}$

$\begin{array}{llll}39 \mathrm{~B} & 0.918127 & 0.652659 & 2.330318\end{array}$

$\begin{array}{llll}40 B & 1.517299 & -2.363228 & 0.460949\end{array}$

\section{$E 41 / \varepsilon=-219.743606$}

atom $\mathrm{x} / \sigma \quad \mathrm{g} / \sigma \quad \mathrm{z} / \sigma$

$\begin{array}{llll}1 \mathrm{~A} & 0.000000 & 0.000000 & 0.000000\end{array}$

$\begin{array}{llll}2 A & 1.081992 & 0.000000 & 0.000000\end{array}$

$\begin{array}{llll}3 A & 0.473128 & -0.979220 & 0.000000\end{array}$

$\begin{array}{lllll}4 \mathrm{~A} & 0.463004 & -0.290261 & -0.881200\end{array}$

$\begin{array}{llll}5 A & 0.515293 & 0.751052 & 0.559339\end{array}$

$\begin{array}{lllll}6 \mathrm{~A} & -0.498483 & 0.287537 & -0.931038\end{array}$

$\begin{array}{llll}7 A & -0.519820 & 0.943526 & -0.027687\end{array}$

$\begin{array}{lllll}8 \mathrm{~A} & 0.471263 & 0.764049 & -0.565254\end{array}$

$\begin{array}{llll}9 A & 0.475434 & -0.314870 & 0.905210\end{array}$ $\begin{array}{llll}10 A & -0.455842 & -0.774861 & -0.557066\end{array}$ \begin{tabular}{|llll}
$11 \mathrm{~A}$ & -0.481762 & 0.258774 & 0.880529
\end{tabular}

$\begin{array}{lllll}12 \mathrm{~A} & -1.075834 & -0.026220 & -0.025846\end{array}$

$\begin{array}{llll}13 \mathrm{~B} & 1.693657 & 1.411446 & -0.154998\end{array}$

$\begin{array}{llll}14 \mathrm{~A} & -0.457947 & -0.804793 & 0.562706\end{array}$

$\begin{array}{llll}15 B & -0.194098 & 1.804861 & -1.296470\end{array}$

$\begin{array}{llll}16 \mathrm{~B} & 1.113657 & -0.701115 & -2.142124\end{array}$

$\begin{array}{llll}17 \mathrm{~B} & 1.727318 & 0.257662 & 1.337594\end{array}$

$\begin{array}{llll}18 \mathrm{~B} & -0.572941 & -2.135140 & -0.149420\end{array}$

$\begin{array}{llll}19 B & -1.787317 & 0.808294 & 1.017424\end{array}$

$\begin{array}{llll}20 B & -1.712935 & -0.773558 & -1.212920\end{array}$

$\begin{array}{lllll}21 \mathrm{~A} & 0.369940 & -1.348402 & -1.025921\end{array}$

$\begin{array}{lllll}22 \mathrm{~B} & -1.793891 & 1.024066 & -0.811389\end{array}$

$\begin{array}{lllll}23 \mathrm{~A} & 1.341832 & -0.843601 & -0.674397\end{array}$

$\begin{array}{llll}24 \mathrm{~A} & -0.329045 & 1.307975 & 0.984838\end{array}$

$\begin{array}{llll}25 \mathrm{~A} & -0.265597 & -0.558813 & -1.612049\end{array}$

$\begin{array}{lllll}26 \mathrm{~A} & 1.368600 & 0.252279 & -1.025952\end{array}$

$\begin{array}{lllll}27 \mathrm{~A} & 0.339379 & 0.454606 & -1.628488\end{array}$

$\begin{array}{llll}28 B & -0.685783 & -0.527208 & 2.044599\end{array}$

$\begin{array}{llll}29 B & 0.525367 & -1.709566 & 1.308909\end{array}$

$\begin{array}{llll}30 \mathrm{~A} & 0.284911 & 0.545308 & 1.603806\end{array}$

$\begin{array}{llll}31 \mathrm{~A} & 0.268213 & 1.694642 & 0.096282\end{array}$

$\begin{array}{llll}32 \mathrm{~B} & 0.886720 & 1.935694 & 1.446129\end{array}$

$\begin{array}{lllll}33 \mathrm{~A} & 1.455574 & -0.916949 & 0.457915\end{array}$

$\begin{array}{llll}34 \mathrm{~B} & -1.891782 & -1.036581 & 0.651107\end{array}$

$\begin{array}{lllll}35 B & 1.306784 & -2.235159 & -0.300494\end{array}$

$\begin{array}{lllll}36 \mathrm{~B} & 2.576977 & -0.221209 & -0.275545\end{array}$

$\begin{array}{llll}37 \mathrm{~B} & -0.916259 & 0.473062 & -2.399076\end{array}$

$\begin{array}{llll}38 \mathrm{~B} & -1.033473 & 2.369064 & 0.291573\end{array}$

$\begin{array}{lllll}39 \mathrm{~B} & 1.463500 & 1.289078 & -2.041858\end{array}$

$\begin{array}{llll}40 \mathrm{~B} & -0.473358 & -1.935055 & -2.055859\end{array}$

$\begin{array}{llll}41 B & -0.661730 & 1.345582 & 2.409737\end{array}$

$E 42 / \varepsilon=-226.250732$

atom $\mathrm{x} / \sigma \quad \mathrm{y} / \sigma$

$\begin{array}{llll}1 \mathrm{~A} & 0.000000 & 0.000000 & 0.000000\end{array}$

$\begin{array}{llll}2 \mathrm{~A} & 1.050909 & 0.000000 & 0.000000\end{array}$

$\begin{array}{llll}3 \mathrm{~A} & -0.477127 & -0.985715 & 0.000000\end{array}$

$\begin{array}{llll}4 \mathrm{~A} & -0.477129 & 0.785095 & -0.596035\end{array}$

$\begin{array}{llll}5 A & -0.450110 & -0.297058 & -0.882552\end{array}$

$\begin{array}{llll}6 \mathrm{~A} & 0.463332 & -0.791460 & 0.583016\end{array}$

$\begin{array}{lllll}7 \mathrm{~A} & 0.463331 & 0.982911 & -0.014218\end{array}$

$\begin{array}{lllll}8 \mathrm{~A} & 0.494308 & 0.289889 & -0.903992\end{array}$

$\begin{array}{lllll}9 \mathrm{~A} & 0.494308 & -0.777509 & -0.544718\end{array}$

$\begin{array}{lllll}10 \mathrm{~B} & 1.702632 & -0.438857 & -1.303839\end{array}$

$\begin{array}{llll}11 \mathrm{~A} & -1.050319 & -0.009988 & -0.029671\end{array}$

$\begin{array}{llll}12 \mathrm{~A} & 0.426126 & 0.301174 & 0.894783\end{array}$

$\begin{array}{llll}13 A & -0.506991 & -0.313871 & 0.891411\end{array}$

$\begin{array}{llll}14 \mathrm{~A} & -0.506992 & 0.789001 & 0.520197\end{array}$

$\begin{array}{llll}15 B & -1.083222 & -0.724309 & -2.151905\end{array}$

$\begin{array}{lllll}16 \mathrm{~B} & 0.584004 & -2.159101 & -0.180108\end{array}$

$\begin{array}{lllll}17 \mathrm{~B} & 0.584001 & 1.610761 & -1.449001\end{array}$

$\begin{array}{llll}18 \mathrm{~B} & -1.952954 & -0.832149 & 0.692378\end{array}$

$\begin{array}{llll}19 B & -1.952956 & 1.081443 & 0.048283\end{array}$

$\begin{array}{llll}20 B & 0.326213 & -0.327006 & 2.164419\end{array}$

$\begin{array}{llll}21 \mathrm{~B} & 0.326212 & 1.569217 & 1.526172\end{array}$

$\begin{array}{llll}22 \mathrm{~A} & -0.329399 & -1.347349 & -1.035138\end{array}$

$\begin{array}{lllll}23 A & -0.329400 & 0.447207 & -1.639166\end{array}$

$\begin{array}{llll}24 \mathrm{~B} & -0.461654 & -1.746785 & 1.284684\end{array}$

$\begin{array}{llll}25 B & -0.461657 & 2.168080 & -0.033016\end{array}$

$\begin{array}{llll}26 \mathrm{~A} & -1.339771 & -0.843669 & -0.686243\end{array}$

$\begin{array}{lllll}27 A & -1.339772 & 0.257004 & -1.056718\end{array}$

$\begin{array}{llll}28 A & 1.334126 & -0.270999 & 1.007946\end{array}$

$\begin{array}{llll}29 A & 1.334125 & 0.825322 & 0.638936\end{array}$

$\begin{array}{lllll}30 \mathrm{~A} & 0.276253 & -0.537816 & -1.597839\end{array}$

$\begin{array}{llll}31 \mathrm{~B} & 2.533818 & 0.084229 & 0.250242\end{array}$

$\begin{array}{lllll}32 \mathrm{~A} & 1.446805 & 0.841671 & -0.525009\end{array}$

$\begin{array}{llll}33 \mathrm{~A} & 1.446807 & -0.987827 & 0.090780\end{array}$

$\begin{array}{llll}34 \mathrm{~B} & -1.277778 & 0.586521 & 1.742548\end{array}$

$\begin{array}{llll}35 \mathrm{~B} & -1.274262 & 1.580491 & -1.646451\end{array}$

$\begin{array}{llll}36 \mathrm{~B} & -1.274259 & -2.254388 & -0.355673\end{array}$

$\begin{array}{llll}37 \mathrm{~B} & 1.426220 & 2.177949 & 0.125928\end{array}$

$\begin{array}{llll}38 \mathrm{~B} & 1.426224 & -1.658533 & 1.417245\end{array}$

$\begin{array}{llll}39 B & 0.620612 & 0.242099 & -2.751931\end{array}$ 
$\begin{array}{llll}40 \mathrm{~B} & 0.620612 & -1.856845 & -2.045452\end{array}$ $\begin{array}{llll}41 B & 1.919457 & 0.664572 & 1.974431\end{array}$ $\begin{array}{llll}42 B & -2.669721 & -0.339221 & -1.007809\end{array}$

$E 43 / \varepsilon=-233.255889$

\begin{tabular}{rrrr} 
atom & $\mathrm{X} / \sigma$ & \multicolumn{1}{c}{$y / \sigma$} & \multicolumn{1}{c}{$z / \sigma$} \\
$1 \mathrm{~A}$ & 0.000000 & 0.000000 & 0.000000 \\
$2 \mathrm{~A}$ & 1.078586 & 0.000000 & 0.000000 \\
$3 \mathrm{~A}$ & 0.534401 & -0.993548 & 0.000000 \\
4B & -0.881798 & 1.427298 & -0.000002 \\
5A & 0.565285 & -0.309707 & 0.896296 \\
$6 \mathrm{~A}$ & 0.565290 & -0.309705 & -0.896297 \\
$7 \mathrm{~A}$ & 0.528554 & 0.940833 & 0.000001 \\
8A & 1.630218 & 0.998897 & 0.000004 \\
$9 \mathrm{~A}$ & -0.025714 & 0.614715 & -0.895947 \\
$10 \mathrm{~A}$ & -0.025719 & 0.614712 & 0.895947 \\
$11 \mathrm{~A}$ & -0.360798 & -0.848862 & -0.563807 \\
$12 \mathrm{~A}$ & -0.360802 & -0.848864 & 0.563800 \\
$13 \mathrm{~A}$ & -0.932880 & 0.059096 & 0.560602 \\
$14 \mathrm{~A}$ & -0.932876 & 0.059098 & -0.560610 \\
$15 \mathrm{~A}$ & 1.089745 & 0.623343 & -0.883941 \\
$16 \mathrm{~A}$ & 1.089739 & 0.623341 & 0.883944 \\
$17 \mathrm{~B}$ & 0.438248 & -1.590283 & 1.519865 \\
$18 \mathrm{~B}$ & 0.438256 & -1.590281 & -1.519869 \\
$19 \mathrm{~B}$ & 0.556709 & 1.992868 & -1.009237 \\
$20 \mathrm{~B}$ & 0.556702 & 1.992863 & 1.009246 \\
$21 \mathrm{~A}$ & 2.001665 & 0.112516 & 0.553365 \\
$22 \mathrm{~A}$ & 2.001669 & 0.112517 & -0.553356 \\
$23 \mathrm{~A}$ & 1.448697 & -0.861168 & 0.554648 \\
$24 \mathrm{~A}$ & 1.448700 & -0.861168 & -0.554645 \\
$25 \mathrm{~B}$ & 0.578450 & 0.538988 & 2.249024 \\
$26 \mathrm{~B}$ & 0.578460 & 0.539003 & -2.249023 \\
$27 \mathrm{~B}$ & -0.567927 & -2.167764 & -0.000006 \\
$28 \mathrm{~B}$ & 2.803126 & -1.013407 & 0.000007 \\
$29 \mathrm{~B}$ & 2.215930 & 1.270659 & -1.512057 \\
$30 \mathrm{~B}$ & 2.215919 & 1.270652 & 1.512074 \\
$31 \mathrm{~B}$ & 1.342162 & -2.233219 & 0.000000 \\
$32 \mathrm{~A}$ & -0.351055 & -0.269429 & 1.477050 \\
$33 \mathrm{~A}$ & -0.351045 & -0.269424 & -1.477057 \\
$34 \mathrm{~A}$ & -1.286745 & -0.835882 & -0.000007 \\
$35 \mathrm{~B}$ & -1.576354 & -1.043943 & -1.404201 \\
$36 \mathrm{~B}$ & -1.576364 & -1.043947 & 1.404184 \\
$37 \mathrm{~B}$ & 1.998193 & -0.567233 & -1.845684 \\
$38 \mathrm{~B}$ & 1.998190 & -0.567243 & 1.845690 \\
$39 \mathrm{~B}$ & 3.176144 & 0.825028 & 0.000011 \\
$40 \mathrm{~B}$ & -1.240218 & 0.879221 & -1.736723 \\
$41 \mathrm{~B}$ & -1.240227 & 0.879216 & 1.736717 \\
$42 \mathrm{~B}$ & -2.296232 & 0.218891 & -0.000010 \\
$43 \mathrm{~B}$ & 2.092040 & 2.391950 & 0.000011 \\
& &
\end{tabular}

$E 44 / \varepsilon=-240.201249$

$\begin{array}{cccc}\text { IA } & 0.000000 & 0.000000 & 0.000000\end{array}$

2A $\quad 1.104889 \quad 0.000000 \quad 0.000000$

3A $\quad 0.552444 \quad-0.940171 \quad 0.000000$

$\begin{array}{llll}4 \mathrm{~A} & 0.552445 & -0.289602 & 0.894457\end{array}$

$\begin{array}{lllll}5 A & 0.552444 & 0.304356 & -0.869573\end{array}$

$\begin{array}{lllll}6 \mathrm{~A} & 0.552444 & 0.921042 & -0.021701\end{array}$

$\begin{array}{llll}7 \mathrm{~B} & 0.552445 & 2.197061 & 0.508959\end{array}$

$\begin{array}{llll}8 B & 0.552440 & 0.192544 & -2.247007\end{array}$

$\begin{array}{lllll}9 \mathrm{~A} & 0.013361 & -0.638030 & -0.903101\end{array}$

$\begin{array}{llll}10 \mathrm{~A} & 1.091527 & 0.662656 & 0.885189\end{array}$

$\begin{array}{llll}11 \mathrm{~A} & 1.091526 & -0.638030 & -0.903101\end{array}$

$\begin{array}{llll}12 \mathrm{~A} & 0.013362 & 0.662655 & 0.885190\end{array}$

$\begin{array}{llll}13 \mathrm{~A} & -0.527912 & 0.317093 & -0.912382\end{array}$

$\begin{array}{lllll}14 \mathrm{~A} & 1.632800 & 0.965694 & -0.020634\end{array}$

$\begin{array}{llll}15 A & -0.527912 & 0.965693 & -0.020632\end{array}$

$\begin{array}{llll}16 \mathrm{~A} & 1.632799 & 0.317093 & -0.912383\end{array}$

$\begin{array}{llll}17 \mathrm{~B} & -0.464913 & -0.257572 & 1.951901\end{array}$

$\begin{array}{llll}18 \mathrm{~B} & -0.464915 & -1.936333 & -0.356196\end{array}$

$\begin{array}{llll}19 \mathrm{~B} & 1.569804 & -0.257571 & 1.951900\end{array}$

$\begin{array}{llll}20 B & 1.569803 & -1.936332 & -0.356198\end{array}$ $\begin{array}{llll}21 B & -1.892333 & 0.575401 & -0.418509\end{array}$
22B

$23 \mathrm{~A}$

24A

0.010285

1.258773

$-0.915548$

$\begin{array}{lll}0.010285 & 1.258773 & -0.915548\end{array}$

$\begin{array}{llll}25 \mathrm{~A} & -0.905016 & -0.519524 & -0.309868\end{array}$

$\begin{array}{llll}26 \mathrm{~A} & 2.009906 & 0.134773 & 0.589710\end{array}$

$\begin{array}{llll}27 \mathrm{~A} & -0.905016 & 0.134772 & 0.589712\end{array}$

$\begin{array}{llll}28 \mathrm{~A} & 2.009905 & -0.519523 & -0.309870\end{array}$

$\begin{array}{llll}29 A & -0.362517 & -0.823366 & 0.598863\end{array}$

$\begin{array}{lllll}30 A & 1.467407 & -0.823366 & 0.598863\end{array}$

$\begin{array}{llll}31 B & 0.552445 & -1.745948 & 1.269889\end{array}$

$\begin{array}{llll}32 \mathrm{~B} & -1.159359 & -0.707798 & -1.761343\end{array}$

$\begin{array}{llll}33 \mathrm{~B} & -1.159357 & 1.457677 & 1.215931\end{array}$

$\begin{array}{llll}34 \mathrm{~B} & 2.264243 & -0.707797 & -1.761346\end{array}$

$\begin{array}{llll}35 B & 2.264246 & 1.457679 & 1.215929\end{array}$

$\begin{array}{llll}36 \mathrm{~B} & 0.552445 & 1.297448 & 2.114413\end{array}$

$\begin{array}{lllll}37 \mathrm{~B} & 0.552443 & -1.611953 & -1.885663\end{array}$

$\begin{array}{lllll}38 \mathrm{~B} & 2.100764 & 1.181634 & -2.006823\end{array}$

$\begin{array}{lllll}39 B & -0.995884 & 2.273220 & -0.506014\end{array}$

$\begin{array}{llll}40 \mathrm{~B} & 2.100772 & 2.273221 & -0.506018\end{array}$

$\begin{array}{llll}41 \mathrm{~B} & -0.995882 & 1.181635 & -2.006819\end{array}$

$\begin{array}{lllll}42 \mathrm{~B} & 0.552443 & 2.347847 & -1.707659\end{array}$

$\begin{array}{llll}43 B & -1.812493 & -1.016549 & 0.739371\end{array}$

$\begin{array}{llll}\text { 44B } & 2.917382 & -1.016547 & 0.739369\end{array}$

$E 45 / \varepsilon=-247.677700$

atom $\mathrm{X} / \sigma$ $y / \sigma$

$\begin{array}{llll}1 \mathrm{~A} & 0.000000 & 0.000000 & 0.000000\end{array}$

$\begin{array}{llll}2 \mathrm{~A} & 1.106159 & 0.000000 & 0.000000\end{array}$

$\begin{array}{llll}3 \mathrm{~A} & 0.545095 & -0.962529 & 0.000000\end{array}$

$\begin{array}{lllll}4 \mathrm{~A} & 0.548248 & -0.319578 & -0.844954\end{array}$

$\begin{array}{llll}5 \mathrm{~B} & 0.525911 & -0.306559 & -2.223057\end{array}$

$\begin{array}{llll}6 \mathrm{~A} & -0.544020 & -0.944249 & -0.016519\end{array}$

$\begin{array}{lllll}7 A & 0.553558 & 0.938689 & -0.016520\end{array}$

$\begin{array}{llll}8 \mathrm{~A} & 0.002383 & -0.635128 & 0.882606\end{array}$

$\begin{array}{lllll}9 \mathrm{~A} & 0.553833 & 0.310906 & 0.882605\end{array}$

$\begin{array}{llll}10 \mathrm{~A} & 1.633718 & -0.952306 & -0.017745\end{array}$

$\begin{array}{lllll}11 \mathrm{~A} & 1.097423 & -0.639696 & 0.879452\end{array}$

$\begin{array}{llll}12 \mathrm{~A} & -0.537400 & -0.317285 & -0.915111\end{array}$

$\begin{array}{llll}13 \mathrm{~A} & 0.011265 & 0.623971 & -0.915112\end{array}$

$\begin{array}{llll}14 A & -0.004102 & -1.252277 & -0.917210\end{array}$

$\begin{array}{llll}15 A & 1.087650 & 0.620667 & -0.917210\end{array}$

$\begin{array}{lllll}16 A & 1.086026 & -1.258071 & -0.917559\end{array}$

$\begin{array}{llll}17 A & 1.629887 & -0.325056 & -0.917560\end{array}$

$\begin{array}{llll}18 \mathrm{~B} & -0.441958 & 0.257621 & 1.954310\end{array}$

$\begin{array}{llll}19 \mathrm{~B} & -1.906933 & -0.558866 & -0.402222\end{array}$

$\begin{array}{llll}20 \mathrm{~B} & -0.453404 & 1.934721 & -0.402223\end{array}$

$\begin{array}{llll}21 \mathrm{~A} & -0.904514 & 0.527246 & -0.319484\end{array}$

$\begin{array}{llll}22 \mathrm{~A} & -0.908604 & -0.101218 & 0.585814\end{array}$

$\begin{array}{llll}23 \mathrm{~A} & -0.359669 & 0.840503 & 0.585813\end{array}$

$\begin{array}{llll}24 \mathrm{~B} & 0.541089 & -1.436084 & 1.967977\end{array}$

$\begin{array}{llll}25 \mathrm{~B} & 1.516252 & 0.236846 & 1.967977\end{array}$

$\begin{array}{llll}26 \mathrm{~B} & -0.930374 & -2.290266 & -0.419239\end{array}$

$\begin{array}{llll}27 \mathrm{~B} & 1.534408 & 1.938168 & -0.419242\end{array}$

$\begin{array}{lllll}28 \mathrm{~B} & 1.997740 & -2.305802 & -0.430077\end{array}$

$\begin{array}{lllll}29 B & 2.990850 & -0.602083 & -0.430078\end{array}$

$\begin{array}{llll}30 \mathrm{~B} & -1.269132 & -1.345124 & 1.245558\end{array}$

$\begin{array}{llll}31 B & 0.545057 & 1.767192 & 1.245557\end{array}$

$\begin{array}{llll}32 \mathrm{~A} & 2.015431 & 0.525751 & -0.324542\end{array}$

$\begin{array}{lllll}33 \mathrm{~A} & 0.535684 & -2.012815 & -0.324541\end{array}$

$\begin{array}{llll}34 \mathrm{~A} & 1.479442 & 0.844256 & 0.588892\end{array}$

$\begin{array}{llll}35 A & -0.005589 & -1.703375 & 0.588893\end{array}$

$\begin{array}{llll}36 \mathrm{~A} & 1.084751 & -1.709772 & 0.585984\end{array}$

$\begin{array}{llll}37 \mathrm{~A} & 2.022309 & -0.101354 & 0.585984\end{array}$

$\begin{array}{llll}38 \mathrm{~B} & -1.172966 & 0.683728 & -1.773009\end{array}$

$\begin{array}{llll}39 B & 2.351208 & -1.370535 & 1.238696\end{array}$

$\begin{array}{llll}40 \mathrm{~B} & 0.527550 & -2.277123 & -1.805624\end{array}$

$\begin{array}{llll}41 \mathrm{~B} & 2.241411 & 0.663074 & -1.805626\end{array}$

$\begin{array}{llll}42 \mathrm{~B} & 0.536910 & 1.532012 & -1.952624\end{array}$

$\begin{array}{llll}43 \mathrm{~B} & -1.068507 & -1.222144 & -1.952622\end{array}$

$\begin{array}{llll}44 \mathrm{~B} & 2.121297 & -1.236520 & -1.974527\end{array}$

$\begin{array}{llll}45 B & -1.803502 & 1.051272 & 0.731993\end{array}$

$E 46 / \varepsilon=-255.625219$

\begin{tabular}{|c|c|c|c|}
\hline & $X I$ & $y / \sigma$ & $2 t$ \\
\hline & 000000 & 00000 & \\
\hline 2 & 033 & 000000 & \\
\hline & 50015 & -096 & \\
\hline & 16 & & \\
\hline & 018 & 283 & \\
\hline & 016 & 983 & \\
\hline & & & \\
\hline & 1.638951 & -0.946925 & \\
\hline & -0.538922 & -0.9 & \\
\hline A & 1.096775 & -0.6 & \\
\hline & 0.003255 & -0.637436 & -0.8 \\
\hline & 0.012373 & 285 & \\
\hline & 1.087663 & 0.625283 & \\
\hline & 1.633717 & -0.317915 & \\
\hline & -0.533685 & -0.317911 & \\
\hline ה נס & 1.095349 & -1.252687 & \\
\hline$n$ & 0.0 & 684 & \\
\hline J & -0.4 & 099 & \\
\hline L & 1.539104 & 0.255096 & -1.95 \\
\hline ID & -0.453561 & 1.936697 & 0.3968 \\
\hline & 601 & 694 & 0.2 \\
\hline & 3.0 & -0 & 0.4 \\
\hline & -1.9 & -0 & 0.4 \\
\hline & -0.9 & 277 & 0.3 \\
\hline & 2.0 & 272 & 0 \\
\hline & -0.2 & 0.8 & \\
\hline & & 0.8 & \\
\hline & -0.9 & -0.1 & -0 \\
\hline & & -0.1 & -0 \\
\hline & 0.5 & & -1 \\
\hline & 0.55 & -1.4 & -1.97 \\
\hline & 2.016650 & -2.2 & 0.4 \\
\hline & -0.916626 & -2.293158 & 0.4254 \\
\hline & 2.36 & -1.351215 & -1.2424 \\
\hline & -1.262902 & -1.351209 & -1.2424 \\
\hline & 0.550012 & -2.011320 & 0.3247 \\
\hline & 0.004350 & -1.705332 & -0.5885 \\
\hline & 1.095677 & -1.705334 & -0.5885 \\
\hline & 2.263818 & 0.685622 & 1.7765 \\
\hline & -1.163783 & 0.685629 & 1.776 \\
\hline & 0.550012 & -2.268624 & 1.809 \\
\hline & 0.550019 & 1.547035 & 1.937 \\
\hline & -1.047052 & -1.218155 & 1.961 \\
\hline & 2.147080 & -1.218162 & 1.961 \\
\hline & -1.80 & 1.04 & -0.731 \\
\hline & 2.90 & 1.045941 & -0.731 \\
\hline
\end{tabular}

$E 47 / \varepsilon=-263.555682$

atom $\quad \mathrm{x} / \sigma \quad \mathrm{y} / \sigma$

$\begin{array}{llll}1 \mathrm{~A} & 0.000000 & 0.000000 & 0.000000\end{array}$

$\begin{array}{llll}2 \mathrm{~A} & 1.102045 & 0.000000 & 0.000000\end{array}$

$\begin{array}{llll}3 A & 0.551023 & -0.954400 & 0.000000\end{array}$

$\begin{array}{llll}4 \mathrm{~A} & 0.551023 & -0.318133 & 0.847406\end{array}$

$\begin{array}{llll}5 A & -0.538988 & -0.947453 & 0.017680\end{array}$

$\begin{array}{llll}6 \mathrm{~A} & 1.641035 & -0.947451 & 0.017679\end{array}$

$\begin{array}{llll}7 \mathrm{~A} & 0.551021 & 0.940504 & 0.017679\end{array}$

$\begin{array}{llll}8 \mathrm{~B} & 0.551024 & -0.318132 & 2.224727\end{array}$

$\begin{array}{lllll}9 \mathrm{~A} & 1.096796 & -0.633236 & -0.884966\end{array}$

$\begin{array}{llll}10 \mathrm{~A} & 0.005248 & -0.633237 & -0.884966\end{array}$

$\begin{array}{lllll}11 \mathrm{~A} & 0.551021 & 0.312072 & -0.884966\end{array}$

$\begin{array}{llll}12 \mathrm{~A} & 1.089804 & 0.622407 & 0.914846\end{array}$

$\begin{array}{llll}13 \mathrm{~A} & 0.012240 & 0.622406 & 0.914847\end{array}$

$\begin{array}{llll}14 \mathrm{~A} & 1.634946 & -0.321803 & 0.914846\end{array}$

$\begin{array}{llll}15 A & -0.532899 & -0.321805 & 0.914847\end{array}$

$\begin{array}{llll}16 \mathrm{~A} & 1.096164 & -1.255002 & 0.914846\end{array}$

$\begin{array}{llll}17 A & 0.005884 & -1.255003 & 0.914847\end{array}$

$\begin{array}{llll}18 B & 0.551022 & -1.457329 & -1.958478\end{array}$

$\begin{array}{llll}19 B & 1.537591 & 0.251465 & -1.958479\end{array}$

$\begin{array}{llll}20 \mathrm{~B} & -0.435552 & 0.251463 & -1.958477\end{array}$

$\begin{array}{llll}21 B & -1.900125 & -0.573297 & 0.402997\end{array}$

$\begin{array}{llll}22 \mathrm{~B} & -0.895525 & -2.313311 & 0.402996\end{array}$

$\begin{array}{llll}23 \mathrm{~B} & -0.453579 & 1.932203 & 0.402995\end{array}$ 


$\begin{array}{lrrr}24 B & 3.002171 & -0.573291 & 0.402993 \\ 25 B & 1.997575 & -2.313308 & 0.402995 \\ 26 B & 1.555619 & 1.932205 & 0.402993 \\ 27 A & 2.007079 & 0.522523 & 0.320149 \\ 28 A & -0.905035 & 0.522520 & 0.320152 \\ 29 A & 0.551024 & -1.999443 & 0.320151 \\ 30 A & 1.463210 & 0.839163 & -0.587668 \\ 31 A & -0.907320 & -0.106804 & -0.587665 \\ 32 A & -0.361169 & 0.839162 & -0.587667 \\ 33 A & 1.097176 & -1.686760 & -0.587667 \\ 34 A & 2.009364 & -0.106801 & -0.587668 \\ 35 A & 0.004870 & -1.686761 & -0.587666 \\ 36 B & -1.255279 & -1.361004 & -1.245781 \\ 37 B & 2.357323 & -1.361000 & -1.245784 \\ 38 B & 0.551019 & 1.767603 & -1.245784 \\ 39 B & -1.163214 & 0.671580 & 1.780590 \\ \text { 40B } & 2.265259 & 0.671585 & 1.780586 \\ 41 B & 0.551026 & -2.297563 & 1.780589 \\ 42 B & 2.150247 & -1.241442 & 1.946399 \\ 43 B & -1.048198 & -1.241447 & 1.946401 \\ 44 B & 0.551022 & 1.528491 & 1.946399 \\ 45 B & -1.805626 & 1.042472 & -0.731834 \\ 46 B & 2.907664 & 1.042479 & -0.731841 \\ 47 B & 0.551025 & -3.039353 & -0.731837\end{array}$

$E 48 / \varepsilon=-270.247871$

\begin{tabular}{|c|c|c|c|}
\hline tom & $\mathrm{x} / \sigma$ & $y / \sigma$ & $z / \sigma$ \\
\hline $1 \mathrm{~A}$ & 0.000000 & 0.000000 & 0.000000 \\
\hline $2 \mathrm{~A}$ & 1.084436 & 0.000000 & 0.000000 \\
\hline $3 A$ & 0.542218 & -0.947195 & 0.000000 \\
\hline $4 \mathrm{~A}$ & 0.542218 & -0.310199 & -0.894961 \\
\hline $5 A$ & 0.542218 & 0.311706 & 0.894518 \\
\hline $6 \mathrm{~A}$ & 0.542218 & 0.947270 & 0.001569 \\
\hline $7 A$ & 1.632022 & -0.942960 & -0.001016 \\
\hline $8 \mathrm{~A}$ & -0.547587 & -0.309772 & -0.890627 \\
\hline $9 \mathrm{~A}$ & -0.547587 & -0.942960 & -0.001016 \\
\hline $10 \mathrm{~A}$ & 1.632022 & -0.309772 & -0.890627 \\
\hline $11 \mathrm{~A}$ & 1.086951 & -0.631246 & 0.891165 \\
\hline $12 \mathrm{~A}$ & -0.002516 & -0.631246 & 0.891165 \\
\hline $13 \mathrm{~A}$ & -0.002516 & 0.635293 & -0.888284 \\
\hline $14 \mathrm{~A}$ & 1.086951 & 0.635293 & -0.888284 \\
\hline $15 A$ & -0.001363 & -1.255487 & -0.893603 \\
\hline $16 \mathrm{~A}$ & 1.085799 & -1.255486 & -0.893603 \\
\hline 17B & -0.444585 & 0.259253 & 1.968303 \\
\hline 18B & 1.529021 & 1.944662 & -0.399648 \\
\hline 19B & 1.529021 & 0.259252 & 1.968303 \\
\hline $20 B$ & -0.444586 & 1.944662 & -0.399647 \\
\hline $21 \mathrm{~B}$ & -1.904203 & -0.572263 & -0.407313 \\
\hline $22 B$ & 2.988638 & -0.572263 & -0.407313 \\
\hline $23 \mathrm{~A}$ & 1.449101 & 0.843924 & 0.600671 \\
\hline $24 \mathrm{~A}$ & -0.364666 & 0.843924 & 0.600671 \\
\hline $25 \mathrm{~A}$ & 1.995720 & -0.100808 & 0.597876 \\
\hline $26 \mathrm{~A}$ & -0.911285 & 0.531892 & -0.291048 \\
\hline $27 \mathrm{~A}$ & -0.911285 & -0.100808 & 0.597876 \\
\hline $28 \mathrm{~A}$ & 1.995720 & 0.531892 & -0.291048 \\
\hline 29B & 0.542218 & 1.773267 & 1.262138 \\
\hline 30B & 0.542217 & -1.421412 & 1.983410 \\
\hline 31B & 0.542217 & 1.408532 & -1.992577 \\
\hline $32 B$ & -0.937162 & -2.274548 & -0.430663 \\
\hline 33B & -0.937162 & -1.151810 & -2.008077 \\
\hline 34B & 2.021598 & -1.151810 & -2.008077 \\
\hline 35B & 2.021598 & -2.274548 & -0.430663 \\
\hline 36B & -1.272019 & 0.745310 & -1.682755 \\
\hline 37B & -1.272019 & -1.345875 & 1.255299 \\
\hline $38 \mathrm{~B}$ & 2.356453 & -1.345876 & 1.255299 \\
\hline $39 B$ & 2.356454 & 0.745310 & -1.682756 \\
\hline $40 \mathrm{~A}$ & 1.089072 & -1.694781 & 0.601854 \\
\hline $41 \mathrm{~A}$ & -0.004637 & 0.013637 & -1.798423 \\
\hline $42 \mathrm{~A}$ & -0.004637 & -1.694781 & 0.601854 \\
\hline $43 \mathrm{~A}$ & 1.089072 & 0.013637 & -1.798423 \\
\hline $14 \mathrm{~A}$ & 0.542218 & -2.008301 & -0.291788 \\
\hline $15 \mathrm{~A}$ & 0.542218 & -0.933400 & -1.801993 \\
\hline & 0.542219 & -2.3 & \\
\hline
\end{tabular}

$\begin{array}{|llll|}47 \mathrm{~B} & -1.810970 & 1.050468 & 0.747679\end{array}$ $\begin{array}{llll}48 \mathrm{~B} & 2.895405 & 1.050467 & 0.747679\end{array}$

$E 49 / \varepsilon=-278.200778$

$\begin{array}{cccc}\text { atom } & \mathrm{x} / \sigma & y / \sigma & z / \sigma \\ 1 \mathrm{~A} & 0.000000 & 0.000000 & 0.000000\end{array}$

$\begin{array}{llll}2 \mathrm{~A} & 1.086440 & 0.000000 & 0.000000\end{array}$

$\begin{array}{llll}3 \mathrm{~A} & 0.543219 & -0.940884 & 0.000000\end{array}$

$\begin{array}{llll}4 \mathrm{~A} & 0.543218 & -0.313626 & 0.895587\end{array}$

$\begin{array}{llll}5 \mathrm{~A} & 0.543220 & 0.945848 & 0.000630\end{array}$

$\begin{array}{llll}6 \mathrm{~A} & 1.633958 & -0.943367 & 0.000635\end{array}$

$\begin{array}{llll}7 A & -0.547519 & -0.943366 & 0.000631\end{array}$

$\begin{array}{lllll}8 \mathrm{~A} & 0.543221 & 0.314218 & -0.893804\end{array}$

$\begin{array}{llll}9 \mathrm{~A} & -0.000511 & -0.627553 & -0.893803\end{array}$

$\begin{array}{llll}10 \mathrm{~A} & 1.086953 & -0.627554 & -0.893802\end{array}$

$\begin{array}{llll}11 \mathrm{~A} & -0.001863 & -1.258073 & 0.890447\end{array}$

$\begin{array}{llll}12 \mathrm{~A} & 1.088298 & -1.258073 & 0.890449\end{array}$

$\begin{array}{llll}13 \mathrm{~A} & 1.088593 & 0.630651 & 0.890445\end{array}$

$\begin{array}{llll}14 \mathrm{~A} & 1.633674 & -0.313456 & 0.890448\end{array}$

$\begin{array}{llll}15 A & -0.002157 & 0.630651 & 0.890444\end{array}$

$\begin{array}{llll}16 \mathrm{~A} & -0.547238 & -0.313456 & 0.890445\end{array}$

$\begin{array}{llll}17 \mathrm{~B} & 0.543222 & -1.450105 & -1.970543\end{array}$

$\begin{array}{lllll}18 \mathrm{~B} & 1.527437 & 0.254605 & -1.970545\end{array}$

$\begin{array}{lllll}19 B & -0.440991 & 0.254605 & -1.970548\end{array}$

$\begin{array}{llll}20 \mathrm{~B} & -1.902405 & -0.583929 & 0.403944\end{array}$

$\begin{array}{llll}21 B & -0.445503 & 1.939497 & 0.403941\end{array}$

$\begin{array}{llll}22 \mathrm{~B} & -0.913683 & -2.296447 & 0.403949\end{array}$

$\begin{array}{llll}23 \mathrm{~B} & 1.531942 & 1.939496 & 0.403945\end{array}$

$\begin{array}{llll}\text { 24B } & 2.988842 & -0.583930 & 0.403952\end{array}$

$\begin{array}{llll}25 \mathrm{~B} & 2.000119 & -2.296448 & 0.403954\end{array}$

$\begin{array}{llll}26 \mathrm{~A} & -0.365408 & 0.841851 & -0.600333\end{array}$

$\begin{array}{llll}27 \mathrm{~A} & 1.451851 & 0.841851 & -0.600330\end{array}$

$\begin{array}{llll}28 A & -0.911769 & -0.104472 & -0.600332\end{array}$

$\begin{array}{llll}29 A & 1.089580 & -1.678266 & -0.600326\end{array}$

$\begin{array}{llll}30 \mathrm{~A} & -0.003140 & -1.678266 & -0.600328\end{array}$

$\begin{array}{llll}31 \mathrm{~A} & 1.998211 & -0.104473 & -0.600328\end{array}$

$\begin{array}{llll}32 \mathrm{~A} & -0.912456 & 0.526808 & 0.291123\end{array}$

$\begin{array}{lllll}33 \mathrm{~A} & 0.543218 & -1.994496 & 0.291130\end{array}$

$\begin{array}{llll}34 \mathrm{~A} & 1.998894 & 0.526807 & 0.291127\end{array}$

$\begin{array}{llll}35 \mathrm{~B} & 0.543222 & 1.772439 & -1.259059\end{array}$

$\begin{array}{llll}36 \mathrm{~B} & -1.263368 & -1.356664 & -1.259056\end{array}$

$\begin{array}{llll}37 \mathrm{~B} & 2.349811 & -1.356666 & -1.259050\end{array}$

$\begin{array}{llll}38 \mathrm{~B} & -0.938639 & -1.169173 & 1.997861\end{array}$

$\begin{array}{llll}39 B & 2.025070 & -1.169173 & 1.997866\end{array}$

$\begin{array}{llll}\text { 40B } & 0.543216 & 1.397475 & 1.997859\end{array}$

$\begin{array}{llll}\text { 41B } & 0.543216 & -2.409819 & 1.684611\end{array}$

$\begin{array}{llll}42 \mathrm{~B} & 2.358574 & 0.734473 & 1.684608\end{array}$

$\begin{array}{llll}43 \mathrm{~B} & -1.272141 & 0.734473 & 1.684602\end{array}$

$\begin{array}{llll}44 \mathrm{~A} & 0.543216 & -0.945653 & 1.799539\end{array}$

$\begin{array}{llll}45 \mathrm{~A} & 1.090569 & 0.002390 & 1.799538\end{array}$

$\begin{array}{llll}46 \mathrm{~A} & -0.004137 & 0.002390 & 1.799536\end{array}$

$\begin{array}{llll}47 \mathrm{~B} & -1.811842 & 1.046069 & -0.747539\end{array}$

$\begin{array}{llll}48 \mathrm{~B} & 2.898285 & 1.046067 & -0.747529\end{array}$

$\begin{array}{llll}49 \mathrm{~B} & 0.543220 & -3.033024 & -0.747524\end{array}$

$E 50 / \varepsilon=-286.133713$

$\begin{array}{cccc}\text { atom } & \mathrm{X} / \sigma & y / \sigma & z / \sigma \\ 1 \mathrm{~A} & 0.000000 & 0.000000 & 0.000000\end{array}$

$\begin{array}{llll}2 \mathrm{~A} & 1.088368 & 0.000000 & 0.000000\end{array}$

$\begin{array}{llll}3 \mathrm{~A} & 0.544184 & -0.942555 & 0.000000\end{array}$

$\begin{array}{lllll}4 \mathrm{~A} & 0.544184 & -0.314184 & -0.888649\end{array}$

$\begin{array}{llll}5 \mathrm{~A} & 0.544184 & 0.314372 & 0.892225\end{array}$

$\begin{array}{llll}6 A & -0.002812 & -1.258548 & -0.889930\end{array}$

$\begin{array}{lllll}7 A & 1.091179 & -1.258549 & -0.889930\end{array}$

$\begin{array}{lllll}8 \mathrm{~A} & 0.544184 & 0.945988 & -0.001014\end{array}$

$\begin{array}{lllll}9 \mathrm{~A} & 1.088530 & -0.628464 & 0.892224\end{array}$

$\begin{array}{llll}10 \mathrm{~A} & 1.088530 & 0.631710 & -0.889928\end{array}$

$\begin{array}{llll}11 \mathrm{~A} & -0.000163 & 0.631711 & -0.889928\end{array}$

$\begin{array}{llll}12 \mathrm{~A} & -0.000163 & -0.628464 & 0.892224\end{array}$

$\begin{array}{llll}13 \mathrm{~A} & 1.635525 & -0.944271 & -0.001016\end{array}$

$\begin{array}{llll}14 \mathrm{~A} & -0.547158 & -0.944271 & -0.001016\end{array}$

$\begin{array}{lllll}15 A & 1.635525 & -0.315713 & -0.889929\end{array}$ $\begin{array}{llll}16 A & -0.547158 & -0.315713 & -0.889929\end{array}$ \begin{tabular}{|llll}
$17 B$ & 1.530300 & 0.255149 & 1.966333
\end{tabular}

$\begin{array}{llll}18 \mathrm{~B} & -0.441933 & 0.255149 & 1.966333\end{array}$

$\begin{array}{llll}19 \mathrm{~B} & 1.530300 & 1.938927 & -0.414885\end{array}$

$\begin{array}{llll}20 B & 0.544183 & -1.452855 & 1.966332\end{array}$

$\begin{array}{llll}21 B & -0.441933 & 1.938927 & -0.414885\end{array}$

$\begin{array}{lllll}22 B & 0.544183 & 1.369594 & -2.025207\end{array}$

$\begin{array}{lllll}23 \mathrm{~B} & -1.900127 & -0.586737 & -0.414887\end{array}$

$\begin{array}{llll}24 \mathrm{~B} & -0.914010 & -2.294742 & -0.414889\end{array}$

$\begin{array}{lllll}25 B & -0.914010 & -1.156071 & -2.025209\end{array}$

$\begin{array}{lllll}26 \mathrm{~B} & 2.988494 & -0.586737 & -0.414887\end{array}$

$\begin{array}{lllll}27 B & 2.002376 & -2.294742 & -0.414889\end{array}$

$\begin{array}{lllll}28 \mathrm{~B} & 2.002376 & -1.156071 & -2.025210\end{array}$

$\begin{array}{lllll}29 \mathrm{~A} & 0.544183 & -1.996687 & -0.296991\end{array}$

$\begin{array}{lllll}30 A & -0.912906 & 0.527067 & -0.296989\end{array}$

$\begin{array}{llll}31 \mathrm{~A} & 2.001274 & 0.527067 & -0.296989\end{array}$

$\begin{array}{lllll}32 \mathrm{~A} & 1.090977 & -1.680997 & 0.595919\end{array}$

$\begin{array}{lllll}33 \mathrm{~A} & 0.544183 & -0.945565 & -1.783499\end{array}$

$\begin{array}{lllll}34 \mathrm{~A} & 1.090977 & 0.001509 & -1.783499\end{array}$

$\begin{array}{llll}35 A & -0.002610 & -1.680996 & 0.595919\end{array}$

$\begin{array}{llll}36 \mathrm{~A} & -0.366113 & 0.842758 & 0.595920\end{array}$

$\begin{array}{llll}37 \mathrm{~A} & 2.001274 & -0.104316 & 0.595920\end{array}$

$\begin{array}{llll}38 \mathrm{~A} & 1.454480 & 0.842758 & 0.595921\end{array}$

$\begin{array}{llll}39 A & -0.912906 & -0.104316 & 0.595920\end{array}$

$\begin{array}{llll}40 A & -0.002610 & 0.001509 & -1.783498\end{array}$

$\begin{array}{llll}41 \mathrm{~B} & 0.544184 & 1.773226 & 1.253862\end{array}$

$\begin{array}{llll}42 \mathrm{~B} & -1.263568 & -1.357891 & 1.253860\end{array}$

$\begin{array}{llll}43 \mathrm{~B} & -1.263568 & 0.729523 & -1.698184\end{array}$

$\begin{array}{llll}44 \mathrm{~B} & 2.351935 & -1.357891 & 1.253860\end{array}$

$\begin{array}{llll}45 B & 2.351935 & 0.729523 & -1.698185\end{array}$

$\begin{array}{lllll}46 \mathrm{~B} & 0.544183 & -2.401594 & -1.698187\end{array}$

$\begin{array}{llll}47 \mathrm{~B} & -1.812835 & 1.046641 & 0.740089\end{array}$

$\begin{array}{llll}48 \mathrm{~B} & 2.901203 & 1.046641 & 0.740089\end{array}$

$\begin{array}{llll}49 \mathrm{~B} & 0.544182 & -3.035836 & 0.740086\end{array}$

$\begin{array}{lllll}50 \mathrm{~B} & 0.544182 & -0.314181 & -3.108910\end{array}$

$s=1.8$

$E 5 / \varepsilon=-9.234711$

$\begin{array}{cccc}\text { atom } & x / \sigma & y / \sigma & z / \sigma \\ \text { 1A } & 0.000000 & 0.000000 & 0.000000 \\ \text { 2A } & 1.123913 & 0.000000 & 0.000000 \\ \text { 3A } & 0.561956 & -0.973337 & 0.000000 \\ \text { 4B } & 0.561956 & -0.324446 & 1.425019 \\ \text { 5B } & 0.561956 & -0.324446 & -1.425019\end{array}$

$E 6 / \varepsilon=-13.373777$

\begin{tabular}{cccc} 
atom & $\mathrm{X} / \sigma$ & $y / \sigma$ & \multicolumn{1}{c}{$z / \sigma$} \\
1A & 0.000000 & 0.000000 & 0.000000 \\
2A & 1.138310 & 0.000000 & 0.000000 \\
3B & 0.569155 & -1.447142 & 0.000000 \\
4B & 0.569155 & 0.000000 & -1.447142 \\
5B & 0.569155 & 0.000000 & 1.447142 \\
6B & 0.569155 & 1.447142 & 0.000000
\end{tabular}

$E 7 / \varepsilon=-17.140302$

$\begin{array}{llll}\text { atom } \quad \mathrm{x} / \sigma & y / \sigma & \mathrm{z} / \sigma\end{array}$

$\begin{array}{llll}1 \mathrm{~A} & 0.000000 & 0.000000 & 0.000000\end{array}$

$\begin{array}{llll}2 B & 1.500436 & 0.000000 & 0.000000\end{array}$

$\begin{array}{llll}3 B & 0.000000 & -1.500436 & 0.000000\end{array}$

$\begin{array}{llll}4 \mathrm{~B} & 0.000000 & -0.000003 & 1.500436\end{array}$

$\begin{array}{llll}5 \mathrm{~B} & 0.000000 & 0.000000 & -1.500436\end{array}$

$\begin{array}{llll}6 B & 0.000000 & 1.500436 & 0.000003\end{array}$

$\begin{array}{llll}7 \mathrm{~B} & -1.500436 & -0.000001 & 0.000000\end{array}$

\section{$E 8 / \varepsilon=-21.521513$}

$\begin{array}{llll}\text { atom } & \mathrm{x} / \sigma & \mathrm{y} / \sigma & \mathrm{z} / \sigma\end{array}$

$\begin{array}{llll}1 \mathrm{~A} & 0.000000 & 0.000000 & 0.000000\end{array}$

$\begin{array}{llll}2 B & 1.545837 & 0.000000 & 0.000000\end{array}$

$\begin{array}{llll}3 B & -1.158888 & -1.023030 & 0.000000\end{array}$

$\begin{array}{llll}4 B & 0.205433 & -0.507000 & 1.445810\end{array}$

$\begin{array}{lllll}5 B & 0.181520 & -0.516040 & -1.445811\end{array}$

$\begin{array}{lllll}6 \mathrm{~A} & 0.398252 & -1.052915 & -0.000001\end{array}$ 
$\begin{array}{llll}7 B & 0.241249 & 1.356174 & -0.711700\end{array}$ $\begin{array}{llll}8 B & -1.078370 & 0.857041 & 0.711706\end{array}$

$E 9 / \varepsilon=-26.756037$

\begin{tabular}{cccc} 
atom & $\mathrm{X} / \sigma$ & \multicolumn{1}{c}{$y / \sigma$} & \multicolumn{1}{c}{$z / \sigma$} \\
1A & 0.000000 & 0.000000 & 0.000000 \\
2B & 1.548819 & 0.000000 & 0.000000 \\
3B & 0.151097 & -1.541431 & 0.000000 \\
4B & 0.210451 & 1.172601 & -0.998247 \\
5B & -1.146478 & -0.323841 & 0.998247 \\
6B & 0.210451 & 1.172601 & 0.998247 \\
7B & -1.146478 & -0.323842 & -0.998247 \\
8A & 0.406857 & -0.368926 & 0.983734 \\
9A & 0.406857 & -0.368926 & -0.983734
\end{tabular}

$E 10 / \varepsilon=-32.012806$

\begin{tabular}{rcrr} 
atom & $x / \sigma$ & $y / \sigma$ & \multicolumn{1}{c}{$z / \sigma$} \\
1A & 0.000000 & 0.000000 & 0.000000 \\
2B & 1.561100 & 0.000000 & 0.000000 \\
3B & 0.191721 & -1.549282 & 0.000000 \\
4B & 0.246484 & 1.161961 & -1.012977 \\
5B & -1.122894 & -0.387321 & 1.012977 \\
6B & 0.246484 & 1.161961 & 1.012977 \\
7B & -1.122894 & -0.387321 & -1.012977 \\
8A & -0.846201 & 0.747938 & 0.000000 \\
9A & 0.423100 & -0.373970 & 0.978059 \\
10A & 0.423100 & -0.373970 & -0.978059
\end{tabular}

$E 11 / \varepsilon=-36.270022$

$\begin{array}{cccc}1 \mathrm{~A} & \mathrm{X} / \sigma & y / \sigma & z / \sigma \\ & 0.000000 & 0.000000 & 0.000000\end{array}$

$\begin{array}{llll}2 \mathrm{~A} & 1.115837 & 0.000000 & 0.000000\end{array}$

$\begin{array}{llll}3 \mathrm{~A} & 0.563637 & -0.963018 & 0.000000\end{array}$

$\begin{array}{llll}4 \mathrm{~A} & 0.011437 & -0.646385 & 0.909476\end{array}$

$\begin{array}{llll}5 \mathrm{~A} & 0.563637 & 0.316633 & 0.909477\end{array}$

$\begin{array}{lllll}6 \mathrm{~B} & 0.530937 & -0.304441 & -1.405353\end{array}$

$\begin{array}{llll}7 \mathrm{~B} & -0.963650 & -1.179196 & -0.174557\end{array}$

$\begin{array}{llll}8 B & 0.530937 & 1.427316 & -0.174555\end{array}$

$\begin{array}{llll}9 B & -0.963650 & 0.552562 & 1.056243\end{array}$

$\begin{array}{llll}10 \mathrm{~B} & -1.140458 & 0.653945 & -0.920113\end{array}$

$\begin{array}{llll}\text { 11B } & 1.514179 & -0.868237 & 1.221628\end{array}$

$E 12 / \varepsilon=-41.305141$

\begin{tabular}{rrrr} 
atom & $x / \sigma$ & $y / \sigma$ & \multicolumn{1}{c}{$z / \sigma$} \\
1A & 0.000000 & 0.000000 & 0.000000 \\
2A & 1.073230 & 0.000000 & 0.000000 \\
3B & 0.536613 & -1.459962 & 0.000000 \\
4A & 0.536616 & 0.604067 & -0.765074 \\
5B & 0.536616 & -0.097163 & 1.448691 \\
6B & 1.543136 & -0.427382 & -1.387976 \\
7B & -0.469908 & -0.427379 & -1.387976 \\
8A & 0.536616 & 0.929763 & 0.302368 \\
9B & -0.852703 & 1.223350 & -0.302978 \\
10B & 1.925937 & 1.223346 & -0.302978 \\
11B & 2.332630 & -0.634646 & 0.526392 \\
12B & -1.259402 & -0.634638 & 0.526396
\end{tabular}

$E 13 / \varepsilon=-46.632565$

$\begin{array}{llll}1 \mathrm{~A} & 0.000000 & 0.000000 & 0.000000\end{array}$

2A $\quad 1.096848 \quad 0.000000 \quad 0.000000$

3A $0.548423 \quad-0.949898 \quad 0.000000$

$\begin{array}{lllll}4 \mathrm{~A} & 1.100931 & -0.635623 & -0.916547\end{array}$

$\begin{array}{llll}5 A & -0.004085 & -0.635622 & -0.916547\end{array}$

$\begin{array}{lllll}6 \mathrm{~A} & 0.548424 & 0.321351 & -0.916546\end{array}$

$\begin{array}{llll}7 \mathrm{~B} & 0.548424 & -0.316633 & 1.397943\end{array}$

$\begin{array}{llll}8 \mathrm{~B} & 0.548424 & 1.418903 & 0.177106\end{array}$

$\begin{array}{llll}9 B & -0.954595 & -1.184399 & 0.177103\end{array}$

$\begin{array}{llll}\text { 10B } & 2.051442 & -1.184401 & 0.177102\end{array}$

$\begin{array}{llll}11 \mathrm{~B} & 2.086067 & 0.571127 & -0.990498\end{array}$

$\begin{array}{llll}12 \mathrm{~B} & 0.548422 & -2.092149 & -0.990500\end{array}$

$\begin{array}{llll}13 \mathrm{~B} & -0.989219 & 0.571129 & -0.990495\end{array}$
$E 14 / \varepsilon=-53.222502$

$\begin{array}{ccc}\text { atom } & \mathrm{x} / \sigma & y / \sigma\end{array}$

$y / \sigma$

0.000000

$\begin{array}{lllll}2 \mathrm{~A} & 1.563886 & 0.000000 & 0.000000\end{array}$

$\begin{array}{llll}3 \mathrm{~A} & 0.781943 & -0.781943 & 0.000000\end{array}$

$\begin{array}{llll}4 \mathrm{~A} & 0.781943 & 0.781943 & 0.000000\end{array}$

$\begin{array}{llll}5 \mathrm{~A} & 0.781943 & 0.000000 & 0.781943\end{array}$

$\begin{array}{lllll}6 \mathrm{~A} & 0.781943 & 0.000000 & -0.781943\end{array}$

$\begin{array}{lllll}7 \mathrm{~B} & 1.847476 & -1.065533 & -1.065533\end{array}$

$\begin{array}{llll}8 \mathrm{~B} & 1.847476 & 1.065533 & -1.065533\end{array}$

$\begin{array}{llll}9 B & -0.283590 & 1.065533 & 1.065533\end{array}$

$\begin{array}{llll}10 \mathrm{~B} & -0.283590 & 1.065533 & -1.065533\end{array}$

$\begin{array}{llll}11 \mathrm{~B} & -0.283590 & -1.065533 & -1.065533\end{array}$

$\begin{array}{llll}12 \mathrm{~B} & -0.283590 & -1.065533 & 1.065533\end{array}$

$\begin{array}{llll}\text { 13B } & 1.847476 & -1.065533 & 1.065533\end{array}$

$\begin{array}{llll}14 \mathrm{~B} & 1.847476 & 1.065533 & 1.065533\end{array}$

$E 15 / \varepsilon=-58.931166$

atom $\mathrm{x} / \sigma$

$y / \sigma$

$\begin{array}{lll}1 \mathrm{~A} & 0.000000 & 0.000000\end{array}$

$z / \sigma$

$\begin{array}{llll}2 \mathrm{~A} & 1.105864 & 0.000000 & 0.000000\end{array}$

$\begin{array}{llll}3 A & 0.554549 & -0.956771 & 0.000000\end{array}$

$\begin{array}{lllll}4 \mathrm{~A} & 0.003234 & -0.639089 & -0.902491\end{array}$

$\begin{array}{lllll}5 A & 0.554549 & 0.317683 & -0.902490\end{array}$

$\begin{array}{lllll}6 \mathrm{~A} & 1.108564 & -0.638781 & -0.902056\end{array}$

$\begin{array}{llll}7 \mathrm{~B} & -0.945224 & 0.544662 & -1.093846\end{array}$

$\begin{array}{llll}8 \mathrm{~B} & 0.575153 & 1.425879 & 0.150568\end{array}$

$\begin{array}{llll}9 \mathrm{~B} & -0.945223 & -1.212637 & 0.150564\end{array}$

$\begin{array}{llll}10 \mathrm{~B} & 0.575154 & -0.331419 & 1.394978\end{array}$

$\begin{array}{llll}11 B & 2.062066 & -1.188213 & 0.163975\end{array}$

12B $\quad 0.558891 \quad-2.059460-1.066358$

$\begin{array}{lllll}13 B & 0.558891 & -0.322044 & -2.296690\end{array}$

$\begin{array}{llll}14 \mathrm{~B} & 2.062066 & 0.549203 & -1.066356\end{array}$

$\begin{array}{llll}15 B & -1.102983 & 0.635563 & 0.897515\end{array}$

$E 16 / \varepsilon=-64.728570$

atom $\mathrm{x} / \sigma \quad \mathrm{y} / \sigma$

$\begin{array}{lll}1 \mathrm{~A} & 0.000000 & 0.000000\end{array}$

2A $\quad 1.104490 \quad 0.000000$

$\begin{array}{llll}3 A & 0.552245 & -0.957109\end{array}$

$\begin{array}{lll}4 \mathrm{~A} & 0.552244 & 0.309453\end{array}$

$\begin{array}{lll}5 \mathrm{~A} & 1.105409 & -0.643996\end{array}$

$\begin{array}{lll}6 \mathrm{~A} & -0.000920 & -0.643996\end{array}$

$\begin{array}{lll}7 \mathrm{~B} & 0.552244 & 1.435735\end{array}$

$\begin{array}{llll}8 \mathrm{~B} & 0.552245 & -0.353831\end{array}$

$\begin{array}{lll}9 \mathrm{~B} & -0.947824 & 0.538834\end{array}$

10B $\quad-0.947822-1.211085$

$\begin{array}{lll}11 B & 2.052312 & 0.538835\end{array}$

$\begin{array}{lll}12 \mathrm{~B} & 2.052313 & -1.211085\end{array}$

$\begin{array}{llll}13 B & 0.552243 & -0.336557\end{array}$

$\begin{array}{lll}14 \mathrm{~B} & 0.552245 & -2.064734\end{array}$

$\begin{array}{lll}15 \mathrm{~B} & 2.206920 & 0.640517\end{array}$

$\begin{array}{lll}16 \mathrm{~B} & -1.102430 & 0.640517\end{array}$

$z / \sigma$

0.000000

0.000000

0.000000

$-0.905702$

$-0.900585$

$-0.900584$

0.116636

1.396331

$-1.095720$

0.155625

$-1.095722$

0.155623

$-2.296919$

$-1.061122$

0.895718

$E 17 / \varepsilon=-70.600218$

atom $\mathrm{x} / \sigma \quad \mathrm{y} / \sigma$

$\begin{array}{llll}1 \mathrm{~A} & 0.000000 & 0.000000 & 0.000000\end{array}$

$\begin{array}{llll}2 \mathrm{~A} & 1.105626 & 0.000000 & 0.000000\end{array}$

$\begin{array}{llll}3 A & 0.552812 & -0.957500 & 0.000000\end{array}$

$\begin{array}{llll}4 \mathrm{~B} & 0.552812 & -0.319167 & 1.396975\end{array}$

$\begin{array}{lllll}5 A & 1.103751 & -0.637252 & -0.902296\end{array}$

$\begin{array}{llll}6 \mathrm{~A} & 0.001871 & -0.637252 & -0.902295\end{array}$

$\begin{array}{llll}7 \mathrm{~A} & 0.552812 & 0.317004 & -0.902295\end{array}$

$\begin{array}{llll}8 \mathrm{~B} & -0.965809 & -1.195943 & 0.128708\end{array}$

$\begin{array}{llll}9 \mathrm{~B} & 2.071433 & -1.195944 & 0.128705\end{array}$

$\begin{array}{llll}10 \mathrm{~B} & 0.552814 & 1.434386 & 0.128707\end{array}$

11B $\quad 0.552810 \quad-2.048854-1.097590$

$\begin{array}{llll}12 \mathrm{~B} & 2.050765 & 0.545677 & -1.097590\end{array}$

$\begin{array}{lllll}13 \mathrm{~B} & -0.945141 & 0.545677 & -1.097588\end{array}$

$\begin{array}{llll}14 \mathrm{~B} & 0.552810 & -0.319165 & -2.297809\end{array}$

$\begin{array}{llll}15 B & 2.216974 & 0.641635 & 0.890250\end{array}$ \begin{tabular}{|llll}
$16 \mathrm{~B}$ & -1.111347 & 0.641638 & 0.890250
\end{tabular} $\begin{array}{llll}17 \mathrm{~B} & 0.552812 & -2.240774 & 0.890249\end{array}$

$E 18 / \varepsilon=-76.485964$

atom $\mathrm{x} / \sigma \quad \mathrm{y} / \sigma$

$\begin{array}{lll}1 \mathrm{~A} & 0.000000 & 0.000000\end{array}$

$z / \sigma$

$\begin{array}{llll}3 \mathrm{~A} & 0.553180 & -0.957072 & 0.000000\end{array}$

$\begin{array}{lllll}4 \mathrm{~A} & 0.553179 & 0.325196 & -0.900131\end{array}$

$\begin{array}{llll}5 B & 0.553180 & 1.423012 & 0.172226\end{array}$

$\begin{array}{llll}6 B & 0.553180 & -0.321532 & 1.396868\end{array}$

$\begin{array}{lllll}7 \mathrm{~A} & 1.101865 & -0.633289 & -0.902144\end{array}$

$\begin{array}{lllll}8 \mathrm{~A} & 0.004493 & -0.633289 & -0.902143\end{array}$

$\begin{array}{lllll}9 \mathrm{~B} & 2.070080 & 0.524238 & -1.087681\end{array}$

$\begin{array}{llll}10 \mathrm{~B} & -0.963721 & -1.201093 & 0.123477\end{array}$

$\begin{array}{llll}11 \mathrm{~B} & -0.963722 & 0.524239 & -1.087680\end{array}$

$\begin{array}{llll}12 \mathrm{~B} & 2.070082 & -1.201094 & 0.123473\end{array}$

$\begin{array}{llll}13 \mathrm{~B} & 0.553178 & -0.337353 & -2.297932\end{array}$

$\begin{array}{llll}14 \mathrm{~B} & 0.553179 & -2.046588 & -1.098077\end{array}$

15B $-1.119049 \quad 0.628629 \quad 0.895501$

$\begin{array}{llll}16 \mathrm{~B} & 2.225409 & 0.628628 & 0.895500\end{array}$

$\begin{array}{llll}17 \mathrm{~B} & 0.553181 & -2.242392 & 0.889292\end{array}$

$\begin{array}{llll}18 \mathrm{~B} & 0.553179 & 1.598307 & -1.806815\end{array}$

\section{$E 19 / \varepsilon=-82.446070$}

atom $\mathrm{x} / \sigma \quad y / \sigma$

$\begin{array}{llll}1 \mathrm{~A} & 0.000000 & 0.000000 & 0.000000\end{array}$

$\begin{array}{llll}2 \mathrm{~A} & 1.104730 & 0.000000 & 0.000000\end{array}$

$\begin{array}{llll}3 \mathrm{~A} & 0.550209 & -0.957967 & 0.000000\end{array}$

$\begin{array}{llll}4 \mathrm{~A} & -0.004312 & -0.636981 & 0.902590\end{array}$

$\begin{array}{llll}5 A & 0.550209 & 0.320986 & 0.902589\end{array}$

$\begin{array}{llll}6 \mathrm{~B} & -0.957755 & -1.191999 & -0.164504\end{array}$

$\begin{array}{lllll}7 \mathrm{~B} & 0.556629 & 1.424189 & -0.164506\end{array}$

$\begin{array}{llll}8 \mathrm{~B} & -0.957755 & 0.554398 & 1.067972\end{array}$

$\begin{array}{lllll}9 B & 0.556630 & -0.322209 & -1.396982\end{array}$

$\begin{array}{lllll}10 \mathrm{~A} & 1.096198 & -0.634537 & 0.899127\end{array}$

$\begin{array}{llll}11 \mathrm{~B} & 2.069913 & 0.518533 & 1.087281\end{array}$

$\begin{array}{llll}12 \mathrm{~B} & 0.581272 & -0.336470 & 2.298806\end{array}$

$\begin{array}{llll}13 \mathrm{~B} & 0.581272 & -2.053177 & 1.087283\end{array}$

$\begin{array}{llll}14 \mathrm{~B} & 2.069913 & -1.198173 & -0.124242\end{array}$

$\begin{array}{lllll}15 B & -1.108959 & 0.641920 & -0.909592\end{array}$

$\begin{array}{llll}16 \mathrm{~B} & 2.225258 & 0.631297 & -0.894536\end{array}$

$\begin{array}{lllll}17 \mathrm{~B} & 0.560861 & -2.244050 & -0.894534\end{array}$

$\begin{array}{llll}18 \mathrm{~B} & -1.103541 & -1.280607 & 1.814598\end{array}$

$\begin{array}{llll}19 B & 0.560859 & 1.594739 & 1.814595\end{array}$

$E 20 / \varepsilon=-89.054680$

$\begin{array}{lll}\text { atom } \quad \mathrm{x} / \sigma & \mathrm{y} / \sigma\end{array}$

$\begin{array}{llll}1 \mathrm{~A} & 0.000000 & 0.000000 & 0.000000\end{array}$

$\begin{array}{lllll}2 \mathrm{~A} & 1.088685 & 0.000000 & 0.000000\end{array}$

$\begin{array}{llll}3 \mathrm{~A} & 0.544342 & -0.942829 & 0.000000\end{array}$

$\begin{array}{llll}4 \mathrm{~B} & -1.035919 & -1.226638 & 0.000000\end{array}$

$\begin{array}{llll}5 B & 2.124601 & -1.226641 & 0.000000\end{array}$

$\begin{array}{llll}6 \mathrm{~B} & 0.544344 & 1.510452 & 0.000001\end{array}$

$\begin{array}{llll}7 A & 1.091792 & -0.630347 & 0.911055\end{array}$

8A $\quad 1.091792 \quad-0.630347 \quad-0.911055$

$\begin{array}{llll}9 A & -0.003109 & -0.630346 & -0.911055\end{array}$

$\begin{array}{llll}10 A & -0.003109 & -0.630346 & 0.911055\end{array}$

$\begin{array}{lllll}11 \mathrm{~A} & 0.544343 & 0.317866 & 0.911054\end{array}$

$\begin{array}{lllll}12 \mathrm{~A} & 0.544342 & 0.317866 & -0.911054\end{array}$

$\begin{array}{llll}13 \mathrm{~B} & -0.969733 & 0.559878 & -1.031320\end{array}$

$\begin{array}{llll}14 \mathrm{~B} & -0.969733 & 0.559878 & 1.031320\end{array}$

$\begin{array}{llll}15 B & 2.058419 & 0.559874 & 1.031321\end{array}$

$\begin{array}{llll}16 \mathrm{~B} & 2.058418 & 0.559876 & -1.031320\end{array}$

$\begin{array}{lllll}17 \mathrm{~B} & 0.544340 & -2.062580 & -1.031321\end{array}$

$\begin{array}{llll}18 \mathrm{~B} & 0.544340 & -2.062581 & 1.031320\end{array}$

$\begin{array}{llll}19 B & 0.544341 & -0.314272 & -2.303205\end{array}$

$\begin{array}{llll}20 B & 0.544341 & -0.314273 & 2.303205\end{array}$

$E 21 / \varepsilon=-94.850253$

$\begin{array}{llll}\text { atom } & \mathrm{X} / \sigma & \mathrm{y} / \sigma & \mathrm{z} / \sigma\end{array}$ $\begin{array}{llll}1 \mathrm{~A} & 0.000000 & 0.000000 & 0.000000\end{array}$ 


$\begin{array}{rrrr}2 A & 1.084632 & 0.000000 & 0.000000 \\ 3 A & 0.542316 & -0.945400 & 0.000000 \\ 4 B & 0.542316 & 1.499873 & -0.029780 \\ 5 A & 0.542314 & 0.317776 & 0.922584 \\ 6 B & 2.124237 & -1.231648 & 0.003753 \\ 7 B & -1.039606 & -1.231647 & 0.003746 \\ 8 A & 1.089953 & -0.629799 & 0.906819 \\ 9 A & -0.005325 & -0.629799 & 0.906818 \\ 10 A & -0.006452 & -0.640595 & -0.908629 \\ 11 A & 1.091087 & -0.640596 & -0.908627 \\ 12 B & -0.975361 & 0.555857 & 1.025969 \\ 13 B & 2.059988 & 0.555856 & 1.025975 \\ 14 A & 0.542318 & 0.302664 & -0.920601 \\ 15 B & 0.542314 & -2.067200 & 1.025756 \\ 16 B & 2.057776 & 0.547656 & -1.034804 \\ 17 B & -0.973139 & 0.547657 & -1.034809 \\ 18 B & 0.542318 & -2.071535 & -1.027016 \\ 19 B & 0.542311 & -0.421060 & 2.307605 \\ \text { 20B } & 0.542320 & -0.342910 & -2.304920 \\ 21 B & 0.542312 & 1.514912 & 1.937818\end{array}$

$E 22 / \varepsilon=-100.718586$

\begin{tabular}{rrrr} 
atom & $x / \sigma$ & $y / \sigma$ & \multicolumn{1}{c}{$z / \sigma$} \\
1A & 0.000000 & 0.000000 & 0.000000 \\
2A & 1.083967 & 0.000000 & 0.000000 \\
3A & 0.530704 & -0.945164 & 0.000000 \\
4B & 0.552938 & 1.500214 & 0.027551 \\
5B & -1.037397 & -1.216630 & 0.027550 \\
6A & -0.011848 & -0.624701 & -0.925082 \\
7A & 0.538909 & 0.316182 & -0.925082 \\
8B & -0.981216 & 0.574368 & -1.002103 \\
9B & 2.124209 & -1.243430 & -0.009049 \\
$10 B$ & 0.526630 & -2.063382 & -1.030503 \\
11B & 2.057004 & 0.551023 & -1.030502 \\
12A & 1.084282 & -0.634696 & -0.891631 \\
13A & 1.097639 & -0.642515 & 0.902076 \\
14A & 0.553462 & 0.306145 & 0.920548 \\
15A & 0.004029 & -0.632478 & 0.920548 \\
16B & 0.688731 & -0.403155 & -2.314261 \\
17B & -0.956848 & 0.560102 & 1.048167 \\
18B & 0.543106 & -2.070536 & 1.025481 \\
19B & 2.071307 & 0.540160 & 1.025482 \\
20B & 0.575232 & -0.336719 & 2.306780 \\
21B & 0.524132 & 1.515661 & -1.937176 \\
22B & -1.064970 & -1.199071 & -1.937177
\end{tabular}

$E 23 / \varepsilon=-107.744490$

\begin{tabular}{crrr} 
atom & $x / \sigma$ & \multicolumn{1}{c}{$y / \sigma$} & \multicolumn{1}{c}{$z / \sigma$} \\
$1 \mathrm{~A}$ & 0.000000 & 0.000000 & 0.000000 \\
2A & 1.172287 & 0.000000 & 0.000000 \\
3A & 0.586143 & -0.898535 & 0.000000 \\
4A & 0.586143 & 0.449266 & 0.778155 \\
5A & 0.586143 & 0.449268 & -0.778154 \\
$6 \mathrm{~B}$ & -0.968149 & -1.190651 & 0.000000 \\
$7 \mathrm{~B}$ & -0.968149 & 0.595323 & 1.031136 \\
8B & -0.968150 & 0.595325 & -1.031133 \\
9B & 2.140434 & 0.595327 & -1.031134 \\
$10 \mathrm{~B}$ & 2.140435 & -1.190652 & -0.000002 \\
$11 \mathrm{~B}$ & 2.140435 & 0.595325 & 1.031134 \\
$12 \mathrm{~A}$ & 0.047706 & -0.542019 & 0.938801 \\
$13 \mathrm{~A}$ & 1.124582 & -0.542019 & 0.938801 \\
$14 \mathrm{~A}$ & 1.124580 & 1.084035 & 0.000001 \\
$15 \mathrm{~A}$ & 1.124580 & -0.542017 & -0.938802 \\
$16 \mathrm{~A}$ & 0.047705 & -0.542017 & -0.938802 \\
$17 \mathrm{~A}$ & 0.047704 & 1.084035 & 0.000002 \\
$18 \mathrm{~B}$ & 0.586142 & -1.983780 & -1.056589 \\
$19 \mathrm{~B}$ & 0.586143 & -1.983782 & 1.056588 \\
20B & 0.586144 & 0.076853 & 2.246299 \\
21B & 0.586141 & 0.076859 & -2.246299 \\
22B & 0.586142 & 1.906921 & 1.189713 \\
23B & 0.586140 & 1.906924 & -1.189708
\end{tabular}

$\begin{array}{llll}23 \mathrm{~B} & 0.586140 & 1.906924 & -1.189708\end{array}$
$E 24 / \varepsilon=-113.721598$

$\mathrm{x} / \sigma \quad \mathrm{s} / \sigma$

$\begin{array}{llll}\text { IA } & 0.000000 & 0.000000 & 0.000000\end{array}$

$\begin{array}{llll}2 \mathrm{~A} & 1.175892 & 0.000000 & 0.000000\end{array}$

$\begin{array}{llll}3 A & 0.588254 & -0.896377 & 0.000000\end{array}$

$\begin{array}{lllll}4 \mathrm{~A} & 0.588254 & 0.450739 & 0.774807\end{array}$

$\begin{array}{lllll}5 \mathrm{~A} & 0.588988 & 0.447671 & -0.778343\end{array}$

$\begin{array}{llll}6 \mathrm{~B} & -0.962894 & -1.192211 & -0.008864\end{array}$

$\begin{array}{llll}7 \mathrm{~B} & -0.962893 & 0.607159 & 1.026061\end{array}$

$\begin{array}{llll}8 \mathrm{~A} & 0.052179 & -0.540338 & 0.939457\end{array}$

$\begin{array}{llll}9 \mathrm{~B} & -0.966126 & 0.593740 & -1.032304\end{array}$

$\begin{array}{llll}10 B & 2.146794 & -1.186055 & -0.000576\end{array}$

$\begin{array}{llll}11 B & 2.146793 & 0.596901 & 1.024909\end{array}$

$\begin{array}{llll}12 \mathrm{~B} & 2.143681 & 0.594999 & -1.034493\end{array}$

$\begin{array}{llll}13 \mathrm{~A} & 1.127058 & -0.541130 & 0.940835\end{array}$

$\begin{array}{lllll}14 \mathrm{~A} & 0.052466 & -0.541022 & -0.939845\end{array}$

$\begin{array}{lllll}15 \mathrm{~A} & 0.052466 & 1.084430 & -0.004951\end{array}$

$\begin{array}{llll}16 \mathrm{~A} & 1.128966 & -0.541807 & -0.938910\end{array}$

$\begin{array}{lllll}17 A & 1.128966 & 1.084017 & -0.003802\end{array}$

18B $\quad 0.607095 \quad-1.992752 \quad 1.037950$

$\begin{array}{llll}19 B & 0.607093 & 0.104868 & 2.244417\end{array}$

$\begin{array}{llll}\text { 20B } & 0.592342 & -1.982343 & -1.057523\end{array}$

$\begin{array}{llll}21 B & 0.592342 & 1.910909 & 1.181719\end{array}$

$\begin{array}{lllll}22 B & 0.589480 & 1.904391 & -1.195336\end{array}$

$\begin{array}{llll}23 \mathrm{~B} & 0.589480 & 0.075603 & -2.247180\end{array}$

$\begin{array}{llll}24 B & -0.926933 & -1.142309 & 1.986073\end{array}$

$E 25 / \varepsilon=-119.874315$

$\begin{array}{cccc}\text { atom } & \mathrm{x} / \sigma & y / \sigma & z / \sigma \\ 1 \mathrm{~A} & 0.000000 & 0.000000 & 0.000000\end{array}$

$\begin{array}{llll}2 \mathrm{~A} & 1.180185 & 0.000000 & 0.000000\end{array}$

$\begin{array}{llll}3 \mathrm{~A} & 0.590092 & -0.893890 & 0.000000\end{array}$

$\begin{array}{lllll}4 \mathrm{~A} & 0.590092 & 0.454618 & 0.769650\end{array}$

$\begin{array}{lllll}5 \mathrm{~A} & 0.590092 & 0.444630 & -0.779041\end{array}$

$\begin{array}{llll}6 \mathrm{~B} & -0.965041 & -1.187961 & -0.004100\end{array}$

$\begin{array}{llll}7 \mathrm{~B} & 2.145225 & 0.607708 & 1.020765\end{array}$

$\begin{array}{llll}8 \mathrm{~B} & -0.965042 & 0.607708 & 1.020763\end{array}$

$\begin{array}{lllll}9 B & 2.145225 & -1.187962 & -0.004098\end{array}$

$\begin{array}{llll}10 \mathrm{~A} & 0.055021 & -0.537680 & 0.942072\end{array}$

$\begin{array}{llll}11 \mathrm{~A} & 1.125161 & -0.537680 & 0.942072\end{array}$

$\begin{array}{llll}12 \mathrm{~B} & 0.590091 & -2.006373 & 1.011808\end{array}$

$\begin{array}{llll}13 \mathrm{~B} & 0.590091 & 0.149230 & 2.242101\end{array}$

$\begin{array}{lllll}14 \mathrm{~B} & -0.965973 & 0.591347 & -1.036106\end{array}$

$\begin{array}{llll}15 B & 2.146159 & 0.591348 & -1.036105\end{array}$

$\begin{array}{lllll}16 \mathrm{~A} & 0.051644 & -0.542299 & -0.938846\end{array}$

$\begin{array}{llll}17 \mathrm{~A} & 1.128541 & 1.084162 & -0.010558\end{array}$

$\begin{array}{lllll}18 A & 1.128542 & -0.542299 & -0.938846\end{array}$

$\begin{array}{lllll}19 A & 0.051643 & 1.084162 & -0.010558\end{array}$

$\begin{array}{lllll}\text { 20B } & 0.590092 & -1.980678 & -1.060282\end{array}$

$\begin{array}{llll}21 \mathrm{~B} & 0.590091 & 1.920257 & 1.166144\end{array}$

$\begin{array}{llll}22 \mathrm{~B} & 0.590093 & 0.070261 & -2.247843\end{array}$

$\begin{array}{lllll}23 \mathrm{~B} & 0.590093 & 1.899683 & -1.203717\end{array}$

$\begin{array}{llll}24 \mathrm{~B} & -0.902841 & -1.140087 & 1.997551\end{array}$

$\begin{array}{llll}25 B & 2.083022 & -1.140087 & 1.997553\end{array}$

$E 26 / \varepsilon=-125.890569$

atom $\mathrm{x} / \sigma \quad \mathrm{y} / \sigma \quad \mathrm{z} / \sigma$

$\begin{array}{llll}1 \mathrm{~A} & 0.000000 & 0.000000 & 0.000000\end{array}$

$\begin{array}{llll}2 \mathrm{~A} & 1.183360 & 0.000000 & 0.000000\end{array}$

$\begin{array}{llll}3 \mathrm{~A} & 0.591769 & -0.891974 & 0.000000\end{array}$

$\begin{array}{llll}4 \mathrm{~B} & -0.959959 & -1.188960 & -0.004592\end{array}$

$\begin{array}{lllll}5 \mathrm{~A} & 0.592849 & 0.453360 & -0.769845\end{array}$

$\begin{array}{llll}6 \mathrm{~A} & 0.591793 & 0.446292 & 0.775816\end{array}$

$\begin{array}{llll}7 \mathrm{~B} & -0.962697 & 0.606709 & -1.022543\end{array}$

$\begin{array}{llll}8 \mathrm{~B} & 2.150928 & -1.183434 & 0.003436\end{array}$

$\begin{array}{llll}9 B & -0.961029 & 0.604304 & 1.030338\end{array}$

$\begin{array}{lllll}10 B & 2.148770 & 0.607930 & -1.022999\end{array}$

$\begin{array}{llll}11 \mathrm{~A} & 0.059396 & -0.536248 & -0.943714\end{array}$

$\begin{array}{llll}12 \mathrm{~A} & 1.129372 & -0.537288 & -0.942384\end{array}$

$\begin{array}{llll}13 \mathrm{~A} & 0.055151 & -0.540611 & 0.940155\end{array}$

$\begin{array}{llll}14 \mathrm{~B} & 0.595181 & -2.004166 & -1.014130\end{array}$

$\begin{array}{lllll}15 B & 0.595034 & 0.149800 & -2.243009\end{array}$ \begin{tabular}{|llll}
$16 B$ & 2.152216 & 0.592798 & 1.030320
\end{tabular}

$\begin{array}{lllll}17 A & 1.130241 & -0.540981 & 0.940859\end{array}$

$\begin{array}{llll}18 \mathrm{~A} & 0.056378 & 1.084690 & 0.005424\end{array}$

$\begin{array}{llll}19 \mathrm{~A} & 1.132827 & 1.084142 & 0.007218\end{array}$

$\begin{array}{llll}20 B & 0.612055 & -1.988971 & 1.042957\end{array}$

$\begin{array}{llll}21 B & 0.610820 & 0.098972 & 2.246111\end{array}$

22B $\quad 0.594049 \quad 1.918889-1.170578$

$\begin{array}{llll}23 B & 0.596091 & 1.904389 & 1.195230\end{array}$

$\begin{array}{llll}24 \mathrm{~B} & -0.896597 & -1.139340 & -2.002345\end{array}$

25B $\quad 2.088137 \quad-1.139290 \quad-1.998319$ $\begin{array}{llll}26 B & -0.924935 & -1.144759 & 1.987535\end{array}$

\section{$E 27 / \varepsilon=-133.907416$}

atom $\mathrm{x} / \sigma \quad \mathrm{y} / \sigma \quad \mathrm{z} / \sigma$

$\begin{array}{llll}1 \mathrm{~A} & 0.000000 & 0.000000 & 0.000000\end{array}$

$\begin{array}{llll}2 \mathrm{~A} & 1.097842 & 0.000000 & 0.000000\end{array}$

$\begin{array}{llll}3 \mathrm{~A} & 0.000000 & -1.097842 & 0.000000\end{array}$

$\begin{array}{llll}4 \mathrm{~A} & -0.548921 & 0.548921 & -0.776292\end{array}$

$\begin{array}{llll}5 \mathrm{~A} & 0.000000 & 1.097842 & 0.000000\end{array}$

$\begin{array}{llll}6 \mathrm{~A} & 0.548921 & -0.548921 & 0.776292\end{array}$

$\begin{array}{llll}7 A & -0.548921 & -0.548921 & -0.776292\end{array}$

$\begin{array}{llll}8 \mathrm{~A} & 0.548921 & 0.548921 & 0.776292\end{array}$

$\begin{array}{llll}9 \mathrm{~A} & -0.548921 & 0.548921 & 0.776292\end{array}$

$\begin{array}{llll}10 \mathrm{~A} & 0.548921 & -0.548921 & -0.776292\end{array}$

$\begin{array}{llll}11 \mathrm{~A} & -0.548921 & -0.548921 & 0.776292\end{array}$

$\begin{array}{llll}12 \mathrm{~A} & 0.548921 & 0.548921 & -0.776292\end{array}$

$\begin{array}{llll}13 \mathrm{~A} & -1.097842 & 0.000000 & 0.000000\end{array}$

$\begin{array}{llll}14 \mathrm{~B} & 0.000000 & 0.000000 & 2.105807\end{array}$

15B $\quad 1.489031-1.489031 \quad 0.000000$

$\begin{array}{llll}16 \mathrm{~B} & -1.489031 & 1.489031 & 0.000000\end{array}$

$\begin{array}{llll}17 \mathrm{~B} & 1.489031 & 1.489031 & 0.000000\end{array}$

$\begin{array}{llll}18 \mathrm{~B} & -1.489031 & -1.489031 & 0.000000\end{array}$

$\begin{array}{llll}19 B & 0.000000 & 0.000000 & -2.105807\end{array}$

$\begin{array}{llll}20 \mathrm{~B} & 1.872748 & 0.000000 & 1.324233\end{array}$

$\begin{array}{llll}21 \mathrm{~B} & 1.872748 & 0.000000 & -1.324233\end{array}$

$\begin{array}{llll}22 \mathrm{~B} & 0.000000 & -1.872748 & 1.324233\end{array}$

$\begin{array}{llll}23 \mathrm{~B} & 0.000000 & 1.872748 & 1.324233\end{array}$

$\begin{array}{lllll}24 \mathrm{~B} & 0.000000 & -1.872748 & -1.324233\end{array}$

$\begin{array}{llll}25 \mathrm{~B} & -1.872748 & 0.000000 & 1.324233\end{array}$

$\begin{array}{lllll}26 \mathrm{~B} & 0.000000 & 1.872748 & -1.324233\end{array}$

$\begin{array}{lllll}27 \mathrm{~B} & -1.872748 & 0.000000 & -1.324233\end{array}$

$E 28 / \varepsilon=-138.966341$

$\begin{array}{llll}\text { atom } & \mathrm{x} / \sigma & \mathrm{g} / \sigma & \mathrm{z} / \sigma\end{array}$

$\begin{array}{llll}1 \mathrm{~A} & 0.000000 & 0.000000 & 0.000000\end{array}$

$\begin{array}{llll}2 \mathrm{~A} & 1.097418 & 0.000000 & 0.000000\end{array}$

$\begin{array}{llll}3 B & 1.523756 & -1.445742 & 0.000000\end{array}$

$\begin{array}{lllll}4 \mathrm{~B} & 1.523756 & 1.445742 & -0.000001\end{array}$

$\begin{array}{llll}5 \mathrm{~A} & 0.545234 & 0.544878 & 0.781901\end{array}$

$\begin{array}{lllll}6 \mathrm{~A} & 0.545234 & 0.544877 & -0.781901\end{array}$

$\begin{array}{llll}7 \mathrm{~A} & 0.545235 & -0.544877 & -0.781901\end{array}$

$\begin{array}{lllll}8 \mathrm{~A} & 0.545234 & -0.544877 & 0.781901\end{array}$

$\begin{array}{llll}9 \mathrm{~A} & -0.552280 & 0.551385 & -0.771643\end{array}$

$\begin{array}{llll}10 \mathrm{~A} & -0.552281 & -0.551385 & 0.771642\end{array}$

$\begin{array}{llll}11 \mathrm{~A} & -0.552281 & 0.551386 & 0.771641\end{array}$

$\begin{array}{llll}12 \mathrm{~A} & -0.552280 & -0.551385 & -0.771643\end{array}$

$\begin{array}{llll}13 \mathrm{~A} & 0.012151 & -1.097330 & 0.000000\end{array}$

$\begin{array}{llll}14 \mathrm{~A} & 0.012151 & 1.097330 & -0.000001\end{array}$

$\begin{array}{lllll}15 A & -1.098924 & 0.000000 & -0.000001\end{array}$

$\begin{array}{llll}16 \mathrm{~B} & -1.476579 & -1.501475 & -0.000001\end{array}$

$\begin{array}{llll}17 \mathrm{~B} & -1.476580 & 1.501474 & -0.000003\end{array}$

$\begin{array}{llll}18 \mathrm{~B} & -0.061421 & -0.000001 & -2.111577\end{array}$

$\begin{array}{llll}19 B & -0.061424 & 0.000002 & 2.111575\end{array}$

$\begin{array}{llll}20 \mathrm{~B} & 1.798220 & -0.000001 & -1.457157\end{array}$

$\begin{array}{llll}21 B & 1.798219 & 0.000000 & 1.457157\end{array}$

$\begin{array}{llll}22 \mathrm{~B} & 0.022439 & -1.871621 & -1.321647\end{array}$

$\begin{array}{llll}23 \mathrm{~B} & 0.022438 & -1.871619 & 1.321648\end{array}$

$\begin{array}{llll}24 \mathrm{~B} & 0.022439 & 1.871620 & -1.321649\end{array}$

$\begin{array}{llll}25 \mathrm{~B} & 0.022438 & 1.871622 & 1.321644\end{array}$

$\begin{array}{llll}26 \mathrm{~B} & -1.896143 & -0.000001 & -1.296418\end{array}$

$\begin{array}{llll}27 \mathrm{~B} & -1.896146 & 0.000001 & 1.296413\end{array}$

$\begin{array}{llll}28 \mathrm{~A} & 2.210264 & 0.000000 & 0.000000\end{array}$ 


\begin{tabular}{|c|c|c|c|}
\hline \multicolumn{4}{|c|}{$E 29 / \varepsilon=-145.342393$} \\
\hline atom & $\mathrm{x} / \sigma$ & $y / \sigma$ & $z / \sigma$ \\
\hline $1 \mathrm{~A}$ & 0.000000 & 0.000000 & 0.000000 \\
\hline $2 \mathrm{~A}$ & 1.103368 & 0.000000 & 0.000000 \\
\hline $3 A$ & 0.555143 & -0.953540 & 0.000000 \\
\hline $4 \mathrm{~A}$ & 0.555141 & -0.319172 & 0.898536 \\
\hline $5 B$ & -0.076024 & -1.717836 & 1.212792 \\
\hline $6 B$ & 1.446314 & 0.930003 & 1.212795 \\
\hline $7 B$ & 1.446321 & -0.831541 & -1.282305 \\
\hline $8 \mathrm{~A}$ & -0.012390 & -0.626320 & -0.898115 \\
\hline $9 A$ & -0.556447 & -0.309619 & 0.890809 \\
\hline $10 \mathrm{~A}$ & 0.535037 & 0.325834 & -0.898113 \\
\hline $11 \mathrm{~A}$ & 0.535034 & 0.955372 & -0.006418 \\
\hline $12 \mathrm{~A}$ & -0.556444 & -0.943063 & -0.006421 \\
\hline $13 \mathrm{~A}$ & -0.012396 & 0.636663 & 0.890811 \\
\hline $14 \mathrm{~A}$ & -0.559802 & 0.951491 & -0.003571 \\
\hline $15 \mathrm{~A}$ & -1.103943 & 0.005054 & -0.003573 \\
\hline $16 \mathrm{~A}$ & -0.559799 & 0.321849 & -0.895414 \\
\hline 17B & -1.510631 & 0.868512 & 1.202048 \\
\hline $18 \mathrm{~B}$ & -0.032385 & 1.729136 & -1.220772 \\
\hline $19 \mathrm{~B}$ & -1.510622 & -0.842003 & -1.220778 \\
\hline $20 B$ & -0.058962 & -2.149079 & -0.799361 \\
\hline 21B & 1.827583 & 1.132237 & -0.799357 \\
\hline $22 \mathrm{~B}$ & -0.058971 & 0.033901 & 2.292676 \\
\hline 23B & -0.042652 & 0.024526 & -2.299334 \\
\hline 24B & -1.901037 & -1.057410 & 0.746522 \\
\hline $25 B$ & -0.042663 & 2.174907 & 0.746530 \\
\hline $26 B$ & -1.887349 & 1.085104 & -0.766092 \\
\hline $27 B$ & 1.612160 & -0.926891 & 1.861979 \\
\hline $28 \mathrm{~B}$ & 1.612164 & -2.064824 & 0.250181 \\
\hline $29 B$ & 2.595573 & -0.354357 & 0.250182 \\
\hline
\end{tabular}

$E 30 / \varepsilon=-151.524277$

\begin{tabular}{rrrr} 
atom & \multicolumn{1}{c}{$\mathrm{X} / \sigma$} & $y / \sigma$ & \multicolumn{1}{c}{$z / \sigma$} \\
1A & 0.000000 & 0.000000 & 0.000000 \\
2B & 2.094427 & 0.000000 & 0.000000 \\
3B & 0.027929 & -2.094241 & 0.000000 \\
4B & 0.027928 & -0.027558 & 2.094059 \\
5A & -0.001465 & -0.781642 & 0.771423 \\
6A & 0.781552 & 0.011889 & 0.771423 \\
7A & 0.781553 & -0.771199 & -0.022038 \\
8A & -0.774554 & -0.778587 & -0.002954 \\
9A & -0.003038 & 0.774583 & 0.778557 \\
10A & -0.774555 & -0.007292 & 0.778558 \\
11A & 0.768189 & 0.013282 & -0.784761 \\
12A & -0.003037 & -0.768298 & -0.784761 \\
13A & 0.768188 & 0.784868 & -0.002954 \\
14A & -0.783140 & 0.002053 & -0.772806 \\
15A & -0.012497 & 0.783043 & -0.772806 \\
16A & -0.783141 & 0.772765 & 0.008115 \\
17B & -0.022739 & 0.022438 & -2.108977 \\
18B & -0.022742 & 2.109089 & 0.005314 \\
19B & -2.109205 & -0.005388 & 0.005315 \\
20B & 1.309517 & -1.292169 & -1.373808 \\
21B & -1.339103 & -1.327491 & 1.310134 \\
22B & 1.309515 & 1.356687 & 1.310134 \\
23A & 1.023152 & -1.009597 & 0.996399 \\
24B & -1.320938 & 1.303437 & 1.347578 \\
25B & 1.312577 & 1.338559 & -1.321058 \\
26B & -1.320935 & -1.330311 & -1.321059 \\
27B & -1.344727 & 1.326911 & -1.309566 \\
28B & 0.437000 & -1.978874 & 1.953003 \\
29B & 1.984526 & -0.410571 & 1.953003 \\
30B & 1.984527 & -1.958237 & 0.384838 \\
& & &
\end{tabular}

$E 31 / \varepsilon=-156.759040$

$\begin{array}{cccc}\text { atom } & \mathrm{x} / \sigma & y / \sigma & z / \sigma \\ \text { 1A } & 0.000000 & 0.000000 & 0.000000 \\ \text { 2B } & 1.483113 & 0.000000 & 0.000000 \\ \text { 3A } & -0.745299 & -0.775715 & 0.000000 \\ \text { 4A } & -0.745299 & -0.013269 & 0.775602 \\ \text { 5A } & -0.745300 & 0.775715 & 0.000001\end{array}$
6A $-0.745300 \quad 0.013269 \quad-0.775601$

$\begin{array}{llll}7 A & 0.019510 & -0.796374 & -0.810116\end{array}$

$\begin{array}{llll}8 \mathrm{~A} & 0.019511 & 0.796373 & 0.810115\end{array}$

$\begin{array}{llll}9 A & 0.315270 & -1.006262 & 0.215514\end{array}$

$\begin{array}{llll}10 A & 0.315270 & 0.232697 & -1.002428\end{array}$

$\begin{array}{lllll}11 \mathrm{~A} & 0.315270 & 1.006262 & -0.215515\end{array}$

$\begin{array}{llll}12 \mathrm{~A} & 0.315270 & -0.232697 & 1.002428\end{array}$

13B $-1.786635-1.052941-1.071110$

$\begin{array}{llll}14 \mathrm{~B} & -1.786636 & 1.052940 & 1.071110\end{array}$

$\begin{array}{llll}15 B & -0.569351 & -2.257340 & 0.065430\end{array}$

$\begin{array}{llll}16 \mathrm{~B} & -0.569351 & 0.104039 & -2.255890\end{array}$

$\begin{array}{llll}17 \mathrm{~B} & -0.569350 & -0.104039 & 2.255891\end{array}$

$\begin{array}{llll}18 \mathrm{~B} & -0.569351 & 2.257340 & -0.065431\end{array}$

$\begin{array}{llll}19 B & -1.925242 & -1.029988 & 1.012516\end{array}$

$\begin{array}{llll}20 B & -1.925242 & 1.029987 & -1.012516\end{array}$

$\begin{array}{llll}21 \mathrm{~A} & -0.414059 & 1.046430 & -1.028680\end{array}$

$\begin{array}{llll}22 \mathrm{~A} & -0.414059 & -1.046431 & 1.028680\end{array}$

$\begin{array}{llll}23 \mathrm{~A} & -1.518940 & 0.000000 & 0.000000\end{array}$

$\begin{array}{llll}24 \mathrm{~B} & 0.997459 & -1.499364 & 1.473929\end{array}$

$\begin{array}{llll}25 \mathrm{~B} & 0.997458 & 1.499363 & -1.473930\end{array}$

$\begin{array}{lllll}26 \mathrm{~B} & 1.278800 & -0.493121 & -1.906255\end{array}$

$\begin{array}{llll}27 \mathrm{~B} & 1.278800 & 1.897540 & 0.525659\end{array}$

$\begin{array}{llll}28 \mathrm{~B} & 1.278801 & 0.493121 & 1.906254\end{array}$

$\begin{array}{lllll}29 \mathrm{~B} & 1.278800 & -1.897540 & -0.525660\end{array}$

$\begin{array}{llll}30 \mathrm{~B} & -0.185063 & 1.852530 & 1.884499\end{array}$

$\begin{array}{llll}31 B & -0.185063 & -1.852531 & -1.884499\end{array}$

$E 32 / \varepsilon=-163.768277$

atom $\mathrm{x} / \sigma \quad \mathrm{y} / \sigma$

$\begin{array}{cccc}1 \mathrm{~A} & 0.000000 & 0.000000 & 0.000000\end{array}$

$\begin{array}{llll}2 B & 1.775560 & 0.000000 & 0.000000\end{array}$

$\begin{array}{llll}3 \mathrm{~B} & -1.772348 & -0.060145 & 0.000000\end{array}$

$\begin{array}{lllll}4 \mathrm{~A} & 0.561860 & 0.299996 & -0.894499\end{array}$

$\begin{array}{lllll}5 A & 0.561860 & 0.300044 & 0.894482\end{array}$

$\begin{array}{lllll}6 \mathrm{~A} & 0.548791 & 0.948054 & -0.000026\end{array}$

$\begin{array}{llll}7 A & -0.566451 & 0.938983 & -0.000026\end{array}$

$\begin{array}{lllll}8 \mathrm{~A} & 0.551368 & -0.764814 & -0.554809\end{array}$

$\begin{array}{lllll}9 \mathrm{~A} & 0.551367 & -0.764785 & 0.554850\end{array}$

$\begin{array}{llll}10 \mathrm{~A} & -0.551307 & 0.296861 & -0.896907\end{array}$

$\begin{array}{llll}11 \mathrm{~A} & -0.551307 & 0.296909 & 0.896890\end{array}$

$\begin{array}{llll}12 \mathrm{~A} & -0.556146 & -0.760238 & -0.568150\end{array}$

$\begin{array}{llll}13 A & -0.556146 & -0.760207 & 0.568190\end{array}$

$\begin{array}{llll}14 \mathrm{~B} & -0.033819 & 2.446862 & -0.000066\end{array}$

$\begin{array}{llll}15 B & 0.037402 & 0.785055 & 2.318609\end{array}$

$\begin{array}{llll}16 \mathrm{~B} & 0.037401 & 0.784930 & -2.318652\end{array}$

$\begin{array}{llll}17 \mathrm{~A} & -0.005561 & 1.238880 & 0.890559\end{array}$

$\begin{array}{llll}18 \mathrm{~A} & -0.005561 & 1.238832 & -0.890627\end{array}$

$\begin{array}{llll}19 B & -1.477699 & 1.515727 & 1.115029\end{array}$

$\begin{array}{llll}20 B & -1.477698 & 1.515668 & -1.115112\end{array}$

$\begin{array}{llll}21 \mathrm{~A} & 0.008939 & -0.452112 & 1.454691\end{array}$

$\begin{array}{llll}22 \mathrm{~A} & 0.008940 & -0.452190 & -1.454667\end{array}$

$\begin{array}{llll}23 \mathrm{~B} & 1.457384 & 1.568493 & -1.092306\end{array}$

$\begin{array}{llll}24 \mathrm{~B} & 1.457384 & 1.568553 & 1.092220\end{array}$

$\begin{array}{llll}25 \mathrm{~B} & 1.466818 & -0.583443 & -1.814311\end{array}$

$\begin{array}{llll}26 \mathrm{~B} & 1.466817 & -0.583347 & 1.814342\end{array}$

$\begin{array}{llll}27 \mathrm{~B} & 1.437379 & -1.910512 & 0.000051\end{array}$

$\begin{array}{llll}28 \mathrm{~B} & -0.002313 & -1.953199 & 1.451987\end{array}$

$\begin{array}{llll}29 B & -0.002312 & -1.953277 & -1.451883\end{array}$

$\begin{array}{llll}30 \mathrm{~A} & -0.036920 & -1.527302 & 0.000041\end{array}$

$\begin{array}{llll}31 \mathrm{~B} & -1.441149 & -0.504701 & 1.871198\end{array}$ $\begin{array}{llll}32 \mathrm{~B} & -1.441147 & -0.504803 & -1.871173\end{array}$

\section{$E 33 / \varepsilon=-172.578008$}

at

$\begin{array}{llll}10 A & 0.557435 & 0.477557 & -0.813713\end{array}$

$\begin{array}{llll}11 \mathrm{~A} & -0.557434 & -0.864640 & 0.377606\end{array}$

$\begin{array}{llll}12 \mathrm{~A} & 0.557436 & 0.921460 & 0.202732\end{array}$

$\begin{array}{lllll}13 \mathrm{~A} & -0.557434 & 0.477557 & -0.813713\end{array}$

$\begin{array}{llll}14 \mathrm{~B} & 0.000001 & -2.235288 & 0.976196\end{array}$

$\begin{array}{llll}15 \mathrm{~B} & 0.000000 & 1.234592 & -2.103630\end{array}$

$\begin{array}{llll}16 \mathrm{~B} & 0.000003 & -1.619160 & -1.824225\end{array}$

$\begin{array}{llll}17 \mathrm{~B} & 0.000001 & 2.382181 & 0.524108\end{array}$

$\begin{array}{llll}18 B & 0.000001 & 0.237677 & 2.427547\end{array}$

$\begin{array}{llll}19 \mathrm{~A} & 0.000001 & 1.009433 & 1.137274\end{array}$

$\begin{array}{llll}20 A & 0.000001 & -0.148174 & -1.513404\end{array}$

$\begin{array}{llll}21 \mathrm{~A} & 0.000001 & 1.393544 & -0.608590\end{array}$

$\begin{array}{lllll}22 \mathrm{~A} & 0.000001 & -1.485121 & -0.326746\end{array}$

$\begin{array}{llll}23 \mathrm{~A} & 0.000001 & -0.769680 & 1.311464\end{array}$

$\begin{array}{llll}24 \mathrm{~B} & -1.458213 & 1.749620 & -0.764097\end{array}$

$\begin{array}{lllll}25 \mathrm{~B} & 1.458214 & 1.749620 & -0.764098\end{array}$

$\begin{array}{llll}26 \mathrm{~B} & -1.458212 & 1.267361 & 1.427870\end{array}$

$\begin{array}{llll}27 \mathrm{~B} & -1.458212 & -0.966348 & 1.646569\end{array}$

$\begin{array}{llll}28 \mathrm{~B} & -1.458212 & -0.186037 & -1.900107\end{array}$

$\begin{array}{llll}29 B & -1.458211 & -1.864597 & -0.410237\end{array}$

$\begin{array}{llll}30 \mathrm{~B} & 1.458214 & 1.267361 & 1.427869\end{array}$

$\begin{array}{llll}31 \mathrm{~B} & 1.458214 & -0.966348 & 1.646568\end{array}$

$\begin{array}{lllll}32 \mathrm{~B} & 1.458215 & -0.186033 & -1.900107\end{array}$

$\begin{array}{lllll}33 \mathrm{~B} & 1.458215 & -1.864597 & -0.410236\end{array}$

\section{$E 34 / \varepsilon=-179.450528$}

$\begin{array}{llll}\text { atom } \quad \mathrm{x} / \sigma \quad \mathrm{z} / \sigma & \mathrm{z} / \sigma\end{array}$

$\begin{array}{llll}1 \mathrm{~A} & 0.000000 & 0.000000 & 0.000000\end{array}$

$\begin{array}{llll}2 \mathrm{~B} & 1.727309 & 0.000000 & 0.000000\end{array}$

$\begin{array}{llll}3 \mathrm{~A} & -0.570518 & -0.929829 & 0.000000\end{array}$

$\begin{array}{llll}4 \mathrm{~A} & -0.570518 & 0.752248 & 0.546540\end{array}$

$\begin{array}{llll}5 A & -0.570518 & 0.752248 & -0.546540\end{array}$

$\begin{array}{llll}6 \mathrm{~A} & -0.570518 & -0.287332 & 0.884320\end{array}$

$\begin{array}{llll}7 A & -0.570518 & -0.287332 & -0.884320\end{array}$

$\begin{array}{llll}8 \mathrm{~A} & -1.153850 & 0.000001 & 0.000000\end{array}$

$\begin{array}{llll}9 \mathrm{~A} & 0.521250 & -0.957555 & 0.000000\end{array}$

$\begin{array}{llll}10 \mathrm{~A} & 0.521250 & 0.774678 & -0.562836\end{array}$

$\begin{array}{lllll}11 \mathrm{~A} & 0.521250 & -0.295900 & -0.910689\end{array}$

$\begin{array}{llll}12 \mathrm{~A} & 0.521250 & 0.774678 & 0.562836\end{array}$

$\begin{array}{llll}13 \mathrm{~A} & 0.521250 & -0.295900 & 0.910689\end{array}$

$\begin{array}{llll}14 \mathrm{~B} & -1.601390 & 1.711513 & 0.000000\end{array}$

15B $-1.601391-1.384641-1.006002$

$\begin{array}{llll}16 \mathrm{~B} & -1.601391 & 0.528888 & -1.627745\end{array}$

$\begin{array}{llll}17 \mathrm{~B} & -1.601391 & -1.384642 & 1.006002\end{array}$

$\begin{array}{llll}18 \mathrm{~B} & -1.601391 & 0.528887 & 1.627745\end{array}$

$\begin{array}{llll}19 B & -0.105091 & -2.436410 & 0.000000\end{array}$

$\begin{array}{llll}20 B & -0.105090 & 1.971098 & 1.432086\end{array}$

$\begin{array}{llll}21 B & -0.105089 & -0.752891 & 2.317164\end{array}$

$\begin{array}{llll}22 B & -0.105090 & 1.971098 & -1.432086\end{array}$

$\begin{array}{llll}23 B & -0.105089 & -0.752891 & -2.317164\end{array}$

$\begin{array}{llll}24 \mathrm{~A} & -0.055847 & 1.533886 & 0.000000\end{array}$

$\begin{array}{llll}25 A & -0.055848 & 0.473997 & 1.458811\end{array}$

$\begin{array}{llll}26 \mathrm{~A} & -0.055848 & -1.240939 & 0.901595\end{array}$

$\begin{array}{llll}27 A & -0.055848 & -1.240938 & -0.901595\end{array}$

$\begin{array}{lllll}28 \mathrm{~A} & -0.055848 & 0.473997 & -1.458811\end{array}$

$\begin{array}{lllll}29 B & 1.401632 & 0.593389 & -1.826258\end{array}$

$\begin{array}{llll}30 \mathrm{~B} & 1.401631 & 1.920242 & 0.000000\end{array}$

$\begin{array}{llll}31 \mathrm{~B} & 1.401631 & -1.553508 & -1.128690\end{array}$

$\begin{array}{llll}32 B & 1.401632 & 0.593389 & 1.826258\end{array}$

$\begin{array}{llll}33 \mathrm{~B} & 1.401631 & -1.553509 & 1.128690\end{array}$

$\begin{array}{llll}34 \mathrm{~B} & -2.658391 & 0.000002 & 0.000000\end{array}$

$E 35 / \varepsilon=-185.635002$

atom $\mathrm{x} / \sigma \quad \mathrm{y} / \sigma$

$\begin{array}{llll}1 \mathrm{~A} & 0.000000 & 0.000000 & 0.000000\end{array}$

$\begin{array}{llll}2 \mathrm{~A} & 1.083613 & 0.000000 & 0.000000\end{array}$

$\begin{array}{llll}3 \mathrm{~A} & 0.520798 & -0.950256 & 0.000000\end{array}$

$\begin{array}{llll}4 \mathrm{~A} & 0.520799 & 0.729996 & 0.608352\end{array}$

$\begin{array}{llll}5 \mathrm{~A} & -0.389855 & -0.807551 & 0.608352\end{array}$

$\begin{array}{llll}6 A & -0.389855 & 0.230902 & 0.984334\end{array}$

$\begin{array}{lllll}7 A & -0.017400 & 1.048759 & -0.272062\end{array}$

$\begin{array}{lllll}8 \mathrm{~A} & -0.928054 & -0.488787 & -0.272063\end{array}$

$\begin{array}{llll}9 A & -0.928054 & 0.549666 & 0.103920\end{array}$ 


$\begin{array}{rrrr}\text { 10A } & 0.545415 & 0.318763 & -0.880415 \\ \text { 11A } & -0.017400 & -0.631493 & -0.880415 \\ \text { 12A } & 0.555725 & -0.329143 & 0.909085 \\ \text { 13A } & -0.555724 & 0.329143 & -0.909084 \\ \text { 14B } & 0.311503 & 0.994403 & 2.074490 \\ \text { 15B } & -0.722314 & -0.751091 & 2.074489 \\ \text { 16B } & 1.984252 & -1.175227 & 0.266397 \\ \text { 17B } & 1.984252 & 0.732272 & 0.957028 \\ \text { 18B } & 0.311502 & -2.091997 & 0.957026 \\ \text { 19B } & -1.236174 & -1.175341 & -1.574745 \\ \text { 20B } & 0.436577 & 1.648928 & -1.574743 \\ \text { 21B } & 0.436576 & -0.258573 & -2.265374 \\ \text { 22B } & -2.269991 & 0.165564 & -0.457282 \\ \text { 23B } & -1.236174 & 1.911059 & -0.457281 \\ \text { 24B } & 2.109199 & 0.412722 & -1.139926 \\ \text { 25B } & 0.651779 & -2.047985 & -1.139927 \\ \text { 26B } & 0.651779 & 2.303061 & 0.435415 \\ \text { 27B } & -1.706377 & -1.678446 & 0.435413 \\ \text { 28B } & -1.706376 & 1.010648 & 1.409028 \\ \text { 29A } & -0.412103 & -1.456169 & -0.275301 \\ \text { 30A } & 1.078901 & 1.061240 & -0.275300 \\ \text { 31A } & -1.333594 & -0.260954 & 0.720748 \\ \text { 32A } & -0.412102 & 1.294890 & 0.720749 \\ \text { 33A } & 1.078901 & -0.639008 & -0.890893 \\ \text { 34B } & 1.300890 & -0.770490 & 2.128064 \\ \text { 35B } & -1.300889 & 0.770489 & -2.128064\end{array}$

$E 36 / \varepsilon=-191.280568$

$\begin{array}{llll}1 \mathrm{~A} & 0.000000 & 0.000000 & 0.000000\end{array}$

$\begin{array}{lllll}2 \mathrm{~A} & 1.084726 & 0.000000 & 0.000000\end{array}$

$\begin{array}{llll}3 \mathrm{~A} & 0.551281 & -0.934195 & 0.000000\end{array}$

$\begin{array}{llll}4 \mathrm{~A} & 0.524831 & 0.319712 & 0.893850\end{array}$

$\begin{array}{llll}5 A & -0.008615 & -0.614483 & 0.893850\end{array}$

$\begin{array}{lllll}6 A & -0.931626 & -0.089907 & -0.551401\end{array}$

$\begin{array}{llll}7 \mathrm{~A} & -0.396040 & 0.848035 & -0.551400\end{array}$

$\begin{array}{lllll}8 \mathrm{~A} & 0.512180 & 0.331316 & -0.894432\end{array}$

$\begin{array}{llll}9 A & -0.025038 & -0.609484 & -0.894432\end{array}$

$\begin{array}{llll}10 \mathrm{~A} & -0.391578 & 0.847381 & 0.548378\end{array}$

$\begin{array}{llll}11 \mathrm{~A} & -0.928795 & -0.093419 & 0.548378\end{array}$

$\begin{array}{llll}12 \mathrm{~A} & 0.553454 & 0.966039 & 0.001143\end{array}$

$\begin{array}{llll}13 \mathrm{~A} & -0.550702 & -0.967610 & 0.001142\end{array}$

$\begin{array}{llll}14 \mathrm{~B} & 1.994519 & 0.625164 & 1.025731\end{array}$

$\begin{array}{llll}15 B & 0.475246 & -2.035456 & 1.025729\end{array}$

$\begin{array}{llll}16 \mathrm{~A} & 1.096039 & -0.625862 & 0.910402\end{array}$

$\begin{array}{llll}17 \mathrm{~B} & 0.313832 & 1.607222 & 1.640080\end{array}$

$\begin{array}{llll}18 \mathrm{~B} & 0.438517 & -2.036829 & -1.015158\end{array}$

$\begin{array}{llll}19 B & -1.224688 & -1.087105 & 1.640077\end{array}$

$\begin{array}{llll}\text { 20B } & 1.977036 & 0.657496 & -1.015156\end{array}$

$\begin{array}{llll}21 B & 0.599644 & -0.342411 & 2.330145\end{array}$

$\begin{array}{llll}22 \mathrm{~B} & 2.109324 & -1.204470 & -0.079995\end{array}$

$\begin{array}{llll}23 \mathrm{~B} & -0.724615 & 2.207434 & 0.004246\end{array}$

$\begin{array}{llll}24 \mathrm{~B} & 0.303523 & 1.620344 & -1.637134\end{array}$

$\begin{array}{llll}25 \mathrm{~B} & -1.241227 & -1.084891 & -1.637137\end{array}$

$\begin{array}{llll}26 \mathrm{~B} & -2.269366 & -0.497802 & 0.004243\end{array}$

$\begin{array}{llll}27 \mathrm{~B} & -1.716302 & 0.980049 & -1.425614\end{array}$

$\begin{array}{llll}28 \mathrm{~B} & -1.716400 & 0.980102 & 1.428557\end{array}$

$\begin{array}{llll}29 B & 0.635598 & -0.362938 & -2.326306\end{array}$

$\begin{array}{llll}30 \mathrm{~A} & -1.338405 & 0.764259 & 0.001951\end{array}$

$\begin{array}{llll}31 \mathrm{~A} & -0.420703 & 0.240232 & -1.463121\end{array}$

$\begin{array}{llll}32 \mathrm{~A} & -0.423427 & 0.241786 & 1.448375\end{array}$

$\begin{array}{llll}33 \mathrm{~A} & 1.059904 & -0.605228 & -0.919699\end{array}$

$\begin{array}{llll}34 \mathrm{~B} & 1.306761 & 2.255671 & 0.011728\end{array}$

$\begin{array}{llll}35 \mathrm{~B} & -1.278524 & -2.271794 & 0.011723\end{array}$

$\begin{array}{llll}36 \mathrm{~B} & 2.218434 & -1.266774 & 1.842697\end{array}$

\section{$E 37 / \varepsilon=-198.154921$}

$\begin{array}{cccc}\text { atom } & x / \sigma & y / \sigma & z / \sigma \\ 1 \mathrm{~A} & 0.000000 & 0.000000 & 0.000000 \\ \text { 2B } & 1.746929 & 0.000000 & 0.000000 \\ \text { 3A } & 0.544057 & -0.923272 & 0.000000 \\ \text { 4A } & -0.607940 & -0.893779 & 0.000000 \\ \text { 5A } & -0.567675 & -0.311854 & -0.886891\end{array}$
$\begin{array}{llll}5 A & -0.567675 & -0.311854 & -0.886891\end{array}$ $\begin{array}{llll}7 \mathrm{~A} & -0.553281 & 0.739024 & 0.558396\end{array}$

$\begin{array}{lllll}8 A & -0.553281 & 0.739024 & -0.558395\end{array}$

$\begin{array}{lllll}9 A & 0.515403 & -0.327994 & 0.910551\end{array}$

$\begin{array}{llll}10 A & 0.515403 & -0.327994 & -0.910551\end{array}$

$\begin{array}{llll}11 \mathrm{~A} & -1.163373 & 0.029303 & 0.000000\end{array}$

$\begin{array}{llll}12 \mathrm{~A} & 0.538454 & 0.749012 & 0.574670\end{array}$

$\begin{array}{llll}13 \mathrm{~A} & 0.538454 & 0.749013 & -0.574669\end{array}$

$\begin{array}{llll}14 \mathrm{~B} & -1.636222 & -1.375026 & -1.005754\end{array}$

$\begin{array}{llll}15 B & -1.636221 & -1.375027 & 1.005754\end{array}$

$\begin{array}{llll}16 \mathrm{~A} & -0.060362 & -1.323858 & -0.797418\end{array}$

$\begin{array}{llll}17 A & -0.060362 & -1.323858 & 0.797418\end{array}$

$\begin{array}{llll}18 \mathrm{~B} & -0.109645 & -0.880477 & 2.242826\end{array}$

$\begin{array}{llll}19 \mathrm{~B} & -0.109646 & -0.880476 & -2.242826\end{array}$

$\begin{array}{llll}20 \mathrm{~B} & -1.574619 & 1.700580 & 0.000001\end{array}$

$\begin{array}{llll}21 \mathrm{~B} & 1.423159 & -1.553854 & -1.116263\end{array}$

$\begin{array}{llll}22 \mathrm{~B} & 1.423159 & -1.553855 & 1.116262\end{array}$

$\begin{array}{llll}23 B & -1.591660 & 0.517878 & -1.629801\end{array}$

$\begin{array}{llll}24 \mathrm{~B} & -1.591660 & 0.517877 & 1.629802\end{array}$

$\begin{array}{llll}25 \mathrm{~B} & -0.070962 & 1.935598 & 1.440736\end{array}$

$\begin{array}{llll}26 \mathrm{~B} & -0.070962 & 1.935599 & -1.440734\end{array}$

$\begin{array}{lllll}27 \mathrm{~A} & -0.050034 & 0.432977 & -1.475952\end{array}$

$\begin{array}{llll}28 A & -0.050034 & 0.432976 & 1.475953\end{array}$

$\begin{array}{llll}29 A & -0.033605 & 1.506322 & 0.000001\end{array}$

$\begin{array}{llll}30 \mathrm{~A} & 0.443253 & -1.997331 & 0.000000\end{array}$

$\begin{array}{llll}31 \mathrm{~B} & 1.406518 & 0.513658 & -1.847409\end{array}$

$\begin{array}{llll}32 \mathrm{~B} & 1.406518 & 0.513658 & 1.847409\end{array}$

$\begin{array}{llll}33 \mathrm{~A} & -0.633986 & -1.963823 & 0.000000\end{array}$

$\begin{array}{llll}34 \mathrm{~B} & 1.413783 & 1.907569 & 0.000001\end{array}$

$\begin{array}{llll}35 \mathrm{~B} & -0.172411 & -2.769706 & -1.218540\end{array}$

$\begin{array}{llll}36 \mathrm{~B} & -0.172411 & -2.769707 & 1.218539\end{array}$ $\begin{array}{llll}37 \mathrm{~B} & -2.668959 & 0.017330 & 0.000000\end{array}$

\section{$E 38 / \varepsilon=-204.430886$}

$\begin{array}{llll}1 \mathrm{~A} & 0.000000 & 0.000000 & 0.000000\end{array}$

$\begin{array}{llll}2 \mathrm{~A} & 1.071855 & 0.000000 & 0.000000\end{array}$

$\begin{array}{llll}3 A & 0.467541 & -0.964510 & 0.000000\end{array}$

$\begin{array}{llll}4 \mathrm{~A} & -0.016280 & -0.624047 & 0.893326\end{array}$

$\begin{array}{lllll}5 A & 0.554448 & 0.286857 & -0.893326\end{array}$

$\begin{array}{llll}6 \mathrm{~A} & -0.016279 & -0.624047 & -0.893326\end{array}$

$\begin{array}{lllll}7 \mathrm{~A} & 0.554448 & 0.286857 & 0.893326\end{array}$

$\begin{array}{llll}8 \mathrm{~A} & -0.345189 & 0.850352 & 0.564991\end{array}$

$\begin{array}{lllll}9 A & -0.345189 & 0.850352 & -0.564991\end{array}$

$\begin{array}{llll}10 A & -0.915760 & -0.060303 & -0.564991\end{array}$

$\begin{array}{llll}11 \mathrm{~A} & -0.915760 & -0.060303 & 0.564991\end{array}$

$\begin{array}{llll}12 \mathrm{~A} & 0.581684 & 0.963454 & 0.000000\end{array}$

$\begin{array}{llll}13 \mathrm{~A} & -0.613235 & -0.943685 & 0.000000\end{array}$

$\begin{array}{llll}14 \mathrm{~B} & 2.033215 & 0.564558 & -1.018635\end{array}$

$\begin{array}{llll}15 \mathrm{~B} & 0.378867 & -2.075849 & 1.018636\end{array}$

$\begin{array}{llll}16 \mathrm{~B} & 2.033214 & 0.564559 & 1.018637\end{array}$

$\begin{array}{llll}17 \mathrm{~B} & 0.378868 & -2.075849 & -1.018636\end{array}$

$\begin{array}{llll}18 A & 1.120299 & -0.701924 & -0.798832\end{array}$

$\begin{array}{lllll}19 A & 1.120298 & -0.701923 & 0.798832\end{array}$

$\begin{array}{llll}20 \mathrm{~B} & 0.747926 & -0.468612 & -2.237235\end{array}$

$\begin{array}{llll}21 B & 0.747925 & -0.468612 & 2.237235\end{array}$

$\begin{array}{llll}22 \mathrm{~B} & -2.258043 & -0.427412 & 0.000000\end{array}$

$\begin{array}{llll}23 \mathrm{~B} & -0.600349 & 2.218338 & 0.000000\end{array}$

$\begin{array}{llll}24 \mathrm{~B} & -1.245774 & -1.056051 & 1.641769\end{array}$

$\begin{array}{llll}25 B & 0.406884 & 1.581658 & -1.641769\end{array}$

$\begin{array}{llll}26 \mathrm{~B} & -1.245773 & -1.056052 & -1.641769\end{array}$

$\begin{array}{llll}27 \mathrm{~B} & 0.406883 & 1.581658 & 1.641769\end{array}$

$\begin{array}{llll}28 \mathrm{~B} & -1.637504 & 1.025980 & -1.439470\end{array}$

$\begin{array}{lllr}29 B & -1.637504 & 1.025980 & 1.439469\end{array}$

$\begin{array}{llll}30 \mathrm{~A} & -0.366632 & 0.229713 & 1.481240\end{array}$

$\begin{array}{llll}31 \mathrm{~A} & -0.366631 & 0.229713 & -1.481241\end{array}$

$\begin{array}{lllr}31 \mathrm{~A} & -0.366631 & 0.229713 & -1.481241 \\ 32 \mathrm{~A} & -1.284659 & 0.804903 & 0.000000\end{array}$

$\begin{array}{llll}33 \mathrm{~A} & 1.393853 & -1.509317 & 0.000001\end{array}$

$\begin{array}{llll}34 \mathrm{~A} & 1.966157 & -0.595897 & 0.000001\end{array}$

$\begin{array}{llll}35 \mathrm{~B} & 2.335362 & -1.463221 & 1.230918\end{array}$

$\begin{array}{llll}36 \mathrm{~B} & 2.335365 & -1.463222 & -1.230914\end{array}$

$\begin{array}{llll}37 \mathrm{~B} & -1.353439 & -2.242214 & 0.000000\end{array}$

$\begin{array}{llll}38 \mathrm{~B} & 1.427288 & 2.195944 & 0.000000\end{array}$
$E 39 / \varepsilon=-210.627234$

$\begin{array}{ccc}\text { atom } & \mathrm{X} / \sigma & y / \sigma \\ 1 \mathrm{~A} & 0.000000 & 0.0000\end{array}$

$\begin{array}{llll}1 \mathrm{~A} & 0.000000 & 0.000000 & 0.000000\end{array}$

$\begin{array}{llll}2 \mathrm{~B} & 1.750821 & 0.000000 & 0.000000\end{array}$

$\begin{array}{llll}3 B & -1.636644 & -1.699792 & 0.000000\end{array}$

$\begin{array}{llll}4 \mathrm{~B} & -1.636642 & -0.499806 & 1.624652\end{array}$

$\begin{array}{llll}5 A & -0.611200 & -0.718792 & 0.530909\end{array}$

$\begin{array}{llll}6 \mathrm{~A} & 0.547820 & -0.739308 & 0.546061\end{array}$

$\begin{array}{lllll}7 \mathrm{~A} & -0.566254 & -0.776173 & -0.528786\end{array}$

$\begin{array}{llll}8 \mathrm{~A} & -0.566253 & 0.277185 & 0.897346\end{array}$

$\begin{array}{lllll}9 \mathrm{~A} & -0.550916 & 0.263697 & -0.889129\end{array}$

$\begin{array}{llll}10 A & -0.550915 & 0.927361 & 0.009400\end{array}$

$\begin{array}{llll}11 \mathrm{~A} & 0.516868 & 0.278096 & 0.926699\end{array}$

$\begin{array}{llll}12 \mathrm{~A} & 0.516867 & -0.803962 & -0.538290\end{array}$

$\begin{array}{lllll}13 A & -1.162345 & 0.025503 & -0.018836\end{array}$

$\begin{array}{lllll}14 \mathrm{~A} & 0.540766 & 0.260930 & -0.906462\end{array}$

$\begin{array}{llll}15 A & 0.540767 & 0.943114 & 0.017139\end{array}$

$\begin{array}{llll}16 \mathrm{~B} & 1.424100 & -1.910420 & 0.025821\end{array}$

$\begin{array}{llll}17 \mathrm{~B} & 1.424100 & -0.586421 & 1.818373\end{array}$

$\begin{array}{llll}18 \mathrm{~A} & -0.059817 & -0.589647 & 1.423652\end{array}$

$\begin{array}{llll}19 A & -0.059818 & -1.534097 & 0.144970\end{array}$

$\begin{array}{llll}20 B & -0.107530 & 0.622908 & 2.326381\end{array}$

$\begin{array}{lllll}21 \mathrm{~B} & -0.107533 & -2.040380 & -1.279420\end{array}$

$\begin{array}{llll}22 \mathrm{~B} & -1.572730 & 1.368112 & -1.010502\end{array}$

$\begin{array}{lllll}23 B & -1.589256 & -0.549164 & -1.620942\end{array}$

$\begin{array}{llll}24 \mathrm{~B} & -1.589252 & 1.387812 & 1.001512\end{array}$

$\begin{array}{llll}25 \mathrm{~A} & 0.429990 & -1.597508 & 1.179937\end{array}$

$\begin{array}{llll}26 \mathrm{~A} & -0.638301 & -1.579719 & 1.166799\end{array}$

$\begin{array}{llll}27 \mathrm{~B} & -0.172406 & -1.471159 & 2.636658\end{array}$

$\begin{array}{lllll}28 \mathrm{~B} & -0.172408 & -2.952680 & 0.630839\end{array}$

$\begin{array}{llll}29 B & -0.066837 & 0.701404 & -2.309129\end{array}$

$\begin{array}{llll}30 \mathrm{~B} & -0.066833 & 2.413290 & 0.008577\end{array}$

$\begin{array}{llll}31 \mathrm{~A} & -0.048036 & 1.225866 & 0.929805\end{array}$

$\begin{array}{llll}32 \mathrm{~A} & -0.048039 & -0.528248 & -1.445075\end{array}$

$\begin{array}{lllll}33 \mathrm{~A} & -0.030778 & 1.212180 & -0.895331\end{array}$

$\begin{array}{lllll}34 \mathrm{~B} & 1.408895 & -0.686099 & -1.789873\end{array}$

$\begin{array}{llll}35 \mathrm{~B} & 1.408897 & 1.509008 & 1.182061\end{array}$

$\begin{array}{llll}36 \mathrm{~B} & 1.416927 & 1.533836 & -1.132915\end{array}$

$\begin{array}{llll}37 \mathrm{~B} & -2.667659 & 0.012585 & -0.009291\end{array}$

$\begin{array}{llll}38 \mathrm{~B} & 1.322890 & -2.593547 & 1.915622\end{array}$

$\begin{array}{llll}39 B & -1.691512 & -2.486499 & 1.836558\end{array}$

$E 40 / \varepsilon=-216.945765$

$\begin{array}{cccc}\text { atom } & \mathrm{x} / \sigma & y / \sigma & z / \sigma \\ 1 \mathrm{~A} & 0.000000 & 0.000000 & 0.000000\end{array}$

$\begin{array}{llll}2 \mathrm{~B} & 2.343246 & 0.000000 & 0.000000\end{array}$

$\begin{array}{llll}3 \mathrm{~B} & 1.452637 & -1.838654 & 0.000000\end{array}$

$\begin{array}{llll}4 \mathrm{~B} & -0.614446 & -0.837399 & 2.100482\end{array}$

$\begin{array}{llll}5 \mathrm{~B} & 0.276164 & 1.001259 & 2.100480\end{array}$

$\begin{array}{llll}6 \mathrm{~A} & 0.170361 & -0.082519 & 1.054752\end{array}$

$\begin{array}{llll}7 A & 0.930296 & -0.450617 & 0.282540\end{array}$

$\begin{array}{llll}8 \mathrm{~A} & -0.553544 & -0.724510 & 0.596882\end{array}$

$\begin{array}{lllll}9 A & 0.939109 & 0.537750 & -0.128423\end{array}$

$\begin{array}{lllll}10 \mathrm{~A} & 0.160227 & -1.070247 & -0.128422\end{array}$

$\begin{array}{llll}11 \mathrm{~A} & 0.225338 & 0.883487 & 0.596880\end{array}$

$\begin{array}{llll}12 \mathrm{~A} & -0.341686 & -0.463024 & -0.911312\end{array}$

$\begin{array}{llll}13 \mathrm{~A} & 0.151496 & 0.555146 & -0.911313\end{array}$

$\begin{array}{lllll}14 \mathrm{~A} & -1.055015 & -0.117501 & -0.186458\end{array}$

$\begin{array}{llll}15 A & -0.561833 & 0.900669 & -0.186459\end{array}$

$\begin{array}{llll}16 \mathrm{~A} & 0.735322 & -0.356176 & -0.775222\end{array}$

$\begin{array}{llll}17 A & -0.760438 & 0.368342 & 0.744704\end{array}$

$\begin{array}{lllll}18 A & 0.455740 & -1.104462 & 0.974968\end{array}$

$\begin{array}{llll}19 \mathrm{~A} & 1.149153 & 0.327083 & 0.974967\end{array}$

$\begin{array}{llll}20 \mathrm{~B} & -0.436173 & -2.272211 & 0.653877\end{array}$

$\begin{array}{llll}21 B & 1.512519 & 1.750851 & 0.653873\end{array}$

$\begin{array}{lllll}22 \mathrm{~B} & 0.017579 & -0.008517 & -2.296378\end{array}$

$\begin{array}{lllll}23 B & -2.060036 & 0.997839 & -0.185197\end{array}$

$\begin{array}{lllll}24 \mathrm{~B} & 0.019390 & -1.833130 & -1.414628\end{array}$

$\begin{array}{llll}25 \mathrm{~B} & 1.450404 & 1.121189 & -1.414630\end{array}$

$\begin{array}{llll}26 \mathrm{~B} & -0.618059 & 2.123111 & 0.687250\end{array}$

$\begin{array}{llll}27 \mathrm{~B} & -2.049071 & -0.831207 & 0.687252\end{array}$ $\begin{array}{llll}28 \mathrm{~A} & 0.845640 & -0.409610 & 1.818478\end{array}$ 


\begin{abstract}
$\begin{array}{llll}29 A & 1.554719 & -0.753076 & 1.097942\end{array}$
$\begin{array}{llll}30 \mathrm{~B} & 2.211081 & 0.315351 & 2.025601\end{array}$

$\begin{array}{llll}31 B & 1.123264 & -1.930440 & 2.025604\end{array}$

$\begin{array}{llll}32 \mathrm{~B} & -1.800747 & -0.727487 & -1.425338\end{array}$

$\begin{array}{lllll}33 \mathrm{~B} & -0.545501 & 1.863960 & -1.425340\end{array}$

$\begin{array}{llll}34 \mathrm{~A} & -0.908416 & -1.207063 & -0.318590\end{array}$

$\begin{array}{lllll}35 \mathrm{~A} & 0.383983 & 1.461087 & -0.318592\end{array}$

$\begin{array}{lllll}36 \mathrm{~A} & -0.920501 & 0.445871 & -1.118404\end{array}$

$\begin{array}{llll}37 \mathrm{~B} & -1.705471 & 0.826096 & 1.807926\end{array}$

$\begin{array}{llll}38 \mathrm{~B} & 1.772612 & -0.858619 & -1.726351\end{array}$

$\begin{array}{llll}39 B & 0.907560 & -0.439601 & 3.347184\end{array}$

$\begin{array}{llll}40 \mathrm{~B} & 2.919532 & -1.414164 & 1.302704\end{array}$
\end{abstract}

$E 41 / \varepsilon=-223.258093$

atom $\mathrm{x} / \sigma$

ylo

$\begin{array}{llll}1 \mathrm{~A} & 0.000000 & 0.000000 & 0.000000\end{array}$

$\begin{array}{llll}2 \mathrm{~A} & 1.094698 & 0.000000 & 0.000000\end{array}$

$\begin{array}{llll}3 A & 0.550723 & -0.948256 & 0.000000\end{array}$

$\begin{array}{llll}4 \mathrm{~A} & 0.550722 & -0.293426 & -0.901715\end{array}$

$\begin{array}{llll}5 A & 0.545886 & 0.313295 & 0.879071\end{array}$

$\begin{array}{lllll}6 A & 0.545886 & 0.932871 & 0.025903\end{array}$

$\begin{array}{llll}7 \mathrm{~A} & 1.090124 & -0.648204 & 0.910058\end{array}$

$\begin{array}{lllll}8 \mathrm{~A} & 1.090123 & 0.664815 & -0.897995\end{array}$

$\begin{array}{lllll}9 \mathrm{~A} & 0.012126 & 0.663778 & -0.893212\end{array}$

$\begin{array}{llll}10 A & 0.012127 & -0.643977 & 0.907593\end{array}$

$\begin{array}{llll}11 \mathrm{~A} & -0.538857 & 0.963299 & 0.014652\end{array}$

$\begin{array}{llll}12 \mathrm{~A} & -0.538857 & 0.312011 & 0.911488\end{array}$

$\begin{array}{llll}13 B & -0.495981 & -2.009435 & 0.355615\end{array}$

14B $\quad-0.495982 \quad-0.283633-2.020850$

$\begin{array}{llll}15 B & 1.602652 & -0.288549 & -2.018917\end{array}$

$\begin{array}{llll}16 \mathrm{~B} & 1.602653 & -2.009116 & 0.350340\end{array}$

$\begin{array}{llll}17 \mathrm{~B} & -1.964974 & 0.581746 & 0.422470\end{array}$

$\begin{array}{llll}18 B & 0.662669 & 0.191892 & 2.315067\end{array}$

$\begin{array}{llll}19 \mathrm{~B} & 0.662667 & 2.260823 & -0.533890\end{array}$

$\begin{array}{llll}20 A & -0.904369 & -0.525966 & 0.303786\end{array}$

$\begin{array}{llll}21 \mathrm{~A} & -0.904370 & 0.126121 & -0.594152\end{array}$

$\begin{array}{llll}22 \mathrm{~A} & -0.360797 & -0.829287 & -0.602232\end{array}$

$\begin{array}{llll}23 \mathrm{~A} & 1.462754 & -0.829212 & -0.602178\end{array}$

$\begin{array}{llll}24 \mathrm{~A} & 2.001984 & -0.520755 & 0.303415\end{array}$

$\begin{array}{lllll}25 \mathrm{~A} & 2.001984 & 0.127383 & -0.589084\end{array}$

$\begin{array}{llll}26 \mathrm{~B} & 1.356552 & 1.746319 & 1.268187\end{array}$

$\begin{array}{llll}27 \mathrm{~B} & 0.549457 & -1.816407 & -1.319084\end{array}$

$\begin{array}{llll}28 \mathrm{~A} & 1.625118 & 0.308006 & 0.917633\end{array}$

$\begin{array}{llll}29 \mathrm{~A} & 1.625117 & 0.967903 & 0.008941\end{array}$

$\begin{array}{llll}30 \mathrm{~B} & 3.040487 & 0.618663 & 0.449276\end{array}$

$\begin{array}{llll}31 \mathrm{~B} & -1.213657 & -0.753452 & 1.792687\end{array}$

$\begin{array}{llll}32 \mathrm{~B} & -1.213660 & 1.471554 & -1.271193\end{array}$

$\begin{array}{llll}33 \mathrm{~A} & -0.009298 & 1.263179 & 0.917329\end{array}$

$\begin{array}{lllll}34 \mathrm{~B} & 2.352885 & 1.449188 & -1.265641\end{array}$

$\begin{array}{llll}35 \mathrm{~B} & 2.352887 & -0.755093 & 1.769697\end{array}$

$\begin{array}{llll}36 \mathrm{~B} & 0.557717 & 1.337412 & -2.209958\end{array}$

$\begin{array}{llll}37 \mathrm{~B} & 0.557720 & -1.687651 & 1.955615\end{array}$

$\begin{array}{llll}38 \mathrm{~B} & -1.870562 & -1.053427 & -0.765001\end{array}$

$\begin{array}{llll}39 \mathrm{~B} & 2.984438 & -1.032670 & -0.749931\end{array}$

$\begin{array}{llll}40 \mathrm{~B} & -1.002219 & 1.198903 & 2.083826\end{array}$

$\begin{array}{llll}41 B & -1.002221 & 2.352535 & 0.495252\end{array}$

$E 42 / \varepsilon=-230.236470$

\begin{tabular}{|c|c|c|c|}
\hline tom & $\mathrm{x} / \sigma$ & $y / \sigma$ & $z / \sigma$ \\
\hline $1 \mathrm{~A}$ & 0.000000 & 0.000000 & 0.000000 \\
\hline $2 A$ & 1.123900 & 0.000000 & 0.000000 \\
\hline $3 \mathrm{~A}$ & 0.582476 & -0.914367 & 0.000000 \\
\hline $4 \mathrm{~A}$ & 0.582474 & 0.777239 & -0.481629 \\
\hline $5 \mathrm{~A}$ & 0.744658 & 0.813152 & 0.617477 \\
\hline $6 \mathrm{~A}$ & 0.744660 & -0.365955 & 0.953190 \\
\hline $7 \mathrm{~A}$ & 0.687468 & -0.273582 & -0.960891 \\
\hline $8 \mathrm{~A}$ & -0.225629 & -0.783477 & -0.736218 \\
\hline $9 A$ & -0.225630 & 0.278185 & -1.038491 \\
\hline $10 \mathrm{~B}$ & 0.674822 & -1.952381 & 1.078036 \\
\hline $11 B$ & 0.674816 & 2.227422 & -0.112026 \\
\hline $12 \mathrm{~B}$ & 0.659293 & 1.113254 & -1.949763 \\
\hline & 0.659297 & -1.973307 & -1.0 \\
\hline & 2750110 & -0.030299 & $\$ 427$ \\
\hline
\end{tabular}

$\begin{array}{llll}14 \mathrm{~B} & 2.759148 & -0.030299 & -0.106427\end{array}$ $\begin{array}{llll}16 \mathrm{~A} & -0.999333 & 0.481942 & -0.315683\end{array}$

$\begin{array}{lllll}17 A & -0.999332 & -0.575950 & -0.014482\end{array}$

$\begin{array}{llll}18 \mathrm{~A} & -0.253423 & -0.735382 & 0.758216\end{array}$

$\begin{array}{llll}19 A & -0.253426 & 1.024475 & 0.257155\end{array}$

$\begin{array}{llll}20 B & -0.197937 & -0.642089 & -2.255181\end{array}$

$\begin{array}{llll}21 \mathrm{~A} & -0.074741 & 0.299614 & 1.052323\end{array}$

$\begin{array}{llll}22 \mathrm{~B} & -0.117939 & -0.570741 & 2.275207\end{array}$

$\begin{array}{llll}23 \mathrm{~B} & -0.117943 & 1.683577 & 1.633364\end{array}$

$\begin{array}{llll}24 \mathrm{~A} & 1.603833 & 0.981942 & 0.004034\end{array}$

$\begin{array}{llll}25 A & 1.603836 & -0.832552 & 0.520652\end{array}$

$\begin{array}{llll}26 \mathrm{~A} & 1.653745 & 0.251928 & 0.884828\end{array}$

$\begin{array}{lllll}27 \mathrm{~A} & 1.538605 & -0.819670 & -0.570431\end{array}$

$\begin{array}{lllll}28 \mathrm{~A} & 1.538603 & 0.396280 & -0.916633\end{array}$

$\begin{array}{llll}29 B & -1.395373 & -1.785313 & -1.006109\end{array}$

$\begin{array}{llll}30 \mathrm{~B} & -1.395376 & 0.987609 & -1.795610\end{array}$

$\begin{array}{llll}31 B & 1.795795 & -0.579355 & -2.034851\end{array}$

$\begin{array}{llll}32 \mathrm{~A} & 0.865707 & 0.471077 & 1.654538\end{array}$

$\begin{array}{llll}33 \mathrm{~B} & 1.868375 & 1.646870 & 1.363568\end{array}$

$\begin{array}{llll}34 \mathrm{~B} & 1.868379 & -0.681643 & 2.026537\end{array}$

$\begin{array}{llll}35 \mathrm{~A} & -0.333004 & 1.318317 & -0.783317\end{array}$

$\begin{array}{llll}36 \mathrm{~A} & -0.332999 & -1.533211 & 0.028563\end{array}$

$\begin{array}{llll}37 \mathrm{~B} & -1.455296 & 1.933197 & 0.024790\end{array}$

$\begin{array}{llll}38 \mathrm{~B} & -1.455289 & -1.630222 & 1.039359\end{array}$

$\begin{array}{lllll}39 \mathrm{~A} & -1.130970 & -0.308866 & -1.084808\end{array}$

$\begin{array}{llll}40 \mathrm{~B} & -2.466915 & -0.129332 & -0.454236\end{array}$

$\begin{array}{llll}41 \mathrm{~B} & 2.360727 & 1.681463 & -1.099492\end{array}$

$\begin{array}{llll}42 \mathrm{~B} & 2.360732 & -2.008431 & -0.048917\end{array}$

\section{$E 43 / \varepsilon=-237.073585$}

\section{$\begin{array}{llll}\text { atom } \quad \mathrm{x} / \sigma & y / \sigma & \mathrm{z} / \sigma\end{array}$}

$\begin{array}{llll}1 \mathrm{~A} & 0.000000 & 0.000000 & 0.000000\end{array}$

$\begin{array}{lllll}2 \mathrm{~A} & 1.096375 & 0.000000 & 0.000000\end{array}$

$\begin{array}{llll}3 A & 0.560099 & -0.922144 & 0.000000\end{array}$

$\begin{array}{llll}4 \mathrm{~B} & -0.870880 & 0.529779 & 1.386183\end{array}$

$\begin{array}{lllll}5 \mathrm{~A} & 0.541201 & -0.357098 & -0.968401\end{array}$

$\begin{array}{llll}6 \mathrm{~B} & 1.977234 & 0.490110 & 1.411713\end{array}$

$\begin{array}{lllll}7 \mathrm{~B} & 0.573804 & -2.039840 & -0.946402\end{array}$

$\begin{array}{llll}8 \mathrm{~A} & 0.542545 & 0.707399 & -0.606445\end{array}$

$\begin{array}{llll}9 \mathrm{~A} & 0.553655 & 0.324284 & 0.887372\end{array}$

$\begin{array}{lllll}10 \mathrm{~B} & 0.464088 & 0.906079 & -2.082968\end{array}$

$\begin{array}{llll}11 \mathrm{~A} & -0.001730 & -0.646496 & 0.880957\end{array}$

$\begin{array}{llll}12 \mathrm{~A} & 1.130873 & -0.652597 & 0.877390\end{array}$

$\begin{array}{lllll}13 \mathrm{~A} & -0.361886 & 0.210781 & -1.008381\end{array}$

$\begin{array}{lllll}14 \mathrm{~A} & -0.345986 & -0.837391 & -0.617806\end{array}$

$\begin{array}{llll}15 \mathrm{~A} & 1.460696 & -0.794846 & -0.648150\end{array}$

$\begin{array}{llll}16 \mathrm{~A} & -0.933973 & 0.544418 & -0.150476\end{array}$

$\begin{array}{llll}17 A & -0.005796 & 1.063046 & 0.241759\end{array}$

$\begin{array}{llll}18 A & -0.921244 & -0.515952 & 0.242226\end{array}$

$\begin{array}{llll}19 A & 2.044085 & -0.486020 & 0.204987\end{array}$

$\begin{array}{llll}20 B & 0.749899 & 2.233797 & -0.614844\end{array}$

$\begin{array}{llll}21 \mathrm{~A} & 1.122747 & 1.049026 & 0.253325\end{array}$

$\begin{array}{llll}22 \mathrm{~A} & 2.015031 & 0.568174 & -0.151114\end{array}$

$\begin{array}{lllll}23 \mathrm{~A} & 1.414011 & 0.261359 & -1.012137\end{array}$

$\begin{array}{llll}24 \mathrm{~B} & 0.595872 & -2.098357 & 1.210040\end{array}$

$\begin{array}{llll}25 \mathrm{~B} & -0.518388 & -0.816013 & -2.104064\end{array}$

$\begin{array}{llll}26 \mathrm{~B} & 1.560258 & -0.741311 & -2.138087\end{array}$

$\begin{array}{llll}27 \mathrm{~B} & 0.567655 & 1.877908 & 1.386015\end{array}$

$\begin{array}{llll}28 B & 0.538184 & -0.464262 & 2.291793\end{array}$

$\begin{array}{llll}29 B & 2.717524 & -1.665907 & -0.644810\end{array}$

$\begin{array}{llll}30 \mathrm{~B} & -1.617979 & 0.935981 & -1.551269\end{array}$

$\begin{array}{lllll}31 \mathrm{~B} & -1.582202 & -1.763817 & -0.553302\end{array}$

$\begin{array}{llll}32 \mathrm{~B} & 2.416112 & 1.228442 & -1.500766\end{array}$

$\begin{array}{llll}33 \mathrm{~A} & 1.487111 & -1.454212 & 0.201013\end{array}$

$\begin{array}{llll}34 \mathrm{~B} & -1.264367 & -1.378399 & 1.453857\end{array}$

$\begin{array}{llll}35 \mathrm{~A} & -0.343874 & -1.478282 & 0.241361\end{array}$

$\begin{array}{llll}36 \mathrm{~A} & 2.369478 & -0.208260 & -0.860339\end{array}$

$\begin{array}{llll}37 \mathrm{~A} & -1.277969 & -0.312831 & -0.790536\end{array}$

$\begin{array}{llll}38 \mathrm{~A} & -0.369642 & 1.263684 & -0.789519\end{array}$

$\begin{array}{llll}39 \mathrm{~B} & -1.202868 & 2.038710 & 0.190199\end{array}$

$\begin{array}{llll}\text { 40B } & 2.456306 & -1.385763 & 1.371257\end{array}$

$\begin{array}{llll}41 \mathrm{~B} & 3.452093 & 0.125436 & 0.151447\end{array}$

$\begin{array}{llll}42 B & -2.354925 & 0.046930 & 0.203611\end{array}$
43B $\quad 2.434316 \quad 1.984165 \quad 0.318821$

$E 44 / \varepsilon=-244.756631$

$\begin{array}{llll}\text { atom } \mathrm{x} / \sigma & \mathrm{y} / \sigma & \mathrm{z} \sigma\end{array}$

$\begin{array}{llll}1 \mathrm{~A} & 0.000000 & 0.000000 & 0.000000\end{array}$

$\begin{array}{llll}2 \mathrm{~A} & 1.100400 & 0.000000 & 0.000000\end{array}$

$\begin{array}{llll}3 A & 0.553444 & -0.969252 & 0.000000\end{array}$

$\begin{array}{lllll}4 \mathrm{~B} & 0.653308 & -0.367093 & -2.274355\end{array}$

$\begin{array}{lllll}5 \mathrm{~A} & 0.548761 & 0.944750 & -0.023252\end{array}$

$\begin{array}{lllll}6 \mathrm{~A} & 0.548637 & -0.312745 & -0.847779\end{array}$

$\begin{array}{llll}7 \mathrm{~A} & 0.551327 & 0.311736 & 0.889624\end{array}$

$\begin{array}{llll}8 \mathrm{~A} & -0.547181 & -0.943105 & -0.015191\end{array}$

$\begin{array}{llll}9 \mathrm{~A} & -0.003111 & -0.621731 & 0.892704\end{array}$

$\begin{array}{lllll}10 \mathrm{~A} & 1.094218 & 0.630612 & -0.923892\end{array}$

$\begin{array}{llll}11 \mathrm{~A} & 1.094748 & -0.654998 & 0.877881\end{array}$

$\begin{array}{lllll}12 \mathrm{~A} & 0.011218 & 0.628590 & -0.924479\end{array}$

$\begin{array}{llll}13 \mathrm{~A} & -0.535419 & -0.324028 & -0.920562\end{array}$

$\begin{array}{lllll}14 \mathrm{~A} & 1.649028 & -0.309492 & -0.920590\end{array}$

$\begin{array}{llll}15 \mathrm{~B} & 1.174981 & -2.377874 & 0.053152\end{array}$

$\begin{array}{lllll}16 \mathrm{~A} & 1.665786 & -0.936321 & -0.033524\end{array}$

$\begin{array}{llll}17 \mathrm{~B} & -0.472157 & 0.280889 & 2.024300\end{array}$

$\begin{array}{llll}18 \mathrm{~B} & 1.548746 & 0.261562 & 2.029951\end{array}$

$\begin{array}{lllll}19 B & -0.481478 & 2.001060 & -0.416243\end{array}$

$\begin{array}{llll}20 \mathrm{~B} & 1.576490 & 2.007555 & -0.413529\end{array}$

$\begin{array}{lllll}21 \mathrm{~B} & -1.974445 & -0.573710 & -0.414000\end{array}$

$\begin{array}{lllll}22 A & 0.010063 & -1.266215 & -0.910006\end{array}$

$\begin{array}{llll}23 A & -0.901498 & 0.525092 & -0.319941\end{array}$

$\begin{array}{lllll}24 \mathrm{~A} & 1.999078 & 0.539121 & -0.305460\end{array}$

$\begin{array}{llll}25 \mathrm{~A} & -0.358361 & 0.843871 & 0.587007\end{array}$

$\begin{array}{llll}26 \mathrm{~A} & -0.912272 & -0.097972 & 0.587010\end{array}$

$\begin{array}{llll}27 \mathrm{~A} & 1.448384 & 0.850609 & 0.592117\end{array}$

$\begin{array}{llll}28 \mathrm{~A} & 1.997867 & -0.091642 & 0.603428\end{array}$

$\begin{array}{llll}29 B & 0.544365 & 1.837058 & 1.291902\end{array}$

$\begin{array}{llll}30 \mathrm{~A} & 1.094132 & -1.239724 & -0.936805\end{array}$

$\begin{array}{lllll}31 \mathrm{~B} & 3.119225 & -0.491468 & -0.344577\end{array}$

$\begin{array}{llll}32 \mathrm{~B} & -1.337141 & -1.364900 & 1.295521\end{array}$

$\begin{array}{lllll}33 \mathrm{~B} & -0.884608 & -2.384977 & -0.397761\end{array}$

$\begin{array}{lllll}34 \mathrm{~B} & 0.547652 & -1.490860 & 2.021588\end{array}$

$\begin{array}{llll}35 \mathrm{~B} & 2.354032 & 0.693511 & -1.802219\end{array}$

$\begin{array}{llll}36 \mathrm{~B} & -1.224263 & 0.710857 & -1.812610\end{array}$

$\begin{array}{llll}37 \mathrm{~B} & 2.392673 & -1.399174 & 1.270041\end{array}$

$\begin{array}{lllll}38 \mathrm{~B} & 0.560193 & 1.564644 & -2.033068\end{array}$

$\begin{array}{lllll}39 \mathrm{~B} & -1.078850 & -1.258692 & -2.020322\end{array}$

$\begin{array}{lllll}40 \mathrm{~B} & 0.551223 & -2.360219 & -1.838959\end{array}$

$\begin{array}{llll}41 \mathrm{~A} & -0.012849 & -1.671833 & 0.617488\end{array}$

$\begin{array}{llll}42 \mathrm{~B} & -1.856658 & 1.084533 & 0.755187\end{array}$

$\begin{array}{llll}43 \mathrm{~B} & 2.291219 & -1.389015 & -1.840921\end{array}$

$\begin{array}{llll}\text { 44B } & 2.919721 & 1.126199 & 0.807909\end{array}$

$E 45 / \varepsilon=-251.897352$

atom $\mathrm{x} / \sigma \quad \mathrm{y} / \sigma \quad \mathrm{z} / \sigma$

$\begin{array}{cccc}1 \mathrm{~A} & 0.000000 & 0.000000 & 0.000000\end{array}$

$\begin{array}{llll}2 \mathrm{~A} & 1.103619 & 0.000000 & 0.000000\end{array}$

$\begin{array}{llll}3 A & 0.547040 & -0.958501 & 0.000000\end{array}$

$\begin{array}{llll}4 \mathrm{~B} & 0.536492 & -0.311528 & -2.277691\end{array}$

$\begin{array}{llll}5 A & 0.548165 & -0.318307 & -0.848450\end{array}$

$\begin{array}{llll}6 \mathrm{~A} & -0.546218 & -0.947970 & -0.017571\end{array}$

$\begin{array}{lllll}7 A & 0.552568 & 0.944281 & -0.017569\end{array}$

$\begin{array}{llll}8 \mathrm{~A} & 0.553357 & 0.312505 & 0.889358\end{array}$

$\begin{array}{llll}9 A & 0.002874 & -0.635498 & 0.889357\end{array}$

$\begin{array}{lllll}10 A & 1.640379 & -0.952530 & -0.017978\end{array}$

$\begin{array}{llll}11 \mathrm{~A} & 1.098919 & -0.638117 & 0.887469\end{array}$

$\begin{array}{lllll}12 \mathrm{~A} & 0.007954 & 0.629675 & -0.920609\end{array}$

$\begin{array}{llll}13 \mathrm{~A} & -0.542934 & -0.319026 & -0.920610\end{array}$

$\begin{array}{llll}14 \mathrm{~A} & 1.088863 & 0.627220 & -0.923080\end{array}$

$\begin{array}{lllll}15 A & -0.005017 & -1.256584 & -0.923081\end{array}$

$\begin{array}{lllll}16 A & 1.092822 & -1.260594 & -0.922659\end{array}$

$\begin{array}{lllll}17 A & 1.636522 & -0.324272 & -0.922659\end{array}$

$\begin{array}{llll}18 \mathrm{~B} & -0.465327 & 0.270202 & 2.026641\end{array}$

$\begin{array}{llll}19 B & -1.981040 & -0.566043 & -0.397218\end{array}$

$\begin{array}{llll}20 B & -0.490350 & 2.001120 & -0.397217\end{array}$

$\begin{array}{llll}21 \mathrm{~A} & -0.904198 & 0.525046 & -0.312204\end{array}$ $\begin{array}{llll}22 \mathrm{~A} & -0.906583 & -0.104775 & 0.591655\end{array}$ 


$\begin{array}{rrrr}\text { 23A } & -0.358377 & 0.839306 & 0.591655 \\ 24 \mathrm{~B} & 0.543669 & -1.483559 & 2.029638 \\ \text { 25B } & 1.557963 & 0.263186 & 2.029639 \\ \text { 26B } & -0.951388 & -2.367693 & -0.403963 \\ \text { 27B } & 1.584771 & 1.999902 & -0.403960 \\ \text { 28B } & 2.027896 & -2.378698 & -0.410649 \\ \text { 29B } & 3.071098 & -0.582168 & -0.410648 \\ \text { 30B } & -1.321849 & -1.376208 & 1.296831 \\ \text { 31B } & 0.540030 & 1.830189 & 1.296833 \\ \text { 32B } & -1.238452 & 0.719140 & -1.799655 \\ \text { 33B } & 2.406958 & -1.397665 & 1.291628 \\ \text { 34A } & 0.539752 & -2.004699 & -0.317037 \\ \text { 35A } & 2.008636 & 0.524909 & -0.317036 \\ \text { 36A } & 1.472531 & 0.844093 & 0.594451 \\ \text { 37A } & -0.003197 & -1.697302 & 0.594449 \\ \text { 38A } & 2.016855 & -0.101506 & 0.592219 \\ \text { 39A } & 1.087871 & -1.701337 & 0.592218 \\ \text { 40B } & 2.312276 & 0.703891 & -1.826929 \\ \text { 41B } & 0.534814 & -2.357128 & -1.826931 \\ \text { 42B } & 0.540179 & 1.605143 & -2.011384 \\ \text { 43B } & -1.126320 & -1.264785 & -2.011386 \\ \text { 44B } & 2.198617 & -1.276684 & -2.021370 \\ \text { 45B } & -1.854172 & 1.076672 & 0.775017\end{array}$

$E 46 / \varepsilon=-260.391381$

atom $x / \sigma$

$\begin{array}{cccc}1 \mathrm{~A} & 0.000000 & 0.000000 & 0.000000\end{array}$

2A $\quad 1.099820 \quad 0.000000 \quad 0.000000$

$\begin{array}{llll}3 A & 0.549910 & -0.958060 & 0.000000\end{array}$

$\begin{array}{llll}4 \mathrm{~B} & 0.549909 & -0.303028 & 2.278093\end{array}$

$\begin{array}{llll}5 \mathrm{~A} & 0.549910 & -0.316959 & 0.849198\end{array}$

$\begin{array}{llll}6 \mathrm{~A} & 0.549910 & 0.945359 & 0.017924\end{array}$

$\begin{array}{lllll}7 A & 0.549911 & 0.310582 & -0.891240\end{array}$

$\begin{array}{llll}8 \mathrm{~A} & -0.543076 & -0.949407 & 0.017949\end{array}$

$\begin{array}{lllll}9 A & 1.642897 & -0.949407 & 0.017951\end{array}$

$\begin{array}{llll}10 A & 0.002670 & -0.637289 & -0.889242\end{array}$

$\begin{array}{lllll}11 \mathrm{~A} & 1.097152 & -0.637289 & -0.889241\end{array}$

$\begin{array}{llll}12 \mathrm{~A} & 0.009660 & 0.630614 & 0.921041\end{array}$

$\begin{array}{llll}13 \mathrm{~A} & 1.090159 & 0.630614 & 0.921042\end{array}$

$\begin{array}{llll}14 \mathrm{~A} & -0.540085 & -0.318944 & 0.920292\end{array}$

$\begin{array}{llll}15 A & 1.639904 & -0.318944 & 0.920293\end{array}$

$\begin{array}{llll}16 \mathrm{~A} & 0.000849 & -1.256334 & 0.922815\end{array}$

$\begin{array}{llll}17 \mathrm{~A} & 1.098970 & -1.256334 & 0.922815\end{array}$

$\begin{array}{llll}18 \mathrm{~B} & 1.563387 & 0.269978 & -2.027533\end{array}$

$\begin{array}{llll}19 B & -0.463564 & 0.269978 & -2.027534\end{array}$

$\begin{array}{llll}\text { 20B } & 1.587737 & 2.003140 & 0.393835\end{array}$

$\begin{array}{llll}21 B & -0.487917 & 2.003140 & 0.393834\end{array}$

$\begin{array}{llll}22 \mathrm{~B} & -1.976569 & -0.568074 & 0.400749\end{array}$

$\begin{array}{llll}23 \mathrm{~B} & 3.076389 & -0.568074 & 0.400753\end{array}$

$\begin{array}{llll}24 \mathrm{~A} & -0.902297 & 0.526369 & 0.312316\end{array}$

$\begin{array}{llll}25 \mathrm{~A} & 2.002118 & 0.526369 & 0.312318\end{array}$

$\begin{array}{llll}26 \mathrm{~A} & -0.357971 & 0.840727 & -0.593600\end{array}$

$\begin{array}{lllll}27 \mathrm{~A} & 1.457792 & 0.840727 & -0.593599\end{array}$

$\begin{array}{llll}28 \mathrm{~A} & -0.905410 & -0.104983 & -0.591304\end{array}$

$\begin{array}{llll}29 \mathrm{~A} & 2.005232 & -0.104983 & -0.591303\end{array}$

$\begin{array}{lllll}30 \mathrm{~B} & 0.549911 & 1.829720 & -1.299578\end{array}$

$\begin{array}{lllll}31 \mathrm{~B} & 0.549912 & -1.480610 & -2.030971\end{array}$

$\begin{array}{llll}32 \mathrm{~B} & -0.941458 & -2.369271 & 0.407887\end{array}$

$\begin{array}{llll}33 \mathrm{~B} & 2.041278 & -2.369271 & 0.407889\end{array}$

$\begin{array}{llll}34 \mathrm{~B} & 2.416917 & -1.379354 & -1.294602\end{array}$

$\begin{array}{llll}35 \mathrm{~B} & -1.317095 & -1.379353 & -1.294605\end{array}$

$\begin{array}{llll}36 \mathrm{~B} & 2.331637 & 0.721361 & 1.801483\end{array}$

$\begin{array}{llll}37 \mathrm{~B} & -1.231819 & 0.721361 & 1.801481\end{array}$

$\begin{array}{llll}38 \mathrm{~A} & 0.549910 & -2.003433 & 0.317241\end{array}$

$\begin{array}{llll}39 A & 1.095919 & -1.698720 & -0.594082\end{array}$

$\begin{array}{llll}40 \mathrm{~A} & 0.003902 & -1.698720 & -0.594083\end{array}$

$\begin{array}{llll}41 \mathrm{~B} & 0.549909 & -2.351316 & 1.829217\end{array}$

$\begin{array}{llll}42 \mathrm{~B} & 0.549909 & 1.611394 & 2.004639\end{array}$

43B $\quad 2.213044 \quad-1.262051 \quad 2.015113$

$\begin{array}{llll}44 \mathrm{~B} & -1.113226 & -1.262051 & 2.015111\end{array}$

$\begin{array}{llll}45 \mathrm{~B} & -1.854466 & 1.074753 & -0.774170\end{array}$

$\begin{array}{llll}46 \mathrm{~B} & 2.954288 & 1.074753 & -0.774167\end{array}$
$E 47 / \varepsilon=-268.881577$

atom $\mathrm{x} / \sigma \quad \mathrm{y} / \sigma$

$\begin{array}{llll}1 \mathrm{~A} & 0.000000 & 0.000000 & 0.000000\end{array}$

$\begin{array}{llll}2 \mathrm{~A} & 1.100898 & 0.000000 & 0.000000\end{array}$

$\begin{array}{llll}3 A & 0.550449 & -0.953405 & 0.000000\end{array}$

$\begin{array}{lllll}4 \mathrm{~B} & 0.550452 & -0.317801 & -2.278481\end{array}$

$\begin{array}{lllll}5 A & 0.550449 & -0.317802 & -0.849884\end{array}$

$\begin{array}{lllll}6 \mathrm{~A} & 0.550449 & 0.944742 & -0.018265\end{array}$

$\begin{array}{lllll}7 \mathrm{~A} & -0.542946 & -0.949074 & -0.018265\end{array}$

$\begin{array}{lllll}8 \mathrm{~A} & 1.643844 & -0.949074 & -0.018263\end{array}$

$\begin{array}{lllll}9 \mathrm{~A} & 1.096874 & -0.633280 & 0.891088\end{array}$

$\begin{array}{llll}10 \mathrm{~A} & 0.550448 & 0.313156 & 0.891087\end{array}$

$\begin{array}{llll}11 \mathrm{~A} & 0.004023 & -0.633280 & 0.891087\end{array}$

$\begin{array}{lllll}12 \mathrm{~A} & 1.640087 & -0.322301 & -0.920691\end{array}$

$\begin{array}{lllll}13 \mathrm{~A} & 1.099165 & -1.259206 & -0.920691\end{array}$

$\begin{array}{lllll}14 \mathrm{~A} & 1.091371 & 0.628102 & -0.920692\end{array}$

$\begin{array}{lllll}15 A & 0.009527 & 0.628102 & -0.920692\end{array}$

$\begin{array}{lllll}16 \mathrm{~A} & 0.001734 & -1.259206 & -0.920692\end{array}$

$\begin{array}{lllll}17 \mathrm{~A} & -0.539188 & -0.322302 & -0.920692\end{array}$

$\begin{array}{llll}18 \mathrm{~B} & -0.462954 & 0.267286 & 2.028388\end{array}$

$\begin{array}{llll}\text { 19B } & 0.550447 & -1.487975 & 2.028389\end{array}$

$\begin{array}{llll}\text { 20B } & 1.563849 & 0.267287 & 2.028389\end{array}$

$\begin{array}{lllll}21 B & -0.936395 & -2.376220 & -0.397579\end{array}$

$\begin{array}{llll}22 \mathrm{~B} & -1.975615 & -0.576238 & -0.397580\end{array}$

$\begin{array}{llll}23 \mathrm{~B} & -0.488772 & 1.999052 & -0.397579\end{array}$

$\begin{array}{llll}24 \mathrm{~B} & 1.589669 & 1.999053 & -0.397578\end{array}$

$\begin{array}{lllll}25 B & 3.076514 & -0.576237 & -0.397576\end{array}$

$\begin{array}{lllll}26 \mathrm{~B} & 2.037294 & -2.376220 & -0.397576\end{array}$

$\begin{array}{llll}27 A & -0.903832 & 0.521828 & -0.312594\end{array}$

$\begin{array}{lllll}28 A & 0.550449 & -1.997061 & -0.312593\end{array}$

$\begin{array}{lllll}29 A & 2.004730 & 0.521828 & -0.312593\end{array}$

$\begin{array}{llll}30 \mathrm{~A} & 1.097338 & -1.684112 & 0.593096\end{array}$

$\begin{array}{llll}31 \mathrm{~A} & -0.359367 & 0.838975 & 0.593094\end{array}$

$\begin{array}{llll}32 \mathrm{~A} & 1.460263 & 0.838975 & 0.593095\end{array}$

$\begin{array}{lllll}33 \mathrm{~A} & 2.007153 & -0.108267 & 0.593095\end{array}$

$\begin{array}{llll}34 \mathrm{~A} & 0.003558 & -1.684112 & 0.593095\end{array}$

$\begin{array}{llll}35 \mathrm{~A} & -0.906256 & -0.108267 & 0.593094\end{array}$

$\begin{array}{llll}36 \mathrm{~B} & -1.308341 & -1.390974 & 1.297244\end{array}$

$\begin{array}{llll}37 \mathrm{~B} & 0.550448 & 1.828544 & 1.297244\end{array}$

$\begin{array}{llll}38 \mathrm{~B} & 2.409237 & -1.390973 & 1.297247\end{array}$

$\begin{array}{llll}39 \mathrm{~B} & 2.332738 & 0.711202 & -1.803622\end{array}$

$\begin{array}{llll}40 \mathrm{~B} & -1.231837 & 0.711202 & -1.803624\end{array}$

$\begin{array}{llll}41 \mathrm{~B} & 0.550451 & -2.375810 & -1.803622\end{array}$

$\begin{array}{lllll}42 \mathrm{~B} & 2.211247 & -1.276663 & -2.008361\end{array}$

$\begin{array}{llll}43 \mathrm{~B} & -1.110344 & -1.276662 & -2.008365\end{array}$

$\begin{array}{llll}44 \mathrm{~B} & 0.550450 & 1.599920 & -2.008364\end{array}$

$\begin{array}{llll}45 \mathrm{~B} & -1.855642 & 1.071354 & 0.773208\end{array}$

$\begin{array}{llll}46 \mathrm{~B} & 0.550448 & -3.096115 & 0.773210\end{array}$

$\begin{array}{llll}47 \mathrm{~B} & 2.956538 & 1.071356 & 0.773210\end{array}$

$E 48 / \varepsilon=-275.324355$

atom $\mathrm{x} / \sigma \quad \mathrm{y} / \sigma$

$\begin{array}{llll}1 \mathrm{~A} & 0.000000 & 0.000000 & 0.000000\end{array}$

$\begin{array}{llll}2 \mathrm{~A} & 1.085833 & 0.000000 & 0.000000\end{array}$

$\begin{array}{llll}3 \mathrm{~A} & 0.542917 & -0.946590 & 0.000000\end{array}$

$\begin{array}{lllll}4 \mathrm{~A} & 0.542917 & -0.311201 & -0.893972\end{array}$

$\begin{array}{llll}5 A & 0.542917 & 0.313530 & 0.898385\end{array}$

$\begin{array}{llll}6 \mathrm{~A} & 0.542917 & 0.951523 & 0.000748\end{array}$

$\begin{array}{lllll}7 \mathrm{~A} & 1.088867 & -0.631998 & 0.896287\end{array}$

$\begin{array}{lllll}8 A & -0.003033 & 0.638690 & -0.891531\end{array}$

$\begin{array}{lllll}9 A & -0.003034 & -0.631998 & 0.896287\end{array}$

$\begin{array}{llll}10 \mathrm{~A} & 1.088867 & 0.638690 & -0.891531\end{array}$

$\begin{array}{llll}11 \mathrm{~A} & -0.551242 & -0.946114 & -0.001054\end{array}$

$\begin{array}{llll}12 \mathrm{~A} & -0.551241 & -0.312039 & -0.893177\end{array}$

$\begin{array}{llll}13 \mathrm{~A} & 1.637076 & -0.946114 & -0.001053\end{array}$

$\begin{array}{lllll}14 \mathrm{~A} & 1.637076 & -0.312039 & -0.893176\end{array}$

$\begin{array}{llll}15 A & -0.004238 & -1.259899 & -0.895471\end{array}$

$\begin{array}{llll}16 \mathrm{~A} & 1.090073 & -1.259899 & -0.895471\end{array}$

$\begin{array}{llll}17 \mathrm{~B} & -0.472406 & 0.275810 & 2.032999\end{array}$

$\begin{array}{llll}18 \mathrm{~B} & 1.558239 & 2.010667 & -0.407890\end{array}$

$\begin{array}{lllll}19 \mathrm{~B} & -0.472404 & 2.010667 & -0.407891\end{array}$

$\begin{array}{llll}20 B & 1.558238 & 0.275810 & 2.032999\end{array}$ $\begin{array}{lrrr}21 \mathrm{~B} & -1.975328 & -0.584153 & -0.415187\end{array}$ $\begin{array}{llll}22 \mathrm{~B} & 3.061163 & -0.584153 & -0.415185\end{array}$

$\begin{array}{llll}23 \mathrm{~A} & -0.364290 & 0.844720 & 0.600383\end{array}$

$\begin{array}{llll}24 \mathrm{~A} & 1.450123 & 0.844720 & 0.600383\end{array}$

$\begin{array}{llll}25 A & -0.911576 & -0.101867 & 0.597862\end{array}$

$\begin{array}{lllll}26 \mathrm{~A} & 1.997409 & 0.531140 & -0.292757\end{array}$

$\begin{array}{llll}27 A & -0.911575 & 0.531140 & -0.292758\end{array}$

$\begin{array}{lllll}28 \mathrm{~A} & 1.997409 & -0.101867 & 0.597863\end{array}$

$\begin{array}{llll}29 B & 0.542917 & 1.836142 & 1.305034\end{array}$

$\begin{array}{lllll}30 \mathrm{~B} & 0.542916 & -1.474942 & 2.037895\end{array}$

$\begin{array}{lllll}31 \mathrm{~B} & 0.542918 & 1.439714 & -2.062934\end{array}$

$\begin{array}{lllll}32 \mathrm{~B} & 2.053528 & -2.343541 & -0.425782\end{array}$

$\begin{array}{lllll}33 \mathrm{~B} & -0.967693 & -1.172578 & -2.073293\end{array}$

$\begin{array}{lllll}34 \mathrm{~B} & 2.053529 & -1.172578 & -2.073292\end{array}$

$\begin{array}{lllll}35 \mathrm{~B} & -0.967693 & -2.343542 & -0.425783\end{array}$

$\begin{array}{llll}36 \mathrm{~B} & -1.326520 & 0.775746 & -1.726477\end{array}$

$\begin{array}{llll}37 \mathrm{~B} & 2.412355 & -1.375472 & 1.300222\end{array}$

$\begin{array}{llll}38 \mathrm{~B} & -1.326521 & -1.375473 & 1.300220\end{array}$

$\begin{array}{llll}39 \mathrm{~B} & 2.412356 & 0.775746 & -1.726475\end{array}$

$\begin{array}{lllll}\text { 40B } & 0.542918 & -2.458591 & -1.747438\end{array}$

$\begin{array}{llll}41 \mathrm{~A} & 1.090535 & -1.692777 & 0.601268\end{array}$

$\begin{array}{llll}42 \mathrm{~A} & 1.090536 & 0.011328 & -1.796355\end{array}$

$\begin{array}{llll}43 \mathrm{~A} & -0.004700 & 0.011328 & -1.796355\end{array}$

$\begin{array}{llll}44 \mathrm{~A} & -0.004701 & -1.692778 & 0.601267\end{array}$

$\begin{array}{lllll}45 \mathrm{~A} & 0.542918 & -0.936357 & -1.799041\end{array}$

$\begin{array}{lllll}46 \mathrm{~A} & 0.542917 & -2.006875 & -0.292855\end{array}$

$\begin{array}{llll}47 \mathrm{~B} & -1.864210 & 1.080244 & 0.767780\end{array}$

$\begin{array}{llll}48 \mathrm{~B} & 2.950043 & 1.080244 & 0.767782\end{array}$

\section{$E 49 / \varepsilon=-283.859545$}

atom $\mathrm{x} / \sigma \quad \mathrm{y} / \sigma$

$\begin{array}{llll}1 \mathrm{~A} & 0.000000 & 0.000000 & 0.000000\end{array}$

$\begin{array}{llll}2 \mathrm{~A} & 1.087342 & 0.000000 & 0.000000\end{array}$

$\begin{array}{lllll}3 A & 0.543671 & -0.941666 & 0.000000\end{array}$

$\begin{array}{lllll}4 \mathrm{~A} & 0.543671 & -0.313888 & -0.894504\end{array}$

$\begin{array}{llll}5 A & 1.088825 & -0.628633 & 0.898124\end{array}$

$\begin{array}{llll}6 \mathrm{~A} & -0.001483 & -0.628633 & 0.898124\end{array}$

$\begin{array}{lllll}7 \mathrm{~A} & 0.543671 & 0.315601 & 0.898124\end{array}$

$\begin{array}{lllll}8 \mathrm{~A} & 0.543671 & 0.950169 & -0.000832\end{array}$

$\begin{array}{llll}9 \mathrm{~A} & -0.551035 & -0.945917 & -0.000832\end{array}$

$\begin{array}{lllll}10 A & 1.638377 & -0.945917 & -0.000832\end{array}$

$\begin{array}{llll}11 \mathrm{~A} & 1.092015 & -1.261502 & -0.893173\end{array}$

$\begin{array}{lllll}12 A & -0.551158 & -0.314961 & -0.893173\end{array}$

$\begin{array}{llll}13 A & -0.004672 & -1.261502 & -0.893173\end{array}$

$\begin{array}{lllll}14 \mathrm{~A} & 1.638501 & -0.314961 & -0.893172\end{array}$

$\begin{array}{llll}15 A & 1.090157 & 0.634798 & -0.893172\end{array}$

$\begin{array}{llll}16 \mathrm{~A} & -0.002815 & 0.634798 & -0.893172\end{array}$

$\begin{array}{llll}17 \mathrm{~B} & -0.471061 & 0.271967 & 2.034171\end{array}$

$\begin{array}{llll}18 B & 0.543671 & -1.485599 & 2.034172\end{array}$

$\begin{array}{llll}19 B & 1.558402 & 0.271967 & 2.034172\end{array}$

$\begin{array}{llll}20 B & -0.957295 & -2.354295 & -0.411290\end{array}$

$\begin{array}{llll}21 \mathrm{~B} & -0.472890 & 2.006190 & -0.411290\end{array}$

$\begin{array}{llll}22 B & 2.044637 & -2.354295 & -0.411290\end{array}$

$\begin{array}{llll}23 B & 1.560232 & 2.006190 & -0.411290\end{array}$

$\begin{array}{llll}24 \mathrm{~B} & -1.973856 & -0.593560 & -0.411290\end{array}$

$\begin{array}{llll}25 B & 3.061198 & -0.593559 & -0.411290\end{array}$

$\begin{array}{llll}26 \mathrm{~A} & 1.452380 & 0.842722 & 0.600101\end{array}$

$\begin{array}{llll}27 A & -0.365038 & 0.842722 & 0.600101\end{array}$

$\begin{array}{llll}28 \mathrm{~A} & 1.090971 & -1.679159 & 0.600101\end{array}$

$\begin{array}{llll}29 A & -0.003629 & -1.679159 & 0.600101\end{array}$

$\begin{array}{llll}30 \mathrm{~A} & 1.999680 & -0.105229 & 0.600101\end{array}$

$\begin{array}{llll}31 \mathrm{~A} & -0.912338 & -0.105229 & 0.600101\end{array}$

$\begin{array}{lllll}32 \mathrm{~A} & -0.912507 & 0.526837 & -0.292581\end{array}$

$\begin{array}{lllll}33 \mathrm{~A} & 1.999849 & 0.526837 & -0.292581\end{array}$

$\begin{array}{lllll}34 \mathrm{~A} & 0.543671 & -1.995338 & -0.292582\end{array}$

$\begin{array}{llll}35 \mathrm{~B} & 0.543671 & 1.834412 & 1.303080\end{array}$

$\begin{array}{llll}36 \mathrm{~B} & -1.316812 & -1.388039 & 1.303080\end{array}$

$\begin{array}{llll}37 \mathrm{~B} & 2.404154 & -1.388038 & 1.303080\end{array}$

$\begin{array}{llll}38 \mathrm{~B} & 0.543671 & 1.430877 & -2.066478\end{array}$

$\begin{array}{lllll}39 \mathrm{~B} & -0.967339 & -1.186270 & -2.066478\end{array}$

$\begin{array}{lllll}40 \mathrm{~B} & 2.054682 & -1.186270 & -2.066479\end{array}$

$\begin{array}{lllll}41 \mathrm{~B} & 0.543671 & -2.473726 & -1.727603\end{array}$

$\begin{array}{llll}42 \mathrm{~B} & 2.414146 & 0.766031 & -1.727602\end{array}$

$\begin{array}{llll}\text { 43B } & -1.326804 & 0.766031 & -1.727602\end{array}$ 
44A $\quad 0.543671 \quad-0.946457 \quad-1.797280$ $\begin{array}{llll}45 \mathrm{~A} & -0.004150 & 0.002397 & -1.797280\end{array}$ $\begin{array}{llll}46 \mathrm{~A} & 1.091492 & 0.002397 & -1.797280\end{array}$ $\begin{array}{llll}47 \mathrm{~B} & 0.543671 & -3.095039 & 0.767489\end{array}$ $\begin{array}{llll}\text { 48B } & -1.864877 & 1.076687 & 0.767489\end{array}$ $\begin{array}{llll}\text { 49B } & 2.952218 & 1.076688 & 0.767490\end{array}$

$E 50 / \varepsilon=-292.387819$

$\begin{array}{llll}1 \mathrm{~A} & 0.000000 & 0.000000 & 0.000000\end{array}$

$\begin{array}{llll}2 \mathrm{~A} & 1.088858 & 0.000000 & 0.000000\end{array}$

$\begin{array}{llll}3 A & 0.544429 & -0.942979 & 0.000000\end{array}$

$\begin{array}{llll}4 \mathrm{~A} & 0.544429 & -0.314326 & 0.889049\end{array}$

$\begin{array}{lllll}5 \mathrm{~A} & 1.090108 & -0.629374 & -0.896595\end{array}$

$\begin{array}{llll}6 \mathrm{~A} & 1.090107 & 0.635527 & 0.892244\end{array}$

$\begin{array}{llll}7 A & -0.001250 & -0.629374 & -0.896595\end{array}$

$\begin{array}{llll}8 A & -0.005329 & -1.261824 & 0.892245\end{array}$

$\begin{array}{llll}9 \mathrm{~A} & 0.544429 & 0.315769 & -0.896595\end{array}$

$\begin{array}{llll}10 A & 1.639865 & -0.946777 & 0.001156\end{array}$

$\begin{array}{llll}11 \mathrm{~A} & 1.094186 & -1.261824 & 0.892245\end{array}$

$\begin{array}{llll}12 \mathrm{~A} & -0.551007 & -0.316681 & 0.892244\end{array}$

$\begin{array}{llll}13 \mathrm{~A} & 0.544429 & 0.950574 & 0.001154\end{array}$

$\begin{array}{llll}14 \mathrm{~A} & 1.639865 & -0.316681 & 0.892245\end{array}$

$\begin{array}{llll}15 A & -0.001250 & 0.635527 & 0.892244\end{array}$

$\begin{array}{llll}16 \mathrm{~A} & -0.551007 & -0.946777 & 0.001155\end{array}$

$\begin{array}{llll}17 \mathrm{~B} & 0.544429 & -1.487370 & -2.030477\end{array}$

$\begin{array}{lllll}18 \mathrm{~B} & 1.560314 & 0.272194 & -2.030477\end{array}$

$\begin{array}{llll}19 B & 0.544429 & 1.418565 & 2.079129\end{array}$

$\begin{array}{llll}20 \mathrm{~B} & -0.471456 & 0.272194 & -2.030478\end{array}$

$\begin{array}{llll}21 B & -0.956298 & -1.180769 & 2.079131\end{array}$

$\begin{array}{llll}22 B & -0.471456 & 2.005085 & 0.420196\end{array}$

$\begin{array}{llll}23 \mathrm{~B} & 1.560313 & 2.005085 & 0.420197\end{array}$

$\begin{array}{llll}24 \mathrm{~B} & 2.045154 & -1.180770 & 2.079132\end{array}$

$\begin{array}{llll}25 \mathrm{~B} & -0.956297 & -2.353813 & 0.420199\end{array}$

$\begin{array}{llll}26 \mathrm{~B} & 3.061040 & -0.594249 & 0.420199\end{array}$

$\begin{array}{llll}27 \mathrm{~B} & -1.972182 & -0.594249 & 0.420197\end{array}$

$\begin{array}{llll}28 B & 2.045154 & -2.353814 & 0.420200\end{array}$

$\begin{array}{llll}29 \mathrm{~A} & -0.913188 & -0.104956 & -0.596319\end{array}$

$\begin{array}{lllll}30 \mathrm{~A} & 1.091917 & -1.681346 & -0.596318\end{array}$

$\begin{array}{lllll}31 \mathrm{~A} & -0.365700 & 0.843321 & -0.596319\end{array}$

$\begin{array}{llll}32 \mathrm{~A} & 2.002047 & -0.104956 & -0.596318\end{array}$

$\begin{array}{llll}33 \mathrm{~A} & -0.913188 & 0.527230 & 0.297725\end{array}$

$\begin{array}{llll}34 \mathrm{~A} & 2.002046 & 0.527230 & 0.297726\end{array}$

$\begin{array}{llll}35 \mathrm{~A} & 0.544429 & -1.997438 & 0.297727\end{array}$

$\begin{array}{llll}36 \mathrm{~A} & 0.544429 & -0.946510 & 1.783961\end{array}$

$\begin{array}{llll}37 \mathrm{~A} & 1.454558 & 0.843322 & -0.596319\end{array}$

$\begin{array}{llll}38 \mathrm{~A} & 1.091917 & 0.001767 & 1.783960\end{array}$

$\begin{array}{llll}39 A & -0.003059 & -1.681346 & -0.596318\end{array}$

$\begin{array}{llll}40 A & -0.003060 & 0.001768 & 1.783960\end{array}$

41B $\quad 0.544429 \quad 1.835215 \quad-1.297695$

$\begin{array}{llll}42 \mathrm{~B} & -1.317129 & 0.760446 & 1.742217\end{array}$

$\begin{array}{llll}\text { 43B } & -1.317129 & -1.389098 & -1.297693\end{array}$

$\begin{array}{llll}44 \mathrm{~B} & 2.405987 & 0.760446 & 1.742218\end{array}$

$\begin{array}{llll}45 \mathrm{~B} & 2.405987 & -1.389098 & -1.297692\end{array}$

$\begin{array}{llll}46 \mathrm{~B} & 0.544428 & -2.463867 & 1.742219\end{array}$

$\begin{array}{llll}47 \mathrm{~B} & 0.544429 & -0.314323 & 3.173848\end{array}$

$\begin{array}{lllll}48 \mathrm{~B} & 2.954388 & 1.077066 & -0.761600\end{array}$

$\begin{array}{llll}49 B & -1.865530 & 1.077066 & -0.761601\end{array}$

$\begin{array}{lllll}50 B & 0.544429 & -3.097109 & -0.761598\end{array}$

$s=1.9$

$E 5 / \varepsilon=-9.251172$

$\begin{array}{cccc}\text { atom } & \mathrm{x} / \sigma & y / \sigma & z / \sigma \\ 1 \mathrm{~A} & 0.000000 & 0.000000 & 0.000000\end{array}$

$\begin{array}{llll}2 \mathrm{~A} & 1.123881 & 0.000000 & 0.000000\end{array}$

3A $\quad 0.561940 \quad-0.973310 \quad 0.000000$

$\begin{array}{llll}4 \mathrm{~B} & 0.561940 & -0.324440 & -1.485951\end{array}$

$\begin{array}{llll}5 B & 0.561940 & -0.324440 & 1.485951\end{array}$

$E 6 / \varepsilon=-13.439465$

atom $\mathrm{x} / \sigma \quad \mathrm{y} / \sigma \quad \mathrm{z} / \sigma$ $\begin{array}{llll}1 \mathrm{~A} & 0.000000 & 0.000000 & 0.000000\end{array}$

$\begin{array}{llll}2 \mathrm{~A} & 1.131135 & 0.000000 & 0.000000\end{array}$

$\begin{array}{llll}3 \mathrm{~B} & 0.565569 & -1.514218 & 0.000000\end{array}$

$\begin{array}{llll}4 \mathrm{~B} & 0.565569 & 0.000000 & 1.514218\end{array}$

$\begin{array}{llll}5 \mathrm{~B} & 0.565569 & 1.514218 & 0.000000\end{array}$

$\begin{array}{lllll}6 B & 0.565569 & 0.000000 & -1.514218\end{array}$

$E 7 / \varepsilon=-17.672694$

$\begin{array}{ccc}\text { atom } & x / \sigma & y / \sigma \\ 1 \mathrm{~A} & 0.000000 & 0.00000\end{array}$

$\begin{array}{llll}1 \mathrm{~A} & 0.000000 & 0.000000 & 0.000000\end{array}$

$\begin{array}{llll}2 B & 1.562405 & 0.000000 & 0.000000\end{array}$

$\begin{array}{llll}3 B & 0.000000 & -1.562405 & 0.000000\end{array}$

$\begin{array}{llll}4 \mathrm{~B} & 0.000000 & 0.000000 & 1.562405\end{array}$

$\begin{array}{llll}5 B & 0.000000 & 1.562405 & 0.000000\end{array}$

$\begin{array}{llll}6 \mathrm{~B} & 0.000000 & 0.000000 & -1.562405\end{array}$

$\begin{array}{llll}7 \mathrm{~B} & -1.562405 & 0.000000 & 0.000000\end{array}$

\section{$E 8 / \varepsilon=-21.634684$}

\begin{tabular}{cccc} 
atom & $x / \sigma$ & $y / \sigma$ & \multicolumn{1}{c}{$z / \sigma$} \\
1A & 0.000000 & 0.000000 & 0.000000 \\
2B & 1.617923 & 0.000000 & 0.000000 \\
3B & -1.210707 & -1.073251 & 0.000000 \\
4B & 0.186920 & -0.542957 & -1.512593 \\
5B & 0.220297 & -0.530298 & 1.512592 \\
6A & 0.399619 & -1.053226 & -0.000001 \\
7B & 0.248859 & 1.420621 & -0.745382 \\
8B & -1.128594 & 0.897978 & 0.745388
\end{tabular}

$E 9 / \varepsilon=-26.868864$

atom $\mathrm{x} / \sigma \quad \mathrm{y} / \sigma \quad \mathrm{z} / \sigma$

$\begin{array}{llll}1 \mathrm{~A} & 0.000000 & 0.000000 & 0.000000\end{array}$

$\begin{array}{llll}2 \mathrm{~B} & 1.621957 & 0.000000 & 0.000000\end{array}$

$\begin{array}{llll}3 \mathrm{~B} & 0.172073 & -1.612804 & 0.000000\end{array}$

$\begin{array}{llll}\text { 4B } & 0.216387 & 1.226774 & 1.047547\end{array}$

$\begin{array}{llll}5 B & -1.196896 & -0.345312 & -1.047545\end{array}$

$\begin{array}{llll}6 B & 0.216389 & 1.226775 & -1.047547\end{array}$

$\begin{array}{llll}7 \mathrm{~B} & -1.196894 & -0.345316 & 1.047549\end{array}$

$\begin{array}{lllll}8 \mathrm{~A} & 0.408694 & -0.367410 & -0.983815\end{array}$

$\begin{array}{llll}9 A & 0.408695 & -0.367411 & 0.983815\end{array}$

$E 10 / \varepsilon=-32.132430$

$\begin{array}{llll}\text { atom } & \mathrm{x} / \sigma & \mathrm{y} / \sigma & \mathrm{z} / \sigma\end{array}$

$\begin{array}{cccc}1 \mathrm{~A} & 0.000000 & 0.000000 & 0.000000\end{array}$

$\begin{array}{llll}2 B & 1.635017 & 0.000000 & 0.000000\end{array}$

$\begin{array}{llll}3 B & 0.209709 & -1.621513 & 0.000000\end{array}$

$\begin{array}{llll}4 B & -1.173836 & -0.405379 & -1.063514\end{array}$

$\begin{array}{llll}5 B & 0.251472 & 1.216135 & -1.063513\end{array}$

$\begin{array}{llll}6 \mathrm{~B} & 0.251472 & 1.216135 & 1.063513\end{array}$

$\begin{array}{llll}7 \mathrm{~B} & -1.173836 & -0.405379 & 1.063514\end{array}$

$\begin{array}{lllll}8 A & 0.423561 & -0.372309 & -0.976758\end{array}$

$\begin{array}{llll}9 \mathrm{~A} & 0.423561 & -0.372309 & 0.976758\end{array}$

$\begin{array}{llll}10 A & -0.847122 & 0.744619 & 0.000000\end{array}$

\section{$E 11 / \varepsilon=-36.549295$}

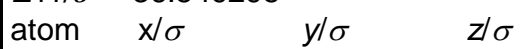

$\begin{array}{llll}1 \mathrm{~A} & 0.000000 & 0.000000 & 0.000000\end{array}$

$\begin{array}{llll}2 \mathrm{~A} & 1.120471 & 0.000000 & 0.000000\end{array}$

$\begin{array}{llll}3 \mathrm{~A} & 0.027058 & -1.120144 & 0.000000\end{array}$

$\begin{array}{lllll}4 \mathrm{~A} & 0.573765 & -0.560072 & -0.782668\end{array}$

$\begin{array}{lllll}5 \mathrm{~A} & 0.573764 & -0.560072 & 0.782668\end{array}$

$\begin{array}{llll}6 \mathrm{~B} & -0.994581 & -0.580585 & 1.110193\end{array}$

$\begin{array}{llll}7 B & -0.994581 & -0.580584 & -1.110194\end{array}$

$\begin{array}{llll}8 \mathrm{~B} & 0.556396 & 1.008312 & -1.110192\end{array}$

$\begin{array}{llll}9 \mathrm{~B} & 0.556397 & 1.008311 & 1.110194\end{array}$

$\begin{array}{llll}10 B & -1.207030 & 1.178224 & 0.000001\end{array}$

$\begin{array}{llll}11 \mathrm{~B} & 1.576504 & -1.538883 & 0.000000\end{array}$

$E 12 / \varepsilon=-41.889065$

$\begin{array}{llll}\text { atom } & \mathrm{x} / \sigma & \mathrm{y} / \sigma & \mathrm{z} / \sigma\end{array}$

$\begin{array}{llll}1 \mathrm{~A} & 0.000000 & 0.000000 & 0.000000\end{array}$

$\begin{array}{llll}2 A & 1.082056 & 0.000000 & 0.000000\end{array}$

$\begin{array}{llll}\text { 3B } & 0.541029 & -1.523346 & 0.000000\end{array}$

$\begin{array}{llrr}4 \mathrm{~A} & 0.541027 & 0.607566 & -0.759451\end{array}$ $\begin{array}{llll}5 B & 0.541029 & -0.087182 & 1.521290\end{array}$

$\begin{array}{lllll}6 B & 1.597495 & -0.418365 & -1.446637\end{array}$

$\begin{array}{llll}7 \mathrm{~B} & -0.515441 & -0.418367 & -1.446636\end{array}$

$\begin{array}{lllll}8 \mathrm{~A} & 0.541027 & 0.923992 & 0.310233\end{array}$

$\begin{array}{lllll}9 \mathrm{~B} & 1.954644 & 1.304390 & -0.287458\end{array}$

$\begin{array}{llll}10 \mathrm{~B} & -0.872591 & 1.304388 & -0.287459\end{array}$

$\begin{array}{llll}11 B & -1.326162 & -0.627849 & 0.559569\end{array}$

$\begin{array}{llll}12 B & 2.408220 & -0.627847 & 0.559566\end{array}$

$E 13 / \varepsilon=-47.448527$

$\begin{array}{llll}\text { atom } & \mathrm{x} / \sigma & \mathrm{y} / \sigma & \mathrm{z} / \sigma\end{array}$

$\begin{array}{llll}1 \mathrm{~A} & 0.000000 & 0.000000 & 0.000000\end{array}$

$\begin{array}{llll}2 \mathrm{~A} & 1.099049 & 0.000000 & 0.000000\end{array}$

$\begin{array}{lllll}3 \mathrm{~A} & 0.549524 & -0.951804 & 0.000000\end{array}$

$\begin{array}{lllll}4 \mathrm{~A} & 1.102304 & -0.636417 & -0.914313\end{array}$

$\begin{array}{lllll}5 A & -0.003258 & -0.636417 & -0.914312\end{array}$

$\begin{array}{lllll}6 \mathrm{~A} & 0.549523 & 0.321028 & -0.914313\end{array}$

$\begin{array}{llll}7 \mathrm{~B} & 0.549525 & -0.317267 & 1.461358\end{array}$

$\begin{array}{llll}8 \mathrm{~B} & -1.005170 & -1.214872 & 0.202459\end{array}$

$\begin{array}{llll}9 \mathrm{~B} & 0.549524 & 1.477939 & 0.202454\end{array}$

10B $\quad 2.104219 \quad-1.214872 \quad 0.202453$

$\begin{array}{llll}11 \mathrm{~B} & 0.549522 & -2.146348 & -1.024026\end{array}$

$\begin{array}{lllll}12 \mathrm{~B} & -1.034507 & 0.597270 & -1.024026\end{array}$

$\begin{array}{llll}13 B & 2.133552 & 0.597271 & -1.024030\end{array}$

$E 14 / \varepsilon=-54.331902$

atom $\quad \mathrm{x} / \sigma \quad y / \sigma \quad z / \sigma$

$\begin{array}{llll}1 \mathrm{~A} & 0.000000 & 0.000000 & 0.000000\end{array}$

$\begin{array}{llll}2 \mathrm{~A} & 1.564018 & 0.000000 & 0.000000\end{array}$

$\begin{array}{llll}3 \mathrm{~A} & 0.782009 & -0.782008 & 0.000000\end{array}$

$\begin{array}{llll}4 \mathrm{~A} & 0.782009 & 0.000000 & 0.782008\end{array}$

$\begin{array}{llll}5 A & 0.782009 & 0.782008 & 0.000000\end{array}$

$\begin{array}{lllll}6 \mathrm{~A} & 0.782009 & 0.000000 & -0.782008\end{array}$

$\begin{array}{llll}7 \mathrm{~B} & -0.319972 & 1.101982 & 1.101982\end{array}$

$\begin{array}{lllll}8 B & -0.319972 & -1.101982 & -1.101982\end{array}$

$\begin{array}{lllll}9 B & 1.883991 & -1.101982 & -1.101982\end{array}$

10B $\quad-0.319972 \quad-1.101982 \quad 1.101982$

$\begin{array}{llll}11 \mathrm{~B} & 1.883991 & 1.101982 & 1.101982\end{array}$

12B $\quad-0.319972 \quad 1.101982-1.101982$

$\begin{array}{llll}13 \mathrm{~B} & 1.883991 & 1.101982 & -1.101982\end{array}$

14B $\quad 1.883991 \quad-1.101982 \quad 1.101982$

$E 15 / \varepsilon=-59.981696$

atom $\mathrm{x} / \sigma \quad \mathrm{y} / \sigma$

$\begin{array}{llll}1 \mathrm{~A} & 0.000000 & 0.000000 & 0.000000\end{array}$

$\begin{array}{llll}2 \mathrm{~A} & 1.111168 & 0.000000 & 0.000000\end{array}$

$\begin{array}{llll}3 A & 0.018393 & -1.111016 & 0.000000\end{array}$

$\begin{array}{lllll}4 \mathrm{~A} & 0.564781 & -0.555508 & -0.779184\end{array}$

$\begin{array}{llll}5 \mathrm{~A} & 0.564780 & -0.555508 & 0.779184\end{array}$

$\begin{array}{llll}6 \mathrm{~A} & 1.123499 & -1.105054 & 0.000000\end{array}$

$\begin{array}{llll}7 \mathrm{~B} & -0.980898 & -0.608006 & 1.121302\end{array}$

$\begin{array}{llll}8 \mathrm{~B} & -0.980897 & -0.608006 & -1.121303\end{array}$

$\begin{array}{llll}9 \mathrm{~B} & 0.591683 & 0.990827 & 1.121303\end{array}$

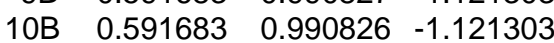

$\begin{array}{llll}11 \mathrm{~B} & 0.586711 & -2.117647 & 1.098326\end{array}$

$\begin{array}{llll}12 B & 2.127067 & -0.551577 & 1.098327\end{array}$

$\begin{array}{lllll}13 \mathrm{~B} & 0.586712 & -2.117647 & -1.098325\end{array}$

$\begin{array}{lllll}14 \mathrm{~B} & 2.127069 & -0.551578 & -1.098324\end{array}$

$\begin{array}{llll}15 B & -1.171161 & 1.151929 & 0.000000\end{array}$

$E 16 / \varepsilon=-65.711712$

atom $\mathrm{x} / \sigma \quad \mathrm{y} / \sigma$

$\begin{array}{llll}1 \mathrm{~A} & 0.000000 & 0.000000 & 0.000000\end{array}$

$\begin{array}{llll}2 \mathrm{~A} & 1.114201 & 0.000000 & 0.000000\end{array}$

$\begin{array}{llll}3 A & 0.557100 & -0.957730 & 0.000000\end{array}$

$\begin{array}{lllll}4 \mathrm{~A} & 0.557101 & 0.293209 & 0.911743\end{array}$

$\begin{array}{lllll}5 A & 1.110589 & -0.655981 & 0.900025\end{array}$

$\begin{array}{llll}6 \mathrm{~A} & 0.003614 & -0.655980 & 0.900026\end{array}$

$\begin{array}{lllll}7 \mathrm{~B} & 0.557100 & 1.503697 & -0.090051\end{array}$

$\begin{array}{lllll}8 \mathrm{~B} & 0.557100 & -0.374631 & -1.459063\end{array}$

$\begin{array}{llll}9 B & -0.992154 & 0.546102 & 1.148509\end{array}$

10B $\quad-0.992154 \quad-1.260551 \quad-0.168262$ 
$\begin{array}{llll}11 B & 2.106357 & 0.546101 & 1.148508\end{array}$

$\begin{array}{llll}12 B & 2.106355 & -1.260551 & -0.168265\end{array}$

$\begin{array}{llll}13 \mathrm{~B} & 0.557102 & -0.369864 & 2.364437\end{array}$

$\begin{array}{llll}\text { 14B } & 0.557102 & -2.137670 & 1.075980\end{array}$

$\begin{array}{llll}15 B & 2.282020 & 0.681973 & -0.935689\end{array}$

$\begin{array}{llll}16 \mathrm{~B} & -1.167820 & 0.681972 & -0.935688\end{array}$

\section{$E 17 / \varepsilon=-71.481302$}

\begin{tabular}{rrrr} 
atom & $x / \sigma$ & $y / \sigma$ & \multicolumn{1}{c}{$z / \sigma$} \\
1A & 0.000000 & 0.000000 & 0.000000 \\
2A & 1.114445 & 0.000000 & 0.000000 \\
3A & 0.557222 & -0.965138 & 0.000000 \\
4B & 0.557223 & -0.321713 & -1.457634 \\
5A & 1.106448 & -0.638808 & 0.904592 \\
6A & 0.007994 & -0.638808 & 0.904591 \\
7A & 0.557221 & 0.312481 & 0.904591 \\
8B & 0.557222 & 1.502800 & -0.118718 \\
9B & 2.137297 & -1.233969 & -0.118713 \\
10B & -1.022852 & -1.233968 & -0.118718 \\
11B & -0.986589 & 0.569607 & 1.148560 \\
12B & 0.557220 & -2.104349 & 1.148564 \\
13B & 2.101030 & 0.569608 & 1.148564 \\
14B & 0.557219 & -0.321711 & 2.368524 \\
15B & 2.300517 & 0.684778 & -0.930082 \\
16B & 0.557223 & -2.334696 & -0.930082 \\
17B & -1.186071 & 0.684779 & -0.930087
\end{tabular}

$E 18 / \varepsilon=-77.246409$

\begin{tabular}{crrr} 
atom & \multicolumn{1}{c}{$\mathrm{x} / \sigma$} & \multicolumn{1}{c}{$y / \sigma$} & \multicolumn{1}{c}{$z / \sigma$} \\
1A & 0.000000 & 0.000000 & 0.000000 \\
2A & 1.113254 & 0.000000 & 0.000000 \\
3A & 0.556628 & -0.965603 & 0.000000 \\
4A & 0.556626 & 0.334386 & -0.905855 \\
5B & 0.556628 & -0.332165 & 1.458397 \\
$6 \mathrm{~B}$ & 0.556628 & 1.483186 & 0.193427 \\
7A & 1.101903 & -0.629779 & -0.903794 \\
8A & 0.011349 & -0.629779 & -0.903793 \\
9B & 2.131310 & -1.245347 & 0.113402 \\
$10 \mathrm{~B}$ & 2.131307 & 0.537649 & -1.129020 \\
$11 \mathrm{~B}$ & -1.018053 & -1.245347 & 0.113408 \\
12B & -1.018055 & 0.537649 & -1.129017 \\
13B & 0.556626 & -2.101455 & -1.146192 \\
14B & 0.556625 & -0.347541 & -2.368350 \\
15B & -1.200775 & 0.658978 & 0.945696 \\
16B & 2.314031 & 0.658977 & 0.945693 \\
17B & 0.556630 & -2.343790 & 0.930669 \\
18B & 0.556625 & 1.684733 & -1.876479
\end{tabular}

$E 19 / \varepsilon=-83.597114$

$\begin{array}{rrrr}\text { atom } & x / \sigma & y / \sigma & z / \sigma \\ \text { 1A } & 0.000000 & 0.000000 & 0.000000 \\ \text { 2A } & 1.093952 & 0.000000 & 0.000000 \\ \text { 3A } & 0.546976 & -0.947390 & 0.000000 \\ \text { 4B } & -1.095636 & -1.264158 & -0.008058 \\ \text { 5B } & 2.189588 & -1.264160 & -0.008057 \\ \text { 6B } & 0.546977 & 1.580929 & -0.008056 \\ \text { 7A } & 0.000156 & -0.631502 & -0.910475 \\ \text { 8A } & 1.093797 & -0.631503 & -0.910475 \\ \text { 9A } & 0.546977 & 0.315618 & -0.910474 \\ \text { 10B } & -1.008130 & 0.582047 & -1.100913 \\ \text { 11B } & 2.102085 & 0.582045 & -1.100912 \\ \text { 12B } & 0.546976 & -2.111479 & -1.100915 \\ \text { 13A } & 0.546976 & 0.316233 & 0.920837 \\ \text { 14A } & 1.094330 & -0.631813 & 0.920837 \\ \text { 15A } & -0.000379 & -0.631813 & 0.920837 \\ \text { 16B } & 2.127316 & 0.596612 & 1.036929 \\ \text { 17B } & -1.033364 & 0.596613 & 1.036927 \\ \text { 18B } & 0.546976 & -2.140618 & 1.036926 \\ \text { 19B } & 0.546978 & -0.315794 & -2.368135\end{array}$

$E 20 / \varepsilon=-90.753192$

atom $\mathrm{x} / \sigma \quad \mathrm{y} / \sigma$ $z / \sigma$
2A

$\begin{array}{llll}3 \mathrm{~A} & 0.548165 & -0.949450 & 0.000000\end{array}$

$\begin{array}{lllll}4 \mathrm{~B} & 0.548164 & 1.582194 & -0.000002\end{array}$

$\begin{array}{llll}5 \mathrm{~B} & 2.192468 & -1.265822 & -0.000002\end{array}$

$\begin{array}{llll}6 \mathrm{~B} & -1.096138 & -1.265823 & 0.000003\end{array}$

$\begin{array}{lllll}7 A & 1.096016 & -0.632787 & -0.907570\end{array}$

$\begin{array}{lllll}8 \mathrm{~A} & 0.000314 & -0.632786 & 0.907570\end{array}$

$\begin{array}{lllll}9 \mathrm{~A} & 1.096019 & -0.632786 & 0.907569\end{array}$

$\begin{array}{llll}10 A & 0.548163 & 0.316122 & -0.907570\end{array}$

$\begin{array}{llll}11 \mathrm{~A} & 0.548166 & 0.316123 & 0.907570\end{array}$

$\begin{array}{llll}12 \mathrm{~A} & 0.000311 & -0.632787 & -0.907569\end{array}$

$\begin{array}{llll}13 \mathrm{~B} & -1.012034 & 0.584298 & 1.079087\end{array}$

$\begin{array}{llll}14 \mathrm{~B} & -1.012037 & 0.584297 & -1.079085\end{array}$

$\begin{array}{llll}15 B & 0.548167 & -2.118046 & 1.079087\end{array}$

$\begin{array}{lllll}16 \mathrm{~B} & 0.548164 & -2.118047 & -1.079085\end{array}$

$\begin{array}{llll}17 \mathrm{~B} & 2.108362 & 0.584298 & -1.079089\end{array}$

$\begin{array}{llll}18 B & 2.108366 & 0.584299 & 1.079084\end{array}$

$\begin{array}{llll}19 B & 0.548168 & -0.316483 & 2.362757\end{array}$

$\begin{array}{llll}20 \mathrm{~B} & 0.548161 & -0.316486 & -2.362757\end{array}$

$E 21 / \varepsilon=-96.404037$

3A

$\begin{array}{llll}1 \mathrm{~A} & 0.000000 & 0.000000 & 0.000000\end{array}$

$\begin{array}{llll}2 \mathrm{~A} & 1.089331 & 0.000000 & 0.000000\end{array}$

$\begin{array}{llll}3 A & 0.544665 & -0.953798 & 0.000000\end{array}$

$\begin{array}{llll}4 \mathrm{~B} & 0.544668 & 1.568893 & -0.046102\end{array}$

$\begin{array}{lllll}5 A & 0.544666 & 0.324401 & 0.925481\end{array}$

$\begin{array}{llll}6 \mathrm{~B} & 2.188512 & -1.276941 & 0.004810\end{array}$

$\begin{array}{llll}7 \mathrm{~B} & -1.099185 & -1.276937 & 0.004810\end{array}$

$\begin{array}{llll}8 \mathrm{~B} & -1.027177 & 0.571777 & 1.065438\end{array}$

$\begin{array}{llll}\text { 9B } & 2.116510 & 0.571772 & 1.065437\end{array}$

$\begin{array}{llll}10 A & 1.091105 & -0.630809 & 0.903413\end{array}$

$\begin{array}{llll}11 \mathrm{~A} & -0.001776 & -0.630808 & 0.903413\end{array}$

$\begin{array}{lllll}12 \mathrm{~A} & -0.004605 & -0.647401 & -0.904668\end{array}$

$\begin{array}{lllll}13 \mathrm{~A} & 1.093934 & -0.647402 & -0.904668\end{array}$

$\begin{array}{lllll}14 \mathrm{~A} & 0.544665 & 0.295040 & -0.920269\end{array}$

$\begin{array}{llll}15 B & 0.544663 & -2.130183 & 1.062884\end{array}$

$\begin{array}{llll}16 \mathrm{~B} & -1.018340 & 0.562511 & -1.088516\end{array}$

$\begin{array}{llll}17 \mathrm{~B} & 2.107672 & 0.562507 & -1.088517\end{array}$

$\begin{array}{lllll}18 B & 0.544663 & -2.130367 & -1.078414\end{array}$

$\begin{array}{llll}19 \mathrm{~B} & 0.544662 & -0.434743 & 2.368615\end{array}$

$\begin{array}{llll}20 \mathrm{~B} & 0.544663 & -0.349900 & -2.365756\end{array}$

$\begin{array}{llll}21 \mathrm{~B} & 0.544669 & 1.600468 & 2.016277\end{array}$

$E 22 / \varepsilon=-102.128446$

$\begin{array}{lll}\text { atom } & \mathrm{x} / \sigma & \mathrm{y} / \sigma\end{array}$

$\begin{array}{lll}1 \mathrm{~A} & 0.000000 & 0.000000\end{array}$

$z / \sigma$

0.000000

$4 \mathrm{~B}-1.100219-1.254103$

$\begin{array}{lll}5 \mathrm{~B} & 0.565861 & 1.569413\end{array}$

0.000000

1.

$\begin{array}{lllll}6 \mathrm{~A} & 0.539929 & 0.319280 & -0.930696\end{array}$

$\begin{array}{lllll}7 \mathrm{~A} & -0.018443 & -0.626996 & -0.930696\end{array}$

$\begin{array}{lllll}8 \mathrm{~B} & -1.033752 & 0.609988 & -1.021570\end{array}$

$\begin{array}{lllll}9 \mathrm{~B} & 2.187366 & -1.290705 & -0.014576\end{array}$

$\begin{array}{llll}10 B & 2.119359 & 0.566848 & -1.059383\end{array}$

$\begin{array}{llll}11 B & 0.528461 & -2.129256 & -1.059382\end{array}$

$\begin{array}{lllll}12 \mathrm{~A} & 1.079868 & -0.637201 & -0.886131\end{array}$

$\begin{array}{llll}13 \mathrm{~A} & 1.101743 & -0.650109 & 0.895463\end{array}$

$\begin{array}{llll}14 A & 0.008328 & -0.635507 & 0.920514\end{array}$

$\begin{array}{llll}15 A & 0.560323 & 0.299962 & 0.920513\end{array}$

$\begin{array}{llll}16 \mathrm{~B} & 0.730889 & -0.431279 & -2.379671\end{array}$

$\begin{array}{llll}17 \mathrm{~B} & -0.990548 & 0.584496 & 1.115693\end{array}$

$\begin{array}{llll}18 \mathrm{~B} & 0.546629 & -2.127107 & 1.081381\end{array}$

$\begin{array}{llll}19 B & 2.126262 & 0.549907 & 1.081381\end{array}$

$\begin{array}{llll}\text { 20B } & 0.584578 & -0.344942 & 2.368634\end{array}$

$\begin{array}{llll}21 \mathrm{~B} & -1.131475 & -1.230850 & -2.020261\end{array}$

$\begin{array}{llll}22 B & 0.530394 & 1.585528 & -2.020262\end{array}$

$z / \sigma$ $\begin{array}{llll}1 \mathrm{~A} & 0.000000 & 0.000000 & 0.000000\end{array}$

$\begin{array}{llll}2 \mathrm{~A} & 1.162754 & 0.000000 & 0.000000\end{array}$

$\begin{array}{llll}3 \mathrm{~A} & 0.581377 & -0.909517 & 0.000000\end{array}$

$\begin{array}{lllll}4 \mathrm{~A} & 0.581377 & 0.454759 & -0.787665\end{array}$

$\begin{array}{llll}5 \mathrm{~A} & 0.581377 & 0.454759 & 0.787665\end{array}$

$\begin{array}{lllll}6 \mathrm{~B} & -1.031964 & 0.615238 & -1.065624\end{array}$

$\begin{array}{llll}7 \mathrm{~B} & 2.194720 & 0.615238 & -1.065624\end{array}$

$\begin{array}{llll}8 \mathrm{~B} & -1.031964 & 0.615238 & 1.065624\end{array}$

$\begin{array}{llll}9 B & 2.194720 & 0.615238 & 1.065624\end{array}$

$\begin{array}{llll}10 \mathrm{~B} & 2.194721 & -1.230476 & 0.000000\end{array}$

$\begin{array}{llll}11 B & -1.031965 & -1.230477 & 0.000000\end{array}$

$\begin{array}{lllll}12 \mathrm{~A} & 1.120326 & -0.539676 & -0.934746\end{array}$

$\begin{array}{lllll}13 A & 0.042429 & -0.539676 & -0.934746\end{array}$

$\begin{array}{llll}14 \mathrm{~A} & 1.120326 & -0.539676 & 0.934746\end{array}$

$\begin{array}{llll}15 A & 0.042429 & -0.539676 & 0.934746\end{array}$

$\begin{array}{llll}16 \mathrm{~A} & 0.042429 & 1.079352 & 0.000000\end{array}$

$\begin{array}{llll}17 \mathrm{~A} & 1.120326 & 1.079352 & 0.000000\end{array}$

$\begin{array}{llll}18 \mathrm{~B} & 0.581378 & -2.025358 & -1.125032\end{array}$

$\begin{array}{lllll}19 \mathrm{~B} & 0.581378 & 0.038371 & -2.316527\end{array}$

$\begin{array}{llll}20 \mathrm{~B} & 0.581378 & -2.025358 & 1.125032\end{array}$

$\begin{array}{llll}21 \mathrm{~B} & 0.581378 & 1.986987 & -1.191492\end{array}$

$\begin{array}{llll}22 B & 0.581378 & 0.038371 & 2.316527\end{array}$

$\begin{array}{llll}23 B & 0.581378 & 1.986987 & 1.191492\end{array}$

$E 24 / \varepsilon=-115.433419$

atom $\quad \mathrm{x} / \sigma \quad \mathrm{y} / \sigma$

$\begin{array}{llll}1 \mathrm{~A} & 0.000000 & 0.000000 & 0.000000\end{array}$

$\begin{array}{llll}2 \mathrm{~A} & 1.168864 & 0.000000 & 0.000000\end{array}$

$\begin{array}{llll}3 \mathrm{~A} & 0.589442 & -0.909949 & 0.000000\end{array}$

$\begin{array}{llll}4 \mathrm{~A} & 0.582339 & 0.447612 & 0.786594\end{array}$

$\begin{array}{lllll}5 A & 0.582338 & 0.447611 & -0.786595\end{array}$

$\begin{array}{llll}6 \mathrm{~B} & -1.027893 & 0.583501 & 1.079633\end{array}$

$\begin{array}{llll}7 B & -1.027896 & 0.583499 & -1.079631\end{array}$

$\begin{array}{llll}8 \mathrm{~A} & 0.030551 & 1.087853 & 0.000000\end{array}$

$\begin{array}{llll}9 \mathrm{~B} & -1.020430 & -1.245038 & 0.000003\end{array}$

$\begin{array}{llll}10 B & 2.203639 & 0.610355 & -1.058998\end{array}$

$\begin{array}{llll}11 B & 2.203641 & 0.610356 & 1.058993\end{array}$

$\begin{array}{llll}12 B & 2.206193 & -1.233796 & -0.000002\end{array}$

$\begin{array}{lllll}13 \mathrm{~A} & 1.109748 & 1.080371 & -0.000002\end{array}$

$\begin{array}{llll}14 \mathrm{~A} & 0.054272 & -0.550172 & 0.930570\end{array}$

$\begin{array}{lllll}15 A & 0.054269 & -0.550173 & -0.930569\end{array}$

$\begin{array}{lllll}16 \mathrm{~A} & 1.131502 & -0.544199 & -0.931913\end{array}$

$\begin{array}{llll}17 \mathrm{~A} & 1.131504 & -0.544198 & 0.931913\end{array}$

$\begin{array}{llll}18 \mathrm{~B} & 0.605191 & 1.954005 & -1.238290\end{array}$

$\begin{array}{llll}19 \mathrm{~B} & 0.605195 & 1.954008 & 1.238287\end{array}$

$\begin{array}{llll}20 \mathrm{~B} & 0.595392 & 0.018791 & -2.316060\end{array}$

$\begin{array}{llll}21 B & 0.595396 & 0.018795 & 2.316060\end{array}$

$\begin{array}{lllll}22 \mathrm{~B} & 0.597067 & -2.031980 & -1.122231\end{array}$

$\begin{array}{llll}23 \mathrm{~B} & 0.597070 & -2.031979 & 1.122232\end{array}$

$\begin{array}{llll}24 \mathrm{~B} & -1.018548 & 2.370061 & 0.000000\end{array}$

\section{$E 25 / \varepsilon=-121.508101$}

atom $\quad \mathrm{x} / \sigma$

$y / \sigma$

$\begin{array}{llll}1 \mathrm{~A} & 0.000000 & 0.000000 & 0.000000\end{array}$

$\begin{array}{lllll}2 \mathrm{~A} & 1.175604 & 0.000000 & 0.000000\end{array}$

$\begin{array}{llll}3 \mathrm{~A} & 0.587803 & -0.910182 & 0.000000\end{array}$

$\begin{array}{lllll}4 \mathrm{~A} & 0.587802 & 0.438496 & -0.786185\end{array}$

$\begin{array}{llll}5 \mathrm{~A} & 0.587802 & 0.438496 & 0.786185\end{array}$

$\begin{array}{lllll}6 B & 2.205660 & 0.582696 & -1.071142\end{array}$

$\begin{array}{llll}7 \mathrm{~B} & 2.205662 & 0.582696 & 1.071140\end{array}$

$\begin{array}{llll}8 \mathrm{~B} & -1.030057 & 0.582691 & 1.071143\end{array}$

$\begin{array}{llll}9 B & -1.030059 & 0.582692 & -1.071141\end{array}$

$\begin{array}{llll}10 \mathrm{~A} & 1.125697 & 1.088745 & 0.000000\end{array}$

$\begin{array}{llll}11 \mathrm{~A} & 0.049904 & 1.088744 & 0.000000\end{array}$

$\begin{array}{llll}12 \mathrm{~B} & 0.587799 & 1.908842 & -1.307812\end{array}$

$\begin{array}{llll}13 \mathrm{~B} & 0.587800 & 1.908842 & 1.307812\end{array}$

14B $\quad-1.026504 \quad-1.247450 \quad 0.000001$

$\begin{array}{lllll}15 B & 2.202112 & -1.247446 & -0.000003\end{array}$

$\begin{array}{lllll}16 \mathrm{~A} & 0.048955 & -0.554850 & 0.927199\end{array}$

$\begin{array}{llll}17 \mathrm{~A} & 0.048953 & -0.554850 & -0.927199\end{array}$

$\begin{array}{lllll}18 \mathrm{~A} & 1.126651 & -0.554848 & -0.927200\end{array}$

$\begin{array}{llll}19 \mathrm{~A} & 1.126653 & -0.554849 & 0.927199\end{array}$

$\begin{array}{llll}20 B & 0.587801 & -0.014058 & -2.317151\end{array}$ 
$\begin{array}{llll}21 B & 0.587805 & -0.014058 & 2.317150\end{array}$

$\begin{array}{llll}22 \mathrm{~B} & 0.587803 & -2.038553 & -1.118988\end{array}$

$\begin{array}{llll}23 \mathrm{~B} & 0.587806 & -2.038555 & 1.118985\end{array}$

$\begin{array}{llll}24 \mathrm{~B} & -0.954232 & 2.383332 & 0.000001\end{array}$

$\begin{array}{llll}25 B & 2.129827 & 2.383337 & -0.000001\end{array}$

$E 26 / \varepsilon=-127.784324$

$\begin{array}{llll}1 \mathrm{~A} & 0.000000 & 0.000000 & 0.000000\end{array}$

$\begin{array}{llll}2 \mathrm{~A} & 1.157525 & 0.000000 & 0.000000\end{array}$

$\begin{array}{llll}3 \mathrm{~A} & 0.578761 & -1.002446 & 0.000000\end{array}$

$\begin{array}{llll}4 \mathrm{~A} & 0.578761 & -0.334149 & -0.945115\end{array}$

$\begin{array}{lllll}5 \mathrm{~A} & 1.461061 & -0.843547 & -0.596477\end{array}$

$\begin{array}{lllll}6 \mathrm{~A} & 0.578762 & 0.684644 & -0.596477\end{array}$

$\begin{array}{llll}7 A & 0.578762 & -0.334148 & 0.844315\end{array}$

$\begin{array}{lllll}8 A & -0.303541 & -0.843545 & -0.596476\end{array}$

$\begin{array}{lllll}9 A & 1.525257 & 0.212309 & -1.009090\end{array}$

$\begin{array}{llll}10 \mathrm{~A} & -0.367734 & 0.212311 & -1.009089\end{array}$

$\begin{array}{lllll}11 \mathrm{~A} & 0.578759 & -1.427068 & -1.009089\end{array}$

$\begin{array}{llll}12 \mathrm{~A} & 1.525257 & -0.880609 & 0.536531\end{array}$

$\begin{array}{llll}13 \mathrm{~A} & -0.367735 & -0.880607 & 0.536532\end{array}$

$\begin{array}{llll}14 \mathrm{~A} & 0.578763 & 0.758770 & 0.536531\end{array}$

$\begin{array}{lllll}15 \mathrm{~B} & 0.578762 & 0.884099 & -2.119940\end{array}$

16B $1.633793 \quad-0.943276-2.119940$

$\begin{array}{llll}17 \mathrm{~B} & -1.662598 & -0.258346 & -0.182680\end{array}$

$\begin{array}{lllll}18 \mathrm{~B} & 1.633793 & -2.313125 & -0.182681\end{array}$

$\begin{array}{llll}19 \mathrm{~B} & 1.765088 & 0.350775 & 1.540182\end{array}$

$\begin{array}{llll}20 \mathrm{~B} & -0.476277 & -2.313122 & -0.182680\end{array}$

$\begin{array}{llll}21 B & -0.607561 & 1.569026 & -0.182681\end{array}$

$\begin{array}{lllll}22 B & 1.765089 & 1.569024 & -0.182683\end{array}$

$\begin{array}{llll}23 \mathrm{~B} & -0.476277 & -0.943273 & -2.119939\end{array}$

$\begin{array}{llll}24 \mathrm{~B} & 2.820121 & -0.258351 & -0.182682\end{array}$

$\begin{array}{llll}25 \mathrm{~B} & -0.607562 & 0.350778 & 1.540184\end{array}$

$\begin{array}{llll}26 \mathrm{~B} & 0.578760 & -1.703998 & 1.540184\end{array}$

$E 27 / \varepsilon=-135.837752$

$\begin{array}{cccc}\text { atom } & \mathrm{x} / \sigma & y / \sigma & z / \sigma \\ 1 \mathrm{~A} & 0.000000 & 0.000000 & 0.000000\end{array}$

$\begin{array}{llll}2 \mathrm{~A} & 1.100073 & 0.000000 & 0.000000\end{array}$

$\begin{array}{llll}3 \mathrm{~A} & 0.550037 & -0.952692 & 0.000000\end{array}$

$\begin{array}{llll}4 \mathrm{~A} & -0.550037 & 0.317563 & -0.898206\end{array}$

$\begin{array}{llll}5 A & -0.550036 & -0.317564 & 0.898206\end{array}$

$\begin{array}{llll}6 \mathrm{~A} & 0.550037 & 0.317564 & -0.898206\end{array}$

$\begin{array}{llll}7 \mathrm{~A} & 0.000000 & 0.635128 & 0.898206\end{array}$

$\begin{array}{llll}8 \mathrm{~A} & -0.550037 & 0.952691 & 0.000000\end{array}$

$\begin{array}{llll}9 A & -0.550037 & -0.952692 & 0.000000\end{array}$

$\begin{array}{llll}10 A & 0.550037 & -0.317564 & 0.898206\end{array}$

$\begin{array}{llll}11 \mathrm{~A} & 0.550037 & 0.952691 & 0.000000\end{array}$

$\begin{array}{llll}12 \mathrm{~A} & 0.000000 & -0.635128 & -0.898206\end{array}$

$\begin{array}{llll}13 \mathrm{~A} & -1.100073 & 0.000000 & 0.000000\end{array}$

$\begin{array}{llll}14 \mathrm{~B} & -1.540941 & 0.889663 & 1.258173\end{array}$

$\begin{array}{llll}15 B & 0.000000 & 1.779325 & -1.258173\end{array}$

$\begin{array}{llll}16 \mathrm{~B} & -1.540941 & -0.889663 & -1.258172\end{array}$

$\begin{array}{llll}17 \mathrm{~B} & 0.000001 & -1.779326 & 1.258173\end{array}$

$\begin{array}{llll}18 \mathrm{~B} & 1.540941 & 0.889663 & 1.258173\end{array}$

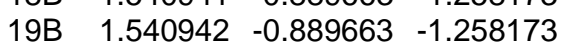

$\begin{array}{lllll}20 B & 1.931098 & 1.114919 & -0.788368\end{array}$

$\begin{array}{llll}21 B & 0.000000 & -2.229839 & -0.788368\end{array}$

$\begin{array}{llll}22 \mathrm{~B} & 1.931098 & -1.114920 & 0.788368\end{array}$

$\begin{array}{llll}23 \mathrm{~B} & 0.000000 & 2.229839 & 0.788368\end{array}$

$\begin{array}{llll}24 \mathrm{~B} & -1.931097 & -1.114920 & 0.788368\end{array}$

$\begin{array}{llll}25 \mathrm{~B} & 0.000000 & -0.000001 & -2.365102\end{array}$

$\begin{array}{llll}26 \mathrm{~B} & 0.000001 & 0.000000 & 2.365102\end{array}$

$\begin{array}{llll}27 \mathrm{~B} & -1.931098 & 1.114919 & -0.788368\end{array}$

$E 28 / \varepsilon=-140.858567$

$\begin{array}{cccc}\text { atom } & x / \sigma & y / \sigma & z / \sigma \\ \text { 1A } & 0.000000 & 0.000000 & 0.000000 \\ \text { 2A } & 1.100343 & 0.000000 & 0.000000 \\ \text { 3B } & 1.577099 & -1.492933 & 0.000000 \\ \text { 4B } & 1.577099 & 1.492933 & 0.000000 \\ \text { 5A } & -0.555523 & 0.553027 & 0.772386\end{array}$

$\begin{array}{llll}6 A & -0.555523 & 0.553027 & -0.772386\end{array}$

$\begin{array}{llll}7 A & -0.555523 & -0.553028 & 0.772386\end{array}$

$\begin{array}{lllll}8 A & -0.555523 & -0.553028 & -0.772386\end{array}$

$\begin{array}{lllll}9 \mathrm{~A} & 0.544966 & -0.544689 & 0.785701\end{array}$

$\begin{array}{llll}10 A & 0.544965 & -0.544689 & -0.785701\end{array}$

$\begin{array}{llll}11 \mathrm{~A} & 0.544966 & 0.544689 & 0.785701\end{array}$

$\begin{array}{llll}12 \mathrm{~A} & 0.544965 & 0.544689 & -0.785701\end{array}$

$\begin{array}{llll}13 \mathrm{~A} & 0.013684 & -1.098421 & 0.000000\end{array}$

$\begin{array}{llll}14 \mathrm{~A} & 0.013683 & 1.098421 & 0.000000\end{array}$

$\begin{array}{llll}15 \mathrm{~A} & -1.100941 & -0.000001 & 0.000000\end{array}$

$\begin{array}{llll}16 \mathrm{~B} & -1.519732 & 1.563355 & 0.000000\end{array}$

$\begin{array}{llll}17 \mathrm{~B} & -1.519731 & -1.563357 & 0.000000\end{array}$

$\begin{array}{llll}18 \mathrm{~B} & -0.090050 & 0.000000 & -2.187161\end{array}$

$\begin{array}{llll}19 \mathrm{~B} & -0.090048 & 0.000000 & 2.187161\end{array}$

$\begin{array}{llll}20 B & 1.844755 & 0.000000 & -1.516981\end{array}$

$\begin{array}{llll}21 B & 1.844756 & 0.000000 & 1.516980\end{array}$

$\begin{array}{llll}22 \mathrm{~B} & 0.033112 & -1.928359 & 1.363730\end{array}$

23B $\quad 0.033112 \quad-1.928359-1.363730$

$\begin{array}{llll}24 \mathrm{~B} & 0.033111 & 1.928359 & -1.363730\end{array}$

$\begin{array}{llll}25 B & 0.033111 & 1.928359 & 1.363730\end{array}$

$\begin{array}{llll}26 \mathrm{~B} & -1.974771 & -0.000001 & 1.314023\end{array}$

$\begin{array}{llll}27 \mathrm{~B} & -1.974772 & -0.000001 & -1.314021\end{array}$

$\begin{array}{llll}28 \mathrm{~A} & 2.222730 & 0.000000 & -0.000001\end{array}$

$E 29 / \varepsilon=-146.434540$

atom $\quad \mathrm{x} / \sigma \quad \mathrm{yl}$

$\begin{array}{llll}1 \mathrm{~A} & 0.000000 & 0.000000 & 0.000000\end{array}$

$\begin{array}{llll}2 \mathrm{~A} & 1.120537 & 0.000000 & 0.000000\end{array}$

$\begin{array}{llll}3 \mathrm{~A} & 0.591990 & -0.951394 & 0.000000\end{array}$

$\begin{array}{lllll}4 \mathrm{~B} & 1.528408 & -0.849107 & -1.296302\end{array}$

$\begin{array}{llll}5 \mathrm{~B} & 0.092298 & -1.746727 & 1.273001\end{array}$

$\begin{array}{llll}6 \mathrm{~B} & 1.531824 & 0.844447 & 1.273000\end{array}$

$\begin{array}{lllll}7 \mathrm{~A} & 0.515005 & -0.286111 & 0.897577\end{array}$

$\begin{array}{lllll}8 \mathrm{~A} & 0.540695 & 0.947367 & 0.015992\end{array}$

$\begin{array}{llll}9 A & -0.518710 & -0.959580 & 0.015993\end{array}$

$\begin{array}{llll}10 A & 0.543741 & 0.328984 & -0.891316\end{array}$

$\begin{array}{lllll}11 \mathrm{~A} & 0.007938 & -0.635470 & -0.891315\end{array}$

$\begin{array}{llll}12 \mathrm{~A} & -0.566562 & 0.945979 & -0.001779\end{array}$

$\begin{array}{llll}13 \mathrm{~A} & -1.102505 & -0.018728 & -0.001778\end{array}$

$\begin{array}{lllll}14 A & -0.556447 & 0.309134 & -0.895672\end{array}$

$\begin{array}{llll}15 A & -0.034176 & 0.648075 & 0.904110\end{array}$

$\begin{array}{llll}16 \mathrm{~A} & -0.568305 & -0.313365 & 0.904111\end{array}$

$\begin{array}{llll}17 \mathrm{~B} & -1.535515 & -0.913341 & -1.244601\end{array}$

$\begin{array}{llll}18 \mathrm{~B} & -0.035752 & 1.786258 & -1.244604\end{array}$

$\begin{array}{llll}19 \mathrm{~B} & -1.581817 & 0.878780 & 1.236916\end{array}$

$\begin{array}{llll}20 \mathrm{~B} & -0.002697 & -2.227593 & -0.791315\end{array}$

$\begin{array}{lllll}21 B & 1.889918 & 1.179147 & -0.791316\end{array}$

$\begin{array}{llll}22 \mathrm{~B} & -1.889997 & -1.169903 & 0.803894\end{array}$

$\begin{array}{llll}23 \mathrm{~B} & -0.005193 & 2.222777 & 0.803890\end{array}$

$\begin{array}{lllll}24 \mathrm{~B} & -0.029831 & 0.016571 & -2.362223\end{array}$

$\begin{array}{llll}25 B & -0.046317 & 0.025732 & 2.372851\end{array}$

$\begin{array}{llll}26 \mathrm{~B} & -1.949620 & 1.083112 & -0.805433\end{array}$

$\begin{array}{llll}27 \mathrm{~A} & 1.433542 & -0.796405 & 0.721804\end{array}$

$\begin{array}{lllll}28 \mathrm{~B} & 2.858044 & -0.367774 & 0.216498\end{array}$

$\begin{array}{lllll}29 B & 1.822189 & -2.232331 & 0.216497\end{array}$

\section{$E 30 / \varepsilon=-153.819680$}

$\begin{array}{lll}\text { atom } & \mathrm{x} / \sigma & \mathrm{y} / \sigma\end{array}$

$\begin{array}{llll}1 \mathrm{~A} & 0.000000 & 0.000000 & 0.000000\end{array}$

$\begin{array}{llll}2 B & 2.164714 & 0.000000 & 0.000000\end{array}$

$\begin{array}{llll}3 B & 0.039654 & -2.164351 & 0.000000\end{array}$

$\begin{array}{llll}4 \mathrm{~B} & 0.039654 & -0.038933 & 2.164001\end{array}$

$\begin{array}{llll}5 A & 0.005029 & -0.784704 & 0.770713\end{array}$

$\begin{array}{lllll}6 \mathrm{~A} & 0.784664 & -0.770420 & -0.023210\end{array}$

$\begin{array}{llll}7 \mathrm{~A} & 0.784664 & 0.009348 & 0.770713\end{array}$

$\begin{array}{llll}8 \mathrm{~A} & -0.773189 & -0.782853 & -0.001679\end{array}$

$\begin{array}{llll}9 \mathrm{~A} & -0.773189 & -0.012403 & 0.782757\end{array}$

$\begin{array}{llll}10 A & 0.768558 & 0.787400 & -0.001680\end{array}$

$\begin{array}{llll}11 \mathrm{~A} & -0.001762 & 0.773287 & 0.782756\end{array}$

$\begin{array}{llll}12 \mathrm{~A} & -0.001763 & -0.768719 & -0.787242\end{array}$

$\begin{array}{llll}13 \mathrm{~A} & 0.768558 & 0.015844 & -0.787242\end{array}$

$\begin{array}{lllll}14 \mathrm{~A} & -0.015548 & 0.785954 & -0.771941\end{array}$ $\begin{array}{lll}15 A & -0.786107 & 0.771836\end{array}$ \begin{tabular}{|llll}
$16 \mathrm{~A}$ & -0.786107 & 0.001148 & -0.771940
\end{tabular}

$\begin{array}{llll}17 \mathrm{~B} & -0.026233 & 2.180918 & 0.013477\end{array}$

$\begin{array}{llll}18 \mathrm{~B} & -0.026234 & 0.025757 & -2.180807\end{array}$

$\begin{array}{llll}19 \mathrm{~B} & -2.181033 & -0.013722 & 0.013479\end{array}$

$\begin{array}{llll}20 \mathrm{~B} & 1.356529 & -1.331903 & -1.410495\end{array}$

$\begin{array}{llll}21 \mathrm{~B} & 1.356529 & 1.386308 & 1.357060\end{array}$

$\begin{array}{llll}22 \mathrm{~B} & -1.361225 & -1.381696 & 1.357062\end{array}$

$\begin{array}{llll}23 \mathrm{~A} & 1.034531 & -1.015750 & 0.997639\end{array}$

$\begin{array}{llll}24 \mathrm{~B} & -1.358183 & 1.333529 & 1.400360\end{array}$

$\begin{array}{llll}25 \mathrm{~B} & -1.358185 & -1.376145 & -1.358502\end{array}$

$\begin{array}{lllll}26 \mathrm{~B} & 1.351035 & 1.383164 & -1.358503\end{array}$

$\begin{array}{lllll}27 \mathrm{~B} & -1.392766 & 1.367481 & -1.343098\end{array}$

$\begin{array}{lllll}28 \mathrm{~B} & 0.532738 & -2.063788 & 2.026992\end{array}$

$\begin{array}{llll}29 \mathrm{~B} & 2.073201 & -0.494842 & 2.026992\end{array}$

$\begin{array}{llll}30 \mathrm{~B} & 2.073202 & -2.035565 & 0.458299\end{array}$

$E 31 / \varepsilon=-158.954495$

$\begin{array}{llll}\text { atom } & \mathrm{x} / \sigma & \mathrm{y} / \sigma & \mathrm{z} / \sigma\end{array}$

$\begin{array}{llll}1 \mathrm{~A} & 0.000000 & 0.000000 & 0.000000\end{array}$

2B $\quad 1.543352 \quad 0.000000 \quad 0.000000$

$\begin{array}{llll}3 \mathrm{~A} & -0.747400 & -0.780149 & 0.000000\end{array}$

$\begin{array}{llll}4 \mathrm{~A} & -0.747400 & -0.002455 & 0.780146\end{array}$

$\begin{array}{llll}5 \mathrm{~A} & -0.747404 & 0.002451 & -0.780140\end{array}$

$\begin{array}{llll}6 \mathrm{~A} & -0.747404 & 0.780145 & 0.000007\end{array}$

$\begin{array}{llll}7 A & 0.020882 & -0.807899 & -0.810446\end{array}$

$\begin{array}{llll}8 \mathrm{~A} & 0.020884 & 0.807898 & 0.810447\end{array}$

$\begin{array}{llll}9 \mathrm{~A} & 0.315865 & -1.012115 & 0.221644\end{array}$

$\begin{array}{llll}10 A & 0.315865 & -0.224831 & 1.011412\end{array}$

$\begin{array}{llll}11 \mathrm{~A} & 0.315861 & 1.012115 & -0.221645\end{array}$

$\begin{array}{lllll}12 \mathrm{~A} & 0.315860 & 0.224832 & -1.011412\end{array}$

$\begin{array}{llll}13 \mathrm{~B} & -1.861373 & 1.084131 & 1.087562\end{array}$

14B $-1.861372-1.084141-1.087550$

$\begin{array}{llll}15 B & -0.594432 & -0.120525 & 2.327816\end{array}$

$\begin{array}{llll}16 \mathrm{~B} & -0.594442 & 2.328179 & -0.113187\end{array}$

$\begin{array}{llll}17 \mathrm{~B} & -0.594442 & 0.120520 & -2.327811\end{array}$

$\begin{array}{llll}18 \mathrm{~B} & -0.594431 & -2.328183 & 0.113191\end{array}$

$\begin{array}{llll}19 B & -1.999873 & 1.048128 & -1.044831\end{array}$

$\begin{array}{llll}20 B & -1.999862 & -1.048139 & 1.044844\end{array}$

$\begin{array}{llll}21 \mathrm{~A} & -0.429040 & 1.030115 & -1.026875\end{array}$

$\begin{array}{llll}22 A & -0.429030 & -1.030119 & 1.026880\end{array}$

$\begin{array}{llll}23 \mathrm{~A} & -1.514301 & -0.000004 & 0.000005\end{array}$

$\begin{array}{llll}24 \mathrm{~B} & 0.972441 & -1.555388 & 1.550496\end{array}$

$\begin{array}{llll}25 B & 0.972424 & 1.555392 & -1.550500\end{array}$

$\begin{array}{lllll}26 \mathrm{~B} & 1.338852 & -0.502769 & -1.980719\end{array}$

$\begin{array}{llll}27 \mathrm{~B} & 1.338851 & 1.979124 & 0.509008\end{array}$

$\begin{array}{llll}28 B & 1.338857 & 0.502774 & 1.980718\end{array}$

$\begin{array}{llll}29 B & 1.338857 & -1.979122 & -0.509013\end{array}$

$\begin{array}{lllll}30 \mathrm{~B} & -0.186526 & -1.924618 & -1.930689\end{array}$ $\begin{array}{llll}31 \mathrm{~B} & -0.186527 & 1.924615 & 1.930690\end{array}$

$E 32 / \varepsilon=-165.730628$

atom $\quad \mathrm{x} / \sigma \quad \mathrm{y} / \sigma$

$\begin{array}{llll}1 \mathrm{~A} & 0.000000 & 0.000000 & 0.000000\end{array}$

$\begin{array}{llll}2 \mathrm{~B} & 1.813105 & 0.000000 & 0.000000\end{array}$

$\begin{array}{llll}3 \mathrm{~A} & -0.562109 & -0.935866 & 0.000000\end{array}$

$\begin{array}{llll}4 \mathrm{~A} & -0.562112 & 0.778000 & -0.520151\end{array}$

$\begin{array}{lllll}5 A & -0.561621 & -0.269047 & -0.886493\end{array}$

$\begin{array}{lllll}6 \mathrm{~A} & 0.537965 & 0.798645 & -0.523522\end{array}$

$\begin{array}{llll}7 A & 0.537968 & -0.954898 & 0.008671\end{array}$

$\begin{array}{lllll}8 A & 0.536777 & -0.274936 & -0.905902\end{array}$

$\begin{array}{lllll}9 \mathrm{~A} & -1.186081 & -0.008649 & -0.028490\end{array}$

$\begin{array}{llll}10 \mathrm{~A} & 0.512890 & -0.289379 & 0.919410\end{array}$

$\begin{array}{llll}11 \mathrm{~A} & 0.512889 & 0.751573 & 0.603486\end{array}$

$\begin{array}{llll}12 \mathrm{~A} & -0.597832 & -0.294047 & 0.867977\end{array}$

$\begin{array}{llll}13 A & -0.597833 & 0.726864 & 0.558135\end{array}$

14B $\quad-0.124993 \quad-0.723918-2.385271$

$\begin{array}{lllll}15 B & -1.641712 & -1.404045 & -1.027013\end{array}$

$\begin{array}{lllll}16 \mathrm{~B} & -1.641716 & 0.596393 & -1.634137\end{array}$

$\begin{array}{llll}17 \mathrm{~B} & -0.032390 & -2.493702 & -0.011346\end{array}$

$\begin{array}{llll}18 \mathrm{~B} & -0.032402 & 2.066755 & -1.395424\end{array}$

$\begin{array}{llll}19 B & -0.205093 & -0.487973 & 2.348765\end{array}$

$\begin{array}{llll}20 B & -0.205096 & 1.711097 & 1.681357\end{array}$

$\begin{array}{llll}21 \mathrm{~A} & -0.038986 & 0.509334 & -1.429866\end{array}$ 
$\begin{array}{llll}22 \mathrm{~A} & -0.038983 & -1.218133 & -0.905588\end{array}$ $\begin{array}{llll}23 \mathrm{~B} & -1.586316 & -1.555098 & 1.051189\end{array}$ $\begin{array}{llll}24 \mathrm{~B} & -1.586323 & 1.877024 & 0.009557\end{array}$ $\begin{array}{llll}25 \mathrm{~A} & -0.048574 & 1.535190 & 0.084390\end{array}$ $\begin{array}{llll}26 \mathrm{~A} & -0.048570 & -1.229328 & 0.923409\end{array}$ $\begin{array}{llll}27 \mathrm{~B} & 1.447512 & 0.628857 & -1.890842\end{array}$ $\begin{array}{llll}28 \mathrm{~B} & 1.447515 & -1.573698 & -1.222382\end{array}$ $\begin{array}{llll}29 B & 1.429785 & 1.997896 & 0.178875\end{array}$ 30B $\quad 1.429789-1.561463 \quad 1.259134$ $\begin{array}{llll}31 B & -1.893633 & 0.431475 & 1.421696\end{array}$ $\begin{array}{llll}32 \mathrm{~B} & 1.498091 & 0.575988 & 1.897836\end{array}$

\section{$E 33 / \varepsilon=-174.297678$}$$
\text { atom } \quad \mathrm{x} / \sigma \quad \mathrm{y} / \sigma
$$

$\begin{array}{llll}1 \mathrm{~A} & 0.000000 & 0.000000 & 0.000000\end{array}$ $\begin{array}{llll}2 \mathrm{~B} & 1.806439 & 0.000000 & 0.000000\end{array}$ $\begin{array}{llll}3 A & -0.573899 & -0.929597 & 0.000000\end{array}$ $\begin{array}{llll}4 \mathrm{~A} & -0.573899 & -0.287262 & -0.884098\end{array}$ $\begin{array}{lllll}5 A & -0.573899 & -0.287262 & 0.884098\end{array}$ $\begin{array}{llll}6 \mathrm{~A} & -0.573900 & 0.752058 & -0.546403\end{array}$ $\begin{array}{llll}7 A & -0.573900 & 0.752058 & 0.546403\end{array}$ 8A $\quad 0.526471 \quad-0.959169 \quad 0.000000$ $\begin{array}{lllll}9 A & 0.526471 & -0.296400 & -0.912223\end{array}$ $\begin{array}{llll}10 A & 0.526471 & -0.296400 & 0.912223\end{array}$

$\begin{array}{llll}11 \mathrm{~A} & 0.526470 & 0.775983 & -0.563786\end{array}$

$\begin{array}{llll}12 \mathrm{~A} & 0.526470 & 0.775983 & 0.563785\end{array}$

$\begin{array}{llll}13 \mathrm{~A} & -1.180166 & -0.000001 & 0.000000\end{array}$ $\begin{array}{llll}14 \mathrm{~B} & -0.109663 & 2.029753 & 1.474703\end{array}$ $\begin{array}{llll}15 B & -0.109663 & 2.029752 & -1.474704\end{array}$ $\begin{array}{llll}16 \mathrm{~B} & -0.109662 & -0.775299 & 2.386119\end{array}$ $\begin{array}{llll}17 \mathrm{~B} & -0.109662 & -0.775300 & -2.386119\end{array}$ $\begin{array}{llll}18 B & -0.109662 & -2.508916 & 0.000000\end{array}$ $\begin{array}{llll}19 B & -1.645968 & 1.767782 & 0.000000\end{array}$ $\begin{array}{llll}20 B & -1.645966 & -1.430169 & 1.039077\end{array}$ 21B $\quad-1.645966 \quad-1.430170-1.039077$ $\begin{array}{lllll}22 \mathrm{~B} & -1.645968 & 0.546272 & -1.681262\end{array}$ $\begin{array}{llll}23 \mathrm{~B} & -1.645967 & 0.546273 & 1.681262\end{array}$ $\begin{array}{llll}24 \mathrm{~A} & -0.052949 & 0.473064 & 1.455947\end{array}$

$\begin{array}{llll}25 A & -0.052949 & 0.473064 & -1.455947\end{array}$ $\begin{array}{llll}26 \mathrm{~A} & -0.052949 & 1.530872 & 0.000000\end{array}$ $\begin{array}{llll}27 \mathrm{~A} & -0.052948 & -1.238504 & 0.899824\end{array}$ $\begin{array}{llll}28 A & -0.052948 & -1.238504 & -0.899824\end{array}$ $\begin{array}{llll}29 B & 1.437110 & -1.615414 & 1.173664\end{array}$ 30B $\quad 1.437110 \quad-1.615413 \quad-1.173664$ $\begin{array}{llll}31 B & 1.437109 & 0.617030 & -1.899031\end{array}$ $\begin{array}{llll}32 \mathrm{~B} & 1.437109 & 0.617031 & 1.899031\end{array}$ $\begin{array}{llll}33 \mathrm{~B} & 1.437109 & 1.996759 & 0.000000\end{array}$

$E 34 / \varepsilon=-182.112852$

atom $\quad x / \sigma \quad 0.00000$

$\begin{array}{cccc}\text { 1A } & 0.000000 & 0.000000 & 0.000000\end{array}$ $\begin{array}{llll}2 \mathrm{~B} & 1.810719 & 0.000000 & 0.000000\end{array}$ $\begin{array}{llll}3 \mathrm{~A} & -0.572256 & -0.932108 & 0.000000\end{array}$ $\begin{array}{llll}4 \mathrm{~A} & -0.572256 & -0.288037 & 0.886487\end{array}$ $\begin{array}{llll}5 \mathrm{~A} & -0.572256 & -0.288037 & -0.886487\end{array}$ $\begin{array}{llll}6 \mathrm{~A} & -0.572256 & 0.754091 & -0.547879\end{array}$ $\begin{array}{llll}7 \mathrm{~A} & -0.572256 & 0.754091 & 0.547879\end{array}$ $\begin{array}{lllll}8 A & 0.529639 & 0.774862 & -0.562971\end{array}$ $\begin{array}{lllll}9 A & 0.529639 & -0.295971 & -0.910906\end{array}$ $\begin{array}{llll}10 A & 0.529639 & -0.295971 & 0.910906\end{array}$ $\begin{array}{llll}11 \mathrm{~A} & 0.529639 & 0.774863 & 0.562970\end{array}$ $\begin{array}{llll}12 \mathrm{~A} & 0.529639 & -0.957783 & 0.000000\end{array}$ $\begin{array}{llll}13 \mathrm{~A} & -1.159435 & 0.000000 & 0.000000\end{array}$ $\begin{array}{lllll}14 \mathrm{~B} & -1.630023 & 0.551639 & -1.697771\end{array}$ 15B $\quad-1.630023 \quad-1.444210-1.049280$ $\begin{array}{llll}16 \mathrm{~B} & -1.630023 & 1.785142 & 0.000000\end{array}$ $\begin{array}{llll}17 \mathrm{~B} & -1.630023 & -1.444210 & 1.049280\end{array}$ $\begin{array}{llll}18 \mathrm{~B} & -1.630023 & 0.551639 & 1.697771\end{array}$ $\begin{array}{llll}19 \mathrm{~B} & -0.100720 & -0.775411 & 2.386470\end{array}$ $\begin{array}{llll}20 \mathrm{~B} & -0.100718 & -2.509282 & 0.000000\end{array}$ $\begin{array}{llll}21 B & -0.100719 & 2.030052 & 1.474919\end{array}$ $\begin{array}{llll}22 B & -0.100719 & 2.030052 & -1.474920\end{array}$ $\begin{array}{llll}23 \mathrm{~B} & -0.100720 & -0.775411 & -2.386470\end{array}$

$\begin{array}{|llll|}24 A & -0.047348 & 0.472737 & 1.454935\end{array}$ $\begin{array}{llll}25 \mathrm{~A} & -0.047348 & 1.529809 & 0.000000\end{array}$ $\begin{array}{lllll}26 \mathrm{~A} & -0.047348 & 0.472737 & -1.454935\end{array}$ $\begin{array}{lllll}27 \mathrm{~A} & -0.047348 & -1.237641 & -0.899199\end{array}$ $\begin{array}{llll}28 \mathrm{~A} & -0.047348 & -1.237641 & 0.899199\end{array}$ $\begin{array}{llll}29 B & 1.442393 & -1.614632 & 1.173100\end{array}$ $\begin{array}{llll}30 \mathrm{~B} & 1.442393 & -1.614632 & -1.173100\end{array}$ $\begin{array}{lllll}31 \mathrm{~B} & 1.442393 & 0.616734 & -1.898115\end{array}$ $\begin{array}{llll}32 \mathrm{~B} & 1.442393 & 0.616734 & 1.898115\end{array}$ $\begin{array}{llll}33 \mathrm{~B} & 1.442393 & 1.995795 & 0.000000\end{array}$ $\begin{array}{llll}34 \mathrm{~B} & -2.735158 & 0.000000 & 0.000000\end{array}$

\section{$E 35 / \varepsilon=-189.341608$}

atom $\mathrm{x} / \sigma \quad \mathrm{yl}$

$\begin{array}{llll}1 \mathrm{~A} & 0.000000 & 0.000000 & 0.000000\end{array}$

$\begin{array}{llll}2 \mathrm{~A} & 1.086526 & 0.000000 & 0.000000\end{array}$

$\begin{array}{llll}3 \mathrm{~A} & 0.540692 & -0.942439 & 0.000000\end{array}$

$\begin{array}{llll}4 \mathrm{~A} & 0.524337 & 0.325605 & 0.894199\end{array}$

$\begin{array}{llll}5 A & -0.021497 & -0.616834 & 0.894199\end{array}$

$\begin{array}{llll}6 \mathrm{~A} & -0.385306 & 0.852444 & 0.552645\end{array}$

$\begin{array}{lllll}7 A & 0.524336 & 0.325605 & -0.894199\end{array}$

$\begin{array}{llll}8 \mathrm{~A} & -0.021498 & -0.616834 & -0.894199\end{array}$

$\begin{array}{llll}9 A & -0.931140 & -0.089995 & 0.552646\end{array}$

$\begin{array}{llll}10 A & -0.385306 & 0.852444 & -0.552645\end{array}$

$\begin{array}{llll}11 \mathrm{~A} & -0.931140 & -0.089995 & -0.552645\end{array}$

$\begin{array}{llll}12 \mathrm{~A} & 0.562868 & 0.971848 & 0.000000\end{array}$

$\begin{array}{llll}13 \mathrm{~A} & -0.562867 & -0.971848 & 0.000000\end{array}$

$\begin{array}{llll}14 \mathrm{~B} & 2.052297 & 0.639398 & -1.057471\end{array}$

$\begin{array}{llll}15 B & 2.052297 & 0.639398 & 1.057471\end{array}$

$\begin{array}{llll}16 \mathrm{~B} & -1.273887 & -1.090230 & -1.711024\end{array}$

$\begin{array}{llll}17 \mathrm{~B} & -2.349621 & -0.467196 & 0.000000\end{array}$

$\begin{array}{llll}18 \mathrm{~B} & 0.311723 & 1.647489 & 1.711024\end{array}$

$\begin{array}{llll}\text { 19B } & 0.466687 & -2.098322 & -1.057471\end{array}$

$\begin{array}{llll}20 \mathrm{~B} & -0.764012 & 2.270524 & 0.000000\end{array}$

$\begin{array}{llll}21 \mathrm{~B} & -1.273886 & -1.090230 & 1.711025\end{array}$

$\begin{array}{llll}22 \mathrm{~B} & 0.466688 & -2.098322 & 1.057471\end{array}$

$\begin{array}{llll}23 \mathrm{~B} & 0.311722 & 1.647490 & -1.711024\end{array}$

$\begin{array}{llll}24 \mathrm{~B} & -1.752264 & 1.014862 & 1.471205\end{array}$

$\begin{array}{llll}25 \mathrm{~B} & -1.752264 & 1.014862 & -1.471204\end{array}$

$\begin{array}{llll}26 \mathrm{~B} & 0.669306 & -0.387642 & -2.380458\end{array}$

$\begin{array}{llll}27 \mathrm{~B} & 2.165919 & -1.254438 & 0.000000\end{array}$

$\begin{array}{llll}28 \mathrm{~B} & 0.669307 & -0.387642 & 2.380458\end{array}$

$\begin{array}{llll}29 A & -1.327606 & 0.768912 & 0.000000\end{array}$

$\begin{array}{llll}29 A & -0.410252 & 0.237607 & 1.459110\end{array}$

$\begin{array}{llll}31 \mathrm{~A} & -0.410252 & 0.237607 & -1.459109\end{array}$

$\begin{array}{llll}32 \mathrm{~A} & 1.074057 & -0.622063 & -0.901779\end{array}$

$\begin{array}{llll}33 \mathrm{~A} & 1.074057 & -0.622063 & 0.901779\end{array}$

$\begin{array}{llll}34 \mathrm{~B} & -1.346032 & -2.324064 & 0.000000\end{array}$ $\begin{array}{llll}35 \mathrm{~B} & 1.346033 & 2.324065 & 0.000000\end{array}$

\section{$E 36 / \varepsilon=-194.828824$}

$$
\text { atom } \mathrm{x} / \sigma \quad \mathrm{y} / \sigma
$$

$\begin{array}{llll}1 \mathrm{~A} & 0.000000 & 0.000000 & 0.000000\end{array}$

$\begin{array}{llll}2 \mathrm{~A} & 1.087917 & 0.000000 & 0.000000\end{array}$

$\begin{array}{llll}3 A & 0.549502 & -0.938941 & 0.000000\end{array}$

$\begin{array}{llll}4 \mathrm{~A} & 0.528338 & 0.320878 & 0.895243\end{array}$

$\begin{array}{llll}5 A & -0.010077 & -0.618063 & 0.895243\end{array}$

$\begin{array}{lllll}6 \mathrm{~A} & -0.393249 & 0.853576 & -0.551748\end{array}$

$\begin{array}{lllll}7 \mathrm{~A} & -0.935318 & -0.091738 & -0.551748\end{array}$

$\begin{array}{lllll}8 \mathrm{~A} & 0.513355 & 0.335865 & -0.895085\end{array}$

$\begin{array}{lllll}9 A & -0.030580 & -0.612702 & -0.895085\end{array}$

$\begin{array}{llll}10 \mathrm{~A} & -0.388193 & 0.852838 & 0.547256\end{array}$

$\begin{array}{llll}11 \mathrm{~A} & -0.932128 & -0.095729 & 0.547256\end{array}$

$\begin{array}{llll}12 \mathrm{~A} & 0.560555 & 0.971335 & 0.002228\end{array}$

$\begin{array}{llll}13 A & -0.555190 & -0.974411 & 0.002228\end{array}$

$\begin{array}{llll}14 \mathrm{~B} & 2.056862 & 0.631166 & 1.059433\end{array}$

$\begin{array}{llll}15 B & 0.494178 & -2.094002 & 1.059434\end{array}$

$\begin{array}{llll}16 \mathrm{~B} & 2.037483 & 0.657187 & -1.063852\end{array}$

$\begin{array}{llll}17 \mathrm{~B} & 0.307881 & 1.648989 & 1.703249\end{array}$

$\begin{array}{llll}18 \mathrm{~B} & -1.267671 & -1.098619 & 1.703249\end{array}$

$\begin{array}{lllll}19 B & 0.461930 & -2.090418 & -1.063853\end{array}$

$\begin{array}{llll}20 \mathrm{~B} & 0.608431 & -0.348890 & 2.400713\end{array}$ $\begin{array}{llll}21 \mathrm{~B} & 2.168114 & -1.243256 & -0.094544\end{array}$ $\begin{array}{llll}22 \mathrm{~B} & -2.352258 & -0.475906 & 0.001620\end{array}$

$\begin{array}{llll}23 \mathrm{~B} & -1.281386 & -1.089972 & -1.711613\end{array}$

$\begin{array}{llll}24 \mathrm{~B} & 0.293492 & 1.656459 & -1.711612\end{array}$

$\begin{array}{llll}25 B & -0.777380 & 2.270525 & 0.001620\end{array}$

$\begin{array}{llll}26 \mathrm{~A} & 1.098715 & -0.630033 & 0.912581\end{array}$

$\begin{array}{llll}27 \mathrm{~B} & -1.765561 & 1.012421 & -1.466458\end{array}$

$\begin{array}{llll}28 \mathrm{~B} & -1.763504 & 1.011241 & 1.470548\end{array}$

$\begin{array}{lllll}29 B & 0.651942 & -0.373840 & -2.393801\end{array}$

$\begin{array}{llll}30 A & -1.334244 & 0.765092 & 0.002012\end{array}$

$\begin{array}{lllll}31 \mathrm{~A} & -0.420646 & 0.241210 & -1.459607\end{array}$

$\begin{array}{llll}32 \mathrm{~A} & -0.422651 & 0.242360 & 1.442757\end{array}$

$\begin{array}{lllll}33 \mathrm{~A} & 1.053466 & -0.604087 & -0.918809\end{array}$

$\begin{array}{lllll}34 \mathrm{~B} & -1.332442 & -2.329237 & 0.002002\end{array}$

$\begin{array}{llll}35 \mathrm{~B} & 1.337266 & 2.326471 & 0.002002\end{array}$

$\begin{array}{llll}36 \mathrm{~B} & 2.316720 & -1.328469 & 1.924244\end{array}$

$E 37 / \varepsilon=-200.863152$

$\begin{array}{llll}\text { atom } & \mathrm{x} / \sigma & \mathrm{y} / \sigma & \mathrm{z} / \sigma\end{array}$

$\begin{array}{llll}1 \mathrm{~A} & 0.000000 & 0.000000 & 0.000000\end{array}$

$\begin{array}{llll}2 \mathrm{~B} & 1.826224 & 0.000000 & 0.000000\end{array}$

$\begin{array}{llll}3 \mathrm{~A} & -0.612460 & -0.892002 & 0.000000\end{array}$

$\begin{array}{lllll}4 \mathrm{~A} & 0.543091 & -0.928664 & -0.000003\end{array}$

$\begin{array}{lllll}5 A & -0.571739 & -0.310856 & -0.889898\end{array}$

$\begin{array}{llll}6 \mathrm{~A} & -0.571736 & -0.310862 & 0.889900\end{array}$

$\begin{array}{llll}7 \mathrm{~A} & -0.553685 & 0.743844 & 0.557725\end{array}$

$\begin{array}{llll}8 \mathrm{~A} & -0.553687 & 0.743849 & -0.557716\end{array}$

$\begin{array}{lllll}9 \mathrm{~A} & 0.517880 & -0.337383 & 0.913238\end{array}$

$\begin{array}{lllll}10 \mathrm{~A} & 0.517878 & -0.337376 & -0.913240\end{array}$

11B $\quad-1.698540 \quad-1.401488-1.038181$

$\begin{array}{llll}12 \mathrm{~B} & -1.698536 & -1.401496 & 1.038178\end{array}$

$\begin{array}{llll}13 \mathrm{~A} & 0.549068 & 0.744969 & 0.575654\end{array}$

$\begin{array}{llll}14 \mathrm{~A} & 0.549067 & 0.744974 & -0.575647\end{array}$

$\begin{array}{llll}15 A & -1.172111 & 0.033019 & 0.000003\end{array}$

$\begin{array}{llll}16 A & -0.066255 & -1.336133 & -0.799076\end{array}$

$\begin{array}{llll}17 A & -0.066252 & -1.336140 & 0.799070\end{array}$

$\begin{array}{llll}18 \mathrm{~B} & -0.118386 & -0.897743 & 2.312147\end{array}$

$\begin{array}{llll}19 B & -0.118392 & -0.897725 & -2.312150\end{array}$

$\begin{array}{llll}20 \mathrm{~B} & -1.580504 & 1.801104 & 0.000011\end{array}$

$\begin{array}{llll}21 \mathrm{~B} & -1.612279 & 0.561162 & -1.704080\end{array}$

$\begin{array}{llll}22 B & -1.612275 & 0.561149 & 1.704091\end{array}$

$\begin{array}{llll}23 B & 1.467250 & -1.616332 & -1.150305\end{array}$

$\begin{array}{llll}24 \mathrm{~B} & 1.467252 & -1.616341 & 1.150293\end{array}$

$\begin{array}{llll}25 \mathrm{~B} & -0.049942 & 1.995859 & -1.483907\end{array}$

$\begin{array}{llll}26 \mathrm{~B} & -0.049939 & 1.995847 & 1.483926\end{array}$

$\begin{array}{llll}27 \mathrm{~A} & -0.013455 & 1.500642 & 0.000007\end{array}$

$\begin{array}{lllr}28 A & -0.039711 & 0.434380 & -1.467984\end{array}$

$\begin{array}{llll}29 A & -0.039707 & 0.434369 & 1.467990\end{array}$

$\begin{array}{lllll}30 \mathrm{~A} & 0.433172 & -2.001507 & -0.000007\end{array}$

$\begin{array}{lllll}31 \mathrm{~B} & 1.445217 & 0.522460 & -1.924642\end{array}$

$\begin{array}{llll}32 \mathrm{~B} & 1.445221 & 0.522445 & 1.924646\end{array}$

$\begin{array}{llll}33 \mathrm{~A} & -0.642221 & -1.958981 & -0.000005\end{array}$

$\begin{array}{llll}34 \mathrm{~B} & 1.465195 & 1.984001 & 0.000008\end{array}$

$\begin{array}{llll}35 \mathrm{~B} & -0.196814 & -2.871227 & 1.203537\end{array}$

$\begin{array}{llll}36 \mathrm{~B} & -0.196817 & -2.871215 & -1.203558\end{array}$

$\begin{array}{llll}37 \mathrm{~B} & -2.752467 & 0.075879 & 0.000006\end{array}$

$E 38 / \varepsilon=-208.347221$

$\begin{array}{llll}\text { atom } & \mathrm{x} / \sigma & \mathrm{y} / \sigma & \mathrm{z} / \sigma\end{array}$

$\begin{array}{llll}1 \mathrm{~A} & 0.000000 & 0.000000 & 0.000000\end{array}$

$\begin{array}{llll}2 \mathrm{~A} & 1.074152 & 0.000000 & 0.000000\end{array}$

$\begin{array}{llll}3 \mathrm{~A} & 0.469631 & -0.966048 & 0.000000\end{array}$

$\begin{array}{llll}4 \mathrm{~A} & -0.017516 & -0.626767 & -0.894968\end{array}$

$\begin{array}{lllll}5 A & 0.556031 & 0.289783 & -0.894968\end{array}$

$\begin{array}{lllll}6 \mathrm{~A} & 0.556030 & 0.289783 & 0.894968\end{array}$

$\begin{array}{llll}7 \mathrm{~A} & -0.017517 & -0.626767 & 0.894967\end{array}$

$\begin{array}{llll}8 \mathrm{~A} & -0.919763 & -0.064810 & -0.562252\end{array}$

$\begin{array}{llll}9 \mathrm{~A} & -0.343842 & 0.855534 & -0.562252\end{array}$

$\begin{array}{llll}10 A & -0.919765 & -0.064810 & 0.562249\end{array}$

$\begin{array}{llll}11 \mathrm{~A} & -0.343843 & 0.855534 & 0.562250\end{array}$

$\begin{array}{llll}12 \mathrm{~B} & 2.088919 & 0.591897 & 1.051301\end{array}$

$\begin{array}{llll}13 B & 0.380971 & -2.137473 & -1.051300\end{array}$

$\begin{array}{llll}14 \mathrm{~B} & 0.380968 & -2.137473 & 1.051300\end{array}$

$\begin{array}{llll}15 B & 2.088921 & 0.591896 & -1.051299\end{array}$ 


$\begin{array}{rrrr}\text { 16A } & 0.589637 & 0.969472 & 0.000000 \\ \text { 17A } & -0.614108 & -0.954158 & -0.000001 \\ \text { 18A } & 1.127026 & -0.705256 & 0.802614 \\ \text { 19A } & 1.127027 & -0.705257 & -0.802613 \\ \text { 20B } & 0.753510 & -0.471522 & -2.306584 \\ \text { 21B } & 0.753506 & -0.471520 & 2.306584 \\ \text { 22B } & -0.669441 & 2.282091 & -0.000002 \\ \text { 23B } & -2.345107 & -0.395684 & -0.000003 \\ \text { 24B } & 0.387988 & 1.619933 & 1.715125 \\ \text { 25B } & -1.287271 & -1.057192 & 1.715124 \\ \text { 26B } & 0.387991 & 1.619932 & -1.715128 \\ \text { 27B } & -1.287267 & -1.057193 & -1.715128 \\ \text { 28B } & -1.692399 & 1.059048 & 1.476880 \\ \text { 29B } & -1.692396 & 1.059048 & -1.476885 \\ \text { 30A } & -0.372649 & 0.233191 & 1.471665 \\ \text { 31A } & -0.372646 & 0.233190 & -1.471667 \\ \text { 32A } & -1.282693 & 0.802668 & -0.000002 \\ \text { 33A } & 1.966306 & -0.595979 & 0.000001 \\ \text { 34A } & 1.395689 & -1.507848 & 0.000001 \\ \text { 35B } & 2.407984 & -1.506839 & 1.232414 \\ \text { 36B } & 2.407987 & -1.506840 & -1.232409 \\ \text { 37B } & -1.432923 & -2.288397 & -0.000003 \\ \text { 38B } & 1.431604 & 2.289223 & -0.000001\end{array}$

$E 39 / \varepsilon=-214.480766$

$\begin{array}{cccc}\text { atom } & \mathrm{X} / \sigma & y / \sigma & z / \sigma \\ 1 \mathrm{~A} & 0.000000 & 0.000000 & 0.000000\end{array}$

$\begin{array}{llll}2 \mathrm{~B} & 2.407432 & 0.000000 & 0.000000\end{array}$

$\begin{array}{llll}3 B & 1.473740 & -1.903633 & 0.000000\end{array}$

$\begin{array}{lllll}4 \mathrm{~A} & 0.929934 & -0.456113 & -0.296730\end{array}$

$\begin{array}{lllll}5 A & 0.166272 & -0.081553 & -1.057052\end{array}$

$\begin{array}{llll}6 \mathrm{~A} & 0.942644 & 0.533719 & 0.125997\end{array}$

$\begin{array}{llll}7 A & 0.155023 & -1.072102 & 0.125996\end{array}$

$\begin{array}{llll}8 \mathrm{~A} & -0.566651 & -0.718580 & -0.596591\end{array}$

$\begin{array}{llll}9 \mathrm{~A} & 0.221321 & 0.887957 & -0.596591\end{array}$

$\begin{array}{llll}10 A & -1.058185 & -0.105701 & 0.192148\end{array}$

$\begin{array}{llll}11 \mathrm{~A} & -0.564200 & 0.901446 & 0.192148\end{array}$

$\begin{array}{llll}12 \mathrm{~A} & -0.333453 & -0.461744 & 0.918027\end{array}$

$\begin{array}{llll}13 \mathrm{~A} & 0.160988 & 0.546334 & 0.918027\end{array}$

$\begin{array}{llll}14 \mathrm{~B} & -0.670112 & -0.837311 & -2.156671\end{array}$

$\begin{array}{llll}15 B & 0.251871 & 1.042450 & -2.156671\end{array}$

$\begin{array}{llll}16 \mathrm{~A} & 0.748216 & -0.366984 & 0.768446\end{array}$

$\begin{array}{llll}17 \mathrm{~A} & -0.767070 & 0.376232 & -0.748507\end{array}$

$\begin{array}{lllll}18 \mathrm{~A} & 1.150209 & 0.330027 & -0.985665\end{array}$

$\begin{array}{llll}19 A & 0.443153 & -1.111537 & -0.985665\end{array}$

$\begin{array}{llll}20 \mathrm{~B} & 1.542512 & 1.813600 & -0.659897\end{array}$

$\begin{array}{llll}21 B & -0.489803 & -2.329931 & -0.659897\end{array}$

$\begin{array}{llll}22 \mathrm{~B} & -0.012864 & 0.006310 & 2.379426\end{array}$

$\begin{array}{llll}23 \mathrm{~B} & 1.486485 & 1.182259 & 1.453100\end{array}$

$\begin{array}{llll}24 \mathrm{~B} & -0.024879 & -1.899146 & 1.453100\end{array}$

$\begin{array}{llll}25 \mathrm{~B} & -2.124102 & 1.041827 & 0.272709\end{array}$

$\begin{array}{llll}26 \mathrm{~B} & -0.621312 & 2.214514 & -0.657081\end{array}$

$\begin{array}{llll}27 \mathrm{~B} & -2.131431 & -0.864351 & -0.657082\end{array}$

$\begin{array}{llll}28 \mathrm{~A} & 1.556665 & -0.763512 & -1.117439\end{array}$

$\begin{array}{llll}29 B & -1.850296 & -0.736596 & 1.487183\end{array}$

$\begin{array}{llll}30 \mathrm{~B} & -0.550232 & 1.914004 & 1.487183\end{array}$

$\begin{array}{llll}31 \mathrm{~A} & 0.380920 & 1.451060 & 0.328705\end{array}$

$\begin{array}{llll}32 \mathrm{~A} & -0.914215 & -1.189491 & 0.328705\end{array}$

$\begin{array}{llll}33 \mathrm{~A} & -0.909376 & 0.446030 & 1.127208\end{array}$

$\begin{array}{lllll}34 \mathrm{~A} & 0.834628 & -0.409367 & -1.830944\end{array}$

$\begin{array}{lllll}35 \mathrm{~B} & 2.231261 & 0.313381 & -2.115212\end{array}$

$\begin{array}{llll}36 \mathrm{~B} & 1.118094 & -1.956170 & -2.115212\end{array}$

$\begin{array}{llll}37 \mathrm{~B} & 1.802247 & -0.883964 & 1.803079\end{array}$

$\begin{array}{lllll}38 \mathrm{~B} & -1.812271 & 0.888881 & -1.797123\end{array}$

$\begin{array}{llll}39 \mathrm{~B} & 3.006139 & -1.474448 & -1.353025\end{array}$

$E 40 / \varepsilon=-220.807300$

atom $\mathrm{X} / \sigma$

$$
y / \sigma
$$

$z / \sigma$

$\begin{array}{llll}1 \mathrm{~A} & 0.000000 & 0.000000 & 0.000000\end{array}$

$\begin{array}{llll}2 \mathrm{~B} & 2.404690 & 0.000000 & 0.000000\end{array}$

$\begin{array}{llll}3 B & -0.682101 & -2.305921 & 0.000000\end{array}$

$\begin{array}{llll}4 B & 0.242964 & -1.614871 & 1.765132\end{array}$

$\begin{array}{lllll}5 B & 1.479624 & -0.691051 & -1.765133\end{array}$

$\begin{array}{llll}6 \mathrm{~A} & 0.928930 & -0.444735 & -0.312717\end{array}$ $\begin{array}{llll}7 A & 0.162973 & -1.016927 & 0.312717\end{array}$

$\begin{array}{lllll}8 A & 0.157080 & -0.272663 & -1.043983\end{array}$

$\begin{array}{lllll}9 \mathrm{~A} & -0.567345 & -0.813829 & -0.452462\end{array}$

$\begin{array}{llll}10 \mathrm{~A} & 0.941331 & 0.313196 & 0.452461\end{array}$

$\begin{array}{llll}11 \mathrm{~A} & 0.216907 & -0.227970 & 1.043982\end{array}$

$\begin{array}{llll}12 \mathrm{~A} & -1.057663 & 0.144432 & -0.171304\end{array}$

$\begin{array}{llll}13 A & -0.329601 & 0.688315 & -0.765795\end{array}$

$\begin{array}{llll}14 A & -0.566552 & 0.511306 & 0.765794\end{array}$

$\begin{array}{llll}15 A & 0.161510 & 1.055190 & 0.171303\end{array}$

$\begin{array}{llll}16 \mathrm{~A} & -0.769515 & -0.556585 & 0.621188\end{array}$

$\begin{array}{llll}17 A & 0.752000 & 0.580030 & -0.621188\end{array}$

$\begin{array}{llll}18 \mathrm{~A} & 0.438338 & -1.321793 & -0.672447\end{array}$

$\begin{array}{llll}19 \mathrm{~A} & 1.143165 & -0.795267 & 0.672446\end{array}$

$\begin{array}{llll}20 \mathrm{~B} & -0.490833 & -1.455916 & -1.933092\end{array}$

$\begin{array}{llll}21 B & 1.535343 & 0.057696 & 1.933091\end{array}$

$\begin{array}{llll}22 \mathrm{~B} & 1.485911 & 1.785561 & 0.571593\end{array}$

$\begin{array}{llll}23 \mathrm{~B} & -2.133707 & -0.918399 & -0.571596\end{array}$

$\begin{array}{llll}24 B & -0.629390 & 0.205370 & 2.298820\end{array}$

$\begin{array}{llll}25 \mathrm{~B} & -0.018407 & 0.661791 & -2.298822\end{array}$

$\begin{array}{llll}26 \mathrm{~B} & -0.010200 & 2.220525 & -0.863993\end{array}$

$\begin{array}{llll}27 \mathrm{~B} & -2.126429 & 0.639642 & 0.863990\end{array}$

$\begin{array}{llll}28 \mathrm{~A} & 0.831645 & -1.862524 & 0.293332\end{array}$

$\begin{array}{lllll}29 A & 1.550123 & -1.325800 & -0.293334\end{array}$

$\begin{array}{llll}30 \mathrm{~B} & 2.245157 & -1.807966 & 1.095545\end{array}$

$\begin{array}{llll}31 \mathrm{~B} & 1.096856 & -2.665779 & -1.095547\end{array}$

$\begin{array}{llll}32 \mathrm{~B} & -0.551942 & 2.088315 & 1.234588\end{array}$

$\begin{array}{llll}33 \mathrm{~B} & -1.845981 & 1.121630 & -1.234591\end{array}$

$\begin{array}{llll}34 \mathrm{~A} & 0.376552 & 0.839430 & 1.229128\end{array}$

$\begin{array}{llll}35 \mathrm{~A} & -0.911763 & -0.122979 & -1.229129\end{array}$

$\begin{array}{llll}36 \mathrm{~A} & -0.908451 & 1.216084 & -0.000001\end{array}$

$\begin{array}{llll}37 \mathrm{~B} & 1.804948 & 1.360313 & -1.478497\end{array}$

$\begin{array}{llll}38 \mathrm{~B} & -1.816424 & -1.344954 & 1.478494\end{array}$

$\begin{array}{lllll}39 \mathrm{~B} & 2.988624 & -1.823609 & -0.852325\end{array}$

$\begin{array}{llll}\text { 40B } & 0.900970 & -3.383146 & 0.852324\end{array}$

\section{$E 41 / \varepsilon=-226.951993$}

atom $\mathrm{x} / \sigma \quad \mathrm{g} / \sigma$

$\begin{array}{llll}1 \mathrm{~A} & 0.000000 & 0.000000 & 0.000000\end{array}$

$\begin{array}{llll}2 \mathrm{~A} & 1.067006 & 0.000000 & 0.000000\end{array}$

$\begin{array}{llll}3 \mathrm{~A} & -0.902651 & -0.568966 & 0.000000\end{array}$

$\begin{array}{llll}4 \mathrm{~A} & 0.462818 & -0.174529 & -0.945432\end{array}$

$\begin{array}{llll}5 A & -0.298462 & -0.394437 & 0.945432\end{array}$

$\begin{array}{llll}6 \mathrm{~A} & 0.551667 & -0.836210 & 0.447892\end{array}$

$\begin{array}{llll}7 A & -0.020794 & -1.001574 & -0.447892\end{array}$

$\begin{array}{llll}8 A & -0.372793 & 0.672881 & 0.761949\end{array}$

$\begin{array}{lllll}9 A & 0.530669 & 0.933860 & 0.136403\end{array}$

$\begin{array}{lllll}10 A & -0.946895 & 0.507043 & -0.136403\end{array}$

$\begin{array}{llll}11 \mathrm{~A} & -0.043433 & 0.768022 & -0.761948\end{array}$

$\begin{array}{llll}12 \mathrm{~B} & -0.646669 & 0.370252 & 2.275996\end{array}$

$\begin{array}{llll}13 B & 0.349630 & 0.658049 & -2.275996\end{array}$

$\begin{array}{llll}14 \mathrm{~B} & -2.357727 & -0.124013 & -0.401457\end{array}$

$\begin{array}{llll}15 \mathrm{~B} & 2.060688 & 1.152314 & 0.401457\end{array}$

$\begin{array}{llll}16 \mathrm{~B} & 0.382561 & -1.416753 & -1.909905\end{array}$

$\begin{array}{llll}17 \mathrm{~B} & 0.431831 & -1.402521 & 1.909904\end{array}$

$\begin{array}{llll}18 \mathrm{~B} & -1.275574 & -1.895732 & -0.761833\end{array}$

$\begin{array}{llll}19 B & 2.089966 & -0.923543 & 0.761833\end{array}$

$\begin{array}{lllll}20 B & 0.679877 & -2.353609 & 0.000000\end{array}$

$\begin{array}{llll}21 \mathrm{~A} & -0.617025 & -0.161502 & -0.958537\end{array}$

$\begin{array}{llll}22 \mathrm{~A} & 0.608102 & 0.192395 & 0.958537\end{array}$

$\begin{array}{llll}23 \mathrm{~A} & 1.115318 & -0.924305 & -0.542127\end{array}$

$\begin{array}{llll}24 \mathrm{~A} & -0.450648 & -1.376659 & 0.542127\end{array}$

$\begin{array}{llll}25 \mathrm{~A} & 1.101109 & 0.675445 & -0.828365\end{array}$

$\begin{array}{llll}26 \mathrm{~A} & -1.291672 & -0.015747 & 0.828365\end{array}$

$\begin{array}{llll}27 \mathrm{~B} & -1.755309 & 1.484749 & 0.847662\end{array}$

$\begin{array}{llll}28 \mathrm{~B} & 0.693212 & 2.192043 & -0.847661\end{array}$

$\begin{array}{llll}29 B & 0.344814 & 1.952757 & 1.312702\end{array}$

$\begin{array}{llll}30 \mathrm{~B} & -1.332980 & 1.468101 & -1.312701\end{array}$

$\begin{array}{llll}31 \mathrm{~A} & -0.423339 & 1.465525 & 0.000000\end{array}$

$\begin{array}{llll}32 \mathrm{~A} & 1.957480 & -0.094150 & -0.588504\end{array}$

$\begin{array}{llll}33 \mathrm{~A} & -1.605757 & -1.123447 & 0.588504\end{array}$

$\begin{array}{llll}34 \mathrm{~A} & -1.034190 & -0.958340 & 1.482890\end{array}$

$\begin{array}{llll}35 \mathrm{~A} & 1.385913 & -0.259256 & -1.482890\end{array}$

$\begin{array}{llll}36 \mathrm{~B} & 2.372281 & 0.978068 & -1.696587\end{array}$ \begin{tabular}{|llll}
$37 \mathrm{~B}$ & -2.528410 & -0.437571 & 1.696587
\end{tabular}

$\begin{array}{llll}38 \mathrm{~B} & 2.395531 & -1.478548 & -1.257948\end{array}$

$\begin{array}{llll}39 \mathrm{~B} & -1.238123 & -2.528185 & 1.257948\end{array}$

$\begin{array}{lllll}40 \mathrm{~B} & 1.448897 & 0.409340 & 2.263387\end{array}$

$\begin{array}{llll}41 B & -1.443991 & -0.426316 & -2.263387\end{array}$

\section{$E 42 / \varepsilon=-233.679219$}

atom $\mathrm{X} / \sigma$

$y / \sigma$

$\begin{array}{llll}1 \mathrm{~A} & 0.000000 & 0.000000 & 0.000000\end{array}$

$\begin{array}{llll}2 \mathrm{~A} & 1.121913 & 0.000000 & 0.000000\end{array}$

$\begin{array}{llll}3 \mathrm{~A} & 0.586700 & -0.916266 & 0.000000\end{array}$

$\begin{array}{lllll}4 \mathrm{~A} & 0.586699 & 0.781839 & -0.477778\end{array}$

$\begin{array}{llll}5 \mathrm{~A} & 0.751320 & 0.822430 & 0.621834\end{array}$

$\begin{array}{llll}6 \mathrm{~A} & 0.751320 & -0.377520 & 0.959451\end{array}$

$\begin{array}{lllll}7 A & 0.709937 & -0.272895 & -0.969919\end{array}$

$\begin{array}{llll}8 \mathrm{~B} & 0.682761 & 2.297003 & -0.115002\end{array}$

$\begin{array}{llll}9 B & 0.682761 & -2.019971 & 1.099620\end{array}$

$\begin{array}{llll}\text { 10B } & 2.848720 & -0.018287 & -0.064993\end{array}$

11B $\quad 0.696312 \quad-2.041388-1.081226$

$\begin{array}{llll}12 \mathrm{~B} & 0.696312 & 1.178099 & -1.987058\end{array}$

$\begin{array}{lllll}13 \mathrm{~A} & -0.209649 & 0.276431 & -1.048260\end{array}$

$\begin{array}{llll}14 \mathrm{~A} & -0.209648 & -0.782479 & -0.750325\end{array}$

$\begin{array}{llll}15 \mathrm{~B} & -1.569161 & 0.335707 & 1.193157\end{array}$

$\begin{array}{llll}16 \mathrm{~A} & -0.991193 & -0.580175 & -0.022222\end{array}$

$\begin{array}{lllll}17 \mathrm{~A} & -0.991193 & 0.483469 & -0.321488\end{array}$

$\begin{array}{llll}18 A & -0.254768 & 1.027827 & 0.271361\end{array}$

$\begin{array}{llll}19 A & -0.254768 & -0.735533 & 0.767500\end{array}$

$\begin{array}{llll}20 B & -0.180280 & -0.656004 & -2.331557\end{array}$

$\begin{array}{llll}21 B & -0.096842 & 1.713376 & 1.716521\end{array}$

$\begin{array}{llll}22 \mathrm{~B} & -0.096843 & -0.566939 & 2.358109\end{array}$

$\begin{array}{llll}23 \mathrm{~A} & -0.061812 & 0.297950 & 1.058966\end{array}$

$\begin{array}{llll}24 \mathrm{~A} & 1.608698 & -0.838612 & 0.518435\end{array}$

$\begin{array}{llll}25 A & 1.608698 & 0.985910 & 0.005088\end{array}$

$\begin{array}{lllll}26 \mathrm{~A} & 1.560407 & 0.402879 & -0.905331\end{array}$

$\begin{array}{lllll}27 A & 1.560406 & -0.815847 & -0.562431\end{array}$

$\begin{array}{llll}28 \mathrm{~A} & 1.651174 & 0.246917 & 0.877585\end{array}$

$\begin{array}{lllll}29 B & -1.417819 & 1.018590 & -1.862283\end{array}$

$\begin{array}{lllll}30 \mathrm{~B} & -1.417815 & -1.840222 & -1.057932\end{array}$

$\begin{array}{llll}31 \mathrm{~B} & 1.991415 & -0.685303 & 2.046559\end{array}$

$\begin{array}{llll}32 B & 1.991416 & 1.651919 & 1.388959\end{array}$

$\begin{array}{lllll}33 \mathrm{~B} & 1.919514 & -0.578809 & -2.057190\end{array}$

$\begin{array}{llll}34 \mathrm{~B} & -1.481693 & -1.704188 & 1.064941\end{array}$

$\begin{array}{llll}35 \mathrm{~B} & -1.481691 & 2.009470 & 0.020070\end{array}$

$\begin{array}{llll}36 \mathrm{~A} & 0.895715 & 0.465010 & 1.652725\end{array}$

$\begin{array}{llll}37 A & -0.336288 & -1.520090 & 0.020463\end{array}$

$\begin{array}{llll}38 A & -0.336288 & 1.307745 & -0.775175\end{array}$

$\begin{array}{llll}39 A & -1.119405 & -0.306656 & -1.089905\end{array}$

$\begin{array}{lllll}40 \mathrm{~B} & -2.537271 & -0.139946 & -0.497374\end{array}$

$\begin{array}{llll}41 \mathrm{~B} & 2.467779 & 1.737095 & -1.077912\end{array}$

$\begin{array}{llll}42 B & 2.467779 & -2.044309 & -0.013980\end{array}$

\section{$E 43 / \varepsilon=-240.538283$}

atom $\mathrm{X} / \sigma$

$y / \sigma$

$\begin{array}{lll}1 \mathrm{~A} & 0.000000 & 0.000000\end{array}$

$z / \sigma$

$\begin{array}{llll}3 \mathrm{~A} & 0.580176 & -0.912895 & 0.000000\end{array}$

$\begin{array}{llll}4 \mathrm{~A} & 0.580174 & 0.789469 & 0.458384\end{array}$

$\begin{array}{llll}5 \mathrm{~A} & 0.737823 & 0.789551 & -0.648207\end{array}$

$\begin{array}{lllll}6 \mathrm{~A} & 0.737825 & -0.357323 & -0.957018\end{array}$

$\begin{array}{llll}7 A & 0.728109 & -0.260951 & 0.969127\end{array}$

$\begin{array}{lllll}8 B & 2.829952 & -0.019708 & 0.073206\end{array}$

$\begin{array}{lllll}9 \mathrm{~B} & 0.645431 & -2.005917 & -1.113735\end{array}$

$\begin{array}{llll}10 \mathrm{~B} & 0.645424 & 2.293942 & 0.044059\end{array}$

$\begin{array}{llll}11 \mathrm{~B} & 0.683031 & -2.047374 & 1.083227\end{array}$

$\begin{array}{llll}12 \mathrm{~B} & 0.683025 & 1.226651 & 1.964803\end{array}$

$\begin{array}{llll}13 \mathrm{~A} & -0.188254 & 0.283330 & 1.069915\end{array}$

$\begin{array}{llll}14 \mathrm{~A} & -0.188253 & -0.782253 & 0.782993\end{array}$

$\begin{array}{lllll}15 A & -0.259429 & -0.709083 & -0.763414\end{array}$

$\begin{array}{lllll}16 A & -0.259432 & 0.996538 & -0.304153\end{array}$

$\begin{array}{llll}17 A & -0.956699 & 0.480751 & 0.369969\end{array}$

$\begin{array}{llll}18 A & -0.956698 & -0.601526 & 0.078552\end{array}$

$\begin{array}{llll}19 B & -0.115247 & -0.565753 & -2.384995\end{array}$

$\begin{array}{llll}20 B & -0.115251 & 1.686816 & -1.778462\end{array}$ 


$\begin{array}{lrrr}21 B & -0.174875 & -0.632960 & 2.350706 \\ 22 A & -0.081353 & 0.302926 & -1.125023 \\ 23 A & -1.008771 & 0.174400 & -0.647702 \\ 24 B & -2.421988 & -0.047651 & 0.176950 \\ 25 B & -1.556626 & -1.464835 & -1.104944 \\ 26 B & -1.556632 & 1.821595 & -0.220030 \\ 27 A & 1.593105 & 0.993973 & -0.038649 \\ 28 A & 1.593109 & -0.840176 & -0.532518 \\ 29 A & 1.651037 & 0.240356 & -0.892637 \\ 30 A & 1.552147 & -0.837330 & 0.550764 \\ 31 A & 1.552144 & 0.447572 & 0.896742 \\ 32 B & -1.453384 & 1.050632 & 1.806604 \\ 33 B & -1.453379 & -1.815724 & 1.034800 \\ 34 B & 1.914991 & -0.549079 & 2.039198 \\ 35 B & 1.975247 & -0.701801 & -2.060417 \\ 36 B & 1.975243 & 1.641500 & -1.429452 \\ 37 A & -0.348959 & 1.318403 & 0.738000 \\ 38 A & -0.348955 & -1.510720 & -0.023778 \\ 39 A & 0.897631 & 0.452980 & -1.682293 \\ 40 A & -1.123631 & -0.300386 & 1.115574 \\ 41 B & -1.856944 & 0.506562 & -1.881306 \\ 42 B & 2.445228 & 1.788033 & 1.013708 \\ 43 B & 2.445235 & -2.055284 & -0.021159\end{array}$

$E 44 / \varepsilon=-248.636081$

$\begin{array}{cccc}\text { atom } & \mathrm{x} / \sigma & y / \sigma & z / \sigma \\ 1 \mathrm{~A} & 0.000000 & 0.000000 & 0.000000\end{array}$ $\begin{array}{llll}2 \mathrm{~A} & 1.106139 & 0.000000 & 0.000000\end{array}$

$\begin{array}{llll}3 A & 0.553070 & -0.924395 & 0.000000\end{array}$

$\begin{array}{llll}4 \mathrm{~A} & 0.553070 & 0.704833 & 0.598094\end{array}$

$\begin{array}{lllll}5 \mathrm{~B} & 0.553072 & -2.124273 & 0.946608\end{array}$

$\begin{array}{llll}6 \mathrm{~B} & 0.553072 & 1.007253 & 2.096199\end{array}$

$\begin{array}{llll}7 B & -0.918289 & 0.530072 & -1.443938\end{array}$

$\begin{array}{lllll}8 \mathrm{~B} & 2.024425 & 0.530075 & -1.443942\end{array}$

$\begin{array}{llll}9 \mathrm{~A} & 0.553071 & -0.355266 & 0.967757\end{array}$

$\begin{array}{llll}10 \mathrm{~A} & 0.553069 & 0.325121 & -0.885642\end{array}$

$\begin{array}{llll}11 \mathrm{~A} & -0.343503 & 0.222225 & 1.019991\end{array}$

$\begin{array}{llll}12 \mathrm{~A} & -0.343503 & -0.829387 & 0.633942\end{array}$

$\begin{array}{llll}13 \mathrm{~A} & 1.449645 & 0.222226 & 1.019990\end{array}$

$\begin{array}{llll}14 \mathrm{~A} & 1.449646 & -0.829387 & 0.633940\end{array}$

$\begin{array}{llll}15 \mathrm{~A} & 1.118958 & 1.070558 & -0.250896\end{array}$

$\begin{array}{llll}16 \mathrm{~A} & -0.012820 & 1.070558 & -0.250895\end{array}$

$\begin{array}{llll}17 A & 1.118958 & -0.653948 & -0.883967\end{array}$

$\begin{array}{llll}18 \mathrm{~A} & -0.012820 & -0.653949 & -0.883965\end{array}$

$\begin{array}{llll}19 A & -0.932796 & 0.538796 & 0.159891\end{array}$

$\begin{array}{llll}20 A & 2.038936 & 0.538798 & 0.159887\end{array}$

$\begin{array}{lllll}21 \mathrm{~A} & 2.038936 & -0.514271 & -0.226697\end{array}$

$\begin{array}{llll}22 \mathrm{~A} & -0.932797 & -0.514273 & -0.226693\end{array}$

$\begin{array}{llll}23 \mathrm{~B} & -0.507616 & -0.802824 & 2.186925\end{array}$

$\begin{array}{llll}24 \mathrm{~B} & 1.613763 & -0.802824 & 2.186921\end{array}$

$\begin{array}{llll}25 \mathrm{~B} & 0.553069 & -2.226511 & -1.204099\end{array}$

$\begin{array}{llll}26 \mathrm{~B} & 0.553069 & 2.476737 & 0.522472\end{array}$

$\begin{array}{llll}27 \mathrm{~B} & 2.717377 & 0.968122 & 1.656856\end{array}$

$\begin{array}{llll}28 \mathrm{~B} & 2.717379 & -1.810176 & 0.636935\end{array}$

$\begin{array}{llll}29 B & -1.611234 & 0.968120 & 1.656862\end{array}$

$\begin{array}{llll}30 \mathrm{~B} & -1.611235 & -1.810179 & 0.636943\end{array}$

$\begin{array}{llll}31 \mathrm{~B} & 0.553068 & -0.534256 & -2.363413\end{array}$

$\begin{array}{llll}32 \mathrm{~B} & 0.553067 & 1.936512 & -1.456389\end{array}$

$\begin{array}{lllll}33 \mathrm{~B} & -2.437792 & 0.054514 & -0.148495\end{array}$

$\begin{array}{llll}34 \mathrm{~B} & 3.543931 & 0.054518 & -0.148503\end{array}$

$\begin{array}{llll}35 \mathrm{~B} & -1.365164 & 2.039319 & -0.166887\end{array}$

$\begin{array}{llll}36 \mathrm{~B} & 2.471300 & -1.446963 & -1.446715\end{array}$

$\begin{array}{llll}37 \mathrm{~B} & 2.471302 & 2.039322 & -0.166891\end{array}$

$\begin{array}{llll}38 \mathrm{~B} & -1.365165 & -1.446966 & -1.446708\end{array}$

$\begin{array}{llll}39 \mathrm{~A} & 1.470357 & 1.267108 & 0.772843\end{array}$

$\begin{array}{llll}40 \mathrm{~A} & -0.364216 & 1.267107 & 0.772846\end{array}$

$\begin{array}{llll}41 \mathrm{~A} & 1.470357 & -1.466184 & -0.230554\end{array}$

$\begin{array}{llll}42 \mathrm{~A} & -0.364217 & -1.466185 & -0.230551\end{array}$

$\begin{array}{llll}43 \mathrm{~A} & 2.373466 & -0.299269 & 0.815221\end{array}$ $\begin{array}{llll}44 \mathrm{~A} & -1.267324 & -0.299271 & 0.815227\end{array}$

$E 45 / \varepsilon=-255.883412$

atom $\mathrm{x} / \sigma \quad \mathrm{y} / \sigma$

$z / \sigma$ $\begin{array}{llll}1 A & 0.000000 & 0.000000 & 0.000000\end{array}$

$\begin{array}{llll}2 A & 1.095783 & 0.000000 & 0.000000\end{array}$

$\begin{array}{llll}3 \mathrm{~A} & 0.537665 & -0.924597 & 0.000000\end{array}$

$\begin{array}{llll}4 \mathrm{~A} & 0.537663 & 0.732908 & 0.563671\end{array}$

$\begin{array}{lllll}5 \mathrm{~B} & 2.037670 & 0.492396 & -1.447911\end{array}$

$\begin{array}{llll}6 \mathrm{~A} & 0.513706 & -0.325872 & 0.958246\end{array}$

$\begin{array}{llll}7 \mathrm{~B} & 0.494926 & -2.108463 & 0.956830\end{array}$

$\begin{array}{llll}8 \mathrm{~B} & 0.494922 & 1.088014 & 2.043861\end{array}$

$\begin{array}{lllll}9 \mathrm{~A} & 0.554227 & 0.301635 & -0.886976\end{array}$

$\begin{array}{llll}10 A & -0.013945 & -0.645489 & -0.879416\end{array}$

$\begin{array}{llll}11 \mathrm{~A} & -0.013947 & 1.047791 & -0.303580\end{array}$

$\begin{array}{llll}12 \mathrm{~A} & -0.926961 & -0.495389 & -0.257229\end{array}$

$\begin{array}{llll}13 A & -0.926963 & 0.549498 & 0.098108\end{array}$

$\begin{array}{llll}14 \mathrm{~A} & -0.376907 & -0.814346 & 0.618444\end{array}$

$\begin{array}{llll}15 A & -0.376909 & 0.268485 & 0.986684\end{array}$

$\begin{array}{llll}16 \mathrm{~A} & 1.412113 & 0.252461 & 1.017337\end{array}$

$\begin{array}{llll}17 A & 1.412114 & -0.820327 & 0.652512\end{array}$

$\begin{array}{lllll}18 \mathrm{~A} & 1.117594 & 1.065107 & -0.298653\end{array}$

$\begin{array}{llll}19 A & 1.117596 & -0.662217 & -0.886067\end{array}$

$\begin{array}{llll}20 A & -0.555816 & 0.313256 & -0.921152\end{array}$

$\begin{array}{llll}21 \mathrm{~A} & 2.023640 & 0.538048 & 0.160257\end{array}$

$\begin{array}{llll}22 \mathrm{~A} & 2.023642 & -0.524196 & -0.200982\end{array}$

$\begin{array}{llll}23 B & 1.519989 & -0.750872 & 2.207986\end{array}$

$\begin{array}{llll}24 \mathrm{~B} & 0.379328 & 1.679863 & -1.658940\end{array}$

$\begin{array}{lllll}25 B & 0.379332 & -0.320240 & -2.339117\end{array}$

$\begin{array}{llll}26 \mathrm{~B} & 0.549587 & 2.469619 & 0.278973\end{array}$

$\begin{array}{llll}27 \mathrm{~B} & 0.549593 & -2.127689 & -1.284440\end{array}$

$\begin{array}{llll}28 \mathrm{~B} & -0.595750 & -0.733341 & 2.156424\end{array}$

$\begin{array}{llll}29 B & -1.331768 & 1.943520 & -0.452702\end{array}$

$\begin{array}{llll}30 \mathrm{~B} & -1.331764 & -1.264608 & -1.543695\end{array}$

$\begin{array}{lllll}31 B & -2.374096 & 0.119720 & -0.352058\end{array}$

$\begin{array}{llll}32 \mathrm{~B} & 2.665223 & -1.805841 & 0.721192\end{array}$

$\begin{array}{llll}33 \mathrm{~B} & 2.665219 & 0.991790 & 1.672588\end{array}$

$\begin{array}{llll}34 \mathrm{~B} & -1.681425 & 1.055086 & 1.501340\end{array}$

$\begin{array}{llll}35 \mathrm{~B} & -1.681420 & -1.751627 & 0.546857\end{array}$

$\begin{array}{llll}36 \mathrm{~B} & 3.529631 & 0.033463 & -0.098389\end{array}$

$\begin{array}{lllll}37 \mathrm{~A} & 1.451678 & -1.473720 & -0.207732\end{array}$

$\begin{array}{llll}38 \mathrm{~A} & 1.451675 & 1.294831 & 0.733774\end{array}$

$\begin{array}{llll}39 \mathrm{~A} & -0.384526 & 1.311531 & 0.690854\end{array}$

$\begin{array}{llll}40 A & -0.384522 & -1.460798 & -0.251936\end{array}$

$\begin{array}{llll}41 \mathrm{~A} & -1.301564 & -0.256985 & 0.755671\end{array}$

$\begin{array}{lllll}42 \mathrm{~A} & 2.331643 & -0.287080 & 0.844182\end{array}$

$\begin{array}{llll}43 \mathrm{~B} & 2.524357 & 2.005326 & -0.182489\end{array}$

$\begin{array}{llll}44 \mathrm{~B} & 2.524360 & -1.478323 & -1.367181\end{array}$

$\begin{array}{llll}45 \mathrm{~B} & -1.437441 & 0.731508 & -2.151052\end{array}$

\section{$E 46 / \varepsilon=-263.282585$}

atom $\mathrm{x} / \sigma \quad \mathrm{y} / \sigma$

$\begin{array}{llll}1 \mathrm{~A} & 0.000000 & 0.000000 & 0.000000\end{array}$

$\begin{array}{llll}2 \mathrm{~A} & 1.098640 & 0.000000 & 0.000000\end{array}$

$\begin{array}{llll}3 A & 0.550909 & -0.950532 & 0.000000\end{array}$

$\begin{array}{llll}4 \mathrm{~A} & 0.544366 & -0.313684 & 0.865023\end{array}$

$\begin{array}{lllll}5 A & 0.552416 & 0.949031 & 0.018733\end{array}$

$\begin{array}{llll}6 \mathrm{~A} & -0.544083 & -0.953834 & 0.018732\end{array}$

$\begin{array}{llll}7 \mathrm{~A} & 0.000776 & -0.634276 & -0.896435\end{array}$

$\begin{array}{lllll}8 A & 0.549156 & 0.317384 & -0.896435\end{array}$

$\begin{array}{lllll}9 \mathrm{~A} & 1.097047 & -0.632159 & -0.900113\end{array}$

$\begin{array}{llll}10 \mathrm{~A} & 1.647606 & -0.949411 & 0.005470\end{array}$

$\begin{array}{llll}11 B & 0.867541 & -0.499910 & 2.319711\end{array}$

$\begin{array}{llll}12 \mathrm{~A} & 1.100737 & 0.627811 & 0.923655\end{array}$

$\begin{array}{llll}13 A & 0.008786 & -1.267161 & 0.923655\end{array}$

$\begin{array}{llll}14 \mathrm{~A} & 0.005358 & 0.633009 & 0.919511\end{array}$

$\begin{array}{llll}15 A & -0.544986 & -0.322058 & 0.919511\end{array}$

$\begin{array}{llll}16 \mathrm{~B} & -0.499847 & 0.288030 & -2.092073\end{array}$

$\begin{array}{llll}17 \mathrm{~B} & 0.550112 & -1.527286 & -2.095797\end{array}$

$\begin{array}{llll}18 \mathrm{~B} & 1.597241 & 0.289904 & -2.095797\end{array}$

$\begin{array}{llll}19 \mathrm{~B} & -0.980214 & -2.430577 & 0.426240\end{array}$

$\begin{array}{llll}20 B & 1.611379 & 2.066875 & 0.426241\end{array}$

$\begin{array}{llll}21 B & -2.043811 & -0.602539 & 0.428254\end{array}$

$\begin{array}{llll}22 B & -0.503555 & 2.070421 & 0.428255\end{array}$

$\begin{array}{llll}23 \mathrm{~A} & 1.106221 & -1.265318 & 0.909890\end{array}$

$\begin{array}{llll}24 \mathrm{~A} & 1.649449 & -0.322600 & 0.909891\end{array}$

$\begin{array}{llll}25 A & -0.911609 & -0.110495 & -0.590284\end{array}$ \begin{tabular}{|llll}
$26 A$ & -0.361526 & 0.844120 & -0.590284
\end{tabular}

$\begin{array}{lllll}27 A & 1.461968 & 0.843669 & -0.594554\end{array}$

$\begin{array}{lllll}28 \mathrm{~A} & 0.003168 & -1.687934 & -0.594554\end{array}$

$\begin{array}{lllll}29 A & 1.102500 & -1.686342 & -0.595362\end{array}$

$\begin{array}{llll}30 \mathrm{~A} & 2.011848 & -0.108259 & -0.595362\end{array}$

$\begin{array}{llll}31 \mathrm{~A} & -0.907060 & 0.522680 & 0.310333\end{array}$

$\begin{array}{llll}32 \mathrm{~A} & 2.008035 & 0.523197 & 0.305123\end{array}$

$\begin{array}{llll}33 \mathrm{~A} & 0.554260 & -1.999685 & 0.305123\end{array}$

$\begin{array}{lllll}34 \mathrm{~B} & -1.368964 & -1.428208 & -1.337707\end{array}$

$\begin{array}{llll}35 \mathrm{~B} & 0.549204 & 1.900582 & -1.337706\end{array}$

$\begin{array}{lllll}36 \mathrm{~B} & 2.472060 & -1.424490 & -1.342418\end{array}$

$\begin{array}{lllll}37 \mathrm{~B} & 3.139957 & -0.611104 & 0.439290\end{array}$

$\begin{array}{lllll}38 \mathrm{~B} & 2.103244 & -2.410217 & 0.439290\end{array}$

$\begin{array}{llll}39 \mathrm{~B} & -1.322779 & 0.762231 & 1.822840\end{array}$

$\begin{array}{llll}40 \mathrm{~B} & 0.579904 & -2.504586 & 1.809829\end{array}$

$\begin{array}{llll}41 \mathrm{~B} & 2.457728 & 0.754191 & 1.809829\end{array}$

$\begin{array}{llll}42 \mathrm{~B} & -1.045989 & -1.253533 & 2.131968\end{array}$

$\begin{array}{llll}43 \mathrm{~B} & 0.560032 & 1.533556 & 2.131969\end{array}$

$\begin{array}{llll}44 \mathrm{~B} & -1.919231 & 1.105929 & -0.781073\end{array}$

$\begin{array}{llll}45 \mathrm{~B} & 3.025171 & 1.097889 & -0.783232\end{array}$

$\begin{array}{lllll}46 \mathrm{~B} & 0.567085 & -3.167877 & -0.783233\end{array}$

$E 47 / \varepsilon=-271.372063$

atom $\mathrm{x} / \sigma \quad \mathrm{y} / \sigma$

$\begin{array}{llll}1 \mathrm{~A} & 0.000000 & 0.000000 & 0.000000\end{array}$

$\begin{array}{llll}2 A & 1.102506 & 0.000000 & 0.000000\end{array}$

$\begin{array}{llll}3 \mathrm{~A} & 0.551253 & -0.954799 & 0.000000\end{array}$

$\begin{array}{llll}4 \mathrm{~B} & 0.551250 & -0.318269 & -2.330576\end{array}$

$\begin{array}{lllll}5 A & 0.551253 & -0.318267 & -0.850810\end{array}$

$\begin{array}{lllll}6 \mathrm{~A} & -0.545444 & -0.951445 & -0.020429\end{array}$

$\begin{array}{llll}7 A & 1.647950 & -0.951445 & -0.020430\end{array}$

$\begin{array}{llll}8 \mathrm{~A} & 0.551253 & 0.948090 & -0.020430\end{array}$

$\begin{array}{llll}9 A & 0.002969 & -0.634819 & 0.896663\end{array}$

$\begin{array}{llll}10 A & 1.099538 & -0.634819 & 0.896663\end{array}$

$\begin{array}{llll}11 \mathrm{~A} & 0.551253 & 0.314839 & 0.896663\end{array}$

$\begin{array}{llll}12 \mathrm{~A} & 1.103762 & -1.264169 & -0.929493\end{array}$

$\begin{array}{lllll}13 A & 0.008332 & 0.633172 & -0.929494\end{array}$

$\begin{array}{lllll}14 A & -0.544178 & -0.323804 & -0.929493\end{array}$

$\begin{array}{lllll}15 A & -0.001258 & -1.264169 & -0.929493\end{array}$

$\begin{array}{lllll}16 A & 1.646682 & -0.323804 & -0.929494\end{array}$

$\begin{array}{lllll}17 \mathrm{~A} & 1.094172 & 0.633172 & -0.929494\end{array}$

$\begin{array}{llll}18 B & 1.596471 & 0.285191 & 2.098981\end{array}$

$\begin{array}{llll}19 \mathrm{~B} & 0.551254 & -1.525179 & 2.098983\end{array}$

$\begin{array}{llll}20 B & -0.493963 & 0.285190 & 2.098983\end{array}$

$\begin{array}{lllll}21 \mathrm{~B} & 1.622533 & 2.068271 & -0.403315\end{array}$

$\begin{array}{llll}22 \mathrm{~B} & -0.520029 & 2.068271 & -0.403314\end{array}$

$\begin{array}{llll}23 \mathrm{~B} & -0.979910 & -2.439291 & -0.403312\end{array}$

$\begin{array}{lllll}24 \mathrm{~B} & 3.153695 & -0.583779 & -0.403315\end{array}$

$\begin{array}{lllll}25 B & 2.082414 & -2.439293 & -0.403313\end{array}$

$\begin{array}{llll}26 \mathrm{~B} & -2.051190 & -0.583779 & -0.403312\end{array}$

$\begin{array}{llll}27 \mathrm{~A} & -0.903213 & 0.521469 & -0.311119\end{array}$

$\begin{array}{lllll}28 A & 2.005718 & 0.521470 & -0.311121\end{array}$

$\begin{array}{llll}29 A & 0.551252 & -1.997739 & -0.311119\end{array}$

$\begin{array}{llll}30 \mathrm{~A} & -0.907963 & -0.109538 & 0.595706\end{array}$

$\begin{array}{llll}31 \mathrm{~A} & -0.359120 & 0.841088 & 0.595705\end{array}$

$\begin{array}{llll}32 \mathrm{~A} & 1.461626 & 0.841088 & 0.595704\end{array}$

$\begin{array}{llll}33 \mathrm{~A} & 0.002409 & -1.686350 & 0.595706\end{array}$

$\begin{array}{llll}34 \mathrm{~A} & 1.100097 & -1.686350 & 0.595705\end{array}$

$\begin{array}{lllll}35 \mathrm{~A} & 2.010470 & -0.109537 & 0.595704\end{array}$

$\begin{array}{llll}36 \mathrm{~B} & 2.469011 & -1.425484 & 1.347064\end{array}$

$\begin{array}{llll}37 \mathrm{~B} & -1.366504 & -1.425484 & 1.347066\end{array}$

$\begin{array}{llll}38 \mathrm{~B} & 0.551253 & 1.896170 & 1.347064\end{array}$

$\begin{array}{llll}39 B & 2.395973 & 0.746783 & -1.842095\end{array}$

$\begin{array}{lllll}40 \mathrm{~B} & 0.551251 & -2.448369 & -1.842092\end{array}$

$\begin{array}{llll}41 \mathrm{~B} & -1.293471 & 0.746783 & -1.842092\end{array}$

$\begin{array}{llll}42 \mathrm{~B} & 0.551252 & -3.165093 & 0.805123\end{array}$

$\begin{array}{lllll}43 \mathrm{~B} & -1.914171 & 1.105147 & 0.805124\end{array}$

$\begin{array}{llll}44 \mathrm{~B} & 3.016677 & 1.105148 & 0.805120\end{array}$

$\begin{array}{llll}45 \mathrm{~B} & 0.551251 & 1.682090 & -2.094389\end{array}$

$\begin{array}{lllll}46 \mathrm{~B} & 2.283612 & -1.318447 & -2.094388\end{array}$

$\begin{array}{lllll}47 \mathrm{~B} & -1.181111 & -1.318447 & -2.094387\end{array}$

$E 48 / \varepsilon=-278.085201$ 


\begin{tabular}{|c|c|c|c|}
\hline & $\mathrm{x} / \sigma$ & $y / \sigma$ & \\
\hline $1 \mathrm{~A}$ & 0.000000 & 00000 & 00000 \\
\hline $2 \mathrm{~A}$ & 1.089206 & 0.000000 & 000000 \\
\hline $3 A$ & 0.544602 & -0.946432 & 0.000000 \\
\hline $4 \mathrm{~A}$ & 0.544603 & -0.313460 & -0.893015 \\
\hline $5 A$ & 0.544603 & 0.316231 & 0.902200 \\
\hline $6 \mathrm{~A}$ & 0.544603 & 0.956016 & -0.000426 \\
\hline $7 A$ & 1.092402 & -0.632769 & 0.901784 \\
\hline $8 \mathrm{~A}$ & -0.003197 & 0.641313 & -0.895727 \\
\hline $9 A$ & 1.092403 & 0.641313 & -0.895726 \\
\hline $10 \mathrm{~A}$ & -0.003198 & -0.632769 & 0.901783 \\
\hline $11 \mathrm{~A}$ & 1.644088 & -0.950336 & -0.001148 \\
\hline $12 \mathrm{~A}$ & -0.554883 & -0.950335 & -0.001149 \\
\hline $13 \mathrm{~A}$ & -0.554882 & -0.315836 & -0.896318 \\
\hline $14 \mathrm{~A}$ & 1.644089 & -0.315837 & -0.896317 \\
\hline $15 A$ & -0.006898 & -1.266270 & -0.897536 \\
\hline $16 \mathrm{~A}$ & 1.096104 & -1.266270 & -0.897536 \\
\hline 17B & 1.590508 & 2.078473 & -0.421744 \\
\hline $18 \mathrm{~B}$ & -0.501301 & 2.078473 & -0.421744 \\
\hline $19 B$ & -0.501302 & 0.290451 & 2.100847 \\
\hline $20 B$ & 1.590506 & 0.290450 & 2.100848 \\
\hline $21 B$ & 3.13 & -0.603753 & -0.427940 \\
\hline $22 B$ & -2.048422 & -0.603751 & -0.42 \\
\hline $23 \mathrm{~A}$ & -0.365254 & 131 & 0.5 \\
\hline $24 \mathrm{~A}$ & 1.454459 & 131 & 0.5 \\
\hline $25 A$ & -0.913835 & 0.529484 & -0.296227 \\
\hline $26 \mathrm{~A}$ & -0.9 & -0.104142 & 7711 \\
\hline $27 \mathrm{~A}$ & 2.003041 & -0.104143 & 0.597712 \\
\hline $28 \mathrm{~A}$ & 2.003041 & 0.529484 & -0.296226 \\
\hline $29 B$ & 0.544601 & -1.538850 & 2.091803 \\
\hline $\mathrm{OB}$ & 0.544604 & 1.464073 & -2.144804 \\
\hline $31 \mathrm{~B}$ & 0.544603 & 1.903315 & 1.349078 \\
\hline $32 B$ & 2.083508 & -2.421809 & -0.418512 \\
\hline $33 B$ & -0.994304 & -2.421807 & -0.418513 \\
\hline $34 B$ & 2.083509 & -1.196999 & -2.146508 \\
\hline $35 B$ & -0.994303 & -1.196998 & -2.146510 \\
\hline $36 B$ & -1.386631 & -1.408961 & 1.347734 \\
\hline $37 B$ & 2.475834 & -1.408962 & 1.347736 \\
\hline $38 \mathrm{~B}$ & 2.475836 & 0.805018 & -1.775810 \\
\hline $39 B$ & -1.386628 & 0.805020 & -1.775811 \\
\hline $40 A$ & -0.004645 & 0.006492 & -1.794406 \\
\hline $41 \mathrm{~A}$ & 1.093851 & 0.006491 & -1.794405 \\
\hline $42 \mathrm{~A}$ & -0.004646 & -1.690980 & 0.600435 \\
\hline $43 A$ & 1.093850 & -1.690980 & 0.600435 \\
\hline $44 \mathrm{~A}$ & 0.544602 & -2.006677 & -0.295099 \\
\hline $45 A$ & 0.544603 & -0.943058 & -1.795682 \\
\hline $46 \mathrm{~B}$ & 0.544603 & -2.533080 & -1.795455 \\
\hline 47B & 3.013534 & 1.109481 & 0.786407 \\
\hline $48 \mathrm{~B}$ & -1.924329 & 1.109482 & 0.78640 \\
\hline
\end{tabular}

$E 49 / \varepsilon=-286.985560$

\begin{tabular}{|c|c|c|c|}
\hline tom & $\mathrm{x} / \sigma$ & $y / \sigma$ & $z / \sigma$ \\
\hline $1 \mathrm{~A}$ & 0.000000 & 0.000000 & 0.000000 \\
\hline $2 A$ & 1.090020 & 0.000000 & 0.000000 \\
\hline $3 A$ & 0.545010 & -0.943985 & 0.000000 \\
\hline $4 \mathrm{~A}$ & 0.545010 & -0.314662 & -0.893188 \\
\hline $5 A$ & -0.002377 & -0.630695 & 0.902382 \\
\hline $6 \mathrm{~A}$ & 0.545010 & 0.317406 & 0.902381 \\
\hline $7 \mathrm{~A}$ & 1.092397 & -0.630695 & 0.902382 \\
\hline $8 A$ & 0.545010 & 0.955235 & -0.001274 \\
\hline $9 A$ & -0.554753 & -0.949610 & -0.001274 \\
\hline $10 \mathrm{~A}$ & 1.644773 & -0.949610 & -0.001274 \\
\hline $11 \mathrm{~A}$ & 1.093028 & 0.639394 & -0.896579 \\
\hline $12 \mathrm{~A}$ & 1.645255 & -0.317092 & -0.896579 \\
\hline $13 \mathrm{~A}$ & -0.555236 & -0.317092 & -0.896579 \\
\hline $14 \mathrm{~A}$ & -0.007217 & -1.266288 & -0.896579 \\
\hline $15 \mathrm{~A}$ & 1.097237 & -1.266288 & -0.896579 \\
\hline $16 \mathrm{~A}$ & -0.003009 & 0.639394 & -0.896579 \\
\hline 17B & -0.503624 & 0.290768 & 2.099909 \\
\hline $18 \mathrm{~B}$ & 0.545010 & -1.525519 & 2.099910 \\
\hline $19 B$ & 1.59 & 0.290768 & 2.099909 \\
\hline $20 \mathrm{E}$ & 3.13 & -0.604507 & -0.4 \\
\hline & & 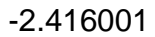 & \\
\hline
\end{tabular}

\begin{tabular}{|llll}
$22 \mathrm{~B}$ & -0.500857 & 2.076522 & -0.424081
\end{tabular} $\begin{array}{llll}23 B & 2.092903 & -2.416001 & -0.424080\end{array}$ $\begin{array}{llll}24 \mathrm{~B} & 1.590876 & 2.076522 & -0.424081\end{array}$ $\begin{array}{llll}25 B & -2.048749 & -0.604508 & -0.424081\end{array}$ $\begin{array}{llll}26 \mathrm{~A} & -0.365528 & 0.845162 & 0.599240\end{array}$ $\begin{array}{llll}27 \mathrm{~A} & 1.455548 & 0.845163 & 0.599240\end{array}$ $\begin{array}{llll}28 \mathrm{~A} & -0.004158 & -1.683123 & 0.599241\end{array}$ $\begin{array}{llll}29 A & 2.004716 & -0.106025 & 0.599240\end{array}$ $\begin{array}{llll}30 A & -0.914696 & -0.106025 & 0.599240\end{array}$ $\begin{array}{llll}31 \mathrm{~A} & 1.094178 & -1.683123 & 0.599241\end{array}$ $\begin{array}{lllll}32 \mathrm{~A} & 0.545010 & -1.999281 & -0.295990\end{array}$ $\begin{array}{lllll}33 \mathrm{~A} & 2.003933 & 0.527648 & -0.295991\end{array}$ $\begin{array}{lllll}34 \mathrm{~A} & -0.913913 & 0.527647 & -0.295991\end{array}$ $\begin{array}{llll}35 \mathrm{~B} & 2.465114 & -1.423233 & 1.347617\end{array}$ $\begin{array}{llll}36 \mathrm{~B} & -1.375094 & -1.423234 & 1.347617\end{array}$ $\begin{array}{llll}37 \mathrm{~B} & 0.545010 & 1.902483 & 1.347617\end{array}$ $\begin{array}{llll}38 \mathrm{~B} & -0.991553 & -1.201798 & -2.146762\end{array}$ $\begin{array}{llll}39 \mathrm{~B} & 0.545009 & 1.459609 & -2.146762\end{array}$ $\begin{array}{lllll}40 B & 2.081573 & -1.201797 & -2.146763\end{array}$ $\begin{array}{llll}41 B & -1.386380 & 0.800426 & -1.776329\end{array}$ $\begin{array}{lllll}42 \mathrm{~B} & 0.545010 & -2.544839 & -1.776328\end{array}$ $\begin{array}{lllll}43 \mathrm{~B} & 2.476399 & 0.800427 & -1.776329\end{array}$ $\begin{array}{lllll}44 \mathrm{~A} & 1.094005 & 0.002301 & -1.794708\end{array}$ $\begin{array}{lllll}45 \mathrm{~A} & 0.545010 & -0.948588 & -1.794708\end{array}$ $\begin{array}{lllll}46 \mathrm{~A} & -0.003986 & 0.002301 & -1.794708\end{array}$ $\begin{array}{llll}47 \mathrm{~B} & -1.923987 & 1.110812 & 0.785082\end{array}$ $\begin{array}{llll}48 \mathrm{~B} & 0.545010 & -3.165613 & 0.785083\end{array}$ $\begin{array}{llll}49 \mathrm{~B} & 3.014007 & 1.110813 & 0.785081\end{array}$

$E 50 / \varepsilon=-295.883575$

atom $x / \sigma \quad y / \sigma$

$\begin{array}{llll}1 \mathrm{~A} & 0.000000 & 0.000000 & 0.000000\end{array}$

$\begin{array}{lllll}2 \mathrm{~A} & 1.090767 & 0.000000 & 0.000000\end{array}$

$\begin{array}{llll}3 \mathrm{~A} & 0.545383 & -0.944632 & 0.000000\end{array}$

$\begin{array}{lllll}4 \mathrm{~A} & 0.545382 & -0.314876 & 0.890607\end{array}$

$\begin{array}{llll}5 A & -0.002210 & -0.631031 & -0.901601\end{array}$

$\begin{array}{lllll}6 \mathrm{~A} & 0.545384 & 0.317430 & -0.901600\end{array}$

$\begin{array}{llll}7 A & 1.092976 & 0.639694 & 0.895475\end{array}$

$\begin{array}{llll}8 A & -0.007503 & -1.266392 & 0.895475\end{array}$

$\begin{array}{lllll}9 A & 0.545383 & 0.955847 & 0.001256\end{array}$

$\begin{array}{llll}10 A & -0.555096 & -0.950239 & 0.001256\end{array}$

$\begin{array}{llll}11 \mathrm{~A} & 1.645863 & -0.950239 & 0.001259\end{array}$

$\begin{array}{llll}12 \mathrm{~A} & 1.098267 & -1.266392 & 0.895476\end{array}$

$\begin{array}{llll}13 A & 1.645861 & -0.317931 & 0.895476\end{array}$

$\begin{array}{llll}14 \mathrm{~A} & -0.555097 & -0.317931 & 0.895474\end{array}$

$\begin{array}{llll}15 A & -0.002213 & 0.639694 & 0.895474\end{array}$

$\begin{array}{llll}16 \mathrm{~A} & 1.092979 & -0.631031 & -0.901600\end{array}$

$\begin{array}{llll}17 \mathrm{~B} & 1.594043 & 2.075363 & 0.425558\end{array}$

$\begin{array}{lllll}18 \mathrm{~B} & 3.139721 & -0.601829 & 0.425561\end{array}$

$\begin{array}{llll}19 B & 2.091060 & -2.418163 & 0.425561\end{array}$

$\begin{array}{llll}20 \mathrm{~B} & -1.000295 & -2.418163 & 0.425557\end{array}$

$\begin{array}{llll}21 B & 0.545380 & 1.469919 & 2.138013\end{array}$

$\begin{array}{llll}22 \mathrm{~B} & -1.000297 & -1.207273 & 2.138013\end{array}$

$\begin{array}{llll}23 B & -0.503279 & 2.075362 & 0.425555\end{array}$

$\begin{array}{llll}24 \mathrm{~B} & -2.048956 & -0.601829 & 0.425555\end{array}$

$\begin{array}{llll}25 B & 2.091057 & -1.207272 & 2.138017\end{array}$

$\begin{array}{lllll}26 \mathrm{~B} & 1.594047 & 0.290568 & -2.098522\end{array}$

$\begin{array}{lllll}27 \mathrm{~B} & 0.545386 & -1.525767 & -2.098522\end{array}$

$\begin{array}{llll}28 B & -0.503275 & 0.290567 & -2.098524\end{array}$

$\begin{array}{llll}29 A & -0.365843 & 0.845076 & -0.597561\end{array}$

$\begin{array}{llll}30 A & -0.914779 & -0.105708 & -0.597561\end{array}$

$\begin{array}{llll}31 \mathrm{~A} & 2.005545 & 0.528149 & 0.298850\end{array}$

$\begin{array}{llll}32 \mathrm{~A} & 0.545383 & -2.000928 & 0.298850\end{array}$

$\begin{array}{llll}33 A & -0.914780 & 0.528148 & 0.298847\end{array}$

$\begin{array}{llll}34 \mathrm{~A} & -0.003555 & 0.002053 & 1.786876\end{array}$

$\begin{array}{lllll}35 \mathrm{~A} & 1.456611 & 0.845077 & -0.597559\end{array}$

$\begin{array}{llll}36 \mathrm{~A} & 1.094317 & 0.002053 & 1.786877\end{array}$

$\begin{array}{llll}37 \mathrm{~A} & 0.545381 & -0.948732 & 1.786877\end{array}$

$\begin{array}{lllll}38 A & 1.094320 & -1.684000 & -0.597558\end{array}$

$\begin{array}{lllll}39 A & -0.003552 & -1.684000 & -0.597559\end{array}$

$\begin{array}{lllll}40 \mathrm{~A} & 2.005547 & -0.105708 & -0.597558\end{array}$

$\begin{array}{llll}41 \mathrm{~B} & 0.545384 & 1.902454 & -1.345240\end{array}$

$\begin{array}{llll}42 \mathrm{~B} & 2.465650 & -1.423543 & -1.345236\end{array}$ \begin{tabular}{|llll}
$43 B$ & 2.465646 & 0.793791 & 1.790544
\end{tabular} 44B $\quad-1.374881-1.423544-1.345240$ $\begin{array}{llll}45 \mathrm{~B} & 0.545381 & -2.532208 & 1.790543\end{array}$ $\begin{array}{llll}46 \mathrm{~B} & -1.374885 & 0.793790 & 1.790539\end{array}$ $\begin{array}{lllll}47 \mathrm{~B} & 0.545383 & -3.166090 & -0.785402\end{array}$ $\begin{array}{llll}48 \mathrm{~B} & -1.923838 & 1.110729 & -0.785408\end{array}$ $\begin{array}{llll}49 \mathrm{~B} & 3.014607 & 1.110729 & -0.785401\end{array}$ $\begin{array}{llll}50 \mathrm{~B} & 0.545378 & -0.314874 & 3.246820\end{array}$ $s=2.0$

$E 5 / \varepsilon=-9.267451$

$\begin{array}{cccc}\text { atom } & x / \sigma & y / \sigma & z / \sigma \\ \text { 1A } & 0.000000 & 0.000000 & 0.000000 \\ \text { 2A } & 1.123849 & 0.000000 & 0.000000 \\ \text { 3A } & 0.561925 & -0.973282 & 0.000000 \\ \text { 4B } & 0.561923 & -0.324428 & 1.546474 \\ \text { 5B } & 0.561923 & -0.324428 & -1.546474\end{array}$

$E 6 / \varepsilon=-13.473670$

$\begin{array}{cccc}\text { atom } & \mathrm{x} / \sigma & y / \sigma & z / \sigma \\ \text { 1A } & 0.000000 & 0.000000 & 0.000000 \\ \text { 2A } & 1.125643 & 0.000000 & 0.000000 \\ \text { 3B } & 0.562820 & -1.581510 & 0.000000 \\ \text { 4B } & 0.562820 & 0.000000 & 1.581510 \\ \text { 5B } & 0.562820 & 1.581510 & 0.000000 \\ \text { 6B } & 0.562820 & 0.000000 & -1.581510\end{array}$

$E 7 / \varepsilon=-18.073138$

\begin{tabular}{cccc} 
atom & $\mathrm{X} / \sigma$ & $y / \sigma$ & \multicolumn{1}{c}{$z / \sigma$} \\
1A & 0.000000 & 0.000000 & 0.000000 \\
2B & 1.626551 & 0.000000 & 0.000000 \\
3B & 0.000000 & -1.626551 & 0.000000 \\
4B & 0.000000 & 0.000000 & -1.626551 \\
5B & 0.000000 & 0.000000 & 1.626551 \\
6B & 0.000000 & 1.626551 & 0.000000 \\
7B & -1.626551 & 0.000000 & 0.000000
\end{tabular}

$E 8 / \varepsilon=-21.814932$

\begin{tabular}{ccrr} 
atom & $\mathrm{x} / \sigma$ & $y / \sigma$ & \multicolumn{1}{c}{$z / \sigma$} \\
1A & 0.000000 & 0.000000 & 0.000000 \\
2B & 1.621114 & 0.000000 & 0.000000 \\
3B & -1.271283 & -1.002600 & 0.000000 \\
4A & 0.328350 & -0.935257 & -0.549449 \\
5A & 0.328349 & -0.935253 & 0.549456 \\
6B & -0.139311 & 0.199148 & -1.640945 \\
7B & -0.139321 & 0.199157 & 1.640942 \\
8B & 0.102540 & 1.665018 & -0.000004
\end{tabular}

$E 9 / \varepsilon=-26.901093$

atom $\mathrm{x} / \sigma \quad \mathrm{y} / \sigma \quad \mathrm{z} / \sigma$

$\begin{array}{llll}1 \mathrm{~A} & 0.000000 & 0.000000 & 0.000000\end{array}$

$\begin{array}{llll}2 B & 1.697957 & 0.000000 & 0.000000\end{array}$

$\begin{array}{llll}3 B & 0.195785 & -1.686632 & 0.000000\end{array}$

$\begin{array}{llll}4 \mathrm{~B} & 0.224619 & 1.280199 & 1.098358\end{array}$

$\begin{array}{llll}5 B & -1.245760 & -0.370736 & 1.098359\end{array}$

$\begin{array}{lllll}6 \mathrm{~B} & -1.245760 & -0.370736 & -1.098359\end{array}$

$\begin{array}{lllll}7 B & 0.224619 & 1.280199 & -1.098358\end{array}$

$\begin{array}{llll}8 \mathrm{~A} & 0.411519 & -0.366513 & 0.981291\end{array}$

$\begin{array}{llll}9 A & 0.411519 & -0.366513 & -0.981291\end{array}$

\section{$E 10 / \varepsilon=-32.187970$}

$\begin{array}{llll}\text { atom } & \mathrm{x} / \sigma & \mathrm{y} / \sigma & \mathrm{z} \sigma\end{array}$

$\begin{array}{llll}1 \mathrm{~A} & 0.000000 & 0.000000 & 0.000000\end{array}$

$\begin{array}{llll}2 B & 1.709625 & 0.000000 & 0.000000\end{array}$

$\begin{array}{llll}3 B & 0.227591 & -1.694408 & 0.000000\end{array}$

$\begin{array}{llll}4 B & 0.256712 & 1.270807 & 1.114436\end{array}$

$\begin{array}{lllll}5 B & 0.256712 & 1.270807 & -1.114436\end{array}$

$\begin{array}{lllll}6 \mathrm{~B} & -1.225321 & -0.423600 & -1.114437\end{array}$

$\begin{array}{llll}7 \mathrm{~B} & -1.225321 & -0.423600 & 1.114437\end{array}$

$\begin{array}{lllll}8 \mathrm{~A} & 0.423317 & -0.370258 & -0.974098\end{array}$

$\begin{array}{llll}9 \mathrm{~A} & 0.423317 & -0.370258 & 0.974098\end{array}$ 
10A $\quad-0.846633 \quad 0.740518 \quad 0.000000$

$E 11 / \varepsilon=-37.111833$

\begin{tabular}{rrrr} 
atom & $\mathrm{x} / \sigma$ & $y / \sigma$ & \multicolumn{1}{c}{$z / \sigma$} \\
1A & 0.000000 & 0.000000 & 0.000000 \\
2B & 1.644798 & 0.000000 & 0.000000 \\
3B & 0.027246 & -1.644572 & 0.000000 \\
4A & 0.356382 & -0.350526 & 0.960456 \\
5A & -0.434601 & 0.427461 & 0.927577 \\
6B & 0.059200 & 1.622244 & -0.102161 \\
7B & -1.621040 & -0.086062 & -0.102163 \\
8B & -0.872109 & -0.822690 & 1.916220 \\
9B & 0.808132 & 0.885617 & 1.916221 \\
10B & 1.345872 & -1.323760 & 1.817044 \\
11B & 0.067497 & -0.066383 & -1.628205
\end{tabular}

$E 12 / \varepsilon=-42.523248$

$\begin{array}{cccc}\text { atom } & \mathrm{x} / \sigma & y / \sigma & z / \sigma \\ 1 \mathrm{~A} & 0.000000 & 0.000000 & 0.000000\end{array}$

$\begin{array}{llll}2 \mathrm{~B} & 1.636574 & 0.000000 & 0.000000\end{array}$

$\begin{array}{llll}3 \mathrm{~B} & -1.635631 & -0.055533 & 0.000000\end{array}$

$\begin{array}{llll}4 \mathrm{~B} & 0.049138 & -0.027010 & -1.635614\end{array}$

$\begin{array}{llll}5 B & -0.875410 & 1.931755 & 0.817724\end{array}$

$\begin{array}{llll}6 \mathrm{~B} & -0.048194 & -0.028524 & 1.635615\end{array}$

$\begin{array}{llll}7 \mathrm{~B} & 0.809359 & 1.960278 & -0.817889\end{array}$

$\begin{array}{lllll}8 \mathrm{~A} & 0.364069 & 0.951709 & 0.391607\end{array}$

$\begin{array}{llll}9 \mathrm{~A} & -0.396152 & 0.938774 & -0.391687\end{array}$

$\begin{array}{llll}10 \mathrm{~B} & 0.027518 & -1.621451 & 0.000068\end{array}$

$\begin{array}{llll}11 B & 1.328363 & 1.779075 & 1.399308\end{array}$

$\begin{array}{llll}12 \mathrm{~B} & -1.387962 & 1.732860 & -1.399457\end{array}$

$E 13 / \varepsilon=-48.110134$

\begin{tabular}{crrr} 
atom & $x / \sigma$ & $y / \sigma$ & \multicolumn{1}{c}{$z / \sigma$} \\
$1 \mathrm{~A}$ & 0.000000 & 0.000000 & 0.000000 \\
2A & 1.102098 & 0.000000 & 0.000000 \\
3A & 0.551049 & -0.954445 & 0.000000 \\
4A & 1.103931 & -0.637354 & -0.912164 \\
5A & -0.001831 & -0.637354 & -0.912165 \\
$6 \mathrm{~A}$ & 0.551050 & 0.320264 & -0.912164 \\
$7 \mathrm{~B}$ & 0.551049 & -0.318148 & 1.525982 \\
8B & -1.057350 & -1.246759 & 0.223601 \\
9B & 0.551048 & 1.539071 & 0.223602 \\
$10 \mathrm{~B}$ & 2.159448 & -1.246758 & 0.223603 \\
$11 \mathrm{~B}$ & 0.551050 & -2.197250 & -1.068597 \\
$12 \mathrm{~B}$ & -1.076300 & 0.621403 & -1.068597 \\
13B & 2.178400 & 0.621403 & -1.068594
\end{tabular}

$E 14 / \varepsilon=-55.233067$

$\begin{array}{cccc}\text { atom } & \mathrm{x} / \sigma & y / \sigma & z / \sigma \\ 1 \mathrm{~A} & 0.000000 & 0.000000 & 0.000000\end{array}$

$\begin{array}{llll}2 \mathrm{~A} & 1.564641 & 0.000000 & 0.000000\end{array}$

$\begin{array}{llll}3 \mathrm{~A} & 0.782320 & -0.782321 & 0.000000\end{array}$

$\begin{array}{llll}4 \mathrm{~A} & 0.782320 & 0.782321 & 0.000000\end{array}$

$\begin{array}{llll}5 \mathrm{~A} & 0.782320 & 0.000000 & 0.782321\end{array}$

$\begin{array}{lllll}6 \mathrm{~A} & 0.782320 & 0.000000 & -0.782321\end{array}$

$\begin{array}{llll}7 \mathrm{~B} & -0.357018 & 1.139338 & 1.139338\end{array}$

$\begin{array}{llll}8 \mathrm{~B} & -0.357018 & -1.139338 & 1.139338\end{array}$

$\begin{array}{llll}9 B & 1.921658 & 1.139338 & -1.139338\end{array}$

$\begin{array}{llll}10 \mathrm{~B} & -0.357018 & 1.139338 & -1.139338\end{array}$

11B $\quad-0.357018 \quad-1.139338-1.139338$

12B $\quad 1.921658 \quad-1.139338-1.139338$

$\begin{array}{llll}13 \mathrm{~B} & 1.921658 & 1.139338 & 1.139338\end{array}$

$\begin{array}{llll}\text { 14B } & 1.921658 & -1.139338 & 1.139338\end{array}$

$E 15 / \varepsilon=-60.758081$

1A $0.000000 \quad 0.000000 \quad 0.000000$

$\begin{array}{llll}2 \mathrm{~A} & 1.115668 & 0.000000 & 0.000000\end{array}$

$\begin{array}{llll}3 \mathrm{~A} & 0.029132 & -1.115288 & 0.000000\end{array}$

$\begin{array}{llll}4 \mathrm{~A} & 0.572400 & -0.557644 & -0.778529\end{array}$

$\begin{array}{llll}5 A & 0.572400 & -0.557644 & 0.778529\end{array}$

$\begin{array}{llll}6 \mathrm{~A} & 1.134497 & -1.105251 & 0.000000\end{array}$ $\begin{array}{llll}8 \mathrm{~B} & -1.021969 & -0.628531 & 1.163348\end{array}$ $\begin{array}{llll}9 \mathrm{~B} & -1.021969 & -0.628531 & -1.163348\end{array}$ $\begin{array}{llll}10 \mathrm{~B} & 0.601631 & 1.038033 & -1.163348\end{array}$ $\begin{array}{llll}11 B & 2.191190 & -0.552243 & -1.133482\end{array}$ $\begin{array}{llll}12 \mathrm{~B} & 2.191190 & -0.552243 & 1.133482\end{array}$ $\begin{array}{llll}13 \mathrm{~B} & 0.609271 & -2.176023 & -1.133483\end{array}$ $\begin{array}{llll}14 \mathrm{~B} & 0.609271 & -2.176023 & 1.133483\end{array}$ $\begin{array}{llll}15 B & -1.249379 & 1.217171 & 0.000000\end{array}$

$E 16 / \varepsilon=-66.356133$

$\begin{array}{cccc}\text { atom } & \mathrm{X} / \sigma & y / \sigma & z / \sigma \\ 1 \mathrm{~A} & 0.000000 & 0.000000 & 0.000000\end{array}$ $\begin{array}{llll}2 \mathrm{~A} & 1.122127 & 0.000000 & 0.000000\end{array}$ $\begin{array}{llll}3 \mathrm{~A} & 0.561064 & -0.958735 & 0.000000\end{array}$ $\begin{array}{llll}4 \mathrm{~A} & 0.561063 & 0.283106 & 0.915982\end{array}$

$\begin{array}{llll}5 \mathrm{~A} & 1.114826 & -0.664241 & 0.900544\end{array}$

$\begin{array}{llll}6 \mathrm{~A} & 0.007300 & -0.664242 & 0.900543\end{array}$

$\begin{array}{lllll}7 \mathrm{~B} & 0.561062 & 1.568986 & -0.078552\end{array}$

$\begin{array}{llll}8 \mathrm{~B} & 0.561065 & -0.388259 & -1.522216\end{array}$

$\begin{array}{llll}9 \mathrm{~B} & -1.041720 & 0.558293 & 1.191037\end{array}$

$\begin{array}{llll}10 \mathrm{~B} & -1.041717 & -1.302788 & -0.181697\end{array}$

$\begin{array}{llll}11 B & 2.163847 & -1.302784 & -0.181694\end{array}$

$\begin{array}{llll}12 B & 2.163844 & 0.558296 & 1.191039\end{array}$

$\begin{array}{llll}13 \mathrm{~B} & 0.561062 & -0.394383 & 2.434069\end{array}$

$\begin{array}{llll}\text { 14B } & 0.561064 & -2.209069 & 1.095556\end{array}$

$\begin{array}{llll}15 B & 2.364129 & 0.728800 & -0.988064\end{array}$

$\begin{array}{llll}16 \mathrm{~B} & -1.242002 & 0.728795 & -0.988067\end{array}$

$E 17 / \varepsilon=-71.965936$

$\begin{array}{lll}\text { atom } & \mathrm{x} / \sigma & \mathrm{yl} \sigma\end{array}$

$\begin{array}{llll}1 \mathrm{~A} & 0.000000 & 0.000000 & 0.000000\end{array}$

$\begin{array}{llll}2 \mathrm{~A} & 1.120768 & 0.000000 & 0.000000\end{array}$

$\begin{array}{llll}3 A & 0.560384 & -0.970615 & 0.000000\end{array}$

$\begin{array}{llll}4 \mathrm{~B} & 0.560384 & -0.323540 & 1.518697\end{array}$

$\begin{array}{lllll}5 A & 1.109113 & -0.640348 & -0.906554\end{array}$

$\begin{array}{lllll}6 \mathrm{~A} & 0.011651 & -0.640348 & -0.906553\end{array}$

$\begin{array}{lllll}7 \mathrm{~A} & 0.560383 & 0.310082 & -0.906554\end{array}$

$\begin{array}{llll}8 \mathrm{~B} & -1.077732 & -1.269304 & 0.121887\end{array}$

$\begin{array}{llll}9 \mathrm{~B} & 0.560384 & 1.567996 & 0.121881\end{array}$

$\begin{array}{llll}10 B & 2.198500 & -1.269305 & 0.121879\end{array}$

$\begin{array}{llll}11 B & 2.154935 & 0.597077 & -1.186666\end{array}$

$\begin{array}{lllll}12 \mathrm{~B} & -1.034171 & 0.597077 & -1.186662\end{array}$

$\begin{array}{llll}13 \mathrm{~B} & 0.560380 & -2.164770 & -1.186664\end{array}$

$\begin{array}{llll}14 \mathrm{~B} & 0.560380 & -0.323538 & -2.440696\end{array}$

$\begin{array}{llll}15 B & 2.390640 & 0.733160 & 0.986977\end{array}$

$\begin{array}{lllll}16 \mathrm{~B} & 0.560386 & -2.436939 & 0.986978\end{array}$

$\begin{array}{llll}17 \mathrm{~B} & -1.269872 & 0.733163 & 0.986981\end{array}$

$E 18 / \varepsilon=-77.549442$

atom $\quad \mathrm{x} / \sigma \quad \mathrm{y} / \sigma$

$\begin{array}{llll}1 \mathrm{~A} & 0.000000 & 0.000000 & 0.000000\end{array}$

$\begin{array}{llll}2 \mathrm{~A} & 1.117155 & 0.000000 & 0.000000\end{array}$

$\begin{array}{llll}3 \mathrm{~A} & 0.558578 & -0.971404 & 0.000000\end{array}$

$\begin{array}{lllll}4 \mathrm{~A} & 0.558578 & 0.337383 & 0.910933\end{array}$

$\begin{array}{lllll}5 B & 0.558578 & 1.544171 & -0.208366\end{array}$

$\begin{array}{llll}6 \mathrm{~B} & 0.558578 & -0.340919 & -1.520413\end{array}$

$\begin{array}{llll}7 \mathrm{~A} & 0.013929 & -0.629846 & 0.904934\end{array}$

$\begin{array}{lllll}8 \mathrm{~A} & 1.103227 & -0.629846 & 0.904934\end{array}$

$\begin{array}{llll}9 B & 2.187557 & 0.561667 & 1.160932\end{array}$

$\begin{array}{llll}10 \mathrm{~B} & -1.070400 & -1.283740 & -0.123494\end{array}$

$\begin{array}{llll}11 B & 2.187557 & -1.283738 & -0.123495\end{array}$

$\begin{array}{llll}12 B & -1.070401 & 0.561667 & 1.160933\end{array}$

$\begin{array}{llll}13 \mathrm{~B} & 0.558579 & -2.164395 & 1.178051\end{array}$

$\begin{array}{llll}14 \mathrm{~B} & 0.558579 & -0.352988 & 2.438813\end{array}$

$\begin{array}{llll}15 B & 2.405585 & 0.703485 & -1.010735\end{array}$

$\begin{array}{llll}16 \mathrm{~B} & -1.288429 & 0.703484 & -1.010733\end{array}$

$\begin{array}{llll}17 \mathrm{~B} & 0.558579 & -2.455498 & -0.993817\end{array}$

$\begin{array}{llll}18 \mathrm{~B} & 0.558579 & 1.784781 & 1.957471\end{array}$

$E 19 / \varepsilon=-84.509377$

atom $\mathrm{x} / \sigma \quad \mathrm{l} / \sigma \quad \mathrm{z} / \sigma$ $\begin{array}{llll}1 \mathrm{~A} & 0.000000 & 0.000000 & 0.000000\end{array}$

$\begin{array}{llll}2 \mathrm{~A} & 1.101571 & 0.000000 & 0.000000\end{array}$

$\begin{array}{llll}3 A & 0.550786 & -0.953989 & 0.000000\end{array}$

$\begin{array}{llll}4 \mathrm{~B} & 0.550784 & 1.654522 & 0.008902\end{array}$

$\begin{array}{llll}5 B & 2.259038 & -1.304254 & 0.008899\end{array}$

$\begin{array}{llll}6 \mathrm{~B} & -1.157464 & -1.304258 & 0.008904\end{array}$

$\begin{array}{llll}7 A & 0.003109 & -0.634199 & 0.908158\end{array}$

$\begin{array}{llll}8 \mathrm{~A} & 1.098466 & -0.634199 & 0.908157\end{array}$

$\begin{array}{lllll}9 \mathrm{~A} & 0.550787 & 0.314408 & 0.908158\end{array}$

$\begin{array}{llll}10 \mathrm{~B} & -1.055684 & 0.609498 & 1.139117\end{array}$

$\begin{array}{llll}11 B & 0.550789 & -2.172990 & 1.139114\end{array}$

$\begin{array}{llll}12 B & 2.157257 & 0.609501 & 1.139113\end{array}$

$\begin{array}{lllll}13 \mathrm{~A} & 1.098758 & -0.634368 & -0.915245\end{array}$

$\begin{array}{lllll}14 \mathrm{~A} & 0.550784 & 0.314749 & -0.915245\end{array}$

$\begin{array}{llll}15 A & 0.002811 & -0.634369 & -0.915244\end{array}$

$\begin{array}{llll}16 \mathrm{~B} & 0.550790 & -0.317998 & 2.431932\end{array}$

$\begin{array}{lllll}17 \mathrm{~B} & 0.550786 & -2.186641 & -1.104902\end{array}$

$\begin{array}{llll}18 \mathrm{~B} & -1.067511 & 0.616325 & -1.104899\end{array}$

$\begin{array}{llll}19 B & 2.169077 & 0.616328 & -1.104903\end{array}$

$E 20 / \varepsilon=-91.988950$

$\begin{array}{llll}\text { atom } \quad \mathrm{x} / \sigma & \mathrm{y} / \sigma & \mathrm{z} / \sigma\end{array}$

$\begin{array}{llll}1 \mathrm{~A} & 0.000000 & 0.000000 & 0.000000\end{array}$

$\begin{array}{llll}2 \mathrm{~A} & 1.103196 & 0.000000 & 0.000000\end{array}$

$\begin{array}{llll}3 \mathrm{~A} & 0.551599 & -0.955394 & 0.000000\end{array}$

$\begin{array}{llll}4 \mathrm{~B} & -1.157130 & -1.304999 & 0.000000\end{array}$

$\begin{array}{llll}5 \mathrm{~B} & 2.260328 & -1.304998 & 0.000000\end{array}$

$\begin{array}{llll}6 B & 0.551599 & 1.654606 & 0.000000\end{array}$

$\begin{array}{lllll}7 A & 1.100080 & -0.635130 & 0.905562\end{array}$

$\begin{array}{lllll}8 \mathrm{~A} & 0.551599 & 0.314868 & -0.905562\end{array}$

$\begin{array}{llll}9 A & 0.551599 & 0.314868 & 0.905562\end{array}$

$10 \mathrm{~A} \quad 1.100080-0.635130 \quad-0.905562$

$\begin{array}{llll}11 \mathrm{~A} & 0.003118 & -0.635130 & 0.905563\end{array}$

$\begin{array}{lllll}12 \mathrm{~A} & 0.003118 & -0.635130 & -0.905563\end{array}$

$\begin{array}{llll}13 \mathrm{~B} & 2.160468 & 0.610417 & 1.125918\end{array}$

$\begin{array}{llll}14 \mathrm{~B} & -1.057270 & 0.610418 & 1.125918\end{array}$

$\begin{array}{llll}15 \mathrm{~B} & -1.057270 & 0.610418 & -1.125918\end{array}$

$\begin{array}{lllll}16 \mathrm{~B} & 2.160468 & 0.610417 & -1.125918\end{array}$

$\begin{array}{llll}17 \mathrm{~B} & 0.551600 & -2.176225 & -1.125919\end{array}$

$\begin{array}{llll}18 B & 0.551600 & -2.176225 & 1.125919\end{array}$

$\begin{array}{lllll}19 B & 0.551600 & -0.318462 & -2.427152\end{array}$

$\begin{array}{llll}20 \mathrm{~B} & 0.551600 & -0.318462 & 2.427152\end{array}$

$E 21 / \varepsilon=-97.424715$

atom $\mathrm{X} / \sigma \quad \mathrm{y} / \sigma$

$\begin{array}{llll}1 \mathrm{~A} & 0.000000 & 0.000000 & 0.000000\end{array}$

$\begin{array}{llll}2 A & 1.095033 & 0.000000 & 0.000000\end{array}$

$\begin{array}{llll}3 \mathrm{~A} & 0.547516 & -0.960634 & 0.000000\end{array}$

$\begin{array}{lllll}4 \mathrm{~B} & 0.547518 & 1.641156 & -0.055345\end{array}$

$\begin{array}{llll}5 \mathrm{~A} & 0.547517 & 0.327565 & 0.923595\end{array}$

$\begin{array}{llll}6 \mathrm{~B} & 2.255127 & -1.318036 & 0.004490\end{array}$

$\begin{array}{llll}7 B & -1.160096 & -1.318034 & 0.004492\end{array}$

$\begin{array}{llll}8 \mathrm{~B} & -1.071832 & 0.596361 & 1.112496\end{array}$

$\begin{array}{llll}9 B & 2.166867 & 0.596359 & 1.112495\end{array}$

$\begin{array}{llll}10 \mathrm{~A} & 1.093797 & -0.632884 & 0.901789\end{array}$

$\begin{array}{llll}11 \mathrm{~A} & 0.001236 & -0.632884 & 0.901789\end{array}$

$\begin{array}{llll}12 \mathrm{~A} & -0.002342 & -0.650473 & -0.902842\end{array}$

$\begin{array}{lllll}13 \mathrm{~A} & 1.097373 & -0.650473 & -0.902843\end{array}$

$\begin{array}{lllll}14 \mathrm{~A} & 0.547516 & 0.292643 & -0.918712\end{array}$

$\begin{array}{lllll}15 B & -1.066298 & 0.584633 & -1.136644\end{array}$

$\begin{array}{llll}16 B & 2.161330 & 0.584630 & -1.136646\end{array}$

$\begin{array}{llll}17 \mathrm{~B} & 0.547516 & -2.193552 & 1.103304\end{array}$

$\begin{array}{llll}18 \mathrm{~B} & 0.547514 & -2.188511 & -1.129710\end{array}$

$\begin{array}{llll}19 B & 0.547516 & -0.425037 & 2.431398\end{array}$

$\begin{array}{lllll}20 \mathrm{~B} & 0.547514 & -0.346643 & -2.431049\end{array}$

$\begin{array}{llll}21 B & 0.547519 & 1.719147 & 2.108114\end{array}$

$E 22 / \varepsilon=-102.921119$

$\begin{array}{llll}\text { atom } & \mathrm{x} / \sigma & \mathrm{y} / \sigma & \mathrm{z} \sigma\end{array}$

$\begin{array}{llll}1 \mathrm{~A} & 0.000000 & 0.000000 & 0.000000\end{array}$

$\begin{array}{llll}2 \mathrm{~A} & 1.094493 & 0.000000 & 0.000000\end{array}$

$\begin{array}{llll}3 A & 0.527952 & -0.958740 & 0.000000\end{array}$ 


$\begin{array}{rrrr}4 B & -1.163485 & -1.293670 & -0.055284 \\ 5 B & 0.571979 & 1.643203 & -0.055286 \\ 6 B & -1.073777 & 0.634518 & 1.072306 \\ 7 A & 0.543579 & 0.322783 & 0.927929 \\ 8 A & -0.020540 & -0.631859 & 0.927929 \\ 9 B & 2.252123 & -1.330831 & 0.016947 \\ 10 B & 2.175936 & 0.595850 & 1.094695 \\ 11 B & 0.527664 & -2.193469 & 1.094697 \\ 12 A & 1.080130 & -0.638274 & 0.887977 \\ 13 A & 1.105085 & -0.653021 & -0.894175 \\ 14 A & 0.563499 & 0.297819 & -0.919488 \\ 15 A & 0.010935 & -0.637268 & -0.919488 \\ 16 B & 0.713185 & -0.421437 & 2.441152 \\ 17 B & -1.036065 & 0.612233 & -1.165363 \\ 18 B & 0.552408 & -2.184504 & -1.135777 \\ 19 B & 2.180018 & 0.569850 & -1.135778 \\ \text { 20B } & 0.581379 & -0.343552 & -2.435477 \\ 21 B & -1.231999 & -1.299281 & 2.107125 \\ 22 B & 0.543845 & 1.705927 & 2.107123\end{array}$

$E 23 / \varepsilon=-110.903815$

\begin{tabular}{rrrr} 
atom & $x / \sigma$ & \multicolumn{1}{c}{$y / \sigma$} & \multicolumn{1}{c}{$z / \sigma$} \\
1A & 0.000000 & 0.000000 & 0.000000 \\
2A & 1.157324 & 0.000000 & 0.000000 \\
3A & 0.578662 & -0.918600 & 0.000000 \\
4A & 0.578662 & 0.459300 & -0.795532 \\
5A & 0.578661 & 0.459300 & 0.795531 \\
6B & 2.250354 & 0.637357 & 1.103931 \\
7B & 2.250356 & -1.274708 & 0.000002 \\
8B & 2.250354 & 0.637356 & -1.103931 \\
9B & -1.093032 & 0.637354 & 1.103930 \\
10B & -1.093031 & 0.637352 & -1.103932 \\
11B & -1.093030 & -1.274710 & 0.000000 \\
12A & 0.038964 & -0.538019 & -0.931874 \\
13A & 1.118361 & -0.538017 & 0.931875 \\
14A & 1.118359 & 1.076036 & 0.000000 \\
15A & 1.118362 & -0.538019 & -0.931874 \\
16A & 0.038964 & -0.538018 & 0.931874 \\
17A & 0.038962 & 1.076036 & -0.000001 \\
18B & 0.578664 & -2.068637 & -1.194791 \\
19B & 0.578663 & -0.000408 & -2.388888 \\
20B & 0.578661 & -0.000403 & 2.388887 \\
21B & 0.578659 & 2.069040 & 1.194092 \\
22B & 0.578663 & -2.068635 & 1.194793 \\
23B & 0.578660 & 2.069039 & -1.194094
\end{tabular}

$E 24 / \varepsilon=-116.521863$

atom $\quad x / \sigma \quad y / \sigma \quad z / \sigma$

$\begin{array}{llll}1 \mathrm{~A} & 0.000000 & 0.000000 & 0.000000\end{array}$

2A $\quad 1.163942 \quad 0.000000 \quad 0.000000$

$\begin{array}{llll}3 A & 0.589907 & -0.920286 & 0.000000\end{array}$

$\begin{array}{llll}4 \mathrm{~A} & 0.577886 & 0.449793 & 0.794824\end{array}$

$\begin{array}{lllll}5 \mathrm{~A} & 0.577886 & 0.449793 & -0.794824\end{array}$

$\begin{array}{lllll}6 \mathrm{~B} & -1.092317 & 0.587993 & -1.120743\end{array}$

$\begin{array}{llll}7 \mathrm{~B} & -1.092317 & 0.587993 & 1.120743\end{array}$

$\begin{array}{llll}8 \mathrm{~A} & 0.013642 & 1.088917 & 0.000000\end{array}$

$\begin{array}{llll}9 B & -1.074354 & -1.302616 & 0.000000\end{array}$

$\begin{array}{llll}10 B & 2.259601 & 0.632406 & -1.095900\end{array}$

$\begin{array}{llll}11 \mathrm{~B} & 2.259601 & 0.632406 & 1.095900\end{array}$

$\begin{array}{llll}12 \mathrm{~B} & 2.266727 & -1.278255 & 0.000000\end{array}$

$\begin{array}{llll}13 \mathrm{~A} & 1.097625 & 1.076077 & 0.000000\end{array}$

$\begin{array}{llll}14 \mathrm{~B} & 0.596919 & 2.027049 & -1.250331\end{array}$

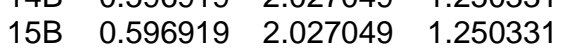

$\begin{array}{llll}16 \mathrm{~A} & 0.054354 & -0.553118 & 0.925885\end{array}$

$\begin{array}{llll}17 \mathrm{~A} & 0.054354 & -0.553118 & -0.925885\end{array}$

$\begin{array}{llll}18 \mathrm{~A} & 1.132788 & -0.543849 & 0.928387\end{array}$

$\begin{array}{llll}19 \mathrm{~A} & 1.132788 & -0.543849 & -0.928387\end{array}$

$\begin{array}{llll}20 \mathrm{~B} & 0.596351 & -0.023869 & 2.388153\end{array}$

$\begin{array}{llll}21 B & 0.596351 & -0.023869 & -2.388153\end{array}$

22B $\quad 0.602664 \quad-2.078344 \quad 1.192753$

23B $\quad 0.602664 \quad-2.078344 \quad-1.192753$

$\begin{array}{llll}24 \mathrm{~B} & -1.138492 & 2.464187 & 0.000000\end{array}$
$E 25 / \varepsilon=-122.388638$

$\begin{array}{lcl}\text { atom } \quad \mathrm{x} / \sigma & \mathrm{y} / \sigma\end{array}$

$\begin{array}{llll}1 \mathrm{~A} & 0.000000 & 0.000000 & 0.000000\end{array}$

$\begin{array}{llll}2 \mathrm{~A} & 1.174028 & 0.000000 & 0.000000\end{array}$

$\begin{array}{llll}3 A & 0.587013 & -0.921645 & 0.000000\end{array}$

$\begin{array}{lllll}4 \mathrm{~A} & 0.587013 & 0.435987 & -0.793776\end{array}$

$\begin{array}{lllll}5 A & 0.587013 & 0.435988 & 0.793776\end{array}$

$\begin{array}{llll}6 \mathrm{~B} & 2.268407 & 0.582869 & -1.111018\end{array}$

$\begin{array}{llll}7 \mathrm{~B} & -1.094381 & 0.582872 & 1.111016\end{array}$

$\begin{array}{llll}8 \mathrm{~B} & 2.268408 & 0.582870 & 1.111016\end{array}$

$\begin{array}{llll}9 B & -1.094381 & 0.582871 & -1.111016\end{array}$

$\begin{array}{llll}10 \mathrm{~A} & 1.128345 & 1.091486 & -0.000001\end{array}$

$\begin{array}{llll}11 \mathrm{~A} & 0.045683 & 1.091486 & 0.000000\end{array}$

$\begin{array}{llll}12 \mathrm{~B} & 0.587015 & 1.956827 & 1.357084\end{array}$

$\begin{array}{llll}13 \mathrm{~B} & 0.587014 & 1.956826 & -1.357086\end{array}$

$\begin{array}{llll}14 \mathrm{~B} & 2.258296 & -1.308027 & 0.000000\end{array}$

$\begin{array}{llll}15 B & -1.084272 & -1.308025 & 0.000000\end{array}$

$\begin{array}{llll}16 \mathrm{~A} & 1.126423 & -0.561612 & -0.920778\end{array}$

$\begin{array}{llll}17 A & 1.126423 & -0.561612 & 0.920778\end{array}$

$\begin{array}{llll}18 \mathrm{~A} & 0.047602 & -0.561611 & 0.920778\end{array}$

$\begin{array}{lllll}19 \mathrm{~A} & 0.047602 & -0.561612 & -0.920778\end{array}$

$\begin{array}{lllll}20 \mathrm{~B} & 0.587012 & -0.072790 & -2.389257\end{array}$

$\begin{array}{llll}21 \mathrm{~B} & 0.587013 & -0.072789 & 2.389256\end{array}$

22B $\quad 0.587012 \quad-2.092176-1.186109$

$\begin{array}{llll}23 \mathrm{~B} & 0.587012 & -2.092176 & 1.186109\end{array}$

$\begin{array}{llll}24 \mathrm{~B} & 2.202560 & 2.473230 & -0.000002\end{array}$

$\begin{array}{llll}25 B & -1.028530 & 2.473233 & 0.000000\end{array}$

\section{$E 26 / \varepsilon=-129.869161$}

\begin{tabular}{|c|c|c|c|}
\hline atom & $\mathrm{x} / \sigma$ & $y / \sigma$ & $z / \sigma$ \\
\hline $1 \mathrm{~A}$ & 0.000000 & 0.000000 & 0.000000 \\
\hline $2 \mathrm{~A}$ & 1.163855 & 0.000000 & 0.000000 \\
\hline $3 A$ & 0.581928 & -1.007928 & 0.000000 \\
\hline $4 \mathrm{~A}$ & 0.581928 & -0.335976 & 0.950283 \\
\hline $5 \mathrm{~A}$ & -0.297632 & -0.843791 & 0.596650 \\
\hline $6 \mathrm{~A}$ & 0.581928 & 0.679653 & 0.596650 \\
\hline $7 \mathrm{~A}$ & 1.461489 & -0.843790 & 0.596649 \\
\hline $8 \mathrm{~A}$ & 0.581928 & -0.335976 & -0.839666 \\
\hline $9 B$ & 1.690844 & -0.976210 & 2.162506 \\
\hline $10 B$ & -1.729053 & -0.282198 & 0.199542 \\
\hline 11B & -0.620138 & 1.638502 & 0.199543 \\
\hline $12 B$ & 0.581928 & 0.944490 & 2.162507 \\
\hline $13 B$ & -0.526988 & -2.364234 & 0.199541 \\
\hline 14B & -0.620137 & 0.358036 & -1.611310 \\
\hline 15B & -0.526989 & -0.976210 & 2.162506 \\
\hline $16 B$ & 0.581928 & -1.723999 & -1.611311 \\
\hline 17B & 1.783993 & 1.638502 & 0.199543 \\
\hline 18B & 1.783992 & 0.358037 & -1.611311 \\
\hline 19B & 1.690844 & -2.364234 & 0.199541 \\
\hline $20 B$ & 2.892909 & -0.282196 & 0.199541 \\
\hline $21 \mathrm{~A}$ & -0.364612 & 0.210508 & 1.010417 \\
\hline $22 \mathrm{~A}$ & 1.528467 & -0.882461 & -0.535276 \\
\hline $23 \mathrm{~A}$ & -0.364611 & -0.882461 & -0.535276 \\
\hline $24 \mathrm{~A}$ & 0.581928 & 0.756994 & -0.535276 \\
\hline $25 \mathrm{~A}$ & 0.581928 & -1.428946 & 1.010417 \\
\hline $26 \mathrm{~A}$ & 1.528468 & 0.210509 & 1.010417 \\
\hline
\end{tabular}

\section{$E 27 / \varepsilon=-136.658297$}

$$
\begin{array}{llll}
\text { atom } & \mathrm{x} / \sigma & \mathrm{y} / \sigma & \mathrm{z} / \sigma
\end{array}
$$

$\begin{array}{llll}1 \mathrm{~A} & 0.000000 & 0.000000 & 0.000000\end{array}$

$\begin{array}{llll}2 A & 1.103094 & 0.000000 & 0.000000\end{array}$

$\begin{array}{llll}3 \mathrm{~A} & 0.000000 & -1.103094 & 0.000000\end{array}$

$\begin{array}{llll}4 \mathrm{~A} & -0.551547 & 0.551547 & -0.780006\end{array}$

$\begin{array}{llll}5 A & -0.551547 & 0.551547 & 0.780006\end{array}$

$\begin{array}{llll}6 \mathrm{~A} & -0.551547 & -0.551547 & 0.780006\end{array}$

$\begin{array}{llll}7 \mathrm{~A} & -1.103094 & 0.000000 & 0.000000\end{array}$

$\begin{array}{lllll}8 \mathrm{~A} & 0.000000 & 1.103094 & 0.000000\end{array}$

$\begin{array}{llll}9 A & -0.551547 & -0.551547 & -0.780006\end{array}$

$\begin{array}{lllll}10 A & 0.551547 & 0.551547 & -0.780006\end{array}$

$\begin{array}{llll}11 \mathrm{~A} & 0.551547 & 0.551547 & 0.780006\end{array}$

$\begin{array}{llll}12 \mathrm{~A} & 0.551547 & -0.551547 & 0.780006\end{array}$

$\begin{array}{llll}13 A & 0.551547 & -0.551547 & -0.780006\end{array}$ \begin{tabular}{|llll}
$14 \mathrm{~B}$ & -1.595543 & 1.595543 & 0.000000
\end{tabular}

$\begin{array}{llll}15 \mathrm{~B} & 0.000000 & 0.000000 & 2.256440\end{array}$

$\begin{array}{llll}16 \mathrm{~B} & -1.595543 & -1.595543 & 0.000000\end{array}$

$\begin{array}{llll}17 \mathrm{~B} & 0.000000 & 0.000000 & -2.256440\end{array}$

$\begin{array}{llll}18 \mathrm{~B} & 1.595543 & 1.595543 & 0.000000\end{array}$

$\begin{array}{llll}19 B & 1.595543 & -1.595543 & 0.000000\end{array}$

$\begin{array}{lllll}20 \mathrm{~B} & 1.993568 & 0.000000 & -1.409666\end{array}$

$\begin{array}{llll}21 B & 0.000000 & -1.993568 & -1.409666\end{array}$

$\begin{array}{lllll}22 \mathrm{~B} & -1.993568 & 0.000000 & -1.409666\end{array}$

$\begin{array}{llll}23 \mathrm{~B} & -1.993568 & 0.000000 & 1.409666\end{array}$

$\begin{array}{llll}24 \mathrm{~B} & 1.993568 & 0.000000 & 1.409666\end{array}$

$\begin{array}{llll}25 \mathrm{~B} & 0.000000 & 1.993568 & 1.409666\end{array}$

$\begin{array}{llll}26 \mathrm{~B} & 0.000000 & 1.993568 & -1.409666\end{array}$

$\begin{array}{llll}27 \mathrm{~B} & 0.000000 & -1.993568 & 1.409666\end{array}$

$E 28 / \varepsilon=-142.460916$

$\begin{array}{lcc}\text { atom } & \mathrm{x} / \sigma & \mathrm{yl} / \mathrm{r}\end{array}$

$\begin{array}{llll}1 \mathrm{~A} & 0.000000 & 0.000000 & 0.000000\end{array}$

$\begin{array}{llll}2 A & 1.084508 & 0.000000 & 0.000000\end{array}$

$\begin{array}{llll}3 \mathrm{~A} & -0.004959 & -1.084497 & 0.000000\end{array}$

$\begin{array}{llll}4 \mathrm{~A} & 0.539774 & -0.542248 & 0.768614\end{array}$

$\begin{array}{lllll}5 \mathrm{~A} & 0.539774 & -0.542248 & -0.768614\end{array}$

$\begin{array}{lllll}6 \mathrm{~A} & -0.523308 & 0.525706 & -0.792733\end{array}$

$\begin{array}{llll}7 A & -0.523308 & 0.525706 & 0.792733\end{array}$

$\begin{array}{llll}8 \mathrm{~A} & -1.085135 & -0.033559 & 0.000000\end{array}$

$\begin{array}{lllll}9 \mathrm{~A} & 0.038520 & 1.084970 & 0.000000\end{array}$

$\begin{array}{llll}10 A & -0.545524 & -0.581447 & 0.796828\end{array}$

$\begin{array}{llll}11 \mathrm{~A} & -0.545524 & -0.581447 & -0.796828\end{array}$

$\begin{array}{llll}12 \mathrm{~A} & 0.583935 & 0.542859 & 0.796828\end{array}$

$\begin{array}{lllll}13 \mathrm{~A} & 0.583935 & 0.542859 & -0.796828\end{array}$

$\begin{array}{lllll}14 \mathrm{~B} & 0.083975 & -0.084359 & -2.247124\end{array}$

$\begin{array}{llll}15 \mathrm{~B} & 0.083975 & -0.084359 & 2.247124\end{array}$

$\begin{array}{llll}16 \mathrm{~B} & -1.508609 & -1.669681 & 0.000000\end{array}$

$\begin{array}{llll}17 \mathrm{~B} & 1.676562 & 1.500959 & 0.000000\end{array}$

$\begin{array}{llll}18 \mathrm{~B} & 2.073422 & -0.372162 & 1.206923\end{array}$

$\begin{array}{llll}19 B & 0.362678 & -2.075102 & 1.206923\end{array}$

$\begin{array}{lllll}20 \mathrm{~B} & 2.073422 & -0.372162 & -1.206923\end{array}$

$\begin{array}{lllll}21 \mathrm{~B} & 0.362678 & -2.075102 & -1.206923\end{array}$

$\begin{array}{llll}22 \mathrm{~B} & -1.556588 & 1.563721 & 0.000000\end{array}$

$\begin{array}{llll}23 \mathrm{~A} & 1.094900 & -1.099918 & 0.000000\end{array}$

$\begin{array}{llll}24 \mathrm{~B} & -1.961833 & -0.036468 & -1.416119\end{array}$

$\begin{array}{llll}25 \mathrm{~B} & -1.961833 & -0.036468 & 1.416119\end{array}$

$\begin{array}{llll}26 \mathrm{~B} & 0.045438 & 1.961646 & 1.416118\end{array}$

$\begin{array}{llll}27 \mathrm{~B} & 0.045438 & 1.961646 & -1.416118\end{array}$

$\begin{array}{llll}28 \mathrm{~B} & 2.241923 & -2.252199 & 0.000000\end{array}$

$E 29 / \varepsilon=-148.254717$

atom $\quad \mathrm{x} / \sigma \quad \mathrm{y} / \sigma$

$\begin{array}{llll}1 \mathrm{~A} & 0.000000 & 0.000000 & 0.000000\end{array}$

$\begin{array}{llll}2 \mathrm{~A} & 1.101142 & 0.000000 & 0.000000\end{array}$

$\begin{array}{llll}3 \mathrm{~A} & 0.550570 & -0.953618 & 0.000000\end{array}$

$\begin{array}{lllll}4 \mathrm{~A} & 0.550570 & -0.317873 & -0.942694\end{array}$

$\begin{array}{llll}5 A & 0.550570 & -0.317873 & 0.942694\end{array}$

$\begin{array}{llll}6 \mathrm{~B} & 1.632073 & -2.315921 & 0.000000\end{array}$

$\begin{array}{llll}7 \mathrm{~B} & -0.639037 & 1.617761 & 0.000000\end{array}$

$\begin{array}{llll}8 \mathrm{~B} & -1.720542 & -0.255461 & 0.000000\end{array}$

$\begin{array}{llll}9 \mathrm{~B} & -0.530938 & -2.315919 & 0.000000\end{array}$

$\begin{array}{llll}10 \mathrm{~B} & 1.740178 & 1.617762 & 0.000000\end{array}$

$\begin{array}{llll}11 B & 2.821682 & -0.255461 & 0.000000\end{array}$

$\begin{array}{llll}12 \mathrm{~A} & -0.343695 & -0.834177 & -0.579217\end{array}$

$\begin{array}{llll}13 A & -0.343695 & -0.834177 & 0.579217\end{array}$

$\begin{array}{lllll}14 \mathrm{~A} & 1.444836 & -0.834178 & -0.579217\end{array}$

$\begin{array}{lllll}15 A & 1.444836 & -0.834178 & 0.579217\end{array}$

$\begin{array}{llll}16 \mathrm{~A} & 0.550571 & 0.714736 & -0.579217\end{array}$

$\begin{array}{llll}17 \mathrm{~A} & 0.550571 & 0.714736 & 0.579217\end{array}$

$\begin{array}{llll}18 \mathrm{~A} & -0.409942 & 0.236679 & 0.974382\end{array}$

$\begin{array}{lllll}19 A & 0.550569 & -1.426976 & -0.974382\end{array}$

$\begin{array}{lllll}20 A & 1.511082 & 0.236679 & 0.974382\end{array}$

$\begin{array}{lllll}21 \mathrm{~A} & -0.409942 & 0.236679 & -0.974382\end{array}$

$\begin{array}{llll}22 \mathrm{~A} & 0.550569 & -1.426976 & 0.974382\end{array}$

$\begin{array}{lllll}23 A & 1.511082 & 0.236679 & -0.974382\end{array}$

$\begin{array}{llll}24 \mathrm{~B} & 1.656062 & -0.956129 & 2.140517\end{array}$

$\begin{array}{llll}25 B & 0.550570 & 0.958639 & 2.140517\end{array}$ 
$\begin{array}{llll}26 \mathrm{~B} & -0.554922 & -0.956129 & 2.140517\end{array}$ $\begin{array}{llll}27 \mathrm{~B} & -0.554922 & -0.956129 & -2.140517\end{array}$ $\begin{array}{llll}28 B & 1.656062 & -0.956129 & -2.140517\end{array}$ $\begin{array}{llll}29 B & 0.550570 & 0.958639 & -2.140517\end{array}$

\section{$E 30 / \varepsilon=-154.825577$}

atom $\mathrm{x} / \sigma \quad \mathrm{yl}$

$\begin{array}{llll}1 \mathrm{~A} & 0.000000 & 0.000000 & 0.000000\end{array}$

$\begin{array}{llll}2 B & 2.235653 & 0.000000 & 0.000000\end{array}$

$\begin{array}{llll}3 B & 0.046579 & -2.235168 & 0.000000\end{array}$

$\begin{array}{llll}4 \mathrm{~B} & 0.046578 & -0.045617 & -2.234703\end{array}$

$\begin{array}{lllll}5 \mathrm{~A} & 0.010091 & -0.787996 & -0.772075\end{array}$

$\begin{array}{lllll}6 \mathrm{~A} & 0.788035 & 0.006329 & -0.772075\end{array}$

$\begin{array}{llll}7 A & 0.788035 & -0.771784 & 0.022085\end{array}$

$\begin{array}{llll}8 \mathrm{~A} & -0.774054 & -0.785121 & 0.001300\end{array}$

$\begin{array}{llll}9 \mathrm{~A} & -0.774054 & -0.014724 & -0.784983\end{array}$

$\begin{array}{llll}10 A & 0.768822 & 0.790243 & 0.001301\end{array}$

$\begin{array}{llll}11 \mathrm{~A} & 0.768822 & 0.017429 & 0.790052\end{array}$

$\begin{array}{lllll}12 \mathrm{~A} & -0.001407 & 0.774192 & -0.784983\end{array}$

$\begin{array}{llll}13 \mathrm{~A} & -0.001407 & -0.769019 & 0.790052\end{array}$

$\begin{array}{lllll}14 \mathrm{~A} & -0.789402 & 0.773122 & -0.014162\end{array}$

$\begin{array}{llll}15 \mathrm{~A} & -0.789402 & 0.001620 & 0.773250\end{array}$

$\begin{array}{llll}16 \mathrm{~A} & -0.018068 & 0.789196 & 0.773251\end{array}$

$\begin{array}{llll}17 \mathrm{~B} & -0.031946 & 0.031285 & 2.258136\end{array}$

$\begin{array}{llll}18 \mathrm{~B} & -0.031945 & 2.258304 & -0.014808\end{array}$

$\begin{array}{llll}19 \mathrm{~B} & -2.258480 & -0.015115 & -0.014809\end{array}$

$\begin{array}{llll}20 B & 1.399217 & -1.370363 & 1.461085\end{array}$

$\begin{array}{llll}21 B & 1.399217 & 1.432813 & -1.399897\end{array}$

22B $\quad-1.403349 \quad-1.428767 \quad-1.399898$

$\begin{array}{llll}23 \mathrm{~A} & 1.048506 & -1.026883 & -1.006135\end{array}$

$\begin{array}{llll}24 \mathrm{~B} & 1.392402 & 1.429055 & 1.400182\end{array}$

$\begin{array}{llll}25 B & -1.399737 & 1.370870 & -1.450155\end{array}$

$\begin{array}{llll}26 B & -1.399736 & -1.421876 & 1.400180\end{array}$

$\begin{array}{llll}27 \mathrm{~B} & -1.442857 & 1.413100 & 1.384549\end{array}$

$\begin{array}{lllll}28 \mathrm{~B} & 0.586653 & -2.148610 & -2.105198\end{array}$

$\begin{array}{llll}29 B & 2.160367 & -0.541758 & -2.105198\end{array}$

$\begin{array}{llll}30 \mathrm{~B} & 2.160368 & -2.115816 & -0.498681\end{array}$

\section{$E 31 / \varepsilon=-160.752462$}

\begin{tabular}{|c|c|c|c|}
\hline$n$ & $\mathrm{x} / \sigma$ & $y / \sigma$ & $z / \sigma$ \\
\hline $1 \mathrm{~A}$ & 0.000000 & 00000 & 0.000000 \\
\hline 2B & 2.169144 & 0.000000 & 0.000000 \\
\hline $3 A$ & 0.021713 & -1.157094 & 0.000000 \\
\hline 4B & -0.052768 & -1.538898 & 1.647001 \\
\hline $5 B$ & -0.052768 & -1.538898 & -1.647001 \\
\hline $6 \mathrm{~A}$ & 0.734349 & -0.532364 & 0.587302 \\
\hline $7 A$ & 0.734349 & -0.532364 & -0.587302 \\
\hline $8 B$ & -1.593906 & -1.811639 & 0.000000 \\
\hline $9 A$ & -0.724577 & -0.579148 & 0.546326 \\
\hline $10 \mathrm{~A}$ & -0.724577 & -0.579148 & -0.546326 \\
\hline $11 \mathrm{~A}$ & 0.715223 & 0.587671 & 0.561388 \\
\hline $12 \mathrm{~A}$ & -0.793437 & 0.509931 & 0.539784 \\
\hline $13 \mathrm{~A}$ & -0.793437 & 0.509931 & -0.539784 \\
\hline $14 \mathrm{~A}$ & 0.715223 & 0.587671 & -0.561388 \\
\hline $15 \mathrm{~A}$ & -0.037916 & 0.024589 & -1.139740 \\
\hline $16 \mathrm{~A}$ & -0.037916 & 0.024589 & 1.139740 \\
\hline $17 \mathrm{~A}$ & -0.073411 & 1.121743 & 0.000000 \\
\hline 18B & -1.728584 & -0.069769 & 1.700972 \\
\hline 19B & -1.728584 & -0.069769 & -1.700972 \\
\hline $20 B$ & -0.205394 & 1.613302 & -1.563817 \\
\hline 21B & -0.205394 & 1.613302 & 1.563817 \\
\hline 22B & -1.844272 & 1.595544 & 0.000000 \\
\hline $23 \mathrm{~A}$ & -1.543011 & -0.089916 & 0.000000 \\
\hline $24 B$ & 1.351632 & 0.119410 & 2.011012 \\
\hline $25 B$ & 1.351632 & 0.119410 & -2.011012 \\
\hline $26 \mathrm{~A}$ & 1.089454 & -1.369130 & 0.000000 \\
\hline 27B & 1.315940 & 2.016966 & 0.000000 \\
\hline $28 B$ & 0.411793 & -2.849822 & 0.000000 \\
\hline $29 \mathrm{~B}$ & 2.070957 & -1.840084 & -1.184203 \\
\hline $30 \mathrm{~B}$ & 2.070957 & -1.840084 & 1.184203 \\
\hline 010 & -3.166058 & -0.21807 & 0.0000 \\
\hline
\end{tabular}

$\begin{array}{llll}31 B & -3.166058 & -0.218070 & 0.000000\end{array}$
$E 32 / \varepsilon=-167.803356$

atom $\mathrm{x} / \sigma \quad \mathrm{y} / \sigma$

$\begin{array}{llll}1 \mathrm{~A} & 0.000000 & 0.000000 & 0.000000\end{array}$

$\begin{array}{llll}2 A & 1.041698 & 0.000000 & 0.000000\end{array}$

3B $\quad 1.957366 \quad-1.324903 \quad 0.000000$

$\begin{array}{llll}4 \mathrm{~B} & 1.957359 & 0.857359 & 1.010109\end{array}$

$\begin{array}{llll}5 A & -0.120412 & -0.488530 & 1.055425\end{array}$

$\begin{array}{llll}6 \mathrm{~A} & 0.379764 & -0.975465 & 0.234905\end{array}$

$\begin{array}{lllll}7 A & 0.379759 & 0.452137 & 0.895705\end{array}$

8A $-0.957915 \quad-0.209382$

0.452341

$\begin{array}{lllll}9 \mathrm{~A} & 0.454851 & 0.410719 & -0.887326\end{array}$

$\begin{array}{llll}10 A & 0.530848 & 1.031796 & 0.007314\end{array}$

$\begin{array}{llll}11 \mathrm{~A} & 0.530853 & -0.673257 & -0.781910\end{array}$

$\begin{array}{llll}12 \mathrm{~B} & -0.549176 & 1.725449 & -1.040353\end{array}$

$\begin{array}{llll}13 B & -0.549170 & -0.323388 & -1.988704\end{array}$

$\begin{array}{lllll}\text { 14B } & 0.019419 & -2.273725 & -0.688683\end{array}$

$\begin{array}{llll}15 B & 0.019406 & 1.996390 & 1.287838\end{array}$

$\begin{array}{llll}16 \mathrm{~A} & -0.518323 & -0.789520 & -0.446225\end{array}$

$\begin{array}{llll}17 \mathrm{~A} & -0.518329 & 0.851101 & 0.313175\end{array}$

$\begin{array}{llll}18 \mathrm{~B} & 0.445029 & -1.953130 & 1.540919\end{array}$

$\begin{array}{llll}19 \mathrm{~B} & 0.445022 & 0.089082 & 2.486204\end{array}$

$\begin{array}{llll}20 B & -1.525858 & -0.852329 & 1.841373\end{array}$

$\begin{array}{llll}21 B & 1.218826 & 1.017004 & -2.197154\end{array}$

$\begin{array}{llll}22 \mathrm{~A} & 0.976763 & -0.464923 & 1.004433\end{array}$

$\begin{array}{llll}23 \mathrm{~A} & -0.946244 & 0.241837 & -0.522479\end{array}$

$\begin{array}{llll}24 \mathrm{~B} & -2.083630 & -1.055831 & -0.379527\end{array}$

$\begin{array}{llll}25 B & -2.083638 & 0.972569 & 0.559366\end{array}$

$\begin{array}{llll}26 A & -0.658064 & -1.285810 & 0.506563\end{array}$

$\begin{array}{llll}27 A & -0.658070 & 0.445844 & 1.308099\end{array}$

$\begin{array}{lllll}28 \mathrm{~A} & 1.428810 & 0.856160 & -0.567312\end{array}$

$\begin{array}{lllll}29 A & 1.428814 & -0.121504 & -1.019847\end{array}$

$\begin{array}{llll}\text { 30B } & 1.409670 & -1.270270 & -2.135000\end{array}$

$\begin{array}{lllll}31 B & 1.409659 & 2.449727 & -0.413115\end{array}$

$\begin{array}{lllll}32 \mathrm{~B} & 2.960195 & 0.431293 & -0.931757\end{array}$

\section{$E 33 / \varepsilon=-175.843136$}

\begin{tabular}{|c|c|c|c|}
\hline atom & $\mathrm{x} / \sigma$ & $y / \sigma$ & $z / \sigma$ \\
\hline $1 \mathrm{~A}$ & 0.000000 & 0.000000 & 0.000000 \\
\hline $2 \mathrm{~A}$ & 1.088402 & 0.000000 & 0.000000 \\
\hline $3 A$ & 0.526519 & -0.952573 & 0.000000 \\
\hline $4 \mathrm{~A}$ & -0.382626 & -0.813086 & 0.614090 \\
\hline $5 A$ & 0.526519 & 0.728210 & 0.614090 \\
\hline $6 \mathrm{~A}$ & -0.382626 & 0.225695 & 0.993619 \\
\hline $7 \mathrm{~A}$ & 0.537927 & 0.324702 & -0.888718 \\
\hline $8 \mathrm{~A}$ & -0.023956 & -0.627872 & -0.888718 \\
\hline $9 A$ & -0.023956 & 1.052912 & -0.274628 \\
\hline $10 \mathrm{~A}$ & -0.933101 & -0.488385 & -0.274627 \\
\hline $11 \mathrm{~A}$ & -0.933101 & 0.550396 & 0.104901 \\
\hline $12 \mathrm{~A}$ & 0.575186 & -0.339278 & 0.928614 \\
\hline $13 \mathrm{~A}$ & -0.575186 & 0.339277 & -0.928612 \\
\hline 14B & 0.688191 & -2.164203 & -1.216977 \\
\hline $15 B$ & -1.801708 & 1.062750 & 1.504271 \\
\hline $16 B$ & -1.801707 & -1.782186 & 0.464847 \\
\hline 17B & 0.688192 & 2.439001 & 0.464846 \\
\hline 18B & 2.227034 & 0.444634 & -1.216977 \\
\hline $19 B$ & -1.315199 & 2.037201 & -0.453144 \\
\hline 20B & -2.419200 & 0.165559 & -0.453143 \\
\hline 21B & -1.315198 & -1.265247 & -1.659723 \\
\hline $22 B$ & 0.327750 & -2.234350 & 0.992745 \\
\hline 23B & 0.471114 & -0.277890 & -2.405432 \\
\hline 24B & 2.114062 & -1.246996 & 0.247037 \\
\hline $25 B$ & 0.471116 & 1.763135 & -1.659724 \\
\hline $26 B$ & 2.114061 & 0.794029 & 0.992746 \\
\hline 27B & -0.776252 & -0.803544 & 2.199326 \\
\hline 28B & 0.327748 & 1.068098 & 2.199326 \\
\hline $29 A$ & -1.326042 & -0.264749 & 0.724627 \\
\hline $30 A$ & 1.072791 & 1.061166 & -0.276781 \\
\hline $31 \mathrm{~A}$ & -0.409769 & -1.452255 & -0.276781 \\
\hline $32 \mathrm{~A}$ & -0.409770 & 1.288630 & 0.724627 \\
\hline $33 \mathrm{~A}$ & 1.072791 & -0.632794 & -0.895686 \\
\hline
\end{tabular}

$\mathrm{x} / \sigma$ $y / \sigma$

$\begin{array}{llll}1 \mathrm{~A} & 0.000000 & 0.000000 & 0.000000\end{array}$ $\begin{array}{llll}2 \mathrm{~A} & 1.090209 & 0.000000 & 0.000000\end{array}$

$\begin{array}{llll}3 \mathrm{~A} & 0.527307 & -0.954203 & 0.000000\end{array}$

$\begin{array}{llll}4 \mathrm{~A} & 0.527306 & 0.729546 & 0.615033\end{array}$

$\begin{array}{llll}5 A & -0.383489 & -0.814387 & 0.615033\end{array}$

$\begin{array}{llll}6 \mathrm{~A} & -0.383490 & 0.226228 & 0.995144\end{array}$

$\begin{array}{lllll}7 \mathrm{~A} & 0.536840 & 0.324829 & -0.889270\end{array}$

$\begin{array}{llll}8 \mathrm{~A} & -0.024650 & -0.626980 & -0.889270\end{array}$

$\begin{array}{llll}9 A & -0.024650 & 1.052544 & -0.275780\end{array}$

$\begin{array}{llll}10 \mathrm{~A} & -0.933159 & -0.487514 & -0.275780\end{array}$

$\begin{array}{llll}11 \mathrm{~A} & -0.933160 & 0.550488 & 0.103377\end{array}$

$\begin{array}{llll}12 \mathrm{~A} & 0.568195 & -0.335189 & 0.917634\end{array}$

$\begin{array}{llll}13 \mathrm{~A} & -0.575890 & 0.339728 & -0.930060\end{array}$

$\begin{array}{llll}14 \mathrm{~B} & -0.806177 & -0.801582 & 2.194456\end{array}$

$\begin{array}{llll}15 B & 0.311657 & -2.250341 & 0.973101\end{array}$

$\begin{array}{llll}16 B & 0.311654 & 1.093311 & 2.194456\end{array}$

$\begin{array}{llll}17 \mathrm{~B} & 2.120347 & 0.815660 & 0.973099\end{array}$

$\begin{array}{llll}18 B & 2.120348 & -1.250832 & 0.218260\end{array}$

$\begin{array}{llll}19 B & 0.683191 & 2.442703 & 0.456429\end{array}$

$\begin{array}{llll}20 \mathrm{~B} & -1.807527 & -1.779437 & 0.456429\end{array}$

$\begin{array}{llll}21 B & 2.222542 & 0.447639 & -1.225481\end{array}$

22B $\quad 0.683194 \quad-2.161787 \quad-1.225480$

23B $\quad-1.807529 \quad 1.066295 \quad 1.495905$

$\begin{array}{llll}24 \mathrm{~B} & 0.467808 & -0.275969 & -2.407522\end{array}$

$\begin{array}{llll}25 \mathrm{~B} & 0.467809 & 1.762768 & -1.662820\end{array}$

$\begin{array}{llll}26 \mathrm{~B} & -1.316591 & -1.262057 & -1.662820\end{array}$

$\begin{array}{lllll}27 \mathrm{~B} & -1.316592 & 2.036691 & -0.457868\end{array}$

$\begin{array}{lllll}28 \mathrm{~B} & -2.419411 & 0.167247 & -0.457870\end{array}$

$\begin{array}{llll}29 \mathrm{~A} & -1.327178 & -0.263289 & 0.720794\end{array}$

$\begin{array}{llll}30 A & -0.411480 & 1.288955 & 0.720794\end{array}$

$\begin{array}{lllll}31 \mathrm{~A} & 1.070152 & 1.061510 & -0.279707\end{array}$

$\begin{array}{llll}32 \mathrm{~A} & -0.411478 & -1.450074 & -0.279707\end{array}$

33A $\quad 1.070152 \quad-0.631303 \quad-0.898051$

$\begin{array}{llll}34 \mathrm{~B} & 1.391318 & -0.820762 & 2.246971\end{array}$

$E 35 / \varepsilon=-191.637722$

$\begin{array}{lcr}\text { atom } & \mathrm{x} / \sigma & y / \sigma\end{array}$

0.000000

$z / \sigma$

$\begin{array}{llll}2 \mathrm{~A} & 1.090132 & 0.000000 & 0.000000\end{array}$

$\begin{array}{llll}3 \mathrm{~A} & -0.934806 & -0.560827 & 0.000000\end{array}$

$\begin{array}{llll}4 \mathrm{~A} & -0.934806 & 0.531541 & 0.178857\end{array}$

$\begin{array}{lllll}5 \mathrm{~A} & 0.528155 & 0.936722 & -0.178862\end{array}$

$\begin{array}{llll}6 \mathrm{~A} & -0.381142 & 0.684881 & -0.757665\end{array}$

$\begin{array}{lllll}7 \mathrm{~A} & 0.528155 & -0.830767 & -0.468259\end{array}$

$\begin{array}{lllll}8 \mathrm{~A} & -0.381142 & -0.407487 & -0.936522\end{array}$

$\begin{array}{lllll}9 A & -0.025509 & -0.984107 & 0.468264\end{array}$

$\begin{array}{llll}10 A & -0.025509 & 0.783382 & 0.757661\end{array}$

$\begin{array}{llll}11 \mathrm{~A} & 0.536468 & -0.153340 & 0.936523\end{array}$

$\begin{array}{llll}12 \mathrm{~A} & 0.570686 & 0.158055 & -0.965317\end{array}$

$\begin{array}{llll}13 A & -0.570687 & -0.158055 & 0.965317\end{array}$

$\begin{array}{llll}14 \mathrm{~B} & -0.799448 & 0.378085 & -2.309152\end{array}$

$\begin{array}{llll}15 B & 0.316429 & -1.481896 & -1.953997\end{array}$

$\begin{array}{llll}16 \mathrm{~B} & 0.491026 & 0.735507 & 2.309148\end{array}$

$\begin{array}{llll}17 \mathrm{~B} & 0.491026 & -1.433529 & 1.954004\end{array}$

$\begin{array}{llll}18 \mathrm{~B} & 0.316428 & 2.027678 & -1.379362\end{array}$

$\begin{array}{llll}19 \mathrm{~B} & -1.314500 & 1.575984 & 1.379351\end{array}$

$\begin{array}{llll}20 \mathrm{~B} & -1.314499 & -1.933591 & 0.804716\end{array}$

$\begin{array}{llll}21 B & -2.430375 & -0.073609 & 0.449560\end{array}$

$\begin{array}{llll}22 \mathrm{~B} & 2.121954 & -0.981835 & -0.804709\end{array}$

$\begin{array}{llll}23 B & 2.121953 & 1.187202 & -0.449564\end{array}$

$\begin{array}{llll}24 B & 2.226649 & -0.209907 & 1.282007\end{array}$

$\begin{array}{llll}25 \mathrm{~B} & -1.801398 & 1.665158 & -0.792330\end{array}$

$\begin{array}{llll}26 \mathrm{~B} & -1.801397 & -1.325523 & -1.282004\end{array}$

$\begin{array}{llll}27 \mathrm{~B} & 0.688071 & 2.354647 & 0.792317\end{array}$

$\begin{array}{lllll}28 \mathrm{~B} & 0.688073 & -2.484377 & 0.000007\end{array}$

$\begin{array}{llll}29 \mathrm{~A} & 1.070420 & 0.787647 & 0.761788\end{array}$

$\begin{array}{llll}30 \mathrm{~A} & 1.070421 & -0.989466 & 0.470815\end{array}$

$\begin{array}{llll}31 \mathrm{~A} & -0.408866 & 1.476259 & -0.000005\end{array}$

$\begin{array}{lllll}32 \mathrm{~A} & -0.408864 & -1.399172 & -0.470809\end{array}$

$\begin{array}{lllll}33 \mathrm{~A} & -1.323114 & 0.124730 & -0.761791\end{array}$

$\begin{array}{llll}34 \mathrm{~B} & -1.396719 & -0.386829 & 2.362546\end{array}$

$\begin{array}{llll}35 \mathrm{~B} & 1.396717 & 0.386828 & -2.362546\end{array}$ 
$E 36 / \varepsilon=-196.965963$

atom $x / \sigma$

$\begin{array}{llll}2 \mathrm{~A} & 1.090368 & 0.000000 & 0.000000\end{array}$

$\begin{array}{llll}3 \mathrm{~A} & 0.527269 & -0.954405 & 0.000000\end{array}$

$\begin{array}{llll}4 \mathrm{~A} & -0.027016 & -0.627377 & 0.891386\end{array}$

$\begin{array}{llll}5 \mathrm{~A} & 0.536082 & 0.327030 & 0.891386\end{array}$

6A $\quad-0.381902 \quad-0.813698 \quad-0.616930$

$\begin{array}{llll}7 A & -0.936158 & -0.486686 & 0.274408\end{array}$

$\begin{array}{llll}8 \mathrm{~A} & -0.026700 & 1.054771 & 0.274406\end{array}$

$\begin{array}{lllll}9 A & 0.527556 & 0.727760 & -0.616932\end{array}$

$\begin{array}{llll}10 A & -0.935005 & 0.551651 & -0.107188\end{array}$

$\begin{array}{llll}11 \mathrm{~A} & -0.381870 & 0.225301 & -0.996724\end{array}$

$\begin{array}{llll}12 \mathrm{~A} & -0.570982 & 0.336880 & 0.916189\end{array}$

$\begin{array}{llll}13 \mathrm{~A} & 0.569308 & -0.335892 & -0.917594\end{array}$

$\begin{array}{llll}14 \mathrm{~B} & 0.527444 & -0.311189 & 2.400131\end{array}$

$\begin{array}{llll}15 B & 2.128118 & -1.255588 & -0.174021\end{array}$

$\begin{array}{llll}16 \mathrm{~B} & 0.658566 & -2.184831 & 1.211078\end{array}$

$\begin{array}{llll}17 \mathrm{~B} & 2.230858 & 0.480076 & 1.211074\end{array}$

$\begin{array}{llll}18 \mathrm{~B} & -1.312165 & -1.287821 & 1.652794\end{array}$

$\begin{array}{llll}19 B & 2.124675 & 0.808440 & -0.971682\end{array}$

$\begin{array}{llll}\text { 20B } & 0.492712 & 1.771300 & 1.652790\end{array}$

$\begin{array}{llll}21 B & 0.319797 & -2.250681 & -0.971677\end{array}$

$\begin{array}{llll}22 \mathrm{~B} & 0.323064 & 1.082695 & -2.197804\end{array}$

$\begin{array}{llll}23 \mathrm{~B} & -0.791467 & -0.806345 & -2.197801\end{array}$

$\begin{array}{llll}24 \mathrm{~B} & -2.427695 & 0.159030 & 0.433529\end{array}$

$\begin{array}{llll}25 \mathrm{~B} & -1.313164 & 2.048071 & 0.433526\end{array}$

$\begin{array}{llll}26 \mathrm{~A} & 1.066127 & -0.629013 & 0.893714\end{array}$

$\begin{array}{lllll}27 \mathrm{~B} & 0.688481 & 2.440985 & -0.467429\end{array}$

$\begin{array}{llll}28 \mathrm{~B} & -1.803679 & -1.783023 & -0.467423\end{array}$

$\begin{array}{llll}29 B & -1.799742 & 1.061843 & -1.508692\end{array}$

$\begin{array}{llll}30 A & -1.324249 & -0.265144 & -0.726176\end{array}$

$\begin{array}{lllll}31 \mathrm{~A} & -0.408288 & 1.287335 & -0.726178\end{array}$

$\begin{array}{llll}32 \mathrm{~A} & 1.066086 & 1.061612 & 0.273437\end{array}$

$\begin{array}{llll}33 \mathrm{~A} & -0.413706 & -1.446515 & 0.273440\end{array}$

$\begin{array}{llll}34 \mathrm{~B} & 1.413054 & -0.833705 & -2.226940\end{array}$

$\begin{array}{llll}35 \mathrm{~B} & -1.375866 & 0.811762 & 2.258113\end{array}$

36A $1.837953-1.084389 \quad 1.540725$

\section{$E 37 / \varepsilon=-202.799137$}

\begin{tabular}{crrr|} 
atom & $x / \sigma$ & $y / \sigma$ & \multicolumn{1}{c}{$z / \sigma$} \\
1A & 0.000000 & 0.000000 & 0.000000 \\
2A & 1.076401 & 0.000000 & 0.000000 \\
3A & 0.468938 & -0.965959 & 0.000000 \\
4A & 0.558371 & 0.292678 & 0.897899 \\
5A & 0.558372 & 0.292678 & -0.897899 \\
6A & -0.346353 & 0.858289 & -0.561943 \\
7A & -0.346353 & 0.858289 & 0.561942 \\
8A & -0.015631 & -0.629364 & -0.899278 \\
9A & -0.015631 & -0.629364 & 0.899278 \\
10A & -0.918484 & -0.076048 & -0.564296 \\
11A & -0.918485 & -0.076048 & 0.564295 \\
12B & 2.152211 & 0.607587 & 1.086305 \\
13B & 2.152212 & 0.607587 & -1.086304 \\
14A & 0.592895 & 0.975107 & 0.000000 \\
15A & 1.132596 & -0.712866 & 0.805171 \\
16A & 1.132596 & -0.712866 & -0.805171 \\
17B & 0.765841 & -0.485040 & 2.378062 \\
18B & 0.765842 & -0.485039 & -2.378061 \\
19B & 0.357935 & -2.208748 & 1.073165 \\
20B & 0.357936 & -2.208747 & -1.073165 \\
21A & -0.620627 & -0.977892 & 0.000000 \\
22B & 0.374880 & 1.661253 & -1.785320 \\
23B & 0.374879 & 1.661253 & 1.785321 \\
24B & -0.731730 & 2.343329 & 0.000000 \\
25B & -1.760814 & 1.077550 & 1.512295 \\
26B & -1.760813 & 1.077550 & -1.512296 \\
27B & -2.394793 & -0.463440 & -0.000001 \\
28B & -1.325265 & -1.077687 & 1.792778 \\
29B & -1.325264 & -1.077687 & -1.792779 \\
30A & -0.377224 & 0.234293 & 1.468807 \\
31A & -0.377224 & 0.234294 & -1.468808 \\
\end{tabular}
$\begin{array}{lllll}31 \mathrm{~A} & -0.377224 & 0.234294 & -1.468808\end{array}$

\begin{tabular}{|llll}
$32 \mathrm{~A}$ & -1.287012 & 0.784831 & 0.000000
\end{tabular} $\begin{array}{llll}33 \mathrm{~A} & 1.960932 & -0.606148 & 0.000000\end{array}$ $\begin{array}{llll}34 \mathrm{~A} & 1.387704 & -1.515294 & 0.000000\end{array}$ $\begin{array}{llll}35 B & 2.475387 & -1.574100 & 1.225719\end{array}$ $\begin{array}{llll}36 \mathrm{~B} & 2.475388 & -1.574099 & -1.225717\end{array}$ $\begin{array}{llll}37 \mathrm{~B} & 1.452700 & 2.375498 & 0.000001\end{array}$

\section{$E 38 / \varepsilon=-210.677219$}

atom $\mathrm{x} / \sigma \quad \mathrm{yl}$

$\begin{array}{llll}1 \mathrm{~A} & 0.000000 & 0.000000 & 0.000000\end{array}$

$\begin{array}{llll}2 \mathrm{~A} & 1.076990 & 0.000000 & 0.000000\end{array}$

$\begin{array}{llll}3 A & 0.470478 & -0.968792 & 0.000000\end{array}$

$\begin{array}{lllll}4 \mathrm{~A} & 0.559680 & 0.291320 & -0.897419\end{array}$

$\begin{array}{lllll}5 \mathrm{~A} & 0.559680 & 0.291320 & 0.897419\end{array}$

$\begin{array}{llll}6 \mathrm{~A} & -0.017561 & -0.630714 & 0.897419\end{array}$

$\begin{array}{llll}7 A & -0.017561 & -0.630714 & -0.897419\end{array}$

$\begin{array}{llll}8 \mathrm{~A} & -0.341016 & 0.861064 & 0.561267\end{array}$

$\begin{array}{lllll}9 A & -0.341016 & 0.861064 & -0.561267\end{array}$

$\begin{array}{llll}10 A & -0.923530 & -0.069393 & 0.561267\end{array}$

$\begin{array}{llll}11 A & -0.923530 & -0.069393 & -0.561267\end{array}$

$\begin{array}{llll}12 \mathrm{~B} & 0.389283 & -2.204420 & 1.086116\end{array}$

$\begin{array}{llll}13 B & 0.389283 & -2.204420 & -1.086116\end{array}$

$\begin{array}{llll}14 \mathrm{~B} & 2.153015 & 0.612812 & -1.086116\end{array}$

$\begin{array}{llll}15 B & 2.153015 & 0.612812 & 1.086116\end{array}$

$\begin{array}{llll}16 \mathrm{~A} & -0.616955 & -0.964898 & 0.000000\end{array}$

$\begin{array}{llll}17 A & 0.598449 & 0.976485 & 0.000000\end{array}$

$\begin{array}{llll}18 \mathrm{~A} & 1.135619 & -0.710955 & -0.805030\end{array}$

$\begin{array}{llll}19 A & 1.135619 & -0.710955 & 0.805030\end{array}$

$\begin{array}{llll}20 \mathrm{~B} & 0.765091 & -0.478986 & -2.378095\end{array}$

$\begin{array}{llll}\text { 21B } & 0.765091 & -0.478986 & 2.378095\end{array}$

$\begin{array}{lllll}22 B & 0.380965 & 1.662781 & -1.784535\end{array}$

$\begin{array}{llll}23 \mathrm{~B} & 0.380965 & 1.662781 & 1.784535\end{array}$

$\begin{array}{llll}24 \mathrm{~B} & -1.329312 & -1.069066 & -1.784536\end{array}$

$\begin{array}{llll}25 B & -1.329312 & -1.069066 & 1.784536\end{array}$

$\begin{array}{llll}26 \mathrm{~B} & -0.720311 & 2.351144 & 0.000000\end{array}$

$\begin{array}{llll}27 \mathrm{~B} & -2.429606 & -0.379134 & 0.000000\end{array}$

$\begin{array}{llll}28 \mathrm{~B} & -1.748357 & 1.094562 & -1.518473\end{array}$

$\begin{array}{llll}29 B & -1.748357 & 1.094562 & 1.518473\end{array}$

$\begin{array}{llll}30 \mathrm{~A} & -1.279854 & 0.801254 & 0.000000\end{array}$

$\begin{array}{llll}31 \mathrm{~A} & -0.376420 & 0.235659 & -1.464958\end{array}$

$\begin{array}{llll}32 \mathrm{~A} & -0.376420 & 0.235659 & 1.464958\end{array}$

$\begin{array}{llll}33 \mathrm{~A} & 1.966308 & -0.597748 & 0.000000\end{array}$

$\begin{array}{llll}34 \mathrm{~A} & 1.396666 & -1.507646 & 0.000000\end{array}$

$\begin{array}{llll}35 \mathrm{~B} & 2.492749 & -1.560589 & -1.221309\end{array}$

$\begin{array}{llll}36 \mathrm{~B} & 2.492749 & -1.560589 & 1.221309\end{array}$

$\begin{array}{llll}37 \mathrm{~B} & -1.503313 & -2.352599 & 0.000000\end{array}$

$\begin{array}{llll}38 \mathrm{~B} & 1.459541 & 2.380005 & 0.000000\end{array}$

$E 39 / \varepsilon=-216.589951$

$\begin{array}{cccc}1 \mathrm{~A} & 0.000000 & 0.000000 & 0.000000\end{array}$

$\begin{array}{llll}2 \mathrm{~A} & 1.082588 & 0.000000 & 0.000000\end{array}$

$\begin{array}{llll}3 \mathrm{~A} & 0.552598 & -0.930931 & 0.000000\end{array}$

$\begin{array}{llll}4 \mathrm{~B} & 2.152339 & 0.696257 & 0.936820\end{array}$

$\begin{array}{llll}5 \mathrm{~B} & 0.499925 & -2.206223 & 0.936816\end{array}$

$\begin{array}{llll}6 \mathrm{~A} & 0.534796 & -0.304467 & 0.963348\end{array}$

$\begin{array}{llll}7 \mathrm{~A} & 0.013091 & -0.638980 & -0.893954\end{array}$

$\begin{array}{lllll}8 \mathrm{~A} & 0.556150 & 0.314907 & -0.893953\end{array}$

$\begin{array}{llll}9 A & -0.362595 & -0.823825 & 0.611865\end{array}$

$\begin{array}{llll}10 \mathrm{~A} & 0.523332 & 0.732313 & 0.611867\end{array}$

$\begin{array}{lllll}11 \mathrm{~A} & -0.035041 & 1.046567 & -0.281528\end{array}$

$\begin{array}{llll}12 \mathrm{~A} & -0.917842 & -0.504079 & -0.281530\end{array}$

$\begin{array}{llll}13 \mathrm{~A} & -0.943696 & 0.537257 & 0.091601\end{array}$

$\begin{array}{llll}14 \mathrm{~A} & -0.404492 & 0.230280 & 0.988725\end{array}$

$\begin{array}{llll}15 A & 1.142547 & -0.650464 & -0.883705\end{array}$

$\begin{array}{lllll}16 \mathrm{~A} & -0.553373 & 0.315043 & -0.929067\end{array}$

$\begin{array}{lllll}17 \mathrm{~B} & 0.537995 & -0.306283 & -2.392773\end{array}$

$\begin{array}{llll}18 \mathrm{~B} & 0.343344 & 1.066109 & 2.182851\end{array}$

$\begin{array}{llll}19 \mathrm{~B} & -0.741505 & -0.839437 & 2.182848\end{array}$

$\begin{array}{llll}\text { 20B } & 0.572219 & -2.226569 & -1.298101\end{array}$

$\begin{array}{lllll}21 B & 2.206740 & 0.644480 & -1.298097\end{array}$

$\begin{array}{llll}22 \mathrm{~B} & -1.319580 & -1.275408 & -1.648316\end{array}$ $\begin{array}{llll}23 \mathrm{~B} & 0.423173 & 1.785750 & -1.648311\end{array}$ \begin{tabular}{|llll}
$24 \mathrm{~B}$ & -2.439738 & 0.118842 & -0.406838
\end{tabular} $\begin{array}{lllll}25 \mathrm{~B} & -1.347537 & 2.037300 & -0.406834\end{array}$ $\begin{array}{lllll}26 \mathrm{~A} & 1.426425 & -0.812080 & 0.667219\end{array}$ $\begin{array}{llll}27 \mathrm{~B} & -1.728966 & -1.839662 & 0.473361\end{array}$ $\begin{array}{llll}28 \mathrm{~B} & 0.699411 & 2.425799 & 0.473368\end{array}$ $\begin{array}{llll}29 B & -1.794245 & 1.021482 & 1.525657\end{array}$ $\begin{array}{llll}30 \mathrm{~A} & -0.418621 & 1.284154 & 0.717144\end{array}$ $\begin{array}{llll}31 \mathrm{~A} & -1.317943 & -0.295510 & 0.717142\end{array}$ $\begin{array}{lllll}32 \mathrm{~A} & 1.059945 & 1.059480 & -0.275311\end{array}$ $\begin{array}{llll}33 \mathrm{~A} & -0.370019 & -1.452262 & -0.275315\end{array}$ $\begin{array}{lllll}34 \mathrm{~A} & 1.482506 & -1.473893 & -0.201427\end{array}$ $\begin{array}{lllll}35 \mathrm{~A} & 2.024152 & -0.522486 & -0.201427\end{array}$ $\begin{array}{lllll}36 \mathrm{~B} & 1.438897 & -0.819183 & 2.311081\end{array}$ $\begin{array}{llll}37 \mathrm{~B} & 2.826633 & -1.609233 & 0.754043\end{array}$ $\begin{array}{llll}38 \mathrm{~B} & 2.555041 & -1.454612 & -1.466799\end{array}$ $\begin{array}{lllll}39 B & -1.412234 & 0.804007 & -2.239082\end{array}$

$E 40 / \varepsilon=-223.700145$

$\begin{array}{cccc}\text { atom } & \mathrm{x} / \sigma & y / \sigma & z / \sigma \\ 1 \mathrm{~A} & 0.000000 & 0.000000 & 0.000000\end{array}$

$\begin{array}{llll}2 \mathrm{~A} & 1.078780 & 0.000000 & 0.000000\end{array}$

$\begin{array}{llll}3 \mathrm{~B} & -1.656201 & -0.000017 & 0.000000\end{array}$

$\begin{array}{llll}4 \mathrm{~B} & 2.734981 & 0.000016 & 0.000001\end{array}$

$\begin{array}{lllll}5 A & 0.539383 & 0.999057 & -0.034117\end{array}$

$\begin{array}{llll}6 \mathrm{~A} & 0.539393 & -0.469982 & 0.882268\end{array}$

$\begin{array}{lllll}7 A & 0.539394 & -0.529075 & -0.848150\end{array}$

$\begin{array}{llll}8 \mathrm{~B} & 0.539405 & -2.026431 & 1.375992\end{array}$

$\begin{array}{lllll}9 B & 0.539374 & 2.126936 & -1.214873\end{array}$

10B $\quad 0.539407 \quad-2.115580-1.234542$

$\begin{array}{lllll}11 \mathrm{~B} & 0.539392 & -0.178430 & -2.442936\end{array}$

$\begin{array}{llll}12 B & 0.539372 & 2.204860 & 1.066944\end{array}$

$\begin{array}{llll}13 B & 0.539389 & -0.011358 & 2.449418\end{array}$

$\begin{array}{lllll}14 \mathrm{~A} & 0.539386 & 0.523304 & -0.982366\end{array}$

$\begin{array}{llll}15 A & 0.539385 & 0.589102 & 0.944379\end{array}$

$\begin{array}{llll}16 A & 0.539398 & -1.112406 & 0.037989\end{array}$

$\begin{array}{lllll}17 A & 1.480070 & -0.953429 & -0.507985\end{array}$

$\begin{array}{llll}18 \mathrm{~A} & -0.401290 & 0.916639 & -0.571706\end{array}$

$\begin{array}{llll}19 \mathrm{~A} & 1.480070 & -0.916552 & 0.571846\end{array}$

$\begin{array}{llll}20 A & -0.401290 & 0.953505 & 0.507841\end{array}$

$\begin{array}{lllll}21 \mathrm{~A} & 1.480063 & -0.036947 & -1.079685\end{array}$

$\begin{array}{lllll}22 \mathrm{~A} & -0.401282 & -0.036960 & -1.079685\end{array}$

$\begin{array}{llll}23 \mathrm{~A} & -0.401284 & 0.036782 & 1.079691\end{array}$

$\begin{array}{llll}24 \mathrm{~A} & 1.480062 & 0.036796 & 1.079692\end{array}$

$\begin{array}{lllll}25 \mathrm{~A} & 1.480056 & 0.916651 & -0.571705\end{array}$

$\begin{array}{llll}26 \mathrm{~A} & 1.480055 & 0.953518 & 0.507841\end{array}$

$\begin{array}{llll}27 A & -0.401276 & -0.916565 & 0.571845\end{array}$

$\begin{array}{llll}28 A & -0.401275 & -0.953441 & -0.507985\end{array}$

$\begin{array}{llll}29 B & 2.313243 & 1.184182 & 1.898318\end{array}$

$\begin{array}{llll}30 \mathrm{~B} & -1.234482 & 1.051893 & -1.974679\end{array}$

$\begin{array}{llll}31 \mathrm{~B} & 2.313247 & 1.051920 & -1.974677\end{array}$

$\begin{array}{llll}32 \mathrm{~B} & -1.234484 & 1.184154 & 1.898317\end{array}$

$\begin{array}{llll}33 \mathrm{~B} & -1.234458 & -2.236088 & 0.076361\end{array}$

$\begin{array}{llll}34 \mathrm{~B} & 2.313274 & -2.236061 & 0.076363\end{array}$

$\begin{array}{lllll}35 \mathrm{~B} & -1.342754 & -1.156234 & -1.853511\end{array}$

$\begin{array}{lllll}36 \mathrm{~B} & 2.421554 & -1.156205 & -1.853510\end{array}$

$\begin{array}{llll}37 \mathrm{~B} & 2.421527 & 2.183311 & -0.074558\end{array}$

$\begin{array}{llll}38 \mathrm{~B} & -1.342758 & -1.027094 & 1.928070\end{array}$

$\begin{array}{llll}39 B & 2.421552 & -1.027064 & 1.928072\end{array}$

$\begin{array}{llll}40 \mathrm{~B} & -1.342783 & 2.183282 & -0.074559\end{array}$

$E 41 / \varepsilon=-229.365627$

atom $\quad \mathrm{x} / \sigma \quad \mathrm{y} / \sigma$

$\begin{array}{cccc}1 \mathrm{~A} & 0.000000 & 0.000000 & 0.000000\end{array}$

$\begin{array}{llll}2 \mathrm{~A} & 1.069745 & 0.000000 & 0.000000\end{array}$

$\begin{array}{llll}3 A & 0.456888 & -0.967267 & 0.000000\end{array}$

$\begin{array}{lllll}4 A & -0.291798 & 0.862668 & -0.561259\end{array}$

$\begin{array}{lllll}5 A & -0.904655 & -0.104599 & -0.561259\end{array}$

$\begin{array}{lllll}6 \mathrm{~A} & -0.021966 & -0.625577 & -0.908239\end{array}$

$\begin{array}{lllll}7 \mathrm{~A} & 0.556269 & 0.287047 & -0.908239\end{array}$

$\begin{array}{llll}8 \mathrm{~A} & 0.535947 & 0.303946 & 0.894890\end{array}$

$\begin{array}{llll}9 \mathrm{~A} & -0.370600 & 0.878331 & 0.521191\end{array}$

$\begin{array}{llll}10 A & -0.952474 & -0.040035 & 0.521191\end{array}$ $\begin{array}{llll}11 \mathrm{~A} & -0.045927 & -0.614420 & 0.894890\end{array}$ 


$\begin{array}{lrrr}\text { 12B } & 0.699804 & -0.443392 & -2.379141 \\ \text { 13B } & -0.664869 & 2.382257 & -0.049069 \\ \text { 14B } & 0.355072 & -2.185969 & 1.102301 \\ \text { 15B } & 2.128216 & 0.612568 & 1.102302 \\ \text { 16B } & -2.438014 & -0.416280 & -0.049070 \\ \text { 17B } & -1.322112 & -1.121538 & -1.782907 \\ \text { 18B } & 0.391630 & -2.207360 & -1.076464 \\ \text { 19B } & 2.163171 & 0.588648 & -1.076463 \\ \text { 20B } & 0.449429 & 1.674470 & -1.782906 \\ \text { 21A } & 0.618736 & 0.986454 & 0.015229 \\ \text { 22A } & -0.627694 & -0.980776 & 0.015229 \\ \text { 23A } & -1.295363 & 0.820738 & -0.175580 \\ \text { 24A } & 1.107326 & -0.701597 & 0.814861 \\ \text { 25A } & 1.126396 & -0.713679 & -0.805740 \\ \text { 26A } & -0.460035 & 0.291478 & -1.459702 \\ \text { 27B } & -1.802093 & 1.141801 & 1.347204 \\ \text { 28B } & 0.705509 & -0.447008 & 2.380893 \\ \text { 29B } & -1.375328 & -1.028324 & 1.755814 \\ \text { 30B } & 0.342415 & 1.682774 & 1.755814 \\ \text { 31A } & -0.421618 & 0.267136 & 1.433390 \\ \text { 32A } & 1.380550 & -1.508917 & 0.019256 \\ \text { 33A } & -1.030056 & 1.286847 & -1.210838 \\ \text { 34A } & -1.603508 & 0.381772 & -1.210838 \\ \text { 35A } & 1.954001 & -0.603842 & 0.019256 \\ \text { 36B } & -2.585314 & 1.638047 & -0.818243 \\ \text { 37B } & 2.455471 & -1.555778 & 1.259679 \\ \text { 38B } & 2.484803 & -1.574361 & -1.197167 \\ \text { 39B } & -1.319281 & 0.835893 & -2.765293 \\ \text { 40B } & -1.510482 & -2.380022 & 0.006083 \\ \text { 41B } & 1.506905 & 2.382291 & 0.006084\end{array}$

$E 42 / \varepsilon=-236.995833$

$\begin{array}{cccc}\text { atom } & \mathrm{x} / \sigma & y / \sigma & z / \sigma \\ 1 \mathrm{~A} & 0.000000 & 0.000000 & 0.000000\end{array}$

$\begin{array}{llll}2 \mathrm{~A} & 1.102676 & 0.000000 & 0.000000\end{array}$

3A $\quad 0.551337 \quad-0.901954 \quad 0.000000$

$\begin{array}{lllll}4 \mathrm{~A} & 0.551338 & -0.227921 & -0.872682\end{array}$

$\begin{array}{lllll}5 B & 2.796708 & -0.069194 & -0.053439\end{array}$

$\begin{array}{lllll}6 \mathrm{~B} & -1.694033 & -0.069192 & -0.053444\end{array}$

$\begin{array}{llll}7 \mathrm{~B} & 0.551340 & 0.876783 & -2.159934\end{array}$

$\begin{array}{llll}8 \mathrm{~B} & 0.551334 & -1.868275 & 1.394136\end{array}$

$\begin{array}{lllll}9 \mathrm{~A} & 0.551336 & -0.309888 & 0.916055\end{array}$

$\begin{array}{llll}10 A & -0.363073 & -0.814002 & -0.628712\end{array}$

$\begin{array}{lllll}11 \mathrm{~A} & 1.465748 & -0.814004 & -0.628711\end{array}$

$\begin{array}{lllll}12 \mathrm{~A} & 0.551338 & 0.808018 & -0.531313\end{array}$

$\begin{array}{llll}13 \mathrm{~A} & 0.551337 & -1.312688 & -1.013881\end{array}$

$\begin{array}{llll}14 \mathrm{~A} & 0.551337 & 0.747750 & 0.577541\end{array}$

$\begin{array}{llll}15 B & -0.717849 & -2.106221 & -1.626785\end{array}$

$\begin{array}{llll}16 \mathrm{~B} & 0.551338 & 2.317101 & 0.185988\end{array}$

$\begin{array}{llll}17 \mathrm{~B} & 0.551334 & 0.765472 & 2.194903\end{array}$

$\begin{array}{llll}18 \mathrm{~B} & 1.820522 & -2.106224 & -1.626783\end{array}$

$\begin{array}{llll}19 \mathrm{~A} & -0.391601 & -0.858858 & 0.528089\end{array}$

$\begin{array}{llll}20 A & -0.391599 & 0.293922 & -0.964431\end{array}$

$\begin{array}{llll}21 \mathrm{~A} & 1.494273 & -0.858859 & 0.528092\end{array}$

$\begin{array}{lllll}22 \mathrm{~A} & 1.494276 & 0.293920 & -0.964430\end{array}$

$\begin{array}{llll}23 \mathrm{~A} & -0.018825 & -0.618931 & -1.668935\end{array}$

$24 \mathrm{~A} \quad-0.018828 \quad-1.771171 \quad-0.177111$

$\begin{array}{llll}25 \mathrm{~A} & 1.493832 & 0.281589 & 0.937922\end{array}$

$\begin{array}{llll}26 \mathrm{~A} & -0.391160 & 0.281589 & 0.937920\end{array}$

$\begin{array}{llll}27 \mathrm{~A} & -0.391159 & 0.978638 & 0.035442\end{array}$

$\begin{array}{lllll}28 A & 1.121499 & -1.771173 & -0.177110\end{array}$

$\begin{array}{llll}29 \mathrm{~A} & 1.121502 & -0.618932 & -1.668934\end{array}$

$\begin{array}{llll}30 \mathrm{~A} & 1.493834 & 0.978638 & 0.035444\end{array}$

$\begin{array}{llll}31 \mathrm{~B} & 2.534803 & -0.109965 & -2.225230\end{array}$

$\begin{array}{llll}32 \mathrm{~B} & 2.245213 & -0.631988 & 2.018078\end{array}$

$\begin{array}{llll}33 \mathrm{~B} & -1.142540 & 1.792880 & -1.121437\end{array}$

$\begin{array}{llll}34 \mathrm{~B} & 2.534798 & -2.180803 & 0.455916\end{array}$

$\begin{array}{llll}35 \mathrm{~B} & 2.245218 & 1.792878 & -1.121434\end{array}$

$\begin{array}{llll}36 \mathrm{~B} & -1.142545 & -0.631987 & 2.018074\end{array}$

$\begin{array}{llll}37 \mathrm{~B} & -1.432128 & -2.180802 & 0.455913\end{array}$

$\begin{array}{llll}38 \mathrm{~B} & -1.432124 & -0.109961 & -2.225235\end{array}$

$\begin{array}{llll}39 B & -1.256166 & 1.629185 & 1.258333\end{array}$

$\begin{array}{llll}40 \mathrm{~B} & 0.551334 & -3.298994 & -0.264181\end{array}$ $\begin{array}{|llll|}41 \mathrm{~B} & 0.551338 & -1.089252 & -3.125170\end{array}$ $\begin{array}{llll}\text { 42B } & 2.358838 & 1.629183 & 1.258338\end{array}$

$E 43 / \varepsilon=-243.686941$

$\begin{array}{cccc}\text { atom } & \mathrm{x} / \sigma & y / \sigma & z / \sigma \\ 1 \mathrm{~A} & 0.000000 & 0.000000 & 0.000000\end{array}$

$\begin{array}{llll}2 \mathrm{~A} & 1.127820 & 0.000000 & 0.000000\end{array}$

$\begin{array}{llll}3 \mathrm{~A} & 0.582761 & -0.917202 & 0.000000\end{array}$

$\begin{array}{llll}4 \mathrm{~A} & 0.582758 & 0.795463 & 0.456615\end{array}$

$\begin{array}{lllll}5 \mathrm{~A} & 0.728721 & 0.791130 & -0.649121\end{array}$

$\begin{array}{llll}6 \mathrm{~A} & 0.728724 & -0.362970 & -0.956817\end{array}$

$\begin{array}{llll}7 \mathrm{~B} & 2.914107 & -0.005342 & 0.020053\end{array}$

$\begin{array}{llll}8 \mathrm{~A} & 0.746328 & -0.260504 & 0.977095\end{array}$

$\begin{array}{lllll}9 \mathrm{~B} & 0.632868 & -2.065543 & -1.151945\end{array}$

$\begin{array}{llll}10 B & 0.632858 & 2.364865 & 0.029248\end{array}$

$\begin{array}{llll}\text { 11B } & 0.717648 & -2.111140 & 1.100024\end{array}$

$\begin{array}{llll}12 \mathrm{~B} & 0.717641 & 1.283302 & 2.005018\end{array}$

$\begin{array}{llll}13 \mathrm{~A} & -0.177054 & -0.780636 & 0.790633\end{array}$

$\begin{array}{llll}14 \mathrm{~A} & -0.177056 & 0.283415 & 1.074320\end{array}$

$\begin{array}{llll}15 A & -0.958988 & 0.477475 & 0.374600\end{array}$

$\begin{array}{llll}16 A & -0.958985 & -0.600596 & 0.087175\end{array}$

$\begin{array}{llll}17 A & -0.272106 & -0.711311 & -0.762236\end{array}$

$\begin{array}{llll}18 \mathrm{~A} & -0.272111 & 0.996364 & -0.306953\end{array}$

$\begin{array}{llll}19 \mathrm{~B} & -0.134316 & -0.544523 & -2.467529\end{array}$

$\begin{array}{llll}20 \mathrm{~B} & -0.134321 & 1.700666 & -1.868939\end{array}$

$\begin{array}{llll}21 B & -0.162109 & -0.646837 & 2.426142\end{array}$

$\begin{array}{llll}22 \mathrm{~A} & -0.089847 & 0.299525 & -1.123461\end{array}$

$\begin{array}{llll}23 \mathrm{~B} & -2.493036 & -0.058827 & 0.220619\end{array}$

$\begin{array}{lllll}24 \mathrm{~A} & -1.024768 & 0.173293 & -0.649999\end{array}$

$\begin{array}{llll}25 B & -1.592114 & -1.538006 & -1.120984\end{array}$

$\begin{array}{llll}26 \mathrm{~B} & -1.592122 & 1.891924 & -0.206528\end{array}$

$\begin{array}{llll}27 \mathrm{~A} & 1.590397 & 1.001395 & -0.047346\end{array}$

$\begin{array}{lllll}28 A & 1.590401 & -0.844910 & -0.539591\end{array}$

$\begin{array}{llll}29 B & 2.062884 & -0.696568 & -2.098585\end{array}$

$\begin{array}{lllll}30 \mathrm{~B} & 2.062879 & 1.648867 & -1.473266\end{array}$

$\begin{array}{lllll}31 \mathrm{~A} & 1.637993 & 0.237943 & -0.892468\end{array}$

$\begin{array}{llll}\text { 32B } & -1.487166 & 1.077488 & 1.871107\end{array}$

$\begin{array}{llll}33 \mathrm{~B} & -1.487159 & -1.865987 & 1.086346\end{array}$

$\begin{array}{llll}34 \mathrm{~A} & 1.571668 & 0.453519 & 0.882706\end{array}$

$\begin{array}{lllll}35 \mathrm{~A} & 1.571671 & -0.832764 & 0.539770\end{array}$

$\begin{array}{llll}36 \mathrm{~B} & 2.025957 & -0.549210 & 2.059978\end{array}$

$\begin{array}{llll}37 \mathrm{~A} & -0.351594 & 1.311862 & 0.741556\end{array}$

$\begin{array}{llll}38 A & -0.351589 & -1.506918 & -0.009961\end{array}$

$\begin{array}{llll}39 A & -1.110279 & -0.300615 & 1.127527\end{array}$

$\begin{array}{lllll}40 A & 0.901463 & 0.447719 & -1.679297\end{array}$

$\begin{array}{llll}41 \mathrm{~B} & -1.980036 & 0.505422 & -1.895758\end{array}$

$\begin{array}{llll}42 \mathrm{~B} & 2.532845 & 1.864618 & 0.978621\end{array}$

$\begin{array}{lllll}43 \mathrm{~B} & 2.532854 & -2.104317 & -0.079538\end{array}$

\section{$E 44 / \varepsilon=-250.867906$}

$\begin{array}{lll}\text { atom } & \mathrm{X} / \sigma & \mathrm{y} / \sigma\end{array}$

$\begin{array}{llll}1 \mathrm{~A} & 0.000000 & 0.000000 & 0.000000\end{array}$

$\begin{array}{llll}2 A & 1.083493 & 0.000000 & 0.000000\end{array}$

$\begin{array}{llll}3 A & 0.550094 & -0.928531 & 0.000000\end{array}$

$\begin{array}{llll}4 \mathrm{~B} & 0.564128 & -2.173185 & 0.956467\end{array}$

$\begin{array}{lllll}5 A & 0.533304 & -0.324376 & 0.954530\end{array}$

$\begin{array}{lllll}6 \mathrm{~A} & 0.538165 & 0.745380 & 0.572734\end{array}$

$\begin{array}{lllll}7 A & 0.539360 & 0.307564 & -0.893286\end{array}$

$\begin{array}{llll}8 \mathrm{~A} & -0.008778 & -0.642322 & -0.890478\end{array}$

$\begin{array}{llll}9 \mathrm{~B} & 0.453710 & 1.094603 & 2.134389\end{array}$

$\begin{array}{llll}10 \mathrm{~A} & -0.017143 & 1.051029 & -0.302067\end{array}$

$\begin{array}{llll}11 \mathrm{~A} & -0.927618 & 0.552456 & 0.100917\end{array}$

$\begin{array}{llll}12 \mathrm{~A} & -0.921334 & -0.504809 & -0.264410\end{array}$

$\begin{array}{llll}13 \mathrm{~A} & -0.373290 & 0.255662 & 0.988895\end{array}$

$\begin{array}{llll}14 A & -0.361132 & -0.825272 & 0.613159\end{array}$

$\begin{array}{llll}15 A & 1.098957 & -0.637816 & -0.884750\end{array}$

$\begin{array}{llll}16 \mathrm{~A} & 1.451744 & -0.793522 & 0.643548\end{array}$

$\begin{array}{lllll}17 A & 2.037486 & -0.469528 & -0.212393\end{array}$

$\begin{array}{lllll}18 \mathrm{~B} & 0.532762 & -0.294184 & -2.389974\end{array}$

$\begin{array}{llll}19 \mathrm{~A} & -0.567314 & 0.314306 & -0.920320\end{array}$

$\begin{array}{llll}20 \mathrm{~B} & 0.766309 & 2.411357 & 0.419869\end{array}$

$\begin{array}{llll}21 B & 0.468411 & 1.737362 & -1.683930\end{array}$

$\begin{array}{llll}22 \mathrm{~A} & 1.102737 & 1.035933 & -0.318746\end{array}$ $\begin{array}{llll}23 B & -0.607362 & -0.799764 & 2.204258\end{array}$

$\begin{array}{llll}24 \mathrm{~B} & 0.614846 & -2.203254 & -1.308142\end{array}$

$\begin{array}{llll}25 \mathrm{~A} & 2.001811 & 0.568649 & 0.097725\end{array}$

$\begin{array}{llll}26 \mathrm{~A} & 1.416310 & 0.298115 & 0.992745\end{array}$

$\begin{array}{llll}27 \mathrm{~B} & 1.639791 & -0.718214 & 2.241602\end{array}$

$\begin{array}{lllll}28 \mathrm{~A} & 1.656671 & 0.301581 & -0.933620\end{array}$

29B $-1.325338 \quad-1.290450 \quad-1.623780$

$\begin{array}{llll}30 \mathrm{~B} & -1.308047 & 2.049348 & -0.428210\end{array}$

$\begin{array}{lllll}31 \mathrm{~B} & -2.424563 & 0.136506 & -0.377247\end{array}$

$\begin{array}{llll}32 \mathrm{~B} & 2.474213 & -1.052029 & -1.636235\end{array}$

$\begin{array}{llll}33 \mathrm{~B} & -1.705088 & -1.820330 & 0.534170\end{array}$

$\begin{array}{llll}34 \mathrm{~B} & -1.731220 & 1.055473 & 1.551969\end{array}$

$\begin{array}{llll}35 \mathrm{~B} & 2.531158 & 1.391478 & 1.379462\end{array}$

$\begin{array}{llll}36 \mathrm{~B} & 2.793310 & -1.772489 & 0.620920\end{array}$

$\begin{array}{llll}37 \mathrm{~A} & -0.363238 & -1.465194 & -0.268230\end{array}$

$\begin{array}{llll}38 \mathrm{~A} & -1.292880 & -0.272329 & 0.745836\end{array}$

$\begin{array}{llll}39 A & -0.385327 & 1.309074 & 0.701999\end{array}$

$\begin{array}{lllll}40 \mathrm{~B} & 2.459991 & 1.786447 & -0.882843\end{array}$

$\begin{array}{lllll}41 \mathrm{~A} & 2.360740 & -0.197889 & 0.824796\end{array}$

$\begin{array}{lllll}42 \mathrm{~A} & 1.500249 & -1.421124 & -0.246156\end{array}$

$\begin{array}{llll}43 \mathrm{~B} & 3.600902 & 0.115761 & -0.166513\end{array}$

$\begin{array}{llll}44 \mathrm{~B} & -1.413862 & 0.792943 & -2.235091\end{array}$

$E 45 / \varepsilon=-258.409108$

$\begin{array}{llll}\text { atom } & \mathrm{x} / \sigma & \mathrm{y} / \sigma & \mathrm{z} / \sigma\end{array}$

$\begin{array}{llll}1 \mathrm{~A} & 0.000000 & 0.000000 & 0.000000\end{array}$

$\begin{array}{llll}2 \mathrm{~A} & 1.102108 & 0.000000 & 0.000000\end{array}$

$\begin{array}{lllll}3 A & 0.541497 & -0.927578 & 0.000000\end{array}$

$\begin{array}{llll}4 \mathrm{~A} & 0.541498 & 0.729562 & 0.572835\end{array}$

$\begin{array}{llll}5 \mathrm{~B} & 0.498047 & -2.184455 & 0.959759\end{array}$

$\begin{array}{llll}6 \mathrm{~B} & 0.498049 & 1.125416 & 2.103906\end{array}$

$\begin{array}{llll}7 \mathrm{~B} & 2.087498 & 0.518419 & -1.499720\end{array}$

$\begin{array}{lllll}8 \mathrm{~A} & 0.520580 & -0.331194 & 0.958102\end{array}$

$\begin{array}{llll}9 \mathrm{~A} & 0.554271 & 0.307230 & -0.888777\end{array}$

$10 A \quad-0.017202 \quad 1.051562 \quad-0.295300$

$\begin{array}{llll}11 \mathrm{~A} & -0.017203 & -0.644712 & -0.881663\end{array}$

$\begin{array}{llll}12 \mathrm{~A} & -0.934861 & 0.547314 & 0.099947\end{array}$

$\begin{array}{lllll}13 A & -0.934862 & -0.492197 & -0.259388\end{array}$

$\begin{array}{llll}14 \mathrm{~A} & -0.376319 & 0.257998 & 0.991867\end{array}$

$\begin{array}{llll}15 A & -0.376319 & -0.815458 & 0.620797\end{array}$

$\begin{array}{llll}16 \mathrm{~A} & 1.423066 & -0.823551 & 0.651312\end{array}$

$\begin{array}{llll}17 \mathrm{~A} & 1.423067 & 0.245516 & 1.020864\end{array}$

$\begin{array}{llll}18 \mathrm{~A} & 1.117857 & 1.069217 & -0.292176\end{array}$

$\begin{array}{llll}19 \mathrm{~A} & 1.117856 & -0.660528 & -0.890110\end{array}$

$\begin{array}{llll}20 A & 2.037499 & -0.521510 & -0.207925\end{array}$

$\begin{array}{llll}21 \mathrm{~A} & 2.037500 & 0.538584 & 0.158526\end{array}$

$\begin{array}{llll}22 A & -0.559191 & 0.319410 & -0.924011\end{array}$

$\begin{array}{llll}23 \mathrm{~B} & 1.557898 & -0.784890 & 2.270583\end{array}$

$\begin{array}{llll}24 \mathrm{~B} & 0.369029 & 1.755607 & -1.681055\end{array}$

$\begin{array}{lllll}25 B & 0.369030 & -0.342674 & -2.406382\end{array}$

$\begin{array}{llll}26 \mathrm{~B} & -0.630151 & -0.767463 & 2.220175\end{array}$

$\begin{array}{llll}27 \mathrm{~B} & 0.549778 & 2.541951 & 0.355890\end{array}$

$\begin{array}{llll}28 \mathrm{~B} & 0.549774 & -2.219089 & -1.289892\end{array}$

$\begin{array}{lllll}29 B & -1.399826 & -1.323706 & -1.565733\end{array}$

$\begin{array}{lllll}30 \mathrm{~B} & -1.399824 & 2.008062 & -0.414017\end{array}$

$\begin{array}{lllll}31 \mathrm{~B} & -2.471305 & 0.106692 & -0.308642\end{array}$

$\begin{array}{llll}32 \mathrm{~B} & -1.718021 & -1.816808 & 0.582325\end{array}$

$\begin{array}{llll}33 \mathrm{~B} & -1.718019 & 1.069344 & 1.580001\end{array}$

$\begin{array}{llll}34 \mathrm{~B} & 2.718494 & 1.007735 & 1.735919\end{array}$

$\begin{array}{llll}35 \mathrm{~B} & 2.718492 & -1.864646 & 0.742999\end{array}$

$\begin{array}{lllll}36 \mathrm{~B} & 3.626353 & 0.027361 & -0.079159\end{array}$

$\begin{array}{llll}37 \mathrm{~A} & 1.468656 & 1.286056 & 0.733981\end{array}$

$\begin{array}{lllll}38 \mathrm{~A} & 1.468654 & -1.464792 & -0.216926\end{array}$

$\begin{array}{llll}39 A & -0.391691 & 1.298956 & 0.698937\end{array}$

$\begin{array}{llll}40 A & -0.391692 & -1.453295 & -0.252453\end{array}$

$\begin{array}{llll}41 \mathrm{~A} & -1.301836 & -0.262780 & 0.760191\end{array}$

$\begin{array}{llll}42 \mathrm{~A} & 2.343803 & -0.289873 & 0.838560\end{array}$

$\begin{array}{lllll}43 B & 2.592175 & -1.532370 & -1.412072\end{array}$

$\begin{array}{llll}44 \mathrm{~B} & 2.592180 & 2.077282 & -0.164293\end{array}$

$\begin{array}{llll}45 B & -1.518171 & 0.757392 & -2.191037\end{array}$

$E 46 / \varepsilon=-266.121423$

atom $\mathrm{x} / \sigma \quad \mathrm{y} / \sigma$

$z / \sigma$ 
$\begin{array}{llll}1 \mathrm{~A} & 0.000000 & 0.000000 & 0.000000\end{array}$

$\begin{array}{llll}2 \mathrm{~A} & 1.087002 & 0.000000 & 0.000000\end{array}$

3A $\quad 0.543501-0.928280 \quad 0.000000$

$\begin{array}{llll}4 \mathrm{~A} & 0.543501 & 0.735244 & 0.566675\end{array}$

$\begin{array}{llll}5 B & 0.543502 & 1.132937 & 2.091636\end{array}$

$\begin{array}{lllll}6 \mathrm{~B} & 0.543503 & -2.174196 & 0.965070\end{array}$

$\begin{array}{llll}7 A & 0.543502 & -0.324798 & 0.953474\end{array}$

$\begin{array}{llll}8 A & 0.543500 & 0.303459 & -0.890832\end{array}$

$\begin{array}{llll}9 B & 0.543500 & -0.340175 & -2.382418\end{array}$

$\begin{array}{llll}10 B & 0.543499 & 1.723798 & -1.679331\end{array}$

$\begin{array}{lllll}11 \mathrm{~A} & 1.101110 & -0.646711 & -0.887378\end{array}$

$\begin{array}{llll}12 \mathrm{~A} & -0.014109 & 1.053933 & -0.308057\end{array}$

$\begin{array}{llll}13 \mathrm{~A} & 1.101110 & 1.053933 & -0.308058\end{array}$

$\begin{array}{llll}14 \mathrm{~A} & -0.014109 & -0.646712 & -0.887377\end{array}$

$\begin{array}{llll}15 A & -0.925855 & 0.550857 & 0.105765\end{array}$

$\begin{array}{llll}16 \mathrm{~A} & 2.012856 & 0.550858 & 0.105763\end{array}$

$\begin{array}{llll}17 A & -0.925854 & -0.500871 & -0.252504\end{array}$

$\begin{array}{llll}18 \mathrm{~A} & 2.012857 & -0.500870 & -0.252506\end{array}$

$\begin{array}{llll}19 \mathrm{~A} & -0.360753 & 0.263355 & 0.993949\end{array}$

$\begin{array}{llll}20 A & -0.360752 & -0.815353 & 0.626490\end{array}$

$\begin{array}{llll}21 \mathrm{~A} & 1.447756 & 0.263356 & 0.993948\end{array}$

$\begin{array}{llll}22 \mathrm{~A} & 1.447756 & -0.815353 & 0.626489\end{array}$

$\begin{array}{lllll}23 \mathrm{~A} & -0.566828 & 0.311857 & -0.915486\end{array}$

$\begin{array}{llll}24 \mathrm{~A} & 1.653829 & 0.311858 & -0.915487\end{array}$

$\begin{array}{llll}25 \mathrm{~B} & -0.573403 & -0.757914 & 2.224921\end{array}$

$\begin{array}{llll}26 \mathrm{~B} & 1.660409 & -0.757913 & 2.224920\end{array}$

$\begin{array}{llll}27 \mathrm{~B} & 0.543501 & -2.254567 & -1.279307\end{array}$

$\begin{array}{llll}28 \mathrm{~B} & 0.543500 & 2.566690 & 0.363041\end{array}$

$\begin{array}{llll}29 B & -1.347268 & -1.291447 & -1.605249\end{array}$

$\begin{array}{llll}30 B & 2.434270 & -1.291445 & -1.605251\end{array}$

$\begin{array}{llll}31 B & 2.434268 & 2.002827 & -0.483066\end{array}$

$\begin{array}{llll}32 \mathrm{~B} & -1.347269 & 2.002824 & -0.483064\end{array}$

$\begin{array}{lllll}33 \mathrm{~B} & 3.518331 & 0.124140 & -0.364418\end{array}$

$\begin{array}{lllll}34 \mathrm{~B} & -2.431330 & 0.124136 & -0.364414\end{array}$

$\begin{array}{llll}35 \mathrm{~B} & 2.793254 & 1.081812 & 1.558589\end{array}$

$\begin{array}{llll}36 \mathrm{~B} & -1.706251 & 1.081809 & 1.558593\end{array}$

$\begin{array}{llll}37 \mathrm{~B} & 2.793256 & -1.808298 & 0.574080\end{array}$

$\begin{array}{llll}38 \mathrm{~B} & -1.706250 & -1.808300 & 0.574084\end{array}$

$\begin{array}{llll}39 \mathrm{~A} & 1.466151 & -1.456600 & -0.250950\end{array}$

$\begin{array}{llll}40 \mathrm{~A} & 1.466150 & 1.306894 & 0.690426\end{array}$

$\begin{array}{llll}41 \mathrm{~A} & -0.379149 & 1.306893 & 0.690428\end{array}$

$\begin{array}{llll}42 \mathrm{~A} & -0.379148 & -1.456601 & -0.250949\end{array}$

$\begin{array}{llll}43 \mathrm{~A} & 2.375935 & -0.259821 & 0.762730\end{array}$

$\begin{array}{llll}44 \mathrm{~A} & -1.288932 & -0.259823 & 0.762732\end{array}$

$\begin{array}{llll}45 \mathrm{~B} & 2.448511 & 0.771582 & -2.265043\end{array}$

$\begin{array}{llll}46 \mathrm{~B} & -1.361513 & 0.771579 & -2.265040\end{array}$

\section{$E 47 / \varepsilon=-272.215887$}$$
\text { atom } \quad \mathrm{x} / \sigma \quad \mathrm{s} / \sigma
$$

1A $\quad 0.000000 \quad 0.000000 \quad 0.000000$

$\begin{array}{llll}2 \mathrm{~A} & 1.089437 & 0.000000 & 0.000000\end{array}$

$\begin{array}{llll}3 A & 0.544719 & -0.940223 & 0.000000\end{array}$

$\begin{array}{llll}4 \mathrm{~A} & 0.544719 & 0.948652 & 0.036231\end{array}$

$\begin{array}{llll}5 \mathrm{~A} & 0.544718 & -0.306132 & 0.878324\end{array}$

$\begin{array}{llll}6 \mathrm{~B} & 0.544718 & 0.152533 & 2.378892\end{array}$

$\begin{array}{llll}7 A & -0.571593 & -0.923113 & 0.011584\end{array}$

$\begin{array}{llll}8 \mathrm{~A} & 1.661030 & -0.923113 & 0.011585\end{array}$

$\begin{array}{lllll}9 A & -0.010164 & -0.617578 & -0.905754\end{array}$

$\begin{array}{llll}10 \mathrm{~A} & 1.099602 & -0.617579 & -0.905754\end{array}$

$\begin{array}{llll}11 \mathrm{~A} & 0.544719 & 0.321367 & -0.903647\end{array}$

$\begin{array}{lllll}12 \mathrm{~B} & 0.544719 & 2.129703 & -1.014916\end{array}$

13A $-0.005999 \quad 0.647990 \quad 0.906498$

$\begin{array}{lllll}14 \mathrm{~A} & 1.095435 & 0.647989 & 0.906498\end{array}$

$\begin{array}{llll}15 A & 1.648294 & -0.295147 & 0.929952\end{array}$

$\begin{array}{llll}16 \mathrm{~A} & -0.558858 & -0.295146 & 0.929952\end{array}$

$\begin{array}{llll}17 \mathrm{~B} & -0.540979 & 0.433543 & -2.137938\end{array}$

$\begin{array}{llll}18 \mathrm{~B} & 1.630419 & 0.433542 & -2.137937\end{array}$

$\begin{array}{llll}19 B & 2.140863 & -2.432915 & 0.467479\end{array}$

$\begin{array}{llll}20 \mathrm{~B} & -1.051428 & -2.432914 & 0.467478\end{array}$

$\begin{array}{llll}21 \mathrm{~A} & -0.013855 & -1.238620 & 0.915949\end{array}$

$\begin{array}{lllll}22 \mathrm{~A} & 1.103291 & -1.238620 & 0.915949\end{array}$

$\begin{array}{llll}23 \mathrm{~B} & 0.544720 & -1.484299 & -2.182517\end{array}$

$\begin{array}{llll}24 B & 3.247010 & -0.505109 & 0.390483\end{array}$ $\begin{array}{llll}26 \mathrm{~B} & 0.544718 & 2.148240 & 1.378607\end{array}$

$\begin{array}{llll}27 \mathrm{~A} & 1.099419 & -1.667936 & -0.590560\end{array}$

$\begin{array}{llll}28 A & -0.009982 & -1.667936 & -0.590560\end{array}$

$\begin{array}{llll}29 A & 0.544718 & -1.985423 & 0.318966\end{array}$

$\begin{array}{lllll}30 \mathrm{~A} & 1.433772 & 0.840845 & -0.593879\end{array}$

$\begin{array}{lllll}31 \mathrm{~A} & -0.344335 & 0.840845 & -0.593880\end{array}$

$\begin{array}{llll}32 \mathrm{~A} & -0.901866 & 0.505913 & 0.286420\end{array}$

$\begin{array}{llll}33 \mathrm{~A} & 1.991303 & 0.505913 & 0.286422\end{array}$

$\begin{array}{llll}34 \mathrm{~A} & 2.015190 & -0.083261 & -0.622753\end{array}$

$\begin{array}{llll}35 \mathrm{~A} & -0.925753 & -0.083261 & -0.622755\end{array}$

$\begin{array}{llll}36 \mathrm{~B} & 2.449561 & 1.082991 & 1.700644\end{array}$

$\begin{array}{llll}37 \mathrm{~B} & -1.360126 & 1.082991 & 1.700642\end{array}$

$\begin{array}{lllll}38 \mathrm{~B} & -1.429821 & -1.431597 & -1.402701\end{array}$

$\begin{array}{llll}39 \mathrm{~B} & 2.519260 & -1.431597 & -1.402699\end{array}$

$\begin{array}{llll}\text { 40B } & 0.544717 & -2.395756 & 1.946504\end{array}$

$\begin{array}{llll}41 B & -1.828425 & 1.458728 & -0.621929\end{array}$

$\begin{array}{llll}42 \mathrm{~B} & 2.917863 & 1.458728 & -0.621926\end{array}$

$\begin{array}{llll}43 \mathrm{~A} & -0.413336 & 1.481986 & 0.278757\end{array}$

$\begin{array}{llll}44 \mathrm{~A} & 1.502773 & 1.481985 & 0.278758\end{array}$

$\begin{array}{llll}45 B & 2.264711 & -1.108062 & 2.204562\end{array}$

$\begin{array}{llll}46 \mathrm{~B} & -1.175276 & -1.108061 & 2.204560\end{array}$

$\begin{array}{llll}47 \mathrm{~B} & 0.544718 & -3.199168 & -0.822695\end{array}$

$E 48 / \varepsilon=-279.832502$

$\begin{array}{cccc}\text { atom } & \mathrm{x} / \sigma & y / \sigma & z / \sigma \\ 1 \mathrm{~A} & 0.000000 & 0.000000 & 0.000000\end{array}$

$\begin{array}{llll}2 \mathrm{~A} & 1.081390 & 0.000000 & 0.000000\end{array}$

$\begin{array}{llll}3 \mathrm{~A} & 0.539429 & -0.935779 & 0.000000\end{array}$

$\begin{array}{lllll}4 \mathrm{~A} & 0.525852 & -0.320240 & 0.908849\end{array}$

$\begin{array}{llll}5 A & -0.557075 & -0.944491 & 0.016139\end{array}$

$\begin{array}{llll}6 \mathrm{~A} & 0.509365 & 0.962323 & 0.002111\end{array}$

$\begin{array}{llll}7 A & 1.627452 & -0.977292 & 0.002110\end{array}$

$\begin{array}{lllll}8 \mathrm{~A} & 1.084991 & -0.639609 & -0.907882\end{array}$

$\begin{array}{lllll}9 \mathrm{~A} & 0.529711 & 0.323669 & -0.907882\end{array}$

$\begin{array}{llll}10 \mathrm{~B} & -0.130899 & -0.698822 & 2.426961\end{array}$

$\begin{array}{llll}11 \mathrm{~A} & -0.002063 & -0.624555 & -0.913592\end{array}$

$\begin{array}{llll}12 \mathrm{~B} & -1.553981 & -1.519155 & -1.068914\end{array}$

$\begin{array}{llll}13 \mathrm{~A} & 1.637570 & -0.327264 & 0.885854\end{array}$

$\begin{array}{llll}14 \mathrm{~A} & 1.076936 & 0.645303 & 0.885855\end{array}$

$\begin{array}{llll}15 A & -0.033371 & -1.277774 & 0.892866\end{array}$

$\begin{array}{llll}16 \mathrm{~A} & -0.583015 & -0.324274 & 0.892866\end{array}$

$\begin{array}{llll}17 \mathrm{~A} & -0.021233 & 0.620513 & 0.918469\end{array}$

$\begin{array}{llll}18 \mathrm{~A} & 1.065746 & -1.265138 & 0.918469\end{array}$

$\begin{array}{llll}19 B & -0.626505 & 0.266901 & -2.154271\end{array}$

$\begin{array}{llll}20 B & 0.456403 & -1.611687 & -2.154271\end{array}$

$\begin{array}{llll}21 B & 1.575893 & 0.285056 & -2.171564\end{array}$

$\begin{array}{llll}22 \mathrm{~B} & 1.565034 & 2.146281 & 0.465468\end{array}$

$\begin{array}{llll}23 \mathrm{~B} & 3.181059 & -0.657140 & 0.465467\end{array}$

$\begin{array}{llll}24 \mathrm{~B} & 2.058729 & -2.532591 & 0.439620\end{array}$

$\begin{array}{llll}25 \mathrm{~B} & -0.620365 & 2.115000 & 0.439623\end{array}$

$\begin{array}{llll}26 \mathrm{~B} & -1.682794 & -1.593410 & 1.237894\end{array}$

$\begin{array}{llll}27 \mathrm{~A} & 1.994794 & -0.116054 & -0.589205\end{array}$

$\begin{array}{lllll}28 A & 1.438737 & 0.848574 & -0.589205\end{array}$

$\begin{array}{llll}29 \mathrm{~A} & 1.999714 & 0.529365 & 0.306334\end{array}$

$\begin{array}{llll}30 A & -0.002244 & -1.653190 & -0.612732\end{array}$

$\begin{array}{llll}31 \mathrm{~A} & -0.892280 & -0.109189 & -0.612732\end{array}$

$\begin{array}{llll}32 \mathrm{~A} & -0.887410 & 0.533123 & 0.275881\end{array}$

$\begin{array}{llll}33 \mathrm{~A} & 0.556020 & -1.970884 & 0.275881\end{array}$

$\begin{array}{llll}34 \mathrm{~A} & 1.084552 & -1.702096 & -0.627500\end{array}$

$\begin{array}{lllll}35 A & -0.389929 & 0.855778 & -0.627499\end{array}$

$\begin{array}{llll}36 \mathrm{~B} & -1.681247 & 0.617873 & 1.652720\end{array}$

$\begin{array}{llll}37 \mathrm{~B} & 0.231511 & -2.700303 & 1.652721\end{array}$

$\begin{array}{llll}38 \mathrm{~B} & 2.500670 & -1.473898 & -1.397382\end{array}$

$\begin{array}{llll}39 \mathrm{~B} & 0.517260 & 1.966847 & -1.397380\end{array}$

$\begin{array}{llll}40 B & 0.414746 & 1.423040 & 2.239123\end{array}$

$\begin{array}{llll}\text { 41B } & 1.978711 & -1.290071 & 2.239123\end{array}$

$\begin{array}{llll}42 \mathrm{~B} & 2.510977 & 0.824079 & 1.853819\end{array}$

$\begin{array}{llll}43 \mathrm{~B} & -2.150310 & 0.896420 & -0.652619\end{array}$

$\begin{array}{lllll}44 \mathrm{~B} & 0.237468 & -3.245807 & -0.652619\end{array}$

$\begin{array}{llll}45 \mathrm{~A} & -0.528676 & -2.049299 & 0.241392\end{array}$

$\begin{array}{llll}46 A & -1.498885 & -0.366217 & 0.241392\end{array}$

$\begin{array}{llll}47 \mathrm{~B} & 3.052472 & 1.136224 & -0.798181\end{array}$ \begin{tabular}{|llll}
$48 A$ & 1.114969 & 0.019354 & 1.770586
\end{tabular}

$E 49 / \varepsilon=-287.340181$

$\begin{array}{llll}\text { atom } \mathrm{x} / \sigma & \mathrm{y} / \sigma & \mathrm{z} \sigma\end{array}$

1A $\quad 0.000000 \quad 0.000000 \quad 0.000000$

$\begin{array}{llll}2 \mathrm{~A} & 1.094496 & 0.000000 & 0.000000\end{array}$

$\begin{array}{llll}3 \mathrm{~A} & 0.547248 & -0.947861 & 0.000000\end{array}$

$\begin{array}{lllll}4 \mathrm{~A} & 0.547246 & -0.315952 & -0.891618\end{array}$

$\begin{array}{lllll}5 A & 0.547249 & 0.319489 & 0.905933\end{array}$

$\begin{array}{llll}6 \mathrm{~A} & -0.003062 & -0.633678 & 0.905933\end{array}$

$\begin{array}{llll}7 \mathrm{~A} & 1.097561 & -0.633678 & 0.905931\end{array}$

$\begin{array}{lllll}8 \mathrm{~A} & -0.558477 & -0.954344 & -0.001648\end{array}$

$\begin{array}{lllll}9 \mathrm{~A} & 1.652973 & -0.954344 & -0.001652\end{array}$

$\begin{array}{lllll}10 \mathrm{~A} & 0.547248 & 0.960827 & -0.001647\end{array}$

$\begin{array}{llll}11 \mathrm{~A} & -0.009247 & -1.272308 & -0.899866\end{array}$

$\begin{array}{llll}12 \mathrm{~A} & 1.097227 & 0.644163 & -0.899865\end{array}$

$\begin{array}{llll}13 \mathrm{~A} & 1.653721 & -0.319711 & -0.899868\end{array}$

$\begin{array}{lllll}14 \mathrm{~A} & 1.103739 & -1.272308 & -0.899868\end{array}$

$\begin{array}{llll}15 A & -0.002736 & 0.644163 & -0.899864\end{array}$

$\begin{array}{llll}16 A & -0.559229 & -0.319712 & -0.899864\end{array}$

$\begin{array}{llll}17 \mathrm{~B} & 1.632493 & 0.310606 & 2.167687\end{array}$

$\begin{array}{llll}18 \mathrm{~B} & 0.547253 & -1.569086 & 2.167686\end{array}$

$\begin{array}{llll}19 B & -0.537988 & 0.310606 & 2.167691\end{array}$

$\begin{array}{lllll}20 \mathrm{~B} & 3.221812 & -0.617121 & -0.443436\end{array}$

$\begin{array}{llll}21 \mathrm{~B} & -0.529216 & 2.150873 & -0.443424\end{array}$

$\begin{array}{llll}22 B & 1.623710 & 2.150872 & -0.443428\end{array}$

$\begin{array}{lllll}23 \mathrm{~B} & -2.127318 & -0.617121 & -0.443424\end{array}$

$\begin{array}{lllll}24 \mathrm{~B} & 2.145350 & -2.481609 & -0.443438\end{array}$

$\begin{array}{lllll}25 \mathrm{~B} & -1.050855 & -2.481610 & -0.443430\end{array}$

$\begin{array}{llll}26 \mathrm{~A} & -0.367049 & 0.848997 & 0.597440\end{array}$

$\begin{array}{llll}27 \mathrm{~A} & -0.918778 & -0.106626 & 0.597440\end{array}$

$\begin{array}{llll}28 \mathrm{~A} & 2.013276 & -0.106626 & 0.597434\end{array}$

$\begin{array}{llll}29 \mathrm{~A} & 1.461547 & 0.848997 & 0.597437\end{array}$

$\begin{array}{llll}30 \mathrm{~A} & -0.004480 & -1.690236 & 0.597436\end{array}$

$\begin{array}{llll}31 \mathrm{~A} & 1.098979 & -1.690236 & 0.597433\end{array}$

$\begin{array}{lllll}32 \mathrm{~A} & 0.547247 & -2.006885 & -0.301535\end{array}$

$\begin{array}{lllll}33 A & 2.011637 & 0.529513 & -0.301534\end{array}$

$\begin{array}{lllll}34 \mathrm{~A} & -0.917143 & 0.529513 & -0.301528\end{array}$

$\begin{array}{llll}35 \mathrm{~B} & -1.010252 & -1.215170 & -2.238710\end{array}$

$\begin{array}{llll}36 \mathrm{~B} & 0.547242 & 1.482489 & -2.238709\end{array}$

$\begin{array}{llll}37 \mathrm{~B} & 2.104737 & -1.215169 & -2.238717\end{array}$

$\begin{array}{llll}38 \mathrm{~B} & 2.533448 & -1.462688 & 1.392550\end{array}$

$\begin{array}{llll}39 \mathrm{~B} & 0.547251 & 1.977506 & 1.392560\end{array}$

$\begin{array}{llll}40 \mathrm{~B} & -1.438946 & -1.462687 & 1.392559\end{array}$

$\begin{array}{lllll}41 \mathrm{~B} & -1.453838 & 0.839374 & -1.833115\end{array}$

$\begin{array}{llll}42 \mathrm{~B} & 2.548325 & 0.839374 & -1.833124\end{array}$

$\begin{array}{lllll}43 B & 0.547244 & -2.626601 & -1.833125\end{array}$

$\begin{array}{lllll}44 \mathrm{~A} & 1.098169 & 0.002126 & -1.791686\end{array}$

$\begin{array}{llll}45 \mathrm{~A} & -0.003682 & 0.002126 & -1.791684\end{array}$

$\begin{array}{lllll}46 \mathrm{~A} & 0.547244 & -0.952105 & -1.791687\end{array}$

$\begin{array}{llll}47 \mathrm{~B} & -1.990173 & 1.149027 & 0.800479\end{array}$

$\begin{array}{llll}48 \mathrm{~B} & 0.547251 & -3.245919 & 0.800464\end{array}$

$\begin{array}{llll}\text { 49B } & 3.084672 & 1.149027 & 0.800468\end{array}$

\section{$E 50 / \varepsilon=-296.237038$}

$$
\begin{array}{llll}
\text { atom } & \mathrm{X} / \sigma & \mathrm{y} / \sigma & \mathrm{z} / \sigma
\end{array}
$$

$\begin{array}{llll}1 \mathrm{~A} & 0.000000 & 0.000000 & 0.000000\end{array}$

$\begin{array}{llll}2 \mathrm{~A} & 1.094125 & 0.000000 & 0.000000\end{array}$

$\begin{array}{lllll}3 \mathrm{~A} & 0.547063 & -0.947540 & 0.000000\end{array}$

$\begin{array}{llll}4 \mathrm{~A} & 0.547062 & -0.315847 & 0.893349\end{array}$

$\begin{array}{llll}5 A & 1.097090 & 0.643744 & 0.899425\end{array}$

$\begin{array}{llll}6 \mathrm{~A} & -0.558981 & -0.319304 & 0.899425\end{array}$

$\begin{array}{llll}7 A & 1.097090 & -0.633405 & -0.906736\end{array}$

$\begin{array}{llll}8 \mathrm{~A} & -0.002965 & 0.643744 & 0.899425\end{array}$

$\begin{array}{llll}9 \mathrm{~A} & -0.002964 & -0.633404 & -0.906736\end{array}$

$\begin{array}{llll}10 \mathrm{~A} & 1.653106 & -0.319304 & 0.899425\end{array}$

$\begin{array}{llll}11 \mathrm{~A} & 0.547063 & 0.961302 & 0.001234\end{array}$

$\begin{array}{llll}12 \mathrm{~A} & 1.653106 & -0.954421 & 0.001234\end{array}$

$\begin{array}{llll}13 \mathrm{~A} & -0.558981 & -0.954421 & 0.001233\end{array}$

$\begin{array}{llll}14 \mathrm{~A} & 0.547063 & 0.319271 & -0.906736\end{array}$

$\begin{array}{llll}15 \mathrm{~A} & 1.103078 & -1.271980 & 0.899425\end{array}$

$\begin{array}{lllll}16 \mathrm{~A} & -0.008954 & -1.271979 & 0.899425\end{array}$

$\begin{array}{llll}17 \mathrm{~B} & 1.630882 & 0.309898 & -2.171235\end{array}$ 


\begin{tabular}{lrrr|rrrr|rrrr} 
18B & -2.130643 & -0.610335 & 0.431571 & 30A & 2.011917 & -0.106416 & -0.599655 & $42 \mathrm{~B}$ & 0.547062 & -2.608150 & 1.844240 \\
19B & -1.046826 & -1.236078 & 2.201438 & $31 \mathrm{~A}$ & 1.460861 & 0.848040 & -0.599654 & $43 \mathrm{~B}$ & 2.532256 & 0.830305 & 1.844241 \\
20B & -1.046825 & -2.487563 & 0.431571 & $32 \mathrm{~A}$ & -0.003994 & 0.002306 & 1.792442 & $44 \mathrm{~B}$ & -1.438131 & 0.830305 & 1.844240 \\
21B & 2.140949 & -1.236078 & 2.201439 & $33 \mathrm{~A}$ & 2.011917 & 0.529888 & 0.300216 & $45 \mathrm{~B}$ & 2.532256 & -1.461997 & -1.397567 \\
22B & 2.140950 & -2.487563 & 0.431571 & $34 \mathrm{~A}$ & -0.366735 & 0.848040 & -0.599655 & $46 \mathrm{~B}$ & -1.438130 & -1.461997 & -1.397568 \\
23B & 3.224768 & -0.610335 & 0.431573 & $35 \mathrm{~A}$ & -0.003993 & -1.689162 & -0.599656 & $47 \mathrm{~B}$ & 0.547062 & -0.315847 & 3.330210 \\
24B & -0.536754 & 0.309897 & -2.171236 & $36 \mathrm{~A}$ & 1.098118 & -1.689162 & -0.599655 & $48 \mathrm{~B}$ & 0.547062 & -3.245034 & -0.812290 \\
25B & 0.547063 & -1.567330 & -2.171236 & $37 \mathrm{~A}$ & 0.547062 & -2.007315 & 0.300215 & $49 \mathrm{~B}$ & -1.989688 & 1.148748 & -0.812288 \\
26B & 1.630880 & 2.150359 & 0.431573 & $38 \mathrm{~A}$ & 0.547062 & -0.952151 & 1.792442 & $50 \mathrm{~B}$ & 3.083814 & 1.148748 & -0.812286 \\
27B & 0.547062 & 1.524616 & 2.201440 & $39 \mathrm{~A}$ & 1.098118 & 0.002306 & 1.792443 \\
28B & -0.536755 & 2.150359 & 0.431572 & $40 \mathrm{~A}$ & -0.917792 & 0.529888 & 0.300215 & & & & \\
29A & -0.917791 & -0.106416 & -0.599655 & $41 \mathrm{~B}$ & 0.547063 & 1.976458 & -1.397566 & & & &
\end{tabular}




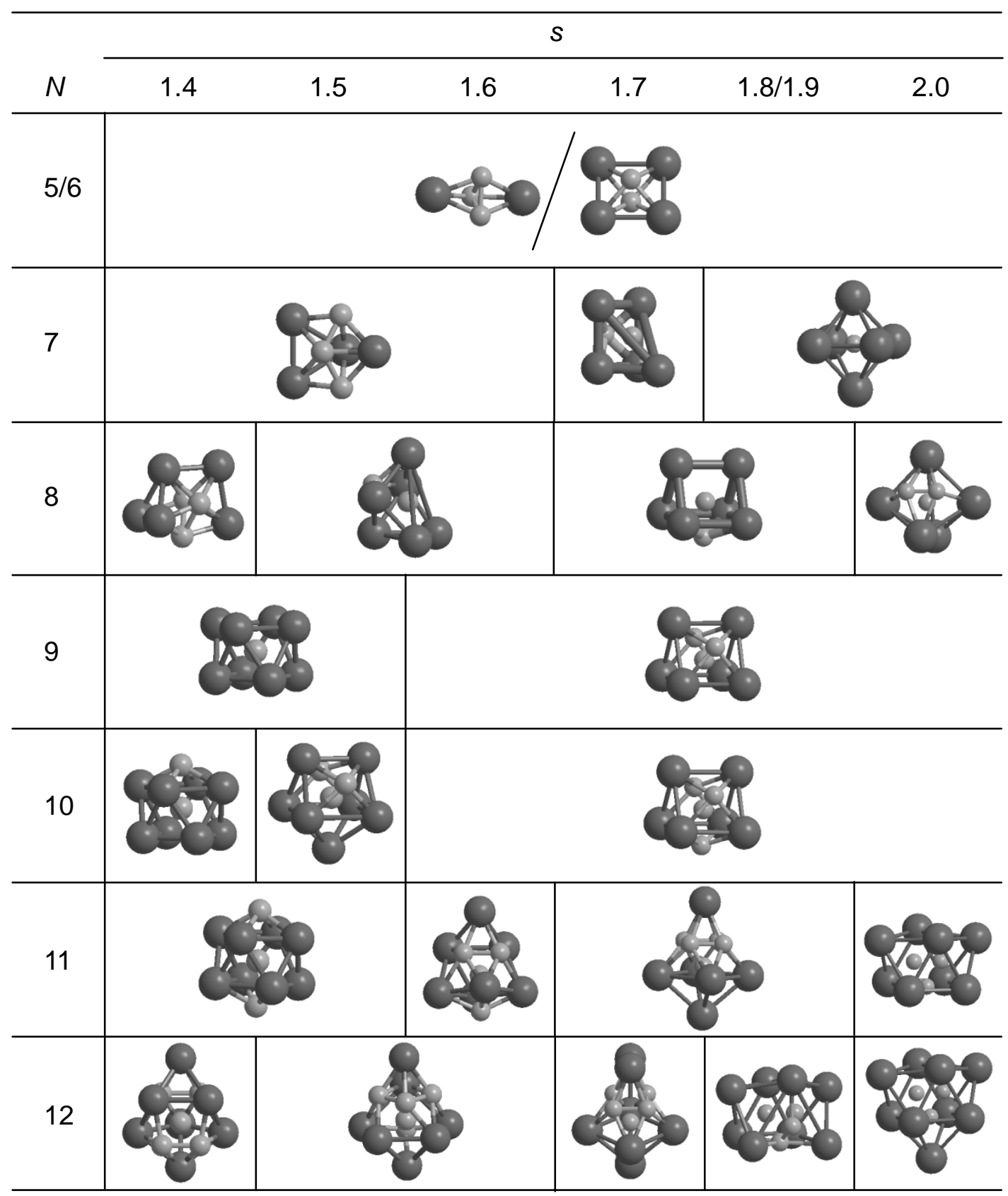

Figure S1 